\title{
Monatsschrift
}

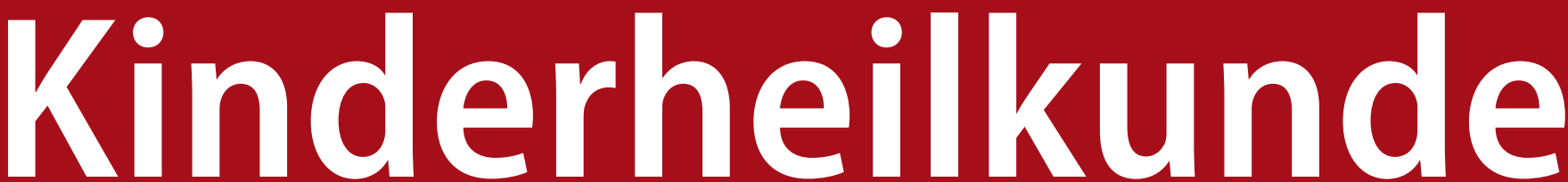

Organ der Deutschen Gesellschaft für Kinder- und Jugendmedizin

Organ der Österreichischen Gesellschaft für Kinder- und Jugendheilkunde

Abstracts der 40. Jahrestagung der Gesellschaft für Neonatologie und Pädiatrische Intensivmedizin (GNPI) 26.-28. Juni 2014, World Conference Center Bonn
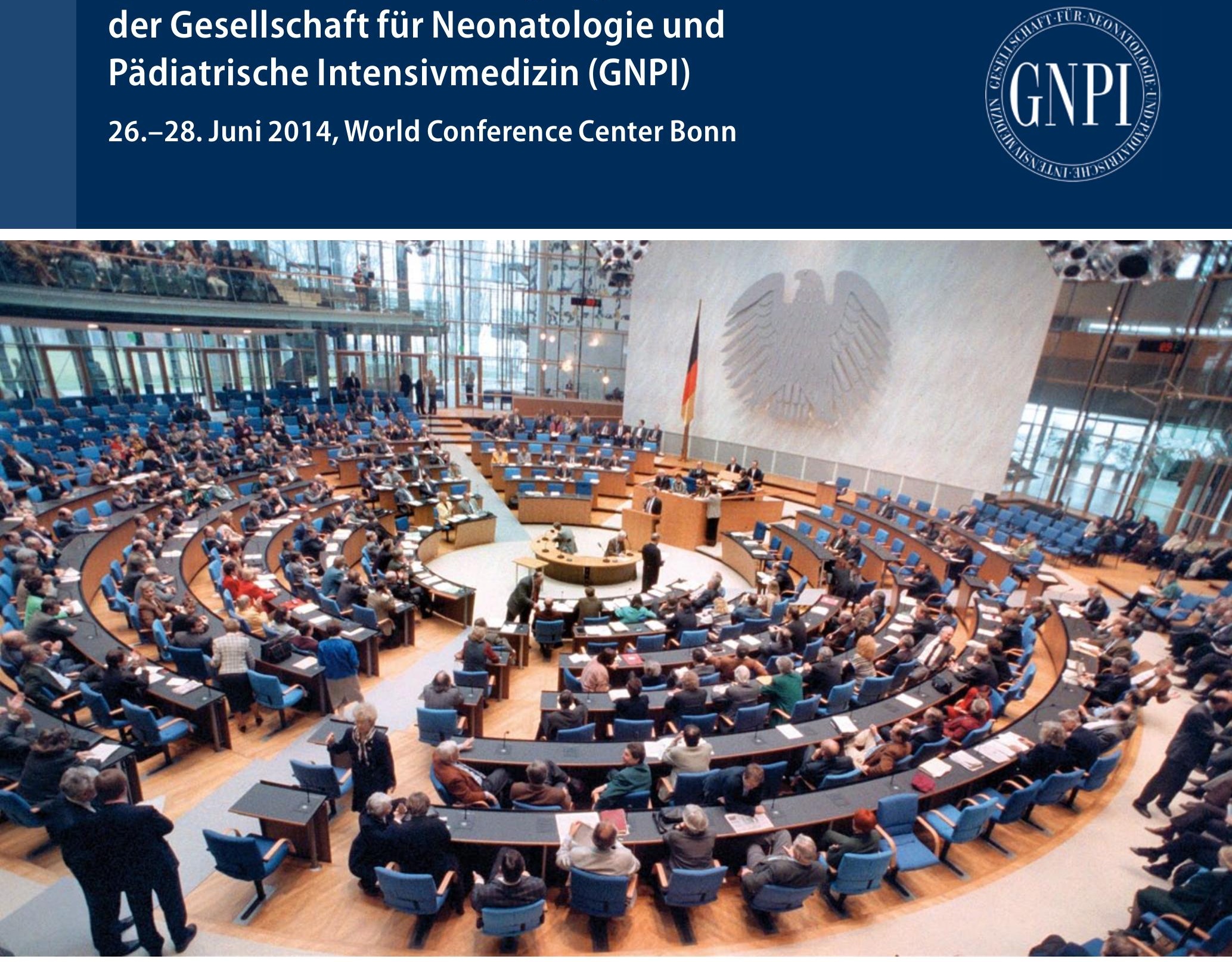

www.MonatsschriftKinderheilkunde.de www.springermedizin.de
Springer Medizin 
Monatsschr Kinderheilkd 2014 · 162:1-103

DOI 10.1007/s00112-014-3151-4

(c) Springer-Verlag Berlin Heidelberg 2014

Titelbild: @ Michael Jung / dpa

\section{Abstracts der 40. Jahrestagung der Gesellschaft für Neonatologie und Pädiatrische Intensivmedizin (GNPI)}

\author{
26. bis 28. Juni 2014, Bonn \\ Wissenschaftliche Leitung: \\ Prof. Dr. Dr. Peter Bartman, Universitätskinderklinik Bonn \\ Prof. Dr. Bernhard Roth, Universitätskinderklinik Köln
}

\section{Vorträge}

\section{Neuste Studienergebnisse}

\section{V01}

Erythropoietin for the repair of cerebral injury in very preterm infants (EpoRepair): eine randomisierte, doppelblinde und multizentrische Interventionsstudie

\section{S. Wellmann', C. Rüegger ', C. Hagmann², B. Koller', C. Bührer' , H. Bucher ${ }^{2}$ 'Universitäts-Kinderspital beider Basel (UKBB), Basel, Schweiz, ${ }^{2}$ Klinik für Neo- natologie, UniversitätsSpital Zürich, Zürich, Schweiz, ${ }^{3}$ Charité - Universitätsme- dizin Berlin, Klinik für Neonatologie, Berlin, Deutschland}

Hintergrund. Frühgeborene Kinder mit perinataler Hirnblutung haben ein signifikant erhöhtes Risiko für neurologische Entwicklungsdefizite. Außer der Drainage eines posthämorrhagischen Hydrocephalus gibt es keine spezifische Intervention zur Verbesserung der neurologischen Entwicklung. Das rekombinant hergestellte humane Hormon Erythropoietin (Epo) ist als Medikament zur Prophylaxe der Frühgeborenanämie zugelassen. Retrospektive Analysen von Epo-behandelten Frühgeborenen zeigen eine verbesserte neurologische Entwicklung insbesondere bei Kindern mit Hirnblutung (Neubauer AP et al., Annals Neurology, 2010). Die erste kürzlich abgeschlossene Epo-Hochdosisstudie bei Frühgeborenen in den ersten Lebenstagen (NCToo413946) ergab ein sehr gutes Sicherheitsprofil (Fauchère JC et al., ESPR Annual Meeting, 2012).

Fragestellung. Verbessert eine Hochdosistherapie mit Epo bei Frühgeborenen nach perinataler Hirnblutung die neurologische Entwicklung? Material und Methoden. Studiendesign: Doppelblind, 1:1 randomisiert, an 11 Perinatalzentren in Deutschland und der Schweiz, die zusammen 1200 Frühgeborene $<32$ Schwangerschaftswochen und/oder einem Geburtsgewicht von weniger als $1500 \mathrm{~g}$ jährlich betreuen. Patienten: 120 Frühgeborene mit sonographisch dokumentierter perinataler höhergradiger Hirnblutung $\left(>^{\circ} \mathrm{I}\right)$ in den ersten 4 Lebenstagen. Intervention: 5 intravenöse Gaben Epo (Recormon) zu je $2000 \mathrm{U} / \mathrm{kg}$ oder Placebo über 3 Wochen verteilt, erste Gabe am 5. Lebenstag. Darreichungsform der Studienmedikamente: Handelsware Recormon oder Placebo. Primärer Endpunkt: Neurologische Entwicklung mit 5 Jahren (Kaufmann-ABC oder SON-R). Sekundäre Endpunkte: 1) Ausmaß der Hirnschädigung mittels konventioneller Magnetresonanztomographie sowie quantitative Messung der Mikrostruktur der weißen Substanz und des Hirnvolumens mittels quantitativer Magnetresonanztomographie am errechneten Geburtstermin. 2) Sicherheitsanalysen zu Retinopathie und anderen unerwünschten Ereignissen. 3) Serielle Schädelultraschalldiagnostik und klinischer posthämorrhagischer Verlauf. 4) Neurologische Entwicklung mit 2 Jahren (BSID-III). Rekrutierungszeitraum: März 2014 bis Mitte 2016. Finanzierung: Schweizer Nationalfonds und weitere unabhängige Drittmittelgeber.

Ergebnisse und Diskussion. Das hier vorgestellte Studienprotokoll wurde bereits von der zuständigen Schweizer Arzneimittelbehörde gebilligt. Da die langfristige neurologische Entwicklung erst im Vorschulalter zuverlässig beurteilt werden kann, ist der primäre Endpunkt dieser Studie mit 5 Lebensjahren und kann entsprechend erst im Jahr 2021 rapportiert werden. Über die sekundären Endpunkte wird früher berichtet und insbesondere dank der rasch fortschreitenden Entwicklung der quantitativen Magnetresonanztomographie können bereits nach Abschluss der Rekrutierung im Jahr 2016 Aussagen über die Hirnschädigungen in beiden Studienarmen gemacht werden.

\section{V02}

Outcome von VLBW-Frühgeborenen nach vorzeitigem Blasensprung - eine Matched-pairs-Analyse des Deutschen Frühgeborenennetzwerks

\section{K. Hanke', C. Härtel', A. Hartz' ${ }^{2}$, E. Herting ${ }^{3}$, W. Göpel ${ }^{\prime}$}

'Universitätsklinikum Schleswig Holstein, Campus Lübeck, Klinik für Kinderund Jugendmedizin, Lübeck, Deutschland, ${ }^{2}$ Universitätsklinikum Schleswig Holstein, Kinderklinik, Lübeck, Deutschland, ${ }^{3}$ Universitätsklinikum SchleswigHolstein Campus Lübeck, Direktor der Klinik für Kinder- und Jugendmedizin, Lübeck, Deutschland

Fragestellung. In der Literatur wird beschrieben, dass der vorzeitige Blasensprung - als Surrogat einer Chorioamnionitis - bei Frühgeborenen mit einer erhöhten Inzidenz für pulmonale und infektiologische Morbidität einher geht. Wir haben Daten von Frühgeborenen mit einem Geburtsgewicht <1500 g („very-low-birth-weight“, VLBW) und einem Gestationsalter $<32$ Wochen in Form einer Matched-pairs-Analyse ausgewertet, um den Einfluss des vorzeitigen Blasensprungs multizentrisch zu untersuchen. 
Material und Methoden. Für die Auswertung unserer prospektiven Beobachtungsstudie des Zeitraums 2009-2012 wurden 3090 VLBW-Frühgeborene ausgewählt, die für einen Paarvergleich mit/ohne vorzeitigen Blasensprung nach „Matching“-Kriterien Gestationsalter pro Woche und Geburtsgewicht in 100-g-Schritten in Frage kamen. VLBW-Frühgeborene mit den Entbindungsgründen EPH-Gestose, HELLP-Syndrom und Plazentalösung wurden ausgeschlossen.

Ergebnisse. VLBW-Frühgeborene mit Blasensprung hatten in unserer Matched-Pairs-Analyse häufiger eine Exposition zu antenatalen (81,1 vs. 51,0\%; $\mathrm{p}<0,0001$ ) und postnatalen Antibiotika (95,5 vs. 90,2\%; $\mathrm{p}<0,0001$ ) im Vergleich zu Frühgeborenen ohne Blasensprung. Der vorzeitige Blasensprung hatte jedoch keinen Einfluss auf Outcome-Parameter einschließlich des Risikos für intrazerebrale Hämorrhagie, Tod, BPD und den kombinierten Endpunkt Tod und BPD sowie Pneumothorax oder Sepsis.

Diskussion. Unsere Analyse einer großen Kohorte zeigt, dass der vorzeitige Blasensprung bei Frühgeborenen nicht mit einer erhöhten Morbidität verbunden ist.

Schlussfolgerung. Unsere Daten bieten die Basis für eine Diskussion zum interdisziplinären Management des vorzeitigen Blasensprungs, da das Gestationsalter das bedeutendste Risiko für ein ungünstiges Outcome darstellt.

\section{Freie Vorträge}

\section{Intensivpflege}

\section{FV01}

Einarbeitungskonzept Neonatologie B2 Universitätskinderkliniken Bern

\section{Zimmermann', A. Frohloff ${ }^{2}$}

'Inselspital Bern, Stationsleitung Neonatologie B2, Bern, Schweiz, ${ }^{2}$ Inselspital Bern, Neonatolgie B2, Bern, Schweiz

Ausgangslage. Die Rekrutierung von Pflegefachpersonen gestaltete sich zunehmend schwieriger. Ein Grund dafür ist eine generalistische Pflegeausbildung in der Schweiz. Diese führte in den letzten Jahren zunehmend zum Verlust von spezifischem pädiatrischem und neonatologischem Fachwissen bei Berufseinsteigern in der Schweiz.

Vorgehen und Ziel. Eine Arbeitsgruppe mit Mitarbeitenden, welche an der Einarbeitung neuer Mitarbeiter beteiligt sind, erarbeitete das Einarbeitungskonzept Neonatologie B2. Ziel der Arbeitsgruppe war die Erstellung eines an die neuen Anforderungen angepasstes Einführungskonzept, welches an die Bedürfnisse der neuen Mitarbeitenden angepasst werden kann.

Inhalt des Konzeptes.

- Rekrutierungsprozess

- Einführung

Praktisch.

- Individuelle Einführung am Patientenbett während 4-12 Wochen

- Zwei spezifische Einführungstage mit der Pflegefachfrau mit Höherer Fachausbildung in Pflege

- Monatliche Instruktionstage mit der Instruktionspflegefachfrau während den ersten Arbeitsmonaten

- Regelmäßige Standort- und Mitarbeitergespräche

Schriftlich. Es gibt definierte Unterlagen, welche am Schnuppertag, bei Anstellung zur Vorbereitung für den Stellenantritt (per E-Mail), sowie zum Stellenantritt zur Einarbeitung in den ersten Wochen abgegeben werden. Das Einarbeitungskonzept Neonatologie B2 Praxis und Theorie ist nach Stufen (in Anlehnung an Patricia Benner, 1994, Stufen zur Pflegekompetenz, From Novice to Expert) aufgebaut.

1. Stufe: Basiswissen/Neuling
2. Stufe: Vertiefung und Aufbau Basiswissen/fortgeschrittenen Anfängerin

3. Stufe: Vertiefung Fachwissen und Sicherheit im Handling/kompetente Pflegende

4. Stufe: Komplexe pflegerische Situationen/erfahrene Pflegende

Fachvertiefende Weiterbildung

Schlussfolgerung.

- Eine individuelle Einführung und Förderung neuer Mitarbeitenden ist mit einem Konzept möglich und kann je nach Vorkenntnisse der neuen Mitarbeitenden individuell gestaltet werden. Das neue Einführungskonzept hat Struktur und gibt allen Beteiligten Orientierung im Prozess der Einführung.

- Neue Mitarbeitende können gut im Team integriert werden.

- Die Einführung sowie die Arbeitsqualität der neuen Mitarbeitenden konnte deutlich gesteigert werden.

- Auswirkungen auf das Team sind erkennbar an den sichtbaren Zeichen: motivierte, engagierte Mitarbeiter, hohe Arbeitszufriedenheit und Identifikation mit der Arbeit und dem Betrieb, gutes Arbeitsklima, sehr geringe Krankheitsausfälle und Fluktuation, hohe Einsatzbereitschaft und Flexibilität, Vertrauen in die Kolleginnen, welcher die Einführung neuer Mitarbeiterinnen gestalten.

- Nachteilig ist der zum Teil lange zeitliche Aufwand, welcher für die Einführung neuer Mitarbeitenden berücksichtigt werden muss. Es lohnt sich, wenn man sieht welchen Nutzen alle davon haben.

\section{Fetale Programmierung}

\section{FV02}

Intrauterine Wachstumsretardierung durch experimentelle uteroplazentare Insuffizienz führt zu einer Aktivierung des Renin-Angiotensin-Aldosteron-Systems (RAAS) in einem kritischen Zeitfenster der Nierenentwicklung

\section{E. Nüsken', M. Wohlfarth' , G. Lippach², J. Dötsch², K. Nüsken}

'Univ.-Kinderklinik, Pädiatrische Nephrologie, Köln, Deutschland, ${ }^{2}$ Universitäts-Kinderklinik, Klinik und Poliklinik für Allgemeine Kinderheilkunde, Köln, Deutschland

Fragestellung. Ein pathologisch verändertes intrauterines Milieu bedingt nicht nur eine IUGR, sondern kann in kritischen Zeitfenstern der Entwicklung zu einer langfristigen Veränderung endokriner Systeme führen (perinatale Programmierung). Unterschiedliche Ursachen einer IUGR führen hierbei aufgrund zum Teil unterschiedlicher Mechanismen zu unterschiedlichen Phänotypen. Nach Niedrigproteindiät wurde eine arterielle Hypertonie in Folge einer dauerhaften Aktivierung des RAAS nachgewiesen. Wir haben daher im Modell der bilateralen Ligatur (LIG) der Aa. und Vv. uterinae (experimentelle uteroplazentare Insuffizienz) die Frage untersucht, ob auch hier eine Aktivierung des RAAS mit dem erhöhten Risiko der Hypertonieentwicklung vorliegt. Material und Methoden. Rattenföten nach LIG oder Scheinoperation (SOP) des Muttertieres wurden mit Föten von unoperierten Kontrolltieren (C) zu den Zeitpunkten $4 \mathrm{~h}(\mathrm{LIG} \mathrm{n}=20$, SOP $\mathrm{n}=24, \mathrm{C} n=12), 24 \mathrm{~h}$ (LIG n=27, SOP n=19, C n=12) und $72 \mathrm{~h}(\mathrm{LIG} n=19, \mathrm{SOP} n=20, \mathrm{C} n=24)$ nach dem Eingriff verglichen (normaler Geburtstermin: 72 h). Alle Föten wurden per Sectio geboren, die Nebennieren und Nieren sofort entnommen und schockgefroren. Zudem wurden bei männlichen Nachkommen nach LIG $(n=6)$, SOP $(n=12)$ und C $(n=6)$ im Alter von 7,15 und 30 Wochen die Aldosteronausscheidung sowie die Natriumausscheidung im Sammelurin bestimmt pro Gruppe. Im Alter von 30 Wochen wurden diese Tiere getötet und die Nieren ebenfalls entnommen und schockgefroren. In den Nebennieren wurde die Gen- und Proteinexpression der Aldosteronsynthase untersucht. In den Nieren wurde die Genexpression von Renin untersucht.

Ergebnisse. Die renale Renin-Genexpression ist 24 und $72 \mathrm{~h}$ nach LIG erhöht, im Alter von 30 Wochen erniedrigt. Die Aldosteronsynthase ist 
auf Gen- und Proteinebene $24 \mathrm{~h}$ nach LIG zunächst erniedrigt und nach $72 \mathrm{~h}$ auf Proteinebene erhöht im Vergleich mit $\mathrm{C}$ bei gleicher Genexpression aller Gruppen. Die Ausscheidung von Aldosteron im Sammelurin ist mit 7 Wochen bei LIG-Tieren erhöht, im Alter von 15 und 30 Wochen unverändert im Vergleich mit C. Passend hierzu ist bei LIG die fraktionierte Natriumexkretion im Alter von 7 Wochen vorübergehend erhöht, bei LIG im Alter von 30 Wochen dann sogar erniedrigt (Signifikanzniveau $\mathrm{p}<0,01$ ).

Diskussion. Eine Hochregulation des RAAS mit vermehrter Salzrückresorption infolge einer erhöhten Aldosteronsekretion ist eine mögliche Ursache für die Entstehung einer Hypertonie. Langfristig ist nach uteroplazentarer Insuffizienz jedoch keine Hochregulation des RAAS nachweisbar. Hingegen ist neonatal bis pubertär eine vorübergehende RAAS-Aktivierung zu beobachten. Da in diesem Zeitraum die Entwicklung der Niere noch nicht abgeschlossen ist und Dysbalancen im RAAS mit einer veränderten Organentwicklung vergesellschaftet sind, sind Auswirkungen auf die renale Struktur zu erwarten.

Schlussfolgerung. Auch im Modell der uteroplazentaren Insuffizienz ist eine RAAS-Aktivierung zu beobachten, allerdings nur vorübergehend in einem kritischen Zeitfenster der Organentwicklung. Die genauen Auswirkungen auf Organstruktur und -funktion sind Gegenstand laufender Studien. Darüber hinaus unterstreicht der Zeitverlauf der Veränderungen die Notwendigkeit longitudinaler Studien.

\section{FV03 \\ Fat and fat-free mass indices for preterm and term infants during first 6 month of life}

\section{Goswami', N. Rochow', G. Fusch', C. Fusch' \\ 'McMaster University, Division of Neonatology, Department of Pediatrics, Hamilton, Ontario, Kanada}

Introduction. Sub-optimal body composition (BC) in neonates, especially fat mass, is related to lifelong morbidity. To assess the nutritional status, reference data of fat mass and fat free mass is needed. However, if absolute values of fat and fat free mass for postmenstrual age are applied to neonates with a different body length then different nutritional status can be interpreted as similar BC. This becomes crucial, when BC of preterm infants are compared to their term counterparts, who usually differ in their mean length. Thus, length normalized reference data is needed.

Objective. To calculate fat mass index (FMI) and fat free mass index (FFMI) for postmenstrual age in preterm and term infants during the first 6 months of corrected age.

Methods. BC data $(\mathrm{n}=853)$ had been taken from 4 longitudinal studies; 396 preterm $(22-36 \mathrm{wks})$ and 133 term infants on fortified breast milk or preterm formula $(80 \mathrm{kcal} / \mathrm{dL})$ and breast milk or term formula $(67 \mathrm{kcal} /$ $\mathrm{dL}$ ) respectively, were analyzed. Time points for measurement were: 1) after reaching full enteral feeding, 2) at term and 3) two further time points until a maximum of 6 months of corrected age. $\mathrm{BC}$ was measured by dual energy X-ray absorptiometry (QDR 1500; Hologic). All measurements were considered as independent data points. $\mathrm{R}$ software (gamlss package) was used for growth data analyses at 30, 40, 52 and 64 wks of post-menstrual age.

Results. Median FMI for both preterm and term infants increased during the analyzed time points (preterm=0.5, 2.1, 4.0, $4.3 \mathrm{~kg} / \mathrm{cm} 2$ at 30 , $40,52,64$ wks respectively; term $=1.7,4.5,5.8 \mathrm{~kg} / \mathrm{cm} 2$ at $40,52,64 \mathrm{wks}$, respectively). Median FFMI for term infants remained stable throughout the time points tested (10.7, 11.0, $10.8 \mathrm{~kg} / \mathrm{cm} 2$ respectively), whereas the median FFMI for preterm infants increased between 30 and 40 wks ( 7.8 to $10.9 \mathrm{~kg} / \mathrm{cm} 2$ ) but remained stable thereafter $(11.4$ and $11.2 \mathrm{~kg} / \mathrm{cm} 2$ between 52 to 64 wks). For both term and preterm infants, the body length increased at the same rate during the first 6 months of corrected age $\left(0.76 \mathrm{~cm} / \mathrm{wk}, \mathrm{R}_{2}=0.7\right)$. However, preterm infants were consistently shorter than their term counterparts.
Conclusions. This study provides large dataset for length normalized BC indices, which could be used to assess nutritional status in neonates. This data is crucial to understanding the effects of postnatal nutrition on the longitudinal change in $\mathrm{BC}$ during the first 6 months of corrected age.

\section{FV04}

Quantifizierung von Dehydroepiandrosteron und Dehydroepiandrosteronsulfat in den Fingernägeln von Früh- und Reifgeborenen zur Beurteilung der fetalen Stressexposition

\section{Schmelter', L. Krebs², S. Fröhlich', P. Verde ${ }^{4}$, D. Herebian 5 , T. Höhn}

${ }^{1}$ Med. Einrichtung der Universität Kinderklinik, Düsseldorf, Deutschland, ${ }^{2}$ Universitäsklinikum Düsseldorf, Frauenklinik, Düsseldorf, Deutschland, ${ }^{3}$ Universitätsklinikum Düsseldorf, Pränatalmedizin, Düsseldorf, Deutschland, ${ }^{4}$ Universitätsklinikum Düsseldorf, KKS, Düsseldorf, Deutschland, ${ }^{5}$ Universitätsklinikum Düsseldorf, Kinderklinik, Düsseldorf, Deutschland

Hintergrund. Maternaler Stress, der mit erhöhten Cortisolspiegeln im mütterlichen Blut einhergeht, führt diaplazentar zu einer Alternierung der fetalen Hypothalamus-Hypophysen-Nebennieren-Achse. Eine frühzeitige Störung dieser Achse geht mit einem erhöhten Lebenszeitrisiko für metabolische kardiovaskuläre und mentale Erkrankungen einher. Dehydroepiandrosteron (DHEA) und DehydroepiandrosteronSulfat (DHEAS) sind Hormone, die die Aktivität der fetalen HPA-Achse wiederspiegeln und eignen sich somit möglicherweise zur Beurteilung der fetalen Stressexposition. DHEA und DHEAS können in menschlichen Nägeln nachgewiesen werden, da sie über die Nagelmatrix in den Nagel inkorporiert werden. Da sowohl das Nagelwachstum als auch die Aktivität der fetalen HPA-Achse schon ab der 8. SSW beginnen, ermöglicht die Untersuchung der Nägel von Neugeborenen eine Beurteilung der fetalen Stressexposition über große Zeiträume der Schwangerschaft. Es konnte bereits gezeigt werden, dass Kinder von stressexpositionierten Müttern im Vergleich zu Kindern von nicht-stressexpositionierten Müttern höhere Konzentrationen von DHEA in ihren Nägeln aufweisen

Methoden. Diese Studie soll die Frage beantworten, ob sich das Stressprofil von Frühgeborenen und intrauterin wachstumsretardierten Kindern von dem reifer Neonaten unterscheidet. Dazu wurde eine Kohorte von 56 am Uniklinikum Düsseldorf geborenen Kindern rekrutiert. Darunter befinden sich 28 Frühgeborene, davon 8 Kinder mit kritischem Geburtsgewicht $(<1500 \mathrm{~g})$, und 9 Kinder mit pränatal diagnostizierter intrauteriner Wachstumsrestriktion. Zur Erfassung des maternalen Stresses wurden zwei Fragebögen, die Prenatal Distress Questionnaire und die Life Experience Survey, herangezogen, die die Mütter während der Schwangerschaft ausfüllten. Nagelmaterial wurde sowohl von Müttern als auch von Kindern untersucht. Die Untersuchung der Materialen erfolgte mittels Ultra Performance Flüssigkeits-Chromatographie gekoppelt mit Tandem Massen Spektrometrie.

Ergebnisse. Erste Datenauswertungen zeigen, dass die Gruppe der reifgeborenen Neonaten die höchsten Werte für DHEA und DHEAS in ihren Nägeln aufweisen. Insbesondere bei Frühgeborenen mit einen Geburtsgewicht unter $1500 \mathrm{~g}$ wurde signifikant weniger DHEA $(p=0,036)$ und DHEAS $(\mathrm{p}=0,004)$ nachgewiesen. Ebenso wurden bei Kindern mit IUGR geringere Hormonkonzentrationen in den Nägeln gemessen. Die weitere Auswertung und statistische Ausarbeitung der Daten steht noch aus. Insbesondere soll hier Bezug zum maternalen Stress und den mütterlichen Hormonkonzentrationen genommen werden. Zur Diskussion stehen vor allem die mögliche Alternierung der fetalen HPA-Achse durch extern zugeführte Kortikosteroide im Rahmen der RDS-Prophylaxe sowie die Beeinflussung der fetalen Hormonproduktion nach Geburt durch externe Stressoren.

Schlussfolgerung. Zusammenfassend können wir zeigen, dass eine Quantifizierung von DHEA und DHEAS auch bei Frühgeborenen und bei SGA-Kindern möglich ist. Beide Kollektive weisen in Relation zu 
Reifgeborenen niedrigere Hormonlevel auf, was dafür spricht, dass beide Gruppen keiner höheren Stressexposition ausgesetzt sind.

\section{FV05 \\ Development of functional connectivity in neonatal brain net- works: improved characterisation using accelerated fMRI acquisi- tion with multiband EPI}

\author{
A. Smith-Collins', K. Luyt' ${ }^{1}$, R. Kauppinen ${ }^{2}$, A. Heep ${ }^{7}$ \\ 'University of Bristol, Neonatal Neuroscience, School of Clinical Sciences, Bristol, \\ United Kingdom, 2University of Bristol, CRIC Bristol, Bristol, United Kingdom
}

Background. Understanding how the neonatal brain develops functional networks and how preterm birth and postnatal experience effects on is of major importance. The analysis of functional MRI images in individuals acquired at rest (rs-fMRI) allows measurement of coordinated neural activity across disparate brain regions (functional connectivity). Most rs-fMRI work has focused on fluctuations in blood oxygenation level dependent (BOLD) fMRI signals occurring at $<0.2 \mathrm{~Hz}$, despite neuronal fluctuations, as measured with electrophysiological means, occurring much more rapidly.

Aims. The aim of the study is to study functional connectivity in the developing brain employing a rapid $(\mathrm{TR}=906 \mathrm{~ms})$ multiband rs-fMRI protocol, probing BOLD fluctuations up to $0.5 \mathrm{~Hz}$ within a neonatal functional MRI protocol.

Methods. 20 preterm infants born $<32$ weeks GA excluding those with major perinatal brain injury were studied with structural and functional MRI protocol on a 3T MRI scanner (Skyra, Siemens) at the Clinical Research and Imaging Center (CRIC), University of Bristol. All studies were performed at term equivalent age in natural sleep. Within the structural and functional MRI protocol a 'multiband' parallel EPI sequence was applied to improve rs-fMRI sampling frequency with multiple brain slices excited simultaneously and spatially distributed receiver coils used to encode anatomical data, allowing accelerated data reconstruction.

Results. We present power spectra, SNR data and comparisons of functional connectivity data at different frequencies identifying unique functional changes at frequencies $>0.2 \mathrm{~Hz}$, which have not been identified nor described within lower frequency fluctuations earlier.

Conclusion. Multiband EPI sequence in neonatal fMRI allows for the first time to identify unique functional brain activity. This has implications for future characterisation of infant brain network development.

\section{FV06}

\section{Der Einfluss maternaler Adipositas auf die Plazenta}

S. Appel', S. Storck', R. Janoschek', M. Handwerk'2, J. Ankerne'2, E. Rother', J. Dötsch

'Univ.-Kinderklinik, Forschungslabor AG Dötsch, Köln, Deutschland, ${ }^{2}$ Univ.-Kinderklinik, Köln, Deutschland, ${ }^{3}$ Klinik und Poliklinik für Kinder- $u$. Jugendmedizin, Uniklinik Köln, Köln, Deutschland, ${ }^{4}$ Universitäts-Kinderklinik, Gebäude ${ }^{44}$ a, Köln, Deutschland, ${ }^{5}$ Universitäts-Kinderklinik, Klinik und Poliklinik für Allgemeine Kinderheilkunde, Köln, Deutschland

Hintergrund. Mütterliche Erkrankungen wie die in ihrer Inzidenz zunehmende Adipositas oder die ebenfalls zunehmende plazentare Dysfunktion beeinflussen die Entwicklung des Kindes im Mutterleib maßgeblich. Es besteht darüber hinaus der Verdacht eines kausalen Zusammenhangs zwischen beiden Erkrankungen, indem mütterliche Adipositas mit einem erhöhten Risiko für eine plazentare Dysfunktion einherzugehen scheint.

Methoden und Ergebnisse. In dieser Studie wurde im Mausmodell untersucht, welchen Einfluss eine durch fettreiche, hochkalorische Diät ausgelöste Adipositas der Mutter auf die Plazenta hat. Am Gestationstag g18/19 erfolgte die Entnahme der Plazenten und auch der Feten. Es zeigte sich, dass sowohl die Plazenten wie auch die Feten von adipösen
Müttern signifikant leichter waren im Vergleich zu normalgewichtigen Müttern. Dies deutet auf einen gestörten Plazentationsprozess und somit auf eine plazentare Dysfunktion hin, die sich wiederum negativ auf die Versorgung des Feten auswirken kann. Das niedrigere Plazentagewicht in adipösen Müttern ist nicht auf ein gestörtes Gleichgewicht zwischen Apoptose und Proliferation in den Plazenten zurückzuführen, wie Western-Blot-Analysen des Apoptosemarkers cleaved-PARP und des Proliferationsmarkers PCNA zeigten. Da ein gestörter Aufbau der Extrazellularmatrix sich negativ auf die Plazentastruktur und somit auf die feto-maternale Grenze auswirkt, die für die Versorgung des Feten mit Nährstoffen essentiell ist, wurde als nächstes in quantitativen TaqMan-PCR-Analysen die Genexpression verschiedener Komponenten der Extrazellularmatrix sowie von Matrix-modellierenden Komponenten analysiert. Jedoch konnte weder bei den Matrix-modellierenden Komponenten noch bei den Matrixkomponenten selbst signifikante Unterschiede zwischen normalgewichtigen und adipösen Müttern detektiert werden. Adipöse Patienten weisen häufig einen erhöhten systemischen Entzündungslevel auf. Greift dieser Prozess in der Schwangerschaft auf die Plazenta über, beeinflusst dies die Funktionalität des Organs. Eine durch die Adipositas ausgelöste plazentare Inflammationsreaktion wurde allerdings in histologischen und TaqMan-PCRAnalysen in unserem Tiermodell ausgeschlossen.

Schlussfolgerung. Zusammenfassend konnten wir bis zum jetzigen Zeitpunkt nicht klären, welche Ursache(n) das geringere Plazentenund Fetengewicht in adipösen Müttern hat.

\section{FV07}

Eine Ernährungsumstellung während Schwangerschaft und Stillzeit verhindert die Ausprägung von Hauptmerkmalen des metabolischen Syndroms in den Nachkommen übergewichtiger Mütter

\section{R. Janoschek', I. Bae-Gartz', C. Vohlen', M. Alejandre Alcazar', J. Dötsch', E. Rother}

'Klinik und Poliklinik für Kinder- u. Jugendmedizin, Uniklinik Köln, Köln, Deutschland

Hintergrund. Die Zahl der übergewichtigen Kinder hat in den letzten Jahrzehnten weltweit dramatisch zugenommen. Der mütterliche Stoffwechsel scheint dabei eine entscheidende Rolle für die Programmierung kindlicher Stoffwechselprozesse zu spielen, so tragen die Nachkommen übergewichtiger Mütter ein besonders hohes Risiko, im späteren Leben ebenfalls übergewichtig zu werden. Die molekularen Mechanismen, denen dieser Effekt zugrunde liegt, sind bislang wenig erforscht. Das Ziel unserer Arbeit war die Ermittlung, ob die Umstellung auf eine ausgewogene Mischkost während Schwangerschaft und Stillzeit das Risiko für ein metabolisches Syndrom in den Nachkommen übergewichtiger Mütter reduziert.

Methoden. Dazu wurden weibliche C57/BL6-Mäuse ab einem Alter von 3 Wochen mit fettreicher Nahrung (HFD) gefüttert, bis sie übergewichtig waren und eine gestörte Glukoseverwertung aufwiesen. Nach der Verpaarung wurden die Muttertiere in zwei Gruppen aufgeteilt. Die HFD-Gruppe erhielt weiterhin fettreiche Kost, während in der sogenannten Interventionsgruppe die fettreiche Nahrung während Schwangerschaft und Stillzeit durch eine ausgewogene Mischkost ersetzt wurde. Die Kontrollgruppe bestand aus Muttertieren, die zu jedem Zeitpunkt mit ausgewogener Mischkost ernährt wurden.

Ergebnisse. Auffallend war, dass die pathologischen Effekte auf das Körpergewicht und den Körperfettanteil am Ende der Stillzeit in den Nachkommen der Interventionsgruppe vollständig aufgehoben waren. Auch die Adipokin-Spiegel im Blut und die Glukoseverwertung der Interventions-Nachkommen waren normalisiert. Zusätzliche Analysen des weißen Fettgewebes ergaben nicht nur eine überwiegend wiederhergestellte Adipokin mRNA-Expression in den Nachkommen der Interventions-Gruppe, sondern offenbarten zusätzlich eine verbesserte Insulin-Sensitivität. Auch die mRNA-Expression von PPAR $\gamma$ und UCP1 war im weißen Fettgewebe der Interventions-Nachkommen deutlich 
gesteigert und deutet darauf hin, dass eine gesunde mütterliche Ernährung während Schwangerschaft und Stillzeit eine potenzielle Rolle bei der Umwandlung von weißem zu braunem Fettgewebe in den Nachkommen spielt.

Schlussfolgerung. Unsere Ergebnisse zeigen, dass eine Verbesserung des mütterlichen Stoffwechsels in der Lage ist, die durch mütterliches Übergewicht und fettreiche Ernährung hervorgerufenen negativen metabolischen Effekte auf die Nachkommen vollständig aufzuheben. Somit bieten die gewonnen Daten eine Grundlage für die Entwicklung wirksamer Gegenmaßnahmen zur Prävention des metabolischen Syndroms in den Nachkommen übergewichtiger Mütter.

\section{Analgosedierung Opiatentzug}

\section{FV08}

Hautleitfähigkeit bei neonatalem Opiat-Abstinenz-Syndrom im Vergleich zu der nichtexponierter Neugeborener

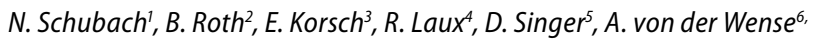
A. Treszl', C. Hünseler ${ }^{8}$

'Diakoniekrankenhaus Klinik f. Kinder- u. Jugendmedizin, Rotenburg, Deutschland, ${ }^{2}$ Universitäts-Kinderklinik Köln, Neonatologie und pädiatrische Intensivmedizin, Köln, Deutschland, ${ }^{3}$ Städt. Krankenanstalten Kinderkrankenhaus, Köln, Deutschland, ${ }^{4}$ AK Barmbek, Neonatologie, Hamburg, Deutschland, ${ }^{5}$ Universitätsklinikum Hamburg-Eppendorf, Sektion Neonatologie und Pädiatrische Intensivmedizin, Hamburg, Deutschland, ${ }^{6}$ Altonaer Kinderkrankenhaus, Hamburg, Deutschland, ${ }^{7}$ Univ.-Kinderklinik Eppendorf, Hamburg, Deutschland, ${ }^{8}$ Univ.-Kinderklinik, Neonatologie und Pädiatrische Intensivmedizin, Köln, Deutschland

Fragestellung. Ziel der Arbeit war es, zu überprüfen ob sich die Hautleitfähigkeit Neugeborener mit Opiat- Entzugs-Syndrom (NAS) von der nicht-opiatexponierter (NE) Neugeborener unterscheidet.

Methodik. Es wurde die Hautleitfähigkeit (SC) von 12 Neugeborenen mit NAS und 12 NE-Neugeborenen in den ersten 6 Lebenswochen untersucht. Acht Untersuchungen wurden wiederholt in einer Ruhephase und einmalig während einer Blutentnahme zum Stoffwechselscreening durchgeführt. Bestimmt und ausgewertet wurden die Frequenz der SC pro Sekunde (NSCF/s), die Amplitude und das mittlere Level.

Ergebnisse. Die NSCF/s der Neugeborenen mit NAS unterschied sich signifikant von der NE-Neugeborener und zeigte höhere Werte $(\mathrm{p}=0,04)$. Das mittlere Level der SC zeigte Interaktionen zwischen den beiden Gruppen über die Zeit (p-Wert für Interaktion =0,02). Die Amplitude unterschied sich nicht. Alle Parameter der SC stiegen mit dem postnatalen Alter an.

Diskussion und Schlussfolgerung. Die NSCF/s und das mittlere Level der SC scheinen geeignet, den Stress im Rahmen des NAS zu reflektieren. Die Messung der SC könnte zukünftig beispielsweise in Therapieoptimierungsstudien eingesetzt werden, um als zusätzlicher Parameter das Ausmaß des Entzugs zu überwachen.

\section{FV09}

\section{Zerebale Oxygenierung während der Intubation Neugeborener}

\section{Schmid', A. Gloßmann', R. Hopfner', H. Hummler' , H. Fuchs ${ }^{2}$}

${ }^{1} K$ linik für Kinder- und Jugendmedizin, Universitätsklinikum Ulm, Sektion Neonatologie und Pädiatrische Intensivmedizin, Ulm, Deutschland, ${ }^{2}$ Zentrum für Kinder- und Jugendmedizin, Klinik für Allgemeine Kinder- und Jugendmedizin, Freiburg, Deutschland

Hintergrund. Die endotracheale Intubation Neugeborener geht einher mit Veränderungen von Sauerstoffsättigung $\left(\mathrm{SpO}_{2}\right)$, Herzfrequenz und Blutdruck. Intubierte Frühgeborene haben ein erhöhtes Risiko für Hirnblutungen (IVH). Dabei ist unklar, ob alleine die Grunderkrankung, die zur Intubationsindikation führt, oder auch die Intubation selbst und die dazu verabreichten Medikamente zu einer zerebralen Minderperfusion führen, die dann das Auftreten der IVH begünstigt. Ziel. Ziel der Studie ist es, die Auswirkung der Analgosedierung und des Intubationsvorgangs auf die zerebrale und somatische Gewebeoxygenierung und das Herzzeitvolumen zu untersuchen.

Methode. Bei 24 Neugeborenen zeichneten wir alle 2 Sekunden $\mathrm{SpO}_{2}$, EKG Herzfrequenz (HF) und zerebrale und renale Gewebesättigung (StO2) mithilfe der Nahinfrarotspektroskopie (NIRS) mit einem Absolutoxymeter (FORE-SIGHT, Casmed) auf. Das Herzzeitvolumen wurde

\begin{tabular}{|c|c|c|}
\hline & Median & Quartilen \\
\hline Gestationsalter (Wochen) & 24,6 & $23,7-26,9$ \\
\hline Geburtsgewicht (g) & 628 & $488-825$ \\
\hline $\begin{array}{l}\text { Postmenstruelles Alter } \\
\text { (Wochen) }\end{array}$ & 26,9 & $26,0-29,4$ \\
\hline Hämatokrit (\%) & 45,0 & $37,0-52,5$ \\
\hline $\mathrm{FiO}_{2}$ vorher/nachher (\%) & $38 / 32$ & $30-79 / 29-40$ \\
\hline $\begin{array}{l}\text { Temperatur }\left({ }^{\circ} \mathrm{C}\right) \text { vorher/ } \\
\text { nachher }\end{array}$ & $36,6 / 36,5$ & $36,2-36,9 / 35,9-36,9$ \\
\hline HF vorher/minimal/nachher & $171 / 118 / 184$ & $\begin{array}{l}166-179 / 83-138 / 178- \\
191\end{array}$ \\
\hline $\mathrm{SpO}_{2}$ vorher/minimal/nachher & $87,8 / 44,0 / 87,9$ & $\begin{array}{l}81,4-93,4 / 9,5- \\
71,5 / 85,9-91,3\end{array}$ \\
\hline $\begin{array}{l}\text { Zerebrale } \mathrm{StO}_{2} \text { vorher/mini- } \\
\mathrm{mal} / \text { nachher }\end{array}$ & $71,7 / 48,0 / 74,6$ & $\begin{array}{l}64,7-78,4 / 38,1- \\
68,0 / 67,0-81,6\end{array}$ \\
\hline $\begin{array}{l}\text { Somatische } \mathrm{StO}_{2} \text { vorher/mini- } \\
\mathrm{mal} / \text { nachher }\end{array}$ & $75,5 / 51,5 / 77,1$ & $\begin{array}{l}64,6-82,3 / 38,6- \\
63,0 / 71,9-80,2\end{array}$ \\
\hline $\begin{array}{l}\text { Schlagindex vorher/minimal/ } \\
\text { nachher }\end{array}$ & $11,6 / 9,9 / 10,5$ & $\begin{array}{l}10,0-14,3 / 8,0-12,1 / 8,6 \\
13,1\end{array}$ \\
\hline $\begin{array}{l}\text { Cardiac index vorher/minimal/ } \\
\text { nachher }\end{array}$ & $1,94 / 1,70 / 1,96$ & $\begin{array}{l}1,72-2,37 / 1,43- \\
1,99 / 1,66-2,29\end{array}$ \\
\hline $\begin{array}{l}\text { Blutdruck (Mitteldruck, inva- } \\
\text { siv) vorher/minimal/nachher }\end{array}$ & $36,6 / 33,0 / 35,7$ & $\begin{array}{l}34,6-43,3 / 27,5- \\
35,3 / 32,2-38,5\end{array}$ \\
\hline $\begin{array}{l}\text { Blutdruck (Systole, invasiv) } \\
\text { vorher/maximal/nachher }\end{array}$ & $47,6 / 75,0 / 46,5$ & $\begin{array}{l}43,5-56,7 / 63,5- \\
78,8 / 40,3-51,3\end{array}$ \\
\hline $\begin{array}{l}\text { Blutdruck (Mitteldruck, nich- } \\
\text { tinvasiv) vorher/minimal/ } \\
\text { nachher }\end{array}$ & $39,4 / 36,0 / 37,6$ & $\begin{array}{l}34,0-49,8 / 31,0- \\
45,3 / 30,0-49,0\end{array}$ \\
\hline $\begin{array}{l}\text { Blutdruck (Systole, nichtinva- } \\
\text { siv) vorher/maximal/nachher }\end{array}$ & $57,3 / 68,0 / 56,0$ & $\begin{array}{l}51,1-66,1 / 60,3- \\
82,3 / 50,4-71,6\end{array}$ \\
\hline
\end{tabular}

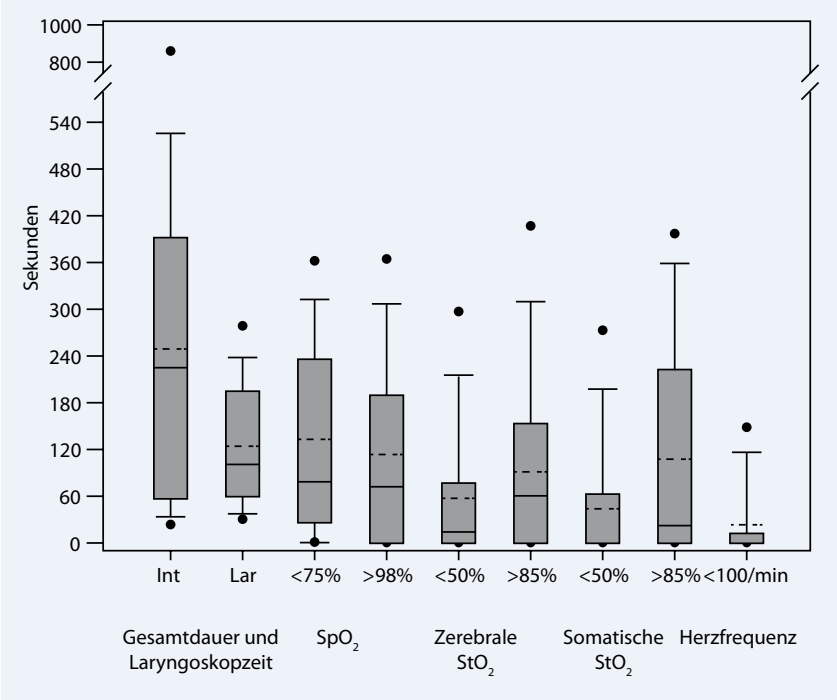

$\Delta$ FVo9-Abb. 1 
kontinuierlich und nichtinvasiv durch Elektroimpedanzkardiometrie bestimmt (ICON, Osypka).

Ergebnis. Patienteneigenschaften und Ergebnisse sind Tab. 1 und Abb. 1 zu entnehmen.

Schlussfolgerung. Während der Intubation kommt es zu Phasen von Hypoxämie, Bradykardie, Hypotension, Hypertension und zu einer deutlichen Beeinträchtigung der zerebralen und somatischen Gewebeoxygenierung. Dies könnte zur Entstehung von Hirnblutungen bei Frühgeborenen beitragen.

\section{FV10 \\ Validation of the Neonatal Pain, Agitation and Sedation Scale for the assessment of sedation in neonatal intensive care patients}

\section{Giordano', P. Deindl', T. Werther', C. Czaba', A. Berger', A. Pollak', M. Weninger ${ }^{1}$, M. Olischar ${ }^{1}$ \\ 'Universitätsklinik für Kinder- und Jugendheilkunde Wien, Abteilung für Neo- natologie, Pädiatrische Intensivmedizin und Neuropädiatrie, Wien, Österreich}

Background. Implementation of sedation protocols based on regular sedation assessment using item-based scales can improve sedative treatment in children. There is a lack of validated tools to assess sedation in neonates.

Objective. To validate the Neonatal Pain, Agitation and Sedation Scale (N-PASS) for the assessment of sedation in preterm and term neonates. Methods. Paired assessments of both the N-PASS and the Nurse Interpretation of Sedation Score (NISS) were performed in 50 sedated neonates at 23 to 44 weeks' postmenstrual age providing a total set of 503 paired observations.

Results. Median N-PASS scores were significantly different for the three NISS categories (over-sedation (-8), adequate sedation $(-2)$, under-sedation (o); $\mathrm{p}<0.0001)$. Inter-observer agreement for the N-PASS sedation subscale was excellent (linearly weighted Cohen's Kappa: 0.93), as was the internal consistency estimated by a Cronbach's alpha of 0.88 , which increased to 0.90 when the vital sign item was excluded from the N-PASS. There was no risk of under-sedation in patients with an N-PASS score $<-5$ and no risk of over-sedation with an N-PASS score $>-2$. The N-PASS reliably detected over-sedation. Detection of undersedation was improved by simultaneous assessment of N-PASS pain scores which were significantly different in patients being considered adequately sedated vs. inadequately sedated (median N-PASS pain subscale score: 2 vs 5 ).

Conclusion. The N-PASS meets the requirements of a valid clinical too to assess sedation in neonates and may facilitate the use of sedation algorithms in the neonatal intensive care unit.

\section{FV11}

\section{Einfluss der Einführung einer systematischen Steuerung der} Analgosedierung auf das Outcome von Very-low-birth-weight-Neugeborenen

\section{P. Deindl', R. Fuiko', T. Waldhör', G. Kappler', L. Unterasinger', V. Giordano', T. Werther', C. Czaba', A. Berger', M. Olischar ${ }^{1}$ \\ ${ }^{1}$ Medizinische Universität Wien, Neonatologie, pädiatrische Intensivmedizin und Neuropädiatrie, Wien, Österreich, ${ }^{2}$ Center of Public Health, Abteilung für Epidemiologie, Wien, Österreich, ${ }^{3}$ Faculty of Psychology, Institute for Applied Psychology: Health, Development, Enhancement, Wien, Österreich}

Hintergrund. Neugeborene auf Intensivstationen sind häufig Stress, Schmerzen und anderen potentiell chronisch schädlichen Einflüsse ausgesetzt. Unbehandelte Schmerzen gehen bei Neugeborenen mit einer erhöhten Morbidität und Mortalität einher. Opiate sind die hauptsächlich genutzte Stoffgruppe, die zur Analgesie und Sedierung von Neugeborenen verwendet wird. Opiate können jedoch kardiorespiratorische Nebenwirkungen wie arterielle Hypotonie, und einen verringerten Atemantrieb bewirken, was u. a. zu längeren Beatmungs- zeiten führen kann. Zusätzlich verlangsamen Opioide die Magen- und Darmpassage, was zu Verzögerungen beim enteralen Nahrungsaufbau und damit zu einer längeren parenteralen Ernährung mit den damit einhergehenden Risiken führen kann. Der potenzielle Einfluss von Opiaten auf die Entwicklung des unreifen menschlichen Gehirns ist noch unklar. Ziel dieser Studie war es, das Kurzzeit-Outcome und die neurologische Entwicklung von Very-low-birth-weight-Neugeborenen (VLBW) vor (Kontrollgruppe) und nach (Interventionsgruppe) Einführung eines standardisierten Protokolls zur Evaluation und Behandlung von Schmerzen und Sedierung zu vergleichen. Der primäre Endpunkt dieser Studie war die entwicklungsneurologische Performance der überlebenden Patienten im korrigierten Alter von 12 Monaten.

Methoden. Opiatexposition, Beatmungsdauer, Katecholamin-Therapie, Dauer der enteralen und parenterale Ernährung, gastrointestinale Komplikationen, Liegedauer von zentralen Kathetern, Auftreten von Bakteriämien, sowie das Wachstum (Kopf, Länge, Gewicht) wurden vor und nach Einführung des Protokolls bei $\mathrm{n}=153$ Patienten verglichen. Die Kinder wurden im korrigieren Alter von 12 Monaten einer standardisierten neurologischen Untersuchung nach Bayley Scales of Infant Development-II unterzogen.

Ergebnisse. Die kumulative mittlere \pm SD Opiatdosis (vorher: $14 \pm 39 \mathrm{mg} /$ $\mathrm{kg}$ vs. nachher: $84 \pm \mathbf{2 2 2} \mathrm{mg} / \mathrm{kg}$ Morphin-Äquivalente; $\mathrm{p}<0,0001)$ stieg nach der Einführung signifikant an. Die Beatmungsdauer, Dauer der Katecholamintherapie, Dauer der parenteralen Ernährung, die Länge des Krankenhausaufenthaltes waren vor und nach Implementierung ähnlich. Die entwicklungsneurologische Performance vor und nach Einführung war ähnlich. Eine multiple lineare Regression identifizierte jedoch die Opiatexposition als einen vom Schweregrad der Erkrankung und anderen Faktoren unabhängigen potentiellen Risikofaktor für einen niedrigeren Mental Developmental Index (estimate -0,15; $p=0,004$ ) und einen niedrigeren Behavior Rating Scale (BRS) Wert (estimate -0,39; $\mathrm{p}=0,012)$. Patienten, die Opiate erhalten hatten im Vergleich zu Patienten ohne Opiatexposition hatten signifikant niedrigere mittlere \pm SD Bayley Mental Developmental Index (MDI) Werte, Psychomotor Developmental Index (PDI) und BRS-Werte (MDI: $77 \pm 14$ vs. $89 \pm 13$; p<0,001; PDI: $79 \pm 19$ vs. $88 \pm 18$, p<0,028; BRS: $61 \pm 31$ vs. $78 \pm 27$; $\mathrm{p}<0,001$; Tab. 1 , Tab. 2).

Schlussfolgerung. Die Einführung eines neonatalen Schmerz- und Sedierungsprotokolls resultierte in einem Anstieg der Opiatexposition ohne das Kurzzeit-Outcome von Very-low-birth-weight-Neugeborenen zu beeinflussen. Die entwicklungsneurologische Performance im korrigierten Alter von 12 Monaten war ähnlich vor und nach Einführung. Die Opiatexposition scheint jedoch ein Risikofaktor für eine schlechtere entwicklungsneurologische Performance im korrigierten Alter von 12 Monaten zu sein.

\section{FV12}

\section{Orale Glukose zur Analgesie beim oropharyngealen Absaugen} Frühgeborener: eine randomisierte Cross-over-Studie

\section{K. Vezyroglou', A. Kribs'², B. Roth'², K. Langhammer', C. Hünseler ${ }^{2}$}

${ }^{1}$ Univ.-Kinderklinik, Köln, Deutschland, ${ }^{2}$ Universitätskinderklinik Köln, Neonatologie und Pädiatrische Intensivmedizin, Köln, Deutschland

Objective. To investigate whether orally applied glucose $20 \%$ reduces the pain response during oropharyngeal suctioning in preterm infants with a birth weight over $1500 \mathrm{~g}$.

Methods. We conducted a randomized controlled trial on 40 preterm infants undergoing oropharyngeal suctioning while on nasal CPAP. The infants were randomly assigned to two groups and treated with glucose or placebo in reversed order before two oropharyngeal suctioning procedures. The Premature Infant Pain Profile (PIPP) score was assessed and compared in a cross-over design to investigate whether there was a significant difference in the patients' pain response.

Results. In Group VP (verum-placebo) receiving glucose before the first and placebo before the second intervention the mean PIPP score after glucose administration was 7.5 (95\% confidence interval 6.1-9.4). The mean PIPP score after placebo was 8.41 (95\% confidence interval 
7.2-9.6). In Group PV (placebo-verum) receiving the study medication in reversed order the mean PIPP scores were 8.44 (95\% confidence interval 7.1-9.8) after glucose and 8.73 (95\% confidence interval 7.6-9.9) after placebo. Comparison of the treatment effects reached no statistic significance $(\mathrm{p}=0.23)$. The mean PIPP score after placebo in both groups was 8.6 (95\% confidence interval 7.8-9.4).

Conclusions. In our study oropharyngeal suctioning of late preterm infants in the first days of life proved only slightly painful as measured by PIPP score. Accordingly patients did not benefit significantly from analgesia with glucose. The oral administration of glucose under nasal CPAP led to no serious adverse events.

\section{FV13}

\section{Messbarer sedierender Effekt von $50 \%$ Xenon mittels aEEG bei gesunden neugeborenen Schweinen}

\section{H. Sabir', H. Gill2, J. Dingley33, X. Liü2, M. Thoresen²}

'Med. Einrichtung der Universität Kinderklinik, Neonatologie und pädiatrische Intensivmedizin, Düsseldorf, Deutschland, ${ }^{2}$ University of Bristol, Child Health, School of Clinical Sciences, Bristol, United Kingdom, ${ }^{3}$ Morriston Hospital, Department of Anaesthetics and Intensive Care, Swansea, United Kingdom

Hintergrund. Die Reduktion der EEG-Hintergrundaktivität durch Inhalationsanästhetika wird genutzt, um die Sedierungstiefe von Kindern zu überwachen. Das Edelgas Xenon ist ein Inhalationsanästhetikum, welches alleine und in Kombination mit therapeutischer Hypothermie nach hypoxisch-ischämischer Hirnschädigung neuroprotektiv ist. In seiner neuroprotektiven Dosierung (50\%) besitzt Xenon jedoch nicht seine volle sedierende Wirkung. Das Ziel dieser Studie ist es den sedierenden Effekt von 50\% inhalativem Xenon mittels aEEG bei neugeborenen gesunden Schweinen zu untersuchen.

Material und Methode. Sechs gesunde neugeborene Schweine (Lebensalter $<24 \mathrm{~h}$ ) erhielten eine i.v.-Fentanyl-Sedierung und wurden für $24 \mathrm{~h}$ mit 50\% Xenon in Raumluft bei normaler Körpertemperatur $\left(\mathrm{NT}=38,5^{\circ} \mathrm{C}\right)$ beatmet. Eine aEEG-Aufzeichung (CFM, Natus, Seattle, USA) erfolgte sowohl zu Beginn der Xenon Zufuhr als auch während der 24-stündigen Xenon Beatmung. aEEG-Aktivitätsänderungen wurden kontinuierlich aufgezeichnet und analysiert.

Ergebnisse. Kurz nach dem Beginn der Xenon-Sedierung änderte sich die aEEG-Aktivität signifikant in 5 Schweinen. Bei einem Schwein blieb die aEEG-Aktivität unverändert. Der mittlere arterielle Blutdruck, die Herzfrequenz und die Sauerstoffsättigung wurden durch die Inhalation von $50 \%$ Xenon nicht beeinflusst. Während der 24-stündigen XenonInhalation war die aEEG-Aktivität konstant. Fentanyl hatte keinen Effekt auf die aEEG-Hintergrundaktivität.

Diskussion. 50\% Xenon reduziert signifikant die aEEG-Aktivität in gesunden neugeborenen Schweinen. Da mittels aEEG-Aktivitätsänderung eine Aussage über das Langzeitoutcome gekühlter Neugeborener nach perinataler Asphyxie getroffen werden kann, ist Vorsicht geboten, wenn diese Kinder zusätzlich mit 50\% Xenon beatmet werden, da das aEEG nicht adäquat beurteilt werden kann.

\section{Hygiene}

\section{FV14}

Erregertypisierung während eines Klebsiella-pneumoniae-Ausbruchs auf einer neonatologischen Intensivstation zeigt nicht-klonalen Ursprung auf

\section{J. Liese', M. Marschal', C. Poets' ${ }^{2}$ C. Gille 2 \\ 'Eberhard-Karls-Universität Tübingen, Institut für Medizinische Mikrobiologie und Hygiene, Tübingen, Deutschland, ${ }^{2}$ Eberhard-Karls-Universität Universi- tätsklinik für Kinderheilkunde und Jugendmedizin, Neonatologie, Tübingen, Deutschland}

Fragestellung. Jedes dritte sehr kleine Frühgeborene erleidet während seines Aufenthalts auf einer neonatologischen Intensivstation mindestens eine nosokomiale Sepsis. Zu den auslösenden Erregern gehören fakultativ pathogene Bakterien aus dem Mikrobiom der Haut und des Gastrointestinaltrakts. Die KRINKO empfiehlt daher wöchentliche Abstriche von Rachen und Anus, damit bei Erkrankung des Patienten adäquat therapiert werden kann und mögliche Übertragungen auf einer Station erkannt werden. Im Herbst 2013 fiel ein gehäufter Nachweis von Klebsiella (K.) pneumoniae auf unserer neonatologischen Intensivüberwachungsstation auf. Handelte es sich bei o. g. Ereignis um einen klonalen Ausbruch oder um den Eintrag verschiedener K.-pneumoniae-Klone?

Material und Methode. Über einen Zeitraum von 3 Monaten, der die Periode des gehäuften Auftretens einschloss, wurden alle K.-pneumoniaeIsolate asserviert. 35 repräsentative Isolate (mindestens eines pro Kind) wurden mittels Rep-PCR (Diversilab ${ }^{\star}$ ) typisiert. Nach Durchführung einer Clusteranalyse wurden Klone mit $>95 \%$ Ähnlichkeit zu einem Cluster zusammengefasst. Bei der Grundannahme der stabilen Besiedlung mit einem Stamm im zeitlichen Verlauf wurden die Patienten den Clustern anhand der Erregertypisierung zugeordnet.

Ergebnisse. Die K.-pneumoniae-Isolate des gesamten Zeitraums ließen sich 11 Clustern zuordnen. Vier Cluster bestanden aus einem einzelnen Isolat, die übrigen umfassten bis zu 8 Isolate mit bis zu 5 von 12 gleichzeitig besiedelten Kindern. Die einzelnen Cluster traten meist zeitlich versetzt auf und wurden durch die Entlassung des letzten noch besiedelten Patienten beendet. Zum Zeitpunkt des gehäuften Auftretens von K. pneumoniae waren vier verschiedene Stämme gleichzeitig nachweisbar. Größere Cluster mit mehreren besiedelten Patienten waren mit einer längeren Nachweisdauer des zugehörigen K.-pneumoniaeStamms verbunden. In 3 Fällen waren Patienten im zeitlichen Verlauf mit Isolaten aus verschiedenen Clustern kolonisiert. Ein Isolat, das bei einer Abklatschuntersuchung der Hände des Personals gefunden wurde, war einem zu diesem Zeitpunkt auf der Station auftretenden Cluster zuzuordnen.

Diskussion. Die beobachtete Häufung von K.-pneumoniae-Nachweisen war nicht monoklonalen Ursprungs. Die stationsspezifische Keimflora scheint durch unterschiedliche Mechanismen zustande zu kommen, dabei scheinen immer wieder neu eingetragene Keime am wichtigsten zu sein. Jedoch scheint auch eine nosokomiale Übertragung stattzufinden, da im Verlauf eines Cluster die Besiedlung von bis zu fünf Kindern nachgewiesen werden konnten. Die Anzucht eines Isolates von den Händen des Personals deutet auf eine Ausbreitung der Stämme durch Kontaktübertragung hin.

Schlussfolgerung. Die Ergebnisse des wöchentlich empfohlenen Screenings müssen kritisch überprüft werden.

\section{FV15}

Sicherheit und Effizienz der Verwendung von Folie bei der Erstversorgung sehr kleiner Frühgeborener

\section{H. Küster', C. Reuter ${ }^{1}$}

'Universitäts- Kinderklinik, Neonatologie, Göttingen, Deutschland

Einleitung. Verschiedene Studien haben in den letzten Jahren gezeigt, dass Folien bei der Erstversorgung einen Temperaturverlust vermeiden helfen. Dies hat nicht nur zu einer raschen Verbreitung des Einsatzes solcher Folien bei der Erstversorgung geführt. Deren Verwendung wird bereits in vielen Leitlinien empfohlen. Eingesetzt wird ein großes Spektrum verschiedener Materialien, ohne dass deren Unterschiede in Bezug auf Durchlässigkeit von Wärme und Feuchte bedacht werden. Zusätzlich unklar bleiben hygienische Aspekte.

Fragestellungen. 1) Sind die verschiedenen, bei der Erstversorgung eingesetzten Folien hygienisch sicher? 2) Gibt es zwischen den verwendeten Folientypen Unterschiede bezüglich der Rückhaltung von Wärme und Feuchte?

Methoden. Für die hygienische Prüfung wurde bei 20 VLBW-Frühgeborenen nach Sektio und einer Versorgung im eigenen Kreißsaal mit sterilen Handschuhen die verwendete Folie nach Eintreffen auf Station unter sterilen Kautelen vom Kind entfernt. Anschließend wurde eine $60 \mathrm{~cm} 2$ große Fläche, die vorher am Rücken des Kindes gewesen war, 
standardisiert mit TWEEN-haltigem Kochsalz abgespült und das Eluate in 3 verschiedenen Medien bei 2 Temperaturen. Zur Testung der Wärmerückhaltung wurden 2 Aluminiumblöcke von $983 \mathrm{~g}$ und $548 \mathrm{~g}$ mit fester zentraler und peripherer Temperatursonde auf $37^{\circ} \mathrm{C}$ gewärmt, mit einer Lage der 7 getesteten Folien (Zelensack, sterile Tüte, sterile Folie, Haushaltsfolie) umwickelt und bei Raumtemperatur die Zeit gemessen, in der der Block um $5^{\circ} \mathrm{C}$ abkühlt. Parallel wurden Raumtemperatur, Luftdruck und Feuchte kontinuierlich gemessen. Getestet wurden die 7 am häufigsten verwendeten Arten an Folie und Beutel. Zur Testung der Feuchterückhaltung wurde ein Waschlappen mit $20 \mathrm{ml}$ Wasser benetzt, in Folie eingewickelt und das Gewicht vor und nach $24 \mathrm{~h}$ bei $37^{\circ} \mathrm{C}$ bestimmt.

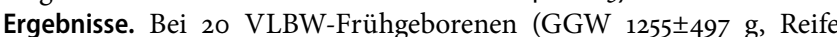
29;5 \pm 3 ; O SSW, 13 männlich) wurden nach einer Kontaktzeit von $53 \pm 10$ Minuten zwischen 1 und 36 (Median 3 ) KFU/m2 gefunden. Es wuchsen in $42 \%$ Staphylokokken, aber auch Pseudomonas, Acinetobacter und Pilze. Die unbenutzte Folie und die Umgebung waren immer steril. Ein Zusammenhang zwischen den Keimen der Mutter und den auf der Folie nachgewiesenen konnte nicht gesichert werden. Die Temperatur des $548 \mathrm{~g}$ Aluminumzylinders fiel ohne Folie binnen 18 Minuten um $5^{\circ} \mathrm{C}$. Die effektivste Folie verlängerte diese Zeit auf 44 Minuten, die am wenigsten effektivste auf nur 28 Minuten. Der Feuchteverlust war zwischen den verglichenen Folien nicht signifikant verschieden.

Schlussfolgerung. Bei der Kreißsaalversorgung werden heutzutage häufig Folien unterschiedlichen Typs verwendet, die zwar primär steril, aber bereits nach kurzer Zeit so stark besiedelt sind, dass von einer längeren Verwendung abgeraten werden muss. Die Folien besitzen eine sehr unterschiedliche Kapazität, Wärme zu halten, was bei der Auswahl des verwendeten Materials berücksichtigt werden sollte.

\section{FV16}

\section{Simulationstrainingsprogramm als zentraler Bestandteils eines "care bundles" zur Senkung der nosokomialen Infektionsrate an der NICU}

\section{Langgartner', A. Berger', N. Haiden', F. Cardona', J. Schwindt', M. Stei-} ner

'Universitätsklinik für Kinder- und Jugendheilkunde, Medizinische Universität Wien, Abt. für Neonatologie, pädiatrische Intensivmedizin und Neuropädiatrie, Wien, Österreich

Fragestellung. Die Senkung der nosokomialen Infektionsrate an der neonatologischen Intensivstation ist ein zentrales Anliegen der Neonatologie. Durch Einführung spezieller Hygienemaßnahmen („care bundles") konnte ein signifikanter Rückgang an nosokomialen Infektionen (NI) erreicht werden. Im Jahr 2010 wurde als zentraler Bestandteil eines neu eingeführten „care bundles“ ein simulationsbasiertes Trainingsprogramm zur einheitlichen Verlegung von Schwemmkathetern (SK) in unserem Simulationszentrum (SZ) erstellt. Ziel dieser Studie war es, $\mathrm{zu}$ untersuchen, ob die Implementierung von Simulationstrainings in „care bundles“ zu einer Senkung an NI führt.

Material und Methode. Zunächst wurde von allen ärztlichen MitarbeiterInnen die SK-Verlegetechnik im SZ gefilmt und analysiert. Aus den unterschiedlichen Verlegetechniken wurde dann eine "best practice“ generiert, welche allen MitarbeiterInnen mittels Lehrvideo präsentiert wurde. Im nächsten Schritt wurde das standardisierte Vorgehen von allen MitarbeiterInnen im SZ trainiert und Fehler mittels Videofilm analysiert. Danach wurde eine Checkliste erstellt, nach der bei Verlegung eines SK vorgegangen werden muss. Die Analyse der Infektionszahlen erfolgte unter Zuhilfenahme der Daten des Neo-KISS (Nationales Referenzzentrum für Surveillance von nosokomialen Infektionen) über einen Zeitraum von 3 Jahren (2010-2012).

Ergebnisse. Die standardisierte Infektionsrate (SIR) für Sepsis konnte von 2,26 im Jahr 2010 auf 1,27 im Jahr 2012 gesenkt werden. Die SIR für ZVK-assoziierte Sepsis wurde von 1,92 auf 1,27 gesenkt.

Diskussion. Der zentrale Teil unseres im Jahr 2010 eingeführten „care bundles“ war das verpflichtende Simulationstraining zur SK-Verlegung.
Unsere Zahlen bestätigen die bekannte Tatsache, dass die Einführung von sogenannten "care bundles“ erheblich zur Reduktion der nosokomialen Infektionsrate beiträgt. Neu ist, dass insbesondere auch Simulationstrainingsprogramme einen wertvollen Beitrag bei der Erstellung von „care bundles“ leisten können.

Schlussfolgerung. Simulationstrainingsprogramme als Teil von „care bundles" können einen wichtigen Beitrag zur Senkung der nosokomialen Infektionsrate an der neonatologischen Intensivstation liefern.

\section{FV17}

\section{Antibiotika-Surveillance in einer Abteilung für Neonatologie}

\section{J. Christoph', E. Kattner' ${ }^{2}$ F. Guthmann' , G. Sperling ${ }^{3}$}

${ }^{1}$ Auf der Bult Kinder- und Jugendkrankenhaus, Neonatologie, Hannover, Deutschland, ${ }^{2}$ ehemals Auf der Bult Kinder- und Jugendkrankenhaus, Neonatologie, Hannover, Deutschland, ${ }^{3}$ Marien-Apotheke Hannover, Krankenhauspharmazie, Hannover, Deutschland

Hintergrund. NEO-KISS ist ein nationales patientenbasiertes Surveillance-System für Frühgeborene mit einem Geburtsgewicht (GG) $<1500 \mathrm{~g}$ bis zu deren Verlegung/Tod/Gewicht $>1800$ g. Unsere Abteilung für Neonatologie nimmt seit dem Jahre 2000 teil, verbunden mit einer Surveillance der Antibiotika-Therapie ab 2002. Mit der Änderung des $\$ 23$ IV Infektionsschutzgesetz am 28.07.2011 ist ab 04.08.2011 der Antibiotika-Verbrauch aufzuzeichnen. Die zusammengefasste Anzahl definierter Tagesdosen („defined daily dose“, DDD) gemäß WHO-Klassifikation ist im Bundesgesundheitsblatt vom Juli 2013 (S. 903-912 u. 996-1002) als Methodik vorgegeben (DDD/10o Patiententage), berechnet aus Gesamtmenge des jeweiligen Wirkstoffes [g]/DDD [g]. Weitere Hinweise hierzu sollen im April-Heft des BGesBl 2014 folgen.

Ziele. Wir prüften die Aussagekraft der DDD-basierten Antibiotika-Anwendungsdichte in der Pädiatrie beispielhaft an unserer Neonatologie.

Methodik. Vergleich der in NEO-KISS dokumentierten Tage mit Antibiotikatherapie (als repräsentativ für alle Säuglinge in der Abteilung angenommen) mit der von der krankenhausversorgenden Apotheke gelieferten Packungsmenge (historisch bedingt nur in den jeweils ersten Halbjahren 2008 bis 2013 exzerpiert) als Basisgröße der AntibiotikaVerbrauchsdichte (pro 100 Patiententage bzw. Neuaufnahmen).

Ergebnisse. Bis 2012 nutzten wir auf unseren neonatologischen Stationen Antibiotika-Durchstichampullen über einen Zeitraum (bis zu 24 Stunden) gemäß Herstellerangaben, ab 2013 nur bis 1 Stunde nach Erstbenutzung. Die Apothekenabgabemengen haben sich deutlich erhöht, teilweise verdoppelt. Die Antibiotika-Anwendungsrate bei den Frühgeborenen unter NEO-KISS-Surveillance hat sich wenig verändert.

Schlussfolgerung. Antibiotika-Anwendungsraten (100 $\times$ Anzahl Antibiotika-Tage/Anzahl Patiententage) sind (vor allem in der Neonatologie) eine wichtige Vergleichsgröße, zusätzlich zur nachzuweisenden Antibiotika-Verbrauchsdichte: $100 \times($ Gesamtmenge des Wirkstoffes in g/DDD in g)/Anzahl Patiententage bzw. pro neu aufgenommene $\mathrm{Pa}$ tienten.

\section{Organersatzverfahren}

\section{FV18}

\section{Langzeiterfahrung mit regionaler Zitrat-Antikoagulation und der} Prismaflex bei pädiatrischen Patienten

\section{S. Rödl', I. Marschitz², S. Ring', C. Cimenti', G. Zobel'}

'Universitätsklinik für Kinder und Jugendheilkunde, Pädiatrische Intensivstation, Graz, Österreich, ${ }^{2}$ Universitätsklinik für Kinder- und Jugendheilkunde Salzburg, Neonatologie, Salzburg, Österreich

Hintergrund. Die regionale Zitrat-Antikoagulation (RCA) wird seit Jahren bei extrakorporalen Therapien bei Erwachsenen eingesetzt. Die Anpassung der Flussverhältnisse an pädiatrische PatientInnen konnte in Einzelfällen mit Hilfe von externen Infusionspumpen und engmaschi- 
gen Laborkontrollen erfolgreich bewerkstelligt werden. Die Prismaflex $^{\oplus}$-Maschine (PF) unterstützt bei allen Filtersets ab der Softwareversion 5.0 die Option einer automatische Zitrat- und Kalziumdosierung. Fragestellung. Vorstellung einer Kombination an Infusionslösungen für die RCA in HDF Filtern für pädiatrische Patienten, die auch für HDF Filter mit kleiner Oberfläche (Prismaflex ${ }^{\otimes} \mathrm{HF}_{20} \mathrm{Set}$ ) kompatibel sind. Patienten und Methoden. Von 9/2010 bis 3/2014 wurden an unserer pädiatrischen Intensivstation für Patienten 3 bis $50 \mathrm{~kg}$ Körpergewicht $(n=17)$ die Nierenersatztherapien bei akutem und chronischem Nierenversagen (NV) mit der PF (SW 5.0, 6.10) und dem HF 20 Set, ST 6o Set und HF 1000 Set durchgeführt. Die RCA erfolgte als Natrium isotone Lösung mit $47,5 \mathrm{mmol} / \mathrm{l}$ Zitrat. Als kalziumfreie Dialysatlösung wurde PrismoCalB22 (Gambro) verwendet. Die Postdilutionssubstitution erfolgte mit Phoxilium (Gambro). Die Kalziumsubstitution wurde mit $10 \%$ (o,23 molarer) Kalziumglukonat-Lösung durchgeführt. Die Steuerung der Flussgeschwindigkeit der Zitrat- und der Kalziumlösung erfolgte automatisiert durch die PF in Abhängigkeit von der eingestellten Zitratdosis pro Liter Blut (mmol/l) und dem vorgewählten Kalzium Ersatz in Prozent vom berechneten Verlust.

Ergebnisse. Insgesamt wurden 13 Patienten mit akutem NV $1600 \mathrm{~h}$ kontinuierlich und 7 Patienten mit chronischem NV über insgesamt 100o-mal ambulant behandelt. Die Kombination der Natrium isotonen Zitratlösung mit 47,5 mmol/l Zitrat, der PrismoCalB22 als Dialysatlösung und Phoxilium als Substitutionslösung ist mit Flussraten und der Filterleistung über den ganzen Bereich des HF 20 Sets kompatibel. Diese Kombination kann für das ST 60 Set und das HF 1000 Set ebenfalls ohne Einschränkung der Flussraten verwendet werden. Die Elektrolyte sind in allen Lösungen in physiologischen Konzentrationen vorhanden, sodass es auch bei langer kontinuierlicher Therapie zu keiner metabolischen Entgleisung kommt. Die Natrium-Konzentration ist in allen Lösungen isoton. Kalium gleicht sich durch 4-mval/l in Dialysat und Substituat aus. Kalzium wird von der Software gesteuert über eine eigene Infusion ausgeglichen. Bikarbonat entsteht durch Metabolisieren von Zitrat und gleicht sich durch physiologische Konzentration in den Lösungen aus. Phosphat wird vorwiegend durch Filtration entfernt, die Substitutionslösung enthält Phosphat in physiologischer Konzentration.

Schlussfolgerung. Die RCA mit der Kombination einer Natrium isotonen Zitratlösung mit 47,5 mmol/1 Zitrat und PrismoCalB22 als Dialysatlösung ist mit allen pädiatrischen Prismaflex ${ }^{-}$-Sets ohne Einschränkungen auf mögliche Flussraten kompatibel. Sowohl bei kontinuierliche als auch intermittierender Behandlung bleiben die Elektrolyte und Metabolik stabil.

\section{FV19}

\section{Surface modification of a polydimethylsiloxane microfluidic oxygenator with dopamine and a covalent antithrombin-heparin complex for the prevention of thrombosis}

\section{J.Leung', L.Berry', N.Rochow ${ }^{3}$, R.Selvaganapathy', C.Fusch ${ }^{5}$,A.Chan' ${ }^{2}$,J.Brash ${ }^{7}$} ${ }^{1}$ McMaster University, Biomedical Engineering, Hamilton, Kanada, ${ }^{2}$ McMaster University, Thrombosis and Atherosclerosis Research Institute, Hamilton, Kana$\mathrm{da},{ }^{3} \mathrm{McM}$ aster University, Division of Neonatology, Department of Pediatrics, Hamilton, Ontario, Kanada, ${ }^{4}$ McMaster University, Mechanical Engineering, Hamilton, Kanada, ${ }^{5}$ McMaster University Medical Center, Dept. of Pediatrics, Division of Neonatology, Hamilton, Kanada

Introduction. Prematurely born infants suffer respiratory insufficiency requiring mechanical ventilation. An alternative to mechanical ventilation is extracorporal membrane oxygenation. Our lab has developed a novel microfluidic oxygenator with polydimethylsiloxane (PDMS) gas transfer membranes [1]. The objective of the work reported here was to modify the PDMS surfaces with a covalent antithrombin-heparin (ATH) complex to prevent coagulation and thrombosis due to bloodmaterial contact. Specifically the ATH was attached to the PDMS using dopamine as a "bio-glue".

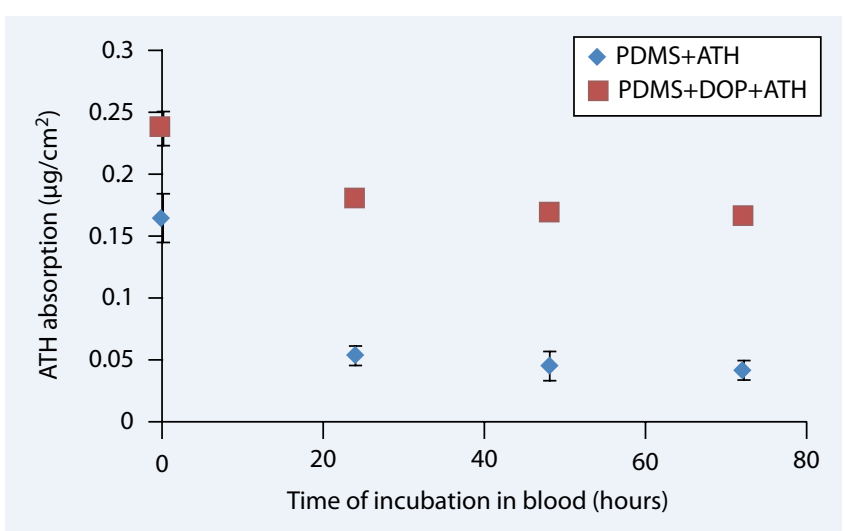

$\triangle$ FV19 - Abb. 1 ATH desorption into blood from PDMS-ATH and PDMS-DOP-ATH surfaces

Materials and methods. PDMS discs were incubated in a solution of dopamine hydrochloride $(1 \mathrm{mg} / \mathrm{mL}$, PBS, $\mathrm{pH}=8.5)$ for $24 \mathrm{~h}$, and then in ATH solution ( $0.1 \mathrm{mg} / \mathrm{mL}, \mathrm{PBS}, \mathrm{pH}=7.4)$ for $3 \mathrm{~h}$. A separate set of PDMS discs were incubated in $0.1 \mathrm{mg} / \mathrm{mL}$ ATH for $3 \mathrm{~h}$. Uptake of ATH on the PDMS and dopamine-PDMS, and adsorption of antithrombin (AT) from plasma (a measure of anticoagulant activity) to the various surfaces was measured using 125I-labelled ATH and AT, respectively. The stability of the ATH on the surfaces was evaluated by incubating in blood for three days and measuring residual radioactivity.

Results and discussion. As shown in Abb. 1, uptake of ATH on PDMS was higher with dopamine as glue. Also, $\sim 74 \%$ of the original ATH was lost from the PDMS+ATH after $3 \mathrm{~h}$ in blood, whereas only $~ 30 \%$ was lost from the PDMS+DOP+ATH. To evaluate the anticoagulant activity of the surfaces as prepared, AT adsorption from plasma was measured. Adsorption was $6.3 \pm 2.7 \mathrm{ng} / \mathrm{cm} 2$ on PDMS $+\mathrm{ATH}$, and $42.3 \pm 2.7 \mathrm{ng} / \mathrm{cm} 2$ on PDMS+DOP+ATH. The ATH surface with dopamine as adhesive thus showed higher AT adsorption, and therefore should have higher anticoagulant activity.

Conclusions. An antithrombin-heparin complex (ATH) was attached to PDMS using dopamine as a bio-glue. The use of dopamine gave surfaces with higher concentration and greater stability of ATH. The bound ATH showed potential for anticoagulant activity through extensive adsorption of antithrombin from plasma.

1. Rochow $\mathrm{N}$ et al (2014) An integrated array of microfluidic oxygenators as a neonatal lung assist device: in vitro characterization and in vivo demonstration. Artifical Organs, in press

\section{FV20}

Pumpless neonatal lung assist device - effects of extracorporeal circulation on cardiovascular function in newborn piglets

\section{N. Rochow', D. Predescu', G. Fusch', S. Monkman' , C. Fusch'}

'McMaster University, Division of Neonatology, Department of Pediatrics, Hamilton, Ontario, Kanada, ${ }^{2}$ McMaster University, Division of Pediatric Cardiology, Hamilton, Kanada

Introduction. A miniaturized lung assist device (LAD) that is perfused like an artificial placenta via umbilical vessels has significant potential to save lives of newborns with respiratory insufficiency. The concept of such LAD was presented recently. Prototype LAD showed feasibility in in vitro and in vivo experiments. The LAD is passively perfused by the newborns own circulation, and extracorporeal flow rates up to $30 \mathrm{~mL} / \mathrm{kg} / \mathrm{min}$ are needed to deliver $40 \%$ of body gas exchange. It is unknown whether this amount of extracorporeal arteriovenous bypass will compromise cardiac function and systemic circulation.

Objective. To test effects of various bypass flow rates on heart rate (HR), blood pressure (BP), cardiac output and lactate production in a piglet model. 
Methods. Newborn piglets ( $\mathrm{n}=7,<2$ days, $1.6-2 \mathrm{~kg}$ ) were anaesthetized (phenobarbital) and ventilated (Servo 300, PIP:15, PEEP:5 cm $\mathrm{H}_{2} \mathrm{O}$, IT:0.5 s, RR:30/min, FiO2:0.3). Maintenance fluids (5\% dextrose at $80 \mathrm{mg} / \mathrm{kg} / \mathrm{d}$ ) were infused. To achieve various high extracorporeal flow rates, the carotic artery and jugular vein were cannulated using low resistance 14 Gauge 1.1" angio catheters and connected to form an extracorporeal bypass. This setup included a mechanical clamp to control blood flow and an ultrasonic inline probe for flow monitoring. HR and BP were measured via femoral artery catheters. Cardiac output (stroke volume, minute volume) was continuously measured throughout the experiment from ECG and velocity time integral at the level of the aortic valve. The echo probe $(12 \mathrm{Mhz})$ from ATL HDI $5000 \mathrm{cv}$ was kept at an identical position throughout the experiment by fixing on a stand.

Results. Baseline HR of piglets $(\mathrm{n}=7)$ was $190 \pm 35 / \mathrm{min}$, BP $85 \pm 10 / 55 \pm 5$ (71 \pm 9$) \mathrm{mmHg}$ [syst./diast. (mean)], and remained stable during the $\sim 3 \mathrm{~h}$ experiment, including shock index (HR/syst.BP). Cardiac output $(n=2)$ increased linearly via increased stroke volume from $198 \mathrm{~mL} / \mathrm{kg} / \mathrm{min}$ to $260 \mathrm{ml} / \mathrm{kg} / \mathrm{min}$ and reflected increasing extracorporeal bypass of up to $60 \mathrm{~mL} / \mathrm{kg} / \mathrm{min}$. Average $\mathrm{pH}$ was 7.47, $\mathrm{pCO}_{2} 40 \mathrm{mmHg}$, pO2 $135 \mathrm{mmHg}$, lactate $1.4 \mathrm{mmol} / \mathrm{L}$. No supportive drugs for BP were needed.

Conclusions. Passive extracorporeal bypass required for LAD of up to $60 \mathrm{~mL} / \mathrm{kg}$ min did not compromise cardiovascular parameters in newborn piglets. The results of this study support the concept of passive perfusion for the "artificial placenta".

\section{FV21}

Einfluss der Hämodialyse auf Kreislaufparameter in der Impedanzkardiographie

\section{Blohm', M. Wilken', A. Lehnhardt', J. Oh², D. Singer'}

'Universitätsklinikum Hamburg Eppendorf, Sektion Neonatologie/Päd. Intensivmedizin, Hamburg, Deutschland, ${ }^{2}$ Universitätsklinikum Hamburg Eppendorf, Pädiatrische Nephrologie, Hamburg, Deutschland

Hintergrund und Fragestellung. Die Impedanzkardiographie (syn. elektrische Velocimetrie, EV) ist ein nichtinvasives kontinuierliches Monitoringverfahren, bei dem aus einer transkutan messbaren Widerstandsänderung gegenüber einem hochfrequenten Wechselstrom verschiedene Kreislaufparameter, wie u. a. Schlagvolumen (SV), Schlagvolumenindex (SVI), Herzindex (CI), sowie Parameter zur thorakalen Flüssigkeit („thoracic fluid content“, TFC) berechnet werden können. Es wurde untersucht, ob die EV den Einfluss der Hämodialyse auf diese Parameter bei pädiatrischen Patienten detektieren kann.

Material und Methoden. Im Rahmen einer Beobachtungsstudie wurden mittels EV (Aesculon', Fa. Osypka Medical, Berlin) vor und nach Dialyse SV, SVI, CI und TFC gemessen. Es wurden 54 Dialysezyklen bei 4 verschiedenen Patienten analysiert. Patientencharakterisierung: Alter im Mittel 12,3 Jahre (5-14 Jahre), Gewicht im Mittel 37,7 kg (16-44 kg), mittlerer BMI 16,1 kg/m2 (13,7-17,6 kg/m2), Flüssigkeitsentzug 0-70 ml/kg Körpergewicht pro Dialyse. Ein Patient mit erhaltener Wasserdiurese wurde ohne Flüssigkeitsentzug dialysiert ( $\mathrm{n}=19$ Dialysezyklen). Die statistische Auswertung erfolgte mit einem gemischten Modell (Mixed Model Analyse).

Ergebnisse. Im Vergleich der Parameter vor und nach Dialyse kam es zu einer signifikanten Abnahme von SV, SVI, CI und TFC ( $\mathrm{p}<0,001)$. Das mittlere SV nahm um durchschnittlich $16,9 \%$ durch die Dialyse ab. Es

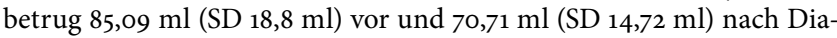
lyse. Auch bei dem Patienten ohne Flüssigkeitsentzug nahm das SV um durchschnittlich $11 \%$ ab. Der SVI nahm um $18 \%$ ab, von durchschnitt-

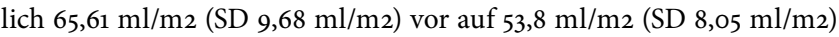
nach Dialyse. Der CI nahm um 11,6\% ab, von 4,67 l/min*m2 (SD o,67 l/ $\min ^{\star}{ }^{2} 2$ ) auf $4,131 / \min ^{\star} m_{2}$ (SD $\left.0,521 / \min ^{\star} \mathrm{m} 2\right)$. Der TFC nahm um $10,8 \%$ von 40,54 (SD 3,32) auf 36,15 (SD 3,43) ab. Relevante Einflussfaktoren in der Mixed Model Analyse waren Messzeitpunkt, Gewicht und Ultrafiltration/kg Körpergewicht.

Diskussion. Die durch die Hämodialyse verursachten Veränderungen der Kreislaufparameter spiegelten sich in den impedanzkardiographischen
Messungen wider. SV, SVI, CI und TFC nahmen erwartungsgemäß mit Flüssigkeitsentzug nach Dialyse ab. Allerdings reagierten diese Parameter auch bei dem ohne Flüssigkeitsentzug dialysierten Patienten in gleicher Weise. Dies könnte entweder durch physiologische Regulationsmechanismen nach Entzug harnpflichtiger Substanzen oder durch den Einfluss einer Änderung der Osmolarität auf die Messmethode EV erklärbar sein. Schlussfolgerung. Bei hämodialysierten Kindern könnte die EV neben dem nicht-invasiven Kreislaufmonitoring einen möglichen Nutzen in der Messung der Flüssigkeitsüberladung oder der Belastung mit osmotisch aktiven harnpflichtigen Substanzen haben, um dadurch Dialyseintervalle zu optimieren.

\section{Hygiene in der Neonatologie und pädiatrischen Intensivmedizin}

\section{FV22}

Nutzen einer aufwendigen mikrobiologischen Surveillance bei Frühgeborenen mit einem Geburtsgewicht unter $1500 \mathrm{Gramm}$ (VLBWI)

\section{W. Lindner', A. Essig'2, H. von Baum², H. Hummler ${ }^{1}$}

'Klinik für Kinder- und Jugendmedizin, Universitätsklinikum Ulm, Sektion Neonatologie und Pädiatrische Intensivmedizin, Ulm, Deutschland, ${ }^{2}$ Universitätsklinikum Ulm, Institut für Medizinische Mikrobiologie und Hygiene, Ulm, Deutschland

Hintergrund und Ziele. Seit 2012 empfiehlt die deutsche KrankenhausInfektions-Kommission (KRINKO) für VLBWI ein mikrobiologisches Screening, um eine Kolonisation mit multiresistenten Erregern zu erkennen. Ausbruchsereignisse können auch durch Pathogene mit normaler Resistenz verursacht werden. Wir untersuchten die postnatale bakterielle Kolonisation bei VLBWI, das endemische Niveau verschiedener klinisch relevanter Pathogene und beschreiben die Auswirkungen auf antibiotische Therapie und Hygienemaßnahmen.

Methodik. Ort: Perinatalzentrum der Universität Ulm. Zeitraum: 7/2012 bis 6/2013. Patienten: Alle VLBWI, die im Studienzeitraum im Zentrum geboren wurden. Die Kolonisation des Respirations- (Rachen-/Trachealsekret) und des Intestinaltraktes (Analabstrich) wurde 1-mal/Woche bis zum Erreichen von $1800 \mathrm{~g}$ untersucht. Eine Differenzierung mit Antibiogramm wurde für S. aureus, Enterobacteriaceae, Pseudomonas spp. und andere gramnegative Nonfermenter (potenzielle nosokomiale Pathogene, PNP) durchgeführt. Nosokomiale Infektionen und die antibiotische Therapie wurde dokumentiert.

Ergebnisse. Insgesamt 839 respiratorische und 661 gastrointestinale Proben von 98 VLBWI wurden untersucht $[4489$ Surveillancetage, Gest.Alter: 28,3 (22,3-34,0) SSW, Geb.-Gew.: 980 (330-1490) g]. PNP wurden früher in den respiratorischen [Tag $7(1-51)]$ als in den gastrointestinalen Proben [Tag 19 (2-53)] gefunden. Im Mittel wurden 2 (o-7) PNP pro VLBWI isoliert. S. aureus wurde früher zu einem vorherrschenden Keim

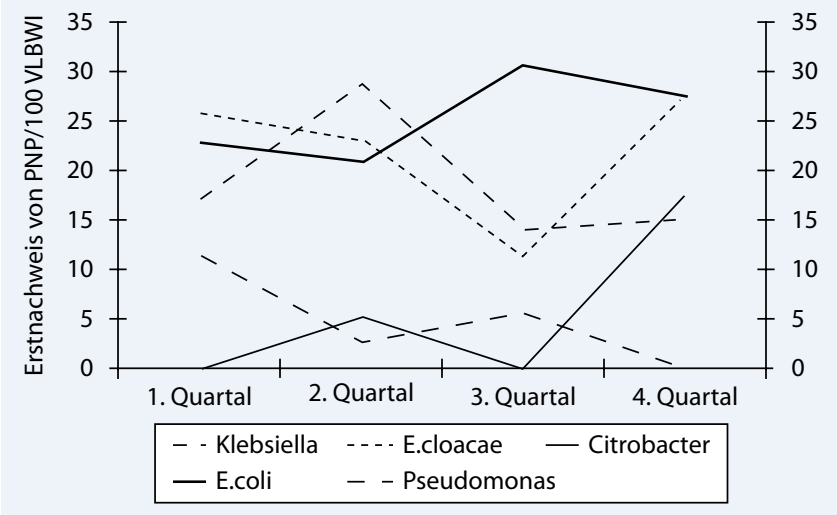

$\Delta \mathrm{FV}_{22}-\mathrm{Abb} .1$ 
als gramnegative Pathogene [7(1-61) vs. 22(2-76) Tage]. 82/98 VLBWI waren mit gramnegativen PNP besiedelt, bei 31 VLWBI gab es mindestens 2 verschiedene Keime. Die Kolonisierung persistierte meistens bis zum Surveillanceende. Acht Species repräsentierten 88\% aller PNP. Das endemische Niveau dieser PNP war in der Studienperiode variabel (Abb. 1). Bei 12/98 VLBWI gab es multiresistente Erreger: S. aureus (MRSA), n=3; E. coli (3MRGN), n=2; Enterobacteriaceae (2MRGN-NeoPäd), n=7. Risikofaktoren waren niedriges Gestationsalter $(\mathrm{p}=0,003)$ und Geburtsgewicht $(\mathrm{p}=0,033)$. Aufgrund der Screeningergebnisse wurden hygienische Barrieremaßnahmen begonnen. Es kam nicht zur Transmission von MRSA oder ESBL-E. coli. V. a. konnatale oder nosokomiale Infektion mit Antibiotikatherapie gab es bei 67 bzw. 37/98 VLBWI. Bei 4/8 Kindern mit Blutkultur positiver Sepsis war der Keim identisch mit einem durch das Screening bereits bekannten Erreger. Das Antibiogramm dieser Erreger wurde bei der empirischen Sepsistherapie berücksichtigt, sodass von Anfang an eine optimale Therapie möglich war.

Schlussfolgerung. Das systematische mikrobiologische Screening der Kolonisation von VLBWI ist hilfreich bei der empirischen Antibiotikatherapie schwerer Infektionen. Die Transmission multiresistenter Erreger kann durch frühe Einrichtung von Barrieremaßnahmen verhindert werden. Die Kenntnis des üblichen endemischen Niveaus lässt das gehäufte Auftreten eines PNP früh erkennen. Dies kann hilfreich sein, drohende Ausbrüche zu verhindern.

\section{Komplementäre Therapie}

\section{FV23}

Ich bin in meinem ganzen Leben noch nie so hilflos gewesen. Die Gefühle und das Erleben von Vätern frühgeborener Kinder während deren stationärer Versorgung auf einer neonatologischen Intensivstation - eine qualitative Untersuchung

\section{J. Binter', T. Garzuly-Rieser \\ 'SALK - Salzburger Universitätsklinikum, Division für Neonatologie, Hallein, Österreich, ${ }^{2}$ SALK - Salzburger Universitätsklinikum, Division für Neonatologie, Salzburg, Österreich}

Hintergrund. In Österreich kommen jährlich rund 6500 Babys als Frühgeburten zur Welt. Der Übergang zur Vaterschaft ist für den Mann ein ausgesprochen intimes und privates Erlebnis, das zwangsläufig Stress und Belastungen mit sich bringt. Die frühzeitige Unterbrechung der Schwangerschaft und der nicht abgeschlossene vorgeburtliche Beziehungsaufbau können in weitreichenden Konsequenzen münden. Im deutschsprachigen Raum wurde mit dieser Studie ein kaum erforschtes Terrain betreten und stellt Väter frühgeborener Kinder in den Mittelpunkt des Interesses. Die Fragen der vorliegenden Studie lauten: Welche Gefühle erfahren Väter und wie erleben sie die Zeit der stationären Versorgung ihrer frühgeborenen Kinder auf einer neonatologischen Intensivstation?

Material und Methode. Das Ziel der Studie bestand darin die Gefühle und Erlebnisse von Vätern frühgeborener Kinder während deren stationärer Versorgung auf einer neonatologischen Intensivstation zu erkunden und transparent darzustellen, sowie für die in der Betreuung involvierten Personen einen Beitrag zum besseren Verständnis von Vätern frühgeborener Kinder zu leisten. Es bot sich an, die Fragen nach dem Erleben mit einem qualitativen Forschungsansatz zu beantworten. Das Ziel bestand darin, ein Phänomen aus der Perspektive der Betroffenen zu erkunden und dessen Bedeutung darzustellen. Emotional bewegte Erlebnisse können nur von den Betroffenen besonders gut geschildert werden. Die Untersuchungsgruppe setzte sich aus einer Gelegenheitsstichprobe von acht Vätern frühgeborener Kinder zusammen und generierte eine zufriedenstellende Sättigung der Daten. Die Datensammlung erfolgte mittels episodischer Interviews. Ein zugrundeliegender Leitfaden beinhaltete die zu besprechenden Themen. Bei der Datenanalyse wurde ein interpretatives-reduktives Verfahren gewählt. In der deskriptiven Vorgehensweise wurden nur sichtbare Bedeutungen und das tatsächlich Gesagte und Niedergeschriebene interpretiert. Der Text wurde reduziert und in Kategorien zusammengefasst sowie miteinander in einen Kontext gebracht.

Ergebnisse. Die Betroffenen erlebten ein Wechselbad der Gefühle, wobei die Dominanz von negativen Emotionen allgegenwärtig war. Väter nehmen Belastungen in sehr unterschiedlichen Bereichen war. Die Dimensionen reichten vom Aussehen und Zustand des Kindes, Sorge um die Partnerin, Als Mann stark sein müssen, Erhaltene Informationen, Das Personal der Station, Sich selbst belasten, Angriffe auf die Alltagsroutine, einen Schuldigen haben wollen bis hin zum Schaudern vor der Intensivstation. Dem gegenüber zeigten Väter auch deutlich positive Aspekte auf. Diese Dimensionen reichten hier vom Kind - Nähe spüren, richtig informiert, durch das Personal der Station umfassend betreut sein, Unterstützung im Alltag erleben bis hin zum Wohlfühlambinente - Intensivstation.

Diskussion und Schlussfolgerung. Eine Vielzahl von identifizierten Belastungen und positiven Erfahrungen wirkten auf Väter frühgeborener Kinder ein. Die Ergebnisse der Studie stellen wichtige Erkenntnisse für den klinischen Alltag dar. Es ist daher von Bedeutung, dass alle in der Betreuung involvierten Personen hierfür sensibilisiert werden. Im Besonderen wird eine maximale Integration der Väter in die Betreuung ihrer frühgeborenen Kinder und die Bildung von Kernteams gefordert.

\section{FV24}

\section{Aromatherapie in der Neonatologie: Literaturübersicht und Erklärungsansätze}

\section{Thiel}

'Sana-Klinikum Remscheid, Klinik für Kinder und Jugendliche, Remscheid, Deutschland

Hintergrund und Zielsetzung. Unter den neonatologischen Behandlungsmethoden sind neben den konventionellen Verfahren wie Beatmung bzw. deren Optimierung und medikamentöse Therapien auch Maßnahmen wie Känguru-Pflege und Minimal-Handling etabliert. Zahlreiche alternative und komplementäre Verfahren werden zwar eingesetzt haben aber oft keine gute Evidenz oder sind gar nicht untersucht. Wir führten eine systematische Literaturrecherche zum Thema Aromatherapie durch, um deren Evidenz abschätzen zu können.

Methodik. Systematische Literaturrecherche nach den PRISMA-Kriterien. Englisch und Deutsch, Aromatherapie und Neonates/Neonatologie/Früh- und Neugeborene, klinische Studien.

Ergebnisse. Es wurden fünf Studien gefunden, die die Einschlusskriterien erfüllten. Bei den untersuchten Substanzen handelte es sich um Vanillin und Lavendel. Der klinische Kontext waren Apnoen/Bradykardien einerseits sowie Schmerz(-Score) und Stress andererseits. Alle Studien zeigten positive Ergebnisse. Zudem sind keine Nebenwirkungen beschrieben.

Diskussion. Es liegen Daten zur Aromatherapie bei Früh- und Neugeborenen vor, die positive Effekte auf Stress, Schmerzscore sowie Apnoen und Bradykardie bei Neonaten zeigen konnten. Als Erklärungsansatz für diese Effekte auch beim unreifen Gehirn könnte dienen, dass ontogenetisch alte Hirnabschnitte (u. a. das Riechhirn) weitreichende Beziehung zum limbischen System und zum autonomen Nervensystem haben bzw. dass es sich ja dabei auch bereits um ,ältere“ Systeme handelt. Diese physiologischen Grundlagen können erklären, warum Geruchssensationen entsprechende, auch vegetative, Reaktionen hervorrufen können. Der unterlagernde Gedanke ist, dass diese „alten“ Hirnabschnitte und seine Reaktionsweisen bereits bei Frühgeborenen vorhanden und in ihrer Reaktionsweise ähnlich sind. Die Ergebnisse reichen für eine allgemeingültige Empfehlung nicht aus. Immerhin liefern sie hinreichend Anlass, sich mit der Thematik weiter auseinander zu setzen.

Schlussfolgerung. Die wenigen vorliegenden Daten sind vielversprechend und können zum Anlass genommen werden weitere Studien durchzuführen. 
FV25

Pflege von Kindern mit komplexen angeborenen Fehlbildungen am Beispiel von Kindern mit intrauterin bekanntem Neuralrohrdefekt

\section{Ditscheid', B. Wiebe', M. Messing-Jünger' ${ }^{2}$ M. Ehlen}

'Asklepios Klinik St. Augustin, Neonatologie und Pädiatrische Intensivmedizin, St. Augustin, Deutschland, ${ }^{2}$ Asklepios Kinderklinik Sankt Augustin, Abteilung für Kinderneurochirurgie, Sankt Augustin, Deutschland

Hintergrund. Viele medizinische Fachdisziplinen arbeiten in der Behandlung von Kindern mit komplexen angeborenen Fehlbildungen von der pränatalen Diagnostik über das perinatale Management bis zur Entlassung und weiter in der ambulanten Betreuung Hand in Hand zusammen. Dabei spielt neben der ärztlichen Versorgung die kompetente Betreuung der pflegerisch häufig sehr aufwändigen Patienten durch geschulte Pflegekräfte die entscheidende Rolle. Auch die Wichtigkeit des Pflegepersonals in einem umfassenden familienzentrierten Betreuungskonzept wird in der Regel unterschätzt.

Methoden. Am Beispiel von Kindern mit angeborenen Neuralrohrdefekten werden die Aufgaben und das an unserem Zentrum etablierte Therapiekonzept aus der Sicht der neonatologischen Pflegefachkraft detailliert erläutert. Hauptaugenmerk liegt dabei auf der Darstellung der Phase der postnatalen Betreuung bis zur ersten Entlassung der Kinder in die häusliche Umgebung. Insbesondere die zentrale Rolle im Aufbau der Eltern-Kind-Bindung vom ersten Bonding im Kreißsaal bis zur selbstverantwortlichen Pflege des Kindes durch die Eltern am Entlassungstag erfordert ein hohes Maß an Kompetenz und Einfühlungsvermögen in die besonderen Ansprüche der Kinder und deren Eltern.

Schlussfolgerung. Fast 40 Kinder mit intrauterin bekannten Neuralrohrdefekten wurden in den letzten 7 Jahren in unserem Zentrum entbunden. Vorgestellt wird ein einheitliches Pflege- und Therapiekonzept, welches auch die unterschiedlichen Ausprägungen und Lokalisationen der Erkrankung berücksichtigt. Herausgehoben wird hierbei die zentrale Bedeutung einer kompetenten pflegerischen Versorgung für die bestmögliche Versorgung und Betreuung der Kinder und deren Familien.

\section{FV26 \\ Elternberatung in der neonatologischen Intensivversorgung - Evaluation eines Beratungsmodells}

\section{Thierfelder \\ ${ }^{1}$ Charite - Universitätsmedizin Berlin Institut für Gesundheits- und Pflegewis- senschaft, Berlin, Deutschland}

Hintergrund. Im Jahr 1994 wurde an der Charité aus der Gesundheitsund Kinderkrankenpflege eine Initiative gestartet, die das Ziel verfolgt, Eltern zu früh geborener Kinder in dieser Krisensituation edukativ zu unterstützen. Die Unterstützung umfasst neben Informationsangeboten auch beratende und anleitende Interventionen zur Begleitung der Eltern bei der individuellen Versorgung des zu früh geborenen Kindes. Wurde diese Tätigkeit anfangs noch informell in den Stationsablauf integriert, sind heute mehrere Gesundheit- und Kinderkrankenpflegerinnen von der pflegerischen Versorgung entbunden, um die edukative Begleitung der Eltern gewährleisten zu können. Ziel dieses Vorhabens war es, die gesammelten Erfahrungen zu resümieren und erstmals einer wissenschaftlichen Evaluation zu unterziehen.

Methoden. Gefragt wurde, welchen konkreten edukativen Unterstützungsbedarf Eltern zu früh geborener Kinder haben, welche Angebote sie sich zur Beantwortung desselben wünschen und ob ihre Erwartungen in der bisherigen Praxis berücksichtigt sind oder eine Anpassung erfolgen muss. Kontrastierend wurde die Perspektive der Anbieter des Unterstützungsangebots - der Gesundheits- und Kinderkrankenpflegerinnen - in die Untersuchung einbezogen. Zur Beantwortung dieser Fragestellungen wurde eine qualitative Befragung relevanter Akteure mittels Interviews durchgeführt. Im Mittelpunkt stand hierbei die Befragung von Eltern zu früh geborener Kinder $(n=12)$ in verschiedenen Phasen der Krisensituation (präpartal, postpartal-stationär, postpartal- ambulant) und der anbietenden Gesundheits- und Krankenpflegerinnen $(n=12)$. Die erhobenen Daten wurden inhaltanalytisch ausgewertet und mit dem vorhandenen Konzept abgeglichen.

Ergebnisse. Die Ergebnisse zeigen, dass Eltern das Ereignis Frühgeburt als eine lang anhaltende, hochbelastete Phase erleben, die von Hilflosigkeit, Orientierungslosigkeit und hoher Unsicherheit geprägt ist. Die Bedürfnisse, die aus diesem Erleben erwachsen, unterscheiden sich in überdauernde und phasenabhängige Bedürfnisse. Zentral sind hierbei die ausgeprägten Bedürfnisse nach umfassender Information und Aufklärung sowie kontinuierlicher Kommunikation mit und zwischen den involvierten professionellen Akteuren. Edukative Interventionen schienen insgesamt einen positiven Einfluss auf elterliche Bewältigungsprozesse zu haben.

Schlussfolgerung. In der neonatologischen Intensivversorgung muss der Fokus auf krankheitsbezogene Belastungen des Kindes um die alltagsbezogene Belastungen des Systems Familie erweitert werden. Hierfür ist es notwendig, objektive Bedarfsbestimmungen durch professionelle Akteure um subjektive Unterstützungsbedürfnisse der Eltern zu ergänzen. Insbesondere edukative Interventionen gewinnen hierbei an Bedeutung, da sie auf die Befriedigung zentraler elterlicher Bedürfnisse wie informiert sein und einbezogen werden zielen und somit zunehmend zum eigenständigen Umgang mit der Krisensituation Frühgeburt befähigen.

\section{FV27}

\section{Szenen der Eltern-Kind-Interaktion in der Versorgungssituation} Baden

\section{Sommerfeld', J. Bonitz', A. Freymann', F. Fischer', J. Reichert', M. Rüdiger}

'Universitätsklinikum Carl Gustav Carus Kinderklinik, Neonatologie/Pädiatrische Intensivmedizin, Dresden, Deutschland

Hintergrund. Die Anleitung der Eltern in der selbstständigen Pflege und Versorgung ihres Kindes entspricht einer Grundforderung der familienzentrierten Pflege; sie sollen als die "natürlichen“ Bezugspersonen des Kindes weitgehend alle kindesbezogenen Handlungen der Pflege ihres Kindes selbst übernehmen. Hiermit verbindet sich auch die Erwartung, dass die Eltern-Kind-Interaktion sowie der Eltern-Kind-Bindungsprozess unterstützt werden. Um für die Eltern den Lern- und Aneignungsprozess eigener Pflege- und Versorgungsleistungen sichtbar zu machen und die Entwicklung ihrer Selbstkompetenzen zu unterstützen, bietet sich ein gezieltes Feedback an. Hierfür hat sich die Anleitungssituation Baden bewährt, die videographiert und gemeinsam mit den Eltern nachbereitet wird, wobei nicht alle Videosequenzen für ein positiv gerichtetes Feedback und das selbstentdeckende Lernen der Eltern geeignet sind. Eine Auswahl erweist sich somit als notwendig.

Fragestellung. Welche Szenen eignen sich für die Rückmeldung einer gelungenen Eltern-Kind-Interaktion bzw. zur Einschätzung von elterlichen Kompetenzen im Videofeedback?

Methodik. Zur Vorbereitung des videogestützten Feedbacks werden fünf Videosequenzen nach definierten Kriterien anhand von Szenenbeurteilungsbögen ausgewählt. 77 Szenenbeurteilungsbögen (anfallende Stichprobe, Kinder mit GG $\leq 1500 \mathrm{~g}$ und oder $\mathrm{GA} \leq 32+\mathrm{o} S \mathrm{SW}$ ) gingen in eine deskriptive Auswertung ein.

Ergebnisse. Die Auswertung ergab, dass einzelne Videosequenzen ausgewählter Versorgungshandlungen mehrheitlich für die Rückmeldung einer gelungenen Eltern-Kind-Interaktion ausgewählt wurden. In unterschiedlichem Ausmaß zeigen die gewählten Sequenzen die Offenheit des Kindes zur Interaktion, Signale seiner psychophysischen Regulation in Anforderungssituationen sowie den damit im Zusammenhang stehenden Unterstützungsangeboten der Eltern.

Diskussion. Trotz theoriegeleiteter Vorauswahl der Videosequenzen für ein positiv gerichtetes Feedback und das selbstentdeckende Lernen der Eltern zeigen sich zwischen den gewählten Videosequenzen Unterschiede in Bezug auf die Interaktionsoffenheit des Kindes, seine 
psychophysische Regulation sowie den elterlichen Unterstützungsangeboten. Diese Kenntnisse sind hilfreich bei der Planung des Feedbacks und dem Anregen des selbstentdeckenden Lernens der Eltern in der Situation des videogestützten Feedbacks.

Schlussfolgerung. Die Qualität eines positiv gerichteten, videogestützten Feedbacks und des selbstentdeckenden Lernens der Eltern kann durch eine theoriegeleitete Vorauswahl von Videosequenzen sowie die Kenntnis ihres Informationsgehalts beeinflusst werden.

\section{FV28 \\ Beeinflusst viszerale osteopathische Behandlung des Abdomens die Mekoniumausscheidung beim Frühgeborenen}

\section{N. Haiden', B. Pimpel', A. Kreissl', A. Berger'}

'Universitätsklinik für Kinder- und Jugendheilkunde Wien, Klinische Abteilung für Neonatologie, Pädiatrische Intensivmedizin und Neuropädiatrie, Wien, Österreich

Ziel. Frühgeborene mit einem Geburtsgewicht unter $1500 \mathrm{~g}$ haben aufgrund ihrer Unreife eine verzögerte Mekoniumausscheidung, die mit einem erschwerten enteralen Nahrungsaufbau und nachfolgenden Problemen assoziiert ist. Der alternativmedizinische Ansatz der viszeralen Osteopathie könnte die Mekoniumausscheidung verkürzen und den Nahrungsaufbau beschleunigen.

Methoden. Im Rahmen einer prospektiven, randomisierten, kontrollierten Studie wurden Frühgeborene mit einem Geburtsgewicht unter $1500 \mathrm{~g}$ und einem Gestationsalter von unter 32 Schwangerschaftswochen 3- mal in der ersten Lebenswoche mit viszeralen osteopathischen Techniken behandelt und mit einer Kontrollgruppe, die keine Behandlung erhielt verglichen.

Ergebnisse. Die Mekoniumpassage dauerte 7,5 Tage (95\% CI 6-9 Tage, $\mathrm{n}=20$ ) in der Interventionsgruppe und 6 Tage (95\% CI 5-9 Tage, $\mathrm{n}=21$ ) in der Kontrollgruppe $(p=0,11)$; Die osteopathische Behandlung war mit einem um 12 Tage längeren Nahrungsaufbau $(\mathrm{p}=0,02)$ und einem um 44 Tage längeren Krankenhausaufenthalt assoziiert. Die Behandlung selbst wurde von allen Patienten gut toleriert.

Schlussfolgerung. Die Behandlung mit viszeralen osteopathischen Techniken hatte keinen Einfluss auf die Mekoniumausscheidung bei Frühgeborenen unter $1500 \mathrm{~g}$ Geburtsgewicht. Allerdings hatten die Kinder einen langsameren Nahrungsaufbau und einen längeren Spitalsaufenthalt als die Kinder in der Kontrollgruppe, was als negativer Effekt der Behandlung interpretiert werden muss. Weitere klinische Studien mit anderen osteopatischen therapeutischen Ansätzen sind unbedingt erforderlich, da die Anwendung von viszeralen osteopathischen Techniken in dieser Patientengruppe derzeit nicht empfohlen werden kann.

\section{Angeborene Immunität}

\section{FV29 \\ RAGE steuert reifealterabhängig die Leukozytenadhäsion von Früh- geborenen}

\section{K. Buschmann', N. Braach', R. Tschada', M. Metzger', N. Kuß', H. Hudalla',} L. Koch', J. Pöschl', D. Frommhold'

'Universitätsklinikum Heidelberg, Zentrum für Kinder- Jugendmedizin, Klinik Kinderheilkunde IV/Neonatologie/Angelika-Lautenschläger-Klinik, Heidelberg, Deutschland

Fragestellung. Das unreife Immunsystem mit eingeschränkter Leukozytenrekrutierung ist ein Grund für die hohe Morbidität und Mortalität von Früh- und Neugeborenen mit bakteriellen Infektionen. Der receptor for advanced glycation end products (RAGE) ist als Ligand des $\beta_{2}$-Integrins macrophage-1-antigen (MAC1) an der Leukozytenrekrutierung beteiligt. Aufgrund seiner hohen fetalen Expression wurde die Bedeutung von RAGE für die Leukozytenadhäsion untersucht.

Material und Methoden. Neutrophile Granulozyten wurden aus Nabelschnurblut von Früh- und Neugeborenen (<30 SSW, 30-35 SSW, >35
SSW) isoliert und fluoreszenzmarkiert. Unter konstantem Leukozytenstrom wurde in Mikroflusskammern mit P-Selektin, Interleukin 8 und ICAM1- bzw. sRAGE-Beschichtung die Leukozytenadhäsion fluoreszenzmikroskopisch untersucht. Ferner wurden die Plasmakonzentration von löslichem RAGE (sRAGE) mittels ELISA und die Expression der MAC1 und lymphocyte function associated antigen (LFA1) durchflusszytometrisch analysiert und die Ergebnisse mit denen von gesunden erwachsenen Probanden verglichen.

Ergebnisse. Im Flusskammermodell war eine gute Adhäsion neonataler und adulter Leukozyten auf P-Selektin, IL8 und ICAM1 zu beobachten. Diese war vergleichbar hoch bei P-Selektin/IL8/sRAGE Beschichtung. Sowohl die RAGE- als auch die ICAM1-abhängige Adhäsion von Leukozyten stieg allerdings erst mit zunehmender Reife des Feten auf das adulte Niveau an. So betrug die Zahl adhärenter Leukozyten bei sehr unreifen Frühgeborenen $7,9 \pm 1,5$ bzw. $6,9 \pm 1,5$ und $8,1 \pm 1,8$ bzw. $9,5 \pm 1,7$ bei unreifen Frühgeborenen sowie bei reifen Neugeborenen 11,6 $\pm 0,8$ bzw. $10,5 \pm 0,8$ Zellen (für sRAGE bzw ICAM1). Die sRAGE-Plasmakonzentration korrelierte invers mit dem Gestationsalter und erreichte die niedrigsten Spiegel bei Erwachsenen. Die Expression der an RAGE und ICAM1 bindenden Integrine MAC1 und LFA1 veränderte sich während der fetalen Entwicklung jedoch nicht.

Diskussion. Die vorliegenden Ergebnisse zeigen, dass RAGE die Leukozytenadhäsion auch schon bei kleinen Frühgeborenen vermitteln kann. Die Rekrutierungsfähigkeit der Leukozyten reift allerdings (in Übereinstimmung mit eigenen und anderen In-vitro- und In-vivo-Beobachtungen) erst im Laufe der Schwangerschaft aus. Da die sRAGE/ RAGE-Expression in der Fetalzeit sehr hoch war, die der $\beta_{2}$-Integrine aber alterskonstant blieb, ist RAGE eine große Bedeutung für die Leukozytenrekrutierung und damit der zellulären Abwehr von sehr kleinen Frühgeborenen zuzuschreiben. Weitere Studien müssen den Stellenwert von RAGE bei neonatalen Infektionen auch im Hinblick auf die Möglichkeit neuer therapeutischer Optionen, wie z. B. löslichem RAGE beleuchten.

Schlussfolgerung. RAGE nimmt eine Schlüsselrolle bei der Leukozytenrekrutierung von Früh- und Neugeborenen ein.

\section{FV30}

RAGE reguliert die Aktivierung und das antiinflammatorische Signaling von Protein C

N. Braach', K. Buschmann', J. Pflaum', L. Koch', H. Hudalla', K. Staudacher', H. Wang' ${ }^{2}$, B. Isermann', P. Nawroth', J. Pöschl', D. Frommhold'

'Universitätsklinikum Heidelberg, Zentrum für Kinder- Jugendmedizin, Klinik Kinderheilkunde IV/Neonatologie/ Angelika-Lautenschläger-Klinik, Heidelberg, Deutschland, ${ }^{2}$ Medizinische Fakultät, Institut für Klinische Chemie und Pathobiochemie, Magdeburg, Deutschland, ${ }^{3}$ Universitätsklinikum Heidelberg - Medizinische Klinik, Innere Medizin I und Klinische Chemie, Heidelberg, Deutschland

Fragestellung. Der receptor for advanced glycation endproducts (RAGE) vermittelt eine Vielzahl von Krankheiten, u. a. akute und chronische Entzündungen. Da Protein C (PC) u. a. auch anti-inflammatorische Eigenschaften besitzt, war es das Ziel die Rolle von RAGE bei der Vermittlung dieser PC-Wirkungen zu beleuchten.

Methoden. Dazu sollte das anti-inflammatorische Potential von PC (Leukozytenrekrutierung/Überleben) bei lokaler und systemischer Entzündung in WT und RAGE defizienten Mäusen untersucht werden. Ferner sollten zugrunde liegende Mechanismen, wie RAGE abhängige PC-Aktivierung und -Signaling aufgeklärt werden.

Ergebnisse und Diskussion. Durch die Applikation von PC (10o U/kg), konnte die Leukozyteninfiltration in entzündliches Gewebe von WTMäusen (TNFa-/Trauma-induzierter Muskelentzündung, LPS-induzierten akutem Lungenversagen) inhibiert und das Überleben beim LPS-induzierten SIRS verlängert werden. In Abwesenheit von RAGE konnte PC diese anti-inflammatorischen Effekte nicht auslösen. Hingegen wurde durch aktiviertes PC ( $24 \mu \mathrm{g} / \mathrm{kg} / \mathrm{h}$ für $3 \mathrm{~h}$ ) im TNFa-Entzündungsmodell 
die Leukozytenrekrutierung sowohl in WT- als auch in RAGE-/--Mäusen blockiert. Die nachfolgende Untersuchung des PC-Aktivierungsprozesses zeigte, dass RAGE sowohl in vivo als auch in vitro an der Aktivierung von PC beteiligt ist. Eine mögliche Ursache dafür ist die verringerte endotheliale Expression von Thrombomodulin und endothelialem PCRezeptor in Abwesenheit von RAGE. Ferner scheinen in der TNFa-induzierten Aktivierung intrazellulärer Signalwege und der nachfolgenden Adhäsionsmolekül-Expression die immunmodulatorischen Funktionen von RAGE und PC miteinander verbunden zu sein.

Schlussfolgerung. RAGE steuert anti-inflammatorische Eigenschaften und Aktivierung von PC.

\section{FV32}

\section{LFA-1 vermittelt anti-inflammatorische Eigenschaften von Protein C}

N. Braach', K. Buschmann', E. Ryschich'2 , H. Wang ${ }^{3}$, R. Pick ${ }^{4}$, B. Isermann ${ }^{3}$, B. Walzog ', J. Pöschl', D. Frommhold'

'Universitätsklinikum Heidelberg, Zentrum für Kinder- Jugendmedizin, Klinik Kinderheilkunde IV/Neonatologie/ Angelika-Lautenschläger-Klinik, Heidelberg, Deutschland, ${ }^{2}$ Universitätsklinikum Heidelberg, Klinik für Allgemeine, Viszerale und Transplantationschirurgie, Heidelberg, Deutschland, ${ }^{3}$ Medizinische Fakultät, Institut für Klinische Chemie und Pathobiochemie, Magdeburg, Deutschland, ${ }^{4}$ Ludwig-Maximilians-Universität, Walter-Brendel-Zentrum für Experimentelle Medizin, München, Deutschland

Fragestellung. Das $\beta_{2}$-Integrin LFA-1 vermittelt die feste Adhäsion der Leukozyten an das Endothel. Da Protein C (PC) die Leukozytenadhäsion bei entzündlichen Prozessen inhibiert und mit Integrinen interagieren kann, sollte geklärt werden ob und wie LFA-1 die PC-induzierte Hemmung der Leukozytenadhäsion und andere anti-inflammatorische Effekte beeinflusst.

Methoden. Die Wirkung von PC auf die Leukozytenrekrutierung bei lokaler und systemischer Entzündung wurde im Mausmodell u. a. intravitalmikroskopisch unter Antikörperblockade bzw. Knockout von LFA-1 untersucht. Darüber hinaus wurde das Überleben von WT- und LFA-1-/--Mäusen bei systemischer Entzündung in Abhängigkeit von PC beobachtet. Die mögliche Interaktion von PC mit LFA-1 und zugrunde liegende Mechanismen wurden beleuchtet.

Ergebnisse und Diskussion. Während die PC-Behandlung (100 U PC/ $\mathrm{kg}, 3 \mathrm{~h}$ ) die TNFa- und Trauma-induzierte Leukozytenadhäsion in WTMäusen reduzierte, versagte PC in Abwesenheit von LFA-1 (Knockout). Diese Ergebnisse konnten in weiteren Entzündungsmodellen (LPS-induziertes akutes Lungenversagen, LPS-induziertes SIRS) bestätigt werden. Nach LFA-1-Antikörperblockade hingegen konnte PC die Leukozytenadhäsion in WT-Mäusen effektiv inhibieren. Im Gegensatz zum Zymogen PC war eine Behandlung mit aktiviertem PC (aPC, $24 \mu \mathrm{g} / \mathrm{kg} / \mathrm{h}$ für $3 \mathrm{~h}$ ) in LFA-1 defizienten -Mäusen anti-inflammatorisch wirksam, was auf eine Beteiligung von LFA-1 im PC-Aktivierungsprozess hinweist. Die insuffiziente PC-Aktivierung in LFA-1-defizienten Mäusen, erhärtete diesen Verdacht. Weitere Untersuchungen deuteten auf eine Kolokalisation von LFA-1 und PC/PC-bindenden Molekülen, jedoch ohne direkte Bindung von PC an LFA-1. Somit ist eine Interaktion von LFA-1 mit Schlüsselmolekülen der PC-Aktivierung sehr wahrscheinlich.

Schlussfolgerung. Bestimmte anti-inflammatorische Eigenschaften von PC werden durch LFA-1 vermittelt.

\section{FV33}

Mechanismen der fetomaternalen Toleranz: myeloide Suppressorzellen akkumulieren in der Plazenta und hemmen die T-Zellantwort

\section{K. Hofstädter', N. Köstlin', B. Spring', C. Poets', C. Gille}

'Eberhard-Karls-Universität Universitätsklinik für Kinderheilkunde und Jugendmedizin, Neonatologie, Tübingen, Deutschland

Hintergrund. Während der Schwangerschaft müssen mütterliches und fetales Immunsystem eine Balance zwischen Toleranz gegenüber einem semiallogenen Organismus und gleichzeitig funktionierender Infektab- wehr halten. Im Falle eines Ungleichgewichts kann es zu Komplikationen wie Abort oder Frühgeburtlichkeit kommen. Myeloide Suppressorzellen (MDSC) sind myeloide Vorläuferzellen, die vor allem im Rahmen pathologischer Prozesse wie Tumorerkrankungen stark vermehrt sind und die T-Zellantwort hemmen. Unsere Vorergebnisse zeigen, dass MDSC auch im Nabelschnurblut und im Blut gesunder Schwangerer in erhöhter Zahl vorkommen. Inwieweit MDSC eine Rolle für die Aufrechterhaltung der fetomaternalen Toleranz spielen ist bisher unklar. Hypothese. MDSC akkumulieren in der Plazenta und hemmen die Aktivierung von T-Zellen.

Methoden. Mononukleäre Zellen wurden aus peripherem Blut Schwangerer am Tag der Entbindung (S-PBMC), dem zugehörigem Nabelschnurblut (CBMC) und der zugehörigen Plazenta $(\mathrm{PlaC})$ isoliert und der Anteil an CD66b+/CD33+/CD14-/HLA-DR-granulozytären MDSC (GRMDSC) sowie die die Expression der Enzyme Arginase I (ARG1) und induzierbare NO-Synthetase (iNOS) durchflusszytometrisch ermittelt. Die Produktion von reaktiven Sauerstoffspezies (ROS) vor und nach Stimulation mit Phorbol-Myristat-Acetat (PMA) wurde durchflusszytometrisch mittels Dihydrorhodamin (DHR) bestimmt. Zur Anreicherung von GRMDSC wurden CD66b-positive Zellen mittels „magnetic activated cell sorting" (MACS) isoliert. Isolierte MDSC aus PlaC wurden zu Carboxyfluorescein Succinimidyl Ester (CFSE)-markierten PBMC gegeben. Nach 96 h Stimulation mit anti-CD3 $\left(\mathrm{OKT}_{3}\right)$ und Interleukin-2 (IL-2) wurde die CD4- und CD8-T-Zell-Proliferation durchflusszytometrisch gemessen. Als Vergleich dienten PBMC ohne Zugabe von MDSC.

Ergebnisse. Der Anteil an GR-MDSC war in PlaC ca. 1,5-fach höher als in CBMC $(3,4 \pm 1,7 \%$ vs. $2,0 \pm 1,5 \%)$ und ca. 2 -fach höher als in S-PBMC $(1,7 \pm 0,7 \%)$. GR-MDSC aus PlaC exprimierten die Effektorenzyme ARG1 und iNOS. Sowohl die basale (23,9 $\pm 4,7$ MFI vs. $16,5 \pm 3,5$ MFI) als auch die PMA-induzierte ROS-Produktion $(388,8 \pm 338,3$ vs. $82,0 \pm 33,6)$ war in GRMDSC aus PlaC deutlich höher als in GR-MDSC aus S-PBMC. Die OKT3und IL-2-induzierte CD4-T-Zell-Proliferation betrug in PBMC ohne Zugabe von GR-MDSC $79,6 \pm 8,0 \%$ und wurde durch Zugabe von GR-MDSC aus $\mathrm{PlaC}$ konzentrationsabhängig auf $42,9 \pm 19,1 \%$ und $62,2 \pm 16,1 \%$ gehemmt. Die CD8-T-Zellproliferation wurde konzentrationsabhängig von $81,0 \pm 14,6 \%$ auf $44,9 \pm 19,9 \%$ bzw. $66,2 \pm 14,7 \%$ gehemmt.

Schlussfolgerung. GR-MDSC reichern sich im Bereich der fetomaternalen Grenzschicht an und exprimieren die Effektorenzyme ARG1 und iNOS. Sie haben ein deutlich höheres Potential ROS zu produzieren als GR-MDSC aus peripherem Blut und hemmen in vitro die $\mathrm{CD}_{4}$ - sowie die CD8-T-Zell-Proliferation. Die Akkumulation und funktionelle Aktivierung von GR-MDSC in der Plazenta könnte eine wichtige Rolle für die Aufrechterhaltung der fetomaternalen Toleranz spielen.

\section{FV34}

Die Überexpression von BCL-2 führt zu verlängertem Überleben von myeloiden Suppressorzellen nach Phagozytose von Escherichia coli

\section{J. Schwarz', A. Leiber', N. Köstlin', B. Spring', C. Poets', C. Gille}

'Eberhard-Karls-Universität Universitätsklinik für Kinderheilkunde und Jugendmedizin, Neonatologie, Tübingen, Deutschland

Hintergrund. Myeloide Suppressorzellen (MDSC) sind Vorläuferzellen von Granulozyten, die nach bisherigen Erkenntnissen Immunantworten hemmen. Unsere Vorergebnisse zeigen, dass ihre Anzahl auch im Nabelschnurblut (NSB) stark erhöht ist. Wie es zur Akkumulation von MDSC kommt und ob sie neben der Immunsuppression auch an der Abwehr von Bakterien beteiligt sind ist unklar. Auch ist nicht bekannt, ob sie sich hinsichtlich ihrer Apoptoseeigenschaften von reifen Granulozyten unterscheiden.

Hypothese. MDSC aus NSB können Bakterien phagozytieren und zeigen eine verminderte Apoptose.

Methoden. In einem In-vitro-Modell mit granulozytären MDSC (GMDSC) aus dem NSB gesunder Termingeborener wurde die Phagozytose von "green fluorescence protein“(GFP)-exprimierenden Escherichia coli (E. coli) mit der Phagozytoseleistung von Granulozyten aus dem 
Nabelschnurblut des gleichen Kindes mittels Durchflusszytometrie (FACS), Konfokal- und Elektronenmikroskopie verglichen. Anschließend wurde die Apoptoserate mittels Annexin-V/7-AAD Färbung ermittelt. Die Expression von pro- (Bax) und anti-apoptotischen (BCL-2, BCL-XL) Bcl-Proteinen wurde mittels FACS bestimmt.

Ergebnisse. G-MDSC waren phagozytotisch aktiv und zeigten die gleiche Phagozytoseleistung wie Granulozyten ( $38 \pm 19 \%$ vs. $39 \pm 11 \%, n=10-$ 14, p>0,05). Die postphagozytäre Apotptoserate von G-MDSC war nach 16 Stunden deutlich geringer als bei reifen Granulozyten (20 $\pm 13 \%$ vs. $59 \pm 20 \%, \mathrm{n}=5, \mathrm{p}<0,05)$. Sowohl der Anteil an BCL-2-exprimierenden Zellen ( $83 \% \pm 14 \%$ vs. $60 \pm 18 \%, n=5, p<0,05)$ als auch die Stärke der BCL2-Expression (20 \pm 10 MFI vs. $10 \pm 1$ MFI, $n=5, p<0,05)$ war bei G-MDSC im Vergleich zu reifen Granulozyten vor Kontakt mit Bakterien erhöht. Nach Phagozytose von E. coli sank der Anteil an BCL-2-exprimierenden Granulozyten auf $36 \pm 14 \%$ ( $n=5, p<0,05$ vs. ohne E. coli), während der Anteil an G-MDSC nicht abnahm $(75 \pm 19 \%, n=5, p>0,05$ vs. ohne E. coli). Auch die BCL-XL Expression nahm nach Phagozytose von E. coli in Granulozyten nicht signifikant ab, in G-MDSC blieb sie unverändert. Die Expression des pro-apoptotischen Proteins Bax unterschied sich nicht zwischen G-MDSC und reifen Granulozyten.

Schlussfolgerung. Wir konnten erstmals zeigen, dass G-MDSC aus NSB mit reifen Granulozyten vergleichbare Phagozytosefähigkeiten haben. Im Gegensatz zu reifen Granulozyten zeigen G-MDSC jedoch eine verzögerte postphagozytäre Apoptose. Sie könnten damit für die primäre Immunantwort bei bakterieller Infektion in der Neonatalzeit wichtig sein. Ob das verlängerte Überleben der G-MDSC zu einer Immunsuppression beim Neugeborenen beiträgt muss weiter untersucht werden.

\section{Neonatale Hämodynamik}

\section{FV35}

Entwicklung der linksventrikulären 2D Speckle Tracking Parameter bei Frühgeborenen während der Neonatalperiode

\section{Czernik', S. Rhode', S. Helfer', G. Schmalisch', C. Bührer', L. Schmitz²}

${ }^{1}$ Charité - Universitätsmedizin Berlin, Klinik für Neonatologie, Berlin, Deutschland, ${ }^{2}$ Charité - Universitätsmedizin Berlin, Klinik für Pädiatrie m. S. Kardiologie, Berlin, Deutschland

Hintergrund. Frühgeborene (FG) können aufgrund einer bronchopulmonalen Dysplasie (BPD) oder eines hämodynamisch signifikanten Duktus arteriosus (hsPDA) einer erhöhten kardialen Belastung ausgesetzt sein. Konventionelle echokardiographische Parameter spiegeln die kardiale Belastung nicht immer zufriedenstellend wider. In mehreren Studien hat sich die neue echokardiographische Technik 2 D Speckle Tracking (2DSTE) in der kardialen Funktionsdiagnostik von Kindern und Erwachsenen bewährt. Dagegen gibt es bisher nur wenige Studien, in denen diese neue echokardiographische Methode bei Neugeborenen bzw. Frühgeborenen untersucht wurde.

Fragestellung. Wir untersuchten die Entwicklung der linken Herzfunktion von FG mit Hilfe der 2DSTE basierten Peak-systolic-Strain (LSS) und Strain-rate (LSSR) in den ersten 28 Lebenstagen mit der Fragestellung der Reproduzierbarkeit der neuen Parameter und ob Unterschiede bei FG mit und ohne BPD bzw. hsPDA zu finden sind.

Material und Methoden. Messung der LSS und LSSR der einzelnen Segmente in der linken Herzwand sowie im Septum an den Lebenstagen (LT) 1, 7, 14 und 28 von 119 FG unter 1500 g Geburtsgewicht. Körpergewicht, Herzfrequenz, Blutdruck, Fractional shortening (FS), HZV sowie das Vorliegen eines hämodynamisch signifikanten (hs)PDA und die Entwicklung einer BPD wurden dokumentiert.

Ergebnisse. Die globale LSSR des linken Ventrikels nahm während der ersten 28 Lebenstage signifikant zu. FG, die eine BPD bzw. einen hsPDA entwickelten, zeigten signifikante Unterschiede in der globalen LSSR an LT 1 und 7 und einen signifikant Unterschied im mittleren Segment der linken Herzwand an LT 1,7 und 14, wobei die Unterschiede bezogen auf den hsPDA geringer ausfielen. Bei den konventionellen Parametern ergaben sich signifikante Unterschiede beim Blutdruck, FS und HZV.
Unterschiede in LSS waren nicht zu finden. Die Variationskoeffizienten für die Intra- und Inter-Operator-Variabilität lagen zwischen 2,0\% und $25,1 \%$, wobei die weniger gute Reproduzierbarkeit in den apikalen Segmenten zu finden war.

Diskussion. Aufgrund der hohen Korrelation der Krankheitsbilder und der klinischen Parameter war eine exakte kausale Zuteilung ihres Einflusses auf LSS oder LSSR nicht möglich. BPD und hsPDA haben aber offensichtlich einen signifikanten Einfluss auf LSSR.

Schlussfolgerung. Trotz akzeptabler Reproduzierbarkeit der 2DSTE Parameter, konnten nur Unterschiede in LSSR und nicht LSS bei FG mit BPD bzw. hsPDA festgestellt werden. Die klinischen Vorteile gegenüber den konventionellen Parametern sowie die klinische Anwendbarkeit der Methode müssen noch weiter untersucht werden.

\section{FV36}

Vitalparameter und pH-Wert zeigen signifikante Unterschiede in den ersten Lebenstagen bei ELBW-Kindern mit späterer Ductusligatur

M. Steiner', V. Swoboda', U. Salzer-Muhar', S. Baumgartner', K. KlebermaßSchrehof', M. Langgartner', T. Waldhör ${ }^{3}$, A. Berger'

'Universitätsklinik für Kinder- und Jugendheilkunde, Medizinische Universität Wien, Abt. für Neonatologie, pädiatrische Intensivmedizin und Neuropädiatrie, Wien, Österreich, ${ }^{2}$ Universitätsklinik für Kinder- und Jugendheilkunde, Medizinische Universität Wien, Abt. für Kardiologie, Wien, Österreich, ${ }^{3}$ Center of Public Health, Abteilung für Epidemiologie, Wien, Österreich

Fragestellung. Im frühen postpartalen Verlauf ist es schwierig, zwischen Frühgeborenen zu unterscheiden, die einen spontanen Verschluss des Ductus arteriosus (PDA) erfahren, von denjenigen mit Ibuprofen-induziertem PDA-Verschluss und denjenigen mit Ibuprofen-Resistenz und notwendiger Ductusligatur. Eine frühere und genauere Einschätzung des PDA-Verlaufes wäre die Grundlage eines optimierten, individualisierten Therapieansatzes. In dieser Studie wurde der Verlauf von Vitalparametern und Blutgaswerten in den ersten Lebenstagen im Hinblick auf den späteren Verlauf des PDA untersucht.

Material und Methode. In einer retrospektiven Studie von prospektiv akquirierten, elektronisch gespeicherten Daten von Frühgeborenen unter 28 Schwangerschaftswochen (SSW) wurden Vitalparameter, arterielle Blutgasanalysen und echokardiographische Parameter in den ersten Lebenstagen im Hinblick auf den späteren Verlauf des PDA analysiert (Spontanverschluss vs. medikamentöser Verschluss vs. Ligatur).

Ergebnisse. Die Studienpopulation umfasste 104 Kinder. In einer „Timetrend“-Analyse zeigten sich innerhalb der Subgruppe von Kindern unter 26 SSW die Ibuprofen zum PDA-Verschluss erhalten hatten bei denjenigen Kindern, die nicht auf Ibuprofen ansprachen und eine spätere Ligatur benötigten signifikant unterschiedliche Verläufe der Vitalparameter in den ersten fünf Lebenstagen mit geringeren Sauerstoffsättigungswerten $(p=0,019)$ und Blutdruckwerten $(p=0,034)$, sowie größeren Schwankungsbreiten der Herzfrequenz $(\mathrm{p}=0,040)$ im Vergleich $z u$ den Kindern mit Ansprechen auf Ibuprofen. Ein metabolisch bedingter, niedrigerer arterieller $\mathrm{pH}$-Wert in den ersten 48 Lebensstunden war die wichtigste Einflussgröße und stellte auch in der multivariablen logistischen Regressionsanalyse einen unabhängigen Prädiktor einer späteren Ductusligatur dar (7,23 $\pm 0,03$ vs. 7,28 $\pm 0,05 ; \mathrm{p}=0,004)$.

Diskussion. Wir konnten zeigen, dass Kinder unter 26 SSW, die einer späteren Ductusligatur bedürfen, bereits in den ersten Lebenstagen signifikante Unterschiede im Verlauf von Vitalparametern und arteriellem $\mathrm{pH}$-Werten aufweisen und dass ein niedrigerer $\mathrm{pH}-$ Wert in den ersten 48 Lebensstunden unabhängig vom Gestationsalter einen Prädiktor einer späteren Ductusligatur darstellt.

Schlussfolgerung. Unsere Ergebnisse geben zusätzlichen Einblick in die komplexe Pathophysiologie des PDA und deuten an, dass eine Kombination aus klinischen Parametern mit anderen Variablen, die hinweisend auf eine Ibuprofen-Resistenz des PDA sind, die Grundlage für eine verbesserte und frühzeitige Risikostratifizierung des PDA-Verlaufes sein könnte. 


\section{FV37}

\section{Quantifizierung von S-100b, NSE und MBP im Urin Frühgeborener}

\section{Langer', F. Pillekamp' ', P. Verde'2, E. Mayatepek', T. Höhn}

'Klinik für Allgemeine Pädiatrie, Neonatologie und Kinderkardiologie, Heinrich-Heine-Universität, Düsseldorf, Deutschland, ${ }^{2}$ Koordinierungszentrum für Klinische Studien, Heinrich-Heine-Universität, Düsseldorf, Deutschland, ${ }^{3}$ Med. Einrichtung der Universität Kinderklinik, Allgemeine Pädiatrie, Düsseldorf, Deutschland

Fragestellung. Der persistierende Ductus arteriosus (PDA) ist eine der häufigsten funktionellen kardiovaskulären Störungen bei Frühgeborenen. Eine Einschätzung der hämodynamischen Relevanz für das Kreislaufsystem ist nach wie vor schwer. Aus diesem Hintergrund, hat unsere Studie versucht, nichtinvasiv zu gewinnende Biomarker zu identifizieren, welche die zerebrale Perfusion widerspiegeln.

Methode. Urinkonzentrationen von S1ooB, Neuronen-spezifischer Enolase (NSE) und Myelin basic Protein (MBP) von 50 Frühgeborenen $<1500$ g wurden mit Hilfe der ELISA-Methode gemessen und mit dem Status des PDA am 2.-4. Lebenstag (LT) korreliert. Die Urinsammlung erfolgte am o.-1., 2.-4. sowie 6.-8. LT. 26 der 50 Kinder hatten zu diesem Zeitpunkt einen PDA. Sechs Kinder erhielten eine Intervention mit Ibuprofen nach aktuellem klinischem Standard.

Ergebnisse. Die Urinkonzentrationen von S10oB und NSE unterschieden sich zu keinem Messzeitpunkt zwischen den Kindern mit und ohne PDA. Der t-Test wurde nach Transformation der Daten mit dem natürlichen Logarithmus berechnet. Die deskriptive Statistik ist in der Originalskala widergegeben. S1oo zeigte keinen Unterschied zwischen den beiden Gruppen (S10o o-1 LT: $\mathrm{p}=0,7769$, kein PDA: MW \pm SEM: $587,5 \pm 181,2 \mathrm{pg} / \mathrm{ml}, \mathrm{n}=19$, PDA: MW \pm SEM: 553,5 $\pm 170,7 \mathrm{pg} / \mathrm{ml}, \mathrm{n}=25,2-4$ LT: $\mathrm{p}=0,0,6201$, kein PDA: MW \pm SEM: 499,4 $\pm 182,5 \mathrm{pg} / \mathrm{ml}, \mathrm{n}=22$, PDA: 403,5 $\pm 70,41 \mathrm{pg} / \mathrm{ml}, \mathrm{n}=24,6-8 \mathrm{LT}: \mathrm{p}=0,8274$, kein PDA: MW \pm SEM: $479,2 \pm 101,6 \mathrm{pg} / \mathrm{ml}, \mathrm{n}=22$, PDA: $442,5 \pm 85,82 \mathrm{pg} / \mathrm{ml}, \mathrm{n}=22$ ). NSE zeigte ebenfalls keinen Unterschied zwischen den beiden Gruppen. MBP zeigte einen Unterschied am 0.-1. LT und am 2.-4. LT, jedoch nicht mehr am 6. -8. LT. (MBP o-1 LT: $\mathrm{p}=0,0041$, kein PDA: MW \pm SEM: $223,1 \pm 55,9 \mathrm{pg} /$ $\mathrm{ml}, \mathrm{n}=21$, PDA: MW \pm SEM: $85,22 \pm 14,5 \mathrm{pg} / \mathrm{ml}, \mathrm{n}=25,2-4$ LT: $\mathrm{p}=0,0054$, kein PDA: MW \pm SEM: $197,7 \pm 43,18 \mathrm{pg} / \mathrm{ml}, \mathrm{n}=22$, PDA: 68,98 $\pm 8,965 \mathrm{pg} /$ $\mathrm{ml}, \mathrm{n}=25,6-8$ LT: $\mathrm{p}=0,9578$, kein PDA: MW \pm SEM 159,9 $\pm 43,18 \mathrm{pg} / \mathrm{ml}$, $\mathrm{n}=22$, PDA: MW \pm SEM $152,4 \pm 30,89 \mathrm{pg} / \mathrm{ml}, \mathrm{n}=24$ ). Die Intervention mit Ibuprofen sowie die Ligatur beeinflussten die Proteinkonzentrationen ebenfalls nicht.

Schlussfolgerung. Ein klinisch hämodynamisch relevanter PDA bei Frühgeborenen $<1500 \mathrm{~g}$ führt in der ersten Lebenswoche nicht zu einer Erhöhung der Konzentrationen von S10oB, NSE und MBP im Urin. Damit besteht kein Hinweis auf einen relevanten zerebralen Zelluntergang soweit dieser anhand obiger Parameter messbar wäre.

\section{FV38 \\ Management des Ductus arteriosus bei extrem kleinen Frühgebo- renen}

\section{Knüpfer', F. Sieg', F. Pulzer', C. Gebauer', A. Bläser', U. Thome \\ 'Universitätsklinikum Leipzig, Klinik und Poliklinik für Kinder und Jugendliche der Universität, Abteilung Neonatologie, Leipzig, Deutschland}

Hintergrund. Das Management des persistierenden Ductus arteriosus (PDA) bei Frühgeborenen ist uneinheitlich. Wir untersuchten, ob die Anwendung einer Richtlinie bestehend aus engmaschiger sonographischer Kontrolle, kombiniert mit einer Therapie bei hämodynamisch wirksamen DA die Behandlung der Kinder optimieren kann.

Methoden. Retrospektiv wurden die Daten aller ELBW-Kinder untersucht, die zwischen 2008 und 2012 im Universitätsklinikum Leipzig geboren wurden. Sonographische Untersuchungen wurden alle $24 \mathrm{~h}$ bis zum 5. und am 7. Lebenstag oder bis zum Verschluss durchgeführt. Eine Behandlung mit Ibuprofen in Standarddosis erfolge nur bei Nachweis eines hämodynamisch wirksamen DA. Im Verlauf erfolgten weite- re Echokontrollen, um eine Wiedereröffnung des PDA nachweisen zu können.

Ergebnisse. Für die Studie auswertbar waren 232 Kinder. Ein Spontanverschluss wurde bei $68,3 \%(n=148)$ beobachtet. Diese Kinder waren signifikant älter (26,5 \pm 2 vs. $25,8 \pm 1,7 \mathrm{SSW}$; $\mathrm{p}=0,023)$ und hatten eine höhere Flüssigkeitszufuhr ( $147 \pm 44$ vs. $162 \pm 40 \mathrm{ml} ; \mathrm{p}=0,001)$ und Flüssigkeitsbilanz ( $76 \pm 37$ vs. $90 \pm 37 \mathrm{ml} ; \mathrm{p}=0,007)$ pro Tag bis zum Tag des Verschlusses. Der DA von $32,7 \%(n=84)$ wurde als hämodynamisch signifikant eingestuft und mit Ibuprofen behandelt. In 36 Fällen wurde nach initialem Verschluss eine Wiedereröffnung diagnostiziert (20 nach Spontanverschluss und 16 nach Ibuprofenbehandlung). Von diesen verschlossen sich 10 DA erneut ohne Therapie, 19 wurden sekundär erfolgreich mit Ibuprofen oder Indomethazin verschlossen. 6 Kinder wurden mit einem offenen, hämodynamisch nicht relevanten DA entlassen. Nur bei einem Kind musste bei hämodynamischer Relevanz ein interventioneller Verschluss erfolgen (Coil). Eine chirurgische Intervention war nicht nötig. Schlussfolgerung. Auch bei ELBW-Kindern verschließt sich der DA weit häufiger spontan als in der Literatur angegeben. Sonographische Untersuchungen in den hier angegebenen Intervallen sind geeignet, um die Entwicklung des Ductus Arteriosus zu verfolgen und die Behandlungsnotwendigkeit zu bestimmen. Mit unserem Vorgehen können die Patienten selektiert werden, die eine Behandlung benötigen. Besonders wichtig ist uns, dass damit chirurgische Interventionen weitgehend vermieden werden.

\section{FV39}

Weder echokardiographische Parameter (EchoP) noch NT-proBNP erlauben eine verlässliche Abschätzung der zerebralen Sauerstoffsättigung bei Frühgeborenen mit Verdacht auf persistierenden Ductus Arteriosus Botalli (PDA)

\section{Schwarz', A. Preusche', W. Baden ${ }^{2}$, M. Wolf ${ }^{3}$, C. Poets ${ }^{1}$, A. Franz}

'Eberhard-Karls-Universität Universitätsklinik für Kinderheilkunde und Jugendmedizin, Neonatologie, Tübingen, Deutschland, ${ }^{2}$ Eberhard-Karls-Universität Universitätsklinik für Kinderheilkunde und Jugendmedizin, Kinderkardiologie, Tübingen, Deutschland, ${ }^{3}$ Universität Zürich UZH, Biomedical Optics Research Laboratory, Zürich, Schweiz

Hintergrund. Die Entscheidung zum PDA-Verschluss bei Frühgeborenen (FG) wird oft aufgrund von klinischen Kriterien und EchoP zur Einschätzung des Links-Rechts-Shunts (LRS) getroffen und durch die NT-proBNP Konzentration ergänzt. Es ist jedoch unklar, ob und welche dieser Parameter die Patienten erkennen lassen, bei denen der PDA zu einer reduzierten zerebralen Sauerstoffsättigung $\left(\mathrm{rcStO}_{2}\right)$ oder einer erhöhten zerebralen Sauerstoffausschöpfung (rcFtOE) führt.

Fragestellung. Korreliert die $\mathrm{rcStO}_{2}$ oder die rcFtOE mit EchoP, Hämoglobin ( $\mathrm{Hb})$ oder dem NT-proBNP bei FG mit einem PDA?

Material und Methoden. Bei FG mit einem Geburtsgewicht $<1501 \mathrm{~g}$ mit klinischem Verdacht auf einen PDA wurden prospektiv folgende EchoP gemessen: LA/Ao (Linkes Atrium/Aortenwurzel), LVPEP/LVET (linksventrikuläre Präejektionsperiode/linksventrikuläre Ejektionszeit), VTI_Ao/VTI_Pa (Volumen-Zeit-Integral Aorta/VTI Pulmonalarterie), Resistance Index im Truncus coeliacus (RI_TC) und der Arteria cerebri anterior (RI_ACA), Durchmesser des PDA an der engsten Stelle (Dia). Außerdem wurden NT-proBNP (Roche), Hb-konzentration (SYSMEX), sowie $\mathrm{rcStO}_{2}$ und rcFtOE mittels FD-NIRS (ISS Oxiplex) gemessen. Alle Angaben als Median (IQR; n). Die statistische Auswertung erfolgte mittels Spearman Korrelationskoeffizienten.

Ergebnisse. Gestationsalter bei Geburt: $28,7(28,5-29,8 ; 19)$ Wochen und postnatales Alter 8 (5-17)Tage. Ein PDA mit LRS wurde farbdopplersonographisch in 12/19 Messungen festgestellt. Die $\mathrm{rcStO}_{2}$ betrug in der Gesamtgruppe $56(53-58 ; 19) \%$ und die rcFtOE 0,40 (0,38-0,44; 19). Bei Kindern mit LRS über den PDA betrug die $\mathrm{rcStO}_{2} 57$ (55-59; 12)\% und die rcFtOE $0,39(0,38-0,43 ; 12)$. Als einziger Parameter korrelierte die Hb-Konzentration $\left[13,9(11,4-15,2 ; 19)\right.$ signifikant $(\mathrm{p}<0,05)$ mit $\mathrm{rcStO}_{2}$ $(\mathrm{r}=0,709 ; \mathrm{p}=0,001)$ und $\mathrm{rcFtOE}(\mathrm{r}=-0,753 ; \mathrm{p}=0,0002)]$. Die Messwer- 
te für die Parameter betrugen für LA/Ao [1,33 (1,23-1,69; 19)], LVPEP/ LVET [0,29 (24-32; 19)], VTI_Ao/VTI_Pa [0,94 (o,88-1,29; 19)], RI_TC [0,82 (o,73-1; 19)], RI_ACA [0,82 (o,77-0,84; 19], Dia [1,9 mm (1,3-2,0; 6), und NT-proBNP [7050 pg/ml (3695-15770; 14)].

Diskussion. Unsere Ergebnisse zeigen einen signifikanten Zusammenhang zwischen Sauerstofftransportkapazität und zerebraler Oxygenierung (rcOXY). Bisherige Studien konnten Veränderungen der rcOXY vor und nach Ductusverschluss (medikamentös oder chirurgisch) und damit einen Zusammenhang zwischen PDA und rcOxy zeigen. Durch die bisher verwendeten Parameter kann jedoch nicht auf eine beeinträchtigte rcOXY geschlossen werden.

Schlussfolgerung. Sollten nur Kinder mit einem PDA behandelt werden, bei denen der LRS zu einer eingeschränkten rcOXY führt, kann auf eine NIRS-Messung nicht verzichtet werden. Bei relevantem LRS über den PDA muss ggf. an die Anwendung höherer Transfusionsgrenzen gedacht werden.

\section{Mit Unterstützung der Else-Kröner-Fresenius-Stiftung.}

\section{FV40}

Gefäßringe, -schlingen und andere -anomalien und ihre potenziell lebensbedrohenden Komplikationen vom Neugeborenen bis zum Erwachsenen: Diagnostik mittels MRT, CT und Tracheobronchoskopie

J.Eichhorn', A. Helling', P. Jahn', M. Treichel', J.Schenk', F. Long ', T.Loukanov 'Klinikum Leverkusen, Klinik für Kinder und Jugendliche, Leverkusen, Deutschland, ${ }^{2}$ Klinikum d. Ruprecht-Karl- Universität, Kinderklinik, Heidelberg, Deutschland, ${ }^{3}$ Radiologische Universitätsklinik, Pädiatrische Radiologie, Heidelberg, Deutschland, ${ }^{4}$ Nationswide Children's Hospital, Pediatric Radiology, Columbus, Ohio, USA, ${ }^{5}$ Chirurgische Universitätsklinik, Kinderherzchirurgie, Heidelberg, Deutschland

Zielstellung. Vaskuläre Ringe, Schlingen und andere Anomalien gehören zu den seltenen angeborenen kardiovaskulären Malformationen (kvM). Sie sind jedoch regelmäßig Ursache für eine Tracheomalazie, welche dann von erheblicher prognostischer Relevanz ist. Ziel der Studie was die Genauigkeit von MRT- und CT-Angiographie bei der Diagnostik von kvM, trachealen Pathologien und topographischen Verbindungen zu untersuchen.

Material und Methoden. In den letzten 12 Jahren wurden 114 Patienten mit Verdacht auf eine kvM [Median (range) 1,2 J (1 Wo. bis $56 \mathrm{~J}$.)] mittels MRT ( $\mathrm{n}=74)$ oder CT (40; nur die kleinsten und kritisch kranken Patienten) untersucht. Der kvM-Typ und seine Ausprägung und Lagebeziehung zu Ösophagus, Trachea und Bronchien wurde untersucht und

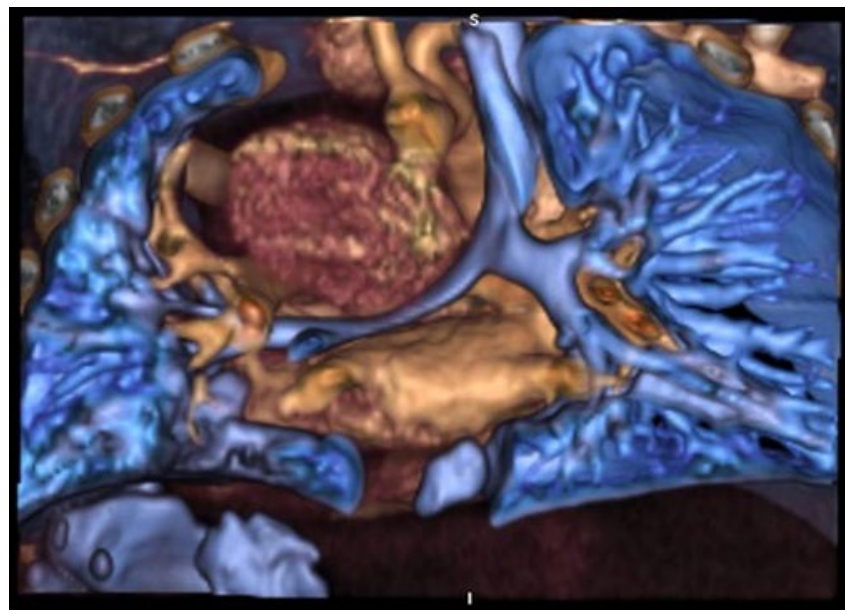

$\Delta$ FV40 - Abb. 1 Aneursyma des Ductus arterios Botalli mit Trachealdeviation und Kompression des linken Hauptbronchus (3D-rekonstruktion aus einer CT bei einem 6 Wochen alten Säugling: koronarer Schnitt, Ansicht von dorsal) mit den Ergebnissen der Herzkatheteruntersuchung (wenn aufgrund assoziierter Herzfehler durchgeführt; $n=10$ ), Tracheobronchoskopie und den intraoperativen Befunden (wenn eine bedeutsame kvM mittels MRT oder CT bestätigt wurde) verglichen.

Ergebnisse. Bei 69 von 114 Patienten wurde eine kvM diagnostiziert (doppelter Aortenbogen (Aobo), $\mathrm{n}=14$; rechter Aobo + aberrante A. subclavia sin. + linksseitiges Ductusligament, $n=29$; Pulmonary Sling, $\mathrm{n}=5$; absent pulmonary valve 4 andere, $\mathrm{n}=17$ ). Bei allen Patienten wurden die Ergebnisse von MRT/CT durch Bronchoskopie und intraoperativen Befund bestätigt. Obwohl MRT und CT eine Schwäche bei der Beurteilung von atretischen Segmenten aufwiesen, gab es keinen falsch positiven Fall. Das CT hat die bessere räumliche Auflösung $(0,5 \mathrm{~mm})$ und den besseren Luft/Weichteil-Kontrast, damit wurden virtuelle Bronchoskopien möglich.

Schlussfolgerung. MRT und CT erlauben eine nichtinvasive Bildgebung von Ringen und Schlingen einschließlich der potenziellen Kompressionen von Trachea, Bronchien und Ösophagus selbst bei kleinsten Kindern. Dreidimensionale Rekonstruktionen erleichtern die präoperative Planung. MRT und CT können hier als Methoden der 1. Wahl angesehen werden und sollten den Vorrang vor der Herzkatheteruntersuchung haben (Abb. 1).

\section{Infektiologie}

\section{FV41}

Fetale pulmonale Entzündungsmarker und oxidativer Stress nach antenataler Ureaplasmeninfektion im Chorioamnionitismodell im Schaf werden nicht durch mütterliche Medroxyprogesterongabe beeinflusst

M. Hütten', S. Kallapur' ${ }^{2}$ A. Jobe' ${ }^{2}$, J. Newnham ${ }^{3}$, M. Kemp ${ }^{3}$, T. Wolfs', U. Pecks ${ }^{5}$, T. Orlikowsky ${ }^{6}$, G. Buonocore', B. Kramer

'Universitätsklinik für Kinder- und Jugendmedizin, Sektion für Neonatologie und Department of Pediatrics, Maastricht University Medical Center, Sektion für Neonatologie, Aachen/Maastricht, Deutschland, ${ }^{2}$ Division of Pulmonary Biology, Cincinnati Children's Hospital Medical Center, University of Cincinnati and School of Women's and Infant's Health, The University of Western Australia, Subiaco, Ohio/Perth, USA, ${ }^{3}$ School of Women's and Infant's Health, The University of Western Australia, Subiaco, Perth, Australien, ${ }^{4}$ Maastricht University Medical Center, Department of Pediatrics, Maastricht, Niederlande, ${ }^{5}$ Universitätsklinikum der RWTH Aachen, Frauenklinik für Geburtsmedizin, Aachen, Deutschland, ${ }^{6}$ Universitätsklinik für Kinder- und Jugendmedizin, Sektion für Neonatologie, Aachen, Deutschland, ${ }^{7}$ Siena University of Siena, Departments of Pediatrics, Siena, Italien

Fragestellung. Chorioamnionitis kann mit dem fetalen Ammnioninfektionssyndrom und Frühgeburtlichkeit assoziiert sein. Das Steroidhormon Progesteron senkt in präventiver Gabe im Risikokollektiv die Rate der Frühgeburten. Dabei unterstützt Progesteron die Aufrechterhaltung der Schwangerschaft sowohl durch endokrine als auch immunmodulatorische Wirkung. Der Einfluss von Progesteron auf die fetale Immunantwort bei Vorliegen einer Chorioamnionitis ist ungeklärt. Wir untersuchten daher im Schafmodell, ob die durch intraamniotische Ureaplasmenexposition (UP) ausgelöste fetale pulmonale Entzündungsreaktion und der oxidative Stress im Feten durch Progesteron mit und ohne Gabe von maternalem Betamethason (Beta) moduliert werden.

Material und Methode. 31 trächtigen Schafen wurden intraamniotisch UP Serovar 6 („UP“) oder Kulturmedium („Kontrolle“) an Tag 83 \pm 2 der Schwangerschaft (Termin: 150 Tage) sowie Medroxyprogesteronacetat ("MPA" und "UP+MPA") oder $\mathrm{NaCl}$ intramuskulär an Tag 112 verabreicht. Eine fünfte Gruppe erhielt zusätzlich 7 Tage vor Schnittentbindung an Tag 124 Betamethason i.m. ("UP+MPA+Beta"). Untersucht wurden Entzündungszellen in bronchoalveolärer Lavage (BAL), T-Zellpopulation im posterioren mediastinalen Lymphknoten, statische Lun- 
genkapazität sowie Produkte nicht-enzymatischer Lipidperoxidation (Iso- und Neuroprostan, Iso- und Neurofuran) in Lunge und Leber. Ergebnisse. In den Gruppen „UP“ und „UP+MPA“ zeigte sich ein signifikanter Anstieg der Entzündungszellen im Vergleich zu „MPA“ und Kontrollgruppe. Dieser Anstieg verringerte sich jedoch in der „UP+MPA+Beta“-Gruppe um bis zu 6o\% (p<0,05). Die statische Lungencompliance war lediglich in der „UP+MPA+Beta“ Gruppe bis auf das doppelte erhöht ( $\mathrm{p}<0,05$ vs. Kontrolle). Tiere der „UP+MPA+Beta"-Gruppe zeigten in der Lunge erhöhte Werte für IsoP ( $\mathrm{p}<0,05$ vs. UP+MPA), IF und NP ( $<<0,05$ vs. MPA). In der Leber dieser Tiere fand sich eine bis zu 2,5-fache Erhöhung von NF und IF ( $\mathrm{p}<0.05$ vs. Kontrolle, vs. „UP“ und vs. „MPA“). Feten in der „UP+MPA“ Gruppe zeigten bis zu 2,2-fach erhöhte Werte für IsoP, IF und NF in der Leber ( $<<0,05$, p=0,094 bzw. $p=0,090$ vs. „UP“)

Diskussion und Schlussfolgerung. MPA schränkt die fetale pulmonale Entzündungsreaktion bei Chorioamnionitis im Schafmodell nicht ein. Die zusätzliche maternale Gabe von Beta, die die klinische Situation bei drohender Frühgeburt widerspiegelt, zeigt die in der Literatur für Beta beschriebenen Effekte in Hinsicht auf Immunmodulation und erhöhten oxidativen Stress. Weitere Untersuchungen zu systemischen Effekten von Progesteron im Feten sind notwendig.

\section{FV42 \\ Die Inzidenz von Early-Onset-Sepsis bei Extrem-Frühgeborenen mit günstigem Risikoprofil}

\section{A. Eccles', M. Hewson ${ }^{2}$}

'Zentrum für Kinderheilkunde der Justus-v.-Liebig-Universität, Neonatologische Intensivstation, Giessen, Deutschland, ${ }^{2}$ Wellington Regional Hospital, Neonatal Intensive Care Unit, Wellington, New Zealand

Hintergrund. Die empirische Antibiose zur Behandlung von Early-Onset-Sepsis (EOS) bei Extrem-Frühgeborenen bleibt ein therapeutisches Dilemma. Die Prävalenz in dieser Gruppe liegt bei 2-3\%; Morbidität und Mortalität sind hoch. Häufig sind die Symptome nicht von denen der Frühgeburtlichkeit selbst abgrenzbar. Behandlung mit Antibiotika trägt jedoch Risiken wie Ototoxizität und nekrotisierende Enterokolitis mit sich und sollte vermieden werden, wenn das Risiko einer Infektion gering ist. Bekannte Risikofaktoren für EOS sind z. B. Chorioamnionitis, vorzeitige Wehentätigkeit, vorzeitiger Blasensprung und vaginale Entbindung. Der Einfluss einer maternalen Präeklampsie auf das Infektionsrisiko bleibt umstritten. Wir untersuchten die Inzidenz von EOS in Frühgeborenen unter 28 SSW unter Berücksichtigung ihres Risikoprofils und analysierten zudem den Einfluss von Geburtsgewicht, Gestationsalter und mütterlichem SS-Hypertonus.

Methoden. Wir analysierten die Geburtskohorte der Extrem-Frühgeborenen (<28 SSW) der Jahre 2007 bis 2011 aus der Datenbank des Australia and New Zealand Neonatal Network (ANZNN). EOS wurde definiert als das Vorliegen einer positiven Blut- oder Liquorkultur innerhalb der ersten 48 Lebensstunden, wenn durch die behandelnden Ärzte eine Indikation für eine fünftägige Antibiose gestellt wurde. Wachstum von Hautflora inklusive Koagulase-negative Staphylokokken wurde als Kontamination gewertet. Anhand des Risikoprofiles wurden drei Gruppen zur Analyse erstellt. Die absolute EOS Inzidenz und das Keimspektrum wurden ermittelt. Weitere Einflussfaktoren wurden mittels logistischer Regression untersucht.

Ergebnisse. Es wurden 4203 Frühgeborene untersucht. Die absolute EOS Inzidenz war 2,8\%. Frühgeborene mit einem vorzeitigen Blasensprung $>24$ Stunden $(n=955)$ hatten eine EOS-Inzidenz von $5,6 \%$ mit vorwiegend E. coli (39\%). Frühgeborene mit vorzeitiger Wehentätigkeit und/oder mit vorzeitigem Blasensprung zwischen 0,5 und 24 Stunden $(n=2247)$ hatten eine EOS Inzidenz von 2,3\% mit vorwiegend GBS (35\%). Frühgeborene, die per Kaiserschnitt aus intakten Eihäuten ohne vorzeitige Wehentätigkeit entbunden wurden, $(\mathrm{n}=1001)$ hatten eine EOS Inzidenz von $1,2 \% \mathrm{mit}$ variablen Keimen, u. a. Enterokokken (25\%), S. aureus (25\%) und Pseudomonas (16\%). Gestationsalter, nicht aber Geburtsgewicht, hatte einen signifikanten Einfluss auf die EOS Inzidenz in der logistischen Regression unter Beachtung des Risikoprofils. Maternale SS-Hypertension erhöhte das Risiko für eine EOS um den Faktor 2,5 (95\% CI 1,1-5,6).

Diskussion. Das Risiko einer EOS bei Extrem-Frühgeborenen ist durch die Abwesenheit perinataler Risikofaktoren stark vermindert, aber keinesfalls abwesend. Immunsuppression durch maternale Präeklampsie spielt möglicherweise eine wichtige Rolle. In der Hälfte der Infektionen in der Gruppe mit günstigem Risikoprofil wurden Keime nachgewiesen, die nicht klassischerweise als EOS-Keime mit vertikaler Transmission betrachtet werden. Eine mögliche Erklärung wäre das Auftreten von sehr frühen nosokomialen Infektionen.

\section{FV43}

\section{Ein Amnioninfektionsmausmodell zur In-vivo-Untersuchung der} Leukozytenrekrutierung während der fetalen Entwicklung

\section{Frommhold', K. Buschmann', H. Hudalla', J. Pöschl' \\ 'Universität Heidelberg, Klinik für Neonatologie, Heidelberg, Deutschland}

Fragestellung. Schwere bakterielle Infektionen sind eine der führenden Todesursachen bei Frühgeborenen. Ein Grund dafür ist die Unreife des Immunsystems, wie z. B. die zelluläre Abwehr, die erst spät in der fetalen Entwicklung ausreift. Mit einem neuartigen Mausmodell für das Amnioninfektionssysndrom (AIS) soll die Leukozytenrekrutierung in vivo bei unreifen Mausfeten in der Inflammation beobachtet werden. Material und Methoden. Vergleichbar mit der neonatalen Early-onset-Sepsis sollte in der Maus im Gestationsstadium E13-E18 (normale Gestationsdauer bis E21) ein Amnioninfektionssyndrom durch intraperitoneale LPS Injektion ausgelöst werden. Zur Einschätzung des Entzündungsausmaßes im Feten könnte ein neu entwickeltes Verfahren dienen, bei dem in-vivo nach Uterotomie unter physiologischen Bedingungen Leukozytenrekrutierungsvorgänge LysEGFP positive Mausfeten mikroskopisch beobachtet werden. Zudem sollte das reifealterspezifische Überleben der Feten sowie die Histologie fetaler Organe beurteilt werden.

Ergebnisse. Obgleich technisch anspruchsvoll, gelang es, unter Narkose bei den LPS vorbehandelten, schwangeren Mäusen die unreifen Mausfeten der Stadien E13-E18 für eine Intravitalmikroskopie zu präparieren. Diese zeigte, dass in mittleren Schwangerschaftsstadien E13/E14 (ca. 24-28 humane SSW), das LPS-induzierte Leukozytenrollen, -adhäsion und -infiltration in fetale Organe noch deutlich eingeschränkt war. Erst im Verlauf der späteren Gestation mit E17/E18 (ca. 32-36 humanen SSW) reift diese Fähigkeit der zellulären Immunantwort graduell aus. Mit diesen Befunden korreliert auch die Überlebens- bzw. Frühgeburtsrate. Somit konnte durch die intraperitoneale LPS Injektion der schwangeren Maus gestationsaltersabhängig eine Inflammation ausgelöst werden, die einem Amnioninfektionssyndrom ähnelt.

Diskussion. Nach Etablierung dieses AIS-Modells in der Maus könnten künftig reifealterabhängig Substanzen zur fetalen Immunmodulation untersucht werden. Zur Trennung maternaler und fetaler Mechanismen könnten ferner plazentagängige mit nicht plazentagängigen Medikamenten verglichen werden. Durch Einbeziehung von genveränderten Mäusen ergibt sich die Möglichkeit, Mechanismen und Moleküle, die die fetale Inflammation vermitteln, aufzuklären. Dieses Modell könnte auch als Grundlage dienen, neue Strategien zur Behandlung von Entzündungen bei Früh- und Neugeborenen zu entwickeln.

Schlussfolgerung. Ein neues Mausmodell für Amnionsinfektionssyndrom ermöglicht die Untersuchung der Immunmodulation bei inflammatorischen Prozessen unreifer Feten. 


\section{FV44}

\section{Präklinische Testung und erste Erfahrungen mit der Etablierung} eines Screenings auf konnatale CMV-Infektionen

\section{K. Künzl' , F. Kipfmueller' , A. Eis-Hübinger ${ }^{2}$, P. Bartmann ${ }^{1}$, A. Müller ${ }^{1}$ \\ 'Universitätsklinik Zentrum f. Kinderheilkunde, Neonatologie, Bonn, Deutsch- land, ${ }^{2}$ Institut für Virologie, Bonn, Deutschland}

Fragestellung. Die Benutzung von Zellstoff-Binden zur Testung auf CMV-positiven Urin könnte eine zeitsparende Alternative zu Urinbeuteln sein. Bedeutung hätte dies für ein bundesweites Screening auf konnatale CMV-Infektion. Untersucht wurde ein möglicher Virusverlust durch Zellstoffbinden, sowie die klinische Praktikabilität anhand von 500 Neugeborenen.

Methodik. Untersucht wurde die CMV-Viruslast von CMV-positivem Urin vor Befeuchtung einer Zellstoff-Binde, sowie nach $15 \mathrm{~min}, 12 \mathrm{~h}$ und nach 7 Tagen Einwirkdauer. Zur Untersuchung des Einflusses von Mekonium auf die Virusdetektion wurde CMV-positiver Urin im Verhältnis 80:1 mit Mekonium vermischt und nach o und 3 Tagen analysiert. Weiterhin wurde untersucht ob sich CMV-DNA auch nach Trocknen der Binde nachweisen lässt. Dazu wurden getrocknete Binden mit $3 \mathrm{ml}$ Aqua angefeuchtet und zentrifugiert. Der gewonnene Überstand wurde mittels real-time PCR auf CMV-Viruslast getestet. In einer klinischen Testungsphase wurde die Methodik bei 500 Neugeborenen angewandt. Ergebnisse. Die Viruslast nahm im Verlauf der Einwirkdauer initial ab, war aber bei zunehmender Lagerungsdauer gleichbleibend (Baseline: 4,67 Mio.; 15 min: 3,12 Mio.; 12 h: o,71 Mio.; 7 Tage: o,72 Mio. Kopien). Durch Beimengung von Mekonium verringerte sich die Viruslast von initial 217.0oo Kopien auf 46.300 Kopien, nach 3-tägiger Lagerung auf 24.960 Kopien. Aus trocknen Binden konnte CMV ab einer initialen Viruslast von 4315 Kopien immer nachgewiesen werden, allerdings war die Viruslast zu $63 \%<1000$ Kopien. Von 500 untersuchten Neugeborenen waren 2 Kindern CMV-positiv. 22\% der Binden waren partiell durch Mekonium verunreinigt. Aus 73,6\% der Binden eine ausreichende Menge Urin ohne Anfeuchtung gewonnen werden.

Diskussion. Bei ausreichend hoher initialer Viruslast, wie sie bei konnataler CMV typischerweise vorkommt, lassen sich Zellstoffbinden problemlos verwenden. Auch Mekoniumbeimengungen scheinen nur eine geringe Bedeutung zu haben. Ein Trocknen der Binden sollte verhindert werden, daher ist ein zeitnahes Verarbeiten der Binden, sowie ggf. eine Kühlung empfehlenswert.

Schlussfolgerung. Die Verwendung von Zellstoff-Binden zum Screening auf CMV-Infektion von Neugeborenen erscheint praktikabel.

\section{FV45}

\section{Positive Blutstrominfektionen (Candida) und seltene Erreger (Aspergillus fumigatus) auf einer neonatologischen Intensivstation}

\section{F. Pulzer', N. Lippmann², C. Gebauer', A. Bläser', M. Knüpfer', U. Thome'} 'Universitätskinderklinik, Neonatologie, Leipzig, Deutschland, ${ }^{2}$ Uniklinikum Leipzig, Institut für medizinische Mikrobiologie und Infektionsepidemiologie, Leipzig, Deutschland

Einleitung. Neueste Studien berichten über eine fallende Inzidenz an invasiven Candidainfektionen bei Früh- und Neugeborenen und eine Assoziation mit der Verweildauer an zentralvenösen Zugängen $(>2 \mathrm{Wo}-$ chen). Wir stellen unsere langjährigen Beobachtungen dar und diskutieren die Ergebnisse anhand der Literatur.

Methodik. Während einer 11-jährigen Beobachtungszeit (01.01.2001 bis 31.12.2012) wurden 6730 Patienten auf unserer neonatologischen Intensivstation betreut. Wir führten eine retrospektive Analyse aller positiven Blutkulturen und Abstriche in Bezug auf Mykosen durch.

Ergebnisse. Von den 2637 durchgeführten Blutkulturen waren lediglich $6(<0,1 \%)$ für Candida positiv (5-mal Cand. albicans, 1-mal Cand. lusitaniae). Diese Fälle traten zwischen 2002-2006 auf. In 3 Fällen (50\%) kam es zum vorzeitigen Blasensprung und bei 2 dieser Fälle war Cand. albicans bereits pränatal im mütterlichen Scheidenabstrich nachweisbar.
Alle Patienten mit Cand. albicans Infektion waren extrem Frühgeborene <27. SSW (24.3 SSW bis 26.5 SSW) welche auch mit zentralvenösen $\mathrm{Zu}$ gängen versorgt waren. Hiervon verstarb 1 Patient (13. Lebenstag). Der Patient mit Nachweis von Cand. lusitaniae war ein reifes Neugeborenes (38.o SSW). In 2 Fällen (33\%) handelte es sich um eine frühe Infektion $(<3$. Lebenstag), die übrigen Infektionen traten in der zweiten Lebenswoche auf. Persistierende Candidämien wurden nicht gefunden. Die antimykotische Therapie erfolgte (teils einzeln bzw. in Kombination) mit Fluconazol (67\%), Amphotericin B (33\%) und Caspofungin (17\%). Wir führten keine antimykotische Prophylaxe mit Fluconazol durch. Im gleichen Zeitraum gelang bei insgesamt 402 Patienten ein positiver Pilznachweis in unterschiedlichen Materialien. In 2 Fällen (1-mal Mundschleimhaut, 1-mal Wundabstriche) wurde Aspergillus fumigatus gefunden, wobei ein Patient (Z. n. Teilresektion eines riesigen Neuroblastoms mit Chemotherapie) trotz Antimykose mit Amphotericin B und Caspofungin verstarb. Schlussfolgerung. Extreme Frühgeburtlichkeit mit der Notwendigkeit zu längerdauernden intensivtherapeutischen Maßnahmen (zentrale Katheter) ist eine der Hauptursachen für systemische Pilzinfektionen in der Neonatologie. Pränatale Scheidenabstriche im Sinne einer Präventionsstrategie können helfen Risikopatienten frühzeitig zu identifizieren bzw. bieten die Möglichkeit zu einer raschen Therapieoptimierung.

\section{FV46}

Detektion von Infektionen bei beatmeten Frühgeborenen mit der „elektronischen Nase“

\section{T. Rogosch', N. Herrmann ${ }^{2}$, E. Domann ${ }^{3}$, A. Hattesohl', A. Koczulla ${ }^{4}$, R. Maier ${ }^{5}$, M. Zemlin ${ }^{6}$}

'Universitätsklinikum Gießen und Marburg GmbH, Standort Marburg, Klinik für Kinder- und Jugendmedizin, Marburg, Deutschland, ${ }^{2}$ Klinik für Kinder- und Jugendmedizin, Marburg, Deutschland, ${ }^{3}$ Institut für Medizinische Mikrobiologie, Gießen, Deutschland, ${ }^{4}$ Klinik für Innere Medizin, Schwerpunkt Pneumologie, Marburg, Deutschland, ${ }^{5}$ Universitätsklinikum Marburg, Zentrum für Kinder und Jugendmedizin, Marburg, Deutschland, ${ }^{6}$ Klinik für Kinder- und Jugendmedizin der Philipps-Universität Marburg, Neonatologie, Marburg, Deutschland

Einleitung. Infektionen beeinflussen entscheidend die Mortalität und Langzeitmorbidität von Frühgeborenen. Die Diagnose einer Infektion erfolgt über die klinische Symptomatik und über die Analytik von Entzündungsparametern im Blut. Ziel ist es, eine antibiotische Behandlung so bald wie möglich aber auch nicht unnötig zu beginnen. Da invasive Untersuchungen Frühgeborene beeinträchtigen und Laborergebnisse oft erst nach Stunden zur Verfügung stehen, besteht Bedarf, die Infektionsdiagnostik mit einer nichtinvasiven Methodik zu ergänzen, die sofortig zuverlässige Ergebnisse liefert.

Fragestellung. Unterscheiden sich die Profile volatiler Substanzen („smell prints“) im Trachealaspirat von Frühgeborenen mit und ohne Infektion?

Material und Methoden. Wir haben 39 Trachealaspiratproben von beatmeten Frühgeborenen $(<37 \mathrm{SSW})$ und untersucht. Eine Infektion wurde diagnostiziert, wenn die CDC-Definition „laboratory-confirmed bloodstream infection" erfüllt war. Dies umfasst: klinische Symptomatik, C-reaktives Protein $>10 \mathrm{mg} / \mathrm{l}$, Interleukin- $8>70 \mathrm{pg} / \mathrm{ml}$, und/oder Verhältnis unreifer zu gesamten Neutrophilen $\geq 0,20$. „Smell prints“ der volatilen organischen Substanzen wurden mittels elektronischer Nase (Cyranose 320) gemessen. Die Mikroflora im Trachealaspirat wurde mit konventioneller Mikrobiologie und zusätzlich durch Amplifikation der 16s rDNA charakterisiert.

Ergebnisse. Die lineare Diskriminanz-Analyse grenzt die „smell prints“ von Frühgeborenen mit systemischer Infektion $(n=8)$ klar von denen von Frühgeborenen ohne Infektion ab. Dies ist unabhängig von einem positiven $(n=15, p<0,0001$, Cross validation value $[\mathrm{CVV}] 62,5 \%$, Mahalanobis Distanz $[M D] 2,39)$ oder negativen Keimnachweis $(n=16$; p<0,0001; CVV 70,0\%; MD 2,55). Die Gruppen mit und ohne Keimbesiedlung unterscheiden sich nicht signifikant von einander $(\mathrm{p}=0,11)$. Die Messung ist in weniger als 5 Minuten durchführbar. 
Diskussion. Wir haben erstmals nachgewiesen, dass „smell prints“ direkt am Trachealaspirat gemessen und zur nichtinvasiven Diagnostik genutzt werden können. Trachealaspirat ist bei beatmeten Patienten einfach zu gewinnen, da Sekret aus klinischen Gründen routinemäßig entfernt werden muss. Um den Einfluss des Gestationsalters, Geschlechts oder andere Variablen zu untersuchen, sind noch größere Studien nötig. Allerdings wurden in unserer Studie Proben von Patienten, die im Verlauf eine Infektion entwickelten bzw. besiedelt wurden, jeweils richtig eingeordnet. Dies zeigt, dass die Infektion das Hauptkriterium für die Zuordnung ist und potenzielle interindividuelle Unterschiede wahrscheinlich keinen großen Einfluss auf das Ergebnis haben. Schlussfolgerung. "Smell prints“ von volatilen organischen Substanzen im Trachealaspirat maschinell beatmeter Frühgeborener erlauben zuverlässig und schnell die Unterscheidung von Kindern mit und ohne Infektion. Die „elektronische Nase“ könnte sich als wertvolle, nichtinvasive Ergänzung der Diagnostik in der Neonatologie eignen.

\section{Neonatale Chirurgie}

\section{FV47}

Konsensus-Definitionen, -Grenzwerte und -Therapieempfehlungen des pädiatrischen Leitlinien-Kommittees der WSACS zu intraabdomineller Hypertonie (IAH) und abdominellem Kompartmentsyndrom (AKS)

\section{T. Kaussen', M. Sasse \\ 'Kinderklinik der Med. Hochschule, Klinik für pädiatrische Kardiologie und Intensivmedizin, Hannover, Deutschland}

Fragestellung. Nachdem bis dato keinerlei evidenzbasierte Definitionen und Empfehlungen zur Diagnostik und Therapie von IAH und AKS im Kindes- und Jugendalter existierten, wurde im Anschluss an deren 7. Weltkongress seitens der World Society of the Abdominal Compartment Syndrome (www.WSACS.org) 2012 eine internationale pädiatrische Expertenkommission gebildet, die die Erarbeitung entsprechender Leitlinien zur Aufgabe hatte. Europäische Vertreter waren die o. g. Autoren.

Methodik. Im Rahmen systematischer Recherchen wurde relevante Literatur mit Bezug zu pädiatrischem IAH und AKS identifiziert, aus der Konsensus-Empfehlungen und Management-Statements unter Nutzung einer modifizierten Delphi-Methodik nach dem GRADE-Modell [Evidenzgrade A (hoch) bis D (niedrig)] entwickelt wurden.

Ergebnisse. Die Ergebnisse wurden zusammen mit den überarbeiteten Konsensus-Leitlinien für Erwachsene 2013 in Intensive Care Medicine publiziert [ICM 2013 Jul;39(7):1190-1206]. Neben allgemeinen Begriffsdefinitionen, Risikofaktoren und kritischen IAD-Grenzwerten (IAD: intraabdomineller Druck) wurden Empfehlungen in Bezug auf ein standardisiertes IAH- und AKS-Monitoring sowie -Management inklusive medizinischer, interventioneller und chirurgischer Therapieoptionen erstmalig auch für das Kindes- und Jugendalter formuliert. Während im Erwachsenenalter ein IAD von $12 \mathrm{mmHg}$ als grenzwertig gilt, ist bei Kindern spätestens ab einem IAD von $10 \mathrm{mmHg}$ von einer IAH auszugehen. Das zusätzliche Auftreten einer neuen oder aggravierten Organdysfunktion markiert den Übergang in das Vollbild eines AKS, dessen Diagnose die schnellstmögliche abdominelle Druckentlastung zur Folge haben sollte. Reicht selbst eine dekompressive Laparotomie zur dauerhaften IAD-Reduktion nicht aus, muss ein prophylaktisches Open-abdomen-Konzept erwogen werden. Das Konzept des abdominellen Perfusionsdruckes (APD = MAD-IAD) kann die Abschätzung des pathogenetischen Einflusses einer IAH im klinischen Alltag erleichtern.

Diskussion. Die bis dato unter neonatologischen und pädiatrischen Intensivisten verbreitete Unsicherheit und Zurückhaltung in Bezug auf regelmäßige IAD-Messungen und die Einleitung v. a. invasiv-dekomprimierender Therapiemaßnahmen wurde im Rahmen von Befragun- gen in der Vergangenheit häufig mit dem Fehlen von Richtlinien und Definitionen begründet. Die erstmalige Publikation von IAD-Grenzwerten und Management-Empfehlungen stellt demnach einen wesentlichen Behandlungsfortschritt und eine therapeutische Entscheidungshilfe dar, die eine signifikante Reduktion der Morbidität und Mortalität von IAH und AKS zur Folge haben sollte.

Schlussfolgerung. Da die Evidenz der zugrunde liegenden Literatur als relativ niedrig einzustufen ist, sind dringend gut konzipierte und möglichst multizentrische Grundlagen- und Interventionsstudien notwendig, die eine kritische Reevaluation der Konsensus-Ergebnisse ermöglichen.

\section{FV48}

Epidemiologie, Therapie und Outcome des angeborenen Chylothorax (ACT) in Deutschland

\section{A. Bialkowski', C. Poets' ${ }^{2}$ A. Franz ${ }^{2}$}

${ }^{1}$ Kinderklinik Tübingen, Neonatologie, Tübingen, Deutschland, ${ }^{2}$ EberhardKarls-Universität Universitätsklinik für Kinderheilkunde und Jugendmedizin, Neonatologie, Tübingen, Deutschland

Hintergrund. Der ACT ist eine seltene Erkrankung unklarer Ursache. Das therapeutische Vorgehen variiert und beinhaltet eine total-parenterale Ernährung, Diät mit mittelkettigen Fettsäuren, Octreotid- oder Somatostatingabe und chirurgische Interventionen. Keiner dieser Therapieansätze wurde bislang prospektiv evaluiert.

Ziel. Erhebung von Inzidenz, therapeutischem Vorgehen und Outcome des ACT im Jahr 2012 in Deutschland.

Methoden. ACT wurde definiert als nicht-traumatischer, $>60 \%$ Lymphozyten enthaltender Pleuraerguss innerhalb der ersten 28 Lebenstage. Mittels einer ESPED (Erhebungseinheit für seltene pädiatrische Erkrankungen in Deutschland)-Umfrage erhielten alle pädiatrischen Kliniken $(\mathrm{n}=432)$ im Jahr 2012 monatliche Abfragekarten mit der Bitte, alle Fälle mit ACT zu melden. Gemeldete Fälle wurden auf Grundlage eines Fragebogens sowie des Arztbriefs analysiert. Die Ethikkommission Tübingen stimmte dieser Untersuchung zu. Daten sind als Median (Range) oder $\mathrm{n} / \mathrm{N}$ dargestellt.

Ergebnisse. Insgesamt wurden 37 Fälle gemeldet, von denen 28 die Einschlusskriterien erfüllten. Ein ausgefüllter Fragebogen oder Arztbrief lag in 27 Fällen vor (11 Mädchen). Eine vollständige Meldung vorausgesetzt, betrug die Inzidenz des ACT in Deutschland 2012 1:24.0oo. Das Gestationsalter bei Geburt betrug 33,2 (24,7-39,1) Wochen, das Geburtsgewicht $2500 \mathrm{~g}$ (740-3940 g). Bei 9/27 wurde eine zugrundeliegende syndromale Erkrankung vermutet oder bestätigt, meist ein Noonan-Syndrom (5/9). Pränatal wurde bei 12/27 Kindern interventiert. Postnatal benötigten 23 eine mechanische Beatmung, weitere 3 CPAP, nur 1 Kind blieb spontan atmend. Die Beatmungsdauer betrug 8 (2-105) Tage. $17 / 27$ wurden mit Inotropika/Vasopressoren für 4 (1-36) Tage behandelt. 25/27 erhielten eine Pleuradrainage über 11 (1-36) Tage. Das kumulative Punktatvolumen betrug $1800 \mathrm{ml}$ (30-10.200 ml). 24 Patienten erhielten während des Aufenthaltes eine Diät mit mittelkettigen Fettsäuren. Acht wurden mit Octreotid für 21 (10-45) Tage, Beginn am 8. (4.-17.) Lebenstag und 2 mit Somatostatin behandelt (1 Kind erhielt beides). $18 / 27$ überlebten ohne weitere Folgeschäden, 6 hatten bei Entlassung Probleme bedingt durch ihre Grundkrankheit und 3 verstarben mit 2, 37 bzw. 144 Tage. Die Dauer des Krankenhausaufenthaltes bei den Überlebenden betrug 51 (20-127). Kinder, die mit Octreotid behandelt wurden hatten ein ähnliches Outcome wie solche, die kein Octreotid erhielten (Dauer des Krankenhausaufenthaltes bei Überlebenden 59 (28-127) Tage vs. 37 (20-75) Tage, $\mathrm{p}=0,08$; Mortalitätsrate $\mathrm{o} / 9$ vs. $3 / 18, \mathrm{p}=0,31$ ).

Schlussfolgerung. Der ACT hat eine günstige Prognose wenn er nicht mit genetischen oder metabolischen Erkrankungen assoziiert ist. Längere Krankenhausaufenthalte mit intensivmedizinischer Behandlung sind bei ACT die Regel. Es werden multinationale Studien benötigt, um die Effizienz und Sicherheit der Therapieoptionen prospektiv zu evaluieren. 


\section{FV49}

\section{Multidisziplinäres Management von Patienten mit intrauterin} bekanntem Neuralrohrdefekt

\section{B. Wiebe', C. Ditscheid', M. Messing-Jünger ${ }^{2}$, M. Ehlen ${ }^{7}$}

${ }^{1}$ Asklepios Klinik St. Augustin, Neonatologie und Pädiatrische Intensivmedizin, St. Augustin, Deutschland, ${ }^{2}$ Asklepios Kinderklinik Sankt Augustin, Abteilung für Kinderneurochirurgie, Sankt Augustin, Deutschland

Einleitung. Die erfolgreiche Behandlung komplexer angeborener Fehlbildungen setzt ein standardisiertes multidisziplinäres Vorgehen voraus. Dieser Ansatz beinhaltet eine kompetente pränatale Diagnostik und eine interdisziplinäre gemeinsame pränatale Beratung der Eltern durch $\mathrm{Ge}$ burtshelfer, Kinderneurochirurgen und Neonatologen. Standards bzgl. des geburtshilflichen Vorgehens, der Erstversorgung des Neugeborenen im Kreissaal und der anschließenden neurochirurgisch-intensivmedizinischen Versorgung ergänzen diesen Therapieansatz. Diese Standards sind wichtig sowohl für ein erfolgreiches chirurgisch-intensivmedizinisches Vorgehen, aber auch ebenso essentiell für eine kompetente und komplikationsvermeidende Pflege. Elternschulung, sozialmedizinische Unterstützung und die Anbindung an ein sozialpädiatrisches Zentrum mit enger Zusammenarbeit der Fachdisziplinen Neurochirurgie, Kinderorthopädie, Kinderurologie und Physiotherapie bilden die Grundlage für eine langfristig bestmögliche Versorgung der betroffenen Kinder und deren Familien auch nach dem stationären Aufenthalt.

Methodik und Ergebnisse. In den Jahren 2007 bis 2/2014 wurden insgesamt 39 Patienten mit Neuralrohrdefekten in unserem Zentrum geboren. Die Kaiserschnittentbindung mit 38+o Schwangerschaftswochen ist der bevorzugte und empfohlene Entbindungsmodus. Myelolyse und plastische Deckung erfolgen regelhaft am 1. Lebenstag; 6 Patienten erhielten zeitgleich einen VP-Shunt. Ansonsten wurde die VP-Shuntanlage im Mittel am 9. Tag (2-24 Tage) durchgeführt, bei 5 Patienten lag kein interventionspflichtiger Hydrocephalus vor. Nach einer intensiven Schulungsphase der Eltern, in der Regel auf der Mutter-Kind-Station, konnten die Kinder nach 14 Tagen bis 8 Wochen entlassen werden. Das multidisziplinäre Versorgungsregime von intrauterin bekannten Feten mit Neuralrohrdefekten mit wird dargestellt. Insbesondere das standardisierte Erstversorgungskonzept sowie das postnatale Betreuungskonzept anhand von Checklisten wird detailliert vorgestellt.

Schlussfolgerung. Am Beispiel einer komplexen angeborenen Fehlbildung wird die Wichtigkeit eines multidisziplinären und familienorientierten Versorgungskonzeptes deutlich. Die Besonderheiten der postnatalen Phase müssen dem gesamten neonatologischen Team bekannt sein

\section{FV50}

Der Plethysmographie-Variabilitätsindex als Indikator eines intraoperativen Volumenmangels bei Neu- und Frühgeborenen

\section{S. Bagci', S. Beckené, A. Müller', A. Heydweiller', P. Bartmann', A. Franz ${ }^{3}$}

'Zentrum für Kinderheilkunde am Universitätsklinikum Bonn, Neonatologie, Bonn, Deutschland, ${ }^{2}$ Klinik und Poliklinik für Allgemein-, Viszeral-, Thorax- und Gefäßchirurgie am Universitätsklinikum Bonn, Kinderchirurgie, Bonn, Deutschland, ${ }^{3}$ Eberhard-Karls-Universität Universitätsklinik für Kinderheilkunde und Jugendmedizin, Neonatologie, Tübingen, Deutschland

Einleitung. Volumensubstitution ist Mittel der ersten Wahl zur Behandlung der intraoperativen arteriellen Hypotension bei Neu- und Frühgeborenen (NG/FG). Der Plethysmographie-Variabilitätsindex (PVI) ermöglicht eine nicht invasive, kontinuierliche Quantifizierung der durch intrathorakale Druckschwankungen bedingten, atemabhängigen Variabilität der Pulskurve und könnte deshalb einen Volumenmangel anzeigen.

Fragestellung. Zeigt der PVI bei NG/FG einen intraoperativen Volumenmangel an?

Methoden. Bei 73 NG/FG wurden während einer Operation mit Hilfe eines Radical 7.0 Pulsoximeters (Masimo) kontinuierlich die PVI-
Werte aufgezeichnet. Dreizehn von 73 NG/FG (18\%) wurden aufgrund technischer Fehler während der Überwachung von der Analyse ausgeschlossen. Bei 15 NG/FG, die keine Volumengaben (VG) erhielten, wurde der 95. Perzentilenrang aller PVI-Werte als oberer Grenzwert für den Referenzbereich ermittelt. Nach Ermessen des Arztes wurden VG verabreicht. Ein „tatsächlicher" Volumenmangel (tVM) wurde definiert als Anstieg des mittleren arteriellen Blutdrucks (MAD) um $>10 \%$ nach Volumenbolus. Die Episoden, in denen für $>3$ min keine erneute VG folgte, wurden als effektive Volumengabe (EVG) definiert. Eine initial ineffektive VG (IIEVG) wurde definiert als Episode, bei der innerhalb von $\leq 3$ min erneut mindestens 1 weitere VG notwendig erschien. Angaben erfolgen als Median (Interquartilsabstand).

Ergebnisse. Der ermittelte PVI-Grenzwert betrug 16\% (P95 aller intraoperativer PVI bei Kindern, die keine Kreislaufunterstützung erhielten). Bei $45 \mathrm{NG} / \mathrm{FG}$ erfolgten $175 \mathrm{VG}$, davon waren $106 \mathrm{EVG}$ und 28 IIEVG, die innerhalb von $<3$ min von $1(n=20), 2(n=5), 3(n=1)$ und 4 $(n=2)$ VG gefolgt wurden. Insgesamt erfolgten also $41 / 175$ VG in einem Abstand von $\leq 3$ min vor einer erneuten VG (Intermediate VG; IVG). Alle 106 EVG und alle 28 IIEVG führten (im Falle der IIEVG nach zusätzlichen VG) zu einem Anstieg des MAD um >10\% und erfüllten damit das Kriterium eines tVM. Der mediane PVI-Wert vor VG dieser 134 tVM-Episoden betrug 20\% (17-23\%), nach (letzter) VG fiel der PVI auf 14\% (13-16\%; $\mathrm{p}<0,001)$. Vor 113/134 tVM betrug der maximale PVI $>16 \%$ (Sensitivität für „tatsächlichen“ Volumenmangel: 84\%). In 33/41 (81\%) IVG fiel der PVI nach VG nicht auf Werte $\leq 16 \%$ ab. Dagegen fiel der PVI in $102(76 \%)$ der 134 VG-Episoden, nach denen keine erneute VG innerhalb von $<3$ min erforderlich war, auf Werte $\leq 16 \%$ ( $p<0,001)$. Der mittlere PVI-Wert nach VG war in den 41 IVG Episoden höher als in den 134 Episoden, nach denen keine erneute VG innerhalb von $<3$ min erforderlich war $[18 \%(16-20 \%)$ vs. $14 \%(13-16 \%)$; $\mathrm{p}<0,001)$.

Schlussfolgerung. Der PVI kann bedingt als Indikator für einen intraoperativen Volumenmangel die klinische Einschätzung ergänzen. Die Ergebnisse sind jedoch nicht geeignet, eine generelle Empfehlung zur Verwendung des PVI bei NG/FG auszusprechen.

\section{FV51}

Outcome von Kindern mit gastrointestinalen Fehlbildungen prospektive, Kontrollgruppen-kontrollierte Studie mit retrospektiven Aspekten

\section{N. Doberschütz', A. Allendorf', R. Dewitz'ㄹ, U. Rolle', R. Schlößer}

'Klinikum der J.W.Goethe-Univ. Zentrum der Kinderheilkunde, Neonatologie, Frankfurt, Deutschland, ${ }^{2}$ Klinikum der J.W.Goethe-Univ. Zentrum der Kinderheilkunde, Neurologie, Frankfurt am Main, Deutschland, ${ }^{3}$ Universitätsklinikum der Johann-Wolfgang-Goethe-Universität Frankfurt am Main, Klinik für Kinderchirurgie, Frankfurt, Deutschland, ${ }^{4}$ Klinikum der J.W.Goethe-Universität, Zentrum der Kinderheilkunde, Abteilung für Neonatologie, Frankfurt am Main, Deutschland

Hintergrund. Die Überlebenschance von Kindern mit angeborenen gastrointestinalen Fehlbildungen ist in den letzten Jahrzenten stark angestiegen. Zum Langzeit-Outcome dieser Kinder gibt es wenige Daten. Von Juni 2011 bis Juli 2013 führten wir an unserer Klinik eine prospektive Kontrollgruppen kontrollierte Studie zum entwicklungsneurologischen Outcome von Kindern mit gastrointestinalen Fehlbildungen und Operationen durch.

Patienten. In den Jahrgängen 2008-2011 wurden in unserer Klinik 63 Kinder mit einer solchen Fehlbildung versorgt. 45 Kinder waren $>32$ Schwangerschaftswochen und hatten keine Ausschlusskriterien. Die Eltern von 40 Kindern stimmten der Teilnahme an der Studie zu. Jedem Patient wurde ein Kontrollkind gleichen Geschlechts, Schwangerschaftswoche, Geburtsgewichts und ähnlichem sozioökonomischen Status, Haushaltssprache, Mehrsprachigkeit gegenübergestellt.

Methode. Die Kinder wurden im Alter von 18 bis 36 Monaten mittels Bayley II von der Kinderpsychologin getestet; Anamneseerhebung, körperliche und neurologische Untersuchungen führte die Kinderärz- 
tin durch. Alle Paare wurden im gleichen Testalter untersucht. Der sozioökonomische Status wurde mittels Fragebogen erhoben. Neonatale Daten (Beatmungszeiten, Komplikationen, Ernährung, Temperatur, $\mathrm{CO}_{2}$ usw.) wurden erhoben, Operationsdauer und Narkosezeiten addiert.

Ergebnisse. Der mittlere psychomotorische Index (PDI) lag in der Patientengruppe bei 103 (76-121) und in der Kontrollgruppe bei $106(84-$ 121), auch grob/feinmotorisch ergab sich kein Unterschied zwischen den Gruppen (Patienten 67,95\%/56,35\% vs. Kontrollgruppe 56,35\%/70,98\%). Beim mental developmental index (MDI) lag der mittlere Wert der operierten Kinder bei 102 (70-127), bei den Kontrollkindern bei 110 (68-128) und damit signifikant $(\mathrm{p}=0,021716)$ höher. Dieser Unterschied liegt rein im sprachlichen Bereich: Patienten $56,45 \%$ vs. Kontrollkinder $72,14 \%$ $(\mathrm{p}=0,028747)$. Im nichtsprachlichen Bereich verhielten sich die Gruppen gleich: Patienten $71,66 \%$ vs. $73,33 \%$. Spearman Rank Correlation Coefficient zeigte keine Korrelation zwischen Narkosedauer und MDI.

Diskussion. Der mittlere MDI der Patienten liegt im Durchschnittsbereich der Bevölkerung, ist aber auf Grund der sprachlichen Entwicklung signifikant unter dem der Kontrollgruppe. Deshalb gehen wir davon aus, dass Kinder mit angeborenen gastrointestinalen Fehlbildungen und Operationen in der Neonatalzeit sich normal entwickeln, aber ein höheres Risiko für eine Sprachentwicklungsverzögerung haben. Dies sollte in der Nachsorge berücksichtigt werden.

\section{FV52}

\section{Jahre Erfahrung mit verzögertem Primärverschluss} bei großen Omphalocelen und Riesen-Omphalocelen nach externer Kompression

\section{S. Berger', M. Bürki', V. Engel ${ }^{2}$}

${ }^{1}$ Inselspital, Universität Bern, Kinderchirurgische Klinik, Bern, Schweiz, ${ }^{2}$ Universitätsmedizin Mainz, Kinderchirurgie, Mainz, Deutschland

Ziel. Der Verschluss großer und sehr großer Omphalocelen ist weiterhin eine Herausforderung. Silastic-Silos haben die Überlebenschancen dieser Kinder verbessert, weisen aber eine hohe Rate an oft schwer zu behandelnden Komplikationen auf. Wir haben deshalb die Technik der präoperativen externen Kompression im intakten Omphalocelensack benutzt, um den Einsatz von Silastic-Silos nach Möglichkeit zu vermeiden.

Methoden. Retrospektive Analyse der Patientenkohorten aus 2 Universitätskliniken (Mainz und Bern). Von 1998 bis 2013 wurden 4 Neugeborene mit großen Omphalocelen (Durchmesser $>5 \mathrm{~cm}$ ) und $7 \mathrm{mit}$ Riesenomphalocelen (Durchmesser $>8 \mathrm{~cm}$ ) behandelt. Durch tägliche sterile Verbandswechsel auf der Intensivstation wurde mit einer elastischen Binde der Druck auf den Omphalocelensack langsam erhöht und der Sack hierbei an der Nabelschnur aufgehängt.

Ergebnisse. Die mittlere Dauer der externen Kompression betrug 4,7 $\pm 1,5$ Tage bei den großen Omphalocelen $(n=4)$ und 6,3 $\pm 2,9$ Tage bei den Riesenomphalocelen $(n=7)$. Danach war ein vollständiger Verschluss aller Schichten möglich bei 6 Patienten (alle 4 großen Omphalocelen und 2 der Riesenomphalocelen). Bei 3 Kindern mit Riesenomphalocele konnte nur die Haut verschlossen werden, die 2 übrigen Kinder benötigten einen schrittweisen Verschluss, eines davon mit Silatic-Silo. Insgesamt 4 Patienten entwickelten eine Sepsis im Behandlungsverlauf, unabhängig von der Dauer der externen Kompression. Alle Patienten überlebten. Schlussfolgerung. Die externe Kompression erlaubte den Verschluss der Bauchwand in allen Schichten mit nur einer einzigen Narkose und Operation bei allen Kindern mit großen Omphalocelen innerhalb von 3-6 Tagen nach Geburt. Bei Riesenomphalocelen betrug die Erfolgsrate 2/7 für einen vollständigen Verschluss und $3 / 7$ für einen Hautverschluss innerhalb von 5-7 Tagen nach Geburt. Die externe Kompression im Omphalocelensack erschien sicher für einen Zeitraum von 5-7 Tagen. Danach ist entweder ein Verschluss oder ein Verfahrenswechsel angezeigt. In der aktuellen Fallserie war ein Silastic-Silo nur bei einem Patienten für 10 Tage notwendig.

\section{Ernährung}

\section{FV53}

Zusammensetzung von Frühgeborenen-Muttermilch und Einflussfaktoren auf den Proteingehalt

\section{A. Kreissl', V. Zwiauer', A. Repa', C. Binder', M. Thanhäuser', A. Berger', N. Haiden ${ }^{1}$}

'Universitätsklinik für Kinder- und Jugendheilkunde, Medizinische Universität Wien, Abteilung für Neonatologie, pädiatrische Intensivmedizin und Neuro-

pädiatrie, Wien, Österreich

Fragestellung. Muttermilch (MM) ist aufgrund Ihrer besonderen nutritiven Inhaltstoffe die beste Quelle für die Ernährung von Frühgeborenen. Allerdings enthält $M M$ keine ausreichenden Mengen an Protein, Energie, Kalzium und Phosphor, um das Frühgeborene entsprechend zu versorgen, weswegen eine Fortifizierung empfohlen wird. Insbesondere Protein stellt einen sehr wichtigen Nährstoff für Wachstum und Gehirnentwicklung dar. Eine zu geringe Proteinzufuhr ist beim Frühgeborenen mit schlechter Gewichtszunahme, geringem Längenwachstum und schlechtem entwicklungsneurologischem Outcome assoziiert. Das Ziel dieser Studie war es, die Zusammensetzung und die Variationen von Frühgeborenen-Muttermilch, und die Effekte von Laktationstag und unterschiedlichen Prozessierungsarten auf den Nährstoffgehalt mit dem MIRIS Muttermilch-Analysator zu untersuchen.

Material und Methode. Untersucht wurden Muttermilchproben von Müttern Frühgeborener, die mit einem Geburtsgewicht unter $1500 \mathrm{~g}$ und einem Gestationsalter $<32$ Wochen geboren wurden. Mittels des MIRIS Muttermilch-Analysators wurde die Protein-, Fett-, Laktose-, und Energiezusammensetzung in der Muttermilch nach unterschiedlichen Prozessierungsmethoden untersucht a) frische unpasteurisierte $M M ~ b)$ frische pasteurisierte $M M$ c) aufgetaute unpasteurisierte $M M$ und d) aufgetaute pasteurisierte MM. Des Weiteren wurde der Zusammenhang zwischen Proteingehalt und Laktationstag evaluiert.

Ergebnisse. Insgesamt wurden 83 Muttermilchproben untersucht. Die native Muttermilch setzte sich im Mittelwert aus 1,1 $\pm 0,37$ g Pro-

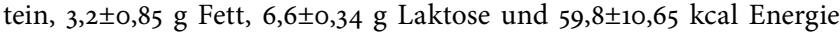
pro $100 \mathrm{ml}$ Frauenmilch zusammen. Die größte Variation zeigte sich bei Protein mit 0,2-2,2 g/100 ml Muttermilch. Der Laktationstag korrelierte signifikant mit dem Proteingehalt ( $\mathrm{p}<0,0001 ; \mathrm{r}=-0,4243$ ). Die Prozessierungsarten von Muttermilch hatten keinen Einfluss auf den Protein- oder Nährstoffgehalt.

Diskussion. Die größte Variabilität im Nährstoffgehalt von Muttermilch wurde im Proteingehalt festgestellt. Diese Studie zeigte, dass sowohl von Mutter zu Mutter große Unterschiede messbar waren, als auch der Laktationstag Einfluss auf den Proteingehalt hat. Der Proteinanteil frühgeborener Frauenmilch nimmt mit der Laktationsperiode signifikant ab. Unterschiedliche Prozessierungsarten beeinflussen den Nährstoffgehalt von Muttermilch nicht.

Schlussfolgerung. Mit dem MIRIS Muttermilch-Analysator kann die Nährstoffzusammensetzung von Muttermilch ermittelt werden. Die Ergebnisse indizieren, dass eine Nährstoffanalyse der Muttermilch bei Frühgeborenen wichtig ist, um die Milch individuell und gezielt anreichern zu können. Die Ermittlung der Muttermilch-Inhaltsstoffe kann somit das Ernährungsmanagement von Frühgeborenen im klinischen Setting verbessern.

\section{FV54}

Target fortification of breast milk with fat, protein and carbohydrates for preterm infants: how should fortifiers be composed?

\section{N. Rochow' , G. Fusch', A. Ali', C. Fusch'}

'McMaster University, Division of Neonatology, Department of Pediatrics, Hamilton, Ontario, Kanada

Background. Target fortification (TFO) is a concept in which breast milk $(\mathrm{BM})$ is 1) analyzed for macronutrient content, 2) enriched with com- 


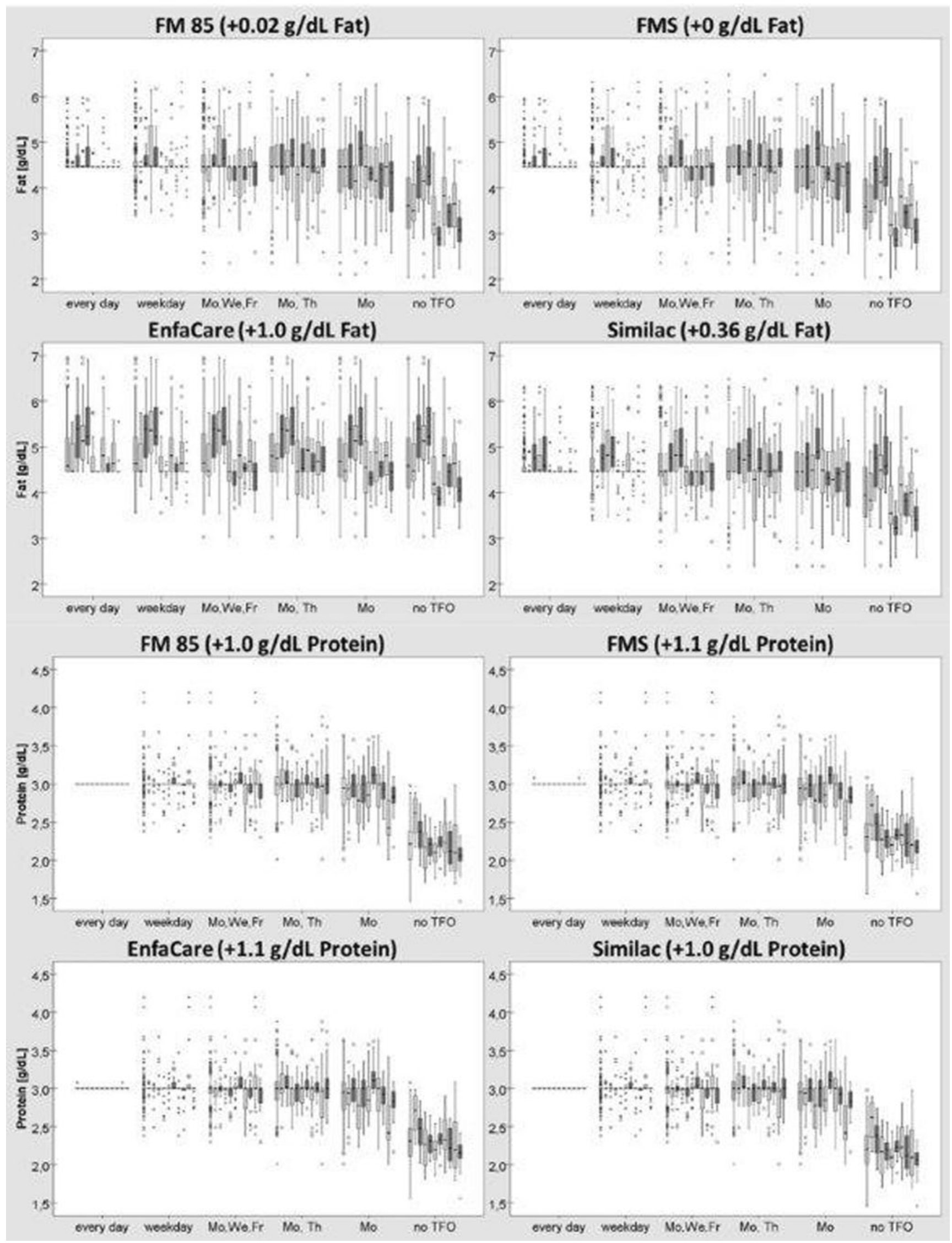

$\Delta \mathrm{FV}_{54}$ - Abb. 1 Variation of fat and protein in target fortified breast milk with Nestles ${ }^{\circ} \mathrm{FM} 85$ for different frequencies of macronutrient measurements compared with routine fortified breast milk. Grouped boxplots show on first boxplot data for all samples (1x $n=210)$ followed by boxplots with data for individual subjects ( $10 \mathrm{x} \mathrm{n}=21)$ 
mercial breast milk fortifier (fixed-dose fortification-FDF) and 3) individually fortified with fat, protein and carbohydrate modular in order to make up to recommended intake according to ESPGHAN. TFO overcomes the uncertainty of macronutrient intake in routine FDF resulting from natural variation in BM and improves growth of VLBW infants. However, commercial fortifiers have different profile of macronutrient composition and will influence TFO.

Objective. To study the variation of macronutrient intake when TFO is done with four different commercial available milk fortifiers and based on various frequencies of milk measurements.

Study design. Data were obtained from a single-center RCT (10 healthy, fully BM fed infants, GA: $26.1 \pm 1.3 \mathrm{wks}$, BW: $890 \pm 210 \mathrm{~g}$, HC: $25.2 \pm 3.9 \mathrm{~cm}$, and their mothers, age: $29 \pm 6 \mathrm{yrs})$. Daily measurements $(\mathrm{n}=210)$ of native BM's fat, protein (near infrared milk analyzer), and lactose(UPLCMS/MS) levels provided the basis to add fat, protein and carbohydrates $(\mathrm{CHO})$ into routinely fortified $\mathrm{BM}$ using modular products to meet ESPGHAN recommendations (fat: 4.2, protein: 3.0, CHO: $8.5 \mathrm{~g} / \mathrm{dL}$ ). Data were analyzed to model the intake of i) calories, ii) macronutrients, iii) gram protein per energy, and iv)\% $\mathrm{CHO} \mathrm{kcal}$ of non-protein energy assuming TFO with varying frequency of $\mathrm{BM}$ measurements $(7,5$, 3,2 and 1 per wk) and compared to routinely fortified BM. The model was employed for 4 different commercially available routine fortifiers (FM85, FMS, Enfamil, Similac).

Results. Measurement $1 / \mathrm{wk}$ increased the median macronutrient intake for all of the milk fortifiers and day-to-day variation was not higher compared to native BM. Day-to-day variation of macronutrients decreased as the frequency of milk analysis increased (Abb. 1). After routine fortification of $\mathrm{BM}$, median carbohydrate level was observed to exceed target levels in FM 85, FMS, Similac fortifier and median fat level in Enfamil fortifier.

Conclusions. Weekly measurements of $\mathrm{BM}$ macronutrient content might provide a reasonable balance between workload and desired clinical outcome. Some fortifiers over fortify due to their different profiles. Hence, either target values for macronutrients need to correct for excess intake by routine fortification in order to achieve the desired intake, or the composition of routine fortifier for use with TFO needs to be reconsidered.

\section{FV55}

Probiotika (Infloran ${ }^{\oplus}$ ) zur Prophylaxe der nekrotisierenden Enterokolitis: Einfluss der enteralen Ernährung auf die Wirksamkeit

\section{Thanhäuser', A. Repa', M. Weber' ', D. Endress' ${ }^{3}$, A. Kreissl', C. Binder', A. Berger', N. Haiden \\ 'Universitätsklinik für Kinder- und Jugendheilkunde, AKH Wien, Abteilung für Neonatologie, pädiatrische Intensivmedizin und Neuropädiatrie, Wien, Österreich, ${ }^{2}$ Medizinische Universität Wien, Universitätsklinik für Radiologie und Nuklearmedizin, Wien, Österreich, ${ }^{3}$ Wilhelminenspital der Stadt Wien, Ab- teilung für Kinder- und Jugendheilkunde, Wien, Österreich}

Hintergrund. Spezielle Probiotika haben bei Frühgeborenen einen protektiven Effekt auf die Entstehung einer nekrotisierender Enterokolitis (NEC). Das Probiotikum Infloran ${ }^{\circledR}$ (Lactobacillus acidophilus, Bifidobacterium infantis) hat in einer Studie an einer chinesischen Very-lowbirth-weight(VLBW)-Population eine Reduktion der NEC Rate um $80 \%$ bewirkt (Lin et al 2005). Infloran ${ }^{\circledR}$ wurde 2010 auch an unserer Abteilung eingeführt.

Ziele. 1. die Veränderung der NEC-Inzidenz bei VLBW des an unserer Abteilung vornehmlich kaukasischen Kollektivs zu analysieren. 2. $\mathrm{Zu}$ klären inwiefern die Nahrung (Muttermilch vs. Formula) einen Effekt des Probiotikums beeinflusst. 3. Einflüsse auf den Schweregrad verbleibender NECFälle zu analysieren.

Material und Methoden. Im Rahmen einer prospektiven Anwendungsbeobachtung erhielten alle Frühgeborenen $<1500 \mathrm{~g}$, die vor der $34+0$ SSW geboren wurden, seit September 2010 das Probiotikum Infloran (Lactobacillus acidophilus, Bifidobacterium infantis). Die Verabrei- chung ( $2 \times 1 \mathrm{Kapsel} / \mathrm{Tag})$ erfolgte mit der ersten enteralen Ernährung bis zur Entlassung bzw. 37+o SSW. Ausschlusskriterien waren Darmfehlbildungen, Tod in der ersten Lebenswoche und Transfer vor der 34 . SSW. Um eine Reduktion der NEC Inzidenz um $65 \%$ zu zeigen, waren 226 Patienten pro Gruppe notwendig ( $\mathrm{a}=0,05$, Power $80 \%$ ). Die NEC Rate wurde mittels logistischer Regression unter Einbeziehung von Gestationswoche und Geburtsgewicht statistisch analysiert.

Ergebnisse. Insgesamt wurden 463 Kindern untersucht (Kontrollgruppe: $n=233$, Interventionsgruppe: $n=230$ ). Nach Einführung von Infloran $^{\circ}$ konnte eine Reduktion der NEC Inzidenz um $32 \%$ gezeigt werden (10,3\% vs. 7\%, p=0,092). Die Subgruppenanalyse getrennt nach Art der Ernährung in den ersten zwei Lebenswochen zeigte, dass ein Absinken der NEC-Rate durch Infloran nur bei Kindern, die auch Muttermilch erhalten haben, zu beobachten war [NEC-Rate keine Probiotika vs. Infloran ${ }^{\infty}$ - Muttermilch: $11,1 \%(n=179)$ vs. 5,4\% $(n=183), p=0,027$; Formula: $7,4 \%(n=54)$ vs. $13,6 \%(n=44), p=0,345]$. Eine Reduktion der Schwere der NEC wurde nicht beobachtet.

Diskussion. Der Effekt von Infloran ${ }^{\circledR}$ war in unserer kaukasischen VLBW-Population schwächer als erwartet und nur bei Kindern, die auch Muttermilch erhalten haben, nachweisbar. Die Wirkungslosigkeit des Präparats Infloran ${ }^{\circledR}$ bei reiner Formulaernährung ist besorgniserregend, weshalb der Einfluss der enteralen Ernährung auf den Effekt von Infloran $^{\oplus}$ bzw. anderer probiotischen Präparate in künftigen Studien mit untersucht werden sollte.

\section{FV56}

Melatoninreiche Ernährung in der Fetalzeit durch Fruchtwasser

S. Bagci', Ö. Altuntas', A. Willruth'², D. Katzer', J. Reinsberg³, P. Bartmann', A. Müller

'Zentrum für Kinderheilkunde am Universitätsklinikum Bonn, Neonatologie, Bonn, Deutschland, ${ }^{2}$ Zentrum für Geburtshilfe und Frauenheilkunde am Universitätsklinikum Bonn, Geburtshilfe und Perinatalmedizin, Bonn, Deutschland, ${ }^{3}$ Zentrum für Geburtshilfe und Frauenheilkunde am Universitätsklinikum Bonn, Gynäkologische Endokrinologie und Reproduktionsmedizin, Bonn, Deutschland

Einleitung. Melatonin (MT), ein lipophiles Neurohormon, besitzt vielfältige biologische Eigenschaften, wie antiinflamatorische Wirkung, Immunstimulation, Stimulierung der Aktivität der antioxidativen Enzyme. Diese Eigenschaften üben nicht nur systemische, sondern auch lokale und intrazelluläre Effekte im Organismus aus. MT zeigt auch einen starken gastroprotektiven Effekt. Die Studien zeigten, dass eine parenterale oder orale Gabe von MT oder Tryptophan als Vorläufer von MT eine Akkumulation von MT im gastrointestinalen Trakt verursacht. Es ist bekannt, dass ein Fetus ab der 16. Gestationswoche das Fruchtwasser (FW) schlucken kann. Obwohl die Melatoninkonzentration (MTK) im FW vorher in zwei Studien untersucht wurde, liegen bisher noch keine Daten über den Melatoninstatus des FWs in der Fetalzeit vor.

Fragestellung. Unterscheidet sich die MTK im FW vor und nach der 37. Schwangerschaftswoche (SSW)? Spiegelt die MTK im FW die MTK der Nabelvene wider?

Methodik. Die Fruchtwasserproben von 75 Schwangeren wurden tagsüber (zwischen 8:00-17:0o Uhr) durch eine Amniozentese entweder im Rahmen einer Pränataldiagnostik oder eines elektiven Kaiserschnittes gewonnen. Direkt im Anschluss an die Geburt wurden die Blutproben aus der Nabelvene gewonnen.

Ergebnisse. Das mediane Gestationsalter (Interquartilsabstand; IQR) war 26 SSW (19,0-34,1) bei der Gruppe <37. SSW (Gruppe 1, n=27) und 38,6 SSW (38-39) bei der Gruppe $\geq 37$. SSW (Gruppe 2, $n=49 ; \mathrm{p}<0,001$ ). Die MTK im FW in der Gruppe 1 war signifikant niedriger als die in der Gruppe 2 [Median (IQR); 10,6 pg/ml (6,1-24,3) vs. 91,6 pg/ml (27,4$172,4) ; \mathrm{p}<0,001]$. Eine Subgruppenanalyse der Gruppe 1 ergab sich, dass die MTK im FW zwischen 30. und 37. SSW höher als die unter 30. SSW war, aber ohne statistische Signifikanz $[22,3 \mathrm{pg} / \mathrm{ml}(8,7-30,7)$ vs. 9,6 pg/ $\mathrm{ml}(5,6-14,9) ; \mathrm{p}=0,097 \mathrm{l}$. Es fand sich statistisch eine signifikante positive Korrelation zwischen der MTK im FW und dem Gestationsalter $(\mathrm{p}=0,015)$. Die MTK in der Nabelvene $(\mathrm{n}=57)$ war signifikant niedriger 

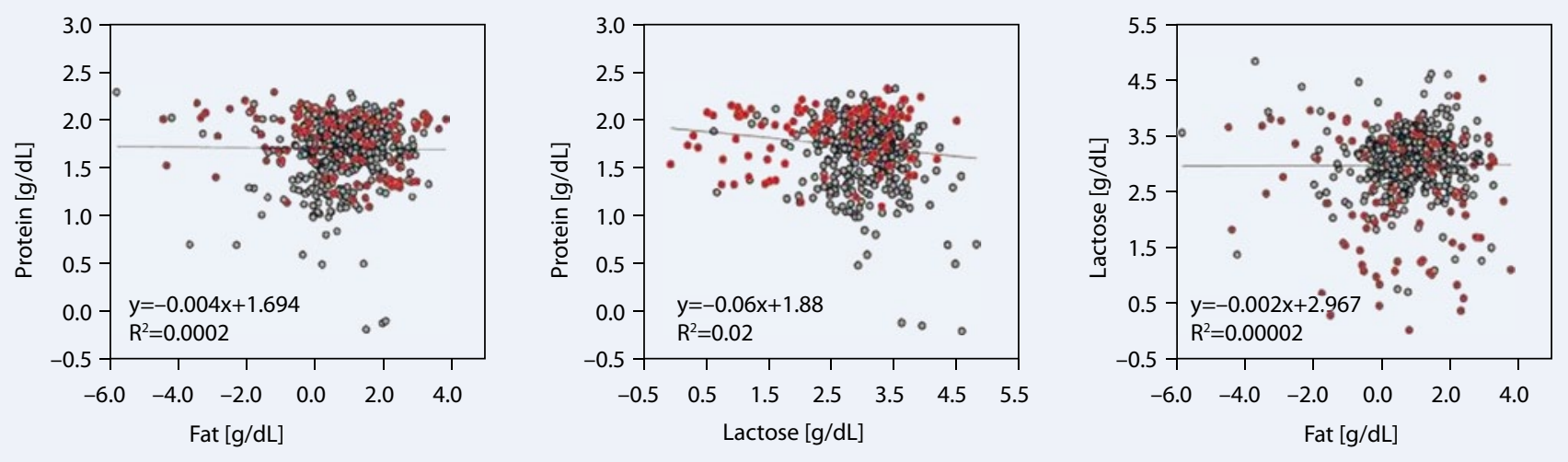

$\Delta F_{58}$ - Abb. 1 Correlation of nutritional deficiencies for fat, lactose, and protein. Differences were calculated between the ESPGHAN recommendations for preterm infants and the measured macro-nutrient concentration in term and preterm breast milk samples $(n=543)$. Pooled milk samples are represented as gray dots, fore, mid and hind milk samples as red dots

als die im FW [11,2 pg/ml (6,1-20,7) vs. 58,4 pg/ml (14,7-135,5); $\mathrm{p}<0,001]$ Es fand sich statistisch keine signifikante Korrelation für die MTK zwischen der Nabelvene und dem FW ( $\mathrm{p}=0,810)$.

Schlussfolgerung. Unsere Ergebnisse zeigten, dass das FW in der Fetalzeit MT enthält und die MTK mit dem Gestationsalter ansteigt. Da MT nicht nur systemische, sondern auch lokale und intrazelluläre Effekte im Organismus ausübt, postulieren wir, dass MT sich positiv auf das gastrointestinale System auswirken kann. Der Gastrointestinaltrakt eines Frühgeborenen ist gegenüber dem reifer Neugeborener kürzer mit dem FW bzw. MT ausgesetzt. Es sollen weitere Studien zeigen, ob das wegen der Frühgeburt entstandene Defizit an Melatonin die Entwicklung des Gastrointestinaltraktes kleiner Frühgeborener beeinflusst und ob eine Supplementierung der Frühgeborenen mit oraler MelatoninGabe notwendig ist.

\section{FV57}

\section{Einfluss der Proteinzufuhr während der ersten 28 Lebenstage auf} das neurokranielle Volumen extrem unreifer Frühgeborener

\section{G. Thein', S. Ifflaender', M. Unrath', M. Rüdiger', W. Burkhardt'}

'Universitätsklinikum Carl Gustav Carus an der TU Dresden, Abteilung für Neonatologie und Pädiatrische Intensivmedizin, Dresden, Deutschland

Hintergrund. Frühgeborene (FG) haben einen besonderen Bedarf an Nährstoffzusammensetzung und Energiedichte der Nahrung. Dies ist nötig, um Wachstumsraten und Organreifung zu gewährleisten, welche den intrauterinen möglichst gleichen sollten. Dabei spielt das Hirnwachstum als Zielgröße eine wichtige Rolle, da es mit der neurologischen Langzeitentwicklung extrem unreifer Frühgeborener assoziiert ist. Insbesondere die Proteinzufuhr ist mit dem Hirnwachstum assoziiert. Aktuelle Richtlinien empfehlen Proteinmengen von 3,5-4 g/kgKG/Tag (parenteral) bzw. 3,8-4,4 g/kgKG/Tag (enteral) für extrem unreife FG. Als einfacher Surrogatparameter für das Hirnwachstum und damit als Maßstab für eine adäquate Ernährung dient derzeit die Messung des Kopfumfanges (KU). Die Kopfumfangsmessung weist aber eine hohe Interobserver Variabilität auf und vernachlässigt das dreidimensionale Wachstum des Gehirns. Die Messung des neurokraniellen Volumens (SV) mittels 3 D Laserscanner erlaubt eine nichtinvasive, genaue und vom Untersucher unabhängige dreidimensionale Annährung an das Hirnwachstum als Zielgröße der Ernährung (Ifflaender et al, PloS One 2013).

Fragestellungen. 1. Entspricht die derzeitige Proteinzufuhr für Frühgeborene am Universitätsklinikum Dresden aktuellen Richtlinien? 2. Erreichen FG adäquate KU und SV im korrigierten Alter (CA) von 36 Schwangerschaftswochen (SSW)? 3. Hat die Proteinzufuhr während der ersten 28 Lebenstage Einfluss auf das SV?

Methoden. Im Rahmen einer Beobachtungsstudie wurden die Daten von Frühgeborenen ( $<32$ SSW, <1500 g), die zwischen März 2011 und Dezem- ber 2013 in einem Level 1 Perinatalzentrum behandelt wurden, analysiert. Menge und Nährstoffzusammensetzung der parenteralen und enteralen Ernährung wurden aus Patientenakten erhoben. Kopfumfänge (KU) und der neurokraniellen Volumina (SV) wurden durch Routineuntersuchungen mittels 3 D Laserscanner im Alter von 36 Wochen \pm 7 Tage CA erfasst und mit denen einer Kontrollgruppe später Frühgeborener verglichen. Quartile (Q1-Q4) nach täglicher Proteinzufuhr bis zum 28. Lebenstag wurden auf Differenzen in KU und SV untersucht.

Ergebnisse. Daten von 92 FG (m 44, w 50; Geburtsgewicht 1048 266 g; GA 28.7 $\pm 1.8 \mathrm{SSW}$ ) wurden analysiert und mit denen einer Kontrollgruppe von 116 Kindern ( $\mathrm{m} \mathrm{53,} \mathrm{w} \mathrm{63,} \mathrm{Geburtsgewicht} \mathrm{2535 \pm 426} \mathrm{g;} \mathrm{GA} 35.9 \pm 0.5$ SSW) verglichen. FG erhielten während der ersten 28 Tage im Median $3,39(2,38-4,44) \mathrm{g} / \mathrm{kgKG} / \mathrm{Tag}$ Protein. Zum Messzeitpunkt unterschieden sich die KU der FG (312 mm, IQR 301-321 ml) signifikant von der Kontrollgruppe (326 mm, IQR 318-333 ml; p<0,001). Die SV (FG $309 \mathrm{ml}$, IQR 209-341 ml; Kontrolle $355 \mathrm{ml}$ IQR 331-385 ml) unterschieden sich ebenfalls signifikant $(\mathrm{p}<0,001)$.Trotz großer interindividueller Unterschiede in der Proteinzufuhr der FG (Q1 2,9 g/kgKG/Tag, Q4 3,9 g/kgKG/Tag), unterschieden sich die Quartile nicht signifikant in KU und SV.

Diskussion. Obwohl die Proteinzufuhr im Median den aktuellen Empfehlungen entsprach, zeigten extrem unreife Frühgeborenen niedrigere Kopfumfänge und neurokranielle Volumina bei 36 SSW CA. Die tägliche Proteinzufuhr zeigte erhebliche interindividuelle Unterschiede, welche jedoch keinen offensichtlichen Einfluss auf das neurokranielle Wachstum hatten.

Die vorliegende Arbeit wurde durch die Else Kröner-Fresenius-Stiftung unterstützt.

\section{FV58}

Target fortification for preterm infants: deficiency of fat, protein or lactose are not related to each other

\section{G. Fusch', N. Rochow', S. Mitra', C. Fusch'}

'McMaster University, Division of Neonatology, Department of Pediatrics, Hamilton, Ontario, Kanada

Background. The routine practice of standard fortification assumes an average content of macro-nutrients. However, breast milk has a high inter- and intra-individual variation in macro-nutrient concentration placing a significant proportion of preterm infants on risk for nutritional deficiency and postnatal growth restriction. Target fortification measures $\mathrm{BM}$ content and adds fat $(\mathrm{F})$, protein $(\mathrm{P})$ and/or carbohydrates (CHO) to achieve a standardized milk composition according to nutritional guidelines. This concept requires real-time milk analysis at bedside. To simplify milk analysis we hypothesize that the concentration of $F$, $\mathrm{P}$ and lactose (L) can be predicted from a key macro-nutrient. 
Objective. To investigate the correlation between deficiencies of macronutrient levels in breast milk.

Methods. Longitudinal set of breast milk samples $(\mathrm{n}=543)$ from 63 mothers of preterm ( 498 samples) and term ( 45 samples) infants. Breast milk levels for F, P, and L were analyzed using validated wet lab methods (ether extraction, elemental analysis, UPLC-MS/MS, respectively). Deficiencies of individual samples for levels of $\mathrm{F}, \mathrm{P}$, and $\mathrm{L}$ were calculated using ESPGHAN recommendations for preterm infants. Differences were plotted for F vs. P, F vs. L, and P vs. L.

Results. Abb. 1 shows the results for all pairs of macro--nutrients. There was no correlation between fat vs. protein, or fat vs. lactose, or protein vs. lactose in this large date set. The maximum $\mathrm{R} 2$ value did not exceed 0.02 . Conclusions. In our study we could not identify a key macro-nutrient which predicts the content of all macro-nutrients in an individual breast milk sample. Therefore, fortification of breast milk targeting F, P and $\mathrm{CHO}$ requires the measurement of all three macro-nutrients.

\section{Qualitätsmanagement}

\section{FV59}

Simulationsbasierter Reanimationsunterricht: Lernerfolg von Medizinstudenten nach unterschiedlichen Lehrmethoden

\section{E. Tutdibi', L. Gortner', M. Wrobel', T. Volk'², E. Reus ${ }^{2}$}

'Unversitätsklinikum des Saarlandes, Klinik für Allgemeine Pädiatrie und Neonatologie, Homburg/Saar, Deutschland, ${ }^{2}$ Universitätsklinikum des Saarlandes, Klinik für Anästhesiologie, Intensivmedizin und Schmerztherapie, Homburg/ Saar, Deutschland

Einleitung. Die Leitlinien des ERC 2010 beinhalten in Sektion 7 die Erstversorgung von Neugeborenen. Um Leitlinien zu unterrichten und den Lernerfolg zu messen gibt es unterschiedliche Lehr-, Lern- und Prüfungsmodelle. Die klassische Unterrichtsform besteht oft aus der 2-Schritt-Methode, dem sog. „see one, do one"-Methode. Diese Lehrform ist zu theorielastig und bietet den Studierenden wenig praktische Übungsmöglichkeiten. Im Bereich der Notfallmedizin stehen zur Lehre und Überprüfung von Lerninhalten moderne Patientensimulatoren in speziell eingerichteten Simulationszentren bereit. Die Vermittlung der theoretischen und praktischen Fertigkeiten findet hierbei oft nach Peyton's 4-Schritt-Methode statt. Die Lernerfolgskontrolle kann dabei mittels OSCE („objective structured clinical examination“) erfolgen. In der folgenden Studie wurde die Umwandlung der Lehrinhalte der Leitlinien in detaillierte Checklisten für einen OSCE und der Evaluation des Lernerfolgs von Medizinstudenten nach beiden Lehrmethoden untersucht. Material und Methoden. 49 freiwillige Studierende der Humanmedizin im 4. klinischen Semester wurden randomisiert in zwei Gruppen (Kontrollen und Peyton) aufgeteilt. Die Kontrollgruppe erhielt eine theoretische Unterweisung in die aktuellen ERC Leitlinien 2010 Sektion 7 in Form eines Frontalunterrichts mit Demonstration der Erstversorgung an einer Puppe. Im Anschluss konnten die Teilnehmer an der Pupper die Einzelschritte üben. In der Studiengruppe wurde als Lehrmethodik das 4-Schritt-Verfahren nach Peyton verwendet: Unterweisung, Demonstration, Anleitung durch Studierenden, Übung an der Puppe. Beide Gruppen durchliefen 3 Tage nach dem Unterricht einen OSCE anhand detaillierter Checklisten mit je 2 möglichen Punkten pro Einzellösung. Im praktischen Teil des OSCE (pOSCE) waren 118 und im theoretischen Teil (tOSCE) 74 Punkte zu erreichen. Die Daten sind als Mittelwerte \pm Standardabweichung angegeben und die Irrtumswahrscheinlichkeit $\mathrm{p}$ wurde auf $<0,05$ festgelegt.

Ergebnisse. Die Studiengruppe erreichte in beiden OSCE eine signifikant höhere Punktzahl als die Kontrollgruppe: $113 \pm 5$ vs. $68 \pm 23$ (pOSCE) und $66 \pm 5$ vs. $40 \pm 23$ (tOSCE).

Schlussfolgerung. Die Kombination aus Peyton's 4-Schritt-Methode und OSCE an Simulatoren anhand detaillierter Checklisten sind ein gutes Mittel, um den Lernerfolg von Medizinstudenten signifikant zu verbessern.

\section{Fetale Programmierung}

\section{FV60}

Die postnatale BMI-Entwicklung eines Neugeborenen bis zum 6. LJ wird chronobiologisch reguliert und hauptsächlich durch den BMI der Mutter determiniert

\section{B. Brune', M. Seewald², T. Brune ${ }^{3}$}

'Universitätsklinikum, Kinderklinik, Magdeburg, Deutschland, ${ }^{2} \mathrm{Hchsch} u l e$ Anhalt, Ökotrophologie, Bernburg, Deutschland, ${ }^{3}$ Klinikum Lippe-Detmold, Kinderklinik, Detmold, Deutschland

Fragestellung. Die pränatale Entwicklung eines Kindes ist stark von der Nährstoffversorgung durch die Mutter abhängig und wird über die Plazenta reguliert, postnatal spielen genetische und Umweltfaktoren eine Rolle. In der vorliegenden Studie wurde untersucht, wie stark der jeweilige Einfluss prä- und postnataler Faktoren hinsichtlich der Entstehung von Übergewicht ist, zu welchem Zeitpunkt sich dieses im Kindesalter manifestiert und ob die postnatale BMI-Entwicklung einem genetisch festgelegten chronobiologischen Anpassungsprozess unterliegt.

Methodik. Von 5433 Kindern, die an den Einschulungsuntersuchungen in Magdeburg teilgenommen hatten, wurden 212 Kinder mit einem extrem niedrigen sowie hohen Geburts-BMI ausgewählt, welche im 6. Lebensjahr die BMI-Perzentilen kreuzten infolge eines extremen postnatalen Anstiegs (von $<10$ auf $>90$ P) oder Abfalls (von $>90$ auf $<10$ P) oder deren BMI sowohl bei der Geburt als auch im Alter von 6 Jahren konstant extrem niedrig bzw. hoch $(<10$ bzw. $>90 \mathrm{P})$ war. Vierzig Kinder mit einem BMI im Normbereich ( $50 \mathrm{P}$ ) sowohl bei der Geburt als auch im Alter von 6 Jahren dienten als Kontrollgruppe. Die jeweiligen Verlaufskurven von Gewicht und Größe in den ersten 6 Jahren wurden bestimmt und der daraus resultierende BMI berechnet. Um Einflüsse auf die BMI-Entwicklung zu identifizieren, untersuchten wir genetische, soziale, Ernährungsund weitere prä- und postnatale Einflussfaktoren.

Ergebnisse. Abb. 1 zeigt, dass in den untersuchten Gruppen während zwei kritischer Zeiträume eine deutliche Entwicklung hin zu einem niedrigen bzw. hohen BMI stattfindet: zum einen in der sehr frühen Kindheit im Alter von ca. 0,5 bis 1,5 Jahren und erneut im Alter von 5 bis 6 Jahren. Bereits im Alter von 1,5 Jahren ist der finale BMI in allen untersuchten Gruppen erreicht. Einziger signifikanter Einflussfaktor auf die kindliche BMI-Entwicklung ist in unserer Studie der mütterliche BMI bei Schwangerschaftsbeginn: Mütter von übergewichtigen 6-jährigen Kindern sind zu diesem Zeitpunkt im Mittel ebenfalls übergewichtig, während Mütter von untergewichtigen 6-jährigen Kindern im Mittel einen BMI im unteren Normbereich aufweisen. Alle anderen untersuchten Faktoren hatten in unserer Studie keinen signifikanten Einfluss auf die postnatale BMI- Entwicklung.

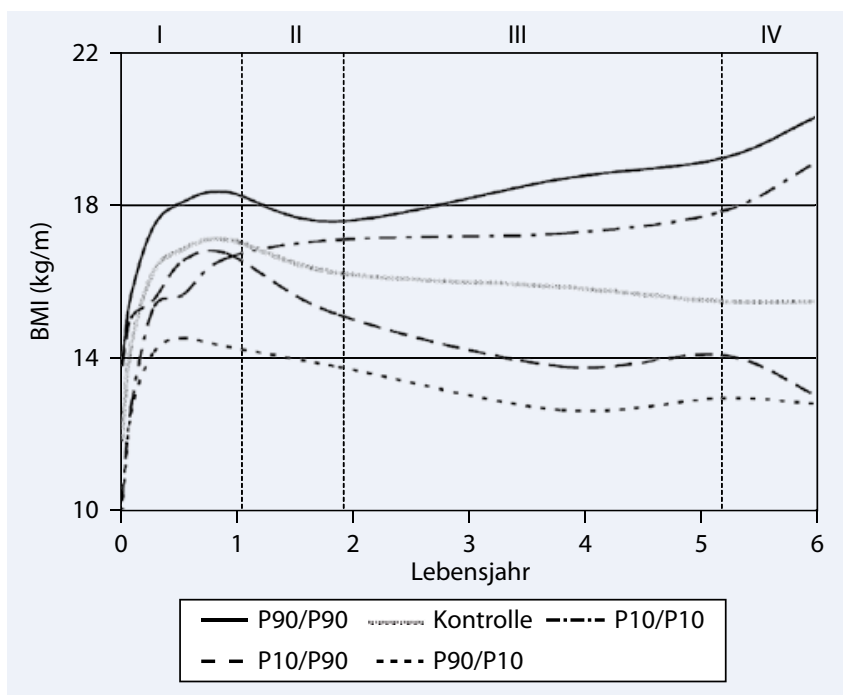

FV6o-Abb 1 
Schlussfolgerung. Unsere Ergebnisse zeigen, dass in allen Extremgruppen das Kreuzen der BMI-Perzentile bereits im ersten Lebensjahr stattfindet und der Trend der BMI-Entwicklung vom mütterlichen BMI bei Schwangerschaftseintritt abhängig ist, während alle anderen untersuchten Faktoren nur einen geringen Einfluss hatten. Diese Beobachtung lässt darauf schließen, dass die postnatale BMI-Entwicklung des Kindes einem chronobiologischem genetischen Programm folgt und hauptsächlich durch den mütterlichen Stoffwechsel programmiert wird.

\section{Erstversorgung}

\section{FV61}

\section{Regionale zerebrale Sauerstoffsättigung in der Adaptationsphase} des Reif- und Frühgeborenen: Spielt das Geschlecht eine Rolle?

\section{Pocivalnik', G. Pichler', A. Avian², C. Binder', B. Schwaberger', E. Beckenbach', E. Ziehenberger', B. Urlesberger' \\ 'Universitätsklinik für Kinder- und Jugendheilkunde Graz, Medizinische Univer- sität Graz, Neonatologie, Graz, Österreich, ${ }^{2}$ Institut für Medizinische Informatik, Statistik und Dokumentation, Medizinische Universität Graz, Graz, Österreich}

Hintergrund und Fragestellung. Das Geschlecht spielt bei Frühgeborenen hinsichtlich Mortalität und Morbidität eine bedeutende Rolle. Rezente Studien, bezugnehmend auf die Sauerstoffversorgung im Rahmen der Erstversorgung von Neugeborenen und Vermeidung von Hyperoxie zeigten einen geschlechtsspezifischen Unterschied zugunsten des weiblichen Geschlechtes. Weibliche Frühgeborene zeigten einen geringeren „oxidativen Stress" und eine höhere Aktivität antioxidativer Enzyme. Ziel dieser Arbeit war die Ermittlung, ob das Geschlech einen Einfluss auf die regionale zerebrale Sauerstoffsättigung ( $\left.\mathrm{crSO}_{2}\right)$ während Adaptationsphase des Neugeborenen nimmt.

Material und Methode. In einer prospektiven Beobachtungsstudie im Zeitraum 2009-2012 wurden bei Reif- und Frühgeborenen mit ungestörter Adaptation (ohne $\mathrm{O}_{2}$-Bedarf, keine respiratorische Unterstützung) während der ersten 15 Lebensminuten $\mathrm{CrSO}_{2}$ Werte mittels Nahinfrarotspektroskopie (NIRS; Invos 5100 cerebral/somatic oximeter monitor; Somanetics Corp, Troy, Michigan) erhoben. Der NIRS Sensor wurde an der linken Stirnseite platziert. Die periphere Sauerstoffsättigung ( $\left.\mathrm{SpO}_{2}\right)$ und Herzfrequenz (HF) wurde kontinuierlich mittels Pulsoximetrie gemessen. Die "cerebral fractional oxygen extraction“ (cFTOE) wurde berechnet. Erhobene Daten wurden für alle StudienGruppen hinsichtlich des Geschlechts analysiert (term/vaginally delivered-VDterm; term/cesarean delivered-CDterm; preterm/cesarean delivered-CDpreterm)

Ergebnisse. Von insgesamt 479 gemessenen Neugeborenen, wurden 374 in die weitere Datenanalyse inkludiert. Bei Reifgeborenen wurde in der Gruppe der Schnittentbindungen (CDterm group, $n=268$ ) ein geschlechtsspezifischer Unterschied erhoben: männliche Neugeborene zeigten significant höhere Werte für $\mathrm{SpO}_{2}(\mathrm{p}=0,009)$ und $\mathrm{CrSO}_{2}$ $(\mathrm{p}=0,009)$; kein Unterschied wurde in der HF ermittelt. FTOE-Werte waren niedriger bei männlichen NG $(\mathrm{p}=0,055)$. In beiden anderen Gruppen, reife vaginal Geborene (VDterm group, $n=80$ ) und Frühgeborene (CDpreterm group, $n=26$ ), wurden keine signifikanten Unterschiede ermittelt. Die durchgeführte Power-Analyse zeigte für diese beiden Gruppen jedoch eine zu geringe Anzahl an Neugeborenen um aussagekräftige Ergebnisse zu erzielen.

Schlussfolgerung. $\mathrm{crSO}_{2}$ und $\mathrm{SpO}_{2}$ zeigten einen signifikanten Unterschied hinsichtlich des zugrunde liegenden Geschlechts bei reifen Neugeborenen nach Schnittentbindung. Männliche Neugeborene zeigten im Vergleich zu weiblichen NG signifikant höhere Sauerstoffsättigungswerte.

\section{FV62}

Veränderungen des zerebralen Blutvolumens in der Adaptationsphase von Neugeborenen

\section{B. Schwaberger', G. Pichler', C. Binder', N. Baik', B. Urlesberger ${ }^{1}$}

${ }^{1}$ Medizinische Universität Graz, Klinische Abteilung für Neonatologie, Graz, Österreich

Fragestellung. Nahinfrarotspektroskopie (NIRS) ermöglicht eine kontinuierliche, nichtinvasive Messung der Konzentrationsänderungen von oxygeniertem $\left(\mathrm{O}_{2} \mathrm{Hb}\right)$ und desoxygeniertem Hämoglobin $(\mathrm{HHb})$ im Gehirn von Neugeborenen. Veränderungen im totalen Hämoglobin $\left(\mathrm{cHb}=\mathrm{O}_{2} \mathrm{Hb}+\mathrm{HHb}\right)$ zeigen das Verhalten des zerebralen Blutvolumens (CBV). Wie sich das CBV während der postpartalen Adaptationsphase von Neugeborenen verhält, ist noch nicht gut untersucht und wurde nun bei Reifgeborenen evaluiert.

Material und Methode. Diese Single-Center-Studie wurde als prospektive Beobachtungsstudie durchgeführt. Inkludiert wurden Reifgeborene nach Schnittentbindung, die eine komplikationslose Adaptation ohne Notwendigkeit einer Atemunterstützung zeigten. Für die NIRS-Messungen wurde ein ,NIRO 200-NX' (Hamamatsu; Japan) verwendet. Neben den Parametern $\mathrm{cHb}, \mathrm{O}_{2} \mathrm{Hb}$ und $\mathrm{HHb}$ wird außerdem der sog. „tissue oxygenation index“ ( $\mathrm{TOI}=\mathrm{O}_{2} \mathrm{Hb} / \mathrm{cHb}^{*}{ }_{100}$ ) ausgegeben, welcher der regionalen Sauerstoffsättigung ( $\left.\mathrm{rSO}_{2}\right)$ entspricht. Der NIRS-Sensor wurde rechts frontal mit elastischer Binde fixiert. Der Verlauf des zerebralen $\mathrm{CBV}$ und korrespondierende Veränderungen der $\mathrm{rSO}_{2}$ während der unmittelbaren postnatalen Adaptationsphase wurden über 15 Minuten aufgezeichnet. Außerdem wurden die Herzfrequenz (HF) und die präduktale arterielle Sauerstoffsättigung $\left(\mathrm{SpO}_{2}\right)$ mittels Pulsoxymetrie aufgezeichnet.

Ergebnisse. 92 Reifgeborene (49 weibliche) mit einem mittleren Gestationsalter von $38+6$ Wochen $( \pm 7$ Tage) und Geburtsgewicht von $3245 \mathrm{~g}$ $( \pm 441)$ wurden inkludiert.

cHb: Das cHb [Median (25; 75. Perzentile)] nahm von Lebensminute (min) 2 bis $3 \mathrm{um}-0,2(-4,8 ; 3,7) \mu \mathrm{M}$, bis min $4 \mathrm{um}-1,1(-5,9 ; 3,8) \mu \mathrm{M}$, bis min 5 um $-1,0(-8,4 ; 4,6) \mu \mathrm{M}$, bis min 10 um $-7,8(-18,0 ;-0,7) \mu \mathrm{M}$ und bis min 15 um $-16,0(-28,1 ;-2,5) \mu \mathrm{M}$ ab. Es zeigte sich eine Abnahme des cHb, als Hinweis auf ein Absinken des CBV während der Adaptationsphase.

rSO2: Die zerebrale rSO2 [Median (25.; 75. Perzentile)] stieg von 56 (47; $63) \%$ nach 2 min, $56(50 ; 63) \%$ nach $3 \mathrm{~min}, 60(53 ; 71) \%$ nach $4 \mathrm{~min}, 63$ (57; $73) \%$ nach $5 \mathrm{~min}, 74(67 ; 79) \%$ nach $10 \mathrm{~min}$, auf $72(66 ; 77) \%$ nach $15 \mathrm{~min}$. Die zerebrale $\mathrm{rSO}_{2}$ zeigte einen langsamen Anstieg ab der 2. min bis zur 7. min und erreichte ab der 8. min ein Steady-State.

$\mathrm{SpO}_{2}$ und HF: Die präduktale $\mathrm{SpO}_{2}$ [Median (25.; 75. Perzentile)] stieg von 76 (70; 81)\% nach $2 \mathrm{~min}, 75(69 ; 80) \%$ nach $3 \mathrm{~min}, 78(67 ; 85) \%$ nach 4 min, 83 ( $72 ; 90) \%$ nach 5 min, 95 (92; 98)\% nach 10 min, auf 96 (93; 99)\% nach 15 min an. Die HF [Median (25.; 75. Perzentile)] zeigte Werte von $114(85 ; 155)$ Schlägen pro Minute (bpm) nach 2 min, $132(92 ; 167) \mathrm{bpm}$ nach $3 \mathrm{~min}, 155(115 ; 168) \mathrm{bpm}$ nach $4 \mathrm{~min}, 155(141 ; 164)$ bpm nach 5 min $152(142 ; 164)$ bpm nach 10 min, und $151(142 ; 163)$ bpm nach 15 min.

Diskussion. Das Absinken des cHb, welches einen Marker für das CBV darstellt, ist gut vereinbar mit dem von Noori S. (J Pediatr 2012) gezeigten Abfall des zerebralen Blutflusses in der Adaptationszeit bei $\mathrm{Zu}$ nahme der zerebralen Sauerstoffversorgung und Zunahme des LinksRechts-Shunts über den Ductus arteriosus botalli.

Schlussfolgerung. In gesunden Reifgeborenen zeigt sich postpartal bei einer HF im Normbereich eine Abnahme des CBV. Diese Beobachtung kann zu einem besseren Verständnis der kardiozirkulatorischen Prozesse in der Adaptationsphase von Neugeborenen beitragen. 


\section{FV63}

Blutdruck bei reifen Neugeborenen und Frühgeborenen unmittelbar nach der Geburt

\section{G. Pichler', P. Cheung', C. Binder', M. O'Reilly², B. Schwaberger', K. Aziz'², B. Urlesberger', G. Schmölzer ${ }^{2}$}

'Universitäts Klinik für Kinder- und Jugendheilkunde Graz, Abteilung für Neonatologie, Graz, Österreich, ${ }^{2}$ Department of Neonatology, Royal Alexandra Hospital, Edmonton, Canada, Edmonton, Kanada

Fragestellung. Nichtinvasive Blutruckmessungen stellen eine Möglichkeit dar Informationen über den kardiozirkulatorischen Zustand eines Neugeborenen unmittelbar nach der Geburt zu erheben und sind daher von wachsendem Interesse. Ziel der Studie war es, in einer größeren Gruppe von Neugeborenen den Blutdruck unmittelbar nach der Geburt zu messen und Blutdruck-Normwerte zu bestimmen.

Material und Methode. In einer prospektiven 2-Zentren-Beobachtungsstudie (Graz, Edmonton) wurde der Blutdruck nichtinvasiv oszillometrisch bei reifen Neugeborenen und Frühgeborenen jede Minute bis 15 Minuten nach Geburt gemessen. Bei Frühgeborenen wurde zusätzlich der Blutdruck in Minute 20, 25, 30, 45 und 60 nach Geburt gemessen. Der Einfluss des Geschlechtes und eventuelle Atemunterstützung wurde bei allen Neugeborenen analysiert. Bei Frühgeborenen wurden zusätzlich mögliche Zusammenhänge mit dem Gestationsalter, Geburtsmodus, Nabelschnurklemmzeit und dem Auftreten von intraventrikulären Blutungen untersucht.

Ergebnisse. 54 reife Neugeborene (Geburtsgewicht: $3298 \pm 442$ g; Gestationsalter $38 \pm 1$ Wochen) und 54 Frühgeborene (Geburtsgewicht: $1602 \pm 601 \mathrm{~g}$; Gestationsalter $30 \pm 3$ Wochen) wurden inkludiert. Bei reifen Neugeborenen war der Blutdruck konstant in den ersten Lebensminuten und unabhängig von Geschlecht und Atemunterstützung. Bei Frühgeborenen sank der Blutdruck im Laufe der ersten Lebensstunde. Frühgeborene die weiblich waren, vaginal entbunden wurden, eine verzögerte Nabelschnurklemmzeit (6o s) hatten, keine Atemunterstützung benötigten, oder keine höhergradige intraventrikuläre Blutung entwickelten, hatten signifikant höhere Blutdruckwerte während einzelner Zeitpunkte in der ersten Lebensstunde.

Diskussion. In der vorliegenden Studie wurden erstmals Normwerte für den Blutdruck bei reifen Neugeborenen und Frühgeborenen unmittelbar nach der Geburt erhoben. Bei Frühgeborenen in verschiedenen Subgruppen zeigten sich unterschiedliche Blutdruckwerte, als Hinweis eines unterschiedlichen kardiozirkulatorischen Zustands.

Schlussfolgerung. Blutdruckmessungen und die vorliegenden Normwerte könnten in der Zukunft helfen, den kardiozirkulatorischen Zustand eines Neugeborenen besser einzuschätzen, wobei deren Kurz- und Langzeit-Nutzen noch in weiteren Studien untersucht werden muss.

\section{FV64}

\section{Verbessern Blähmanöver die zerebrale Oxygenierung von Früh-} geborenen?

\section{B. Schwaberger', G. Pichler', C. Binder', N. Baik', B. Urlesberger ${ }^{1}$ \\ ${ }^{1}$ Medizinische Universität Graz, Klin. Abteilung für Neonatologie, Graz, Öster- reich}

Fragestellung. Blähmanöver („sustained lung inflations“, SLI) begünstigen die Lungenrekrutierung und stellen eine Beatmungsstrategie bei der Erstversorgung von Frühgeborenen (FG) dar. Nahinfrarotspektroskopie (NIRS) ermöglicht kontinuierliche, non-invasive Messungen der zerebralen regionalen Sauerstoffsättigung ( $\left.\mathrm{rSO}_{2}\right)$. Können SLI die zerebrale $\mathrm{rSO}_{2}$ bei $\mathrm{FG}$ verbessern?

Material und Methode. Diese prospektive, randomisierte Beobachtungsstudie wurde an der Abteilung für Neonatologie in Graz durchgeführt. Inkludiert wurden $\mathrm{FG} \geq 28+0$ und $<34+0$ Schwangerschaftswochen (SSW), welche unmittelbar postpartal eine respiratorische Unterstützung benötigten. Für die NIRS Messungen wurde ein ,NIRO 200-NX (Hamamatsu; Japan) verwendet und in den ersten 15 Lebensminuten der Tissue Oxygenation Index aufgezeichnet, welcher bei frontaler Anlage der NIRS Sensoren der zerebralen $\mathrm{rSO}_{2}$ entspricht. Die Herzfrequenz (HF) und die präduktale arterielle Sauerstoffsättigung ( $\left.\mathrm{SpO}_{2}\right)$ wurden mittels Pulsoxymetrie aufgezeichnet. Die Atemunterstützung wurde mit dem ,Neopuff T-piece Resuscitator' (Fisher\&Paykel, Neuseeland) ausgeführt. Die Sauerstofffraktion ( $\mathrm{FiO}_{2}$ ) wurde mit o,30 voreingestellt und so adaptiert, dass $\mathrm{SpO}_{2}$-Werte über der 10. Perzentile für FG der 32. bis 36. SSW nach Dawson et al. (Pediatrics 2010) erreicht wurden. Hinsichtlich der respiratorischen Unterstützung wurden 2 Gruppen gebildet: SLI-Gruppe: Bis zu 3 SLI über jeweils $15 \mathrm{~s}$ als initiale Beatmungsmanöver, anschließend Unterstützung mit Continuous Positive Airway Pressure (CPAP) oder Positive Pressure Ventilation (PPV). Kontrollgruppe: Je nach Klinik CPAP oder PPV.

Ergebnisse. 40 FG (23 weibliche) mit einem mittleren Gestationsalter von $32+1$ SSW ( \pm 3 Tage) und Geburtsgewicht von $1707 \mathrm{~g}( \pm 470)$ wurden inkludiert. Die demographischen Daten zeigten keine signifikanten Unterschiede zwischen den Gruppen.

$\mathrm{SpO}_{2}$ : Die mittlere $\mathrm{SpO}_{2}$ betrug in der SLI- (Kontroll-)Gruppe 30 s nach Beginn der Beatmung $70(67) \%$, nach 60 s $72(73) \%$, nach 2 min $73(73) \%$, nach 3 min $77(77) \%$, nach 5 min $80(83) \%$ und nach 10 min $87(88) \%$.

$\mathrm{rSO}_{2}$ : Die mittlere zerebrale $\mathrm{rSO}_{2}$ betrug in der SLI-(Kontroll-)Gruppe 30 s nach Beginn der Beatmung 49(47)\%, nach 6o s 54(50)\%, nach 2 min $56(51) \%$, nach $3 \min 56(58) \%$, nach 5 min $61(61) \%$ und nach $10 \min 65(69) \%$. $\mathrm{FiO}_{2}$ : Die mittlere $\mathrm{FiO}_{2}$ betrug in der SLI-(Kontroll-)Gruppe 30 s nach Beginn der Beatmung $0,30(0,29)$, nach $60 \mathrm{~s} 0,30(0,30)$, nach 2 min $0,29(0,30)$, nach $3 \mathrm{~min} 0,29(0,32)$, nach $5 \mathrm{~min} 0,32(0,32)$ und nach $10 \mathrm{~min} 0,29(0,27)$.

HF: Die mittlere HF betrug in der SLI-(Kontroll-)Gruppe $30 \mathrm{~s}$ nach Beginn der Beatmung 121(98)/min, nach 6o s 126(144)/min, nach 2 min

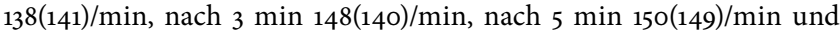
nach $10 \mathrm{~min} 161(152) / \mathrm{min}$.

Bezüglich $\mathrm{rSO}_{2}, \mathrm{SpO}_{2}, \mathrm{FiO}_{2}$ und $\mathrm{HF}$ zeigten sich zwischen den Gruppen keine signifikanten Unterschiede.

Diskussion. Kurzfristige Auswirkungen auf Vitalparameter und die zerebrale $\mathrm{rSO}_{2}$ wurden in dieser Studie untersucht. Diskutiert wird, ob die Anwendung von SLI die Notwendigkeit einer mechanischen Beatmung und die Inzidenz einer bronchopulmonalen Dysplasie günstig beeinflussen kann.

Schlussfolgerung. Die Durchführung von Blähmanövern bei der Erstversorgung von Frühgeborenen $\geq 28+0$ und $<34+0$ SSW zeigte in den ersten 15 Lebensminuten weder signifikanten Unterschiede in der zerebralen Gewebsoxygenierung noch in anderen Vitalparametern (im Vergleich zur Kontrollgruppe).

\section{FV65}

\section{Oropharyngeales Absaugen bei der Routine-Erstversorgung im} Kreißsaal

\section{Konstantelos', J. Dinger', S. Ifflaender', M. Rüdiger'}

'Medizinische Fakultät Carl Gustav Carus an der TU Dresden, Fachbereich Neonatologie und Pädiatrische Intensivmedizin, Dresden, Deutschland

Hintergrund. Oropharyngeales Absaugen wurde in den letzten Empfehlungen zur Erstversorgung der Neugeborenen im Kreißsaal auf Neugeborene ohne Atemantrieb und Verdacht auf Mekonium-Aspiration sowie Neugeborene mit Anpassungsproblemen vor der Applikation einer Atemunterstützung beschränkt.

Fragestellung. Wie oft werden die Neugeborenen bei der Erstversorgung im Kreißsaal abgesaugt und welchen Effekt hat diese Intervention auf die Vitalparameter?

Material und Methode. Es wurden im Zeitraum von Januar 2012 bis April 2013 alle Erstversorgungen von Früh- und Termingeborenen nach Kaiserschnitt im Frühdienst aufgezeichnet. Die Videos wurden nach folgenden Kriterien analysiert und ausgewertet: Zeitpunkt, Dauer und Häufigkeit des Absaugens. Das Absaugen wurde in nasales bzw. orales Absaugen subkategorisiert. Vor und nach dem Absaugen wurden die Vitalparameter (Herzfrequenz - HF, Sättigung - SpO2) aufgezeichnet und auf mögliche Differenzen untersucht. 
Ergebnisse. Insgesamt wurden 346 Videos ausgewertet (260 Reif- und 86 Frühgeborene). Es wurden 23\% der Reif- und 66\% der Frühgeborenen abgesaugt. Die Dauer des Absaugens pro Versuch betrug im Median 9 s (267) für Termingeborene, die eine Atemunterstützung benötigten, 9 s (1-50) für reife Neugeborene mit einer normalen respiratorischen Anpassung und 8 s (1-46) für Frühgeborene. Abgesaugt wurden die Neugeborenen im Median 2- bis 3-mal. In den meisten Fällen (74\%) wurde ausschließlich der Mund abgesaugt. Nur 34\% der Kinder, die eine Atemunterstützung gebraucht haben, wurden vor der Atemunterstützung abgesaugt. Es wurde keine schwere Bradykardie $(<60 / \mathrm{min})$ und nur ein tiefer Sättigungsabfall $(<75 \%)$ beobachtet. Die HF bei den abgesaugten Neugeborenen war leicht erhöht. Außerdem gab es unter den abgesaugten Neugeborenen keine signifikanten Unterschiede bezüglich $\mathrm{SpO}_{2}$, bis auf signifikant niedrigere Werte bei den abgesaugten Termingeborenen ohne Atemunterstützung. Diskussion. Fast ein Drittel aller Neugeborenen wird in unserer Abteilung immer noch abgesaugt, trotz der Tatsache, dass Absaugen nicht mehr in die Routineversorgung gehört. $70 \%$ der Kinder mit Atemunterstützung wurden abgesaugt, allerdings nur ein Drittel der Kinder leitlinienkonform vor dem Anlegen der Atemhilfe. Beschriebene Nebeneffekte des Absaugens (schwere Bradykardien oder tiefe Sättigungsabfälle) wurden nur einmalig beobachtet.

Schlussfolgerung. Videorecording ist eine gute Möglichkeit die Routine-Erstversorgung zu bewerten. Das Absaugen wird bei der Erstversorgung regelmäßig durchgeführt. Allerdings wurden die beschriebenen Nebenwirkungen des Absaugens dabei nicht bestätigt.

Die vorliegende Arbeit wurde durch die Else Kröner-Fresenius-Stiftung unterstützt.

\section{FV66}

Short-term outcome of very low birth weight infants (VLBWI) requiring cardiopulmonary resuscitation in the delivery room

\section{H. Hummler', K. Lang ${ }^{2}$, A. Azpeitia ${ }^{3}$, A. Valls-i-Soler ${ }^{3}$}

${ }^{1} K$ linik für Kinder- und Jugendmedizin, Universitätsklinikum Ulm, Sektion Neonatologie und Pädiatrische Intensivmedizin, Ulm, Deutschland, ${ }^{2}$ Klinik für Kinder- und Jugendmedizin, Universitätsklinikum Ulm, Ulm, Deutschland, ${ }^{3}$ Hospital de Cruces, Dept. of Pediatrics, Barakaldo-Bilbao, Spanien

Background. Recent reports on larger cohorts on the outcome of VLBWI requiring cardio-pulmonary resuscitation including chest compressions (CPR) and/or epinephrine show conflicting results on the risk for mortality and morbidity.

Objectives. To evaluate the relationship of CPR and/or use of epinephrine on mortality and short-term morbidity in a large cohort of VLBWI in the European Neonatal Network (EuroNeoNet) database. We hypothesized that more intense resuscitative efforts in VLBWI immediately after delivery, defined as the use of CPR and/or epinephrine would be associated with increased mortality or with a higher rate of morbidity.

\begin{tabular}{|llll|}
\hline FV66 - Tab. 1 & $\begin{array}{l}\text { No CPR/Epi } \\
(\mathbf{n}=24233)\end{array}$ & $\begin{array}{l}\text { CPR/Epi } \\
(\mathbf{n}=1319)\end{array}$ & $\begin{array}{l}\text { Adjusted OR } \\
(95 \% \text { Cl) }\end{array}$ \\
\hline Death & $3276(13.5 \%)$ & $553(41.9 \%)$ & $3.54(3.08-4.06)$ \\
\hline RDS & $14490(61.3 \%)$ & $1047(83.2 \%)$ & $2.19(1.86-2.58)$ \\
\hline Pneumothorax & $886(3.7 \%)$ & $103(8.1 \%)$ & $1.80(1.44-2.24)$ \\
\hline BPD (O @ @ 36 wks p.m.) & $2090(16.1 \%)$ & $155(29.9 \%)$ & $1.81(1.49-2.20)$ \\
\hline IVH & $5187(25.1 \%)$ & $430(43.1 \%)$ & $1.62(1.41-1.86)$ \\
\hline IVH grade 3/4 & $1860(8.5 \%)$ & $250(22.7 \%)$ & $2.08(1.78-2.45)$ \\
\hline PVL (cystic) & $1197(5.6 \%)$ & $92(8.5 \%)$ & N.S. \\
\hline ROP stage 4/5 & $842(5 \%)$ & $60(8.3 \%)$ & N.S. \\
\hline NEC stage 2/3 & $1488(6.3 \%)$ & $103(8.2 \%)$ & N.S. \\
\hline Focal intestinal perforation & $465(2 \%)$ & $33(2.6 \%)$ & N.S. \\
\hline Data refer to mean \pm SD or median (25th-75th Perc.), N.S. = not significant.
\end{tabular}

Methods. The EuroNeoNet is a voluntary network for Neonatal Intensive Care Units (NICUs) which is collecting a standardized dataset (www.euroneonet.eu) to be used for benchmarking and quality improvement. This dataset includes a standardized report on delivery room interventions. Infants with severe malformations were excluded $(n=783)$. The database includes 25.552 VLBWI born 2006-2010 from 185 European NICUs in 17 countries; 1319 infants did receive CPR and/or epinephrine, 24,233 did not. Results. Infants requiring CPR and/or epinephrine had a lower gestational age, birth weight, lower apgar scores, were more often male, had less often received prenatal steroids, more often single pregnancies and were less often born by cesarean section. After logistic regression adjustment for gestational age, birth weight, gender, prenatal steroids, multiple birth and mode of delivery, rates of death, RDS, pneumothorax, BPD and IVH remained significantly higher in babies exposed to CPR and/ or epinephrine (Tab. 1).

Conclusion. Use of CPR and/or epinephrine in the delivery room was associated with increased neonatal mortality and respiratory and cerebral morbidity.

\section{Pulmologie}

\section{FV67}

Behandlung der pulmonalen Hypertonie bei Patienten mit angeborener Zwerchfellhernie mittels intravenösem Sildenafil

\section{F. Kipfmueller', P. Bartmann', A. Müller ${ }^{1}$}

'Universitätsklinik Zentrum f. Kinderheilkunde, Neonatologie, Bonn, Deutschland

Fragestellung. Welchen Einfluss hat die intravenöse Sildenafiltherapie auf den Verlauf der pulmonalen Hypertonie (PPHN) bei Kindern mit angeborener Zwerchfellhernie (CDH)?

Patienten und Methodik. Retrospektive Analyse aller Patienten mit $\mathrm{CDH}$ die mit intravenösem Sildenafil behandelt wurden. Behandelte Patienten wurden unterteilt in Responder [Verbesserung Oxygenierungsindex (OI)] und Non-Responder (ECMO oder Tod). Verglichen wurden OI und Katecholaminbedarf. Zur Beurteilung der Wirksamkeit wurde echokardiographisch das Ausmaß der PPHN mit dem Quotienten time-to-peak-velocity/rechtsventrikuläre Ejektionszeit (TPV/ RVET) berechnet.

Ergebnisse. Seit September 2012 betreuten wir 29 Kinder mit CDH. Davon wurden 17 mit i.v. Sildenafil behandelt $(60,7 \%)$. Indikation war ein pulmonalarterieller Druck $\geq 0,5$ Systemdruck. Behandlungsbeginn war im Mittel nach 2,4 Stunden. 12 Kinder waren Non-Responder (ECMO: $\mathrm{n}=10$; Tod: $\mathrm{n}=2$ ). Responder hatten einen signifikant niedrigeren OI als Non-Responder vor Behandlungsbeginn $(17,2$ vs. 39,2$)$ und nach $6 \mathrm{~h}$ bzw. vor ECMO (6,3 vs. 57,4). Bei 94\% der Patienten stieg der MAD unter Sildenafiltherapie, jedoch war bei allen Patienten eine Intensivierung der Vasopressoren erforderlich. Die TPV/RVET vor Therapiebeginn war bei Respondern und Non-Respondern vergleichbar (o,239 vs. 0,227), unterschied sich aber nach $24 \mathrm{~h}$ Behandlungsdauer deutlich (o,313 vs. o,249). Kinder mit CDH ohne PPHN hatten eine TPV/RVET von o,319 und 0,371 postpartal und nach $24 \mathrm{~h}$. Von 10 Kindern mit ECMO-Therapie konnten 8 erfolgreich dekanüliert werden. Zwei Kinder verstarben bei Dekanülierung an schwerster Lungenhypoplasie.

Diskussion. Ein frühzeitiger Therapiebeginn mit Sildenafil könnte bei Kindern mit $\mathrm{CDH}$ das vaskuläre Remodelling unterstützen. Bei ca. 30\% der Kinder kam es nach Therapiebeginn zu einer dauerhaften Verbesserung des OI's. $47 \%$ der Kinder benötigten eine passagere ECMO-Therapie, waren aber überwiegend gut zu dekanülieren. Die Berechnung der TPV/RVET ermöglicht eine frühzeitige Risikoabschätzung und könnte besonders unter ECMO der Berechnung einer Trikuspidalinsuffizienz überlegen sein.

Schlussfolgerung. Da in unserer Studie kein Vergleichskollektiv vorliegt, sind Schlüsse zu Wirksamkeit und Risikopotenzial nicht zu ziehen. Eine randomisierte kontrollierte Multizenterstudie ist aktuell in Planung. 


\section{FV68}

Monitoring des Therapieansprechens auf Sildenafil mittels NT-proBNP-Bestimmung im Urin Frühgeborener mit pulmonalarterieller Hypertension

\section{A. Oberthür', H. Niemarkt', G. Malchau', T. Keller', K. Mehler', B. Kramer', E. Rother ${ }^{5}$, B. Roth', A. Kribs \\ 'Uniklinik Köln, Klinik für Kinder- und Jugendmedizin, Neonatologie und Pädiatrische Intensivmedizin, Köln, Deutschland, ${ }^{2}$ Máxima Medical Center, Veldhoven, Neonatologische Intensivstation, Veldhoven, Niederlande, ${ }^{3}$ Uniklinik Köln, Zentrum für Klinische Chemie, Köln, Deutschland, ${ }^{4}$ Maastricht University Medical Center, Department of Pediatrics, Maastricht, Niederlande, ${ }^{5}$ Uniklinik Köln, Klinik für Kinder- und Jugendmedizin, Gebäude 44a, Köln, Deutschland}

Fragestellung. Etwa 25\% der VLBW-Frühgeborenen $(<1500$ g Geburtsgewicht), die an einer bronchopulmonalen Dysplasie (BPD) leiden, entwickeln eine pulmonal-arterielle Hypertension (PAH). Diese schwerwiegende Komplikation kann z. B. mit dem Phosphodiesterasehemmer Sildenafil effektiv behandelt werden, jedoch fehlen gegenwärtig einfache und nichtinvasive Tests, um das Therapieansprechen zu beurteilen und die Therapie optimal steuern zu können. In dieser Arbeit untersuchten wir, ob NT-proBNP-Werte im Urin (uNT-proBNP) VLBW-Frühgeborener mit PAH einen non-invasiven Marker für den Krankheitsverlauf und das Therapieansprechen darstellen können.

Patienten und Methodik. UNT-proBNP wurde bei drei Frühgeborenen $<1000 \mathrm{~g}$ (Gestationsalter zwischen 23 1/7 und 27 6/7 Wochen) mit einem erhöhten $\mathrm{FiO}_{2}$-Bedarf $(>0,5)$ jenseits des 28. Lebenstags und einem Frühgeborenen mit einem Gestationsalter von 33 4/7 Wochen und PPHN bestimmt. Die Messwerte wurden dabei unmittelbar vor Beginn einer Therapie mit Sildenafil und longitudinal über einen Zeitraum von 6-20 Wochen erhoben. Wie von Czernik et al. beschrieben, wurden die uNT-proBNP-Werte zur Korrektur für Konzentrationsunterschiede des Urins auf Urin-Kreatininwerte normalisiert (UNBCR; Czernik et al., Pediatrics, Vol 128, No 3, p 545 ff, 2011). Die resultierenden UNBCRWerte wurden dann mit klinischen Kovariaten (respiratorische Unterstützung, $\mathrm{FiO}_{2}$-Bedarf, echokardiographischen Parametern, medikamentöse Therapie) verglichen, um zu prüfen, ob UNBCR-Werte mit dem Krankheitsverlauf korrelieren bzw. das Ansprechen auf eine medikamentöse Therapie widerspiegeln.

Ergebnisse und Diskussion. In 3 von 4 Fällen war das Vorliegen einer PAH vor Beginn einer klinisch indizierten medikamentösen Therapie mit Sildenafil echokardiographisch zu belegen. Die UNBCR-Werte lagen zu diesem Zeitpunkt zwischen 16,4 und 374,9 ng/mg. Nach Beginn der Therapie mit Sildenafil, bzw. in einem Fall mit Sildenafil und Bosentan, war innerhalb eines Zeitraums von 2-4 Wochen ein Abfall der UNBCR-Werte auf minimal o,6-20,5 ng/mg zu verzeichnen. Der Verlauf der UNBCR-Werte korrelierte dabei jeweils mit klinischen Parametern des Therapieansprechens sowie in drei von vier Fällen mit echokardiographischen Untersuchungsbefunden. Abfallende UNBCR-Werte gingen dabei einer Normalisierung des pulmonal-arteriellen Drucks in der Echokardiographie voraus. Sekundär ansteigende UNBCR-Werte traten bei einem Patienten auf, der nach initialer Stabilisierung eine Therapie-refraktäre PAH entwickelte und an dieser Komplikation verstarb. In einem zweiten Fall fiel eine passagere Erhöhung der UNBCR-Werte 15 Wochen nach Therapiebeginn im Rahmen einer nekrotisierenden Enterokolitis auf.

Schlussfolgerung. UNBCR-Werte spiegeln als einfache und nichtinvasive Parameter den Krankheitsverlauf bzw. das Therapieansprechen Frühgeborener mit PAH wieder. Die UNBCR-Bestimmung könnte daher zukünftig hilfreich in der Therapiesteuerung sein.

\section{FV69}

Plasma Pro-Endothelin-1 als Marker für bronchopulmonale Dysplasie bei sehr frühgeborenen Kindern

P. Baumann', B. Grass', O. Niesse', K. Spanaus', S. Fouzas', C. Bührer', I. Pramana ${ }^{5}$, S. Wellmann

'UniversitätsSpital Zürich (USZ), Klinik für Neonatologie, Zürich, Schweiz, ${ }^{2}$ UniversitätsSpital Zürich (USZ), Institut für Klinische Chemie, Zürich, Schweiz, ${ }^{3}$ Universitätsklinik Patras, Klinik für Neonatologie, Patras, Griechenland, ${ }^{4}$ Charité - Universitätsmedizin Berlin, Klinik für Neonatologie, Berlin, Deutschland, ${ }^{5}$ Universitäts-Kinderspital beider Basel (UKBB), Klinik für Neonatologie, Basel, Schweiz

Hintergrund. Die bronchopulmonale Dysplasie (BPD) stellt die häufigste Morbidität sehr frühgeborener Kinder dar. Die Diagnose einer BPD wird klinisch anhand des zusätzlichen Sauerstoffbedarfs im Alter von 28 Tagen und mit 36 Wochen korrigiertem postmenstruellem Alter (PMA) gestellt. Aktuell gibt es keinen Biomarker, der eine frühzeitige Diagnose erlaubt, um Hochrisikopatienten einer gezielten Therapie zuzuführen. Endothelin-1 (ET-1) ist ein körpereigenes gefäßwirksames Hormon, das in vivo in der multimodalen BPD-Entwicklung eine kausale Rolle spielt. Das bei der Bildung von ET-1 freigesetzte C-terminale Fragment des Vorläufermoleküls proET-1 (CT-ProET-1) ist ein zuverlässiger Surrogatparameter zur Bestimmung des instabilen ET-1.

Fragestellung. Gibt es einen Zusammenhang zwischen CT-proET-1 Plasmaspiegeln und BPD-Entwicklung bei Frühgeborenen mit Geburt vor 32 Schwangerschaftswochen (SSW)?

Material und Methode. Prospektive Querschnittstudie bei 267 Kindern mit Geburt vor 32 SSW mit insgesamt 391 CT-proET-1-Plasmamessungen (automatisierte Immunfluoreszenz), bei Geburt $(n=72)$, am 2. Lebenstag (LT, $n=89)$, am 6. LT ( $\mathrm{n}=49)$, am 28. LT $(\mathrm{n}=106)$ und mit 36 Wochen PMA $(n=75)$. Vergleich der CT-proET-1-Spiegel mit demographischen und klinischen Indizes einschließlich BPD. Statistische Auswertung mittels deskriptiver, nicht-parametrischer Analyse.

Ergebnisse. Die CT-proET-1-Plasmaspiegel betrugen bei Geburt (Median) $151 \mathrm{pmol} / \mathrm{L}$ [Interquartilenabstand (IQR) 118-186], stiegen am 2. LT auf den Maximalwert von $319 \mathrm{pmol} / \mathrm{L}$ (235-382) an und gingen am 6. LT auf $214 \mathrm{pmol} / \mathrm{L}$ (148-293), am 28. LT auf $184 \mathrm{pmol} / \mathrm{L}$ (149-233) und mit 36 SSW auf 150 pmol/L (118-188) zurück. Kinder mit BPD zeigten eine signifikante CT-proET-1 Erhöhung am 2. LT ( $\mathrm{p}=0,001)$, am 6. LT $(\mathrm{p}<0,001)$ und am 28. LT $(\mathrm{p}=\mathrm{o}, 007)$ im Vergleich zu Kindern ohne BPD. Bei Geburt und mit 36 Wochen PMA konnte kein Unterschied nachgewiesen werden. CT-proET-1 korrelierte am 6 . LT signifikant positiv mit der Anzahl an Beatmungstagen (Spearman's Rs o.66; $\mathrm{p}<0,001$ ), CPAPTagen (o,71; $\mathrm{p}<0,001)$ und Tagen Sauerstoffbedarf $(0,75 ; \mathrm{p}<0,001)$. Am 2. und am 28. LT konnte eine moderate Korrelation nachgewiesen werden, bei Geburt und mit 36 Wochen PMA hingegen nicht.

Diskussion. Bei allen frühgeborenen Kindern kommt es unabhängig von der BPD-Entwicklung zu einem Anstieg der CT-proET-1-Spiegel innerhalb der ersten Lebenstage mit nachfolgender Normalisierung. Kinder mit BPD-Entwicklung zeigen nicht nur einen signifikant erhöhten Anstieg der CT-proET-1-Spiegel, sondern auch eine verzögerte Rückbildung.

Schlussfolgerung. Hohe CT-proET-1-Spiegel am Ende der ersten Lebenswoche sind bei Frühgeborenen mit Geburt vor 32 Schwangerschaftswochen mit einer BPD-Entwicklung im weiteren Verlauf assoziiert. 


\section{FV70}

\section{Effect of altered TNF-a signaling on early pulmonary changes in} newborn mice undergoing mechanical ventilation

\section{T. Gimm' ', M. Kossert', M. Koschlig', C. Alvira², H. Ehrhardt' ${ }^{3}$, A. Hilgendorff}

${ }^{1}$ Comprehensive Pneumology Center, Helmholtz Zentrum München, München, Deutschland, '2Dept. of Pediatrics, Stanford University, Stanford, California, USA, ${ }^{3} J u s t u s-L i e b i g$ University, Dept. of General Pediatrics and Neonatology, Giessen, Deutschland, ${ }^{4}$ Ludwig-Maximilian University of Munich, Dept. of Neonatology, Grosshadern, München, Deutschland

Rationale. Chronic lung disease in the premature infant, also known as bronchopulmonary dysplasia (BPD), is characterized by impaired alveolar and vascular development preceded by an increase in lung inflammation and apoptosis. The disease mainly occurs after mechanical ventilation with oxygen rich gas (MV-O2). Here, the pro-inflammatory mediator tumor necrosis factor $\alpha$ (TNF- $\alpha$ ) has been found to be increased in the lung lavage from preterms that later develope BPD. We thus hypothesized, that the absence of TNF- $\alpha$ would significantly reduce the pulmonary inflammatory response following $\mathrm{MV}-\mathrm{O}_{2}$ and thus ameliorate apoptosis and histologic changes induced by ventilation in the developing lung in a mouse model of the disease.

Objective and methods. Five- to six-day-old TNF- $a$ knock-out (TNF- $\alpha-/-$ ) and wild type (WT, TNF- $\alpha+/+$ ) mice received MV with $40 \% \mathrm{O}_{2}$ at 180 breaths/min; controls spontaneously breathed $40 \% \mathrm{O}_{2}$. After $8 \mathrm{~h}$, lungs ( $\mathrm{n}=10-12 /$ group) were harvested for histologic, mRNA and protein analysis. To further confirm obtained effects in vitro, primary pulmonary myofibroblasts (MFB), critically involved in alveolar septation, were isolated from unventilated newborn TNF- $\alpha-/-$ and WT mice and subjected to mechanical stretch (Flexcell $\left.{ }^{\circ}\right)$ as well as cytokine exposure.

Results. Contrary to our expectations, pulmonary chemokine (MCP1, CXCL-1) and cytokine (IL-1b) expression was significantly elevated in ventilated TNF- $\alpha-/-$ as compared to WT mice accompanied by an increased influx of monocytes into the ventilated lung. Furthermore, apoptosis (TUNEL, caspase 3, 8 and 9) was significantly increased in the lungs of mechanically ventilated TNF- $\alpha-/-$ mice. These differences potentially resulted from a significant increase in pulmonary TGF-b signaling in TNF- $\alpha-/-$ mice following $\mathrm{MV}-\mathrm{O}_{2}$. These results were confirmed in vitro by an elevated apoptotic response to TGF- $\beta$ in pulmonary MFB from TNF- $\alpha-/$ - mice in contrast to WT pups. Histological changes were similar in both groups.

Conclusion. These results point towards a critical balance of pulmonary TNF- $\alpha$ and TGF- $\beta$ signaling and underline the importance of both pathways in the induction of early changes in the developing lung undergoing $\mathrm{MV}-\mathrm{O}_{2}$, i.e. pulmonary inflammation and consecutive apoptosis.

\section{FV71}

\section{Early signaling pattern in neonatal chronic lung disease}

\section{A. Höland' ', T. Gimm², H. Ehrhardt' ${ }^{3}$, T. Reicherzer', L. Gortner ${ }^{5}$, M. Scholz ${ }^{6}$, H. Hossain', A. Hilgendorff}

'Institute for Medical Microbiology, Justus-Liebig University, Giessen, Deutschland, ${ }^{2}$ Comprehensive Pneumology Center, Helmholtz Zentrum München, München, Deutschland, ${ }^{3}$ Dept. of Neonatology, Center for Pediatrics and Adolescent Medicine, Justus-Liebig-Univers, Giessen, Deutschland, ${ }^{4}$ Dept. of Neonatology, Grosshadern, Ludwig-Maximilian University of Munich, München, Deutschland, ${ }^{5}$ Dept. of Pediatrics and Neonatology, University of Saarland, Homburg, Deutschland, ${ }^{6}$ Institute for Informatics, Statistics and Epidemiology, University of Leipzig, Leipzig, Deutschland

Rationale. Chronic lung disease in the preterm infant, i.e. bronchopulmonary dysplasia (BPD) is one of the most common complications in this patient cohort. Characterized by impaired alveolar and vascular development, mechanical ventilation along with oxygen toxicity and pre- and postnatal infections are known to increase the risk for the de- velopment of the disease. These risk factors are known to commonly induce pulmonary inflammation, indicated by the pulmonary presence of neutrophils and macrophages. Despite some insights into the pathopysiology, early markers indicating patients at risk are still missing. The purpose of this study was therefore to identify critical pathways and potential early markers that would enable timely diagnosis and potential treatment monitoring in patients with BPD.

Objective and methods. Cord blood (CB) specimen from 22 preterm infants (cohort 1 ) were subjected to microarray-based gene expression analysis (Code Link) to identify critical pathways characterizing patients developing BPD. CB specimen from 110 preterm infants (cohort 2) were available for protein analysis (Luminex ${ }^{\oplus} \mathrm{xAP}^{\oplus}$ technology) to confirm potential early markers at birth indicating disease development. In vitro studies in fibroblasts isolated from tracheal aspirates (TA) of ventilated preterm infants after birth were used for confirmatory analyses.

Results. In a comprehensive gene expression analysis, transcripts were analyzed in correlation with duration of assisted ventilation as well as the need for oxygen supplementation and corrected for the maturity of the preterm infant at birth. This advanced unsupervised clustering (PreAnalytiX; CodeLink human Uniset I 1ok) revealed three main clusters of gene transcripts classified in preterms with no BPD and preterms who developed BPD. Genes correlated with the duration of ventilation and oxygen supply could be attributed to the categories apoptosis and inflammation/infection. Likewise, orthogonal partial least squares discriminance analysis (OPLS-DA) of protein results indicated a separation of $\mathrm{BPD} / \mathrm{no}$ BPD through levels of monocyte chemotactic protein (MCP)-1 and EGF under consideration of gestational age, persistent ductus arteriosus and congenital infection. In line with this, the assessment of both analyses by ingenuity pathway analysis indicated an overlap of signaling pathways in preterms later developing BPD involving monocyte signaling and growth factor expression (epidermal growth factor- (EGF, ErbB), glucocorticoid receptor-, endocytosis- (macropinocytosis, caveolae-mediated), telomerase-, macrophage-stimulating protein- (MSP-Ron), interleukin-8, -6). In vitro analyses confirmed the proliverative effect of EGF on fibroblasts isolated from tracheal aspirates of preterms with BPD.

Conclusion. Gene expression analyses and protein profiling from $\mathrm{CB}$ of preterms allows differentiation for later BPD diagnosis, indicating for increased monocyte signaling and fibroblast activation to play a central role in the pathogenesis of BPD.

\section{FV72}

\section{Effect of TGF- $\beta$ on functional properties of un-stretched and} stretched neonatal lung (myo)fibroblast

\section{P. Oak', N. Jain', T. Reicherzer ${ }^{2}$, M. Koschlig', H. Ehrhardt' ${ }^{3}$, A. Hilgendorff ${ }^{2}$}

${ }^{1}$ Comprehensive Pneumology Center, Helmholtz Zentrum München, München, Deutschland, ' ${ }^{2}$ ept. of Neonatology, Grosshadern, Ludwig-Maximilian's University, München, Deutschland, ${ }^{3}$ Dept. of General Pediatrics and Neonatology, Justus-Liebig-University, Giessen, Deutschland

Introduction. Mechanical ventilation of an immature lung often leads to bronchopulmonary dysplasia (BPD) in preterm infants characterized by impaired alveolarization and vascularization, underlined by altered growth factor signaling. As the interplay of different factors triggering the disease remains unknown, we investigated the effect of stretch and TGF- $\beta$ on functional and signaling properties of PDGF-R- $\alpha$ expressing mouse and human (myo)fibroblast (MFB).

Methods. Primary MFBs isolated from lungs of $\mathrm{P}_{5}-\mathrm{P}_{7} \mathrm{C}_{57} \mathrm{BL} / 6$ mice or from tracheal aspirates of preterm infants $\leq 29$ weeks gestational age were either exposed to in vitro stretch (elongation $\max 10 \% ; 30 \mathrm{cyc} /$ $\mathrm{min}$ ) or $5 \mathrm{ng} / \mathrm{ml} \mathrm{TGF}-\beta$ for $24 \mathrm{~h}$ or both. Additionally, TGF- $\beta$ was added to prestretched MFBs. Functional analyses included proliferation and migration. Growth factor signaling was assessed by immunoblot.

Results. TGF- $\beta$ reduced migration and increased proliferation in prestretched mouse MFBs, whereas stretch alone had no significant effect. In line with reduced migratory capacity, TGF- $\beta$ treatment diminished 
PDGF-Ra expression and signaling in stretched as well as un-stretched MFBs. In human MFBs, a reduction in migration could be confirmed in stretched and prestretched MFBs upon TGF- $\beta$ treatment depending on the disease grade. It was associated with diminished expression and signaling of PDGF-R $\alpha$ in unstretched and stretched human MFBs.

Conclusion. TGF- $\beta$ exhibits stretch-dependant and -independent effects on mouse MFBs, that could be reproduced in primary human lung MFBs depending on disease grade. These findings indicate critical mechanisms in the time course of BPD pathophysiology with potential implications for treatment strategies.

\section{FV72a}

Das Protein DMBT1 beeinflusst die Konzentration von VEGF im Trachealsekret von beatmeten Früh- und Neugeborenen

\section{H. Müller', C. Nagel', C. Weiß3', J. Mollenhauer', J. Pösch/5}

'Zentrum für Kinder- und Jugendmedizin, Universitätsklinikum Essen, Klinik für Kinderheilkunde I, Essen, Deutschland, ${ }^{2}$ Zentrum für Kinder- und Jugendmedizin, Universitätsklinikum Heidelberg, Klinik für Neonatologie, Heidelberg, Deutschland, ${ }^{3}$ Fakultät für Klinische Medizin Mannheim, Universität Heidelberg, Abteilung für Medizinische Statistik, Mannheim, Deutschland, ${ }^{4}$ Lundbeckfonden Center of Excellence NanoCAN and Molecular Oncology, University of Southern, Odense, Dänemark, ${ }^{5}$ Universitätsklinikum Heidelberg, Zentrum für Kinder- Jugendmedizin, Klinik Kinderheilkunde IV/Neonatologie/ AngelikaLautenschläger-Klinik, Heidelberg, Deutschland

Fragestellung. DMBT1 ist ein Protein der angeborenen Immunabwehr das auch von Früh- und Neugeborenen in der Lunge exprimiert und bei Infektionen hochreguliert wird, aber auch bei der Inaktivierung von Surfactant beteiligt ist. DMBT1 hat wichtige Funktionen bei der Angiogenese und kann in vitro VEGF binden. Da die bronchopulmonale Dysplasie (BPD) eine Erkrankung der Frühgeborenen darstellt, bei der die Angiogenese von Bedeutung ist, war es das Ziel, zu untersuchen, ob DMBT1 die Expression von VEGF in der Lunge beeinflusst.

Material und Methoden. In 120 Trachealsekreten von 57 Früh- und Neugeborenen (Mittel: 27,5 SSW, 23-40 SSW) wurden mittels ELISA der DMBT1- und VEGF-Gehalt bestimmt. Zudem wurden A549-Zellen (Pneumozyten Typ II-Zellen) kultiviert, die ein Plasmid mit DMBT1 (DMBT1-Zellen) und ohne DMBT1 (Kontrollzellen) enthielten. Die VEGF- und IL-6-Konzentration im Zellmedium wurde bei den unstimulierten Zellen als auch nach Stimulation mit TNFa, LPS und PMA mittels ELISA gemessen.

Ergebnisse. Der VEGF-Gehalt im Trachealsekret war signifikant mit dem DMBT1-Gehalt $(\mathrm{p}=0,0032)$, mit dem postnatalen Alter $(\mathrm{p}=0,0073)$ und dem Vorliegen einer Infektion/Sepsis $(\mathrm{p}=\mathrm{O}, \mathrm{OoO} 2)$ korreliert. Bei den unstimulierten A549-Zellen führte die DMBT1-Überexpression zu einer signifikant höheren VEGF-Expression $(p=0,0017)$. Nach Stimulation mit TNFa ( $\mathrm{p}=0,0008)$, LPS $(\mathrm{p}=0,0232)$ und PMA $(\mathrm{p}=0,0025)$ zeigten die DMBT1-Zellen jeweils signifikant höhere VEGF-Konzentrationen als die Kontrollzellen. Bei beiden Zelltypen führte die Stimulation mit TNFa und PMA zu einer signifikant höheren VEGF-Expression als bei den jeweiligen unstimulierten Zellen (DMBT1-Zellen: jeweils $\mathrm{p}<0,0001$; Kontrollzellen: $\mathrm{p}=0,0016$ und $\mathrm{p}<0,0001)$. Während die IL6-Konzentration bei den unstimulierten Kontroll- und DMBT1-Zellen ähnlich war $(\mathrm{p}=0,6028)$, zeigten die Kontrollzellen nach Stimulation mit TNFa $(\mathrm{p}=0,0003)$, LPS $(\mathrm{p}=0,0088)$ und PMA $(\mathrm{p}=0,0039)$ jeweils signifikant höhere IL-6-Konzentrationen als die DMBTi-Zellen.

Diskussion. Die vermehrte DMBT1-Expression in Lungenepithelzellen geht mit einer höheren VEGF-Expression einher. VEGF ist wesentlich bei der fetalen Lungenentwicklung als auch beim Erhalt der Lungenmorphologie. Bei der BPD wurde eine verminderte VEGF-Expression in den betroffenen Lungen beschrieben. Die erhöhten IL-6-Konzentrationen bei den Kontrollzellen unterstützen die bisherigen Daten über DMBT1, dass es sowohl pro- als auch anti-inflammatorische Eigenschaften aufweist.

Schlussfolgerung. DMBT1 könnte durch die Beeinflussung von VEGF und IL- 6 bei der BPD eine Rolle spielen.

\section{Beatmung}

\section{FV73}

Einfluss moderater permissiver Hyperkapnie auf die pulmonale Inflammation frühgeborener Kinder mit extrem niedrigem Geburtsgewicht

\section{S. Gentner', U. Uhlig' ${ }^{2}$, H. Hummler ${ }^{3}$, U. Thome ${ }^{4}$}

'Universitätsklinik Ulm, Klinik für Herz-, Thorax- und Gefäßchirurgie, Gefäßchirurgie, Ulm, Deutschland, ${ }^{2}$ Institut für Pharmakologie und Toxikologie, Aachen, Deutschland, ${ }^{3}$ Klinik für Kinder- und Jugendmedizin, Universitätsklinikum Ulm, Sektion Neonatologie und Pädiatrische Intensivmedizin, Ulm, Deutschland, ${ }^{4}$ Universitätsklinikum Leipzig, Klinik und Poliklinik für Kinder und Jugendliche der Universität, Abteilung Neonatologie, Leipzig, Deutschland

Fragestellung. Die beatmungsinduzierte Lungenschädigung bei frühgeborenen Kindern stellt einen zentralen Mechanismus in der Pathogenese der bronchopulmonalen Dysplasie (BPD) dar. In der hier zugrunde liegenden randomisierten, kontrollierten Studie wurde der Hypothese nachgegangen, dass bei Kindern, die mit moderater permissiver Hyperkapnie (PHC) beatmet wurden verglichen mit mild hyperkapnisch (MHC) beatmeten Kindern weniger Lungenschädigungen und pulmonale Entzündungsreaktionen induziert werden. Dies ist unter anderem auf reduzierte Tidalvolumina durch die PHC zurückzuführen. Aufgrund dieser geringeren pulmonalen Entzündungsreaktionen werden im Trachealsekret der mit PHC beatmeten Kinder reduzierte Konzentrationen von proinflammatorischen Zytokinen und saurer Sphingomyelinase (ASMase) erwartet.

Material und Methode. Frühgeborene mit einem Geburtsgewicht von 400-100o g sowie einem Gestationsalter von 23 o/7 bis 28 6/7 Wochen, die innerhalb von $24 \mathrm{~h}$ post partum maschinell beatmet wurden, wurden randomisiert der $\mathrm{PHC}$-Gruppe $\left(\mathrm{PaCO}_{2}\right.$-Zielbereich ansteigend von 55-65 mmHg an Lebenstag 1 auf $65-75 \mathrm{mmHg}$ an Lebenstag 7) bzw. der MHC-Gruppe ( $\mathrm{PaCO}_{2}$-Zielbereich ansteigend von $40-50 \mathrm{mmHg}$ an Lebenstag 1 auf 50-60 mmHg an Lebenstag 7) zugeteilt. Von diesen Kindern wurde an den Lebenstagen 2, 4, 7, 14 und 21 Trachealsekret gesammelt. Dieses wurde hinsichtlich der Parameter IL-1 $\beta$, IL-6, IL8, IL-10, MIP-1a, LTB4, TGF- $\beta$, NPY, Albumin, Nitrat sowie ASMase analysiert und die Messwerte auf das Referenzprotein sIgA normiert. Des Weiteren wurde im postmenstruellen Alter von 36 Wochen \pm 1 Tag das klinische Outcome bezüglich BPD bzw. Tod bestimmt.

Ergebnisse. Für die oben genannten Parameter ergaben sich bezüglich des Haupteffekts des $\mathrm{PaCO}_{2}$-Zielbereiches folgende Signifikanzwerte: IL-1 $\beta(p=0,42), I L-6(p=0,44), I L-8(p=0,91)$, IL-10 $(p=0,87)$, MIP-1 $\alpha$ $(p=0,34)$, LTB $4(p=0,87)$, TGF $-\beta_{1}(p=0,26), N P Y(p=0,47)$, Albumin $(p=0,63)$, Nitrat $(p=0,73)$, ASMase $(p=0,25)$. Es konnte somit in Bezug auf die untersuchten Parameter kein statistisch signifikanter Unterschied zwischen PHC $(n=35)$ und MHC $(n=36)$ ermittelt werden. Auch bezüglich des klinischen Outcomes BPD bzw. Tod ergab sich kein statistisch signifikantes Ergebnis.

Diskussion. Anhand der analysierten Parameter lässt sich statistisch weder ein positiver noch ein negativer Effekt der moderaten permissiven Hyperkapnie nachweisen. Das Ausbleiben der erwarteten Effekte in Bezug auf die Analyse des Trachealsekretes wird durch das klinische Ergebnis bestätigt; zwischen den beiden Gruppen besteht bezüglich des primären Outcomes Tod bzw. BPD kein signifikanter Unterschied.

Schlussfolgerung. Da sich im klinischen Outcome, insbesondere bezüglich der negativen Effekte, zwischen den beiden Gruppen keine signifikanten Unterschiede zeigen, lassen die Ergebnisse vermuten, dass eine moderate permissive Hyperkapnie verglichen mit einer milden hyperkapnischen Beatmung keinen Benefit hat. 


\section{FV74}

Verbesserte CO2-Elimination während nasaler HFOV verglichen mit nasalem CPAP bei Frühgeborenen: Ergebnisse einer retrospektiven Analyse

\section{Stavropoulou', D. Klotz', H. Fuchs', R. Hentschel'}

'Universitätsklinikum Freiburg, Zentrum für Kinder- und Jugendmedizin, Neonatologie/Intensivmedizin, Freiburg, Deutschland

Hintergrund. Die nichtinvasive Unterstützung der Atmung, verglichen mit der invasiven Beatmung, reduziert die Rate an bronchopulmonaler Dysplasie bei kleinen Frühgeborenen und kann, im Vergleich zum CPAP, die Häufigkeit von Apnoen und nach Extubation die Rate an Atemversagen, sowie die Notwendigkeit der Re-Intubation signifikant verringern. Häufig scheitert jedoch eine nichtinvasive Atemunterstützung mit nasalem CPAP aufgrund einer Ventilations- und/oder Oxygenierungsstörung. Nasale Hochfrequenzoszillation (nHFOV) könnte eine Möglichkeit sein, die Effektivität der $\mathrm{CO}_{2}$-Elimination, verglichen mit dem nasalen CPAP, zu verbessern und invasive Beatmung zu vermeiden.

Fragestellung. Verbessert nasaler HFOV die $\mathrm{CO}_{2}$-Elimination, verglichen mit nasalem CPAP, bei sehr kleinen Frühgeborenen, und können dadurch Intubationen vermieden werden.

Material und Methode. Retrospektive Analyse des Verlaufs und Outcome von 11 Frühgeborenen $<34$ SSW, die zwischen 3/2013 und 12/2013 mit nHFOV aufgrund einer $\mathrm{CO}_{2}$-Retention behandelt wurden.

Ergebnisse. Sieben von 11 Frühgeborenen mit einem GA Median von 30 6/7 Median(24-34) SSW wurden bei einem $\mathrm{pCO} 2$ von 68,5 Median(83-52) $\mathrm{mmHg}$ von nCPAP auf nHFOV umgestellt. Nach 2-4 h fiel das $\mathrm{pCO}_{2}$ auf 57 Median (42-60) mmHg und nach $48 \mathrm{~h}$ auf 45 Median (41-48) $\mathrm{mmHg}$. Vier Frühgeborenen mit einem GA von 24 6/7 Median (22-27) SSW wurden direkt nach Extubation auf nHFOV umgestellt. Nach anfänglicher Stabilisierung mussten im Verlauf 3 Kinder 17 (16-19) Tage nach Beginn von nHFOV aufgrund von Apnoen, Bradykardien oder Oxygenierungsstörung letztendlich invasiv beatmet werden. Es wurden keine Nebenwirkungen der Behandlung mit nHFOV beobachtet.

Diskussion. In dieser kleinen Kohorte senkte, wie in anderen publizierten Kohorten, nHFOV den $\mathrm{pCO}_{2}$ bei nicht-invasiv beatmeten Frühgeborenen effektiv. Die Tatsache, dass im Verlauf 3 von 11 Frühgeborenen letztendlich aufgrund von Apnoen und Bradykardien intubiert werden mussten, zeigt, dass möglicherweise die Oxygenierung nicht in gleichem Maß durch nHFOV positiv beeinflusst werden kann, wie die Ventilation.

Schlussfolgerung. Nasale HFOV kann unter bestimmten Umständen nichtinvasiv die $\mathrm{CO}_{2}$-Elimination, verglichen mit nasalem CPAP, verbessern. Randomisierte Studien sind notwendig, um die Sicherheit und den Effekt dieses Beatmungsmodus auf die Intubationsrate in unterschiedlichen Subgruppen von Früh- und Neugeborenen, sowie im Kurz- und Langzeitoutcome zu untersuchen.

\section{FV75}

Nasale Hochfrequenzoszillation in der Neonatologie: eine Umfrage in fünf europäischen Ländern

\section{H. Fischer', K. Bohlin'2, C. Bührer', G. Schmalisch', M. Cremer', C. Czernik' ${ }^{1}$ Charité - Universitätsmedizin Berlin, Klinik für Neonatologie, Berlin, Deutsch- land, ${ }^{2}$ Karolinska University Hospital Huddinge, Dpt. of Neonatology, Stock- holm, Schweden}

Fragestellung. Nasale Hochfrequenzoszillation (nHFOV) ist eine relativ neue Form der nichtinvasiven Beatmung, bei der die Druckoszillationen über ein nasales Interface appliziert werden. Die nHFOV verspricht eine bessere $\mathrm{CO}_{2}$-Abatmung und erfordert dabei keine Synchronisierung mit der Eigenatmung des Patienten. Über den Einsatz von nHFOV in der Neonatologie gibt es jedoch bisher nur wenige Daten. Ziel der vorliegenden Umfrage war daher die Erfassung der bisherigen klinischen Erfahrungen mit nHFOV bei Neugeborenen.
Material und Methode. Zwischen Juni 2013 und Februar 2014 befragten wir alle Perinatalzentren Level I in Deutschland sowie vergleichbare Zentren in Österreich, der Schweiz, den Niederlanden und Schweden. Der Fragebogen enthielt 26 Items. Gefragt wurde nach der technischen Ausstattung sowie nach Indikationen für nHFOV, konkreten nHFOVEinstellungen und Nebenwirkungen.

Ergebnisse. Insgesamt nahmen 172/186 (92\%) aller kontaktierten Zentren an der Umfrage teil. Von den teilnehmenden Neonatologien hatten 30/172 (17\%) Erfahrungen mit nHFOV. In diesen Einrichtungen wurde nHFOV am häufigsten bei Frühgeborenen $<1500$ g eingesetzt $[27 / 30$ (90\%)], häufigste Indikation war nCPAP-Versagen [27/30 (90\%)]. Zumeist wurden binasale Prongs [22/30 (73\%)] und/oder Nasopharyngealtuben $[19 / 30(63 \%)]$ als Interface verwendet. Das Druckniveau (Media$\mathrm{n}$ [range]) bei nHFOV-Beginn war 8 [6-12] $\mathrm{cm} \mathrm{H} \mathrm{H}_{2} \mathrm{O}$, maximal 10 [7-18]

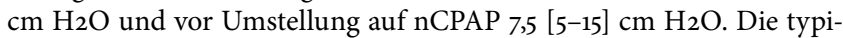
sche nHFOV-Frequenz war 10 [6-13] Hz. Abdominale Distension [11/30 $(37 \%)]$, eingedickte, feste Sekretionen [7/30 (23\%)] und sekretbedingte Obstruktion der oberen Luftwege [8/30 (27\%)] wurden am häufigsten als spezifische nHFOV-Nebenwirkungen genannt.

Diskussion. Aufgrund individueller klinischer Erfahrungen nutzen mehrere der teilnehmenden europäischen Zentren nHFOV als therapeutische Option bei Neugeborenen. Die Umfragedaten zeigen jedoch große Unterschiede hinsichtlich des verwendeten Equipments, der Indikationen und der nHFOV-Einstellungen. Eingedickte Sekretionen könnten auf Optimierungsbedarf bei der Atemgaskonditionierung hindeuten.

Schlussfolgerung. Insgesamt wird nHFOV in der Neonatologie noch relativ selten eingesetzt, da über Nutzen und Nebenwirkungen wenig bekannt ist und klinische Standards fehlen. Neue klinische Studien zur nHFOV sind daher dringend erforderlich.

\section{FV76}

Vergleich von Maske und Prong in der nasalen CPAP-Atemhilfe von Frühgeborenen

\section{Seidel', M. Lange 2, S. Schmidtke 3 , D. Singer}

'Universitätsklinikum Hamburg-Eppendorf, Sektion Neonatologie und Pädiatrische Intensivmedizin, Hamburg, Deutschland, ${ }^{2}$ Universitätsklinikum Hamburg-Eppendorf, Intensivstation für Früh- und Neugeborene, Hamburg, Deutschland, ${ }^{3}$ Asklepios Klinik Barmbek, Abteilung Neonatolgie, Hamburg, Deutschland, ${ }^{4}$ Universitätsklinikum Hamburg-Eppendorf, Sektion Neonatologie und Pädiatrische Intensivmedizin, Hamburg, Deutschland

Hintergrund und Fragestellung. Die nasale $\operatorname{CPAP}($ „continuous positive airway pressure")-Atemhilfe hat sich als nichtinvasives Verfahren zur respiratorischen Unterstützung von Frühgeborenen (FG) etabliert. Nach der Entwicklung von der „mononasalen“ zur „binasalen“ Applikationsform stehen heute verschiedene, alternativ oder alternierend eingesetzte Systeme zur Auswahl. In dieser prospektiven Beobachtungsstudie sollte geprüft werden, ob sich binasale Prongs und Nasenmasken hinsichtlich ihrer technischen Eigenschaften, medizinischen Effektivität und pflegerischen Handhabung unterscheiden.

Material und Methoden. Bei 51 FG der 23.-34. Schwangerschaftswoche (Geburtsgewichte 544-2230 g) wurden insgesamt 58 Beobachtungen à 24 Stunden (mit jeweils 6-stündlichem Wechsel zwischen Maske und Prong) vorgenommen. Dabei wurden stündlich verschiedene Geräteparameter (Atemwegsdruck, Atemgasflow, Druck-Hoch/Tief-Alarme), Vitalparameter (Atem-/Herzfrequenz, Apnoe-/Bradykardiehäufigkeit, $\mathrm{SpO}_{2}, \mathrm{FiO}_{2}$ ) und Einschätzungen des Pflegepersonals (Handhabung, Passform, Abdichtung, Toleranz, Hautzustand; Analogskala mit „Noten“"von 1-5) dokumentiert. Es kamen größenadaptierte Prongs und Masken mit dem zugehörigen CPAP-Generator Medijet ${ }^{\circledR}$ der Fa. Medin Medical Innovations, Puchheim, betrieben an dem Beatmungsgerät Leoni plus der Fa. Heinen \& Löwenstein, Bad Ems, zur Anwendung. Die statistische Auswertung erfolgte mittels eines zweiseitigen Äquivalenztests und einer plurifaktoriellen Varianzanalyse. 
Ergebnisse und Diskussion. Es standen 1232 vollständige Datensätze zur Verfügung. Hinsichtlich der Geräteparameter ergaben sich für Atemwegsdruck (Soll-Ist-Differenz), Atemgasflow und Druck-Tief-Alarme keine signifikanten Unterschiede; lediglich Druck-Hoch-Alarme traten mit der Maske, vermutlich bedingt durch Obstruktion der Nasenöffnungen bei schlechtem Sitz, häufiger auf als mit dem Prong (2,1 $\pm 7,6$ vs. $0,9 \pm 2,2$ h-1; $<<0,05)$. Bei beiden Systemen nahmen sowohl die Soll-IstAbweichungen des Atemwegsdrucks als auch der zur Erhaltung des CPAP notwendige Atemgasflow mit der Dauer der Anwendung signifikant ab. Unter den Vitalparametern zeigte sich in der Atem- und Herzfrequenz wie auch in der Häufigkeit von Entsättigungen die erwartbare Abnahme mit zunehmendem Geburtsgewicht, jedoch - ebenso wie in Apnoe-/Bradykardiehäufigkeit, $\mathrm{SpO}_{2}$ und $\mathrm{FiO}_{2}$ - kein signifikanter Unterschied zwischen Maske und Prong. In den Einschätzungen des Pflegepersonals wurden Handhabung, Passform, Abdichtung wie auch der Hautzustand für beide Systeme nahezu gleich, die Toleranz durch die FG für die Maske tendenziell günstiger bewertet als für den Prong („Note“ 1,5 vs. 1,9).

Schlussfolgerung. Aufgrund dieser für die chronische Intensivbehandlung gültigen Daten sind Maske und Prong in der nasalen CPAP-Atemhilfe von Frühgeborenen nicht nur hinsichtlich ihrer „subjektiven“ pflegerischen Handhabung, sondern auch ihrer „objektiven“ technischen Eigenschaften und medizinischen Effektivität als gleichwertig zu betrachten.

\section{FV77}

Zielwerte von CO2 und Sauerstoffsättigung 1997 und 2011 bei Frühgeborenen unter $1000 \mathrm{~g}$. Eine repräsentative Umfrage in Deutschland, Österreich und der Schweiz

\section{R. Gerull', H. Manser', H. Küster', S. Arenz', T. Arenz ${ }^{3}$, M. Nelle}

'Inselspital Bern, Neonatologie, Bern, Schweiz, ${ }^{2}$ Universitäts- Kinderklinik, Neonatologie, Göttingen, Deutschland, ${ }^{3}$ Inselspital Bern, Abteilung für Neonatologie, Bern, Schweiz, ${ }^{4}$ Medizinische Kinderklinik/Inselspital, Abteilungsleiter Neonatologie, Bern, Schweiz

Einleitung. In den letzten 20 Jahren hat sich die Evidenz zur Behandlung von Frühgeborenen deutlich verbessert. Eine Vielzahl von Studien hat sich mit Zielgrenzen von Sauerstoffsättigung und $\mathrm{pCO}_{2}$ bei beatmeten Frühgeborenen beschäftigt. Es ist nicht bekannt, in welchem Umfang diese Ergebnisse bei den Entscheidungsträgern angekommen ist.

Methoden. 1996 wurde ein Fragebogen über die Routinebehandlung von Frühgeborenen mit einem Geburtsgewicht zwischen $750 \mathrm{~g}$ und $1000 \mathrm{~g}$ entwickelt und 1997 an alle Kliniken, welche Kinder unter $1000 \mathrm{~g}$ behandelten, versendet. Auf diesem Fragebogen basierend wurde 2011 eine online-Folgeumfrage durchgeführt und an alle NICUs, die ELBWFrühgeborene in Deutschland $(n=171)$, Österreich $(n=10)$ und der Schweiz $(n=9)$ behandelten, versandt. Verglichen wurden NICUs zwischen den drei Ländern sowie bezüglich Typ (Universität gegen andere) und Größe der Klinik (definiert als jährliche Aufnahmen von =12, 13-30 und $>30$ ELBW-Frühgeborenen).

Ergebnisse. In den Jahren 1997 und 2011 haben 126 bzw. 129 Kliniken den Fragebogen ausgefüllt. Dies entspricht einem Rücklauf von 63,6\% bzw. 66,2\%. Die Prozentzahl der Kliniken, welche den jeweiligen Wert in ihrem Zielbereich angaben, sind in Tabelle $1\left(\mathrm{pCO}_{2}\right)$ und Tabelle 2 (Sauerstoffsättigung) zusammengefasst. Es zeigt sich eine hoch signifikante Veränderung zur Toleranz eines höheren $\mathrm{pCO}_{2}$ und einer niedrigeren Sauerstoffsättigung. Keine relevanten Unterschiede bestanden zwischen den Ländern, dem Klinik-Typ und zwischen NICUs verschiedener Größe.

Diskussion. Aufgrund des guten Rücklaufs können die Ergebnisse als repräsentativ für Deutschland, Österreich und die Schweiz gelten. In den letzten Jahren hat sich zunehmend herausgestellt, dass eine lungenschonende Beatmung ein besseres pulmonales Outcome zur Folge hat und niedrigere Sauerstoffsättigungen mit einer niedrigeren Rate an Frühgeborenenretinopathien assoziiert sind. Unsere Umfrage zeigt die inner-
FV77 - Tab.1 Anzahl und Prozent der NICUs, die den angegeben $\mathrm{CO}_{2}$ Partialdruck im Zielbereich sahen

\begin{tabular}{|lrrrrrrr}
\hline pCO $_{2}$ & \multicolumn{2}{c}{1997} & \multicolumn{2}{c}{2011} & p-Wert & \multicolumn{1}{c}{$95 \%$ Cl } \\
\hline $30 \mathrm{mmHg}$ & $0 / 125$ & $0 \%$ & $4 / 124$ & $3,2 \%$ & 0,092 & $-0,109,0,580$ \\
\hline $35 \mathrm{mmHg}$ & $10 / 125$ & $8,0 \%$ & $19 / 124$ & $15,3 \%$ & 0,073 & $-0,016,0,343$ \\
\hline $40 \mathrm{mmHg}$ & $67 / 125$ & $53,6 \%$ & $59 / 124$ & $47,6 \%$ & 0,356 & $-0,185,0,065$ \\
\hline $45 \mathrm{mmHg}$ & $88 / 125$ & $70,4 \%$ & $108 / 124$ & $87,1 \%$ & 0,002 & $0,097,0,380$ \\
\hline $50 \mathrm{mmHg}$ & $113 / 125$ & $90,4 \%$ & $112 / 124$ & $90,3 \%$ & 0,992 & $-0,201,0,196$ \\
\hline $55 \mathrm{mmHg}$ & $87 / 125$ & $69,6 \%$ & $117 / 124$ & $94,4 \%$ & $<0,001$ & $0,271,0,528$ \\
\hline $60 \mathrm{mmHg}$ & $67 / 125$ & $53,6 \%$ & $89 / 124$ & $71,8 \%$ & 0,003 & $0,065,0,317$ \\
\hline $65 \mathrm{mmHg}$ & $16 / 125$ & $12,8 \%$ & $56 / 124$ & $45,2 \%$ & $<0,001$ & $0,263,0,504$ \\
\hline $70 \mathrm{mmHg}$ & $13 / 125$ & $10,4 \%$ & $38 / 124$ & $30,6 \%$ & $<0,001$ & $0,160,0,435$ \\
\hline $75 \mathrm{mmHg}$ & $3 / 125$ & $2,4 \%$ & $16 / 124$ & $12,9 \%$ & 0,031 & $-0,004,0,503$ \\
\hline $80 \mathrm{mmHg}$ & $0 / 125$ & $0 \%$ & $19 / 124$ & $15,3 \%$ & 0,002 & $0,371,0,610$ \\
\hline & & & & & &
\end{tabular}

FV77 - Tab.2 Anzahl und Prozent der NICUs, die die angegebene Sauerstoffsättigung im Zielbereich sahen

\begin{tabular}{|lrrrrrrr}
\hline $\begin{array}{l}\text { Sauerstoff- } \\
\text { sättigung }\end{array}$ & 1997 & & 2011 & $\mathrm{p}$-Wert & \multicolumn{2}{c|}{$\mathbf{9 5 \%} \mathrm{Cl}$} \\
\hline $75 \%$ & $0 / 125$ & $0 \%$ & $2 / 125$ & $1,6 \%$ & 0,237 & $-0,276$, & 0,586 \\
\hline $80 \%$ & $2 / 125$ & $1,6 \%$ & $20 / 125$ & $16,0 \%$ & 0,003 & 0,253, & 0,552 \\
\hline $85 \%$ & $50 / 125$ & $40,0 \%$ & $110 / 125$ & $88,0 \%$ & $<0,001$ & 0,405, & 0,619 \\
\hline $90 \%$ & $103 / 125$ & $82,4 \%$ & $104 / 125$ & $83,2 \%$ & 0,769 & $-0,138$, & 0,187 \\
\hline $92 \%$ & $112 / 125$ & $89,6 \%$ & $90 / 125$ & $72,0 \%$ & 0,001 & $-0,417$, & $-0,128$ \\
\hline $94 \%$ & $76 / 125$ & $60,8 \%$ & $30 / 125$ & $24,0 \%$ & $<0,001$ & $-0,488$, & $-0,255$ \\
\hline $96 \%$ & $19 / 125$ & $15,2 \%$ & $3 / 125$ & $2,4 \%$ & 0,004 & $-0,516$, & $-0,192$ \\
\hline $98 \%$ & $0 / 125$ & $0 \%$ & $0 / 125$ & $0 \%$ & 1,000 & & \\
\hline
\end{tabular}

halb von 14 Jahren deutlich gestiegene Toleranz niedrigerer Sauerstoffsättigungen und höherer $\mathrm{pCO}_{2}$-Werte. Dahinter steht $\mathrm{u}$. a. vermutlich das Ziel, kürzere Beatmungszeiten und niedrigere Beatmungsdrucke auf Kosten von niedrigeren Sauerstoffsättigungen und höherem $\mathrm{pCO}_{2}$ zu erreichen, sowie die Rate an Frühgeborenenretinopathie zu senken. Unsere Umfrage bestätigt hochsignifikant, dass das Konzept der „gentle ventilation" "bei den Entscheidungsträgern auf den Intensivstationen angekommen ist. Einschränkend muss festgehalten werden, dass nicht klar ist ob es sich bei den Ergebnissen um theoretisches Wissen handelt oder ob es auch wirklich in der Praxis umgesetzt wird.

Schlussfolgerung. Die Ergebnisse der Umfrage zeigen, dass sich von 1997 bis 2011 die Zielwerte von Sauerstoffsättigung und pCO2 hochsignifikant zu geringerer Sättigung und höherem $\mathrm{pCO}_{2}$ verändert haben. Unsere Umfrage zeigt, dass das Konzept der der „gentle ventilation“ im Sinne einer Toleranz von niedrigeren Sauerstoffsättigungen und permissiver Hyperkapnie den Entscheidungsträgern auf den NICUs bewusst ist.

\section{FV78}

\section{Zuverlässigkeit von Einweg-PEEP-Ventilen bei der manuellen} Beatmung Neugeborener

\section{J. Hartung ', A. te Pas', M. Thio Lluch', G. Schmalisch', C. Roehr}

${ }^{1}$ Charité Universitätsmedizin Berlin, Campus Mitte, Neonatologie/Pädiatrie, Berlin, Deutschland, ' ${ }^{L}$ UUMC, Leiden, Niederlande, ${ }^{3}$ The Royal Women's Hospital, Melbourne, Australien, Division of Neonatal Research, Parkville VIC 3052, Australien

Fragestellung. SIB sind die neben TPR und FIB in den aktuellen Leitlinien empfohlenen Geräte zur manuellen Beatmung Neugeborener [1] und werden in vielen Ländern hierzu am häufigsten eingesetzt [2]. Auch die Anwendung eines PEEP wird in den Leitlinien empfohlenı und kann bei der Beutelbeatmung mit Hilfe eines ansteckbaren PEEP-Ven- 
tils ermöglicht werden. Verschiedene Studien konnten jedoch zeigen, dass der PEEP hiermit nur unzureichend aufgebaut wird $[3,4]$ und dass die Zuverlässigkeit der Ventile durch Autoklavieren weiter abnimmt [5]. Ziel der Studie war es, die Zuverlässigkeit von Einweg-PEEP-Ventilen verschiedener Hersteller als mögliche Alternative zu wieder verwendbaren Ventilen zu untersuchen.

Material und Methode. Zehn neue Einweg-PEEP-Ventile von fünf verschiedenen Herstellern [je 2 identische Ventile von: Laerdal (5$\left.20 \mathrm{cmH}_{2} \mathrm{O}\right)$, $\mathrm{DROH}\left(\mathrm{o}-10 \mathrm{cmH}{ }_{2} \mathrm{O}\right)$, Vital Signs $\left(5-20 \mathrm{cmH}_{2} \mathrm{O}\right)$, medisize $\left(5-2 \mathrm{cmH}_{2} \mathrm{O}\right)$, Ambu $\left.\left(\mathrm{o}-2 \mathrm{O} \mathrm{cmH}_{2} \mathrm{O}\right)\right]$ wurden angesteckt an einen elektromechanisch angesteuerten SIB um ein Modelpüppchen entsprechend einem $1 \mathrm{~kg}$ Frühgeborenen mit Spitzendruck von $2 \mathrm{O} \mathrm{cmH}_{2} \mathrm{O}$ und Beatmungsfrequenz von $60 / \mathrm{min}$ zu beatmen. Der erzeugte PEEP bei Einstellung auf 5 und $10 \mathrm{cmH} 2 \mathrm{O}$ wurde aufgezeichnet und ausgewertet. Ergebnisse. Die Ventile erzeugten im Mittel (SD) bei Einstellung auf $5 \mathrm{cmH}_{2} \mathrm{O}$ einen PEEP von $3,5(1,9) \mathrm{cmH}_{2} \mathrm{O}$, bei Einstellung auf $10 \mathrm{cmH}_{2} \mathrm{O}$ einen PEEP von $5,6(2,9) \mathrm{cmH}_{2} \mathrm{O}$. Ein Ventil konnte bei eingestellten 5 und $10 \mathrm{cmH}_{2} \mathrm{O}$ keinen, das zweite Ventil desselben Herstellers jeweils nur o,o $(0,0)$ und $1,4(0,0) \mathrm{cmH}_{2} \mathrm{O}$ aufbauen. Auch zwischen den zwei Ventilen desselben Herstellers gab es zum Teil deutliche Unterschiede im erzeugten PEEP.

Diskussion. Die untersuchten Einweg-PEEP-Ventile stellen eine Alternative zu mehrfach verwendbaren Ventilen dar. Sie können jedoch ebenfalls nur einen unzuverlässigen PEEP aufbauen.

Schlussfolgerung. Anwender sollten sich der Unzuverlässigkeit von Einweg-PEEP-Ventilen bewusst sein und die Ventile vor dem Einsatz testen.

1. Richmond S, Wyllie J (2010) European resuscitation council guidelines for resuscitation 2010 section 7. Resuscitation of babies at birth. Resuscitation 81:1389-1399

2. Roehr CC, Grobe S, Rudiger M et al (2010) Delivery room management of very low birth weight infants in Germany, Austria and Switzerland - a comparison of protocols. Eur J Med Res 15:493-503

3. Kelm M, Proquitte H, Schmalisch G, Roehr CC (2009) Reliability of two common PEEP-generating devices used in neonatal resuscitation. Klin Padiatr 221:415-418

4. Morley CJ, Dawson JA, Stewart MJ, Hussain F, Davis PG (2010) The effect of a PEEP valve on a Laerdal neonatal self-inflating resuscitation bag. J Paediatr Child Health 46:51-56

5. Hartung JC, Schmölzer G, Schmalisch G, Roehr CC (2013) Repeated thermosterilisation further affects the reliability of positive end-expiratory pressure valves. Journal of Paediatrics and Child Health, 49(9):741-745

\section{Neonatale Neuroprotektion}

\section{FV79 \\ Intranasale Muttermilch in zwei Fällen von schwerer neonataler Hirnschädigung - neuroprotektive Wirkung bei extremen Früh- geborenen?}

T. Keller', A. Oberthür', K. Dinger', K. Mehler', A. Vierzig', B. Roth', A. Kribs'

'Universitätskinderklinik Köln, Neonatologie und Pädiatrische Intensivmedizin, Köln, Deutschland

Hintergrund. Die intraventrikuläre Hämorrhagie (IVH) des Frühgeborenen ist eine schwere Komplikation mit begrenzten therapeutischen Optionen. Neuerdings wird die intranasale (i.n.) Medikamentengabe zunehmend als effiziente Methode erkannt, Wachstumsfaktoren, Hormone und Stammzellen (SZ) unter Umgehung der Blut-Hirn-Schranke ins ZNS zu bringen. Im Mausmodell der neonatalen Hypoxie zeigte sich nach i.n. EGF-Gabe eine verstärkte Neubildung von Oligodendrozyten und eine Verbesserung des funktionellen Outcomes (Scafidi, Nature 2014). Weiterhin ist eine Migration von SZ über die Lamina cribrosa ins ZNS mit neuroprotektivem Effekt belegt (Donega, PloS ONE 2013).
Ebenso verhindern diese im Tiermodell die Entwicklung des posthämorrhagischen Hydrozephalus (Ahn, Stroke 2013). Muttermilch (MM) enthält pluripotente SZ mit der Fähigkeit zur Differenzierung in „neural-like cells" (Patki, Hum Cell 2010) und Wachstumsfaktoren (EGF, VEGF, IGF, Epo) mit z. T. sehr hoher Konzentration (Ballard, Pediatr Clin North Am 2013). Beim Stillen gelangt regelhaft MM in die Nase Neugeborener. Die nasale Verabreichung zur symptomatischen Therapie der akuten Rhinitis im Säuglingsalter ist gängige Praxis (Rose, MoKi 2014).

Methodik. Aufgrund dieser Datenlage verabreichten wir in zwei Fällen schwerer IVH und bei limitierten therapeutischen Optionen nach Aufklärung der Eltern im Rahmen eines individuellen Heilversuchs MM i.n. Nach mikrobiologischem Ausschluss pathogener Keime applizierten wir 3-mal tgl. je $1 \mathrm{gtt}$ MM pro Nasenöffnung. Der Verlauf wurde schädelsonographisch und neuropädiatrisch evaluiert.

Fall 1. Wbl. FG der 25+6.SSW, Apgar 7/8/9, pH 7,25. Am 3. LT IVH III ${ }^{\circ}$ li. mit Infarzierung des parietookzipitalen Marklagers und zunehmender Ventrikelweite sowie IVH II ${ }^{\circ}$ re. Therapie mit Erythropoetin (312 IE/ $\mathrm{kg}$ s.c. 3-mal wöchentlich), zusätzlich MM i.n. (compassionate use). Im Verlauf komplette Resorption der Blutung mit schmalen Seitenventrikeln und klinisch neurologisch bis zum 1. Geburtstag unauffälligem Kind.

Fall 2. Wbl. FG der 25+4.SSW, Apgar 7/7/7, pH 7,39. Am 2. LT IVH II ${ }^{\circ}$ li. und IVH III ${ }^{\circ}$ re. mit großer partiell hämorrhagischer Infarzierung des periventrikulären Marklagers frontal. Bei erweiterten Liquorräumen serielle Lumbalpunktionen. Epo s.c. und MM i.n.. Nach rückläufiger Ventrikelerweiterung Beendigung der Punktionen nach 21 Tagen. Entlassung des Kindes ohne Drainage und klinisch neurologisch unauffällig.

Diskussion und Schlussfolgerung. Die theoretische Grundlage aus dem Nachweis von Wachstumsfaktoren und SZ in MM einerseits, der hohen Effektivität der transnasalen Route ins ZNS andererseits, sowie die Verläufe dieser beiden Patienten führen zu der Hypothese, dass Bestandteile der MM nach intranasaler Applikation bei der schweren Hirnschädigung des Frühgeborenen einen signifikanten neuroprotektiven Effekt entfalten könnten. Die Hypothese der neuroprotektiven Wirkung von MM i.n. bei neonataler schwerer Hirnschädigung sollte in systematischen Studien weiter untersucht werden.

\section{FV80}

MSC-Exosomen: immunmodulatorische und neuroprotektive Effekte im inflammatorisch-induzierten Hirnschädigungsmodell der neugeborenen Ratte

K. Drommelschmidt ${ }^{1}$, M. Serdar ${ }^{1}$, I. Bendix ${ }^{2}$, S. Prager ${ }^{2}$, F. Bertling ${ }^{3}$, M. Keller $^{4}$, A. Ludwig ${ }^{5}$, P. Horn ${ }^{5}$, K. de Miroschedji ${ }^{5}$, B. Giebel' ${ }^{5}$, U. Felderhoff-Müser ${ }^{6}$ ${ }^{1}$ Kinderklinik I, Neonatologie, Essen, Deutschland, ${ }^{2}$ Klinik für Kinder und Jugendmedizin der Universität, Klinik für Kinderheilkunde I, Essen, Deutschland, ${ }^{3}$ Universitätsklinik Essen, Kinderheilkunde I, Essen, Deutschland, ${ }^{4}$ Kinderklinik Dritter Orden Passau, Passau, Deutschland, ${ }^{5}$ Institut für Transfusionsmedizin, Universitätsklinikum Essen, Essen, Deutschland, ${ }^{6}$ Universitätsklinikum Essen, Direktorin der Klinik für Kinderheilkunde I, Zentrum für Kinder- und Jugendmedizin, Essen, Deutschland

Hintergrund und Fragestellung. Das unreife Gehirn ist empfindlich gegenüber Noxen, somit haben Frühgeborene ein erhöhtes Risiko für die Entwicklung von neurologischen Spätschäden wie Zerebralparesen und kognitive Einschränkungen. Die Entwicklung eines neonatalen Hirnschadens ist multifaktoriell und die Inflammation ist ein involvierter Hauptfaktor. Mesenchymale Stammzellen (MSCs) zeigen in vielen tierexperimentellen und klinischen Studien immunmodulatorisches und neuroprotektives Potential. In Tierexperimenten konnte gezeigt werden, dass MSCs nach intravenöser Applikation in der Lunge verbleiben und nicht das Zielgewebe erreichen. Trotzdem sind therapeutische Effekte in den Zielgeweben nachweisbar, so dass ein parakriner Mechanismus wahrscheinlich ist. Der parakrine Mechanismus kann durch Exoso- 
men vermittelt werden. Exosomen sind Mikrovesikel, die eine spezifische Kombination aus Proteinen, Lipiden und RNA enthalten und eine wichtige Rolle in der interzellulären Kommunikation spielen. Das Ziel der Studie ist die Untersuchung der immunmodulatorischen und neuroprotektiven Effekte von MSC-Exosomen im experimentellen Modell der inflammatorisch-induzierten Hirnschädigung der neugeborenen Ratte. Material und Methoden. Wistar Ratten wurden in vier Behandlungsgruppen randomisiert $(\mathrm{NaCl} / \mathrm{NaCl}, \mathrm{NaCl} /$ Exosomen, LPS/ $\mathrm{NaCl}, \mathrm{LPS} /$ Exosomen). LPS (o,25 mg/kg) wurde an $\mathrm{P}_{3}$ appliziert, Exosomen an $\mathrm{P}_{3}$ und $\mathrm{P}_{4}$. An $\mathrm{P}_{5}$ und $\mathrm{P}_{11}$ wurden die Ratten getötet und transkardial perfundiert. Es erfolgte die Aufarbeitung der Gehirne mittels Proteinanalysen und Immunhistochemie.

Ergebnisse. In den molekularbiologischen Untersuchungen ergibt sich für den Zeitpunkt $\mathrm{P}_{5}$ eine signifikant erhöhte Apoptose in der Schadensgruppe (LPS-Applikation) im Vergleich zu den Ratten, die zusätzlich mit Exosomen behandelt wurden. Histologisch zeigen sich Hinweise für einen erhöhten Zelltod (TUNEL) in der LPS-Gruppe und einen geringeren Zelltod in der Gruppe, denen LPS und Exosomen gespritzt wurde. An P11 zeigte sich molekularbiologisch mittels eines Oligodendrozytenreifungsmarkers (MBP)eine signifikant verbesserte Myelinisierung in der Gruppe, die LPS und Exosomen erhalten haben im Vergleich zu den Ratten, die alleinig mit LPS behandelt wurden.

Schlussfolgerung. MSC-Exosomen zeigen immunmodulatorische und neuroprotektive Effekte auf den inflammatorisch-induzierten neonatalen Hirnschaden.

\section{FV81}

Hämatopoetische Stammzellen im Nabelschnurblut: Unterschiede zwischen sehr kleinen Frühgeborenen und Reifgeborenen

\section{Wisgrill', S. Schüller', A. Berger', A. Pollak', A. Spittler², L. Gortner}

'Medizinische Universität Wien, Universitätsklinik für Kinder- und Jugendheilkunde, Wien, Österreich, ${ }^{2}$ Medizinische Universität Wien, Core Facility Flow Cytometry, Wien, Österreich, ${ }^{3}$ Universitätsklinikum des Saarlandes, Klinik für Kinder- und Jugendmedizin, Homburg, Deutschland

Fragestellung. In den letzten Jahren gewann die Stammzelltherapie be neonatalen Erkrankungen immer mehr Aufmerksamkeit. Im Fokus stehen vor allem mesenchymale Stammzellen (MSCs), die effektiv in neonatalen Tiermodellen eingesetzt wurden. Da die Purifikation von MSCs zeitaufwändig und mit Manipulation verbunden ist, werden Alternativen für autologe Therapieansätze gesucht. Hämatopoetische Stammzellen (HSCs) bilden den größten Stammzellpool im Nabelschnurblut und zeigen auch das Potential in nicht- hämatologische Zelllinien zu differenzieren. Die vorliegende In-vitro-Studie untersucht die absolute Zahl sowie das Koloniebildungspotential von HSC Subpopulationen im Nabelschnurblut von sehr unreifen Frühgeborenen und gesunden Reifgeborenen.

Material und Methoden. $\mathrm{CD}_{34}+\mathrm{HSC}-S u b p o p u l a t i o n e n$ wurden im Nabelschnurblut von je 30 Früh- und Reifgeborenen mittels FACS analysiert. Das Koloniebildungspotential wurde mittels MethoCult In-vitroAssays evaluiert. Des Weiteren wurde das Koloniebildungspotential von HSC Subpopulationen, die nach dem Stammzellmarker CD133 oder der Aldehyddehydrogenase (ALDH) Aktivität sortiert wurden, bestimmt. Ergebnisse. Nabelschnurblut von Frühgeborenen enthält eine signifikant höhere HSC-Zahl als von Reifgeborenen, vor allem frühe $\mathrm{CD}_{3} 8$ Progenitor-Zellen sind im Nabelschnurblut von Frühgeborenen deutlich erhöht. Ebenfalls ist die Koloniebildung von HSCs in sehr unreifen Frühgeborenen signifikant erhöht. Absolute HSC-Zahlen und Koloniebildungspotential weisen eine starke Korrelation zwischen Gestationsalter, Geburtsgewicht und dem Alter der Mutter auf. Die Koloniebildung von $\mathrm{CD}_{34+/ C D 133-}$, CD $34+/ \mathrm{CD}_{133}+$ bzw. ALDHhigh HSCs ist bei Frühgeborenen höher als bei Reifgeborenen.

Schlussfolgerung. Im Vergleich zu reifen Neugeborenen enthält Nabelschnurblut von sehr unreifen Frühgeborenen eine signifikant höhere $\mathrm{CD}_{34}+$ Zellzahl und weist eine gesteigerte Koloniebildung auf. Weitere Untersuchungen sind notwendig, um das Potenzial von $\mathrm{CD}_{34}+$ Zellen bei nicht hämatologischen Erkrankungen für autologe regenerative Therapieansätze zu nutzen.

\section{FV82}

Der Einfluss von therapeutischer Hypothermie auf Amplituden-integriertes Elektroenzephalogramm und Outcome bei Neugeborenen mit hypoxisch-ischämischer Enzephalopathie

K. Goeral', V. Giordano', K. Klebermaß-Schrehof', M. Weninger', A. Berger', M. Olischar

'Univ. Klinik für Kinder und Jugendheilkunde, Abt. für Neonatologie, Pädiatrische Intensivmedizin und Neuropädiatrie, Wien, Österreich

Hintergrund. Das Amplituden-integrierte Elektroenzephalogramm (aEEG) stellt bei Neugeborenen mit peripartaler Asphyxie eine verlässliche Methode zur frühzeitigen Vorhersage des entwicklungsneurologischen Outcomes dar. Durch therapeutische Hypothermie wird der positive Vorhersagewert des aEEGs verändert und die Normalisierung der Hintergrundaktivität verzögert (Thoreson et al. 2010).

Methodik. Insgesamt 41 Neugeborene mit peripartaler Asphyxie und daraus resultierender hypoxisch-ischämischer Enzephalopathie (HIE) Grad II nach Sarnat $[\mathrm{n}=24$ Normothermie (NT), $\mathrm{n}=17$ Hypothermie (HT)] wurden in diese retrospektive Analyse inkludiert. Das aEEG der ersten 3 Lebenstage wurde für beide Gruppen analysiert (Hintergrundaktivität, Vorhandensein von Schlaf-Wach Zyklen und Krampfaktivität, zunächst rein deskriptive Analyse, dann Errechnen eines kombinierten aEEG-Scores) und mit klinischen Parametern nach der Geburt, Magnetresonanztomographie und Outcome (Alter bei Untersuchung 6 Monate bis 3 Jahre) korreliert. Anschließend wurde der prognostische Stellenwert des aEEGs im Hinblick auf Outcome zwischen den beiden Gruppen verglichen.

Ergebnisse. Die Rate der auffälligen aEEGs am ersten Lebenstag zeigte keinen signifikanten Unterschied zwischen den beiden Kohorten (pathologisches aEEG: $64 \%$ bei HT, $71 \%$ bei NT). Am dritten Lebenstag war die Rate der auffälligen aEEGs in der HT Gruppe signifikant niedriger als in der Gruppe ohne Kühlung ( $18 \%$ bei HT, $57 \%$ bei NT; $\mathrm{p}=0,045)$. Es zeigte sich ein signifikanter Unterschied im Outcome bei Neugeborenen mit HIE Grad II nach Sarnat in Abhängigkeit davon, ob eine therapeutische HT durchgeführt wurde ( $\mathrm{p}=0,016)$ oder nicht. In der Gruppe der Kinder mit HT zeigten $82 \%$ ein normales Outcome, während dies in der NT Gruppe nur bei 43\% aller Patienten der Fall war. Der positive Vorhersagewert eines kombinierten aEEG-Scores (unter Berücksichtigung von Hintergrundaktivität, Schlaf-Wach Zyklen und Krampfaktivität) war in der nicht gekühlten Kohorte am ersten Lebenstag höher als in der HT Gruppe und stieg in beiden Gruppen vom ersten (HT: 83,3\%, NT: 87,5\%) zum dritten Lebenstag auf $100 \%$ an. In unserer Kohorte der Kinder mit therapeutischer HT korrelierte das fehlende Auftreten von Schlaf-WachZyklen signifikant mit Pathologien in der Magnetresonanztomographie $(p=0,05)$ und einem pathologischen Outcome $(p=0,03)$. Für das Vorhandensein von Krampfaktivität in der ersten Lebenswoche bestand keine Korrelation mit Outcome.

Schlussfolgerung. Diese Analyse unserer Wiener Kohorte mit HIE spiegelt in der Literatur bereits beschriebene Ergebnisse wieder. Der positive Vorhersagewert des aEEGs ist am ersten Lebenstag als Effekt der Ganzkörperkühlung wie auch schon mehrfach beschrieben bei NT höher als bei HT und steigt im Laufe der ersten Tage in beiden Gruppen auf $100 \%$ an. Diese Ergebnisse unterstreichen die Wichtigkeit des Einsatzes des aEEGs als frühen Parameter zur Einschätzung des Outcomes bei Kindern mit HIE.

\section{FV83}

Neuroprotektive Effekte von Hypothermie und Levetiracetam nach Hypoxie-Ischämie im neonatalen Maushirn

\section{K. Straßer', L. Lückemann', B. Reinboth', D. Brait', A. Bertsche², I. Bendix ${ }^{1}$} J. Herz', U. Felderhoff-Müser ${ }^{1}$

'Universitätsklinikum Essen, Kinderklinik ', Neonatologie, Essen, Deutschland, ${ }^{2}$ Universitätsklinikum Leipzig, Klinik für Kinder- und Jugendheilkunde, Leipzig, Deutschland

Hintergrund. Hypoxisch-ischämische Schädigungen des unreifen ZNS stellen während der Perinatalperiode die häufigsten Ursachen entwick- 
lungsneurologischer Langzeitdefizite dar. Die Ganzkörperkühlung ist bisher die einzige standardisierte Therapie bei asphyktischen Neugeborenen, aus der sich positive Effekte auf die neurologische Langzeitentwicklung ergeben. Aktuell ist es jedoch notwendig 8 bis 10 Kinder zu behandeln, um die neurologische Funktion eines Kindes zu verbessern. Um die Effektivität der Hypothermie zu steigern, besteht die Notwendigkeit adjuvante Strategien zu entwickeln.

Fragestellung. Aus diesen Gründen wurden in einer tierexperimentellen Studie neuroprotektive Effekte durch einen kombinierten Therapieansatz von Hypothermie und Levetiracetam nach Hypoxie-Ischämie untersucht.

Material und Methoden. 9 Tage alte $\mathrm{C}_{57} \mathrm{BL} / 6$-Mäuse erhielten entweder eine Schein-OP oder eine Hypoxie-Ischämie (HI, modifiziertes Vanucci-Rice-Modell). Nach HI wurden die Jungtiere in 6 Gruppen randomisiert: 1) keine Behandlung, 2) Hypothermie (Ganzkörperkühlung, 4 Stunden, $32^{\circ} \mathrm{C}$ ), 3) hochdosiert Levetiracetam intraperitoneal (i.p., $70 \mathrm{mg} / \mathrm{kgKG}), 4)$ niedrigdosiert Levetiracetam i.p. (7 mg/kgKG), 5) Kombination von Hypothermie und hochdosiert Levetiracetam i.p. und 6) Kombination von Hypothermie und niedrigdosiert Levetiracetam i.p. Die Hirnhemisphären wurden 24 Stunden nach HI mittels Proteinanalysen und Immunhistochemie bezüglich Faktoren der Apoptose, der Myelinisierung und der neuronalen Vernetzung analysiert. $\mathrm{Ab}$ dem Alter von 4 Wochen wurde die sensomotorische und kognitive Funktion mittels verschiedenen Verhaltenstestungen ermittelt.

Ergebnisse. Nach Hypothermie zeigten sich eine deutliche Verminderung apoptotischer Faktoren sowie eine Verbesserung der Myelinisierung und neuronalen Vernetzung. Unter intraperitonealer Monotherapie mit Levetiracetam sowie in Kombination mit Hypothermie konnte ein Anstieg apoptotischer Faktoren, jedoch kein Einfluss auf myelinisierende Zellen und Neuronen detektiert werden. In den Verhaltenstestungen ergaben sich durch Hypothermie positive Langzeiteffekte.

Diskussion. Die Ganzkörperkühlung führt kurz- und langfristig zur Neuroprotektion im neonatalen Maushirn durch Apoptosereduktion sowie Aufrechterhaltung der Myelinisierung und neuronalen Vernetzung. Eine hochdosierte Therapie mit Levetiracetam führte zu vermehrtem Gewebeverlust durch Anstieg der Apoptose, niedrigdosiert konnte nach Intervention mit Levetiracetam kein additiver Effekt nach hypoxisch-ischämischer Schädigung gezeigt werden.

Schlussfolgerung. Levetiracetam zeigt in der Behandlung der hypoxisch-ischämischen Enzephalopathie keine Wirksamkeit. In der klinisch eingesetzten Dosis hat Levetiracteam keinen schädigenden Effekt auf das sich entwickelnde Gehirn und kann weiterhin als Intervention bei Krampfanfällen eingesetzt werden.

\section{FV84 \\ Therapeutische Hypothermie in einem infektionssensibilisierten neugeborenen Hypoxie-Ischämie-Rattenmodel: Verlust der neuro- protektiven Wirkung}

\section{Osredkar', M. Thoresen', E. Maes', T. Flatebø', M. Elstad', H. Sabir}

'University of Oslo, Department of Physiology, Institute of Basic Clinical Sciences, Oslo, Norwegen, ${ }^{2}$ Med. Einrichtung der Universität Kinderklinik, Neonatologie und pädiatrische Intensivmedizin, Düsseldorf, Deutschland

Hintergrund. Die therapeutische Hypothermie (HT) ist Standardtherapie nach perinataler hypoxisch-ischämischer Hirnläsion (HI). Eine perinatale Infektion erhöht die Vulnerabilität der HI. Die Wirkung von HT nach solcher perinataler Infektion ist bisher nicht bekannt. Diese Studie wurde durchgeführt, um den Einfluss von HT in einem infektionssensibilisierten Model der HI bei neugeborenen Ratten zu untersuchen.

Material und Methode. Sieben Tage alte Ratten erhielten entweder eine intraperitoneale Injektion mit $\mathrm{NaCl}$ o,9\% (Vehikel Gruppe, Veh) oder E.coli Lipopolysaccharid (LPS Gruppe, LPS). Vier Stunden nach der Injektion erhielten alle Ratten eine unilaterale Ligatur der A. carotis und wurden dann für 50 min $8 \%$ Sauerstoff ausgesetzt (HI). Nach der HI wurden die Ratten bezüglich folgender Therapien randomisiert: 1) Vehikel Gruppe für $5 \mathrm{~h}$ bei Normothermie (Veh-NT; n=30), 2) LPS Gruppe für $5 \mathrm{~h}$ bei Normothermie (LPS-NT; $\mathrm{n}=35$ ), 3) Vehikel Gruppe für $5 \mathrm{~h}$ bei HT (Veh-HT; n=29), 4) LPS-Gruppe für 5 h bei HT (LPS-HT; $n=46$ ). Nach 7 Tagen Überleben wurden die Gehirne mittels „brain area loss“ (BAL) und Hippocampusgröße analysiert.

Ergebnisse. In der Veh-NT Gruppe war der BAL $11,2 \pm 14 \%$. In der LPSNT Gruppe war der BAL mit 29,8 $\pm 17 \%$ signifikant höher als in der VehNT Gruppe $(\mathrm{p}=0,002)$. Die Veh-HT Gruppe hatte einen signifikant kleineren BAL $(5,4 \pm 6 \%)$ als die Veh-NT Gruppe $(\mathrm{p}=0,043)$. Die LPS-HT Gruppe hatte einen BAL von $32,5 \pm 16 \%$, welcher signifikant größer war als in der Veh-HT Gruppe.

\section{Poster}

\section{Medizinische Ausbildung und Versorgungsforschung}

\section{P001}

Benchmarking-Projekt Neonatologie Rheinland-Pfalz/Saarland erste Ergebnisse

T. Hoppen', G. Damm², P. Wenzlaff', N. Niemann'2, C. Burmeister³, M. Bücheler', C. von Buch ${ }^{5}$, J. Bensch ${ }^{6}$, U. Kindorff', J. Möller ${ }^{8}$, H. Skopnik ${ }^{9}$

'Gemeinschaftsklinikum Koblenz-Mayen, Kemperhof Koblenz, Klinik für Kinderund Jugendmedizin, Koblenz, Deutschland, ${ }^{2} \mathrm{ZQ}$ Zentrum für Qualität und Management im Gesundheitswesen, ÄK Niedersachsen, Hannover, Deutschland, ${ }^{3}$ SQMed GmbH Geschäftsstelle Qualitätssicherung Rheinland-Pfalz, Mainz, Deutschland, ${ }^{4} \mathrm{QBS}$ Qualitätsbüro im Saarland, Saarbrücken, Deutschland, ${ }^{5} \mathrm{Kin}-$ der- und Jugendmedizin, Diakonie Krankenhaus, Bad Kreuznach, Deutschland, ${ }^{6}$ Vinzentius-Krankenhaus, Kinderabteilung, Landau, Deutschland, ${ }^{7}$ Klinikum Mutterhaus der Borromäerinnen, Abteilung für Kinder- und Jugendmedizin, Trier, Deutschland, ${ }^{8}$ Klinikum Saarbrücken, Zentrum für Kinder- und Jugendmedizin, Saarbrücken, Deutschland, ${ }^{9}$ Klinikum Worms, Klinik für Kinder- und Jugendmedizin, Worms, Deutschland

Hintergrund. Angeregt durch das erfolgreiche bundesweite Projekt „Benchmarking Mukoviszidose“ beschäftigt sich die AG Neonatologie und pädiatrische Intensivmedizin Rheinland-Pfalz und Saarland seit 2010 mit der Benchmarking-Methodik.

Methodik, Indikatoren, erste Ergebnisse. Das Ranking erfolgt auf Grundlage der Neonatalerhebung (2005-2009) und Qualitätssicherung Neonatologie (2010-2012). Unterschiede in beiden Erhebungen bedingen eine geringe methodische Unschärfe. Mittels eines Rankings der adjustierten Qualitätsindikatorraten über 5-8 Jahre werden Kliniken mit „Besten-Ergebnissen“ ermittelt. 12 Klinken aus Rheinland-Pfalz und 3 Kliniken aus dem Saarland nehmen aktiv an dem Projekt seit seiner Startphase teil.

Intra- und periventrikuläre Hirnblutung: Die Auswertung erfolgte für die Geburtsjahrgänge 2006-2010. Für das Ranking wurden die überlebenden Kinder mit einem Geburtsgewicht $<1500$ g und einem Gestationsalter von 24-31 SSW betrachtet. Die risikoadjustierte Raten wurden mittels logistischer Regression berechnet. Unter Anwendung der Regressionsgewichte 2010 für Deutschland bei einer beobachteten Rate von $4,82 \%$ wurde in Rheinland-Pfalz eine adjustierte Rate in 2010 von 2,5\% erhoben.

Pneumothorax: Die Auswertung erfolgte für 2006-2010. Für das Ranking wurden die überlebenden Kinder mit einem Gestationsalter von 24-39 SSW betrachtet. Unter Anwendung der Regressionsgewichte 2010 für Deutschland bei einer beobachteten Rate von $5,72 \%$ wurde in Rheinland-Pfalz eine adjustierte Rate in 2010 von 7,1\% erhoben. Nekrotisierende Enterocolitis: Die Auswertung 2005-2011 ergab: 34.097 versorgte Kinder insgesamt, davon 147 NEC-Fälle $(=0,4 \%)$. 33.841 Überlebende, davon 130 NEC-Fälle $(=0,4 \%)$. 1785 Überlebende <150o g, 24-31 SSW, davon 59 NEC-Fälle $(=3,3 \%)$. Aufgrund der geringen Fallzahl wurde keine Risikoadjustierung mittels logistischer Regression vorgenommen. Die 
berechneten Inzidenzen sind mit den Angaben der aktuellen AWMFLeitlinie vergleichbar.

Bronchopulmonale Dysplasie: Die Auswertung 2005-2012 ergab: 38.431 versorgte Kinder insgesamt, davon 350 BPD-Fälle $(=0,9 \%) \cdot 38.123$ Überlebende, davon 340 BPD-Fälle (=0,9\%). 1999 Überlebende <1500 g, 24-31 SSW, davon 258 BPD-Fälle $(=12,9 \%)$. Die risikoadjustierten Raten wurden mittels logistischer Regression berechnet. Es fanden die Regressionsgewichte 2012 für Deutschland Anwendung (beobachtete Rate $8,0 \%)$.

Aktuell werden die Indikatoren höhergradige Frühgeborenenretinopathie (ROP), Sterblichkeit und Aufnahmetemperatur für die Gestaltung der nächsten Projekttreffen ausgewertet.

Fazit und Ausblick. Dieses Projekt verfolgt einen Ansatz zur systematischen Qualitätsentwicklung in einem festformierten regionalen neonatologischen Netzwerk unter qualifizierter externer Moderation. In einem offenen Benchmarking-Prozess, in dem bei regelmäßigen Treffen eines definierten Teilnehmerkreises ausgewählte Indikatoren der Qualitätssicherung Neonatologie analysiert und klinikinterne Vorgehensweisen der „besten Kliniken“ präsentiert werden, entstehen verbindliche „Best-Practice“-Empfehlungen für die tägliche Routine.

\section{P002}

\section{Effekte eines strukturierten Neugeborenen-Reanimationskurses (ERC-NLS) auf die praktischen Fähigkeiten der Teilnehmer - eine prospektive Observationsstudie}

\section{E. Wilden', U. Kreth ${ }^{2}$, T. Bösing 3 , E. Hamelmann ${ }^{4}$, N. Teig}

'Universitätskinderklinik Bochum, Katholisches Klinikum, Neonatologie und päd. Intensivmedizin, Bochum, Deutschland, ${ }^{2}$ Klinik für Kinder- und Jugendmedizin, AKH Viersen, Viersen, Deutschland, ${ }^{3}$ Klinik für Kinder- und Jugendmedizin, EVK Bielefeld, Bielefeld, Deutschland, ${ }^{4}$ Unikinderklinik Bochum, Katholisches Klinikum, Bochum, Deutschland, ${ }^{5}$ Unikinderklinik Bochum, Katholisches Klinikum, Neonatologie und päd. Intensivmedizin, Bochum, Deutschland

Hintergrund. Die Regionalisierung der Risiko-Geburtshilfe vermindert die Häufigkeit von neonatalen Notfallsituationen in nicht-spezialisierten Geburtskliniken. Dies reduziert die Erfahrung in diesen Häusern, im Falle einer Notfallsituation adäquat zu reagieren. Die systematische Schulung dieser Mitarbeiter mittels strukturierter Curricula ist ein nahelegender Weg dieses Dilemma zu lösen. Wir haben untersucht, ob der Newborn Life Support Kurs (NLS) des European Resuscitation Council (ERC) die praktischen Reanimationsfähigkeiten der Teilnehmer tatsächlich zu verbessern vermag.

Methoden und Probanden. Es wurde untersucht, ob die einzelnen im NLS-Reanimationsalgorithmus des ERC vorgesehenen Interventionen von den Probanden inhaltlich und zeitlich korrekt angewendet wurden. Hierfür wurde mittels eines Neugeborenen-Dummy die Situation einer außerklinischen Geburt eines reifen Neugeborenen mit respiratorischer Anpassungsstörung simuliert, als Beispiel für eine Geburt mit begrenzten diagnostischen und therapeutischen Möglichkeiten. Zwei Prüfer beurteilten systematisch die Reanimationsschritte vor Beginn des Kurses und nach Kursende an Hand eines 42 Prüfungsaspekte umfassenden Fragebogens. Pro Kurs wurden nach dem Zufallsprinzip zwei bis vier Teilnehmer hierfür ausgewählt. Zusätzlich wurde allen Kursteilnehmer ein strukturierter Fragebogen zur subjektiven Beurteilung der Kurseffekte ausgehändigt.

Ergebnisse. Aus 29 Reanimationskursen mit 611 Teilnehmern wurden 61 Teilnehmer (10\%) für die Studie beurteilt. 445 Teilnehmer (73\%) nahmen an der Fragebogenbefragung teil. Die Punktzahl für die praktische Bewältigung der Reanimationssimulation stieg von 25,9 auf 39,1 Punkte $(\mathrm{p}<\mathrm{o}, 001)$ bei einer möglichen Gesamtpunktzahl von 42 Punkten. Die Streuung zwischen den Teilnehmerfertigkeiten (einfache Standardabweichung) reduzierte sich von 6,67 auf 2,57 Punkte ( $\mathrm{p}<0,001)$. Die höchsten Prä-Kurs-Punktzahlen erreichten Pädiater, die niedrigsten Hebammen, nach dem Kurs zeigten sich keine Unterschiede mehr zwischen den Berufsgruppen. Vor der Kursteilnahme wurden 10 Prüfungs- aspekte von weniger als 50\% der Teilnehmer korrekt durchgeführt. Darin beinhaltet waren 5 Aspekte bzgl. der Beatmung (Zahl sowie Dauer der initialen, prolongierten Atemhübe, Zeitdauer der Beatmung, Frequenz der Beatmung, Verhältnis Thoraxkompression - Beatmung).

Schlussfolgerung. Der NLS-Kurs des ERC verbessert effektiv und berufsgruppenunabhängig die praktischen Reanimationsfähigkeiten aller Teilnehmer. Da eine Abnahme dieser Effekte mit der Zeit zu erwarten ist, sollte die Dauer des Interventionserfolges untersucht werden, um das optimale Zeitintervall für eine Wiederholung der Kursteilnahme bestimmen zu können.

\section{$\mathrm{P} 003$}

Paediatric Simulation Training (PST) - ein Peer-Teaching-Projekt zur Verbesserung der Fertigkeiten im Umgang mit Kindernotfällen

\section{Wagner', F. Mölzer', L. Garnys', J. Schwindt'², G. Burda' ${ }^{2}$ K. Göral', K. Kle- bermaß-Schrehof ${ }^{2}$, A. Berger ${ }^{2}$}

${ }^{1}$ Medizinische Universität Wien, Wien, Österreich, ${ }^{2}$ Universitätsklinik für Kinderund Jugendheilkunde Wien, Abteilung für Neonatologie, Pädiatrische Intensivmedizin und Neuropädiatrie, Wien, Österreich

Hintergrund und Fragestellung. Simulationsbasierte Lernmethoden sind ein mittlerweile in vielen Zentren etablierter Teil der Ausbildung spezialisierter Teams [1]. Paediatric Simulation Training (PST) wurde von Studierenden der Medizinischen Universität Wien für Studierende gegründet und zeichnet sich durch qualitatives Peer-Teaching aus. Ziel ist es, Studierenden die Möglichkeit zu bieten, pädiatrische Notfälle regelmäßig in Form von Szenarien zu trainieren. Die teilnehmenden Studierenden bekommen einen Fragebogen vor Beginn und nach Ende des Trainings, um eine laufende Verbesserung des Kurses zu garantieren, sowie den Effekt dieser neuen Lehrmethode bewerten zu können.

Methodik. Mit Hilfe erfahrener, durch pädiatrische FachärztInnen supervidierten TutorInnen werden praxisnahe Szenarien durchgespielt. Es gibt ein zweistufiges Simulationstraining für jeweils acht TeilnehmerInnen pro Kurs. Auf eine kurze Einführung zum Thema Life Support, entsprechend den aktuellen ERC Leitlinien, folgt ein praktisches Training an der Simulationspuppe (Baby/Little Anne). In der zweiten Einheit werden Kindernotfälle im Simulationsraum der Univ. Klinik für Kinder- und Jugendheilkunde Wien, am SimBaby trainiert. Die Szenarien werden aufgezeichnet und anschließend mit Videoanalyse und strukturiertem Debriefing besprochen.

Ergebnisse. Insgesamt haben 47 Studierende von Februar 2013 bis Januar 2014 teilgenommen. Davon haben 26 (55,3\%) einen kompletten Fragebogen retourniert. 15 (57,7\%) TeilnehmerInnen fühlten sich vor dem Training unsicher im Umgang mit kritisch kranken Kindern und 8 (30,8\%) schätzten sich als unsicher in Bezug auf Reanimationsfertigkeiten ein. Es wurde eine statistisch signifikante Verbesserung festgestellt. Nur eine Person fühlte sich unsicher ( $\mathrm{p}=\mathrm{o}, \mathrm{ooo1}$; exakter Test nach Fisher) im Umgang mit kritisch kranken Kindern und ebenso nur eine Person ( $\mathrm{p}=0$,024; exakter Test nach Fisher) unsicher in Bezug auf Reanimationsfertigkeiten. $23(88,5 \%)$ TeilnehmerInnen gaben an, sich wie in einer echten Notfallsituation gefühlt zu haben und alle bewerteten das Simulationstraining als wichtigen Teil ihrer medizinischen Ausbildung.

Diskussion und Schlussfolgerung. Medizinische Simulation ist eine hervorragende Methode zur Verbesserung der medizinischen Ausbildung, sowie zur Erhöhung der PatientInnensicherheit. Wir konnten eine signifikante Steigerung der Fertigkeiten im Umgang mit Kindernotfällen zeigen. Für den weiteren Ausbau der Kurse werden die TeilnehmerInnen ermuntert, nach entsprechender Einschulung, für andere Studierende TutorInnen zu werden. Die zukünftige Evaluation soll auch Fragen in Bezug auf Teamwork und Kommunikation enthalten. Miteinbeziehen von KrankenpflegerInnen soll das interdisziplinäre Teamwork verbessern und wichtiges klinisches Wissen hinzufügen.

1. Bidarkar SS, Wood J, Cohen RC, Holland AJ (2013) Role of simulation for paediatric proceduralists: practice makes perfect or trial and error? Journal of paediatrics and child health 49(2):94-98 
P004

Availability and provision of emergency and basic neonatal care in Namibia, Kenya, Tanzania and Uganda: evidence from the national Service Provision Assessment (SPA) surveys

\section{Krüger', M. Ali ${ }^{2}$}

'St. Franziskus Hospital, Klinik für Kinder- und Jugendliche, Ahlen, Deutschland, ${ }^{2}$ Curtin University, Faculty of Health Sciences, Perth, Australien

Research question. Improving neonatal health is essential for lowering child mortality in developing countries. Little is known about the availability of essential items and provision of emergency and basic neonatal care on a national scale in most of sub-Saharan Africa. Therefore we examined the situation in four of these countries.

Methods. Data from the national SPA surveys of Namibia (NA; 2009), Kenya (KE; 2010), Tanzania (TZ; 2006) and Uganda (UG; 2007) were analyzed with regard to the availability of essential items, organizational aspects and the prevalence of neonatal care practices at different levels of the health system (hospital, health center, dispensary).

Results. Essential items for neonatal care (scissors/blade, cord clamp/ tie, suction apparatus, bag-mask, scale) (NA: $84 \% / \mathrm{KE}: 72 \% / \mathrm{TZ}: 41 \% /$ UG: $55 \%$ ) and measures of infection control (soap, water, sterile gloves, disinfecting solution) (74/69/44/64) were commonly available at hospitals in all countries, except in TZ (41 and 44\%, resp.; p<0.05). At health centers and dispensaries, availability could decrease by $10-30 \%$ (for all countries $\mathrm{p}<0.05$ ). Transport schemes for obstetric emergencies were available at hospitals (97/85/50/89) and to a lesser degree at health centers (61/53/45/16), but were uncommon at dispensaries (10/15/8/12; $\mathrm{p}<0.05)$. Similar findings applied to the provision of Caesarean sections (hospital: 81/55/96/87; health center: 3/36/15/2; dispensary: o/o/o/o; $\mathrm{p}<0.05)$. Trained delivery providers were present onsite 24 hours regularly in hospitals $(95 / 86 / 99 / 88)$, but less so in health centers $(58 / 56 / 72 / 52)$ and dispensaries $(14 / 12 / 12 / 39 ; \mathrm{p}<0.05)$. Up to a third of staff, especially in hospitals, had received training in neonatal resuscitation and basic care in the preceding 36 months (33/35/20/27). Newborn routine suctioning was commonly reported in hospitals (65/17/58/25), as were full immersion baths in NA (86) and UG (29), and prelacteal feeding in NA (16) and TZ (21). Weighing the newborn and rooming-in were routine practices (only in dispensaries <90\%). Except for TZ (38\%), >60\% of births took place in hospitals.

Discussion. Essential items tended to be widely available in hospitals where the organization of care was also better than at other levels. However, harmful practices like routine suctioning, full immersion bath or provision of prelacteal feeding were common. Namibia and Kenya fared better in terms of equipment and service organization, but some harmful practices were more frequent in Namibia than in the other countries. Re-training seemed to improve these practices in Kenya.

Conclusion. Essential items for emergency and routine neonatal care are not routinely available at the various levels of institutional health care, while potentially harmful practices are still common. The findings call for increased efforts to improve the standard of neonatal care in these resource-poor settings.

\section{P005}

Frühkindliche Entwicklung als studentischer "Pate" erleben praxisorientiertes, individuelles Lehrprojekt in der Neonatologie

\section{S. Poralla ${ }^{1}$, A. Müller ${ }^{1}$, T. Dresbach ${ }^{1}$ \\ 'Zentrum für Kinderheilkunde am Universitätsklinikum Bonn, Neonatologie, Bonn, Deutschland}

Projekt. Ein seit zwei Jahren etabliertes Lehrprojekt der Abteilung für Neonatologie am Universitätsklinikum Bonn ermöglicht Medizinstudenten ein gesundes Kind von der Geburt an bis zum Ende des zweiten Lebensjahres zu begleiten. Die Studenten lernen ihr „Patenkind“ direkt nach der Geburt kennen und begleiten die Familie in den ersten zwei Jahren zu allen Kinderarztbesuchen, insbesondere den Vorsorgeunter- suchungen. So erleben die „Paten“ hautnah und aktiv, wie sich ein Kind normal entwickelt - unter motorischen, sprachlichen und psychosozialen Aspekten.

Ablauf. Das erste Kennenlernen findet im Rahmen der U2 statt. Ab diesem Zeitpunkt erfolgt die regelmäßige Kontaktaufnahme zwischen Paten und Patenfamilien in Eigenverantwortung, die jeweiligen Arzttermine werden individuell untereinander abgesprochen. Die Studenten haben einen Arzt als Ansprechpartner, der sie in Kleingruppen in Seminaren auf die jeweils bevorstehende Vorsorgeuntersuchung vorbereitet. Durch gute Kooperation mit den niedergelassenen Kinderärzten wird eine ebenso intensive Betreuung in den Praxen gewährleistet.

Ziel. Das Projekt ermöglicht Studenten bereits in den überwiegend theoretisch ausgerichteten vorklinischen und ersten zwei klinischen Semestern erste Patientenkontakte, ein „Miterleben“ der frühkindlichen Entwicklung, somit erste Kontakte mit dem pädiatrischen Berufsfeld. Nach dem Grundsatz „nur wer ein gesundes Kind kennt, kann auch Krankheiten erkennen" legen die Studenten mit ihren gesammelten praktischen Erfahrungen einen wichtigen Grundstein für ihr späteres Berufsleben.

Erste Erfahrungen. Das Projekt stößt bei Eltern und Studenten auf außerordentlich gute Resonanz. Jedes Semester bewerben sich deutlich mehr Studenten als Plätze zur Verfügung stehen. Die Studenten sind sehr motiviert, engagiert und evaluieren das Projekt mit sehr gut. Aus vielen Patenschaften wird mehr: Besuche zu Hause, Paten als Babysitter, regelmäßiger Kontakt auch zwischen den Terminen der U-Untersuchungen und über die geplanten zwei Jahre hinaus. Um das Gelingen des Projektes und die Zufriedenheit aller beteiligten Personen zu gewährleisten, ist ein hoher personeller Betreuungsaufwand notwendig, der aber insgesamt mehr als gerechtfertigt ist und durch das positive Feedback belohnt wird.

Ausblick. Das Konzept des Projektes lässt sich auch auf andere Bereiche der Pädiatrie anwenden und ermöglicht Studenten Lernen durch aktives Erleben. Fähigkeiten, die so erworben werden können, sind nur schwer durch traditionelle Lehrformen vermittelbar. Profitieren würden Studenten auch von Lehrprojekten in den Fachbereichen Allgemeinpädiatrie, Kardiologie und Onkologie, bei denen Studenten die Möglichkeit erhalten, einen einzelnen Patienten über längere Zeit zu begleiten. Um eine adäquate Umsetzung zu ermöglichen, sind weitere personelle Ressourcen erforderlich.

\section{P006}

Sudden Infant Death (SID) im Kreißsaal - Strategien zur Prävention und Fallpräsentation

\section{N. Linduska', A. Berger ${ }^{1}$}

'Universitätsklinik für Kinder- und Jugendheilkunde Wien, Abteilung für Neonatologie, Pädiatrische Intensivmedizin und Neuropädiatrie, Wien, Österreich

Hintergrund. Sudden Infant Death (SID) oder „apparent life threating events" (ALTE) im Kreißsaal sind selten, wurden zuletzt aber in mehreren Fallserien beschrieben. Wir präsentieren den Fall eines SID im Kreißsaal und analysieren beschriebene Risikofaktoren. Eine Standard Operating Procedure (SOP) zur Senkung des SID Risikos im Kreißsaal wird vorgestellt.

Fallbericht. 3300 g schweres Neugeborenes aus SSW 40, Primiparität, wird nach initial guter Adaptation (Apgar 9/10/10, NS-pH 7,29) in Lebensstunde 3 leblos auf der Mutter aufgefunden. Die Reanimation verläuft frustran, das Kind verstirbt am 2. Lebenstag. Als Risikofaktoren werden Bauchlage, Primiparität und unbeaufsichtigtes Anlegen/Stillen im Kreißsaal gefunden.

Diskussion. Es wird davon ausgegangen, dass durch Aufklärung und Schulung des betreuenden Personals, sowie durch Anleitung der Eltern, insbesondere der Erstgebärenden, zum Offenhalten der Atemwege ihres Kindes während des Bondings, Fälle von plötzlichem Kindstod im Kreißsaal verhindert werden können. Eine entsprechende SOP wird diskutiert. 
P007 - Tab. 1 Patientencharakteristika und wichtige Outcomeparameter

\begin{tabular}{|llll}
\hline Parameter & 2007 & 2008 & 2013 \\
\hline Anzahl (n) & 97 & 91 & 91 \\
\hline Gestationsalter (SSW) & $28^{1 / 7}\left( \pm 2^{3 / 7}\right)$ & $28^{1 / 7}\left( \pm 2^{2 / 7}\right)$ & $28^{2 / 7}\left( \pm 2^{3 / 7}\right)$ \\
\hline Geburtsgewicht (g) & $1.011( \pm 314)$ & $999( \pm 290)$ & $1.032( \pm 275)$ \\
\hline Schwere BPD & $15 \%$ & $19 \%$ & $1 \%$ \\
\hline Beatmungsdauer (Tage) & 5 & 2 & 0,75 \\
\hline ROP (operationspflichtig) & $23 \%$ & $21 \%$ & $9 \%$ \\
\hline IVH $>$ Grad 2 & $13 \%$ & $12 \%$ & $13 \%$ \\
\hline
\end{tabular}

\section{P007}

\section{Möglichkeiten der Qualitätsverbesserung der neonatologischen Versorgung}

\section{Mögel', D. Konstantelos', A. Koch', M. Rüdiger ${ }^{1}$}

${ }^{1}$ Klinik für Kinder- und Jugendmedizin, Universitätsklinikum Carl Gustav Carus, Fachbereich Neonatologie\&Pädiatrische Intensivmedizin, Dresden, Deutschland

Einleitung. In der Vergangenheit konnte die Mortalität sehr unreifer Frühgeborener deutlich gesenkt werden. Die Morbidität blieb in diesem Zeitraum relativ konstant, unterscheidet sich jedoch sehr deutlich zwischen verschiedenen Kliniken. Anzustreben wäre, die Versorgungsqualität auf dem Niveau der besten Kliniken zu nivellieren.

Hypothese. Durch internes Qualitätsmanagement - u. a. im Rahmen von strukturierten, interdisziplinären Perinatalbesprechungen, wie durch den Beschluss des Gemeinsamen Bundesausschusses (GBA) vom 2o.Juni 2013 gefordert - kann sowohl die Prozess- als auch die Ergebnisqualität in der Versorgung sehr unreifer Frühgeborener verbessert werden.

Methoden. Basierend auf Morbiditätsdaten der Jahre 2007/08 wurden Zielstellungen für die Prozess- (Beatmungsdauer, Anzahl Sedierung, Katecholamine, antibiotische Behandlung etc.) und Ergebnisqualität [Inzidenz von bronchopulmonaler Dysplasie (BPD), Hirnblutungen (IVH), Retinopathie (ROP) und Nekrotisierender Enterokolitis (NEC)] definiert. Davon abgeleitet wurden schrittweise Interventionen zur Qualitätsverbesserung implementiert, welche u. a. das Videomonitoring der Erstversorgung mit strukturiertem Auto-Feedback und Perinatalbesprechungen beinhalten.

Ergebnisse. Im Perinatalzentrum Dresden werden jährlich etwa 100 Frühgeborene $<1500 \mathrm{~g}$ betreut. Durch die Implementierung des internen Qualitäts- und Fehlermanagements konnte die Rate für die schwere BPD und die ROP reduziert werden. Als potentieller Einflussfaktor wurde die mittlere Beatmungsdauer gesenkt. Dabei unverändert blieb die Rate an IVH > Grad 2 (Tab. 1).

Diskussion. Durch die eingeführten Maßnahmen konnte die Inzidenz der BPD und ROP signifikant gesenkt werden, die Ergebnisse bezüglich der Hirnblutungsrate sind noch unbefriedigend. In Anlehnung an die Ulmer-Erfahrungen (Hummler et al. 2012) wurde die Perinatalbesprechung umstrukturiert und auf die Vermeidung von IVH fokussiert. Schlussfolgerung. Intrinsisch motiviertes Qualitätsmanagement hat ein großes Potenzial, die Versorgung von sehr unreifen Frühgeborenen zu verbessern und so chronische Erkrankungen zu vermeiden.

Gefördert durch: DSKN - Deutsche Stiftung Kranke Neugeborene.

\section{P008}

Optimierung der Versorgungsmöglichkeiten in außerklinischen Geburtseinrichtungen sowie leitliniengerechte Beratungspflicht der betreuenden Hebammen

\section{Genschaft', E. Robel-Tillig²}

'Städtisches Klinikum St. Georg Kinderklinik, Neonatologie, Leipzig, Deutschland, ${ }^{2}$ Klinikum St. Georg gGmbH, Neonatologie, Leipzig, Deutschland

Einleitung. In den letzten Jahren wurde in Sachsen eine annähernd konstante Zahl der pro Jahr in außerklinischen Geburtseinrichtungen ge- borener Kinder registriert. Die Hebammen einer solchen Einrichtung stellen sich ihrer eigenen Qualitätserfassung. Sollte jedoch ein postnatal in eine Klinik verlegtes Kind Komplikationen erleiden oder versterben, entgeht das der Erfassung.

Ziele. 1. Optimierung der Versorgungsmöglichkeiten kranker Neugeborene in außerklinischen Geburtseinrichtungen. 2. Verpflichtung der betreuenden Hebammen zur leitliniengerechten Aufklärung der Eltern. Methode. Wir beschreiben 5 Kinder, welche in einem Gebärhaus auf die Welt kamen und in unsere Einrichtung verlegt oder von zu Hause aufgenommen wurden.

Ergebnisse. Fall 1: Exitus letalis nach vorzeitiger Plazentalösung und unzureichend adäquater Versorgung. Fall 2: Fehleinschätzung schwerer Atemnot nach Mekoniumaspiration mit Zeitverzug bis zum Ergreifen suffizienter Maßnahmen. Fall 3: B-Streptokokken-Sepsis. In Fällen 1-3 Notarzt-Transport in Babyschale ohne Therapie-/Überwachungsmöglichkeit. Fall 4: B-Streptokokken-Sepsis, Aufnahme von zu Hause, fehlende Aufklärung bei unbekanntem GBS-Status. Fall 5: Massive Hirnblutung mit 5 Wochen nach Gabe einer verdünnten Vitamin-KZubereitung auf Anraten im Gebärhaus.

Diskussion und Schlussfolgerung. Verbesserung des Outcomes betroffener Kinder, Verkürzung des stationären Aufenthaltes mittels Optimierung der Versorgungsmöglichkeiten kranker Neugeborener in außerklinischen Geburtseinrichtungen und leitliniengerechte Beratung durch Hebammen sind anzustreben.

\section{P009}

Einflussfaktoren auf die elterliche Zufriedenheit während des stationären Aufenthaltes von VLBW-Frühgeborenen

\section{Enke', A. Kribs'², C. Woopen ${ }^{1}$}

${ }^{1}$ Uniklinik Köln, Forschungsstelle Ethik, Köln, Deutschland, ${ }^{2}$ Universitätskinderklinik Köln, Neonatologie und Pädiatrische Intensivmedizin, Köln, Deutschland

Fragestellung. Welche Faktoren beeinflussen im komplexen Versorgungsprozess neonatologischer Intensivstationen (NICUs) die Zufriedenheit der Eltern von VLBW Frühgeborenen?

Material und Methode. An fünf deutschen NICUs (Level I) erfolgte eine quantitative, prospektive Befragung deutsch sprechender Eltern von VLBW-Frühgeborenen. Der eigens entwickelte Fragebogen basiert auf validierten Instrumenten zur Erfassung der durch Eltern berichteten Empathie der Ärzte und Pflegekräfte (CARE von Neumann et al. 2008) und der elterlichen Zufriedenheit (Satisfaction-Index von Pfaff et al. 2004). Der Fragebogen wurde entweder zum Zeitpunkt der Verlegung von der NICU oder bei Entlassung ausgegeben und die Erhebungsdaten deskriptiv analysiert (Friedman's Test, Cohen's, Spearman's Rho).

Ergebnisse. 255 Elternteile nahmen teil, 92,1\% Mütter, 7,9\% Väter. In den für die Zufriedenheit relevanten Items berichteten 70,9-93,7\% der Eltern zufrieden oder sehr zufrieden zu sein. Am geringsten war die Zufriedenheit mit den Visiten, dem Vorgehen bei der Verlegung von der NICU und dem Einbezug in die Behandlung. Der Grad berichteter Empathie lag sehr hoch: In Bezug auf die ärztliche Versorgung bei einem Score-Wert von 44/41,6 (Median/Mean; SD=8,1), im Bezug auf die pflegerische Versorgung bei einem Score-Wert von 45/43,2 (Median/ Mean; $\mathrm{SD}=6,8$ ) [Min. (keine Empathie berichtet) =10; Max. (höchstmögliches $\mathrm{Ma}$ an berichteter Empathie) $=50]$. Die Variablen elterlicher Zufriedenheit korrelierten signifikant positiv mit den Empathie-Scores. Die stärksten Korrelationen ergeben sich für die Zufriedenheit mit der Information und dem Einbezug in die Behandlung.

Diskussion. Die Ergebnisse stehen im Einklang mit zahlreichen Arbeiten, die die Effektivität empathischer Kommunikation mit Eltern über den Zustand ihres Kindes belegen. Ergänzend fanden wir eine Korrelation zwischen dem Einbezug der Eltern in die Behandlung ihres Kindes und ihrer Zufriedenheit Dieses Ergebnis deckt sich mit Untersuchungen, die eine Implementierung des Konzeptes der Family-Centered Care (FCC) forcieren. Somit könnte die elterliche Zufriedenheit durch eine Erhöhung entgegengebrachter Empathie und einen höheren Grad des Einbezugs in die Behandlung gesteigert werden. Die Untersuchung 
hat ihre Limitation durch die relativ kleine Stichprobe sowie das erhebliche Überwiegen von Müttern unter den teilnehmenden Eltern.

Schlussfolgerung. In unserer Untersuchung bestimmten der Grad ärztlicher und pflegerischer Empathie und der Einbezug der Eltern in die Behandlung ihres Kindes die elterliche Zufriedenheit. Wegen der genannten Limitationen der Untersuchung sind weitere Studien notwendig. Unsere Folgestudie, Health Services Research in care of VLBW in NICUs - The impact of human and organizational factors on performance (HSRNICU) ${ }^{c}$ greift diese Probleme auf und untersucht die Versorgung in 69 deutschen Kliniken (Level I und II). Im Studiendesign verankerte Maßnahmen stellen den erhöhten Einbezug der Väter Frühgeborener sicher.

\section{P010}

Vereinfachung der Qualitätssicherung durch weniger Arbeit: Neonatalerhebung, NeoKISS, Arztbriefschreibung und Studien aus einem Guss

H. Küster

'Universitäts- Kinderklinik, Neonatologie, Göttingen, Deutschland

Einleitung. Softwarelösungen im Krankenhaus sind oft wenig benutzerfreundlich: unübersichtliche und umständliche Eingabeformulare, mehrfache Erfassung derselben Daten, geringe Überprüfung der Datenqualität, kaum Auswertemöglichkeiten und mangelnde Integration eigener Wünsche kennzeichnen viele der vorhandenen Lösungen. Ein Qualitätsvergleich mit anderen Krankenhäusern ist mit keinem der in Deutschland vertriebenen Systeme möglich.

Fragestellung. Lässt sich eine Software mit folgenden Charakteristika entwickeln: 1) einfache Erfassung der gesetzlich vorgeschrieben Qualitätssicherung, 2) Integration zusätzlicher Datenfelder, die nur nach Bedarf eingeblendet werden 3) Generierung von Arztbriefen aus den eingegeben Daten, 4) Anpassung an lokale Bedürfnisse, 5) Vernetzung mit dem Krankenhausinformationssystem, 6) krankenhausübergreifender Qualitätsvergleich, 7) moderne Sicherheitstechnologie.

Methoden. Basierend auf Neolink20oo - dem seit 1994 kontinuierlich weiterentwickelten Datenerfassungssystem - wurde ein vollständig neues Softwarepaket entwickelt: NIQ ${ }^{\oplus}$. Dieses wurde so ausgelegt, dass sich Module für verschiedene Fachrichtungen, Patientengruppen, Forschungsprojekte oder Qualitätssicherungssysteme frei kombinieren und auswerten lassen.

Ergebnisse. Eine anwenderfreundliche Oberfläche erleichtert Eingabe und Verwertung der Daten aus der Zeit vor, während und nach der stationären Behandlung. Die klare Eingabestruktur, Sichtbarkeit des einzelnen Datenfeldes nur wenn verpflichtend auszufüllen, ausführliche Definitionen jedes Datenfeldes und umfangreiche Fehlerprüfungen mit Farbmarkierung für Fehleingaben helfen, eine hohe Datenqualität zu erreichen. NIQ ${ }^{\circledR}$ ist mehrsprachig und unterstützt den deutschen sowie mehrere internationale Standards der Qualitätssicherung (AQUA, NeoKISS, GNN, VON, ENN). Darüber hinaus können zusätzliche Datenfelder ausgewählt werden. Im Krankenhaus bereits vorhandene Daten können via Schnittstellen übernommen werden. Eine erhebliche Arbeitserleichterung entsteht durch automatische Übernahme der Daten in lokal adaptierbare Arztbriefe und Formulare. Alle Daten können jederzeit vor Ort krankenhausübergreifend analysiert werden. Damit erlaubt $\mathrm{NIQ}^{\circ}$ erstmals einen Online-Vergleich mit der in anderen Kliniken erreichten Qualität. Die Ergebnistabellen bzw. -diagramme sind adaptierbar und exportierbar. Abfragen können gespeichert werden und stehen dann jederzeit für eine schnelle erneute Analyse der Daten zur Verfügung. Eine Zertifizierung dokumentiert den hohen Standard des Datenschutzes und der eingesetzten Technologie.

Schlussfolgerung. NIQ ${ }^{\circledR}$ führt bei hoher Datenqualität zu Arbeitsreduktion und kongruenten Daten durch die einmalige Eingabe mit multipler Ausgabe der Daten sowie Schnittstellen und Adaptierbarkeit. Mit NIQ ${ }^{\circ}$ soll ein europaweiter Datenverbund geschaffen und wichtige Daten von der Schwangerschaft bis zur Einschulung anonym gesammelt und analysiert werden. Mehr Informationen sowie eine kostenlose Testversion unter www.NIQ.eu

Förderung durch EU, BMBF und das Land Mecklenburg-Vorpommern.

\section{Neurologie 1}

\section{P011}

Der Einfluss von antibiotikabeschichteten Ventrikelkathetern auf die Infektionsrate bei Frühgeborenen mit posthämorrhagischem Hydrozephalus - eine retrospektive, deskriptive Analyse

F. Lindinger', C. Czaba', K. Klebermass-Schrehof', M. Olischar', E. Obwegeser $^{1}$, V. Giordano', A. Reinprecht ${ }^{2}$, A. Berger ${ }^{1}$, M. Weninger ${ }^{1}$

'Allgemeines Krankenhaus Wien/Universitätsklinik für Kinder- und Jugendheilkunde, Abteilung für Neonatologie, Wien, Österreich, ${ }^{2}$ Allgemeines Krankenhaus Wien/Universitätsklinik für Neurochirurgie, Abteilung für Neurochirurgie, Wien, Österreich

Einleitung. Die externe Ventrikeldrainage (EVD) ist eine etablierte Therapieoption des posthämorrhagischen Hydrocephalus (PHH) bei Frühgeborenen und wird an der Universitätsklinik für Kinder- und Jugendheilkunde der Medizinischen Universität Wien seit 1982 verwendet. Hauptkomplikationsrisiko ist das Auftreten einer sekundären Ventrikulitis. Um das Infektionsrisiko dafür zu senken, wurden seit dem Jahr 2007 antibiotikabeschichtete Ventrikelkatheter eingesetzt. Das Ziel unserer retrospektiven Studie ist zu erheben, ob der Einsatz von antibiotikabeschichteten Ventrikelkathetern zu einer Reduktion der Infektionsrate geführt hat.

Methodik. Es wurden jene Frühgeborene mit PHH unter der 32. Schwangerschaftswoche inkludiert, bei denen zwischen 2001 und 2011eine EVD angelegt wurde. Folgende Daten wurden erhoben: Geburtsgewicht, Gestationsalter, Grad der intraventrikulären Hirnblutung (IVH), Anzahl der Katheterrevisionen, Anzahl Shuntimplantationen und der Ventrikulitiden. Eine Ventrikulitis wurde nur bei positivem Liquorbefund diagnostiziert. Die statistische Analyse erfolgte mittels T-Test und Chi2-Test.

Ergebnisse. 26 Patienten erhielten die EVD vor 2007 (ohne Beschichtung, Gruppe A), 21 Patienten erhielten die EVD nach 2007 (mit Beschichtung, Gruppe B). Gruppe A: mittleres Geburtsgewicht: $1036 \mathrm{~g}$ $( \pm 474,209)$, mittleres Gestationsalter: 26,7 SSW $( \pm 1,80), 2$ Patienten hatten eine IVH II ${ }^{\circ}(7,7 \%), 16$ Patienten eine IVH III ${ }^{\circ}(61,5 \%)$ und 8 Patienten eine IVH IV ${ }^{\circ}$ (30,8\%), die Mortalität betrug 57,7\%. EVD Revisionen 11 (42\%). Shuntimplantation 8 (31\%) Gruppe B: mittleres Geburtsgewicht: $1115 \mathrm{~g}( \pm 494,902)$, mittleres Gestationsalter: 27,3 ( $\pm 2,32), 1$ Patient hatte eine $\mathrm{IVH} \mathrm{II}^{\circ}(4,8 \%)$, 10 Patienten eine IVH III ${ }^{\circ}(47,6 \%)$ und 10 Patienten eine IVH IV ${ }^{\circ}(47,6 \%)$, die Mortalität betrug $33,3 \%$. EVD Revisionen 7 (33\%). Shuntimplantation 7 (33\%). Die Unterschiede waren statistisch nicht signifikant (Tab. 1).

Schlussfolgerung. Der Einsatz von antibiotikabeschichteten Ventrikelkathetern zur Behandlung des posthämorrhagischen Hydrocephalus hat zu einer deutlichen aber statistisch nicht signifikanten Verminderung des Risikos für das Auftreten einer sekundären Ventrikulitis geführt.

\begin{tabular}{|lllll|}
\hline P011 - Tab. 1 & Häufigkeit Ventrikulitis & & \\
\hline & $\begin{array}{l}\text { Keine } \\
\text { Infektion }\end{array}$ & Infektion & Gesamt & Signifikanz \\
\hline EVD nicht beschichtet & 21 & $5(19,2 \%)$ & 26 & \\
\hline EVD beschichtet & 20 & $1(4,8 \%)$ & 21 & \\
\hline Gesamt & 41 & 6 & 47 & $\mathrm{p}=0,150$ \\
\hline
\end{tabular}

\section{P012}

Joubert-Syndrom mit Molar-tooth-sign - Wie kommt ein Zahn ins Gehirn?

S. Dold?

'Universitäts--Kinderklinik, Ulm, Deutschland

Hintergrund. Das Molar-tooth-sign mit Vermishypo-, oder -aplasie, kräftigen elongierten oberen Kleinhirnstielen, Hypoplasie der mittleren Kleinhirnstiele und konsekutiv backenzahnartiger Konfiguration der Pedunculi cerebelli superiores um den 4 . Ventrikel ist wegweisend 
für die Diagnose des Joubert-Syndroms. Das Joubert-Syndrom gehört zur Gruppe der Ziliopathien und tritt mit einer Prävalenz von 1:80.ooo1:10o.ooo auf. Die Vererbung erfolgt heterogen, autosomal-rezessiv, sowie X-chromosomal. Derzeit sind 22 Gene identifiziert. Abhängig vom Ausmaß der betroffenen Gene ergibt sich das Ausmaß der Erkrankung mit Befall verschiedener Organsysteme und sehr variablen Phenotypen. Der meist temperamentvolle, teilweise aggressive und autistische Charakter kombiniert mit meist schwerer Gangstörung, stellt die Eltern der Betroffenen vor eine immense Herausforderung. Im Säuglingsalter können die Trinkschwäche und die Atemstörungen lebenslimitierend sein. Dies bessert sich mit zunehmendem Lebensalter, zurück bleibt in einigen Fällen eine Schlafapnoe. Im weiteren Verlauf können die chronische Nierenerkrankung und das Ausmaß der Hirnfehlbildungen die Lebenserwartung in einigen Fällen deutlich reduzieren.

Fallbericht. Bei einem reifen Neugeborenes mit pränatal bekannter Vermishypoplasie mit Verdacht auf Dandy-Walker-Malformation, Schizencephalie, Oligohydramion mit polizystischen Nieren und Ovarialzyste zeigte sich postnatal eine erschwerte Adaptation. Bei klinisch auffälligem Äußeren mit tief sitzenden Ohren, Hypotelorismus, hohem Gaumen, großer Fontanelle und dehiszenten Schädelnähten zeigte sich neurologisch ein erhöhter Muskeltonus, ein fehlender Saugreflex, sowie eine arrhythmische, periodische Atmung mit Apnoe- und tachypnoeischen Phasen. In der durchgeführten MRT-Untersuchung zeigten sich zusätzlich zum Molar-tooth-sign eine Thalamusfusion, eine Balkendysplasie, eine Schizencephalie, eine Polymikrogyrie und beidseits fehlende Inselregionen. Klinisch fiel im weiteren Verlauf eine Trinkschwäche, der Verdacht auf eine Laryngomalazie bei auffälligem Stridor, eine schwer einstellbare Hypertonie, eine Hüftdysplasie re., sowie eine $\mathrm{Ne}$ phronophtisis. Es erfolgte die Anleitung der Eltern in das Sondieren und Magensonde anlegen. Der Blutdruck wurde bestmöglich medikamentös eingestellt und die Eltern in einen Heimüberwachungsmonitor und die Reanimationsmaßnahmen eingewiesen. Die Hüftdysplasie wurde mit einer Spreizhose versorgt. Während des stationären Aufenthalts zeigten sich die Retentionsparameter im Normbereich.

Schlussfolgerung. Das hier vorgestellte Kind zeigte in der Gesamtzahl und im Ausmaß der betroffenen Organe einen relativ stark betroffenen Phänotyp. Da es sich bei der Erkrankung um eine bisher nicht gut untersuchte Erkrankung handelt, erscheint es wichtig die Erfahrungen in der Therapie der einzelnen betroffenen Kinder gemeinsam mit anderen Betreuenden zu diskutieren.

\section{P013}

Intrazerebrale Blutung (ICB) bei Reifgeborenen und Frühgeborenen $>32$ SSW: klinische Präsentation, diagnostischer Workup, Therapie und mögliche Ursachen

\section{B. Gehrlein', R. Schlößer², D. Fischer ${ }^{3}$}

'Klinik für Kinder und Jugendmedizin, Klinikum der Goethe Universität Frankfurt, Schwerpunkt Neonatologie, Frankfurt, Deutschland, ${ }^{2}$ Klinikum der J.W.Goethe-Universität, Zentrum der Kinderheilkunde, Abteilung für Neonatologie, Frankfurt am Main, Deutschland, ${ }^{3}$ Klinikum der Johann Wolfgang Goethe-Universität, Zentrum der Kinderheilkunde, Klinik I, Neonatologie, Frankfurt, Deutschland

Hintergrund. Bekannte intrakranielle Blutungen bei Reifgeborenen sind Subduralblutungen (SDB) und intraparenchymale Blutungen(IPB). Bei Frühgeborenen dominieren subarachnoidale Blutungen(SAB), intraventrikuläre Blutungen(IVB)und zerebelläre Blutungen. Subarachnoidalblutungen oder ausschließlich parenchymal ausgedehnte Blutungen außerhalb der Ventrikel definierten wir bei Frühgeborenen als atypische Blutungen.

Material und Methoden. Zwischen Januar 1999 und Dezember 2013 wurden im Zentrum für Kinder-und Jugendmedizin der Goethe Universität Frankfurt 27 Neugeborene retrospektiv bezüglich prä- und perinataler Daten, Alter, Symptome, diagnostischem und therapeutischem Procedere und Outcome analysiert. Der Fokus wurde auf das hämosta- seologische Labor, inklusive des erweiterten Screening thrombophiler Risikofaktoren gesetzt.

Ergebnisse. 15 reife und 12 Frühgeborene wurden untersucht. Der Geburtsmodus war Sectio caeseraea $(n=14)$, Vakuumextraktion $(n=1)$, Forceps $(n=3)$ und via naturalis $(n=9)$. Als Erstsymptomatik wurden Temperaturstörungen $(n=9)$, Blässe $(n=4)$, muskuläre Hypotonie bzw. Apathie $(n=8)$, arterielle Hypotonie $(n=3)$ und/oder zerebrale Krampfanfälle $(n=14)$ beobachtet. Sieben Kinder waren primär asphyktisch oder hatten einen Amnioninfekt. In der Bildgebung zeigte sich bei 12 Neugeborenen ein subdurales Hämatom, bei 4 eine Subarachnoidalblutung, bei 14 eine parenchymatöse und intraventrikuläre Stauungsblutung, bei 1 eine supraventrikuläre Blutung. 4 hatten eine Hypoxie, 3 eine intraparenchymal zerebelläre Blutung, 2 intraparenchymal petechiale Einblutungen, 7 Kinder eine intraparenchymal raumfordernde Blutung, 2 andere intraparenchymale Blutungen. Vier hatten eine supratentorielle Mittellinienverschiebung. Sieben Kinder hatten eine Sinusvenenthrombose. Im hämostaseologischen Workup wurde keine Hämophile, dafür 6 thrombophile Hämostasestörungen nachgewiesen [Protein-S-Mangel $(\mathrm{n}=1)$, Faktor-V-Leiden-Mutation $(\mathrm{n}=3)$, Faktor-XIIMangel ( $n=1)$, Mutation im MTHFR-Gen $(n=2)]$.

Diskussion. In dieser retrospektiven Untersuchung konnten neben den bekannten Risikofaktoren für Hirnblutungen wie Geburtsmodus, Asphyxie oder schweres AIS auch thrombophile Hämostasestörungen als mögliche Ursachen für neonatale ICB gefunden werden. Darüber hinaus trat in einigen Fällen als erstes (und auch alleiniges) Symptom Temperaturinstabilität beim ansonsten unauffälligen reifen Neugeborenen auf.

Schlussfolgerung. Temperaturinstabilität beim reifen Neugeborenen kann hinweisend für eine neonatale ICB sein. Daher sollte in solchen Fällen eine ausführliche Abklärung einschließlich zerebraler Sonographie erfolgen. Bei diagnostizierter ICB muss in der differentialdiagnostischen Abklärung thrombophile Hämostasestörungen berücksichtigt werden.

\section{P014 \\ Risikofaktoren für Hirnblutungen bei Frühgeborenen $<1500 \mathrm{~g}$ Geburtsgewicht}

\section{Mende', M. Schmid', H. Hummler', E. Herting ${ }^{4}$, C. Härtel', W. Göpel'}

'Universitätsklinikum Schleswig-Holstein, Campus Lübeck, Klinik für Kinderund Jugendmedizin, Lübeck, Deutschland, ${ }^{2}$ Universitätsklinik für Kinder- und Jugendmedizin, Sektion Neonatologie und Pädiatrische Intensivmedizin, Ulm, Deutschland, ${ }^{3}$ Klinik für Kinder- und Jugendmedizin, Universitätsklinikum Ulm, Sektion Neonatologie und Pädiatrische Intensivmedizin, Ulm, Deutschland, ${ }^{4}$ Universitätsklinikum Schleswig-Holstein Campus Lübeck, Direktor der Klinik für Kinder- und Jugendmedizin, Lübeck, Deutschland

Fragestellung. In einem kürzlich publizierten Interventionsprogramm zur Prophylaxe von Hirnblutungen bei Frühgeborenen wurden eindrucksvolle Reduktionsraten für alle Hirnblutungsgrade berichtet [1]. Als wesentliche Behandlungsmodifikationen, die zu einer niedrigeren Hirnblutungsrate geführt hatten, wurden genannt: zurückhaltende Indikation zur Entbindung, mehr Sektioentbindungen, Ausstreichen der Nabelschnur und Zeitvorgaben zur Therapie der postnatalen arteriellen Hypotension, zusätzliche Betamethasongabe vor der Entbindung, Zeitvorgaben für die Behandlung des Atemnotsyndroms.

Patienten und Methoden. Analog zu den in der Ulmer Studie genannten Behandlungsmodifikationen untersuchten wir in der GNN-Kohorte (2009-2013) den Einfluss des Gestationsalters (pro Woche), des Entbindungsmodus (Sektio- vs. Spontanentbindung), der postnatalen arteriellen Hypotension (mittlerer arterieller Druck in den ersten $24 \mathrm{~h}$ $<$ Gestationsalter), der antenatalen Steroidtherapie (ja vs. nein) und der Schwere des Atemnotsyndroms (maximaler O2-Bedarf am 1. Lebenstag) auf den Endpunkt intraventrikuläre Hämorrhagie in einer Multivarianzanalyse. Zusätzlich wurden weitere Faktoren wie Geschlecht, Mehrlingsgravidität und Geburtsursachen analysiert. 
Ergebnisse. Von 4766 Frühgeborenen lagen Informationen zu allen Parametern vor. 733 (15\%) hatten eine Hirnblutung. Alle in der Ulmer Studie berücksichtigten Faktoren hatten auch in der auf GNN-Daten basierenden Multivarianzanalyse einen Einfluss auf Hirnblutungen. Protektiv waren: höheres Gestationsalter (OR: o,78; 95\% CI 0,75-0,81 pro Woche; $\mathrm{p}<0,001$ ), antenatale Steroidtherapie (OR: 0,66; 95\% CI 050-0,87; $\mathrm{p}=0,003$ ), Sektioentbindung (OR: 0,7; 95\% CI 0,56-0,91; $\mathrm{p}=0,006)$. Als Risikofaktoren wurden identifiziert: $\mathrm{MAD}<$ Gestationsalter (OR: 1,2; 95\% CI 1,02-1,44; p=0,03); erhöhter Sauerstoffbedarf (OR: 1,21; $95 \%$ CI 1,14-1,28 pro $10 \%$ O2; $<<0,001)$. Zusätzliche signifikante Risikofaktoren waren Mehrlingsgeburt (OR: 1,24; 95\% CI 1,02-1,49; $\mathrm{p}=0,03$ ), 5-Minuten Apgar $<7$ (OR: 1,54; 95\% CI 1,25-1,89; $\mathrm{p} \leq 0,001$ ) und Entbindung aufgrund einer Plazentalösung (OR: 1,43; 95\% CI 1,04-1,95; $\mathrm{p}=0,03)$.

Diskussion. Die Zielpunkte der von den Ulmer Kollegen berichteten Behandlungsmodifikationen waren auch im GNN-Datensatz mit einem erhöhten bzw. erniedrigten IVH-Risiko assoziiert.

Schlussfolgerung. Zurzeit wird im Rahmen des GNN eine Cluster-randomisierte Studie mit dem Ziel der Reduktion von Hirnblutungen vorbereitet. Wir hoffen, mit dieser Studie die Hirnblutungsrate nachhaltig zu senken. Ein weiteres Ziel dieser Studie ist die Identifizierung einzelner Maßnahmen, die die Hirnblutungsrate signifikant reduzieren.

1. Dtsch Arztebl Int 2013; 110:489-496

\section{P015}

\section{Array-basierte genomweite Genotypisierung bei Feten mit zerebra-} len Fehlbildungen

H. Reutter', S. Krutzke', M. Schumann', M. Draaken², A. Hilger', U. Gem-

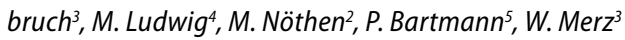

'Universitätsklinik Zentrum f. Kinderheilkunde, Abteilung für Neonatologie, Bonn, Deutschland, ${ }^{2}$ Universitätsklinik Bonn, Institut für Humangenetik, Bonn, Deutschland, ${ }^{3}$ Universitäts-Frauenklinik Bonn, Klinik für Geburtshilfe und Pränataldiagnostik, Bonn, Deutschland, ${ }^{4}$ Universitätsklinik Bonn, Abteilung für Klinische Chemie und Klinische Pharmakologie, Bonn, Deutschland, ${ }^{5}$ Universität Bonn, Zentrum für Kinderheilkunde, Abteilung für Neonatologie, Bonn, Deutschland

Hintergrund. Die genetische Diagnostik angeborener zerebraler Fehlbildungen beschränkt sich während der Schwangerschaft zumeist auf die Durchführung konventioneller Chromosomenanalysen. Die Erfahrungen in der Anwendung neuer Array-basierter Methoden zur Identifizierung genetischer Ursachen sind mangels fehlender systematischer Untersuchungen rudimentär. Hingegen kann der überwiegende Anteil zerebraler Fehlbildungen mittels bildgebender Verfahren pränatal nach anatomischen Gesichtspunkten klassifiziert werden, was dazu geführt hat, dass die meisten der betroffenen Feten aufgrund der assoziierten Entwicklungsstörungen nicht mehr ausgetragen werden.

Fragestellung. Um mehr Erfahrung über den Nutzen von Arrray-basierten Methoden zur Ermittlung von möglichen genetischen Aberrationen bei angeborenen zerebralen Fehlbildungen zu sammeln, wurde ein Kollektiv bestehend aus 68 abortierten Feten mit zerebralen Fehlbildungen untersucht.

Material und Methoden. Alle Feten wurden durch die Abteilung für Geburtshilfe und Pränatalmedizin am Universitätsklinikum Bonn aus über 300 abortierten Feten mit mehrheitlich zerebralen ZNS-Fehlbildungen akquiriert, von denen DNA-Aliquots im Zuge des Fetozids zwischen 2006 und 2012 gewonnen wurden. Für die molekulare Karyotypisierung wurde der Illumina HumanOmniExpress-12v1_H benutzt, der 730.525 Marker enthält. Zur Auswertung der SNP (Single Nucleotide Polymorphism)-Floureszenzintensität benutzten wir QuantiSNP mit dem Objective-Bayes Hidden-Markov Modell, um potentiell ursächliche CNVs zu identifizieren.

Ergebnisse. Die chromosomale Microarray Untersuchung zeigte (Micro-)Abberrationen in 15 Feten mit unauffälligem Karyotyp und unauf- fälliger FISH-Analyse und bestätigte 8 schon im Karyogramm identifizierte strukturelle Aberrationen.

Schlussfolgerung. Unsere vorläufigen Ergebnisse deuten darauf hin, dass Array-basierte Methoden in der pränatalen Diagnostik sinnvoll in der ursächlichen Abklärung bei angeborenen zerebralen ZNS-Fehlbildungen sind.

\section{P016}

Atypische ZNS-Echogenitätserhöhung + klinischer Infektverdacht: Zerebritis durch Citrobacter koseri

\section{Schoberer', K. Heimann', S. Trepels-Kottek', E. Rose', T. Orlikowsky'}

'Universitätsklinikum Aachen, Klinik für Kinder- und Jugendmedizin, Sektion Neonatologie, Aachen, Deutschland

Einleitung. Citrobacter koseri (vormals divs.) ist ein seltener, opportunistischer Erreger der bakteriellen Neugeborenensepsis. Das gramnegative Stäbchen aus der Familie der Enterobacteriacae besitzt in den ersten Lebenswochen einen hohen Tropismus für das ZNS. Infektionen betreffen überwiegend hypotrophe Neugeborene oder Frühgeborene. Die Infektion kann als reine Meningitis ablaufen, typisch ist aber eine Zerebritis mit Abszessbildung, welche in rund 75\% der Fälle beobachtet wird. Die Mortalität liegt bei rund 30\%, bei ca. 80\% der Überlebenden kommt es zu einer Defektheilung mit neurologischen Defiziten.

Kasuistik. Ein AGA-Frühgeborenes mit einem Reifealter 31+5 Schwangerschaftswochen und einem Geburtsgewicht von $1490 \mathrm{~g}$ fällt am 8. Lebenstag klinisch mit Apnoen und Bradykardien auf. Die systemischen laborchemischen Entzündungsparameter (C-reaktives Protein und IL6) sind nicht erhöht. Sonographisch fällt eine Echogenitätserhöhung im linksseitigen parieto-occipitalen Hirnparenchym auf. Im Liquor besteht eine deutliche Erhöhung des IL-6 (1300 ng/l), eine Pleozytose ( $784 \mathrm{M} / \mathrm{l}$, $54 \%$ polymorphkernige, $46 \%$ mononukleäre Zellen), eine Proteinerhöhung auf 1,6 g/l und eine Glucose-Verminderung auf $38 \mathrm{mg} / \mathrm{dl}$. Es wird eine i.v.-antibiotische Therapie mit Cefotaxim (200 mg/kgKG/Tag) und Tobramycin begonnen und über insgesamt 4 Wochen fortgeführt. Der Erregernachweis wird nur aus der Blutkultur geführt, der Liquor bleibt kulturell steril, auch die universelle bakterielle PCR liefert kein Produkt. Serielle MR-Tomographien zeigen im Verlauf porenzephale Läsionen links parieto-occipital, bi-temporal und bi-frontal. Kompliziert wird der Verlauf durch einen sekundären Hirnabszess rechts frontal 4 Wochen nach Ende der antibiotischen Primärtherapie, welcher neurochirurgisch drainiert wird und einen weiteren i.v.-antibiotischen Therapiezyklus über 6 Wochen mit Meropenem erfordert. Die neurologische Entwicklung des Kindes ist bisher (korrigiert 6. Lebensmonat) unauffällig. Diskussion und Schlussfolgerung. In unserem Fall verlief die Neugeborenensepsis mit Citrobacter koseri klinisch subakut und unspezifisch. Wegweisend bei der Diagnosefindung war die Schädelsonographie in Verbindung mit der kulturellen Erregerdifferenzierung. In deutlichem Gegensatz zur milden Klinik steht die erhebliche Gewebsdestruktion durch den Erreger. Hirnabszesse und Defektheilungen sind eine typische Komplikation der Neugeborenen-Infektion mit Citrobacter koseri. Möglicherweise ist Meropenem aufgrund seiner besseren Gewebegängigkeit dem Cefotaxim therapeutisch überlegen, erfolgreiche Behandlungen mit Ciprofloxazin wurden sporadisch publiziert. 


\section{P017}

\section{Posthämorrhagischer Hydrocephalus nach intraventrikulärer} Blutung in der Früh- und Neugeborenenperiode - Ergebnisse nach neuroendoskopischer Lavage

\section{Schulz', C. Bührer', A. Pohl-Schickinger', H. Haberl', U. Thomale'}

'Charité - Universitätsmedizin Berlin, Arbeitsbereich Pädiatrische Neurochirurgie, Berlin, Deutschland, ${ }^{2}$ Charité - Universitätsmedizin Berlin, Klinik für Neonatologie, Berlin, Deutschland

Hintergrund. Intraventrikuläre Blutungen in der Früh- und Neugeborenenperiode können zu einem posthämorrhagischen Hydrocephalus mit sekundärer neurologischer Beeinträchtigung führen.

Methoden. Im Zeitraum von 2010 bis 2012 wurden bei 19 neonatologischen Kindern mit posthämorrhagischen Hydrocephalus eine neuroendoskopischen Operation mit Lavage des Ventrikelsystems und Entfernung des intraventrikulären Hämatoms durchgeführt. Diese Kinder wurden mit einer historischen Kohorte aus den Jahren 2008 bis 2010 verglichen, die mit einem konventionellen Regime mit intermittierender Liquorentlastung über wiederholte Lumbalpunktionen, Punktion eines implantierten Reservoirsystems oder Anlage einer externen Ventrikeldrainage behandelt worden waren.

Ergebnisse. Beide Patientengruppen zeigten keine Unterschiede hinsichtlich Gestationsalter und Geburtsgewicht. In der neuroendoskopischen Gruppe sind keine direkten Komplikationen des operativen Eingriffs beobachtet worden. Nach Lavage des Ventrikelsystems benötigten 11 von 19 Kindern (58\%) die spätere Implantation eines VP-Shunts, in der konventionellen Gruppe betrug die Shuntrate $100 \%(\mathrm{p}<0,05)$. Die Kinder der endoskopischen Gruppe benötigen zudem weniger Operationen insgesamt (median 2 vs. 3,5 pro Patient; $p=0,08$, hatten signifikant weniger Infektionen ( 1 vs. 5 Patienten; $\mathrm{p}<0,05$ ) und weniger häufig die Ausbildung eines multilokulären Hydrocephalus (o vs. 4 Patienten; $\mathrm{p}<0.01$ ). Schlussfolgerung. Die vorgestellte retrospektive Beobachtung demonstriert die Durchführbarkeit und Sicherheit einer neuroendoskopischen Behandlung bei posthämorrhagischem Hydrocephalus in der Neugeborenenperiode; ihre verbesserten Ergebnisse im Vergleich zu einer historischen Kontrollgruppe müssen prospektiv bestätigt werden.

\section{P018}

Vermessung des Corpus callosum mittels 3D-Ultraschall bei Frühgeborenen $<32$ SSW und Korrelation mit dem entwicklungsneurologischen Outcome

\section{K. Klebermass-Schrehof', S. Moerth', R. Fuiko', S. Brandstetter', A. Berger', N. Haiden ${ }^{1}$ \\ 'Universitätsklinik für Kinder- und Jugendheilkunde, Abteilung für Neonatolo- gie, pädiatrische Intensivmedizin und Neuropädiatrie, Wien, Österreich}

Hintergrund. Eine der häufigsten zerebralen Auffälligkeiten bei Frühgeborenen ist eine „Verdünnung“ des Corpus callosum (CC). Dies wird vor allem damit erklärt, dass beim Frühgeborenen immature Oligodendrozyten eine hohe Vulnerabilität auf hypoxisch-ischämische Läsionen aufweisen. Das CC, das beide Hemisphären assoziativ verbindet, ist eine der letzten Strukturen die komplett myelinisieren. Bisher bekannte Daten zeigen einen Zusammenhang zwischen Größe des CC und dem späteren motorischen Outcome. Die Entwicklung von $3 \mathrm{D}$-Ultraschall (3DUS) erweitert die bisherige Methodik des Ultraschalls.

Fragestellung. Im Rahmen dieser Studie haben wir untersucht, ob Veränderungen in Größe, Länge, Umfang und Fläche des CC mit einem auffälligen entwicklungsneurologischen Outcome einhergehen.

Patienten und Methodik. 43 Frühgeborene mit einem Gestationsalter $<32$ Schwangerschaftswochen (SSW) wurden in diese prospektiv-longitudinale Studie inkludiert. Mittels transfontanellärem ${ }_{3} \mathrm{D}$-Ultraschall fanden zu 9 Zeitpunkten (24-26 SSW, 27-28 SSW, 29-30 SSW, 31-32 SSW, 33-34 SSW, 35-36 SSW, 37-43 SSW, 44-50 SSW, 51-55 SSW) Messungen statt und es wurden Breite des Rostrums, des Corpus, des Spleniums und die Länge, der Umfang und die Fläche des Corpus callo- sum vermessen. Das entwicklungsneurologische Outcome im Alter von 5,5 Jahren wurde mittels Kaufmann's Assessment Battery for Children (K-ABC) und Beery-Buktenica Developmental Test of Visual-Motor Integration (VMI) untersucht.

Ergebnisse. Mittels ${ }_{3} D U S$ konnten Referenzwerte für Größe, Länge, Umfang und Fläche des CC zu den verschiedenen Messzeitpunkten erhoben werden. Die Messung zum korrigierten Geburtstermin zeigte eine signifikante Korrelation zwischen der Dicke des Corpus des CC und der visuellen motorischen Integration des VMI $(p=0,04)$ und eine signifikante Korrelation zwischen der Fläche des CC und der Skala des ganzheitlichen Denkens $(\mathrm{p}=\mathrm{o}, \mathrm{o1})$ und der Skala der intellektuellen Fertigkeiten $(\mathrm{p}=\mathrm{o}, \mathrm{O} 4)$ des K-ABC. Einen Trend zeigte die Korrelation zwischen der Fläche des CC und der Fertigkeitenskala des K-ABC $(\mathrm{p}=0,08)$ und der visuellen Perzeption erfasst mittels VMI $(p=0,08)$.

Schlussfolgerung. Die Größe des CC kann mittels ${ }_{3} \mathrm{D}$ US beim Frühgeborenen vermessen werden. Speziell die Fläche des Corpus callosum am korrigierten Geburtstermin korreliert mit dem entwicklungsneurologischen Outcome im Alter von 5,5 Jahren. Hierbei zeigt sich eine Korrelation zum motorischen sowie auch zum kognitiven Outcome.

\section{P019}

Erweiterung der äußeren Liquorräume und Hirngröße bei sehr unreifen Frühgeborenen: Abhängigkeit von typischen Risikofaktoren für eine „white matter disease“ (WMD) und mentale Entwicklung mit 2 Jahren

\section{J. Mütze', P. Jahn², A. Meyer ${ }^{3}$, P. Groneck ${ }^{4}$}

${ }^{1}$ Klinikum Leverkusen, Klinik für Kinder und Jugendliche, Leverkusen, Deutschland, ${ }^{2}$ Klinikum Leverkusen gGmbH, Kinderklinik, Leverkusen, Deutschland, ${ }^{3}$ Klinikum Leverkusen, Radiologische Abteilung, Leverkusen, Deutschland, ${ }^{4}$ Klinikum Leverkusen, Kinderklinik, Leverkusen, Deutschland

Hintergrund. Ein MRT zum Entlassungszeitpunkt zeigt bei sehr unreifen Frühgeborenen (FG) häufig eine Erweiterung der äußeren Liquorräume (EÄL). Es wird vermutet, dass die Ursache dieses Befundes eine Gewebsverminderung aufgrund einer diffusen Entwicklungsstörung der weißen Substanz (WMD) ist.

Methode. FG<1500 g Geburtsgewicht, die über einen Zeitraum von 5 Jahren behandelt wurden und zum Entlassungszeitpunkt ein MRT erhielten, wurden eingeteilt in Gruppen mit und ohne radiologischer Diagnose einer EÄL. Die jeweiligen beiden Gruppen wurden verglichen hinsichtlich der Hirngröße (Messungen nach Tich et al., AJNR 30;125:2009), sowie des Vorkommens klinischer sowie typischer WMDMorbiditätsparameter. Ein Vergleich der kognitiven Funktionen erfolgte mithilfe der Bailey Scales of Infant Development II (BSID).

Patienten. Von 2006 bis 2010 wurden 256 FG behandelt, 220 überlebten, 134 hatten ein auswertbares MRT, 102 FG hatten ein MRT und BSID. Ergebnisse. 52/134 FG (39\%) wiesen eine EÄL auf, die vorwiegend rostral gelegen war. FG mit EÄL waren signifikant unreifer (27,0 vs. 28,2 SSW; $\mathrm{p}<0,01$ ), hatten einen niedrigeren 5-min-Apgar, erhielten mehr Surfactantdosen, benötigten länger eine CPAP-Therapie und erhielten häufiger eine postnatale Steroidbehandlung als FG ohne EÄL. Nach Durchführung einer logistischen Regression war bei Betrachtung der Faktoren Unreife und Steroidtherapie keine Signifikanz mehr nachweisbar. Es zeigte sich kein Unterschied beider Gruppen hinsichtlich folgender WMD-Risikofaktoren: IL- $6 \mathrm{~d}_{1}, \mathrm{~d}_{3}, \max ., \mathrm{CRP} \mathrm{d}_{1}, \mathrm{~d}_{3}, \max ., \mathrm{pCO}_{2}$ min., $\mathrm{PROM}>24 \mathrm{~h}$, histologische Chorioamnionitis, Mehrlinge, niedriger Na-pH, Sepsis, PDA, NEC/FIP mit OP. Während die äußeren Liquorräume der EÄL-Patienten signifikant größer waren als der Kontrollen (rostraler Liquorraum $6,78$ vs. 5,10 mm; $\mathrm{p}<0,001)$ zeigte sich in den übrigen Hirngrößenmesswerten keine Unterschiede. Es zeigten sich keine Unterschiede der kognitiven Funktionen (EÄL MDI 94,o, Kontrollen 89,3; p=0,286).

Schlussfolgerung. Die Erweiterung der äußeren Liquorräume ist häufiger bei zunehmender Unreife und bei unreife-assoziierten Morbiditätsfaktoren. Es fand sich kein Zusammenhang mit typischen WMD-Risikofaktoren. Die Hirngröße sowie die mentale Funktion mit 2 Jahren 
zeigten keinen Unterschied zwischen den Gruppen mit und ohne EÄL. Die EÄL ist somit offensichtlich kein Marker für eine Hirnatrophie bei WMD. Wir spekulieren, dass die EÄL Folge eines lagerungsbedingten rostralen Schädelwachstums ist.

\section{P020 \\ XLAG - eine seltene Differentialdiagnose bei neonatalen Krampf- anfällen}

\section{S. Bode', J. Kirschner', U. Matysiak-Scholze', E. Lausch'3, S. Meyer', K. Wald-} ecker ${ }^{5}$, H. Fuchs'

'Universitäts - Kinderklinik, Pädiatrische Neonatologie und Intensivmedizin, Freiburg, Deutschland, ${ }^{2}$ Universitäts - Kinderklinik, Freiburg, Deutschland, ${ }^{3}$ Universitäts - Kinderklinik Freiburg, Molekulargenetisches Labor der Sektion Pädiatrische Genetik, Freiburg, Deutschland, ${ }^{4}$ Universitätsklinikum des Saarlandes, Klinik für Allgemeine Pädiatrie und Neonatologie, Homburg, Deutschland, ${ }^{5}$ Klinikum Konstanz, Klinik für Kinder und Jugendliche, Konstanz, Deutschland

Hintergrund. Das X-chromosomale Lissencephalie mit abnormen Genitale (XLAG) Syndrom ist gekennzeichnet durch rezidivierende und therapierefraktäre Krampfanfälle. Die Kinder versterben meist im Säuglingsalter.

Fallbericht. Ein 17 Tage altes männliches Neugeborenes wurde bei therapierefraktären Krampfanfällen und MRSA-Sepsis an das Zentrum für Kinder- und Jugendmedizin Freiburg verlegt. Die Schwangerschaft war zunächst unauffällig verlaufen, vier Wochen vor Termin wurde jedoch sonographisch ein Hydrocephalus beobachtet. Retrospektiv hatten die Krampfanfälle bereits als „rhythmische Zuckungen“, die als Schluckauf interpretiert wurden, intrauterin begonnen. Es zeigte sich keine Besserung der Krampfaktivität auf Therapieversuche mit Phenobarbital, Midazolam, Phenytoin, Levetiracetam, Topiramat, Pyridoxalphosphat, Chloralhydrat, Vigabatrin und Lidocain. Eine ausführliche Stoffwechseldiagnostik erbrachte keine Auffälligkeiten. Im EEG fanden sich zahlreiche multifokale epilepsietypische Potentiale. Im MRT konnte eine Agenesie des Balkens und des Gyrus cinguli mit Pachygyrie frontal bis nahezu Agyrie parietal nachgewiesen werden. Die MRSA-Sepsis konnte erfolgreich behandelt werden. Es fielen ein kleiner Gesichtsschädel, eine große Fontanelle und ein Mikrogenitale auf. Die Chromosomenanalyse und ein CGH-Array waren unauffällig. Bei Lissencephalie und Mikropenis wurde die Verdachtsdiagnose eines XLAG-Syndroms gestellt, welche durch Nachweis einer neuen Mutation im ARX Gen (p.Phe376Ser) bestätigt wurde. Die MRSA-Sepsis konnte erfolgreich behandelt werden. Die Familie wünschte sich trotz häufigen rezidivierenden Krampfanfällen eine Versorgung des Kindes zu Hause. Letztendlich verstarb das Kind aber vor Entlassung in einer heimatnahen Klinik an einer nekrotisierenden Enterokolitis.

Diskussion und Schlussfolgerung. Das XLAG-Syndrom ist eine seltene Differentialdiagnose neonataler Krampfanfälle mit infauster Prognose. Eine pränatale Diagnostik wäre möglich.

\section{Hygiene}

\section{P021}

\section{Stellenwert laborchemischer Entzündungsparameter in der Dia-} gnostik nosokomialer Infektionen Neugeborener

\section{Grylli', M. Langgartner', A. Berger}

'Universitätsklinik für Kinder- und Jugendheilkunde Wien, Abteilung für Neonatologie, Pädiatrische Intensivmedizin und Neuropädiatrie, Wien, Österreich

Fragestellung. Ziel dieser retrospektiven Analyse war die Evaluation serieller Messungen immunologischer Infektionsparameter [C-reaktives Protein (CRP), Interleukin-8 (IL-8) und immature to total Neutrophil Ratio (I:T-Ratio)] in Hinblick auf Stellenwert in der frühen Diagnostik nosokomialer Infektionen bei Neugeborenen.
Material und Methode. Untersucht wurden die o. g. immunologischen Marker aller im Zeitraum 2009-2010 an der Neonatologie der Medizinischen Universität Wien stationär aufgenommenen Patienten, bei denen der Verdacht auf nosokomiale Infektion vorlag. Patienten wurden in 3 Gruppen eingeteilt: 1. Blutkultur(BK)-positive Sepsis; 2. keine Infektion, antibiotische Therapie nicht begonnen oder nach max. $72 \mathrm{~h}$ abgesetzt; 3. „klinische Sepsis“: klinische und laborchemische Zeichen einer nosokomialen Infektion, BK negativ, mindestens 5 Tage antibiotische Therapie. Die immunologischen Parameter wurden zum Zeitpunkt o, $24 \mathrm{~h}$ und $48 \mathrm{~h}$ nach Abnahme der Blutkultur erhoben.

Ergebnisse. In Summe lagen 415 Fälle mit Infektionsverdacht vor. 108 Fälle wurden als „BK-positive Sepsis“, 176 Fälle als „keine Infektion“ und 131 Fälle als „klinische Sepsis“klassifiziert. Für den Parameter CRP wurden folgende Sensitivitäten und Spezifitäten jeweils für Gruppe 1 vs. Gruppe $1+3$ ermittelt: $63,7 \% / 72,9 \%$ vs. $58,8 \% / 72,9 \%$ am Tag o, $85 \% / 55,3 \%$ vs. $71,3 \% / 55,3 \%$ nach $24 \mathrm{~h}$, sowie $81,9 \% / 65,5 \%$ vs. $73,2 \% / 65,5 \%$ nach $48 \mathrm{~h}$. Sensitivität/Spezifität für Interleukin 8 in Gruppe 1 vs. Gruppe $1+3$ lagen bei $69,5 / 64,6 \%$ vs. $56,9 \% / 64,6 \%$ am Tag o, bei $59,6 \% / 65,8 \%$ vs. $58,7 \% / 65,8 \%$ nach 24 h und $34,8 \% / 65,3 \%$ vs. $44,1 \% / 65,3 \%$ nach 48 h. Die Werte für I:T Ratio waren zu allen Zeitpunkten von geringem prognostischem Wert. Die berechneten LR+ und LR- für die einzelnen Parameter lagen bei $<5$ und $>0,2$ und waren somit von geringem Wert. Die LR+ für Interleukin 8 kombiniert mit CRP am Tag o lag hingegen bei 5,12 für Gruppe 1 und 3,95 für Gruppe 1+3 sowie bei 10,26 für Gruppe 1 und 8,9 für Gruppe 1+3 $24 \mathrm{~h}$ nach Abnahme der Blutkultur. Serielle CRP Messungen zum Zeitpunkt o und $24 \mathrm{~h}$ oder $48 \mathrm{~h}$ nach Abnahme der Blutkultur ergaben eine LR- von o,24 und eine Sensitivität von 85,1\%.

Diskussion. Die errechneten Werte für Sensitivität und LR+ der einzelnen immunologischen Marker alleine erlauben keine sichere Therapieentscheidung bei klinischem Infektionsverdacht. Im Gegensatz dazu korreliert die Kombination aus erhöhtem CRP und erhöhtem IL-8 bei Infektionsverdacht sehr gut mit dem tatsächlichen Vorliegen einer nosokomialen Infektionsepisode und kann zur Entscheidungsfindung für oder gegen den Beginn einer antibiotischen Therapie herangezogen werden.

Schlussfolgerung. CRP in Kombination mit IL-8 wie auch serielle CRPMessungen sind von guter prognostischer Aussagekraft für die Diagnostik nosokomialer Infektionen bei Neugeborenen.

\section{P022}

Nachweis von probiotischen Keimen in der Blutkultur als Frühsymptom einer nekrotisierenden Enterokolitis?

\section{Stavropoulou', B. Maier' ', P. Henneke ${ }^{3}$, H. Fuchs', R. Hentschel'}

'Universitätsklinikum Freiburg, Zentrum für Kinder- und Jugendmedizin, Neonatologie/Intensivmedizin, Freiburg, Deutschland, ${ }^{2}$ Universitätsklinikum Freiburg, Institut für Medizinische Mikrobiologie und Hygiene, Freiburg, Deutschland, ${ }^{3}$ Universitätsklinikum Freiburg, Zentrum für chronische Immundefizienz, Freiburg, Deutschland

Hintergrund. Nach der aktuellen Studienlage, ist die Gabe von Probiotika zur Prävention der nekrotisierenden Enterokolitis (NEC) bei Frühgeborenen in den meisten Kliniken weit verbreitet. Im Rahmen der regelmäßigen Anwendung erreicht man eine mikrobielle Kolonisation. Diese übt eine regulierende und protektive Rolle auf den Frühgeborenendarm gegenüber einer möglichen Fehlbesiedlung aus. Solch eine Fehlbesiedlung wird unter anderem auch als möglicher Auslöser einer NEC gesehen. Die dafür angewendeten Präparate beinhalten Bifidobakterien und Laktobazillen.

Fragestellung. Kann die mikrobielle Kolonisation mit Bifidobakterien des Darmes verantwortlich für eine Septikämie sein, und lässt der Nachweis von Bifidobakterien in der Blutkultur einen Rückschluss auf eine mögliche Translokation beziehungsweise NEC zu?

Methoden. Wir berichten über 2 Frühgeborene unserer Intensivstation mit einem GA von 29 und 26 SSW und einem GG von $1125 \mathrm{~g}$ und $660 \mathrm{~g}$, bei denen unter laufender probiotischer Prophylaxe mit Infloran ${ }^{\infty}$ im Rahmen einer Sepsis in der Blutkultur Bifidobacterium longum nach- 
gewiesen wurde. Beide Kinder entwickelten im Abstand von 18 und 27 Tagen nach dem positiven BK-Befund eine NEC, beim ersten Kind mit tödlichem Ausgang.

Ergebnisse. In den durchgeführten Subtypisierungen des Erregers aus den Blutkulturen und in der Kultur aus dem klinikintern angewendeten Präparat zur probiotischen Therapie, Infloran', ließ sich mittels MALDI-TOF in allen Proben und Isolaten dieselbe Spezies, Bifidobacterium longum, subspecies infantis nachweisen. Diese ist mit dem probiotischen Erreger aus dem Präparat Infloran identisch.

Diskussion. Wie in vereinzelt publizierten Artikeln berichtet kann im Rahmen einer Septikämie gelegentlich ein probiotischer Keim nach oralen Probiotika nachgewiesen werden. Die Unreife des Frühgeborenendarmes und die immunologischen Prozesse auf der Mucosa intestinalis können analog zu anderen vulnerablen Situationen zu einer Aussaat der kolonisierenden Erreger führen, wobei der kausale Zusammenhang mit der Sepsis nicht zwangsläufig bestehen muss.

Schlussfolgerung. Trotz des weitgehenden Ausschlusses einer Kontamination bei den oben genannten Fällen sollten immer die Hygienestrategien auf einer neonatologischen Intensivstation kritisch hinterfrag und überprüft werden. $\mathrm{Ob}$ positive Blutkulturen für probiotische Erreger mit einer Störung des kapillären Blutflusses einhergehen und somit prädisponierend für die Entstehung einer NEC sind lässt sich bisher nicht beweisen. Jedoch könnte bei einem solchen Blutkulturbefund ein erhöhtes Risiko für eine NEC bestehen.

\section{P023}

\section{Bakterielles Monitoring des CPAP-Equipments bei FG $<1500 \mathrm{~g}$}

\section{T. Winking', J. Wüllenweber ${ }^{2}$, F. Kipp³, E. Rieger-Fackeldey'}

'Universitätsklinikum Münster, Klinik für Kinder- und Jugendmedizin - Allgemeine Pädiatrie -, Neonatologie und Pädiatrische Intensivmedizin, Münster, Deutschland, ${ }^{2}$ Universitätsklinikum Münster, Institut für klinische Mikrobiologie, Münster, Deutschland, ${ }^{3}$ Universitätsklinikum Münster, Institut für Hygiene, Münster, Deutschland

Fragestellung. Wann lässt sich welches bakterielle Wachstum auf dem CPAP-Equipment von VLBW-FG (<1500 g) nachweisen? Korrelation mit den Ergebnissen der Screeningabstriche sowie der Infektionsdiagnostik.

Methodik. Prospektive longitudinale Studie aller beatmeten Frühgeborenen mit Geburtsgewichten <1500 g (VLBW) in der Neonatologie des Universitätsklinikums Münster von September bis Dezember 2012. Material: Abstriche von Wassertopf, Inspirationsschenkel, Konnektor, Prong der CPAP-Beatmung zu den Nutzungstagen 3/7/14. Positive Ergebnisse der routinemäßigen Screeningabstriche von Anus, Rachen und Trachealsekret, sowie der Infektionsdiagnostik.

Ergebnisse. Es konnten 26 Frühgeborene in die Studie eingeschlossen werden. Durchschnittliches Gestationsalter $26+5(23+6$ bis $32+2)$ SSW -gewicht 850 (495-1480) Gramm. Insgesamt 337 Abstriche wurden vom CPAP-Equipment entnommen, ca. 59\% (95\% CI 54; 64) zeigten Keimbesiedlung. Die CPAP-Konnektor-Abstriche wiesen zu ca. zwei Drittel Keimfreiheit auf. Von den CPAP-Prongs wurden 201 Abstriche entnommen, davon waren 1/10 (96\% CI 6; 14) keimfrei, $7 \%$ an Tag 3 und $13 \%$ an Tag 7 der Nutzung. An Tag 3 waren $41 \%$, [24\% grampositiv, $16 \%$ gramnegativ (95\% CI 8; 22), 2\% Pilze] und an Tag 7 67\% [40\% grampositiv, 24\% gramnegativ (95\% CI 15; 32), 3\% Pilze] der Prongs/Masken mit hautflorafernen Keimen kolonisiert. Pseudomonaden waren an Tag 7 (9\%) doppelt so häufig auf den Prongs zu finden wie an Tag 3. Keime der Enterobacteriacea-Spezies und Pilze waren an $\operatorname{Tag} 7(13 \%, 3 \%)$ häufiger zu finden als an Tag 3 (11\%, 2\%). Kolonisierung: In den Screeningabstrichen zeigte sich bei $69 \%(n=18)$ der Studienpatienten eine Kolonisierung; Auftreten nach durchschnittlich 3 Lebenswochen. Bei $72 \%$ dieser Patienten zeigte sich eine identische Kolonisierung des CPAP-Systems (95\% CI 46; 95). Infektionen: Nach NEO-KISS-Kriterien wurden während der CPAPBeatmung 18 Infektionen diagnostiziert bei 16 Patienten. Die Infektionen traten durchschnittlich im Lebensalter von 18 Tagen auf, davon 56\% Sep- titiden $(\mathrm{n}=10), 17 \%(\mathrm{n}=3)$ Harnwegs- und Infektionen ohne Keimnachweis, sowie $11 \%$ Pneumonien $(n=2)$. Bei 90\% der Septitiden wurde KNS isoliert. In den 3 Urinkulturen der HWIs zeigte sich 2-mal C. albicans wie im Routine-Screening. Bei den Pneumonien fand sich E. cloacae im Trachealsekret, in den Routine-, und in den CPAP-Abstrichen.

Schlussfolgerung. Die CPAP-Prongs waren zum größten Teil besiedelt, wobei der Befall mit gramnegativen Keimen, mit der Nutzungsdauer zunahm. Bei den CPAP-Prong-Kolonisierungen konnte in mehr als 4/5 der Fälle eine korrelierende Kolonisierung in den Screeningabstrichen dargestellt werden. Bei Pneumonien und Harnwegsinfekten waren die nachgewiesenen Erreger meist identisch mit denen auf dem Beatmungsequipment und im Routinescreening, nicht jedoch bei Septitiden.

\section{P024}

Hautprobleme extrem unreifer Frühgeborener durch Hautantiseptika

\section{Biermann 1', A. Kribs' 1 B. Roth', I. Tantcheva-Poor ${ }^{2}$}

'Universitätskinderklinik Köln, Neonatologie und Pädiatrische Intensivmedizin, Köln, Deutschland, ${ }^{2}$ Universitätsklinik Köln, Klinik und Poliklinik für Dermatologie und Venerologie, Köln, Deutschland

Einleitung. Die Haut extrem unreifer Frühgeborener (ELGAN) ist wegen struktureller und funktioneller Unreife sehr vulnerabel [1]. Hautantiseptika können zu schweren Hautschäden führen $[2,3]$.

Fragestellung. Erfassung der angewendeten Hautantiseptika an deutschen PNZ Level-1 und Korrelation mit dem Auftreten von Hautschäden. Material und Methode. 166 deutschen PNZ Level-1 wurde ein anonymisierter Fragebogen zugesandt. Der Erhebungszeitraum war April bis Oktober 2013. Erfasst wurden Angaben zur Patientengruppe (FG <100o g), Anwendung von Hautantiseptika und beobachteten Hautkomplikationen in 2011/2012. Deskriptive statistische Auswertung. Ergebnisse. Der Rücklauf verwertbarer Fragebögen betrug 39\% (n=64). Im Jahr 2012 wurden in diesen Zentren 2130 Patienten mit einem Geburtsgewicht $<1000$ erfasst. 701 Patienten wiesen ein Gestationsalter $<26+0$ Wochen auf. Als Hautantiseptika (Mehrfachnennung möglich) verwendeten $58 \%$ der NICUs $(n=37)$ Octenidinhydrochlorid o, $1 \%$ ohne Phenoxyethanol $(\mathrm{OoP}), 56 \%(\mathrm{n}=36)$ Octenidinhydrochlorid mit Phenoxyethanol 2\% (Octenisept $\left.\mathrm{t}^{\circ}\right), 38 \%(\mathrm{n}=24)$ sonstige (u. a. alkoholische Lösungen), $5 \%(\mathrm{n}=3)$ PVPJod $10 \%, 3 \%(n=2)$ Chlorhexidin. Überwiegend wurden Octenisept ${ }^{\oplus}$ oder OoP für die folgenden Prozeduren angewendet: Gefäßpunktionen, kapilläre Butentnahmen, Injektionen, Silastikkatheter, Nabelgefäßkatheter, Pleuradrainagen, Blasenkatheter, Lumbalpunktionen, Rickham-/ Ommaya-Punktionen $27 \%(\mathrm{n}=17)$ der befragten Zentren geben mindestens eine dokumentierte Hautkomplikation an. Von diesen wurde in 9 Fällen Octenisept $^{\oplus}$, in 6 Fällen OoP angewendet. Sämtliche Hautkomplikationen traten in den ersten beiden Lebenswochen auf. In 2 Fällen traten großflächige Nekrosen auf und in 3 Fällen Komplikationen im Sinne einer sekundären mykotischen Infektion. Narbenbildung und Depigmentierung wurden als Spätkomplikationen beobachtet. In $75 \%(n=13)$ der Fälle waren die Hautläsionen periumbilical lokalisiert. Diese Läsionen wurden jeweils in 5 Fällen nach Octenisept ${ }^{\circledR}$ und OoP beobachtet.

Diskussion und Schlussfolgerung. Weiterhin wird Octenisept ${ }^{\oplus}$ trotz der Warnhinweise häufig bei ELGAN als Hautdesinfektionsmittel genutzt. Vom RKI wird die Hautdesinfektion mit OoP empfohlen [4], aber selbst hierbei wurden bei ELGAN Hautläsionen beobachtet, was bisher nicht in der Literatur beschrieben wurde. Häufig geschieht dies bei Anlage von Nabelgefäßkathetern. Als Begleitkomplikationen kann es zu kutanen Mykosen und Spätfolgen wie Narben und Depigmentierungen kommen. Derzeit ist für die spezifischen Verhältnisse der Haut extrem unreifer Frühgeborener kein Hautdesinfektionsmittel verfügbar, welches nicht mit der Gefahr kutaner Komplikationen verbunden ist.

1. Rutter N (1996) European Journal of Pedriatrics 155(Suppl2):18-20

2. Bührer C et al (2002) Journal of Hospital Infection 51:305-307

3. Cucarachi G et al (2010) Minerva Pediatrics 3(Suppl1)157-159

4. KRINKO (2007) Bundesgesundheitsblatt - Gesundheitsforschung - Gesundheitsschutz 50:1256-1303 
P025

\section{Ein seltener Fall von rezidivierender neonataler Herpes-simplex- Infektion}

\section{R. Creutzfeldt', P. Henneke ${ }^{2}$, R. Hentschel ${ }^{1}$}

'Zentrum für Kinder- und Jugendmedizin, Universitätsklinikum Freiburg, Neonatologie/Pädiatrische Intensivmedizin, Freiburg, Deutschland, ${ }^{2}$ Universitätsklinikum Freiburg, Zentrum für chronische Immundefizienz, Freiburg, Deutschland

Einleitung. Die Gesamtinzidenz der neonatalen Herpesinfektion liegt zwischen 1:3000-1:20.000. Sie wird unterteilt in die disseminierte, zentralnervöse und lokale Form. Die disseminierte Form hat mit 30\% die höchste Mortalität, wobei nur $20 \%$ der Überlebenden neurologische Spätfolgen erleiden. Hingegen ist die Mortalität bei zentralnervösem Befall mit 6\% sehr gering, mit neurologischen Komplikationen bei 70\% der Patienten.

Fallbericht. Wir berichten über einen frühgeborenen weiblichen Zwilling $(35+6 / 7$ SSW $)$, der nach vorzeitigem Blasensprung der Zwillingsschwester per sekundärer Sectio geboren wurde. Die primäre Adaptation und die ersten Lebenstage verliefen unauffällig. Ab dem 5. LT verschlechterte sich jedoch der Allgemeinzustand sukzessive, so dass bei ausgeprägter Schläfrigkeit, unzureichendem Trinkverhalten und abdomineller Distension die stationäre Aufnahme zur weiteren Abklärung erfolgte. Differentialdiagnostisch kam neben der Frühgeburtlichkeit primär eine Infektion in Betracht. Die Infektparameter waren inital normal. Sekundär erhöhte Infektparameter, unauffälliger Liquor und massiv erhöhte Transaminasen und des Ferritins waren schwer zu interpretieren. Neben Stoffwechselerkrankungen wurden auch eine virale Infektion und eine neonatale Haemochromatose in Betracht gezogen. Es erfolgte eine i.v.Therapie mit Aciclovir ex juvantibus, vor Auftreten eines Vesikels am Thorax und am linken Fuß. Der Nachweis des Herpes-simplex-Typ-IVirus gelang mit sehr hoher Kopienzahl im Blut, außerdem in den Vesikeln und retrospektiv auch im Nasopharynxsekret. Im Liquor waren nur Spuren des Virus nachweisbar. Beide Eltern und die Großmutter als Kontaktpersonen waren seronegativ und hatten keine frischen Infektionszeichen. Unter Aciclovir-Therapie (insgesamt 21 Tage) kam es zu einer raschen klinischen Besserung. Nach neueren amerikanischen Empfehlungen folgte eine orale Suppressionstherapie mit Aciclovir über 6 Monate, die bei neurologischer Beteiligung in jedem Fall, unter Umständen aber auch bei unklarem Liquorbefund, erfolgen sollte. Ein Monat nach Abschluss der Therapie, erneute stationäre Aufnahme bei zunehmend schläfrigem Kind in reduziertem Allgemeinzustand. Dieses Mal war das Herpes-simplex-Typ-I-Virus nur im ansonsten unauffälligen Liquor nachweisbar. Nach sofortigem Beginn einer i.v. AciclovirTherapie erholte sie sich rasch und komplett. Die Therapie erfolgte über 21 Tage und im Anschluss die erneute Suppression mit Aciclovir über 6 Monate.

Diskussion. Der typische, schwere septische Verlauf bei einer konnatalen Infektion ist bekannt, wie auch die dramatische Symptomatik bei einer HSV-Enzephalitis. Der schleichende Verlauf einer neonatalen HSV-I-Infektion als Differenzialdiagnose zur bakteriellen Infektion ist eine Rarität, aber auch bei fehlenden Zeichen an Haut, Schleimhaut oder Augen in Betracht zu ziehen und sofort zu behandeln. Bei fehlendem Nestschutz (seronegative Mutter) muss das Neugeborene in den ersten Lebenstagen über Besucher oder medizinisches Personal infiziert worden sein. Das Rezidiv ist eine weitere Rarität, die jedoch bei bekannter Persistenz des HSV, insbesondere im Nervensystem, als Möglichkeit in Betracht gezogen werden muss; insbesondere, da die zerebrale Immunität im 1. Lebensjahr noch nicht ganz ausgereift ist. Ein Herpes-spezifischer Immundefekt kann bei HSV-Enzephalitiden zugrunde liegen, jedoch ist der Verlauf dann meist dramatischer.

\section{P026}

Brevundimonas vesicularis als Ursache für eine kongenitale Sepsis

S. Pogodda', U. Holl', J. Hallauer ${ }^{3}$, S. Armbrust ${ }^{1}$

'Klinikum Neubrandenburg, Klinik für Kinder- und Jugendmedizin, Neubrandenburg, Deutschland, ${ }^{2}$ Klinikum Neubrandenburg, Institut für Laboratoriumsdiagnostik, Mikrobiologie und Transfusionsmedizin, Neubrandenburg, Deutschland, ${ }^{3}$ Klinikum Neubrandenburg, Institut für Hygiene, Neubrandenburg, Deutschland

Einleitung. Sepsis ist trotz optimaler Hygienemaßnahmen weiter ein Problem auf neonatologischen Intensivstationen. Die Resistenzlage der Erreger unterliegt einem ständigen Wandel, nicht nur die Nomenklatur hat sich differenziert (2/3/4 MRGN etc.). Durch zunehmende Durchseuchung der Bevölkerung auch mit multiresistenten Erregern, die beim gesunden Menschen problemlos ist, steigt das Risiko auch der vertikalen Transmission. Wir berichten über den Fall einer kongenitalen Brevundimonas-Sepsis.

Fallbeispiel. Reifes Neugeborenes, Geburt am Termin, Mutter Tierärztin. Postnatal klinisch auffällig mit Zeichen der kongenitalen Sepsis. Nachweis von Brevundimonas vesicularis in der Blutkultur, initiale Behandlung mit Ampicillin und Gentamicin, im Verlauf Besserung. Jedoch problematisches Resistogramm mit Resistenz gegen mit Ampicillin. Mutter hatte bis kurz vor der Geburt noch tierärztlich gearbeitet. Diskussion. Brevundimonas ves. ist ein gramnegatives Bakterium, gehört zur Familie der Caulobacteraceae, erstmalig nachgewiesen bei medizinischen Blutegeln (Hirudo medicinalis), zu finden in Isolaten von Wasser, Erde, Ablagerung von Papiermüll etc. Es gibt weltweit wenige Beschreibungen über Infektionen mit diesem Erreger bei immunsupprimierten Patienten die nahezu ausschließlich aus dem asiatischen Raum stammen. Die Resistenzentwicklung von Keimen erfolgt auch zunehmend intestinal über Plasmid-Weitergabe.

Schlussfolgerung. Auch bei einer vermeintlich einfachen kongenitalen Sepsis können Problemkeime eine Rolle spielen. Besondere Aufmerksamkeit sollte Müttern gelten die potenziell mit Problemkeimen Kontakt gehabt haben (Landwirtschaft, Tierhaltung etc.).

\section{P027}

\section{Bakterielles Monitoring der Beatmungsbeutel bei FG $<1500 \mathrm{~g}$}

\section{T. Winking', J. Wüllenweber' ${ }^{2}$, F. Kipp ${ }^{3}$, E. Rieger-Fackeldey ${ }^{1}$}

'Universitätsklinikum Münster, Klinik und Poliklinik für Kinder- und Jugendmedizin - Allgemeine Pädiatrie - Neonatologie und Pädiatrische Intensivmedizin, Münster, Deutschland, ${ }^{2}$ Universitätsklinikum Münster, Institut für klinische Mikrobiologie, Münster, Deutschland, ${ }^{3}$ Universitätsklinikum Münster, Institut für Hygiene, Münster, Deutschland

Fragestellung. Wann lässt sich welches bakterielle Wachstum auf dem Beatmungsbeuteln von VLBW-FG $(<1500 \mathrm{~g})$ nachweisen?

Methodik. Prospektive longitudinale Studie aller beatmeten Frühgeborenen mit Geburtsgewichten $<1500 \mathrm{~g}$ (VLBW) in der Neonatologie des Universitätsklinikums Münster von September bis Dezember 2012. Material: Abstriche von Ambubeutel-Konnektor und -Maske an Tag 3/7/14 auf der intermediate- (IMCU) und den Tagen $3 / 7$ auf der Intensive Care Unit (ICU).

Ergebnisse. Es konnten 26 Frühgeborene in die Studie eingeschlossen werden. Durchschnittliches Gestationsalter $26+5(23+6-32+2)$ SSW, -gewicht 850 (495-1480) Gramm. Insgesamt wurden 266 Abstriche durchgeführt, wovon $81 \%$, (95\% CI 76; 85) keimfrei waren. Auf der IMCU wurden bei 14 Patienten 18 keimfreie Abstriche vom Konnektor an Tag 14 dokumentiert. Auf der ICU waren bei 26 Patienten in 126 Abstrichen $83 \%$ an Tag 3 und $91 \%$ an Tag 7 keimfrei. Bei den Ambubeutel-Masken von 18 Patienten lag auf der IMCU an den Tagen $3 \mathrm{zu} 69 \%, 7 \mathrm{zu} 73 \%$ und $14 \mathrm{zu} 85 \%$ Keimfreiheit vor. Auf der ICU waren bei 71 Abstrichen von 26 Patienten $78 \%$ (95\% CI 63; 90) der Masken an Tag 3 und 66\% (95\% CI 49; 80 ) an Tag 7 keimfrei. An Tag 3 wurden überwiegend Hautkeime isoliert. An Tag 7 lagen neben Hautkeimen zu je $24 \%$ hautfloraferne gram- 
positive und -negative Keime vor. Die isolierten gramnegativen Keime waren Moraxella osloensis und E. cloacae.

Schlussfolgerung. Ambubeutel werden in unserer Neonatologie fast nur noch bei Wechsel der Beatmungsschläuche eingesetzt. Insgesamt zeigte sich in den abgenommenen Abstrichen größtenteils Keimfreiheit, bei stabileren Patienten auf der IMCU ausgeprägter als auf der ICU. Auf der IMCU blieben die Konnektoren keimfrei und die Besiedlung der Masken nahm über die Nutzungsdauer absolut und relativ ab. Dies deutet darauf hin, dass sich die bei anfänglicher Nutzung angesammelten Keime auf der Maske über die weitere Dauer ohne Nutzung bei Keimund nährbodenfreien Lagerung reduzierten. Auf der ICU zeigte sich ebenso größtenteils Keimfreiheit, bei einigen Ambubeuteln jedoch eine Zunahme der Besiedlung mit der Zeit, insbesondere auch von gramnegativen Keimen.

\section{Pädiatrische Intensivmedizin}

\section{P028 \\ Septischer Schock im Ruhrgebiet und Köln - eine retrospektive multizentrische Studie}

\section{Dohna-Schwake}

'Klinik für Kinder und Jugendmedizin der Universität, Essen, Deutschland. Im Namen der Mitglieder des Sepsis-Netzwerkes Ruhr

Hintergrund. Insbesondere in Europa und Deutschland existieren nur sehr spärliche Daten über Epidemiologie, Behandlung, Risikofaktoren und Outcome des septischen Schocks im Kindesalter. Aus diesem Grund haben wir eine multizentrische retrospektive Analyse im Ruhrgebiet und Köln durchgeführt.

Methoden. In 20 Kinderkliniken wurden die Akten von 83 Fällen von Kindern im Alter von 2 Monaten bis 17 Jahren mit septischem Schock ausgewertet. Die Mortalität lag bei $25 \%(n=21)$. Signifikante Risikofaktoren für das Versterben waren PRISM-III-Score, pH, systolischer Blutdruck, Vorliegen einer DIG (disseminierte intravasale Gerinnung) und Anzahl von Organversagen, jedoch nicht Laktat $(\mathrm{p}=0,05)$ und BE (base excess; $\mathrm{p}=0,065$ ).

Ergebnisse. Die Mortalität in Kinderabteilungen, die 10 oder mehr Patienten behandelt hatten, lag bei 17\%, in Abteilungen, die 3-6 Patienten behandelt hatten bei $22 \%$ und stieg auf $61 \%$ an in Abteilungen, die 1 oder 2 Patienten behandelt hatten. In der multiplen Regression konnte die Anzahl der behandelten Kinder als einziger unabhängiger Risikofaktor von allen signifikanten evaluiert werden.

Schlussfolgerung. Die Mortalität des septischen Schocks im Kindesalter ist mit $25 \%$ in Westdeutschland hoch. Da es sich in den Industrienationen um ein seltenes Krankheitsbild handelt, sollten Aspekte der verbesserten Versorgung in Netzwerkbildung und Ausbildung von Kinderärzten liegen. Das kürzlich ins Leben gerufene Sepsis-Netzwerk Ruhr (REDMOPED=Reducing Mortality in Pediatric septic shock) setzt hier an.

po29

\section{Kann man Spiritualität bei Kindern messen? A. Büssing', M. Thiel}

'Professur für Lebensqualität, Spiritualität und Coping, Institut für Integrative Medizin, Universität Witten/Herdecke, Herdecke, Deutschland, ${ }^{2}$ Sana-Klinikum Remscheid, Klinik für Kinder und Jugendliche, Remscheid, Deutschland

Einführung. Daten über spirituelle Bedürfnisse bei Kindern in den USA zeigen u. a., warum diese in Krankenhäusern nicht bedient werden. Für wissenschaftliche Untersuchungen sind verlässliche Messinstrumente wünschenswert. Wir berichten über ein Consensus-Meeting über Spiritualität von Kindern im Krankenhaus, das zum Ziel hatte entsprechende Themen und damit Forschungsansätze zu identifizieren.

Ergebnisse. Wie kann man den vermuteten kindlichen Bedürfnissen mit der erforderlichen Grundhaltung, den inhaltlichen, zeitlichen und personellen Ressourcen begegnen? Folgende Schritte wurden identifiziert:

1. Sensibilisierung des Arztes/der Pflegekraft für das Thema.

2. Voraussetzung, offen für kindliche Bedürfnisse und Signale zu sein, 3. Ist spirituelle Unterstützung eine notwendige Herausforderung oder aufgrund knapper Ressourcen Luxus?

4. Stärkung des traumatisierten Kindes kann Therapieziel sein.

5. Die Auseinandersetzung mit Spiritualität kann Teil des Gesamtbildes „Krankenhaus“ werden.

Offene Fragen. Wie misst man spirituelle Bedürfnisse bei Kindern? - ASP (Aspects of Spirituality, 25 Items) 2.1, getestet bei Kindern/Jugendlichen, nicht bei Kindern.

- SWBQ (Spiritual Well-Being Questionnaire)/SHALOM (Spiritual Health anD Life-Orientation Measures)[2o Items], in Studien (ab 8 Jahre) verwendet, nicht validiert.

- SpNQ (Spiritual Needs Questionnaire), in Studien (ab 11 Jahre) verwendet, nicht validiert.

Der letztgenannte Fragebogen wird in derzeitigen Untersuchungen aus unserer Arbeitsgruppe (Validierungsstudie, M. Down, chronische Krankheiten) verwendet.

Diskussion und Schlussfolgerung. Spiritualität bei Kindern könnte ein Thema für Forschung und Klinik sein. Anerkannte Messinstrumente existieren noch nicht, sind aber Gegenstand aktueller Untersuchungen.

\section{P030}

Kammerflimmern nach Weckergeräusch und 50-minütiger Laienreanimation: Demaskierung eines Long-QT-Syndroms bei einer 14-jährigen Patientin

\section{K. Nilles', D. Fatehpour' ${ }^{2}$ R. Schimpf ${ }^{3}$, M. Borggrefe', T. Hoppen', T. Nüßlein'}

'Gemeinschaftsklinikum Koblenz-Mayen, Kemperhof Koblenz, Klinik für Kinderund Jugendmedizin, Koblenz, Deutschland, ${ }^{2}$ Gemeinschaftskinikum KoblenzMayen, Kemperhof Koblenz, Klinik für Innere Medizin II, Koblenz, Deutschland, 3Universitätsmedizin Mannheim, I. Medizinische Klinik. Kardiologie, Intern. Intensivmedizin, Angiologie und Pneumologie, Mannheim, Deutschland

Einleitung. Das Long-QT-Syndrom (LQTS) zählt zu den genetisch determinierten Ionenkanalerkrankungen der Myokardzellen. Beeinträchtigungen dieser Strukturen führen zu De- und Repolarisationsstörungen der kardialen Zellen und begünstigen die Entstehung von Herzrhythmusstörungen bis hin zu lebensbedrohlichen Arrhythmien wie Tachykardien vom Typ Torsade-de-pointes und Kammerflimmern

Fallbericht. Wir berichten über eine 14-jährige bisher asymptomatische Jugendliche, die frühmorgens durch das Weckersignal erwachte, den Wecker noch ausschaltete und ca. 5 Minuten später von ihren Eltern im Herz-Kreislauf-Stillstand aufgefunden wurde. Die Eltern begannen sofort mit der Laienreanimation. Durch unglückliche Umstände verzögerte sich das Eintreffen des Notarztes. Etwa 45 Minuten nach Auffinden der Patientin traf der Notarzt schließlich am Einsatzort ein, detektierte Kammerflimmern, führte eine erfolgreiche Defibrillation durch und verlegte die Jugendliche im beatmeten Zustand in unsere Klinik. Wir führten eine Hypothermiebehandlung über 24 Stunden durch. Neben anderen Differentialdiagnosen dachten wir frühzeitig an eine angeborene Neigung zu malignen Herzrhythmusstörungen. Das erste Ruhe-EKG bei Aufnahme in der Klinik zeigte eine normale korrigierte QT(QTc)-Zeit von $410 \mathrm{~ms}$. Am Folgetag traten gehäuft ventrikuläre Extrasystolen mit plötzlichem Übergang in eine pulslose ventrikuläre Tachykardie vom Torsade-de-pointes-Typ auf. Im 12-Kanal-EKG zeigte sich nun eine verlängerte QTc-Zeit von 450-550 ms. Damit stellten wir die Diagnose eines LQTS. Nach erfolgter rhythmologischer Stabilisierung der Patientin unter hochnormalen Serummagnesiumspiegeln und Betablockertherapie wurde sie geplant zur weiteren Diagnostik und Therapie an die Universitätsklinik Mannheim verlegt. Aufgrund einer erheblichen posthypoxischen neurologischen Schädigung nach präklinischer Reanimation und schwer einzuschätzender Prognose der sehr 
jungen Patientin erfolgte eine moderierte ethische Fallbesprechung im Team mit Vertretern der Intensivstation, der elektrophysiologischen Abteilung und der Neuropädiatrie. Im Konsens mit den Eltern wurde eine Entscheidung für die sekundärprophylaktische Implantation eines subkutanen Kardioverters/Defibrillators (SICD) getroffen.

Schlussfolgerung. Patienten mit einem Long-QT-Syndrom vom Subtyp 2 werden typischerweise durch besondere Schreckmomente wie akustische Signale auffällig bzw. in Folge getriggerter lebensbedrohlicher Arrhythmien symptomatisch. Aufgrund der bisher fehlenden Symptomatik wurde das familiäre Krankheitsbild erst durch die Reanimation demaskiert. Unerfreulicherweise kam es bei unserer Patientin zu einer Defektheilung nach hypoxischem Hirnschaden. Dennoch konnten durch die Screeninguntersuchungen der Familie und Großfamilie weitere 5 Personen durch Nachweis eines verlängerten QT-Intervalls bzw. molekulargenetisch identifiziert werden, die jetzt alle prophylaktisch mit einem Betablocker therapiert werden.

\section{P031}

\section{Akute Amlodipinintoxikation bei einem 13-jährigen Mädchen: eine therapeutische Herausforderung}

\section{J. Kaiser', L. Berberich', L. Muth', T. Karen', C. Wieg' \\ 'Klinikum Aschaffenburg, Neonatologie und pädiatrische Intensivmedizin, Aschaffenburg, Deutschland}

Einleitung. Die Amlodipinintoxikation stellt eine therapeutische Herausforderung dar, da sie mit schweren kardiogenen, pulmonalen und renalen Nebenwirkungen bis hin zur Letalität einhergehen kann.

Kasuistik. 13-jährige Patientin ohne Vorerkrankungen. In suizidaler Absicht wurden 16-18 Tabletten Amlodipin eingenommen, entsprechend einer potentiell letalen Dosis von $180 \mathrm{mg}(2,9 \mathrm{mg} / \mathrm{kg})$. Der 2 Stunden nach Einnahme alarmierte Notarzt leitete bei zunächst mäßig ausgeprägter Hypotension eine Volumensubstitutionstherapie ein. Nach Eintreffen in der Klinik wurde bei noch stabilen Kreislaufverhältnissen umgehend eine Magenspülung mit $\mathrm{NaCl}$ o,9\% durchgeführt und Aktivkohle via Magensonde verabreicht. Nach anschließender Verlegung auf die pädiatrische Intensivstation wurde bei anhaltender Hypotension die Volumensubstitutionstherapie mit kristalloider Lösung fortgeführt. Im EKG zu diesem Zeitpunkt Bild eines nicht vorbekannten AV-Blocks $\mathrm{I}^{\circ}$ (PQ Zeit $200 \mathrm{~ms}$ ). Bei zunehmender Kreislaufinsuffizienz wurde im Verlauf eine kreislaufunterstützende Therapie mit Noradrenalin und Dopamin notwendig. Unter den Katecholaminen besserten sich die Kreislaufsituation und die dadurch bedingte passagere Oligurie. Als weitere und schwere Komplikation entwickelte die Patientin ca. 6 Stunden nach Medikamenteneinnahme ein nichtkardiogenes Lungenödem mit progredienter respiratorischer Insuffizienz mit Tachydyspnoe und isolierter schwerer Oxygenierungsstörung. Die besondere Herausforderung lag in der der Therapieentscheidung maschineller vs. nichtinvasiver Beatmung. Wir führten erfolgreich eine nichtinvasive Beatmung bei maximalem PEEP $\left(7 \mathrm{cmH}_{2} \mathrm{O}\right)$ und $\mathrm{FiO}_{2}$ von 1,o über 4 Tage durch. Darunter zunehmende Konsolidierung der Ventilation und Oxygenierung. Nach 5 Tagen konnte die Patientin in gutem AZ in unsere Kinderund Jugendpsychiatrie verlegt werden.

Diskussion. Amlodipinintoxikationen können zu einem katecholaminrefraktären Kreislaufschock (periphere arterielle Vasodilatation) sowie zu schweren Bradyarrhythmien (negativ inotrop, dromotrop und chronotrop) und Hypokalzämien führen. Therapeutisch kommen eine primäre Giftelimination mittels Aktivkohle oder die sekundäre Elimination durch eine Hämoperfusion über Kohlefilter in Frage. Einzelfallbeschreibungen existieren zum erfolgreichen Einsatz von Lipidemulsionen, Kalziumglukonatgabe und Hochdosis-Insulingabe bei Vorliegen einer katecholaminrefraktären Kreislaufinsuffizienz. Zur nichtinvasiven Beatmung beim Lungenödem im Kindes- und Jugendalter gibt es bisher nur wenige Daten.

Schlussfolgerung. Die Intoxikation mit Amlodipin ist lebensbedrohlich und bedarf zügig intensivmedizinischer Behandlung. Die nichtinvasive
Beatmung des begleitenden Lungenödems stellt eine erfolgreiche Therapieoption dar.

\section{P032}

Das maligne neuroleptische Syndrom, eine seltene Komplikation in der pädiatrischen Intensivmedizin

\section{F. Ebach', M. Ehlen ${ }^{1}$}

'Asklepios Klinik St. Augustin, Neonatologie/Päd. Intensivmedizin, St. Augustin, Deutschland

Einleitung. Das maligne neuroleptische Syndrom (MNS, syn.: malignes Neuroleptika-Syndrom) stellt eine lebensbedrohliche Komplikationeiner Behandlung mit Neuroleptika dar. Symptome sind in der Regel Dyskinesien und Muskelsteifigkeit, eine ausgeprägte autonome Dysregulation, Bewusstseinsveränderungen, Hyperthermie und eine Rhabdomyolyse mit der Gefahr eines Multiorganversagens. Ursächlich vermutet wird ein Dopaminmangel durch Blockade von dopaminergen D2-Rezeptoren. Berichtet wird über den Krankheitsverlauf bei einem 3-jährigen Jungen, der in der Entwöhnungsphase nach langdauernder Beatmung einige Stunden nach Neuroleptikagabe eine ausgeprägte Rhabdomyolyse, eine Hyperthermie und ein nachfolgendes Multiorganversagen entwickelte.

Kasuistik. 3 8/12 Jahre alter Junge, langwieriger Beatmungsverlauf (24 Tage) bei Pneumothorax und massivem Weichteilemphysem aufgrund einer bronchopleuralen Fistel als Komplikation einer PneumokokkenPneumonie. In der Entwöhnungsphase der Beatmung und nach Extubation Entwicklung eines ausgeprägten Medikamententzugsyndroms (Therapie mit Dexmedetomidin 0,0014 mg/kg/h und Morphin 0,04 mg/ $\mathrm{kg} / \mathrm{h}$ ). Bei unzureichender Wirkung der Sedierung mit ausgeprägtester Agitiertheit einmalige Gabe von Haloperidol (o,25 mg i.v.). Innerhalb von 12 Stunden dann Entwicklung eines generalisierten Krankheitsbildes mit Hyperthermie bis $41,8^{\circ} \mathrm{C}$, Blutdruckabfall sowie kombinierter metabolischer und respiratorischer Azidose. Weitere Symptome dieser akuten Krankheitsphase waren eine muskuläre Dystonie, Bewusstseinsveränderung und Diarrhö. Laborchemisch fanden sich eine CKErhöhung (max. 9140 U/l) sowie ein Anstieg der Transaminasen und des PCT. Zudem bestanden eine ausgeprägte Thormbozytopenie (70oo/ $\mu \mathrm{l})$ und eine mäßige Leukozytose. Das rote Blutbild blieb unauffällig. Im weiteren Verlauf Entwicklung eines Multiorganversagens mit erneuter Beatmungspflichtigkeit und eines oligo-anurischen Nierenversagens aufgrund einer ausgeprägten Rhabdomyolyse. Aufgrund des zeitlichen Zusammenhanges wurde die Verdachtsdiagnose eines MNS gestellt und nach $24 \mathrm{~h}$ eine Therapie mit Dantrolene i.v. (10 $\mathrm{mg} / \mathrm{kg} / \mathrm{Tag}$ in 4 ED) begonnen. Darunter kam das fulminant begonnene Krankheitsbild zum Stillstand, die bis dahin therapeutisch kaum beeinflussbare Hyperthermie besserte sich rasch. Wiederholt abgenommene Blutkulturen blieben steril und eine breite antibiotische Abdeckung bestand bereits vor Beginn der Symptomatik, sonstige Entzündungsparameter zeigten sich nicht. Für eine Maligne Hyperthermie fand sich im kein Trigger, auch Medikamente, die ein Serotonin-Syndrom auslösen können, wurden nicht verabreicht.

Schlussfolgerung. In Zusammenschau der Klinik und Laborwerte erscheint ein malignes neuroleptisches Syndrom als wahrscheinlichste Differentialdiagnose der akuten klinischen Verschlechterung unseres Patienten vorgelegen zu haben. Bei Vorliegen einer rapiden Verschlechterung mit der geschilderten Symptomatik sollte an die Möglichkeit eines MNS gedacht und eine Behandlung eingeleitet werden. 


\section{P033}

Therapie des Pleuraempyems bei Kindern - Stellenwert der frühen Fibrinolyse-Therapie

S. Wien', T. Hoppen², M. Hürtgen³ , T. Nüßlein ${ }^{4}$

${ }^{1}$ Universitätsklinik Zentrum f. Kinderheilkunde, Abteilung für Allgemeine Pädiatrie, Bonn, Deutschland, ${ }^{2}$ Gemeinschaftsklinikum Koblenz-Mayen, Kemperhof Koblenz, Klinik für Kinder- und Jugendmedizin, Koblenz, Deutschland, ${ }^{3}$ Katholisches Klinikum Marienhof/St. Joseph gGmbH, Koblenz, Thoraxchirurgie, Koblenz, Deutschland, ${ }^{4}$ Gemeinschaftsklinikum Kemperhof, Klinik für Kinderund Jugendmedizin, Koblenz, Deutschland

Fragestellung. Die Inzidenz von Pleuraempyemen bei Kindern steigt. Bei geringer Mortalität ist die Inzidenz der Komorbidität hoch. Die Drainage des Pleuraraums mit oder ohne Fibrinolyse sowie die Video-Assisted Thoracoscopic Surgery (VATS) sind etablierte Therapien. Das Vorgehen ist oft abhängig von der Verfügbarkeit der Methoden. Ziel der Studie war die retrospektive Überprüfung beider Behandlungswege in einer Klinik, in der sowohl die konservative als auch die operative Behandlung zur Verfügung stehen.

Material und Methode. Eingeschlossen wurden retrospektiv alle pädiatrischen Patienten, die zwischen Dezember 2007 und April 2011 aufgrund der Diagnose eines Pleuraempyems mittels Fibrinolyse und/oder VATS behandelt worden waren. Der primäre Endpunkt war die Dauer des Klinikaufenthalts. Weiterhin wurden die Zeit von der Aufnahme bis zum ersten Ultraschall des Pleuraraums sowie der Zeitpunkt der ersten Pleuradrainage bzw. Fibrinolyse und/oder VATS erhoben.

Ergebnisse. Es wurden zunächst retrospektiv 5 Kinder im Alter von 3-12 Jahren in die Arbeit eingeschlossen. Patient 1 wurde primär mit VATS, die Patienten 2-4 primär mit Pleuradrainage behandelt. In zwei Fällen wurde die Drainage unmittelbar mit Fibrinolyse kombiniert, in zwei Fällen nach 3 bzw. 4 Tagen ausschließlicher Drainage. Alle vier mittels primärer Drainage/Fibrinolyse behandelten Patienten wurden schließlich einer VATS unterzogen. Die Hospitalisierungsdauer variierte zwischen 9 und 36 Tagen, der Zeitraum von der Aufnahme bis zur Intervention zwischen 3 und 13 Tagen. In allen Fällen war innerhalb von 24 Stunden nach Aufnahme ein Pleura-Ultraschall durchgeführt worden. Patient 6 wurde entsprechend eines 2012 etablierten internen Behandlungsalgorithmus therapiert, der aus den Behandlungsergebnissen der Patienten 1-5 sowie einer Literaturrecherche abgeleitet wurde. Es wurde noch am Aufnahmetag eine Fibrinolyse begonnen. Trotz ausgedehnter Empyeme war eine ausschließliche Fibrinolyse erfolgreich und die Entlassung nach 14 Tagen möglich.

Diskussion. Im Vergleich der Daten der Patienten 1-5 mit denen aktueller Studien fiel eine deutlich längere stationäre Verweildauer auf. Das nach Auswertung der Patienten 1-5 sowie einer Literaturrecherche etablierte Protokoll sieht unter anderem tägliche sonographische Kontrollen bei schwerer Pneumonie sowie den frühzeitigen Beginn einer Pleuradrainage mit sofortiger Fibrinolyse bei Detektion eines Empyems vor. Das gute Ergebnis des Patienten 6 zeigt, dass primär forciertes konservatives Vorgehen der Kombination aus Drainage und nachfolgender VATS überlegen sein kann. Voraussetzung hierfür ist ein dezidierter interner Standard. Schlussfolgerung. Bei Pleuraempyemen im Kindesalter kann die sofortige Fibrinolyse die Dauer der stationären Behandlung signifikant verkürzen und eine VATS ersparen.

\section{P034}

Erfolgreiche Lysetherapie einer fulminanten beidseitigen Lungenembolie bei einer 16-jährigen Kaderathletin

T. Hoppen', S. Schmitt-Kästner', R. Douwa'2, D. Fatehpour ${ }^{2}$, R. Huth ${ }^{3}$, W. Bojara ${ }^{2}$, T. Nüßlein ${ }^{1}$

'Gemeinschaftsklinikum Koblenz-Mayen, Kemperhof Koblenz, Klinik für Kinder- und Jugendmedizin, Koblenz, Deutschland, ${ }^{2}$ Gemeinschaftsklinikum Koblenz-Mayen, Kemperhof Koblenz, Klinik für Innere Medizin II (Schwerpunkt Kardiologie), Koblenz, Deutschland, ${ }^{3}$ Zentrum für Kinder- und Jugendmedizin, Universitätsmedizin Mainz, Interdisziplinäre Kinderintensivstation AE², Mainz, Deutschland

Einleitung. Die Lungenembolie ist eine partielle oder vollständige Verlegung der Lungenarterien durch eingeschwemmte Blutgerinnsel aus der peripheren venösen Strombahn. Die genaue Inzidenz im Kindes- und Jugendalter ist unbekannt.

Fallbeschreibung. Eine 16-jährige Jugendbundesliga-Fußballspielerin wurde mit akuten Thoraxschmerzen, Husten, Ruhedyspnoe und Tachykardie vorgestellt. Ihre körperliche Leistungsfähigkeit sei seit 2 Wochen vermindert. Als Aknetherapeutikum nahm Sie nach ärztlicher Anordnung seit 18 Monaten täglich ein Kombinationspräparat bestehend aus 0,03 mg Ethinylestradiol und $2 \mathrm{mg}$ Dienogest ein. Ein 3/6 Systolikum war auskultierbar, der Blutdruck betrug minimal 99/48 $\mathrm{mmHg}$. Die kapilläre Blutgasanalyse ergab einen $\mathrm{pH}$ von 7,46 bei einem $\mathrm{pCO}_{2}$ von 28 und einem $\mathrm{pO}_{2}$ von $48 \mathrm{mmHg}$. Im EKG zeigte sich ein Rechtstyp. Das Troponin I betrug 1,01 ng/ml, die D-Dimere 1,31 mg/l. Die transthorakale Echokardiographie zeigte eine deutliche rechtsventrikuläre Dysfunktion (Dilatation, Hypokinesie und Druckbelastung) mit einem über die Trikuspidalklappeninsuffizienz abgeschätzten maximalen systolischen pulmonalarteriellen Druck von $46 \mathrm{mmHg}+$ ZVD. Die Verdachtsdiagnose einer Lungenembolie wurde gestellt. In der Mehrschicht-Spiral-Computertomographie-Angiographie kam eine ausgedehnte inkomplette Thrombosierung der linken Arteria pulmonalis sowie rechtsseitig im Aufzweigungsgebiet der Lappenarterien zur Darstellung. Unmittelbar erfolgte eine rekanalisierende Therapie mit einem rekombinantem Gewebe-Plasminogenaktivator (Alteplase: Bolus von $10 \mathrm{mg}$, gefolgt von $50 \mathrm{mg} / 2 \mathrm{~h}$ ). In der Kontroll-Echokardiographie dokumentierte sich eine Befundnormalisierung. Die systemische Antikoagulation wurde über 2 Tage mit unfraktioniertem Heparin (Bolus $5000 \mathrm{E}$, gefolgt von $25 \mathrm{E} /$ $\mathrm{kgKG} / \mathrm{h}$ ) und anschließend mit $2 \mathrm{mg} / \mathrm{kgKG} /$ Tag Enoxaparin auf 2 Gaben unter Faktor Anti Xa-Spiegel-Kontrolle durchgeführt. Nach 7 Tagen wurde eine antithrombotische Sekundärprophylaxe mit Phenprocoumon eingeleitet. Innerhalb von 2 Stunden nach Abschluss der komplikationslosen Lysetherapie kam es zur deutlichen klinischen Besserung. Eine Sauerstoffanreicherung der Atemluft war nicht mehr erforderlich. Die Patientin wurde rasch wieder voll mobilisiert und war bis auf ein intermittierendes Schwindelgefühl beschwerdefrei. Die Thrombophiliediagnostik ist bislang noch nicht abgeschlossen. Weder in der Duplexsonographie des gesamten oberflächlichen und tiefen Venensystems noch in der MR-Phlebographie des Abdomes und der Hüfte (nach Lysetherapie) ließ sich ein Thrombus detektieren.

Schlussfolgerung. Beim hämodynamisch instabilen Kind kann die Echokardiographie und/oder die MS-Spiral-CTA rasch zur Diagnosestellung einer Lungenembolie führen. Im beschriebenen Fall war die Rekanalisierung mittels systemischer Thrombolyse erfolgreich. Neben Auffälligkeiten im Thrombophilie-Screening haben orale Kontrazeptiva einen hohen Stellenwert als erworbene Risikofaktoren für eine Lungenembolie bzw. Venenthrombose. 
P035

Kongenitales kaposiformes Hämangioendotheliom mit KasabachMerritt-Phänomen - Kasuistik

\section{K. Heimann', M. Schoberer', S. Trepels-Kottek', E. Verjans', L. Lassay², T. Orlikowsky'}

'Uniklinik RWTH Aachen, Klinik für Kinder- und Jugendmedizin Sektion Neonatologie, Aachen, Deutschland, ${ }^{2}$ Uniklinik RWTH Aachen, Klinik für Kinder- und Jugendmedizin, Sektion für Pädiatrische Hämatologie, Onkologie und Stammzelltransplantation, Aachen, Deutschland

Einleitung. Kaposiforme Hämangioendotheliome (KHE) sind sehr seltene, niedrigmaligne Gefäßtumoren des Kindesalters. Durch einen mechanischen Thrombozytenverbrauch und eine Induktion der plasmatischen Gerinnung, bezeichnet als Kasabach-Merritt-Phänomen (KMP), können die Tumoren lebensbedrohliche Blutungskomplikationen induzieren. Die Entität ist von den infantilen Hämangiomen distinkt, aber eng mit den sogenannten Büschel-Angiomen („,tufted angioma“, TA) verwandt. Die Raumforderungen sind überwiegend in der Haut bzw. im Unterhautgewebe angelegt, können die Muskulatur und auch die Knochen infiltrieren oder auch ausschließlich dort angelegt sein. Ferner auch im Mediastinum oder Retroperitoneum, zum Teil mit Infiltration der lokalen Organe. Der Aspekt ist rötlich bis livide, der Palpationsbefund prall-induriert oder Plaque-artig. Therapieziel ist neben der Tumorverkleinerung vor allem eine Unterdrückung des KasabachMerritt-Phänomens. Neben Kortikosteroiden und ASS hat sich in der Therapie Vincristin bewährt, in jüngerer Zeit auch Sirolimus. Das Therapieansprechen auf Propranolol ist variabel.

Kasuistik. Das AGA Reifgeborene $(39+4$ Schwangerschaftswochen, 3830 g Geburtsgewicht) wurde postnatal durch unseren Transportdienst aus einer peripheren Geburtsklinik in unsere Einrichtung verlegt. Eine erhebliche, prall-elastische, rötlich-livide Auftreibung des rechten Ober- und Unterarms einschließlich der rechten Schulter (bei normalkalibriger Hand) hatten zu einer protrahierten Entwicklung des Kindes geführt, der Nabelschnur-Arterien-pH zeigte mit 7,12 eine Azidose, während die APGAR-Werte mit 9/9/10 (Vergabe durch Geburtshelfer) für eine gute Adaptation sprachen. Wir beobachteten in den ersten 3 Lebenstagen einen Abfall der Thrombozytenzahl auf 47 G/l, ferner bestand eine erhebliche Erhöhung der D-Dimere (max. $22.691 \mathrm{ng} / \mathrm{ml}$ ). Wir führten über 3 Wochen eine Therapie mit ASS und Prednisolon durch, unter der sich die Thrombozytenzahl und die D-Dimere normalisierten und der Armumfang um $5 \mathrm{~cm}$ abnahm. Dann überlappend Einführung von Sirolimus in die Therapie. Bei sekundärer Größenzunahme des Tumors Erweiterung der Therapie um Vincristin ab der 6. Woche nach histologischer Diagnosesicherung aus einer Biopsie. Ein Therapieversuch mit Propranolol war wirkungslos.

Diskussion und Schlussfolgerung. Unerkannte fetale Hämangioendotheliome können insbesondere bei Lokalisation an der oberen Extremität erhebliche Geburtshindernisse darstellen. Schon in den ersten Lebenstagen sind die Neugeborenen durch Blutungskomplikationen als Folge des Kasabach-Merritt-Phänomens vital gefährdet. Die Therapie mit Steroiden und ASS führt zu einer raschen, aber häufig nicht dauerhaften Erholung der Thrombozytenzahlen. Vincristin hat sich in unserem Fall dem Therapieansprechen auf Sirolimus deutlich überlegen gezeigt, Propranolol war nicht geeignet, die Vincristin-Intervalle zu verlängern. Das Kind wird derzeit hinsichtlich einer laser-chirurgischen Therapieoption evaluiert.

\section{P036}

\section{Posteriores reversibles Enzephalopathie-Syndrom (PRES):} Konsequenzen für die pädiatrische Intensivmedizin

\section{P. Felsecker', A. Vierzig', C. Taylan', B. Roth}

'Univ.-Kinderklinik, Neonatologie und Pädiatrische Intensivmedizin, Köln, Deutschland, ²Univ.-Kinderklinik, Pädiatrische Nephrologie, Köln, Deutschland

Einleitung. Das posteriore reversible Enzephalopathie-Syndrom (PRES) ist eine seltene intensiv-medizinische Erkrankung, die mit Vigilanz- minderung, Sehstörungen, Krampfanfällen und fokalen neurologischen Defiziten einhergehen kann. Als radiologisches Korrelat findet man Veränderungen der weißen und grauen Substanz im Sinne eines vasogenen Ödems meist im posterioren okzipitoparietalen Marklager jedoch auch an anderen Lokalisationen. Die Ätiologie und Pathogenese sind nicht ausreichend geklärt. Eine hypertensiv bedingte Enzephalopathie oder eine neurotoxisch verursachte Endothelschädigung mit Blut-Hirn-Schrankenstörung werden diskutiert. Insbesondere Nierenerkrankungen mit art. Hypertonie und Hypalbuminämie aber auch Autoimmunerkrankungen und immunsuppressive/zytotoxische Therapie gelten als Risikofaktoren. In den letzten Jahren beobachteten wir 3 nephrologische Patienten mit PRES. Am Beispiel des letzten Falls möchten wir über dieses Krankheitsbild berichten.

Fallbeschreibung. 4,5 Jahre alter männlicher Patient wurde bei postinfektiöser Glomerulonephritis anurisch und mit arterieller Hypertonie aufgenommen. RR bis 180/100 $\mathrm{mmHg}$.

Therapie. Dialyse sowie Antihypertensiva Amlodipin, Metoprolol, Dihydralazin. Am 17. Tag der Behandlung traten Erbrechen, progrediente Amaurosis, Somnolenz auf. RR 160//110 mmHg, keine Ödeme jedoch persistierende Hypalbuminämie. Das MRT des Gehirns zeigte ausgedehnte seitensymmetrische FLAIR-Signalanhebung beidseits parietookzipital, die beim Vorliegen der klinischen Symptomatik mit dem Bild einer PRES vereinbar waren. EEG: keine Krampfäquivalente, Enzephalopathie. Bei GCS von 7 Intubation, Analgosedierug und Eskalation der Therapie mit Clonidin und Urapidil-Dauerinfusion. Extubation nach $72 \mathrm{~h}$ bei normotonem Blutdruck und vollständig normalisiertem neurologischem Befund.

Diskussion. Bei nephrologischen Patienten mit art. Hypertonie und neurologischen Symptomen muss ein PRES erwogen werden. Da die Symptome unspezifisch sind sollte rasch zur Diagnosestellung MRT herangezogen werden. Differenzialdiagnosen wie Infarkte, Blutungen, andere Enzephalopathie -Syndrome müssen ausgeschlossen werden um weitere unnötige diagnostische Schritte zu verhindern und rasche Therapie einzuleiten. Diese beruht auf einer unverzüglichen Kontrolle des Blutdrucks und/oder Beseitigung der schädigenden Noxen unter intensivmedizinischer Überwachung. Dies hat eine große Bedeutung für die Prognose der Erkrankung, da die Reversibilität der Befunde alleine von dieser konsequenten Behandlung abhängt. Prompte Therapieeinleitung kann ein Übergang in irreversibles zytotoxisches Ödem und damit bleibende Defekte verhindern.

1. Ozcakar ZB et al (2004) Eur J Pediatr

2. Onder AM et al (2007) Pediatr Nephrol

3. Yamada A et al (2012) Clin Nephrol

4. Gupta Set al (2010) Indian Pediatr

5. Pirker A et al (2011) AJNR Am J Neuroradiol

6. Sfahi Let al (2013) Arch Pediatr 20:633-636

7. Lamy CL et al (2014) Handb Clin Neurol

8. Berrouschot J, Haase U (2010) DIVI

\section{P037}

Intoxikation als seltene Ursache einer Atemstörung bei einem 2-monatigen Säugling

\section{A. Beyersdorff', M. Madi', M. Heckmann'}

'Universitätsmedizin, Kinder- und Jugendmedizin, Greifswald, Deutschland

Einleitung. Eine akute Intoxikation ist bei einem 2 Monate alten Säugling eine extrem seltene Ursache einer Atemstörung.

Fallbericht. Die ambulante Vorstellung des 2 Monate alten, bisher gesunden, eutrophen Säuglings erfolgte wegen Obstipation (teilgestillt, 2 Tage kein Stuhlgang, sonst mehrmals täglich, weich). Bei unauffälligem Abdomenstatus fiel jedoch eine anstoßende Atmung auf. Bei Übernahme auf die ITS zeigte sich ein eingeschränktes Bewusstsein (GCS 6-10) mit Schnappatmung und Miosis, Blutgase normal, BZ $10 \mathrm{mmol} / \mathrm{l}$. Anamnestisch ergab sich zunächst kein Hinweis auf Trauma oder Ingestion. Die Atemunterstützung erfolgte mit Optiflow, und bei V. a. 
Late-onset-Sepsis wurde eine antibiotische Behandlung begonnen. Infektionsparameter einschl. Liquoruntersuchung, Blut-, Urinkultur waren negativ. Ein cMRT zeigte einen Normalbefund. Die erneute Medikamentenanamnese ergab: Sab simplex, Vigantolöl, Carum carvi Zäpfchen (Atropa belladonna) bei Blähungen ein halbes am Vortag und am Tag davor, seit ca. 1 Monat morgens tgl. 5 Tr. BiGaia (Lactobacillus reuteri), am Vortag Verschreibung eines homöopathischen Mittels beim Kinderarzt und am Aufnahmetag Einlauf zum Abführen. Ein toxikologisches Screening im Urin war negativ, Ammoniak normal. Die Augenarztuntersuchung ergab außer der konstant bestehenden Miosis einen Normalbefund. Das EEG zeigte Zeichen des Tiefschlafs ohne Anfallsaktivität. Im weiteren Verlauf HF $80-100 / \mathrm{min}, \mathrm{RR}$ im unteren NB, schnelles Auskühlen des Kindes, Bradypnoe, weiterhin kein Sauerstoffbedarf, Blutgase normal, GCS 10. $7 \mathrm{~h}$ nach Aufnahme berichtete die Mutter, sie habe statt BiGaia-Tropfen fälschlicherweise Alphagan $2 \%$ Augentropfen p.o. gegeben (Brimonidin, alpha-2-Agonist). Die Symptome bei Brimonidinvergiftung sind Bradypnoe bis respiratorische Insuffizienz, Tiefschlaf, teils Krampfanfälle, Hyperglykämie, Miosis, Hypothermie, Bradykardie, Hypotonie. Bei Fehlen eines Antidots ist die Therapie symptomatisch. Die HWZ von Brimonidin ist $2,7 \mathrm{~h}$, das selbstlimitierendes Ende der Symptomatik ist nach 6-24 h zu erwarten. Der Patient erwachte nach $8 \mathrm{~h}$ spontan.

Schlussfolgerung. Auch bei einem 2 Monate alten Säugling muss eine Intoxikation differentialdiagnostisch bei Atem- und Bewusstseinsstörung in Erwägung gezogen werden. Insbesondere zusätzliche Symptome wie Miosis, Hyperglykämie und Temperaturregulationsstörung weisen in diesem Fall darauf hin. Die Medikamentenanamnese muss ggf. mehrfach wiederholt werden, und alle verabreichten Medikamente und Substanzen müssen von den Angehörigen vorgelegt werden.

\section{P038}

Evaluierung der Bilanzgenauigkeit der Prismaflex ${ }^{\circledR}$ Pumpe mit dem Prismaflex ${ }^{\circledR}$ HF 20 Set bei der Nierenersatztherapie von pädiatrischen Patienten

\section{S. Rödl', R. Ulreich', S. Ring', I. Marschitz ${ }^{2}$ \\ ${ }^{1}$ Universitätsklinik für Kinder und Jugendheilkunde, Pädiatrische Intensiv- station, Graz, Österreich, ${ }^{2}$ Universitätsklinik für Kinder- und Jugendheilkunde Salzburg, Neonatologie, Salzburg, Österreich}

Hintergrund. Eine exakte Flüssigkeitsbilanzierung hat für Erfolg und Sicherheit der Nierenersatztherapie gerade bei Säuglingen und Kleinkindern mit niedrigem Körpergewicht höchsten Stellenwert. Die Prismaflex $^{\oplus}$ Pumpe (PF) in Verbindung mit dem Prismaflex HF2o Set (HF2o) ist von Seiten des Füllvolumens und der möglichen Flusseinstellungen für die Behandlung von Säuglingen geeignet. Eine Studie über die erforderliche Bilanzgenauigkeit des Systems im Echtbetrieb liegt jedoch bis dato noch nicht vor.

Fragestellung. Gegenüberstellung von durch Wägung ermittelten Bilanzen und den durch die Maschine berechneten Bilanzen im In-vivound In-vitro-Modell.

Patienten und Methoden. Von 2009 bis 2014 wurden an unserer pädiatrischen Intensivstation 6 Kleinkinder mit chronischem Nierenversagen mittels Hämodiafiltration mit PF und HF2o 1200-mal behandelt. Bei diesen wurde die durch Patientenwägung vor und nach der Behandlung ermittelte Bilanz mit der von der PF gemessenen Bilanz korreliert. Als Ergänzung zu der Patientenstudie, um die nicht dokumentierten Gewichtsänderungen wie Stuhl, feste Nahrung und Naschen auszuschließen, wurde der Patient für den in vitro Test durch einen $250 \mathrm{ml}$ Kochsalzbeutel ersetzt. Die Wägungen dieses Beutels erfolgten stündlich über 24 Stunden unter simulierter Therapie. Insgesamt wurde die in vitro Testanordnung 10-mal unter bettseitigen Bedingungen durchgeführt.

Ergebnisse. Bei dem Vergleich der Patientengewichte mit der von der PF gemessenen Bilanz zeigte sich eine mittlere Differenz von $-7 \pm 4$ g. Wenn man die Stuhl und Ernährungsabweichungen der Patienten mit einbezieht so zeigen sich für die einzelnen Patienten folgende Abweichungen: Pat1: $-21 \pm 17$ g; Pat 2: $-10 \pm 5$ g; Pat 3: $57 \pm 22$ g; Pat 4: $-2 \pm 6$ g; Pat 5: $47 \pm 21 \mathrm{~g}$; Pat 6: $-4 \pm 8 \mathrm{~g}$; Bei dem In-vitro-Test zeigt sich nach 12 Stunden eine mittlere Abweichung von $4( \pm 8)$ g bei einer maximalen Abweichung von $17 \mathrm{~g}$. Nach $24 \mathrm{~h}$ war die mittlere Abweichung $10( \pm 9) \mathrm{g}$ bei einer maximalen Abweichung von $19 \mathrm{~g}$.

Schlussfolgerung. Die gemessenen Gewichtsabweichungen sind mit der notwendigen Präzision zur Behandlung von Säuglingen kompatibel.

\section{Klinische Pharmakologie}

\section{P039a}

Prenatal therapy in developmental disorders: drug targeting via intra-amniotic injection to treat X-linked hypohidrotic ectodermal dysplasia

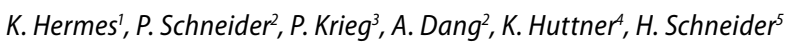

'Universitäts-Kinderklinik, Erlangen, Deutschland, ${ }^{2}$ University of Lausanne, Lausanne, Schweiz, ${ }^{3}$ Deutsches Krebsforschungszentrum, Heidelberg, Deutschland, ${ }^{4}$ Edimer Pharmaceuticals, Cambridge, MA, USA, ${ }^{5}$ Kinder- und Jugendklinik, Universitätsklinikum Erlangen, Experimentelle Perinatalmedizin, Erlangen, Deutschland

Background. Pathologies associated with genetic disorders can irremediably affect fetuses, making early stage therapies desirable. Antenatal maternal drug administration, however, exposes mothers to potential drug toxicity and is limited by the variability in transplacental drug delivery. Alternative approaches to fetal treatment should entail low risk drug delivery with reproducible pharmacokinetics. X-linked hypohidrotic ectodermal dysplasia (XLHED), the most common inherited disorder of ectoderm development, is caused by a lack of the signaling molecule ectodysplasin A1 (EDA1).

Methods. Here we report correction of XLHED in the Tabby mouse model through intra-amniotic injection of an EDA1 replacement molecule. In wild-type mice, a single intra-amniotic injection of Fc-EDA1 at embryonic day 15 resulted in substantial fetal uptake with minimal maternal exposure. In Tabby fetuses, this well-tolerated treatment restored normal ectoderm development in a dose-dependent manner. This approach may represent a novel paradigm for treatment of disorders in early human development.

\section{P039}

Befragung der Mitarbeiter einer Kinderintensivstation zu dem Thema Fehler im Umgang mit i.v.-Arzneimitteln - Vergleich der subjektiven Einschätzung und objektiven Beobachtung

\section{E. Kohl', T. Hermanspann ${ }^{2}$, M. Schoberer', T. Orlikowsky', A. Eisert ${ }^{2}$}

'Universitätsklinikum Aachen, Klinik für Kinder- und Jugendmedizin, Sektion Neonatologie und Pädiatrische Intensivmedizin, Aachen, Deutschland, ${ }^{2} \mathrm{Apo}-$ theke des Universitätsklinikums Aachen, Aachen, Deutschland

Ziel. Mittels einer Mitarbeiterbefragung zum Thema Fehler bei der Herstellung und Applikation von intravenösen Arzneimittel soll die Situation auf Station aus Sicht der Pflegenden abgebildet, Ursachen für Fehler identifiziert und der Nutzen von Strategien zur Fehlerreduktion evaluiert werden. Zusätzlich soll ein Vergleich der Angaben der Mitarbeiter mit den Daten einer Beobachtungsstudie zu diesem Thema die Unterschiede in der subjektiven und objektiven Wahrnehmung untersuchen.

Methode. Befragt werden die Mitarbeiter der Kinderintensivstation des UK Aachen u. a. über ihre generelle Berufserfahrung, Fehler im Umgang mit i.v.-Medikamenten, deren Ursache und welche Maßnahmen sinnvoll sind, um den Ist-Zustand auf Station zu optimieren. Die Ergebnisse einer vorher durchgeführten Beobachtungsstudie werden 
hinzugezogen, um sie mit den Daten der Mitarbeiterbefragung zu vergleichen.

Ergebnisse. Bei den Befragten handelt es sich zu 35\% um Fachpfleger für Intensivmedizin, $72,2 \%$ haben im Rahmen ihrer Ausbildung eine Lehreinheit zu dem Thema Herstellung von Arzneimitteln absolviert und $70 \%$ fühlen sich sicher im Umgang mit i.v.-Medikamenten. Nach Einschätzung des Pflegepersonals sind die häufigsten Fehler im Umgang mit i.v.-Arzneimitteln die fehlerhafte Verdünnung, Verabreichung falscher Dosierungen und unvollständige Etikettierung. Dagegen treten die meisten Fehler nach Datenlage der Studie u. a. durch falsche Applikationsgeschwindigkeit, Abweichung zu dem vom Arzt verordneten Applikationszeitpunkt von mehr als $1 \mathrm{~h}$ und unzureichende Durchmischung auf. Als Ursache für Fehler werden am häufigsten Stress, unzureichende Arbeitsfläche und hohes Verhältnis von Patienten pro Pflegekraft genannt. Einen großen Nutzen sehen die Befragten u. a. in der Bereitstellung von applikationsfertigen Infusionslösungen durch die Apotheke.

Diskussion. Die kritische Selbsteinschätzung des Pflegepersonals ist ein wertvolles Instrument der im Stationsalltag auftretenden Problematik im Umgang mit i.v.-Arzneimitteln. Ähnliche Ergebnisse konnten auf einer „Erwachsenen-Intensivstation“ beobachtet werden [1]. Die Selbsteinschätzung der Pflege unterscheidet sich deutlich von den tatsächlich beobachteten Fehlern. Die Antworten geben Aufschluss darüber, in welchen Bereichen sich die Pflege unsicher fühlt.

Schlussfolgerung. Sinnvolle Maßnahmen sind zu ergreifen, um die Arzneimitteltherapiesicherheit im Bereich der Herstellung und Applikation zu erhöhen. Dazu zählen u. a. Bereitstellung applikationsfertiger Perfusoren, Schaffung optimaler Arbeitsbedingungen, Mitarbeiterschulungen in der Arzneimittelzubereitung und Fortbildungen, um das Risikobewusstsein zu stärken.

1. Literatur beim Verfasser

\section{P040 \\ Maternale Chemotherapie als mögliche Ursache für angeborene Dysphagie}

\section{K. Schneider', N. Hepping', W. Garbe', J. Schmitt ${ }^{2}$}

${ }^{1}$ St. Marien-Hospital Bonn, Neonatologie, Bonn, Deutschland, ${ }^{2}$ St. Marien-Hospital, Kinderklinik, Bonn, Deutschland

Hintergrund. Patientinnen mit schwangerschaftsassoziiertem Brustkrebs werden heute hinsichtlich einer Systemtherapie genauso wie nicht schwangere Patientinnen behandelt. Aktuelle Studien zeigen, dass eine Chemotherapie ab dem 2. Trimenon kein signifikant erhöhtes Risiko für den Fetus darstellt. Jedoch sind die Fallzahlen hier gering, die Therapieprotokolle heterogen.

Kasuistik. Vorgestellt wird ein reifes Neugeborenes mit isolierter, angeborener Dysphagie, die sich bereits pränatal durch ein maternales Polyhydramnion manifestierte. Der Fetus war intrauterin bei metastasiertem Mammakarzinom der Mutter von der 17. bis zur 32. Schwangerschaftswoche (SSW) einer Chemotherapie mit Capecitabin und bis zur 20. SSW einer monoklonalen Antikörpertherapie mit Trastuzumab ausgesetzt. Trotz logopädischer Stimulationstherapie war postnatal bei fehlendem Schluckreflex kein Schluckakt zu erzielen, so dass das Kind dauerhaft oropharyngeal abgesaugt werden musste und die Ernährung nur über eine PEG-Sonde erfolgen konnte. Dennoch bestanden weiterhin sekretbedingte tiefe Desaturationen und Bradykardien sowie chronische Aspirationspneumonien. Die sonstige neurologische Entwicklung verlief altersentsprechend, eine Magnetresonanztomographie des Kopfes erbrachte keine Auffälligkeiten. Ab dem 6. Lebensmonat kam es zum sukzessiven Einsetzen einer suffizienten Schluckfunktion, so dass die PEG-Sonde entfernt und das Kind seit dem 9. Lebensmonat voll oral ernährt werden kann.

Diskussion. Eine isolierte, angeborene Dysphagie bei einem ansonsten neurologisch altersentsprechend entwickelten Neugeborenen ist eine
Rarität. Da in der umfangreichen postnatalen Diagnostik die bereits pränatal diagnostizierte Störung keinem umschriebenen Krankheitsbild zugeordnet werden konnte und es sich um ein passageres Phänomen handelte, ist ein Zusammenhang zwischen maternaler Therapie und neonataler Morbidität naheliegend. Eine Interaktion mit einem neurotoxischen Medikament wie dem Capecitabin muss hier in Betracht gezogen werden.

Schlussfolgerung. Die Embryotoxizität von Chemotherapeutika ist weitreichend erforscht, wenig ist jedoch bekannt über die akuten neurotoxischen Effekte, denen ein Fetus während einer Chemotherapie der Mutter in der Schwangerschaft ausgesetzt ist.

\section{P041}

Konnataler intermittierender AV-Block III ${ }^{\circ}$ nach Retinoideinnahme in der Frühschwangerschaft

\section{S. Nijhof', P. Kutz', T. Menke', C. Roll}

'Vestische Kinder- und Jugendklinik Datteln, Universität Witten/Herdecke, Neonatologie und pädiatrische Intensivmedizin, Datteln, Deutschland, ${ }^{2}$ Vestische Kinder- und Jugendklinik Datteln, Universität Witten/Herdecke, Kinderkardiologie, Datteln, Deutschland

Hintergrund. Retinoide gehören zu den stärksten teratogen wirkenden Arzneimitteln. Nach Exposition im 1. Trimenon kommt es zu einer erhöhten Spontanabortrate und bei 30-50\% der Feten zu Fehlbildungen.

Fallbericht. Wir präsentieren ein weibliches, eutrophes Frühgeborenes (GA 35 Wochen) mit konnatalem intermittierendem AV-Block III'. Wegen Dyskeratosis follicularis (M. Darier) wurde die Mutter bis zur Feststellung der Schwangerschaft in der 12. Woche mit Acitretin $25 \mathrm{mg} / \mathrm{Tag}$ behandelt. Die Entbindung erfolgte wegen fetaler Bradykardie per Sectio, Apgar 9/10/10. In den folgenden Tagen hatte das Kind immer wieder Phasen von Bradyarrythmien mit Herzfrequenzen von minimal bis 55 bpm und das EKG eines AV Block III $^{\circ}$ mit AV-Knoten-Ersatzrhythmus bei stabilem Allgemeinzustand. Die Echokardiographie zeigte lediglich einen ASD II und ein PFO. Ab der zweiten Lebenswoche verkürzten sich die bradykarden Phasen auf wenige Sekunden, sodass bei fehlender Kreislaufbeeinträchtigung und stabilem Allgemeinzustand keine Indikation zur Schrittmacherimplantation bestand. Bei Entlassung (20. Lebenstag) wurde eine Heimmonitorüberwachung initiiert. Diskussion. Die häufigste Ursache des konnatalen AV-Blockes III $^{\circ}$ ist der neonatale Lupus erythematodes durch diaplazentar übertragene maternale Autoantikörper. Weitere Ursachen sind Myokarditiden, strukturelle Defekte und genetische Erkrankungen. In unserem Fall wurden keine mütterlichen Lupus-AK nachgewiesen, keine kardiotropen Viren beim Kind, kein Hinweis auf eine genetische Ätiologie. Nach unserer Kenntnis gibt es bisher keinen berichteten Fall eines isolierten intermittierenden AV-Block III $^{\circ}$ nach Retinoideinnahme in der Frühschwangerschaft. Nach Retinoidexposition in der Schwangerschaft wurden jedoch strukturelle kardiale Veränderungen wie Fallot-Tetralogie, TGA, Septumdefekte und Aortenbogenhypoplasie beschrieben. Acitretin und sein Metabolit Etretinat haben im Vergleich zu Isotretinoin eine wesentlich längere Halbwertszeit (mehrere Monate). Daher wird eine Frist von zwei Jahren zwischen Therapieende und Konzeption empfohlen (bei Isotretinoin nur vier Wochen).

Schlussfolgerung. Wir berichten den ersten Fall eines konnatalen intermittierenden $\mathrm{AV}$-Block $\mathrm{III}^{\circ}$ nach Retinoidexposition in der Frühschwangerschaft, wobei wir die Kausalität zwischen der Retinoidexposition und der Herzrhythmusstörung nicht beweisen können. 
P042

Analyse der Fehler bei Herstellung und Applikation intravenöser Arzneimittelzubereitungen auf einer Kinderintensivstation Aspekte der Arzneimitteltherapiesicherheit (AMTS)

\section{E. Kohl', T. Hermanspann ${ }^{2}$, M. Schoberer', T. Orlikowsky', A. Eisert ${ }^{2}$}

'Universitätsklinikum Aachen, Klinik für Kinder- und Jugendmedizin, Sektion Neonatologie und Pädiatrische Intensivmedizin, Aachen, Deutschland, ${ }^{2}$ Apotheke des Universitätsklinikums Aachen, Aachen, Deutschland

Ziel. Das Ziel der Studie war, ob, welche und wie häufig Fehler bei der Herstellung und Applikation intravenöser Arzneimittel auf der Kinderintensivstation des Universitätsklinikums Aachen beobachtet werden. Es sollten Maßnahmen implementiert werden, die zu einer Fehler- und Risikoreduktion führen.

Methode. Das Pflegepersonal der Kinderintensivstation wurde durch eine Person, die nicht zum pädiatrischen Team gehörte, bei der routinemäßigen Zubereitung und Applikation intravenöser Arzneimitte beobachtet. Abweichungen zu der ärztlichen Verordnung, hausinterner Regelungen und Herstellerangaben wurden als Fehler gewertet.

Ergebnisse. Insgesamt wurden 348 Arzneimittelzubereitungen beobachtet. In 7,5\% der Fälle wurden die Zubereitungen unzureichend durchmischt, in 6,5\% war die Applikationsgeschwindigkeit außerhalb der vorgegebenen Zeit und weitere 6,5\% der Zubereitungen wurden über $1 \mathrm{~h}$ später appliziert als vom Arzt angeordnet. Die aseptische Arbeitsweise und die Etikettierung wiesen nur eine sehr geringe Fehlerhäufigkeit auf. Es wurde immer das richtige Arzneimittel verwendet und in allen beobachteten Fällen vollständig rekonstituiert.

Diskussion. Im Vergleich zu ähnlichen Studien, die über Fehlerraten von bis zu 48\% im Umgang mit intravenösen Arzneimitteln berichten [1], konnte die durchgeführte Beobachtungsstudie zeigen, dass auf der Kinderintensivstation des UK Aachens wenige Fehler bei Herstellung und Applikation von Arzneimitteln auftreten. Dennoch konnten einige Schwachstellen detektiert werden. Maßnahmen wie Mitarbeiterschulungen in der Arzneimittelherstellung, Erstellung von Herstellungsanweisungen und Bereitstellung von applikationsfertigen Perfusoren durch die Apotheke können helfen diese zu beheben.

Schlussfolgerung. Obwohl die AMTS in Bezug auf Herstellung und Applikation auf der Kinderintensivstation als sehr gut bewertet werden kann, muss die Qualität im Rahmen eines Qualitätsmanagements überprüft werden. Eine Schulung für neue Mitarbeiter sollte etabliert werden. Follow-up-Studien werden durchgeführt, um den Erfolg der ergriffenen Maßnahmen zu evaluieren. Die beobachteten Abweichungen und Fehler müssen in einer weiteren Untersuchung auf klinische Relevanz bewertet werden.

1. Taxis K et al (2004) EurJClinPharmacol 59:815-817

\section{P043}

Optimierung der Verordnung parenteraler Ernährung auf einer Kinderintensivstation durch einen klinischen Pharmazeuten - eine retrospektive Analyse

\section{T. Hermanspann', T. Orlikowsky', M. Schoberer ${ }^{3}$, A. Eisert ${ }^{1}$}

'Uniklinik RWTH Aachen, Apotheke, Aachen, Deutschland, ${ }^{2}$ Universitätsklinik für Kinder- und Jugendmedizin, Sektion für Neonatologie, Aachen, Deutschland, ${ }^{3}$ Universitätsklinikum Aachen, Klinik für Neugeborenen- und Konservative Kinderintensivmedizin, Aachen, Deutschland

Fragestellung. Aufgrund von geringen Reserven und hohem Nährstoffbedarf bei Früh- und Neugeborenen, hat die parenterale Ernährung eine große Bedeutung [1]. Durch die Komplexität der Verordnung kann es zum Auftreten von Fehlern kommen [2,3]. Ziel ist es, retrospektiv zu analysieren, welche Fehler bei der Verordnung von parenteraler Ernährung durch einen klinischen Pharmazeuten verhindert werden konnten.

Material und Methode. Im Zeitraum März 2012 bis Juli 2013 wurden die verhinderten fehlerhaften Verordnungen für parenterale Ernährung der Kinderintensivstation der Uniklinik RWTH Aachen gesammelt und in die Kategorien Patientendaten, Zusammensetzung, Dosierung, Indikation, Inkompatibilität, Osmolarität und Konzentrationsbereich eingeteilt. Verordnungsfehler wurden definiert als Abweichung von internen und externen Leitlinien, die sich nach Rücksprache mit dem Arzt als nicht gewollt herausstellten.

Ergebnisse. Von den 2889 Verordnungen, die während des Beobachtungszeitraumes an die Apotheke gesendet wurden, sind 3,8\% fehlerhaft. Über die Hälfte der Fehler treten in der Kategorie Konzentrationsbereich, hier vor allem bei Kalziumglukonat 10\%, auf. Es folgen Dosierungs- und Indikationsfehler mit knapp 12\%. Fehler bei Patientendaten, Zusammensetzung, Inkompatibilität und Osmolarität treten im Bereich o,8-5,0\% auf. Durch die Rücksprache eines klinischen Pharmazeuten mit dem Arzt konnten die Verordnungen entsprechend korrigiert werden.

Diskussion. Eine andere retrospektive Studie weist mit 6,8\% eine ähnliche Fehlerhäufigkeit bei der Verordnung von parenteraler Ernährung auf [2]. Maßnahmen, z. B. die Implementierung von weiteren Warnhinweisen in der Verordnungssoftware, sollten diskutiert werden, um das Auftreten von Verordnungsfehlern zu verhindern. In einem Folgeprojekt soll eine Bewertung der Verordnungsfehler nach klinischer Relevanz durch einen pädiatrischen Facharzt und einen Fachapotheker für klinische Pharmazie durchgeführt werden.

Schlussfolgerung. Eine Zusammenarbeit von Arzt und Apotheker bei der Verordnung von parenteraler Ernährung auf einer Kinderintensivstation ist unabdingbar, um eine sichere Medikation zu gewährleisten.

\section{Koletzko et al (2005) J Pediatr Gastroenterol Nutr 41(Suppl2)S1-87}

2. Brown et al (2007) Am J Perinatol 24(7):417-427

3. Sacks et al (2009) Pharmacotherapy 29(8):966-974

\section{P044}

Populationsbasierte Untersuchung zur Inzidenz des Alkoholkonsums in der Schwangerschaft in Ostvorpommern: die SNiP-Geburtskohorte

\section{A. Lange', L. Fröhlke', T. Ittermann ${ }^{3}$, R. Thyrian ${ }^{4}$, M. Heckmann ${ }^{5}$}

${ }^{1}$ Ernst-Moritz-Arndt Universität, Kinderklinik, Greifswald, Deutschland, ${ }^{2}$ Universitätsmedizin Greifswald, Anästhesiologie und Intensivmedizin, Greifswald, Deutschland, ${ }^{3}$ Community-Medicine SHiP II, Greifswald, Deutschland, ${ }^{4}$ Community-Medicine and Public Health, Greifswald, Deutschland, ${ }^{5}$ Klinik und Poliklinik für Kinder und Jugendmedizin, Abt. Neonatologie u. Päd. Intensivmedizin, Greifswald, Deutschland

Einleitung. Mit einer Häufigkeit von 4-6/10oo Geburten bezogen auf das gesamte Spektrum der alkoholassoziierten Erkrankungen des Neugeborenen (NG) existiert eine relevante gesellschaftliche, medizinische und sozioökonomische Relevanz von potenziell vermeidbaren Schäden. Methoden. Im Zeitraum von 2002-2008 untersuchten wir im Rahmen der SNiP-Studie (Survey of Neonates in Pommerania) 5402 Schwangere hinsichtlich ihres Alkoholkonsums in der Schwangerschaft (AKS) mittels eines anonymisiert und selbst auszufüllenden Fragebogens (AUDIT-C inkl. Abhängigkeitsindex) sowie das neonatale Outcome (Größe, Gewicht, Kopfumfang, Apgar und mögliche Symptome eines fetalen Alkoholsyndroms (FAS) wie Stigmata, muskuläre Hypotonie und SGA). Die statistische Auswertung erfolgte mit SPSS 21 und StataStatistik.

Ergebnisse. Die Antwortrate lag bei 88,6\% $(\mathrm{n}=4785)$. Einen AKS gaben 1077 (22,5\%) Mütter an. 16,4\% tranken etwa einmal im Monat Alkohol, $3,3 \%$ zwei- bis viermal pro Monat, o,2\% zwei- bis dreimal pro Woche. $54 \%$ der Schwangeren, die mehrfachen AKS angaben, hatten Abitur, 38\% einen Realschul- und 6\% einen Hauptschulabschluss. Keinen Alkohol tranken 53\% der Frauen mit Realschulabschluss vs. 29\% der Mütter mit Abitur vs.15\% mit Hauptschulabschluss. Mütter die einen AKS angaben, hatten ein um 1,42 [CI (1,14-1,78)] erhöhtes Risiko für eine Frühgeburt $(<37$ SSW). Bei Müttern die keine Angaben zum AKS gemacht hatten, 
war das Risiko auf o,7 gegenüber der Abstinenzgruppe vermindert [OR 0,71 (CI 0,56-0,89)]. Bei NG ohne Alkoholeinfluss fanden sich mögliche Symptome eines FAS bei 2,9\%, mit Alkoholeinfluss bei 3,7\% [OR 1,21 (CI o,84-1,75)] und bei 5,2\% der NG ohne Angaben zum AKS [OR 1,61 (CI 1,07-2,44)]. In dieser letzten Gruppe fanden sich bei 6,5\% der NG mit einem Kopfumfang <10. Perzentile mindestens ein mögliches Symptom eines FAS. Sowohl bei den absoluten Werten von Geburtsgewicht Körperlänge und Kopfumfang als auch bei den Perzentilen für das Geburtsgewicht $(\mathrm{p}=0,02)$ und den Kopfumfang $(\mathrm{p}=0,01)$ gibt es statistisch signifikante Unterschiede zwischen den Gruppen „kein AKS“ und „keine Angabe AKS“ ( $<<0,05)$. Diese Merkmale lassen sich in den Gestationsgruppen wiederholt nachweisen.

Schlussfolgerung. Über $20 \%$ der Schwangeren gaben an, regelmäßig während der Schwangerschaft Alkohol konsumiert zu haben. Mütter mit höheren Schulabschlüssen gaben häufiger einen Alkoholkonsum an. Mütter mit AKS hatten ein erhöhtes Risiko für eine Frühgeburt. Es fand sich keine signifikante Häufung von Symptomen einer FAS bei den NG, deren Mütter einen AKS angegeben hatten. Signifikant gehäufte Symptome eines FAS fanden sich aber nur bei den NG, deren Mütter keine Angaben zum AKS gemacht hatten. Diese Ergebnisse unterstreichen erneut die schwierige klinische Identifikation von NG mit Alkoholexposition in der Schwangerschaft. Zudem stellen Frauen, die auch bei anonymisierter Befragung keine Angaben zur AKS machten, bzgl. des neonatalen Outcomes eine besondere Risikogruppe dar.

\section{P045}

\section{Neonataler Opiatentzug und Rooming-in: retrospektive Analyse der Erfahrungen eines Zentrums}

\section{Hünseler', M. Brückle', B. Roth' , A. Kribs'}

'Kinderklinik der Uniklinik Köln, Neonatologie und Pädiatrische Intensivmedizin, Köln, Deutschland

Fragestellung. Das neonatale Abstinenzsyndrom (NAS) wurde unter Berücksichtigung des Rooming-in evaluiert.

Methode. Retrospektive Analyse der maternalen und perinatalen Daten der von 2004-2011 in der Kinderklinik der Uniklinik Köln behandelten Kinder mit NAS. Berücksichtigt wurden Therapie des NAS, Dauer der Therapie, Verweildauer im Krankenhaus und Kosten.

Ergebnisse. Es wurden die Daten von 77 Neugeborenen mit NAS durch mütterlichen Opiatkonsum ausgewertet, von denen 65 mit Opiumtinktur behandelt wurden. Kinder mit Rooming-in $(n=24)$ hatten eine um $17 \%$ kürzere Therapiedauer als die Kinder ohne Rooming-in $(\mathrm{n}=53)[27,0$ Tage (IQR 24,0-38,5) vs. 32,5 Tage (IQR 25,0-54,5)] sowie eine kürzere mediane Verweildauer im Krankenhaus [33,0 Tage (IQR 28,0-48,0) vs. 41,5 Tage (IQR 30,3-54,5)]. Die demografischen Daten zwischen beiden Gruppen waren vergleichbar. Die medianen Behandlungskosten für ein Kind mit NAS betrugen 13.457 EUR (IQR 8967-17.494)/Patient [mit Rooming-in: 9547 EUR (IQR 7024-16.135), ohne Rooming-in: 14.486 EUR (IQR 9479-19.352)].

Schlussfolgerung. Rooming-in bei Kindern mit NAS ist möglich und kann die Therapiedauer, die Verweildauer im Krankenhaus und die Kosten verringern. Es entstanden keine größeren Probleme in der Versorgung der Kinder, wenn die Pflege hauptsächlich durch die Eltern erfolgte, allerdings sind eine engmaschige Überwachung der Kinder und die Anleitung der Eltern erforderlich.

1. Hünseler C et al (2013) Neonatal opiate withdrawal and rooming-in: a retrospective analysis of a single center experience. Klin Padiatr 225(5):247-251

\section{P046}

Erfolgreiche Defibrotide-Behandlung einer atypischen pulmonalen Vaskulopathie nach autologer Stammzell-Transplantation bei einem 2-jährigen Jungen mit Neuroblastom Stadium IV

\section{A. Vierzig', F. Brasch', F. Berthold ${ }^{3}$, A. Oberthür', B. Roth'}

'Universitäts-Kinderklinik Köln, Neonatologie und pädiatrische Intensivmedizin, Köln, Deutschland, ${ }^{2}$ Klinikum Bielefeld Mitte, Institut für Pathologie, Bielefeld, Deutschland, ${ }^{3}$ Univ.-Kinderklinik, Kinderonkologie, Köln, Deutschland

Einführung. Pulmonale Komplikationen nach autologer StammzellTransplantation (ASCT) mit einer geschätzten Inzidenz von $30 \%$ und hohen Letalität (24-56\%) stellen eine diagnostische und therapeutische Herausforderung dar. Immunologische, infektiöse und toxische Faktoren tragen zur äußerst komplexen Pathophysiologie der potentiell prognostisch entscheidenden pulmonalen Komplikationen der Tumorerkrankungsbehandlung bei.

Fallbericht. Wir präsentieren den Fall eines 24 Monate alten Jungen mit Neuroblastom Stadium IV, der 2 Tage post-ASCT nach myeloblativer Chemotherapie das klinische Bild einer initialen Polyserositis mit rezidivierendem Lungenödem, konsekutiver pulmonaler Hypertonie und persistierender Beatmungsabhängigkeit entwickelte (Abb. 1). An Tag 26 führten wir eine Lungenbiopsie durch, ab Tag 38 versuchten wir erfolglos via Trachestoma eine Beatmungsentwöhnung zu erreichen. Die durch nosokomiale Infektionen wiederholt exazerbierte Lungenerkrankung

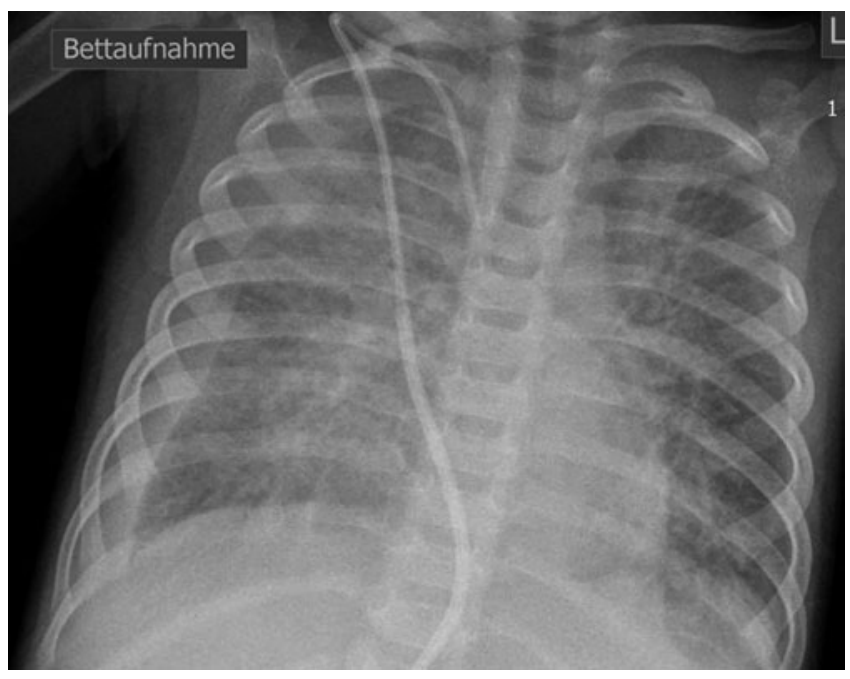

A Po46-Abb. 1 Röntgen-Thorax Tag 3

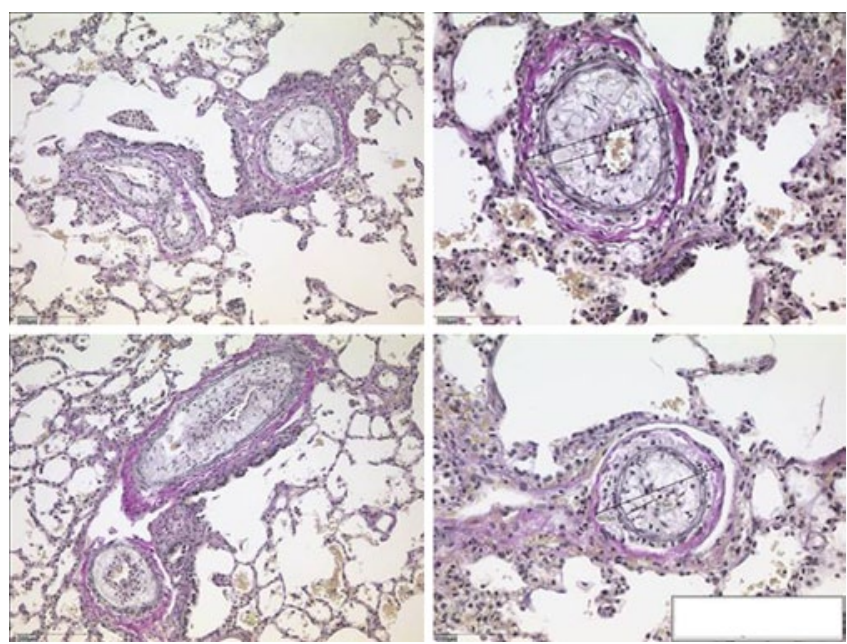

$\triangle$ Po46 - Abb. 2 Histologie Tag 26 
mit persistierender Beatmungs-/PEEP-Abhängigkeit ließ sich erst nach Ausschöpfung der konservativen pulmonal-vasodilatativen, antiinflammatorischen (Steroide) und antithrombotischen (Enoxaparin) Therapie in der Folge eines individuellen Heilversuchs mit Defibrotide nachhaltig sanieren. Neun Tage nach Behandlungsbeginn konnte der Junge endgültig vom Respirator entwöhnt werden, die Therapie wurde über insgesamt 4 Wochen komplikationslos fortgeführt. Im weiteren Verlauf konnte er erfolgreich vom Tracheostoma und den pulmonalen Vasodilatoren entwöhnt und nach Hause entlassen werden. Zwei Jahre nach ASCT trat kein Rezidiv der der Lungenerkrankung oder des Neuroblastoms auf.

Diskussion. Die Ätiologie der pulmonale Erkrankung scheint am ehesten eine Kombination eines akuten toxischen Geschehens (DD: Reaktion auf DMSO i. d. SC-Träger-Lsg.) und einer sich auf dem Boden eines Makrophagen-Aktivierungssyndroms entwickelnden atypischen pulmonalarteriellen „Transplantat“-Vaskulopathie zu sein. Der klinische Verlauf legte eine "pulmonary veno-occlusiv-disease“ nahe. Histologisch zeigte sich eine obliterierende pulmonalarterielle Vaskulopathie (Abb. 2). In der Literatur sind Einzelfälle dieser atypischen Vaskulopathie beschrieben, die nach allogener BMT letal verliefen. Defibrotide ist bei der VOD der Leber ein wirksames Medikament mit antiinflammatorischer, antithrombotischer und mutmaßlich auch endothelprotektiver Aktivität und möglicherweise auch bei anderen Vaskulopathien wirksam und könnte damit eine Rescue-Therapie ansonsten letal oder mit dauerhafter Beatmungspflicht verlaufender Lungenerkrankungen sein.

1. Selby DM et al (1999) Hum Pathol 30:734-740

2. Sreedharan A et al (2006) B M T 37:629-634

3. Trobaugh-Lotrario A et al (2003) J Ped Hematol Oncol 25:405-409

4. Fazekas T et al (2012) Ped Infect Dis J 31:115-119

5. Sirithanakul K et al (2005) Am J Hematol 80:137-146

6. Burcoglu-O'Ral A et al (2002) J Rheumatol 29:2006-2011

\section{P047}

Parenteral verabreichte olivenölbasierte Lipide wirken antiinflammatorisch

\section{K. Buschmann', N. Braach', D. Frommhold', J. Pöschl'}

'Universitätsklinikum Heidelberg, Zentrum für Kinder- und Jugendmedizin, Klinik Kinderheilkunde IV/Neonatologie/ Angelika-Lautenschläger-Klinik, Heidelberg, Deutschland

Fragestellung. Parenterale Ernährung beeinflusst die unspezifische Abwehr und die Funktion von neutrophilen Granulozyten. Aufgrund der widersprüchlichen Datenlage über pro- und antiinflammatorische Wirkungen verschiedener Lipidkompositionen sollte die vorliegende Studie die Wirkung von mittel- und langkettigen Fettsäuren (MCT bzw. LCT), fisch- und olivenölhaltigen Lipidinfusionen auf die Leukozytenrekrutierung in vivo in verschiedenen Entzündungsmodellen in der Maus vergleichen.

Material und Methoden. Intravitalmikroskopisch wurde der dosis- und zeitabhängige Einfluss der i.v.-Infusion von Lipofundin (LCT/MCT), SMOF-Lipids ( $\Omega_{3}$-Fettsäuren, MCT, LCT, Olivenöl) und Clinoleic (Olivenöl, LCT) auf die Trauma- und LPS-induzierte Leukozytenadhäsion untersucht. Diese Versuche wurden durch die Untersuchung der Leukozytenadhäsion in dynamischen Flusskammern und Expressionsanalysen von Adhäsionsmolekülen ergänzt.

Ergebnisse. In klinisch relevanten Dosen wurde schon drei Stunden nach intravenöser Gabe von Clinoleic die Trauma- und die LPS-induzierte Leukozytenrekrutierung signifikant gehemmt. Dieser antiinflammatorischer Effekt war in geringerem Maße auch nach Infusion von SMOF-Lipids, nicht allerdings von Lipofundin zu verzeichnen. Die LPS-induzierte Leukozytenadhäsion in der Flusskammersimulation konnte ebenfalls durch Clinoleic, jedoch nicht durch SMOF-Lipids bzw. Lipofundin reduziert werden.
Diskussion. Antiinflammatorische Effekte im Sinne einer Hemmung der Leukozytenrekrutierung in entzündlich veränderte Gewebe fanden sich im angewandten experimentellen Setting ausschließlich nach Gabe von olivenölbasierten Lipiden. Während nach Lipofundin keine Auswirkungen auf die Leukozytenadhäsion beobachtet wurden, zeigte sich nach SMOF-Lipids ein moderater antiinflammatorischer Effekt. Clinoleic erschien hierbei den SMOF Lipids überlegen. Die weitere mechanistische Abklärung deutet auf eine Beeinflussung leukozytärer Funktionen z. B. aufgrund einer Downregulation leukozytärer Adhäsionsmoleküle durch Clinoleic hin. Künftige Studien sollten klären, ob die Gabe von Clinoleic bei kritisch kranken Patienten nicht nur in Bezug auf die Aufrechterhaltung des Metabolismus, sondern insbesondere auch als supportive Therapie entzündlicher Erkrankungen sinnvoll erscheint.

Schlussfolgerung. Insbesondere im Gegensatz zu Lipofundin und in geringerem Maße auch SMOF Lipids entfaltet das auf LCT und Olivenöl basierende Clinoleic in vivo unter physiologischen Bedingungen einen deutlichen antiinflammatorischen Effekt.

\section{Stoffwechsel}

\section{P048}

\section{Pseudohyperglykämie als Erstmanifestation einer klassischen} Galaktosämie

S. Wiegand', U. Haller', A. Weitkämper', T. Lücke², E. Hamelmann³, N. Teig'

'Klinik für Kinder- und Jugendmedizin der Ruhr-Universität Bochum im St. Josef-Hospital, Abteilung für Neonatologie und pädiatrische Intensivmedizin, Bochum, Deutschland, ${ }^{2}$ Klinik für Kinder- und Jugendmedizin der Ruhr-Universität Bochum im St. Josef-Hospital, Abteilung für Neuropädiatrie, Bochum, Deutschland, ${ }^{3}$ Klinik für Kinder- und Jugendmedizin der Ruhr-Universität Bochum im St. Josef-Hospital, Bochum, Deutschland

Hintergrund. Die klassische Galaktosämie manifestiert sich beim Neugeborenen meist als akutes Leberversagen, so dass bereits vor Eintreffen des Befundes des Stoffwechsel-Screenings Symptome auftreten können. Wir berichten hier über ein asymptomatisches Frühgeborenes mit Galaktosämie, das im Rahmen von Routine-Blutzucker-Kontrollen erstmalig durch eine falsch gemessene Hyperglykämie vor Bestätigung der Diagnose durch das Stoffwechselscreening auffiel.

Falldarstellung. Unsere Patientin ist das erste Kind einer 35-Jährigen 5-Gravida (1-mal Abort, 3-mal Abbruch). Die Geburt erfolgte in der 32+2. SSW per Sectio nach mütterlichem Krampfanfall. Nach unkomplizierter postpartaler Adaptation des hypotrophen Neugeborenen (Geburtsgewicht 1320 g, 3.-10. Perzentile) wurde ab dem ersten Lebenstag ohne Komplikation mit dem oralen Kostaufbau mit Frühgeborenen-Formula begonnen. Bei den routinemäßig durchgeführten Blutzuckerkontrollen fiel eine Diskrepanz zwischen den im Blutgasanalysegerät und den mittels Handheld-Blutzuckermessgerät AccuCheckR bestimmten Werten auf. So wurde 4 Stunden nach der Mahlzeit am 3. Lebenstag ein Blutzuckerwert von $56 \mathrm{mg} / \mathrm{dl} \mathrm{im}$ Blutgasanalysegerät angezeigt, die Messung der gleichen Blutentnahme ergab einen Wert von $190 \mathrm{mg} / \mathrm{dl}$ mit dem AccuCheckR-Gerät. Diese diskrepanten Werte wurden auch an den Folgetagen bei jeder Blutzuckerbestimmung festgestellt. Am fünften Lebenstag wurde die Verdachtsdiagnose einer klassischen Galaktosämie gestellt (Screeninglabor München). Es folgte die Umstellung auf eine galaktosefreie Formulanahrung auf Sojabasis. Die Transaminasen waren nie erhöht. Die diskrepanten Blutzuckerwerte aus den beiden Messverfahren korrelierten mit der erhöhten GalaktoseKonzentration im Blut unserer Patientin (bei Diagnosestellung am 5. Lebenstag: $155,7 \mathrm{mg} / \mathrm{dl}$ ) und näherten sich nach Nahrungsumstellung rasch an. 24 Stunden nach Nahrungsumstellung zeigten sich keine relevanten Messdifferenzen zwischen Blutgasanalyse-Gerät $(65 \mathrm{mg} / \mathrm{dl})$ und AccuCheckR (74 mg/dl). Die Galaktose-Konzentration lag 4 Tage nach der Nahrungsumstellung im Normbereich $(<1,2 \mathrm{mg} / \mathrm{dl})$. 
Schlussfolgerung. Die Blutgasanalyse misst mittels Glukose-Oxidase selektiv die Glukosekonzentration im Blut. Der AccuCheckR erfasst durch die Bestimmung der Hexokinase-Aktivität neben der Glucose auch andere Hexosen. Bei einer mit dieser Methode gemessenen neonatalen Hyperglykämie sollte deshalb stets auf eine mögliche Differenz zu Glukose-Oxidase-basierten Messverfahren geachtet werden und bei einer erheblichen Differenz an das Vorliegen einer Galaktosämie gedacht werden.

\section{P049}

\section{Transkutane Bilirubinmessungen nach Phototherapie bei Reifgebo-} renen und Frühgeborenen

\section{J. Grabenhenrich', L. Grabenhenrich', C. Bührer', M. Berns'}

${ }^{1}$ Charité, Klinik für Neonatologie, Berlin, Deutschland, ${ }^{2}$ Charité, Institut für Sozialmedizin, Epidemiologie und Gesundheitsökonomie, Berlin, Deutschland

Einleitung. Die transkutane Bilirubinmessung ist Bestandteil des Stufenscreenings der neonatalen Hyperbilirubinämie. Nach Phototherapie gilt die Anwendung der unblutigen tcB-Messung jedoch als unzuverlässig, da die Konzentrationskinetik des Bilirubinfarbstoffes im Blut (TSB) und Hautgewebe $(\mathrm{TcB})$ unterschiedlich ist. Die vorliegende Arbeit untersuchte, ob bzw. unter welchen Bedingungen eine transkutane Bilirubinbestimmung nach Fototherapie wieder sicher erfolgen kann.

Methoden. Im Rahmen einer prospektiven Beobachtungsstudie wurden bei Neugeborenen mit einem Geburtsgewicht $\geq 1500$ g und Hyperbilirubinämie parallele Messungen von TcB mittels Jaundice Meter JM103 und TSB vor Therapiebeginn sowie zu verschiedenen Zeitpunkten nach erfolgter Phototherapie durchgeführt und verglichen.

Ergebnisse. Es wurden 86 Neugeborene (47 Frühgeborene, 39 Reifgeborene) mit insgesamt 189 parallelen Vergleichsmessungen rekrutiert. Der mittlere Abstand zwischen den beiden Messmethoden (TcB-TSB, \pm SD) vor Phototherapie $(-0,6 \pm 1,9 \mathrm{mg} / \mathrm{dl})$ stieg in den ersten 8 Stunden nach Phototherapie auf $-2,4 \pm 2,1 \mathrm{mg} / \mathrm{dl}$ und ging danach auf $-1,8 \pm 1,4 \mathrm{mg} / \mathrm{dl}$ (Stunde $8-16),-1,1 \pm 1,6 \mathrm{mg} / \mathrm{dl}$ (Stunde $16-24)$ und $-0,8 \pm 1,8 \mathrm{mg} / \mathrm{dl}(>24$ Stunden nach Phototherapie) zurück.

Schlussfolgerung. Unter Berücksichtigung eines Sicherheitsabstandes von $-7 \mathrm{mg} / \mathrm{dl}$ im Intervall $\mathrm{o}-8 \mathrm{~h}$ und $-5 \mathrm{mg} / \mathrm{dl}$ für $>8 \mathrm{~h}$ nach Phototherapieende erscheint die transkutane Messung ausreichend sicher. Vor einer generellen Anwendung sollten die vorliegenden Studienergebnisse weiter validiert werden.

\section{P050 \\ Effekte von Fototherapie auf Vitalparameter und zerebrale Oxyge- nierung Neugeborener mit einem Gestationsalter von 32 Wochen}

S. Heitmann', W. Pielemeier', S. Supcun-Ritzler', P. Kutz', C. Roll'

'Vestische Kinder- und Jugendklinik Datteln, Universität Witten/Herdecke, Neonatologie und pädiatrische Intensivmedizin, Datteln, Deutschland

Fragestellung. Kommt es unter Fototherapie zu Veränderungen der Vitalparameter und der mit NIRS gemessenen zerebralen Oxygenierung? Treten vermehrt Apnoen und Bradykardien auf?

Patienten und Methoden. Nicht maschinell beatmete Neugeborene mit einem Gestationsalter $>32$ Wochen und fototherapiebedürftiger Hyperbilirubinämie ( $\mathrm{n}=44$; Gestationsalter Median 34 Wochen [Range 32-41], Geburtsgewicht 2180 g [1230-4040], Alter bei Untersuchung 4 Tage [1-7]). Von den 44 Kindern hatten 10 Coffein, 6 CPAP und 3 O2-Supplementierung, ohne eine dieser Maßnahmen waren 35 Kinder. Über insgesamt $12 \mathrm{~h}$ ( $3 \mathrm{~h}$ vor, $6 \mathrm{~h}$ während, $3 \mathrm{~h}$ nach Fototherapie) wurden Herzfrequenz, Atemfrequenz, arterielle Sauerstoffsättigung, tcPCO2, tcPO2, Körpertemperatur, Blutdruck und die regionale zerebrale Sauerstoffkonzentration (rSAT) kontinuierlich aufgezeichnet. Die Anzahl und die Intensität von Herzfrequenz und Sauerstoffsättigungsabfällen und die fraktionelle zerebrale Sauerstoffextraktion (FOE) wurden berechnet. Daten vor, während und nach Fototherapie wurden mit Friedman-Test/Wilcoxon-Test verglichen.
Ergebnisse. Die Herzfrequenz stieg während und nach Fototherapie signifikant an (135,5 - 141,5 - 144 bpm; p<o,oo1), tcPCO2 fiel signifikant $\mathrm{ab}(37-36,5-36 \mathrm{mmHg}$; $=0,003)$. Körpertemperatur, Blutdruck und die NIRS Parameter rSAT und FOE änderten sich nicht signifikant. Die Anzahl und die Intensität von Bradykardien und Sättigungsabfällen nahmen unter Fototherapie nicht zu.

Schlussfolgerung. Bei Reifgeborene und Frühgeborene mit einem Gestationsalter $>32$ Wochen lassen sich physiologische Veränderungen unter Fototherapie nachweisen, die allerdings klinisch nicht relevant sind. Insbesondere treten Apnoen und Bradykardien nicht vermehrt auf, die zerebrale Oxygenierung bleibt unbeeinflusst.

\section{P051}

\section{Antioxidativer Status des Fruchtwassers in der Fetalzeit}

\section{Ö. Altuntas', A. Müller', A. Willruth², D. Katzer', J. Reinsberg', P. Bartmann', S. Bagci'}

'Zentrum für Kinderheilkunde am Universitätsklinikum Bonn, Neonatologie, Bonn, Deutschland, ${ }^{2}$ Zentrum für Geburtshilfe und Frauenheilkunde am Universitätsklinikum Bonn, Geburtshilfe und Perinatalmedizin, Bonn, Deutschland, ${ }^{3}$ Zentrum für Geburtshilfe und Frauenheilkunde am Universitätsklinikum Bonn, Gynäkologische Endokrinologie und Reproduktionsmedizin, Bonn, Deutschland

Einleitung. Das Gleichgewicht zwischen antioxidativen und prooxidativen Faktoren ist für die physiologische Zellfunktion von entscheidender Bedeutung. Ein Mangel an Antioxidantien führt zu Zell und Gewebsschädigung, man spricht von oxidativem Stress. Die Frühgeborenen sind sehr anfällig für oxidativen Stress, da das antioxidative Abwehrsystem des Fetus hauptsächlich erst im letzten Trimester der Schwangerschaft ausreift. Die nekrotisierende Enterokolitis (NEK) ist der häufigste erworbene gastrointestinale Notfall bei Früh- und Neugeborenen. Unabhängig von der Krankheitsursache spielen die freien Radikale in der Pathophysiologie der NEK eine wichtige Rolle. Es ist bekannt, dass ein Fetus ab der 16. Gestationswoche das Fruchtwasser (FW) schlucken kann. Letzte Tierversuche zeigten, dass das FW den frühen Aufbau und die Entwicklung der Funktion der epithelialen Barriere beim Fetus und Neugeborenen beeinflusst.

Fragestellung. Es liegt bisher noch keine Daten über die Konzentration der Superoxid-Dismutase (SOD) und Glutathion-Peroxidase (GPX) des FWs in der Fetalzeit vor. Unterscheidet sich die Konzentration der SOD und GPX im FW vor und nach der 37. Schwangerschaftswoche (SSW)? Methodik. Die Fruchtwasserproben von 70 Schwangeren wurden tagsüber (zwischen 8:00-17:00 Uhr) durch eine Amniozentese entweder im Rahmen einer Pränataldiagnostik oder eines elektiven Kaiserschnittes gewonnen.

Ergebnisse. Das mediane Gestationsalter (Interquartilsabstand, IQR) war 27,8 SSW $(22,0-35,0)$ bei der Gruppe <37. SSW (Gruppe 1, $\mathrm{n}=29$ ) und 38,5 SSW $(38,0-39,0)$ bei der Gruppe $\geq 37$. SSW (Gruppe $2, n=41$; $\mathrm{p}<\mathrm{0}, 001)$. Die SOD-Konzentration im FW in der Gruppe 1 war signifikant höher als die in der Gruppe 2 (median (IQR); 92,6 ng/ml (76,6$136,2)$ vs. $63,3 \mathrm{ng} / \mathrm{ml}(51,1-84,4) ; \mathrm{p}<0,001)$. Statistisch lag eine signifikante negative Korrelation zwischen der SOD-Konzentration im FW und dem Gestationsalter $(\mathrm{p}=0,019)$ vor. Bei $43 \%$ der FW-Proben $(n=30)$ war die GPX-Konzentration nicht nachweisbar. Die mediane GPX-Konzentration in den restlichen FW-Proben war $43,5 \mathrm{ng} / \mathrm{ml}(16,1-118,6)$. Die GPX-Konzentration war statistisch nicht signifikant unterschiedlich zwischen Gruppe 1 und 2 ( $\mathrm{p}=0,419)$.

Schlussfolgerung. Eine plazentare SOD- und GPX-Aktivität ist schon bekannt. Wir konnten in dieser Studie zeigen, dass FW eine gute SODKonzentration darstellt. Im Gegenteil zur SOD war die GPX-Konzentration im FW nicht nachweisbar oder außergewöhnlich niedrig. Es bleibt noch unklar, ob die SOD aus der Plazenta oder vom mütterlichen Blut ins FW freigesetzt wird. Außerdem sollen weitere Studien zeigen, ob die SOD-Aktivität des FWs einen gastroprotektiven Effekt in der Fetalzeit darstellt. 


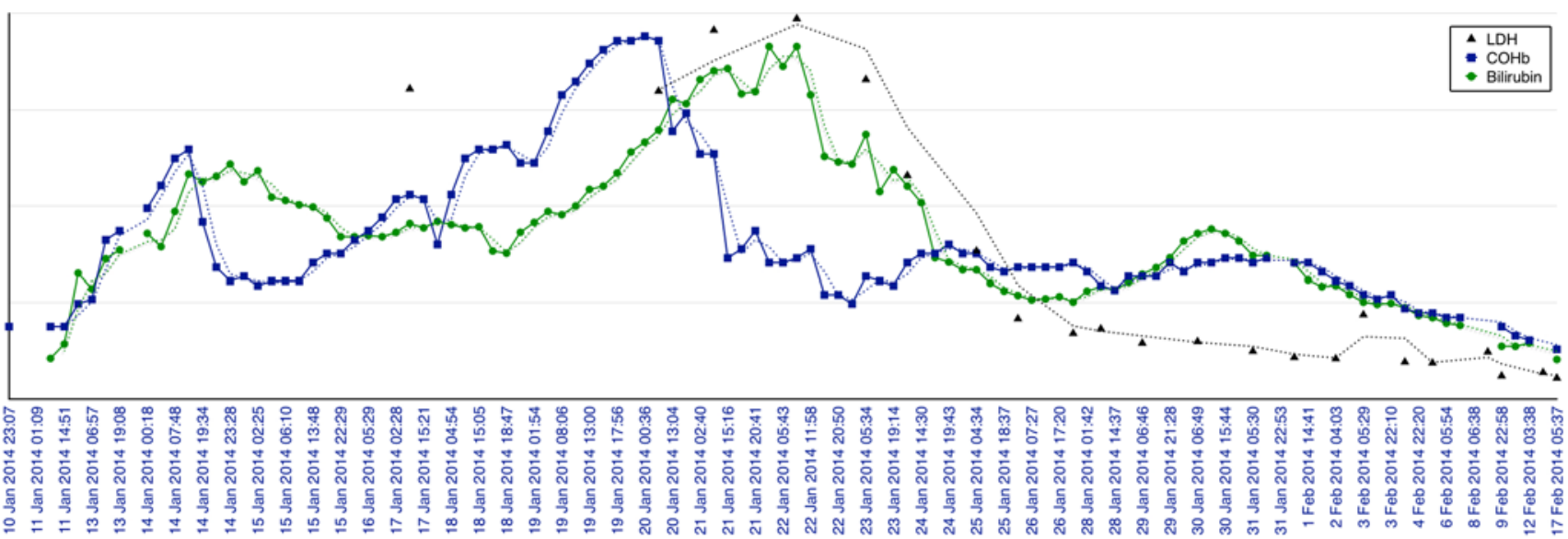

$\triangle$ Po52-Abb. 1

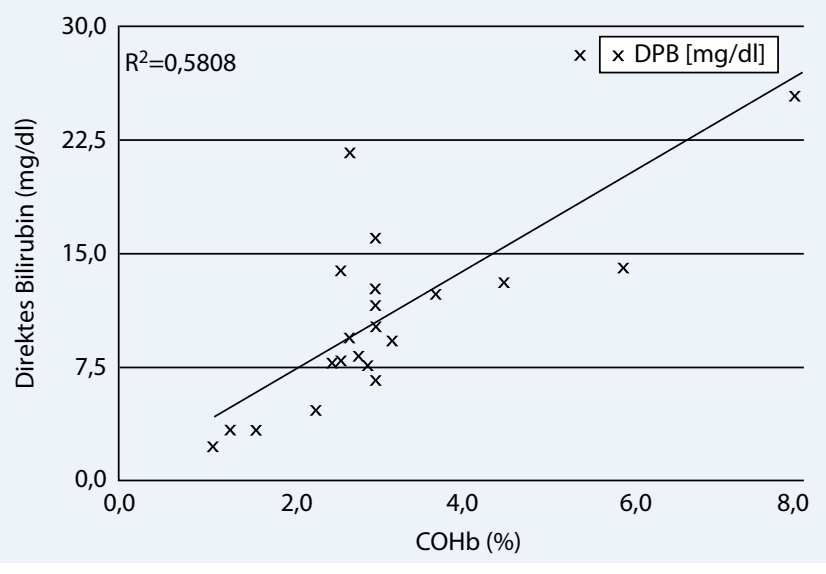

$\triangle$ Po52-Abb. 2

P052

Carboxyhämoglobin - ein vergessener Indikator der Hyperbilirubinämie

\section{Bailey', H. Fuchs'², R. Hentschel'}

'Universitätsklinikum Freiburg, Zentrum für Kinder- und Jugendmedizin, Neonatologie/Intensivmedizin, Freiburg, Deutschland, ${ }^{2}$ Universitäts - Kinderklinik, Freiburg, Deutschland

Hintergrund. Neben dem physiologischen Ikterus ist die akute Hämolyse eine wichtige Ursache des Neugeborenenikterus. Eine schwere Hyperbilirubinämie kann in Einzelfällen zu einem Kernikterus mit gravierenden neurologischen Folgen führen. Neugeborene, die eine hämolytische Erkrankung erleiden, haben hierfür ein höheres Risiko aufgrund des rascheren Anstiegs der Bilirubinproduktion, welches nicht durch den Serumbilirubinwert widergespiegelt wird (Dennery PA et al, NEJM 2001). Als Nebenprodukt der Hämolyse bzw. des Hämabbaus wurde bereits in den 196oer Jahren Kohlenmonoxid (CO) beschrieben, das als Carboxyhämoglobin $(\mathrm{COHb})$ transportiert und in der Lunge abgeatmet wird. $\mathrm{COHb}$ wird in allen modernen BGA-Geräten co-oximetrisch mitgemessen.

Fragestellung. Bei einem Frühgeborenen $(35+6 / 7$ SSW) mit einem außergewöhnlich hohen Bilirubinanstieg im Rahmen eines atypischen HUS (aHUS) wurde der co-oximetrisch gemessene COHb Wert von 3,7\% als stark erhöht beobachtet. Ziel der Untersuchung war es, eine mögliche Assoziation zwischen der Höhe des Bilirubinanstieges und den übrigen Parametern der Hämolyse (LDH, Hb-Abfall) zu finden.

Methode. Analyse der gespeicherten Daten aus dem POCT-Blutgasanalysegerät (Roche cobas b221 V4) über 2 Monate (Speichergrenze). Filterung der Ergebnisse mit einem willkürlichen Cut-off-Wert zur Entdeckung aller Fälle mit $>\mathbf{2} \% \mathrm{COHb}$.
Ergebnisse. Der willkürliche Cut-off-Wert lieferte Daten zu 4 Patienten, welche jeweils gesicherte hämolytische Erkrankungen unterschiedlicher Genese mit der Notwendigkeit der Phototherapie hatten: Ein reifes Neugeborenes mit B-O-Inkompatabilität (max. Bilirubin $20,3 \mathrm{mg} / \mathrm{dl}$, max. $\mathrm{COHb} 2,3 \%$, min $\mathrm{Hb} 17,4 \mathrm{~g} / \mathrm{dl})$, ein eutrophes Frühgeborenes $(34+4 / 7$ SSW) mit Morbus haemolyticus neonatorum (max. Bilirubin 15,2 mg/dl, $\max \mathrm{COHb} 5,0 \%$, min $\mathrm{Hb} 8,6 \mathrm{~g} / \mathrm{dl}$, $\max \mathrm{LDH} 972 \mathrm{U} / \mathrm{l})$ und ein eutrophes Frühgeborenes (25+5/7 SSW) mit biparietalem Kephalhämatom (max. Bilirubin 6,8 g/dl, max. COHb 2,5\%, min Hb 10,o g/dl, max LDH $334 \mathrm{U} / \mathrm{l}$ ), sowie oben erwähnten Patienten mit aHUS (max. Bilirubin 32,o mg/dl, max. $\mathrm{COHb} 8,0 \%$, min $\mathrm{Hb} 5,5 \mathrm{~g} / \mathrm{dl}$, max LDH $3947 \mathrm{U} / \mathrm{l}$ ) In der zeitlichen Aufschlüsselung beim ersten Patienten sieht man einen früheren Anstieg von COHb im Vergleich zu Bilirubin und LDH (Abb. 1, Abb. 2).

Diskussion. Bei der Hämolyse werden $\mathrm{CO}$ und Biliverdin in äquimolaren Anteilen gebildet. Die Umwandlung des Biliverdin zu Bilirubin kann im Serum nachgewiesen werden, doch ist dies nur ein unsicherer Parameter für das Ausmaß der Hämolyse, da Albumin-gebundenes Bilirubin und Gewebsbilirubin nicht nachgewiesen werden können. Hier könnte die $\mathrm{COHb}$ Bestimmung als alternativer Parameter für den zu erwartenden Anstieg der Gesamtbilirubinproduktion bessere und frühere Erkenntnisse zur Hämolyse liefern. Weitere Studien sind erforderlich, um die unterschiedliche Kinetik von $\mathrm{COHb}$, LDH und Bilirubin herauszuarbeiten. Eine frühzeitigere Erkennung einer ablaufenden Hämolyse kann die schwerwiegenden Folgen einer hohen Bilirubinproduktion eventuell verhindern.

\section{P053}

Transkutane vs. kapilläre Bilirubinbestimmung bei Neugeborenen unter Fototherapie

\section{S. Willers', D. Singer?}

UUniversitätsklinikum Hamburg Eppendorf, Sektion Neonatologie und Pädiatrische Intensivmedizin, Hamburg, Deutschland

Hintergrund und Fragestellung. Die transkutane Bilirubinbestimmung ist als Screeningmethode bei ikterischen Neugeborenen vor Beginn der Fototherapie bereits etabliert. Maßgeblich für die Steuerung der Fototherapie bleibt jedoch die Bilirubinkonzentration im (kapillären) Blut. Das fotometrische Bilirubinmessgerät BiliChek ${ }^{\oplus}$ (Philips, Niederlande) wurde von der US-amerikanischen Food and Drug Administration (FDA) auch für Messungen unter laufender Fototherapie zugelassen. Als Voraussetzung wird die Messung in einem unbestrahlten Areal (wiederverschließbares Lochpflaster) angesehen. Wir untersuchten, ob die mit diesem Gerät und dieser Methodik bestimmten transkutanen Bilirubinwerte mit der Konzentration im Blut vor und während Fototherapie korrelieren. Material und Methoden. 80 reife Neugeborene bzw. späte Frühgeborene wurden in die Studie aufgenommen. Bei 60 Kindern wurde einmal und bei 1o Kindern mehrfach zeitgleich der kapilläre (ABL9o FLEX Blutgasanalysator, Radiometer, Dänemark) und transkutane Bilirubinwert vor Beginn der Fototherapie bestimmt. Bei 44 Kindern wurde eine und bei 
14 Kindern mehrere Vergleichsmessungen unter laufender Bestrahlung (mittels des wiederverschließbaren Lochpflasters) gewonnen. Die Auswertung erfolgte mit dem Wilcoxon-Vorzeichen-Rangtest.

Ergebnisse und Diskussion. Während sich vor Fototherapie kein signifikanter Unterschied der Messwerte in Haut und Blut zeigte ( $\mathrm{p}=0,3$, der transkutane Wert war im Mittel um 0,2 mg/dl größer als der Blutwert, 95\% CI $\pm 0,5 \mathrm{mg} / \mathrm{dl}$,) unterschieden sich unter laufender Fototherapie die transkutanen Werte signifikant von den kapillären ( $\mathrm{p}<0,001$, die transkutanen lagen im Mittel 2,5 mg/dl über den kapillären Werten, 95\% CI $\pm 0,7 \mathrm{mg} / \mathrm{dl}$ ). Dies steht im Wiederspruch zu den US-amerikanischen Daten und könnte durch die Verwendung von LED-Kaltlichtlampen an unserer Klinik (im Gegensatz zu konventionellen Fototherapieröhren in der Referenzstudie) bedingt sein. Offenbar führen diese zu einer so effektiven Senkung des Bilirubinspiegels in Haut und Blut, dass die fotometrische Messung in dem unbestrahlten Messfenster regelmäßig zu hohe Werte ergibt ( $83 \%$ der Werte wichen nach oben ab.) Ob bei Messung an der bestrahlten Haut eine bessere Korrelation resultieren würde, bleibt noch zu untersuchen.

Schlussfolgerung. Trotz guter messtechnischer Übereinstimmung zwischen transkutanen (BiliChek ${ }^{\oplus}$, Philips) und kapillären (ABL9o FLEX Blutgasanalysator, Radiometer) Bilirubinwerten kann eine Methodik, bei der die transkutanen Kontrollmessungen an einem unbestrahlten Hautareal durchgeführt werden, zumindest bei Verwendung von Kaltlichtlampen nicht zur Therapiesteuerung unter Fototherapie verwendet werden.

\section{P054}

Schwere Hyperglykämie bei einem reifen Neugeborenen - sind neurologische Folgeschäden durch eine therapeutische Hypothermie vermeidbar?

\section{Niewisch', R. Creutzfeldt', R. Hentschel' \\ 'Universitätskinderklinik Freiburg, Pädiatrische und neonatologische Intensiv- medizin, Freiburg i. Br., Deutschland}

Hintergrund. Eine neonatale Hyperglykämie ist definiert als Blutzucker $>125 \mathrm{mg} / \mathrm{dl}$. Das Auftreten einer neonatalen Hyperglykämie wird vor allem bei Frühgeborenen beobachtet und ist möglicherweise mit einem eingeschränkten neurologischen Outcome assoziiert. Das neurologische Outcome nach einer schweren neonatalen Hyperglykämie bei einem reifen Neugeborenen ist unklar.

Kasuistik. Wir präsentieren den Fall eines reifen Neugeborenen der $41+1 / 7$ SSW mit einer Ösophagusatresie Typ IIIb. Nach der operativen Korrektur trat eine Anastomosen-Insuffizienz auf, so dass eine orale Nahrungskarenz mit parenteraler Ernährung notwendig war. Hierbei kam es zu einer massiven intravenösen Glukose-Überinfusion aufgrund einer fehlerhaft eingestellten Infusionspumpe: Die Infusionsgeschwindigkeit der parenteralen Ernährung war versehentlich um das 10-fache erhöht worden. Es wurden im Verlauf mehrerer Stunden insgesamt 43,4 g Glukose/kgKG verabreicht. Klinisch imponierten plötzlich auftretende Krampfanfälle, die eine Intubation erforderlich machten. Als der Fehler durch einen Zuckerstreifentest bemerkt wurde, zeigten sich in detaillierten Analysen ein Plasma-Glukosewert von $2240 \mathrm{mg} /$ $\mathrm{dl}$ und eine Blutosmolalität von $432 \mathrm{mosm} / \mathrm{kg}$. Das Neugeborene entwickelte ein hyperosmolares Koma mit Status epilepticus, der erst nach mehreren Stunden medikamentös unterbrochen werden konnte. Der Blutzucker wurde durch Beenden der parenteralen Zufuhr, Insulingabe und normotone Flüssigkeitssubstitution bei massiver Diurese über 24 Stunden gesenkt. Nach Beendigung des Status epilepticus zeigte sich eine intraventrikuläre Hämorrhagie bds. (II ${ }^{\circ}$ links, $\mathrm{I}^{\circ}$ rechts). Es erfolgte eine neuroprotektive Hypothermie auf $34^{\circ} \mathrm{C}$ über $72 \mathrm{~h}$ mit anschließend problemloser Aufwärmung in die Normothermie. Es bestanden noch vereinzelte Krampfanfälle, die eine weitere antikonvulsive Therapie notwendig machten. Zunächst bestanden neurologische Auffälligkeiten (reduzierter Muskeltonus, verminderte Vigilanz), die sich jedoch zusehends besserten. Die antikonvulsive Therapie konnte im weiteren Verlauf reduziert und schließlich abgesetzt werden. Im Kontroll-MRT nach 2 Monaten zeigten sich regrediente Blutungsresiduen ohne Hinweis auf darüber hinausgehende zerebrale Schädigungen. 1,5 Jahre nach dem Ereignis besteht aktuell klinisch noch eine leichte muskuläre Hypotonie, die erfolgreich physiotherapeutisch behandelt wird und eine ansonsten altersentsprechende, neurologische Entwicklung.

Schlussfolgerung. Bei parenteral ernährten Neugeborenen ist bei Auftreten von schweren Hyperglykämien die akzidentelle Glukose-Überinfusion eine wichtige Differentialdiagnose. In dem hier präsentierten Fall zeigt sich bei einem reifen Neugeborenen nach schwerster Hyperglykämie eine regelrechte neurologische Entwicklung. Möglicherweise hat die im Anschluss an die Hyperglykämie durchgeführte Hypothermie-Behandlung, die nicht durch eine Leitlinie oder experimentelle Daten gestützt ist, zu dem positiven klinischen Ausgang beigetragen.

\section{P055}

Das Krankheitsbild der maternalen Phenylketonurie, Fallbericht zweier Geschwister nach unzureichender Diäteinhaltung in der Schwangerschaft

\section{S. Henjes', M. Knüpfer', F. Pulzer', C. Gebauer', U. Thome', S. Beblo²}

'Universitätskinderklinik, Neonatologie, Leipzig, Deutschland, ${ }^{2}$ Universität Leipzig, Kinderklinik, Leipzig, Deutschland

Einleitung. Bei der klassischen Phenylketonurie (PKU) handelt es sich um die häufigste angeborene Stoffwechselerkrankung des Aminosäurestoffwechsels. Unbehandelt führt sie zu schwerster psychomotorischer Retardierung. Aufgrund des Defektes der Phenylalanin-Hydroxylase (PAH) kommt es bei der PKU zu einem Aufstau der essentiellen Aminosäure Phenylalanin (Phe) und zum Mangel an Tyrosin, hierdurch entsteht eine Schädigung insbesondere des sich entwickelnden Gehirnes. Seit Mitte der 196oer Jahre wird die PKU in Europa im Neugeborenenscreening erfasst. Bei frühzeitigem postnatalem Beginn einer streng phenylalaninarmen Diät und Substitution eines Aminosäuregemisches entwickeln sich die PKU-Patienten psychomotorisch normal. Diese Diät wird meist nach Abschluss der Myelinisierung nach dem ersten Lebensjahrzehnt etwas gelockert, im Erwachsenenalter sind viele Patienten nicht mehr in Betreuung eines Stoffwechselzentrums. Eine neue Dimension erfährt die Betreuung dieser Erkrankung aber mit dem Eintreten einer Schwangerschaft bei einer Patientin mit PKU. Da die kritische Phase für die Entwicklung des kindlichen Gehirnes bereits in der 5.-8. SSW liegt, ist bei ungeplanter Schwangerschaft eine schlechte Diätführung mit hohen Phe-Spiegeln bereits ab der Konzeption schädlich für den Fetus. Das Krankheitsbild der maternalen PKU (MPKU) mit geistiger Retardierung, Mikrozephalie, Minderwuchs und auch Herzfehlbildungen entspricht damit einer Zweitgenerationserkrankung. Die Kinder sind selbst in der Regel nicht an PKU erkrankt.

Fallbericht. Wir berichten über ein weibliches Neugeborenes einer Mutter mit PKU und schlechter Diätführung, welches in der $39+1$. SSW spontan in unserer Klinik entbunden wurde und das Vollbild einer maternalen PKU (MPKU) zeigt. Bereits pränatal wurde eine Wachstumsretardierung diagnostiziert, die sich postnatal mit einem Geburtsgewicht von $2060 \mathrm{~g}[<3$. PZ $(-0,514 \mathrm{~kg})]$ bestätigte, des Weiteren bestehen eine schwere Mikrozephalie [KU bei Geburt $27 \mathrm{~cm}(<3$. PZ $(-5 \mathrm{~cm})$ ], ein Herzfehler lag nicht vor. Ein älterer Halbbruder mütterlicherseits ist ebenfalls durch MPKU mit Mikrozephalie, Minderwuchs, angeborenem Herzfehler und starker Entwicklungsretardierung betroffen, er begann im Alter von 3 Jahren zu laufen und spricht im Alter von 6 Jahren nur einzelne Wörter. Beide Kinder sind selber nicht an PKU erkrankt. Klassischerweise waren bei unserem Neugeborenen die unmittelbar nach der Geburt gemessenen Phe-Spiegel massiv erhöht $(18,28 \mathrm{mg} /$ $\mathrm{dl}=1108 \mu \mathrm{mol} / \mathrm{l})$, sanken dann ohne Einhaltung einer Phe-reduzierten Ernährung auf Normbereiche ab. Auch im weiteren Verlauf waren wöchentlich kontrollierte Phe-Werte normwertig. Die Münchner funktionelle Entwicklungsdiagnostik für das 1. LJ zeigt bei der Patientin eine Entwicklungsverzögerung, das Perzeptionsalter ist im Alter von 3 Monaten um 2 Monate verzögert. 
Schlussfolgerung. Bereits ab dem frühen Jugendalter ist eine Schulung der Patientinnen mit PKU über Kontrazeption und optimale Stoffwechseleinstellung bereits vor Eintreten einer Schwangerschaft dringend notwendig. Die bereits präkonzeptionelle und pränatale Begleitung und Beratung solcher Frauen stellt eine hohe Herausforderungen an alle beteiligten Fachdisziplinen dar, insbesondere aber bedürfen die PKU- Patientinnen und deren Familien einer guten multidiziplinären Unterstützung in ihrer Lebensführung.

\section{P056}

Postnatale Therapie und Verlauf bei Kindern mit neonataler Hämochromatose - 2 Kasuistiken

\section{A. Hansmann', A. Geipel', H. Fischer ${ }^{3}$, A. Müller ${ }^{4}$}

'Universitätsklinik Zentrum f. Kinderheilkunde, Abteilung für Neonatologie, Bonn, Deutschland, ${ }^{2}$ Universität Bonn, Abteilung für Geburtshilfe und Pränatale Medizin, Bonn, Deutschland, ${ }^{3}$ Universitätklinikum Bonn,, Institut für Pathologie, Bonn, Deutschland, ${ }^{4}$ Zentrum für Kinderheilkunde am Universitätsklinikum Bonn/Neonatologie, Bonn, Deutschland

Fragestellung. Die neonatalen Hämochromatose $(\mathrm{NH})$ ist durch ein in den ersten Lebenstagen auftretendes Leberversagen mit histologisch nachweisbarer Siderose von Leberzellen und extrahepatischem Gewebe charakterisiert. Die Vorstellung von der Ätiologie hat sich in den vergangenen Jahren von einer Eisenspeichererkrankung zu einer "gestational alloimmune liver disease“ (GALD) gewandelt. Dies führte zu neuen präventiven und therapeutischen Optionen. Die Prävention der Erkrankung durch wöchentliche Immunglobulingaben (IVIG) der Schwangeren und die Therapie des Neugeborenen durch Austauschtransfusion und IVIG zeigen hervorragende Ergebnisse. Wir stellen die Klinik zweier Neugeborener mit NH mit sehr unterschiedlichen Verläufen vor. Eine Mutter erhielt während der Schwangerschaft wöchentlich IVIG und die andere nicht.

Kasuistik. Das erste Kind wurde mit einem Gestationsalter von $36+5$ Wochen geboren. Bei drei vorausgehenden unauffälligen Schwangerschaften mit Geburt von gesunden Kindern und einer nachgewiesenen $\mathrm{NH}$ des vierten Geschwisterkindes mit letalem Verlauf hatte die Mutter ab der 22. SSW wöchentlich IVIG erhalten. Postnatal fiel der Patient mit ausgeprägter Hypoglykämie ( $4 \mathrm{mg} / \mathrm{dl}$ ), einer ausgeprägten Gerinnungsstörung und stark erhöhtem Ferritin und AFP auf. Die Therapie erfolgte mit Vitamin K, FFP-Gaben, Eisenchelatbildnern und Antioxydantien sowie Austauschtransfusionen am 2. und 4. Lebenstag mit IVIG-Gaben. Darunter rasche Normalisierung des Gerinnungspotentials und komplikationsfreie Entlassung am 18. Lebenstag. Im Verlauf keine Entwicklungsstörungen und Normalisierung der Laborparameter. Der zweite Patient wurde wegen IUGR und Oligohydramnion per Sektio in der 29+o SSW geboren. Trotz histologischem Nachweis einer $\mathrm{NH}$ mit letalem Ausgang in der Neugeborenenperiode bei dem Kind aus der vorhergehenden Schwangerschaft, war während dieser Schwangerschaft keine IVIG Prävention bei der Mutter durchgeführt worden. In den ersten Lebenstagen entwickelte sich trotz Austauschtransfusion und IVIG Therapie das Vollbild eines Leberversagens. Auch wiederholte Gaben von Vitamin K und häufige FFP-Transfusionen konnten Blutungen in die Seitenventrikel, Nebennieren, Magen und Lungen nicht verhindern. Zusätzlich kam es zu Hypoglykämien, Nierenversagen und bei Lungenhypoplasie zu Ventilations- und Oxygenierungsproblemen. Das Kind verstarb am 9. Lebenstag an Multiorganversagen.

Schlussfolgerung. Die neonatale Hämochromatose ist eine wichtige Differentialdiagnose bei Oligohydramnion, IUGR oder Spätabort sowie be Neugeborenen mit Leberversagen in den ersten Lebenstagen. Mit dem neuen Verständnis der Erkrankung als Alloimmunerkrankung ergeben sich gute Möglichkeiten der Prävention und Therapie, die dem Neonatologen bekannt sein sollten.

\section{P057}

Klinische Effekte der Leberzelltherapie bei Kindern mit Harnstoffzyklusdefekten im Vergleich zu einer Kontrollgruppe

\section{G. Hoffmann', M. Lindner ${ }^{2}$}

'Universitätsklinikum Heidelberg, Zentrum für Kinder- und Jugendmedizin, Heidelberg, Deutschland, ${ }^{2}$ Universitätsklinikum Heidelberg, Klinik Kinderheilkunde I, Heidelberg, Deutschland

Hintergrund. Angeborene neonatal manifestierende Harnstoffzyklusdefekte haben trotz diätetischer und pharmakologischer Therapie eine schlechte Prognose und die einzige kurative Therapie, die frühe orthotope Lebertransplantation (OLT), eine erhöhte Morbidität und Mortalität. Die Leberzelltherapie könnte eine neue Therapieoption darstellen. Methoden. 16 Patienten (OTCD, CPS1D oder ASSD), im Alter von 1 Tag bis 5 Jahren, wurden in klinischen Studien in Deutschland (Selica II/V) und in den USA/Kanada (Selica III) eingeschlossen. 13 Patienten erhielten 0,3×109 humane Leberzellen pro kg Körpergewicht via Pfortaderkatheter und wurden bis zu 2 Jahre nach Gabe oder bis zur OLT verlaufsbeurteilt. Interim-Ergebnisse. Die Leberzelltherapie konnte ohne schwerere leberzellassoziierte Nebenwirkungen durchgeführt werden. Ein Patient verstarb an seiner Grunderkrankung, 12 Kinder erhielten nach 2 bis 20 Monaten eine OLT, wobei 2 der früh transplantierten Kinder an OLTKomplikationen verstarben. Keiner der 9 Patienten, die die Studie protokollgemäß beendeten, verstarb. Die 9 protokollkonform behandelten Patienten wurden mit 19 Kindern einer retrospektiven Beobachtungsstudie verglichen. Die Zeit bis zum Auftreten des ersten hyperammonämischen Ereignisses (HE) war in der Behandlungsgruppe verlängert und diese Patienten zeigten mehr ereignisfreie 6-Monats-Überleben für schwere HEs (Ammoniak $\geq 500 \mu \mathrm{mol} / 1,100 \%$ vs. $39 \%$ ) und für moderate HEs (Ammoniak $\geq 250 \mu \mathrm{mol} / 1,65 \%$ vs. $24 \%$ ). Die Inzidenz schwerer und moderater HEs war in der Behandlungsgruppe im Vergleich zur Beobachtungsgruppe ebenfalls verringert.

Schlussfolgerung. Im Vergleich zu einer historischen Kontrollgruppe zeigten Leberzelltherapie-behandelte Patienten ein deutlich verlängertes ereignisfreies Überleben und weniger schwere hyperammonämische Ereignisse. Diese Daten basieren auf einer Interimsanalyse, in beiden Behandlungsstudien werden weiter Patienten rekrutiert.

\section{P058}

\section{Hyperammonämie und Hämodiafiltration (HDF) bei Neugeborenen}

\section{R. Ulreich', W. Radauer' I. Marschitz' ${ }^{3}$, G. Zobel', S. Rödl}

'Universitätsklinik für Kinder und Jugendheilkunde, Pädiatrische Intensivstation, Graz, Österreich, ${ }^{2}$ Univ. Klinikf. Kinder- u. Jugendheilkunde Salzburg, Pädiatrische Intensivstation, Salzburg, Österreich, ${ }^{3}$ Universitätsklinik für Kinderund Jugendheilkunde Salzburg, Neonatologie, Salzburg, Österreich

Hintergrund. Hyperammonämie des Neugeborenen ist eine lebensbedrohliche Komplikation unterschiedlicher Stoffwechseldefekte. Nur frühzeitiges Senken der neurotoxischen Ammoniakspiegel kann schwere neurologische Beeinträchtigungen verhindern. Die Prismaflex ${ }^{\circ}$ Pumpe in Verbindung mit dem Prismaflex HF2o Set ist von Seiten des Füllvolumens und der möglichen Flusseinstellungen für die Behandlung von Säuglingen geeignet. Eine Studie über die Ammoniakclearance des Systems im Echtbetrieb liegt jedoch bis dato noch nicht vor.

Fragestellung. Ziel der Studie war die Ermittlung von Ammoniakclearance, und deren Korrelation mit Blut- und Dialysatfluss des Prismaflex HF2o Sets.

Material und Methode. Wir berichten von 3 Patienten (Alter 2-4 Tage) mit neonataler Hyperammonämie. Retrospektiv wurden Propionacidämie und Harnstoffzyklus Defekte (CPS-1-Mangel und NAGS-Mangel) diagnostiziert. Alle 3 Patienten wurden klinisch durch eine akute metabolische Enzephalopathie auffällig und mit konventioneller Therapie (aminosäurefreie, hochkalorische, parenterale Ernährung, Na- Benzoat, L-Arginin HCL, L-Carnitin) und HDF mittels Prismaflex HF2o Sets behandelt. Körpergewicht bei HDF Beginn betrug 2,7-2,9 kg. 
Ergebnisse. Die mittleren Ammoniakplasmawerte bei HDF Beginn betrugen $2002 \mu \mathrm{g} / \mathrm{dl}(4904,9-512)$ bei HDF Ende 312 (862-32). Mittlerer Blutfluss betrug $11,1 \mathrm{ml} / \mathrm{kg} / \mathrm{min}(17,9-10,7)$ mittlerer Dialysatfluss $200,8 \mathrm{ml} / \mathrm{kg} / \mathrm{h}(35,7-642,9)$. Die mittlere Ammoniakclearance wurde mit $12,1 \mathrm{ml} / \mathrm{min}(2,4-24,9)$ berechnet. Die Ammoniakclearance sowie die Reduktion der Ammoniakplasmawerte hatten eine direkte Korrelation mit der Höhe des Blut- und Dialysatflusses. Zwei Patienten wurden nach definitiver Diagnosestellung mit spezifischen Langzeittherapien, ohne wesentliche neurologische Beeinträchtigungen, entlassen. Die Behandlung des Patienten mit NAGS-Mangel wurde aufgrund irreversibler zerebraler Schädigung vorzeitig beendet.

Schlussfolgerung. Unsere Erfahrungen zeigen, dass mit Prismaflex HF2o Set bei initial hohen Blut- und Dialysatflüssen eine zufriedenstellende Elimination von Ammoniak möglich ist. Die frühzeitige Entscheidung zu HDF-Behandlung kann neurologische Spätschäden verhindern. Die Einstellungen der Prismaflex-Pumpe in Verbindung mit dem Prismaflex HF 20 Set sind mit den Therapieerfordernissen von Patienten unter $3 \mathrm{~kg}$ Körpergewicht kompatibel.

\section{Hämatologie/Hämostaseologie}

\section{P059}

\section{In-vivo-Beobachtungen zur Thrombozytenfunktion im Mausfeten}

\section{A. Margraf', C. Nußbaum², I. Rohwedder', S. Schmidt', S. Dietzel', L. Kremer $^{3}$, F. Kiefer ${ }^{3}$, E. Quackenbush ${ }^{4}$, U. von Andrian ${ }^{5}$, M. Sperandio ${ }^{7}$} 'Walter-Brendel-Zentrum für Experimentelle Medizin, Ludwig-MaximiliansUniversität, München, Deutschland, ${ }^{2}$ Dr. von Haunersches Kinderspital, Ludwig-Maximilians-Universität, München, Deutschland, ${ }^{3}$ Max-Planck-Institut für molekulare Biomedizin, Münster, Deutschland, ${ }^{4}$ Celldex Therapeutics, Inc., Needham, MA, USA, ${ }^{5}$ Department of Microbiology and Immunobiology, Harvard Medical School, Boston, USA

Fragestellung. Die physiologische Regulation der Thrombozytenfunktion ist essentiell für die Gewährleistung eines intakten Gefäßsystems und das Verhindern von Blutverlusten. Während die Mechanismen im adulten Organismus gut untersucht sind, liefern Studien der letzten Jahre Hinweise auf eine verminderte Aktivität der Thrombozyten in Frühgeborenen. Es bleibt jedoch nach wie vor unklar, in welcher Weise sich die primäre Hämostase im Verlauf der fetalen Reifung entwickelt. Material und Methoden. Die Thrombusformation in fetalen Dottersackgefäßen wurde unter Verwendung eines neu entwickelten in-vivo Mausmodells in Feten der Alter E13.5 bis E17.5 (21d Gestationsdauer) beobachtet. Der zeitliche Verlauf der Thrombusentstehung (Anlagerung, Gefäßverschluss, erneuter Blutfluss) wurde analysiert. Thrombozytenanzahl, -aktivität und funktionelle Bindung von Fibrinogen wurden anhand durchflusszytometrischer Messungen bestimmt. Der P-Selektin Inhalt der Granula wurde mittels Western Blot ermittelt. Die Interaktion adulter Thrombozyten im fetalen Gefäßnetz nachfolgend einer Transfusion wurde anhand Multiphotonenmikroskopie im Dottersack der Mausfeten beobachtet.

Ergebnisse. Thrombozytenanlagerung und Ausbildung eines Gefäßverschlusses waren signifikant reduziert in jungen Feten im Vergleich zu Älteren, während ein vermehrtes Auftreten eines erneuten Durchbrechens des Blutflusses (Reflow) in den jüngeren Feten zu beobachten war. E13.5 Feten benötigten im Vergleich zu älteren Feten eine längere Zeit bis eine Thrombozytenanlagerung oder ein thrombotischer Gefäßverschluss erkennbar wurden. Folgend der Transfusion von adulten Thrombozyten in das fetale Gefäßsystem konnte eine vermehrte Anlagerung der funktionell reifen Thrombozyten beobachtet werden. In der Durchflusszytometrie zeigten sich in fetalen Blutproben signifikant niedrigere Thrombozytenzahlen als in adulten Proben. Weiterhin konnte eine beeinträchtigte Thrombin-vermittelte Thrombozytenaktivierung in Feten im Vergleich zu adulten Kontrollen festgestellt werden. Diskussion und Schlussfolgerung. Wir konnten eine verminderte und verzögerte Thrombusentstehung, sowie eine verminderte Stabilität der gebildeten Thromben beobachten, während sich die Parameter mit der Reifung des Feten bessern. Sowohl erniedrigte Thrombozytenzahlen, als auch veränderte Aktivierungsmuster kommen als Ursache in Frage. Unsere Beobachtungen können nicht nur unser Verständnis der primären Hämostase in Feten ergänzen, sondern auch Hinweise für neue therapeutische Strategien im Hinblick auf neonatologische Erkrankungen, wie den persistierenden Ductus arteriosus oder intraventrikuläre Hämorrhagien, liefern.

\section{P060}

Herstellung von zu Therapiezwecken geeigneten Erythrozytenkonzentraten aus Nabelschnurblut durch Schwerkraftseparation

\section{Weise', O. Oechsle ${ }^{2}$, A. Rabsilber ${ }^{3}$, H. Garritsen ${ }^{4}$, G. Jorch ${ }^{5}$, T. Brune'}

${ }^{1}$ Kinderklinik Klinikum Lippe, Detmold, Deutschland, ${ }^{2} \mathrm{Hemacon} \mathrm{GmbH}$, Düsseldorf, Deutschland, ${ }^{3}$ Universitätsfrauenklinik, Magdeburg, Deutschland, ${ }^{4}$ Institut für Klinische Transfusionsmedizin, Braunschweig, Deutschland, ${ }^{5}$ Universitätskinderklinik, Magdeburg, Deutschland

Einleitung. In verschiedenen Studien konnte gezeigt werden, dass Erythrozythenkonzentrate (EK) allein durch Schwerkraftseparation unter Verwendung von Hohlfaser- Filtersystemen hergestellt werden können. In der vorliegenden Studie haben wir untersucht, ob mit dieser Methode auch fetales Blut trotz des höheren Zellvolumens filtriert und somit Plazentablutkonzentrate hergestellt werden können.

Patienten und Methoden. In die Studie wurden 12 reife Neugeborene nach komplikationslosem Schwangerschaftsverlauf aufgenommen. Plazentablut wurde mittels Standardverfahren entnommen. Der Entnahmebeutel wurde an das Separationssystem konnektiert (Abb. 1), das Blut durch Schwergraft leukozytendepletiert und in EK und Plasma getrennt. Die EK-Beutel enthielten $10 \mathrm{ml}$ SAG-Mannitol als Stabilisator. Die Qualitätsparameter wurden vor und nach 35 Tagen Lagerung bei $+4^{\circ} \mathrm{C}$ bestimmt. Ergebnisse. Die Qualitätsdaten der Plazentablut-EK sind im Vergleich zu herkömmlichen adulten EK in Tab. 1 dargestellt. Die Ergebnisse zeigen nach 35 Tagen Lagerung eine adultem EK vergleichbare Qualität. Schlussfolgerung. Die Verwendung von Plazentablut bei der Behandlung von anämischen Kindern ist in der dritten Welt weit verbreitet.

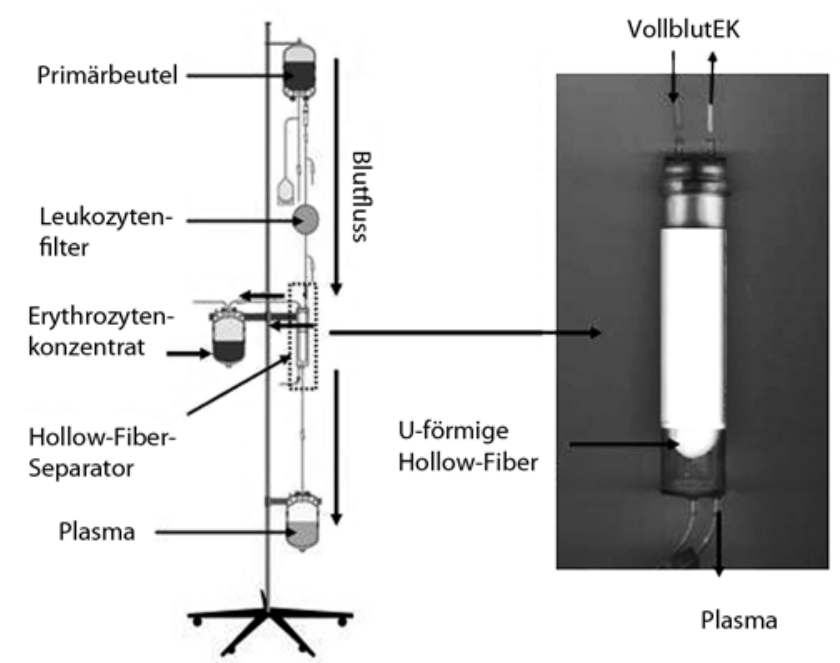

$\Delta$ Po6o-Abb. 1 Schema des Separationssystems

\begin{tabular}{|lll|}
\hline P060 - Tab. 1 & & \\
\hline & Plazentablut-EK & Normale adulte EK \\
\hline Volumen $(\mathrm{ml})$ & $72 \pm 23$ & $271 \pm 7$ \\
\hline Erythroz. $\left(10^{6}\right) / \mathrm{ul}$ & $5,1 \pm 0,6$ & $6,6 \pm 0,3$ \\
\hline Tot.- $\mathrm{Hb}(\mathrm{mmol} / \mathrm{l})$ & $12 \pm 0$ & $11 \pm 0$ \\
\hline Hkt $(\%)$ & $57 \pm 7$ & $56 \pm 1$ \\
\hline Freies $\mathrm{Hb}(\mathrm{mmol} / \mathrm{l})$ & $0,3 \pm 0,0$ & $0,0 \pm, 0,0$ \\
\hline Hämolyse $(\%)$ & $0,0 \pm 0,0$ & $0,0 \pm 0,0$ \\
\hline
\end{tabular}



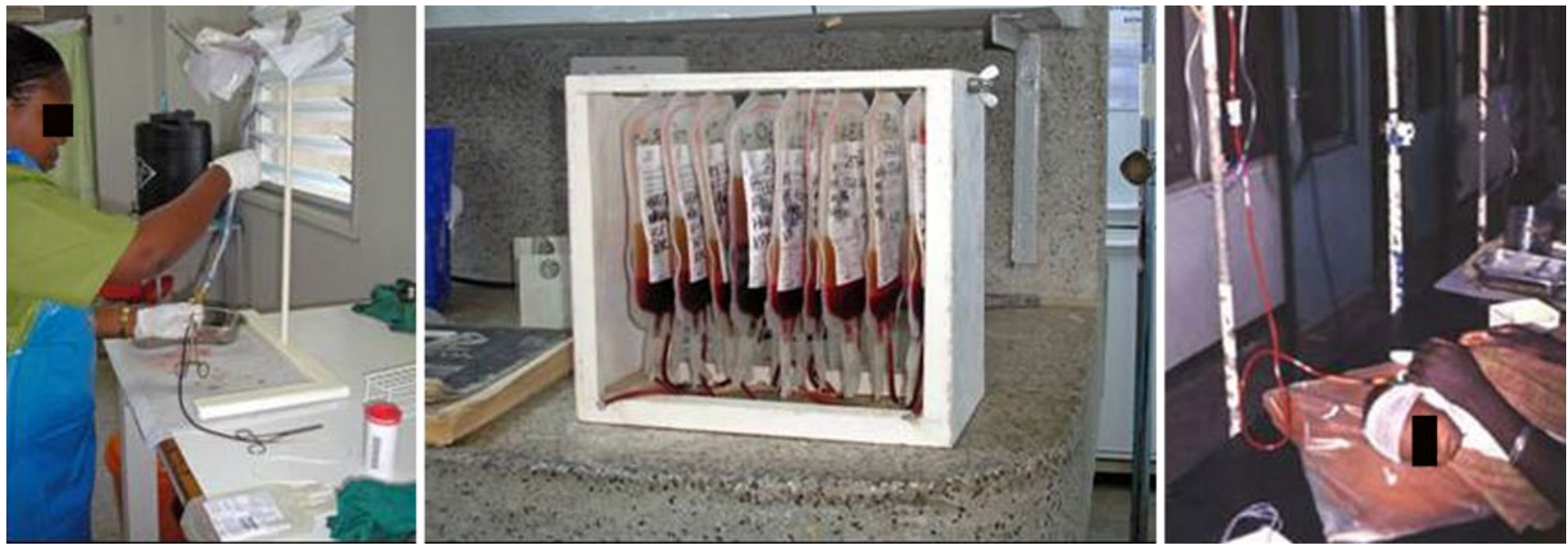

$\Delta$ Po62 - Abb. 1 Einsatz schwerkraftseparierter EK in Kenia

Unsere Gruppe konnte zeigen, dass ca. 40\% aller Neonaten mit angeborenen Fehlbildungen, die zeitnah nach der Geburt operiert werden, mit autologem Plazenblut fremdblutfreigehalten werden können (Brune et al, Transfusion 2003). Mit dem hier vorgestellten System könnte man autologes Plazentablut ohne Blutbankequipment im Kreissaal herstellen und ohne Herstellungserlaubnis dieser Patientengruppe retransfundieren. Eine entsprechende Studie ist in Vorbereitung.

\section{P061}

\section{Akute fetomaternale Transfusionen am Universitätsklinikum Dres-} den - 15 Fälle in 6 Jahren

\section{Mense', S. Gehrisch², M. Rüdiger', J. Dinger ${ }^{1}$}

'Universitätsklinikum Carl Gustav Carus Dresden, TU Dresden, Klinik und Poliklinik für Kinder- und Jugendmedizin, Abt. für Neonatologie \& Pädiatrische Intensivmedizin, Dresden, Deutschland, ${ }^{2}$ Universitätsklinikum Carl Gustav Carus Dresden, TU Dresden, Institut für Klinische Chemie \& Laboratoriumsmedizin, Dresden, Deutschland

Fragestellung. Bei einer fetomaternalen Transfusion kann akut oder chronisch ein pränataler Blutverlust in den mütterlichen Kreislauf auftreten. Die postnatale Präsentation dieser Kinder und ihr kurz- und mittelfristiger Verlauf sollen dargestellt werden.

Material und Methoden. Eine retrospektive Recherche bzgl. der ICD-10 Nummer P50.4 und P61.3 in den Jahren 2007 bis 2013 wurde durchgeführt. In allen Fällen wurden per flowzytometrischem Verfahren fetale Erythrozyten im mütterlichen Blut quantifiziert (Fetal Cell Count ${ }^{\mathrm{N}}$ Kit, IQ Products, Groningen), sodass eine Abschätzung des Shunt-Volumens möglich ist. Prä- und postnataler Verlauf und neurologisches Outcome werden dargestellt.

Ergebnisse. 11 term und 4 präterm geborene Neugeborene wurden im o. g. Zeitraum in der Abteilung behandelt. Der postnatale Zustand war in den meisten Fällen auffällig (1 min APGAR Median 5,5; min. o, max. 9), der Nabelschnur-pH aber normwertig $(7,23 \pm 0,07)$. In allen Fällen bestand eine neonatale Anämie (Hämatokrit $0,19 \pm 0,07$ ) mit einem errechneten Shuntvolumen von $128 \pm 39 \mathrm{ml}$. Die Transfusion von Erythrozytenkonzentraten war in allen Fällen notwendig und in 5/15 Fällen auch von Thrombozytenkonzentraten. Bei 3/15 Patienten trat eine persistierende pulmonale Hypertension des Neugeborenen auf. Die SchädelSonographie zeigte in 6/15 Fällen Auffälligkeiten (Parenchymblutung, Hirnödem oder periventrikuläre Leukomalazie). Zwei Kinder wurden aufgrund neurologischer Defizite in die neuropädiatrische Rehabilitation entlassen, ein Kind musste in Folge eines akuten Nierenversagens in ein Kinder-Dialyse-Zentrum verlegt werden.

Diskussion. Die Patienten dieser Fallserie präsentierten sich mit Symptomen einer akuten Anämie. Ein Hydrops fetalis, wie er bei chronischem fetomaternalem Blutverlust entstehen kann, trat nicht auf. Die
Bestimmung der fetalen Erythrozyten im mütterlichen Blut stellt eine zuverlässige Methode dar, um Patienten mit einer akuten fetomaternalen Transfusion zu identifizieren. Ein unauffälliger Nabelschnur-pH schließt eine fetomaternale Transfusion nicht aus.

Schlussfolgerung. Die fetomaternale Makrotransfusion stellt eine wichtige Differentialdiagnose der neonatalen Anämie dar, die nach der akuten hämodynamischen Stabilisierung mit weiteren Komplikationen einhergehen kann. Die Bestimmung der fetalen Erythrozyten im mütterlichen Blut gehört zur differentialdiagnostischen Abklärung.

\section{P062}

Gepooltes allogenes Plazentablut wird in Entwicklungsländern erfolgreich in der Therapie von anämischen Säuglingen und Kleinkindern eingesetzt

\section{T. Brune', O. Oechsle', G. Jorch ${ }^{3}$, O. Hassall/4}

'Klinikum Lippe, Kinderklinik, Detmold, Deutschland, ${ }^{2} \mathrm{Hemacon} \mathrm{GmbH}$, Düsseldorf, Deutschland, ${ }^{3}$ Universitätskinderklinik, Magdeburg, Deutschland, ${ }^{4}$ Centre for Geographic Medicine Research, Kilifi, Kenya

Hintergrund. Fast alle Frühgeborenen $<28$ SSW werden mindestens 1-mal mit einem EK transfundiert. Bluttransfusionen sind bei sehr unreifen Frühgeborenen mit einer erhöhten Inzidenz der Retinopathia praematurorum verbunden, die genaue Pathogenese ist noch ungeklärt. Das Blut Früh- bzw. Reifgeborener enthält hauptsächlich fetales Hämoglobin $(\mathrm{HbF})$, welches im Vergleich zu adultem Hämoglobin ( $\mathrm{HbA})$ eine höhere Sauerstoffaffinität sowie eine höhere Viskosität besitzt. Dies führt zu einer im Vergleich zum Erwachsenen höheren Sauerstoffaufnahme in der Lunge bei gleichzeitig niedrigerem Sauerstoffdruck im Gewebe. Die bei Transfusionen von HbA-haltigem adultem Blut erhöhte Sauerstoffkonzentration im Gewebe ist für das Frühgeborene unphysiologisch, erschwert die Sauerstoffaufnahme und ist wegen der toxischen Wirkung des Sauerstoffs auf die Retina sowie der geringen Viskosität (Kapillarverstopfung) eventuell sogar schädlich [1, 2]. Der Einsatz von plazentarem Blut zur Transfusion von Frühgeborenen wird in den hoch technologisierten Ländern schon lange diskutiert.

Methoden und Ergebnisse. Studien mit autolog gewonnenem Plazentablut zeigten, dass die Herstellung und Retransfusion von HbF-EK problemlos möglich ist, die Mengen aber nicht ausreichen, um die Frühgeborenen ausschließlich mit autologem HbF-Blut zu transfundieren [3]. Während die Blutbanken in Deutschland bisher wenig Interesse an der Herstellung von allogenen HbF-Blutkonserven entwickelten, wurde dieses Konzept in Entwicklungsländern bereits erfolgreich und mit minimalem technischem Aufwand umgesetzt. In einem Wellcome Trust geförderten Projekt wurden ca. 1000 Kinder mit durch Schwerkraft separiertem und blutgruppenkompatibel gepooltem Plazentablut ohne Nebenwirkungen transfundiert (Abb.1; [4]). 

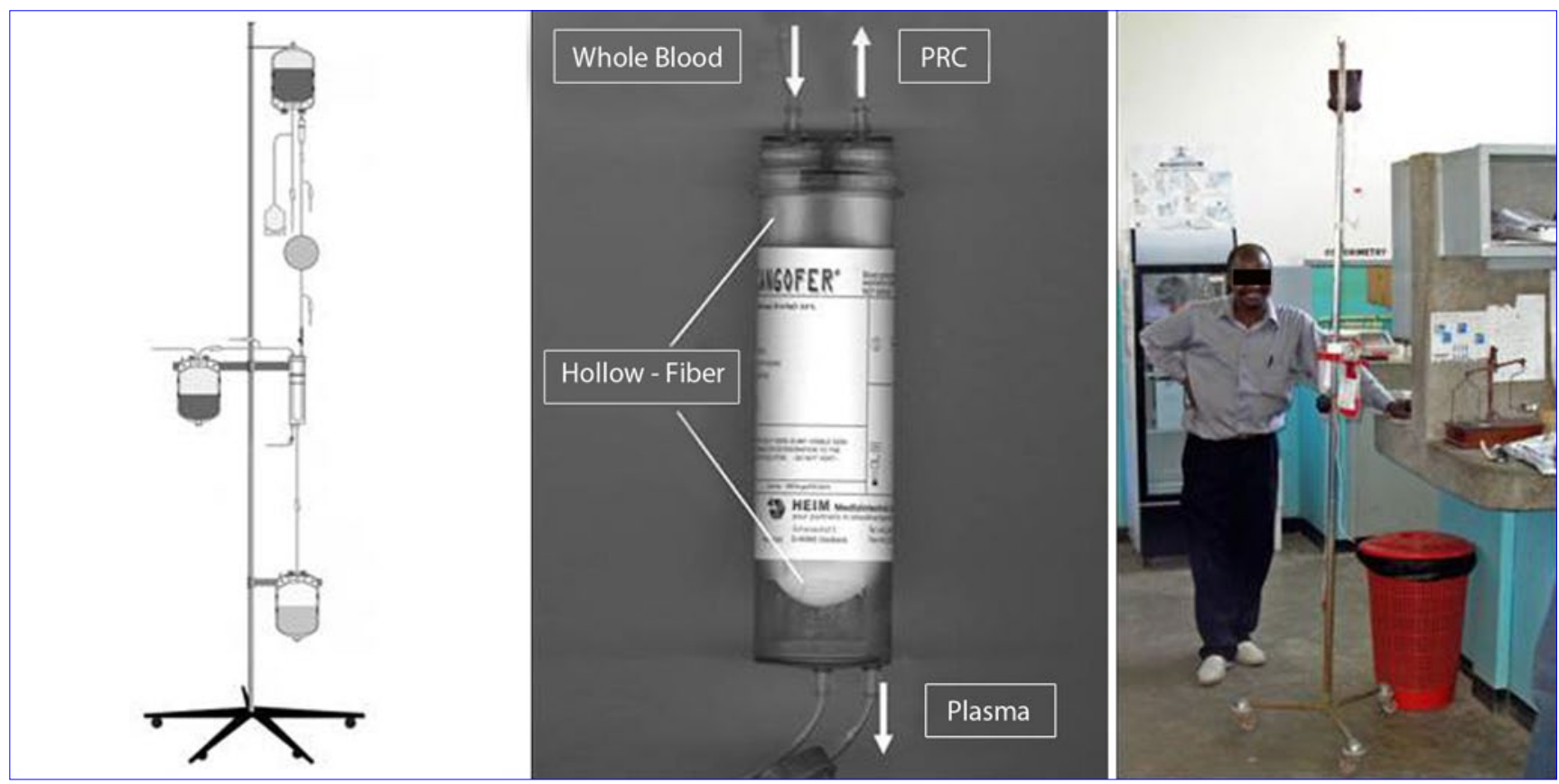

$\Delta$ Po62-Abb. 2 Separation mit Leukozythendepletion und Schwerkraftfiltration

Ergebnisse. Bakteriologische Kontrollen zeigten, dass die Gefahr der bakteriellen Kontamination von plazentar gewonnenem Blut geringer ist als bei konventionell hergestellten Blutkonserven [5]. Erste Versuche mit einem Schwerkraftseparationsfilter einschließlich Leukozythendepletion verliefen ebenfalls erfolgreich (Hemacon; Abb. 2).

Schlussfolgerung. Das Projekt zeigt, dass die Separation von HbF-Blut einfach und schnell durchführbar ist, und auch in großem Stil in Entwicklungsländern eingesetzt wird. Kalkulationen zeigen, dass bei den in der Neonatologie verbrauchten Blutmengen die Herstellung von HbF-Konserven per Schwerkraftseparation den bisherigen Methoden (Zentrifugation) wirtschaftlich gleichwertig bzw. sogar überlegen ist.

1. Gleissner et al (2003) Z Geburtshilfe Neonatol 207(1):24-28

2. Saunders et al (2000) Pediatrics 106:378-379

3. Brune et al (2003) Transfusion 43(9):1210-1216

4. Hassall et al (2003) Lancet 361(9358):678-679

5. Hassall et al (2012) Transfusion 52(7):1542-1551

6. Brune et al (2007) Transfusion 47(12):2271-2275

\section{P063}

Vorgeburtlicher thrombembolischer Carotisverschluss mit Plexusparese

S. Römer', M. Berns', S. Verlohren², L. Dröge', W. Henrich², C. Bührer'

'Charité Universitätsmedizin Berlin, Neonatologie, Berlin, Deutschland, ${ }^{2}$ Charité Universitätsmedizin Berlin, Geburtsmedizin, Berlin, Deutschland

Einleitung. Perinatale thrombembolische arterielle Verschlüsse mit klinischer Symptomatik betreffen meist die A. cerebri media.

Fallbericht. Bei einer 29-jährigen Schwangeren mit Präeklampsie zeigte sich nach 34 Wochen Gestationsdauer ein hochpathologisches Flussprofil in den zerebralen Gefäßen bei normalen Profilen in den Aa. Uterinae und der A. umbilicalis. Wegen zunehmender Präeklampsie-Symptomatik Sectio nach 34 2/7 Wochen, Geburtsgewicht $2300 \mathrm{~g}$, Nabelarterien-pH 7,26, Apgar 9/9/10. Bereits im Kreißsaal schlaffe Lähmung der rechten oberen Extremität, zunehmende Nekrose der Fingerkuppe des rechten Ringfingers. In der Sonographie des Halses Nachweis von Thromben in der A. carotis communis, A. carotis interna und der A. subclavia rechts. In der MRT zusätzlich Verschluss der rechten Ver- tebralarterie im V2- und $\mathrm{V}_{3}$-Segment. Eine Thrombophilie-Suche beim Kind blieb ohne pathologisches Ergebnis. Unter Heparinisierung Rückbildung der Thromben, Besserung der Parese unter Physiotherapie.

Diskussion. Der pränatal physiologische Rechts-Links-Fluss durch das Foramen ovale erlaubt die Passage thromboembolischen Materials aus dem Körper des Kindes und der Plazenta in die Systemzirkulation. In diesem Fall scheint es bereits vor der Geburt zu einem thromboembolischen Carotisverschluss gekommen zu sein. Da die Entwicklung des Kindes völlig atraumatisch erfolgte, liegt es nahe, auch die Ursache der Plexusparese in einer thrombembolisch bedingten Ischämie zu suchen.

\section{P064}

Lysetherapie bei einem 2 Monate alten Säugling mit Nierenarterienthrombose

\section{U. Wördehoff', M. Born'2, H. Hertfelder', A. Müller', S. Bagci ${ }^{5}$}

'Universitätsklinik Zentrum f. Kinderheilkunde, Neonatologie, Bonn, Deutschland, ${ }^{2}$ Radiologische Klinik Universitätsklinikum Bonn, Bonn, Deutschland, ${ }^{3}$ Institut für Experimentelle Hämatologie und Transfusionsmedizin/ Universitätsklinikum Bonn, Bonn, Deutschland, ${ }^{4}$ Universitätsklinikum Bonn, Zentrum für Kinderheilkunde, Abteilung für Neonatologie, Bonn, Deutschland, ${ }^{5}$ Zentrum für Kinderheilkunde der Universität Bonn, Abteilung Neonatologie, Bonn, Deutschland

Einleitung. Eine Nierenarterienthrombose im Neugeborenen- und Säuglingsalter ist ein sehr seltenes Ereignis und bei Neonaten in den meisten beschriebenen Fällen mit einem Nabelarterienkatheter assoziiert. Wir berichten von einem 2 Monate alten Säugling mit arterieller Hypertonie infolge einer Nierenarterienthrombose, die erfolgreich teillysiert wurde.

Kasuistik. Es handelt sich um ein reifes Neugeborenes mit rechtsseitiger Zwerchfellhernie. Nach ECMO-Therapie erfolgt in der 2. Lebenswoche der operative Zwerchfellverschluss. Im Alter von 10 Wochen entwickelt das Kind eine arterielle Hypertonie. Parallel fällt das Kind wenig später durch eine zunehmende Ileussyptomatik auf, bedingt durch eine Rezidivhernie, die operativ versorgt wird. Es wird eine antihypertensive Therapie mit Urapidil eingeleitet und im Verlauf bei weiter ansteigenden Blutdruckwerten mit MAD bis $120 \mathrm{mmHg}$ durch Amlodipin erweitert. Sonographisch fällt eine echogene Veränderung der distalen linken Nierenarterie im Hilusbereich mit pathologischem Flussprofil 
sowie erhöhte D-Dimere auf, passend zu dem Bild einer Nierenarterienthrombose. Nierenwerte und Diurese sind nicht relevant beeinträchtigt. 11 Tage nach den ersten dokumentiert hohen Blutdruckwerten und 5 Tage postoperativ einer Second-look-Operation wird eine systemische Lysetherapie mit rt-PA o,7 mg/kg/Tag begonnen. Innerhalb einer Woche fällt darunter das aktivierte Renin im Serum von $5422 \mathrm{uU} / \mathrm{ml}$ (Norm 11-147 uU/ml) auf $14 \mathrm{uU} / \mathrm{ml} \mathrm{ab}$, sonographisch lässt sich eine verbesserte Nierendurchblutung nachweisen, die Blutdruckwerte sind rückläufig. Nach 10 Tagen wird die Lysetherapie beendet und die Behandlung mit niedermolekularem Heparin weitergeführt. Blutungen oder andere Nebenwirkungen treten unter Lysetherapie nicht auf.

Schlussfolgerung. Eine Nierenarterienthrombose ist im Säuglingsalter ein sehr seltenes Ereignis. Bei neu auftretender arterieller Hypertonie sollte jedoch auch beim Vorliegen einer komplexen Grunderkrankung und vorbestehender kardialer Belastung an eine renale Ursache gedacht werden. Eine Lysetherapie wird üblicherweise bei einer frischen Thrombose in Betracht gezogen. Trotz des relativ späten Beginns der Behandlung mit rt-PA konnte in unserem Fall eine Teil-Lyse des Thrombus mit Normalisierung des Reninwertes erreicht werden.

\section{P065}

Thrombotischer Verschluss der Arteria brachialis bei einem Neugeborenen - seltene Komplikation durch einen periphervenösen Katheter

\section{S. Berzel', W. Garbe', H. Hertfelder', N. Hepping', K. Schneider'}

${ }^{1}$ St. Marien-Hospital Bonn, Neonatologie, Bonn, Deutschland, ${ }^{2}$ Institut für Experimentelle Hämatologie und Transfusionsmedizin/ Universitätsklinikum Bonn, Bonn, Deutschland

Hintergrund. Thrombosen sind in der Pädiatrie im Vergleich zur Erwachsenenmedizin ein seltenes Krankheitsbild. Die Inzidenz für eine Thrombose in der Neonatalperiode wird in der Literatur mit o,5/10.0oo angegeben. Bei stationär behandelten Kindern wird die Inzidenz für eine venöse oder arterielle Thrombose auf 5,3 pro 10.000 Kindern geschätzt, mit erhöhtem Risiko im Säuglings- und Adoleszentenalter. Das höchste Risiko besteht im Zusammenhang mit einem zentralen Gefäßkatheter. Gefäßthrombosen durch einen peripher-venösen Katheter (pVK) hingegen sind in der Neonatologie eine Rarität.

Fallbeschreibung. Beschrieben wird der Fall eines 5 Tage alten, reifen Neugeborenen, bei dem 2 Tage nach Anlage eines pVK in der Ellenbeuge eine Thrombosierung der Arteria brachialis, mit akuter Ischämie der betroffenen Extremität auftrat. Die klinische Diagnose konnte duplexsonographisch bestätigt werden. Aufgrund einer ausreichenden Kollateralperfusion verbesserte sich der klinische Befund des betroffenen Armes rasch. Begleitend erfolgte eine Antikoagulation mit Heparin, ab dem zweiten Tag mit einem niedermolekularen Heparin. Zum Monitoring der antikoagulatorischen Therapie dienten Anti-Xa-Spiegel und D-Dimere.

Diskussion. Aufgrund der zeitlichen Latenz ist davon auszugehen, dass nach intravenöser Lage des Katheters, dieser aufgrund der Position in der Nähe eines großen Gelenkes sekundär zu einer Verletzung der Arterie geführt hat. Wegweisend für das Outcome der Extremität ist die schnelle Diagnosefindung. Dazu gehört neben der typischen klinischen Präsentation auch eine sofortige Bildgebung zur Darstellung des betroffenen Gefäßes. Methodisch stehen der Ultraschall mit Gefäßdoppler, die Angiographie, Magnetresonanztomographie oder Computertomographie zu Verfügung. Außer der Ultraschalldiagnostik sind die angeführten Untersuchungsmethoden wie auch Interventionen vor allem in der Neonatologie, mit erheblichen Risiken, Einschränkungen und potentiellen Nebenwirkungen für den Patienten verbunden. In den ersten Lebenstagen ist die Chance der Rekanalisierung im Vergleich zu anderen Lebensaltern am höchsten, so dass bei Neonaten eine invasive Intervention die Ausnahme bleibt. Ziel der Therapie ist die Revaskularisierung des betroffenen Gefäßes, zumindest aber die Verhinderung eines weiteren Thrombuswachstums. Die aktuell existierenden Therapieempfehlungen für pädiatrische Patienten beruhen auf adaptierten Schemata aus der Erwachsenenmedizin.

Schlussfolgerung. In der Neonatologie ist die Sonographie eine der wichtigsten bildgebenden Untersuchungsverfahren und sollte jederzeit auf der Intensivstation zur Verfügung stehen. In dem geschilderten Fall ermöglichte die rasche Therapie mit an die Neonatologie angepassten antikoagulatorischen Therapieschemata eine rasche Rekanalisierung des betroffenen Gefäßes und die hochauflösenden Duplexsonographie als schonende Untersuchungsmethode die engmaschige Überwachung mit gleichzeitiger Kontrolle des Therapieerfolges (Abb. 1, Abb. 2).

\section{P066}

\section{Experimentelle Hydroxychloroquine - Therapie eines schweren} neonatalen Atemnotsyndroms aufgrund einer neu beschriebenen Surfactant Protein C Mutation

\section{N. Hepping', K. Schneider', M. Griese' ${ }^{2}$, P. Lohse ${ }^{3}$, W. Garbe}

${ }^{1}$ St. Marien-Hospital Bonn, Neonatologie, Bonn, Deutschland, ${ }^{2}$ Dr.-von-Haunerschen Kinderspital, Kinderklinik und Poliklinik, München, Deutschland, ${ }^{3}$ Klinikum der Universität München, ${ }^{3}$ Institut für Klinische Chemie - Großhadern, München, Deutschland

Introduction. SFTPC (surfactant protein C) mutations resulting in SP-C deficiency causing ongoing respiratory failure in the neonatal period represent a rare entity.

Case. We report a full-term female infant who developed respiratory distress and respiratory failure shortly after birth. From the first day of life the infant was mechanically ventilated. Application of exogenous surfactant or cortisone did not lead to any clinical improvement. Genetic analysis identified a novel SFTPC mutation as the cause of her lung disease. The patient was diagnosed as heterozygous for a p.Cys121Gly/ C121G substitution encoded by exon 4, which could not be detected in both parents. Experimental therapy with hydroxychloroquine resulted in a significant clinical improvement within 2 weeks time. Mechanical ventilation was no longer needed, and the patient was discharged without additional oxygen demand. The patient remained well under therapy till the age of 6 months. After that time, the therapy was successfully discontinued.

Conclusion. According to our knowledge, this is the first case report of a severe neonatal respiratory failure due to SP-C deficiency in a neonate who was recovering under hydroxychloroquine therapy. However, a direct relationship between this treatment and the child's clinical improvement cannot be proven, but the close temporal association strongly suggests a causal role of this drug. Additional work is nevertheless needed to assess the general usefulness of hydroxychloroquine in patients with SP-C deficiency.

\section{P067}

\section{Pulmonalarterienembolie bei Neugeborenen}

B. Michael', S. Spinner', F. Löffelbein', I. Dähnert ${ }^{1}$

'Herzzentrum Leipzig GmbH, Klinik für Kinderkardiologie, Leipzig, Deutschland

Einleitung. Die Pulmonalarterienembolie (PAE) bei Kindern ist eine seltene Erkrankung, die häufig stumm und nur in 50\% der Fälle mit typischer Symptomatik verläuft. Am häufigsten findet sie sich in der Neonatalzeit, die Hälfte dieser Kinder sind Frühgeborene. Die Hauptursache liegt in der intensivierten Therapie mit Zentralem Venenkatheter, gefolgt von perinatalen Infektionen. Auch Asphyxie, Hypovolämie und maternaler Diabetes sind als Ursache zu bedenken.

Zielstellung. Überblick über Symptome und Therapie der PAE anhand einer Kasuistik.

Fallbericht. Sekundäre Sectio caesarea in der 38.+4. SSW bei pathol. CTG und Makrosomie. Aufgrund schlechter postnataler Anpassung Reanimation und CPAP-Beatmung. Initial antibiotische Therapie bei V. a. Infektion. Am 9. LT akuter Verfall mit Sättigungsabfall und Ta- 
Tag 0

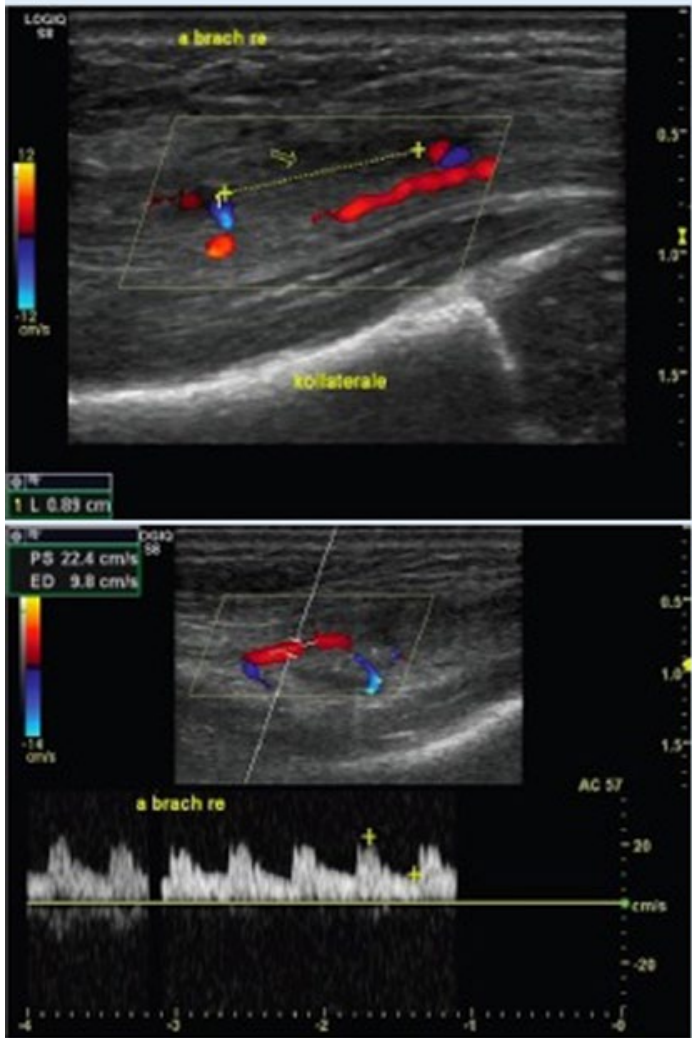

Tag 22

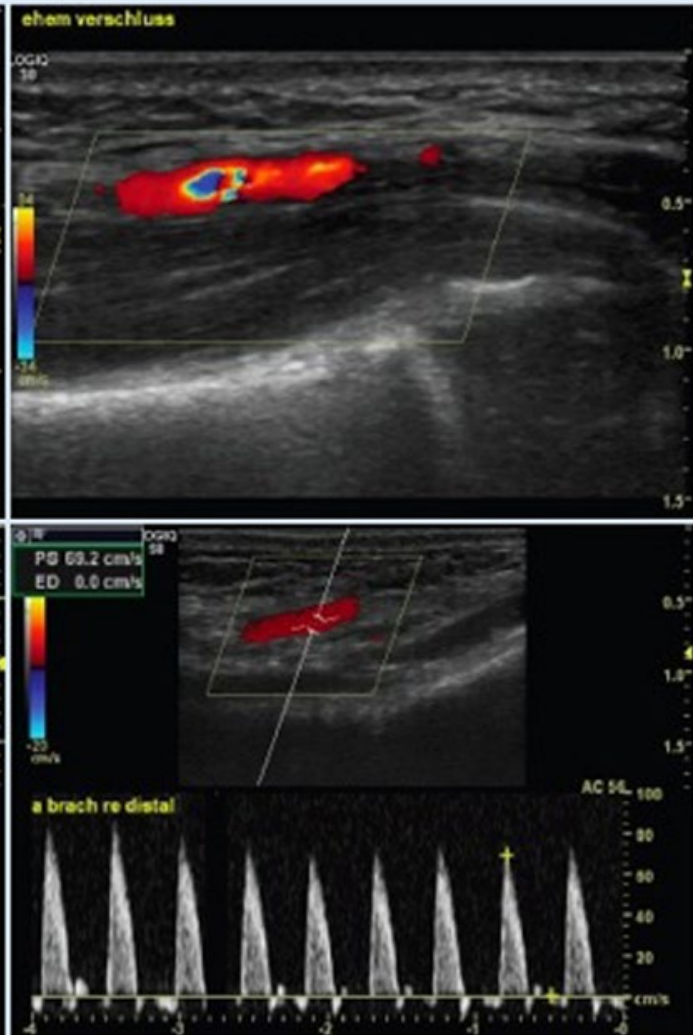

Po65 - Abb. 1
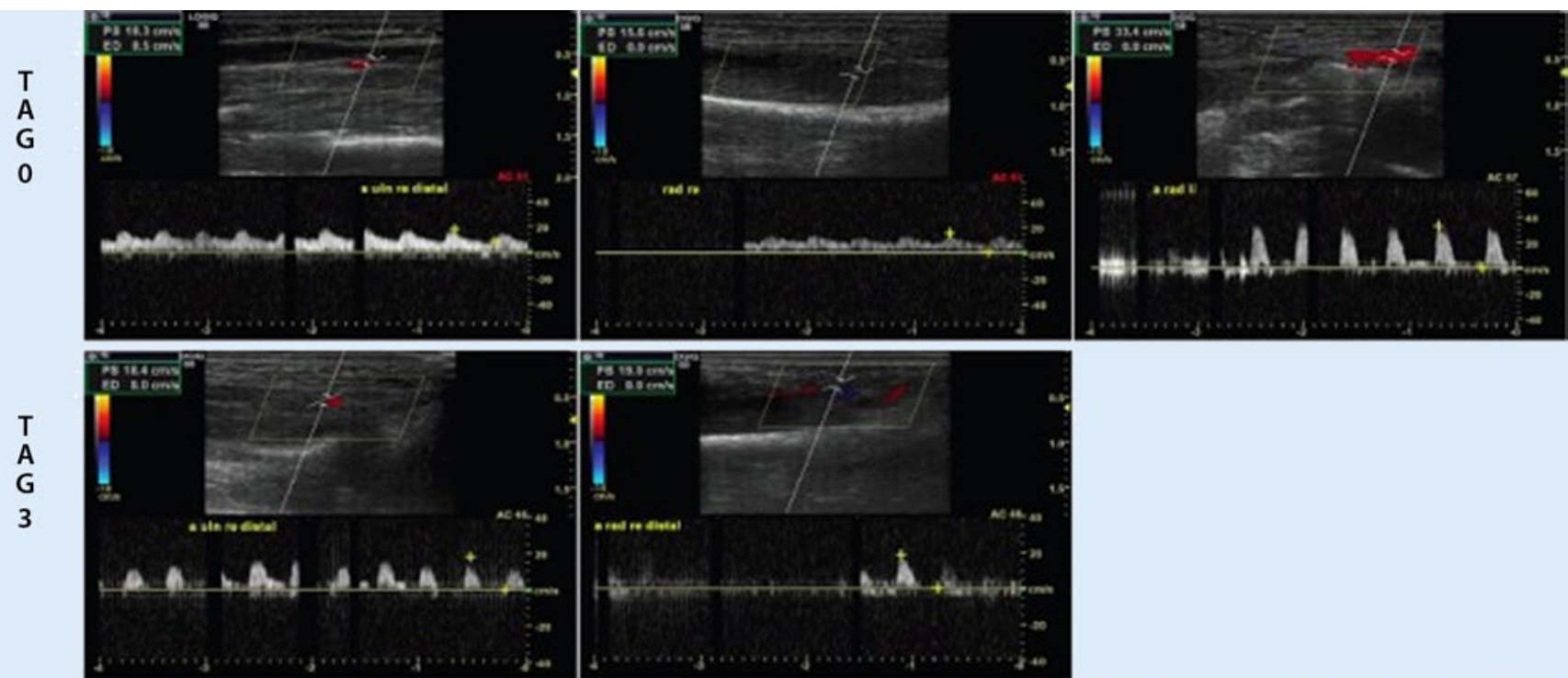

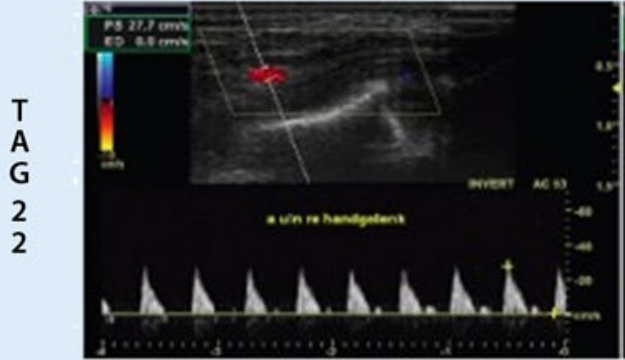

A. ulnaris rechts

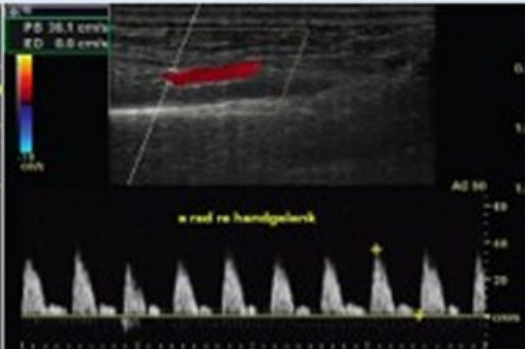

A. radialis rechts

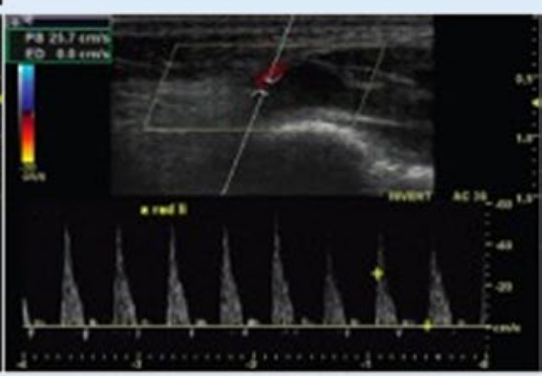

A. radialis links

A Po65-Abb. 2 
chydyspnoe. Mittels Echo Nachweis einer PAE mit akuter Rechtsherzinsuffienz und hochgradiger Trikuspidalinsuffizienz. Unter Heparin-, Minprog- und Dobutamingabe Verlegung des Kindes. Durchführung einer Katheterintervention mit Anlage eines Lysekatheters. Es gelang die Thrombolyse mittels Actilyse und Hochdosis-Heparin. Rückverlegung des Kindes in gutem Allgemeinzustand. Bisher altersentsprechende Entwicklung (über 6 Monate).

Diskussion. Die Symptomatik wurde richtig interpretiert und die Diagnose echokardiographisch richtig gestellt. Durch rasche Einleitung einer antikongestiven Therapie und Verlegung des Kindes wurde ein gutes Ergebnis erreicht.

Schlussfolgerung. Bei Kindern mit o. g. Risikoprofil ist bei einem akuten Verfall mit Sättigungsabfall, Herzinsuffizienz und ggf. Beatmungspflichtigkeit eine PAE in Betracht zu ziehen. Die Echokardiographie dient als diagnostisches Mittel der Wahl.

\section{P068}

\section{Fetomaternale Makrotransfusion bei einem Frühgeborenen}

\section{K. Schneider', W. Garbe', J. Schmitt' ${ }^{2}$, N. Hepping'}

${ }^{1}$ St. Marien-Hospital Bonn, Neonatologie, Bonn, Deutschland, ${ }^{2}$ St. Marien-Hospital, Kinderklinik, Bonn, Deutschland

Einleitung. Die klinischen Zeichen einer fetomaternalen Makrotransfusion $(>30 \mathrm{ml}$ ) sind in Form nachlassender Kindsbewegungen sehr unspezifisch. Allerdings ist eine frühzeitige Diagnosestellung, insbesondere in frühen Gestationswochen entscheidend, um eine rechtzeitige intrauterine Erythrozytentransfusion zu ermöglichen. Bestehen bereits akute CTG-Veränderungen ist unabhängig vom Schwangerschaftsalter eine unmittelbare Sectio indiziert. Eine sofortige postnatale Erythrozytentransfusion ist dann bei hämodynamisch bedeutsamer Anämie unverzichtbar.

Kasuistik. Vorstellung einer Patientin mit $31+3 / 7$ Schwangerschaftswochen aufgrund nachlassender Kindsbewegungen nach bisher unauffälligem Schwangerschaftsverlauf. Bei silentem CTG-Entschluss zur Not-Sectio und Entbindung eines schwer deprimierten weiblichen Frühgeborenen (Apgar 1/6/8, Geburtsgewicht 1595 g). Trotz sofortiger Intubation und Beatmung persistierte bei tachykarder Herzfrequenz von 190/min eine ausgeprägte Blässe, die Nabelschnur stellte sich blutleer dar. Bei einem Hämoglobinwert von 2,5 g/dl wurde umgehend eine Transfusion mit $30 \mathrm{ml}$ eines O-negativen Erythrozytenkonzentrates durchgeführt. Insgesamt waren $105 \mathrm{ml}(65 \mathrm{ml} / \mathrm{kgKG})$ Erythrozytenkonzentrat erforderlich, um einen $\mathrm{Hb}$-Anstieg auf $13,5 \mathrm{mg} / \mathrm{dl}$ zu erreichen. Das maternal gemessene $\mathrm{HbF}$ lag bei $4,22 \%$, was einem übertragenen fetalen Blutvolumen von etwa $150 \mathrm{ml}$ entspricht. Nach schwerem Atemnotsyndrom und persistierender pulmonaler Hypertonie am ersten Lebenstag gestaltete sich der weitere neonatale Verlauf komplikationslos. In den regelmäßigen neurologischen Nachuntersuchungen zeigt das Kind bis heute im Alter von 3 Jahren eine altersentsprechende motorische und kognitive Entwicklung.

Diskussion. Massive fetomaternale Transfusionssyndrome treten selten auf, bedürfen jedoch einer sofortigen Therapie. In Fällen, die vor der 37. Schwangerschaftswoche diagnostiziert werden, sollte eine fetale Erythrozytentransfusion erwogen werden. Sollte diese jedoch wie in dem vorgestellten Fall bei bereits hochgradig pathologischem CTG nicht mehr möglich sein, ist auch bei Frühgeborenen eine Not-Sectio sowie eine sofortige postnatale Transfusion indiziert. Diese muss unter Inkaufnahme aller bekannten Begleitkomplikationen einer frühen Massentransfusion zur hämodynamischen Stabilisierung des Kindes umgehend durchgeführt werden.

Schlussfolgerung. Eine massive fetomaternale Transfusion ist ein seltenes, aber potentiell lebensbedrohliches neonatales Krankheitsbild. Wesentlich ist das Erkennen möglicher Risikofaktoren, um eine adäquate postnatale Versorgung, insbesondere eine sofortige Erythrozytentransfusion ohne Zeitverzug zu gewährleisten.

\section{Pulmologie und Beatmung}

\section{P069}

Genomweite Assoziationsstudie zum Atemnotsyndrom des Frühgeborenen

F. Pagel', M. Preuß', U. Felderhoff-Müser', A. Kribs' ${ }^{3}$, L. Gortner', M. Rüdiger', R. Hentschel' ${ }^{6}$, A. Ziegler', C. Härtel', E. Herting', W. Göpel'

'Klinik für Kinder- und Jugendmedizin, Universität Lübeck, UKSH, Lübeck, Deutschland, ${ }^{2}$ Universitätskinderklinik Essen, Essen, Deutschland, ${ }^{3}$ Universitätskinderklinik Köln, Köln, Deutschland, ${ }^{4}$ Kinderklinik des Universitätsklinikums des Saarlandes, Homburg, Homburg, Deutschland, ${ }^{5}$ Kinderklinik des Universitätsklinikums Carl Gustav Carus, Dresden, Dresden, Deutschland, ${ }^{6} U n i v e r-$ sitätskinderklinik Freiburg, Freiburg, Deutschland, ${ }^{7}$ Institut für Medizinische Biometrie und Statistik, Universität Lübeck, Lübeck, Deutschland

Hintergrund. Trotz großer Fortschritte in der Therapie des Atemnotsyndroms (RDS) zählt diese Erkrankung immer noch zu den häufigsten Todesursachen bei Frühgeborenen weltweit. Wichtige Risikofaktoren für das Auftreten eines RDS sind niedriges Gestationsalter und männliches Geschlecht. Interessanterweise ist das RDS bei europäischen Frühgeborenen deutlich häufiger als bei Asiaten oder Afrikanern. Wir untersuchten in einer genomweiten Assoziationsstudie genetische Risikofaktoren für das Auftreten eines RDS.

Patienten und Methoden. Wir definierten die Therapie mit exogenem Surfactant als sicheres Zeichen für das Vorliegen eines RDS. Frühgeborene mit einem Geburtsgewicht unter 1500 Gramm wurden Chip-genotypisiert (AXIOM „genome wide array"). Mit der Notwendigkeit einer Surfactanttherapie assoziierte Polymorphismen wurden in zwei zusätzlichen Frühgeborenenkohorten repliziert (TaqMan-assay).

Ergebnisse. Von 1272 Chip-genotypisierten Kindern konnten 1257 Datensätze ausgewertet werden. 727 Frühgeborene (59\%) waren mit Surfactant behandelt worden. Von 10 assoziierten Polymorphismen, konnten wir nur einen Polymorphismus in einer 2. Kohorte $(n=3839)$ replizierten. Dieses Ergebnis bestätigte sich in einer dritten Frühgeborenenkohorte $(n=2830)$. Der Polymorphismus befindet sich auf Chromosom 9 und ist mit einer reduzierten RDS-Rate assoziiert (OR 0,7; 95\% CI $0,6-0,8 ; p=3,8 \times 10-8$ korrigiert für das Gestationsalter). Der Effekt des Polymorphismus in Bezug auf das RDS ist etwa vergleichbar mit dem Vorliegen des weiblichen Geschlechts. 20\% aller europäischen Frühgeborenen sind heterozygote, $2 \%$ homozygote Träger des Polymorphismus. Bei Frühgeborenen asiatischer $(n=115)$ bzw. afrikanischer $(n=209)$ Herkunft ist der Polymorphismus deutlich häufiger (40\% heterozygote und $8 \%$ homozygote Merkmalsträger). Auch in diesen Kollektiven ist der Polymorphismus mit einer niedrigeren RDS-Rate assoziiert.

Diskussion. Erstmals konnte ein häufiger Polymorphismus mit Einfluss auf das RDS des Frühgeborenen identifiziert werden.

Schlussfolgerung. Weitere Untersuchungen werden zeigen, ob die beschriebene Assoziation spezifischen Genen zugeordnet werden kann und somit zusätzliche Optionen für die Therapie des Atemnotsyndroms eröffnet.

\section{P070}

Abhängigkeit der BPD-Rate Frühgeborener von Gewichtsverlauf, Flüssigkeits- und Natriumhaushalt in den ersten 10 Lebenstagen

T. Ott ${ }^{1}$, M. Tekaat ${ }^{2}$, E. Rieger-Fackeldey ${ }^{3}$

'Westf. Wilhelms-Univ.- Kinderklinik, Klinik für Kinder- und Jugendmedizin - Allgemeine Pädiatrie - Münster, Deutschland, ${ }^{2}$ Westf. Wilhelms-Univ.- Kinderklinik, Münster, Deutschland, ${ }^{3}$ Universitätsklinikum Münster, Klinik und Poliklinik für Kinder- und Jugendmedizin, Allgemeine Pädiatrie, Neonatologie und Pädiatrische Intensivmedizin, Münster, Deutschland

Fragestellung. Abhängigkeit der BPD-Rate von Gewichtsabnahme sowie von der Menge und Art der Flüssigkeits- und der Höhe der Natriumzufuhr in den ersten 10 Lebenstagen. 
Material und Methoden. Retrospektive Analyse von 215 Frühgeborenen der Jahre 2010 bis 2013 mit einem Geburtsgewicht von unter $1500 \mathrm{~g}$, statistische Auswertung von 165 Frühgeborenen mittels Chi-Quadrat- und Mann-Whitney-Rangsummentest.

Ergebnisse. Frühgeborene mit BPD waren unreifer (25,4 SSW vs. 29,1 SSW; $<<0,001$ ), hatten ein niedrigeres Geburtsgewicht (655 g vs. 1200 g; $\mathrm{p}<0,001)$ und vermehrt weitere Komplikationen wie eine IVH II. oder III. Grades bzw. mit Parenchymbeteiligung (24,0\% vs. $7,8 \%$, RR 2,17, $95 \%$ CI 1,37-3,43), einen behandlungsbedürftigen PDA ( $76,0 \%$ vs. $38,3 \%$, RR 3,21, 95\% CI 1,81-5,68) oder eine NEC Grad II oder höher (10,0\% vs. $1,7 \%$, RR 2,51, 95\% CI 1,48-4,26). BPD-Patienten hatten seltener eine postnatale Gewichtsabnahme $(74,0 \%)$ als Patienten ohne BPD $(84,3 \%)$, dieser Unterschied war statistisch jedoch nicht signifikant (RR o,658, 95\% CI 0,40-1,08). Nach 10 Tagen hatten BPD-Patienten jedoch mehr Gewicht zugenommen als Patienten ohne BPD (6,3\% vs. 2,0\%; $p=0,008)$. Frühgeborene mit BPD hatten in den ersten 10 Lebenstagen signifikant mehr Flüssigkeit erhalten, sowohl in der Gesamtsumme ( $1772 \mathrm{ml} / \mathrm{kg}$ vs. $1558 \mathrm{ml} / \mathrm{kg} ; \mathrm{p}<\mathrm{o}, \mathrm{OO1})$ als auch auf die einzelnen Tage bezogen. Darüber hinaus war die BPD-Rate korreliert mit der Flüssigkeitsmenge durch Volumenboli in den ersten drei (30,3 ml/kg vs. 15,0 ml/kg; p<0,001) und den ersten 10 Lebenstagen $(52,7 \mathrm{ml} / \mathrm{kg}$ vs. $20,1 \mathrm{ml} / \mathrm{kg}$; $\mathrm{p}<0,001)$. Sowohl die Gabe von mehr als $20 \mathrm{ml} / \mathrm{kg}$ Volumen zusätzlich in den ersten 3 Lebenstagen (RR 2,36, 95\% CI 1,40-3,98) als auch mehr als $30 \mathrm{ml} / \mathrm{kg}$ in den ersten 10 Tagen waren in der BPD-Gruppe häufiger (RR 4,15, 95\% CI 2,29-7,53). Ob ein Frühgeborenes überhaupt Bolusgaben erhalten hatte, war stärker mit einer BPD korreliert (RR 12,25, 95\% CI 1,76- 85,48) als Blutprodukte (RR 4,06, 95\% CI 2,34-7,04), Flüssigkeit in Form eines Bilanzausgleichs nicht signifikant ( $R R$ 1,52, 95\% CI o,78-2,94), auch wenn Frühgeborene mit BPD mehr Bilanzausgleich erhalten hatten. Die Natriumzufuhr in der BPD-Gruppe war höher (bis Tag $316,8 \mathrm{mmol} / \mathrm{kg}$ vs. $9,7 \mathrm{mmol} / \mathrm{kg}$; $\mathrm{p}<0,001$, bis Tag $1072,1 \mathrm{mmol} / \mathrm{kg}$ vs. $37,7 \mathrm{mmol} / \mathrm{kg}$; p<o,oo1), ab Tag 4 waren die Natriumwerte höher (z. B. Tag 5144 mmol/l vs. $139 \mathrm{mmol} / \mathrm{l}$; $\mathrm{p}<0,001)$. Ein Natriumwert von über $150 \mathrm{mmol} / \mathrm{l}$ trat bei Frühgeborenen mit BPD in $38 \%$ der Fälle auf, ohne BPD nur in $7 \%$ (RR 3,13, 95\% CI 2,11-4,65). Der enterale Nahrungsaufbau war an Tag 10 bei Frühgeborenen ohne BPD bereits deutlich weiter vorangeschritten $(106 \mathrm{ml} / \mathrm{kg} /$ Tag vs. $51 \mathrm{ml} / \mathrm{kg} /$ Tag; $\mathrm{p}<0,001)$.

Schlussfolgerung. Bei unseren Frühgeborenen unter $1500 \mathrm{~g}$ war eine höhere Flüssigkeits- und Natriumzufuhr signifikant und eine fehlende Gewichtsabnahme nicht signifikant mit der Entstehung einer BPD assoziiert. Diese Punkte sollten bei der Betreuung von Frühgeborenen mit hohem Risiko für eine BPD besonders berücksichtigt werden.

\section{P071}

\section{Einfluss automatischer Steuerung der inspiratorischen Sauer- stoffkonzentration auf die arterielle Sauerstoffsättigung und die zerebrale Gewebeoxygenierung bei Frühgeborenen}

\section{Waitz', M. Schmid', H. Fuchs' ${ }^{2}$, M. Mendler', J. Dreyhaupt ${ }^{3}$, H. Hummler} ${ }^{1}$ Klinik für Kinder- und Jugendmedizin, Universitätsklinikum Ulm, Sektion Neonatologie und pädiatrische Intensivmedizin, Ulm, Deutschland, ${ }^{2}$ UniversitätsKinderklinik, Freiburg, Deutschland, ${ }^{3}$ Universität Ulm, Institut für Epidemiologie und Medizinische Biometrie, Ulm, Deutschland

Background. Intermittent fluctuations of arterial oxygen saturation $(\mathrm{SpO} 2)$ frequently occur in very low birth weight infants (VLBWI). Automated adjustment of inspired oxygen ( $\left.\mathrm{FiO}_{2}\right)$ may reduce their incidence but the effect on fluctuations of cerebral tissue oxygenation $\left(\mathrm{SctO}_{2}\right)$ is unknown.

Objective. To assess the effect of automated control of $\mathrm{FiO}_{2}$ on $\mathrm{SpO}_{2}$ and $\mathrm{SctO}_{2}$ in VLBWI with frequent fluctuations in oxygenation.

Study design. 15 infants (gestational age [median and range]: 25 [23-28] weeks; age: 34 [19-74] days) were assigned in random sequence to automated adjustment of the $\mathrm{FiO}_{2}$ and to manual adjustment (24 h each).

Results. Time within $\mathrm{SpO}_{2}$-range increased during automated $\mathrm{FiO}_{2}-$ control $(76.3 \pm 9.2 \%$ vs. $69.1 \pm 8.2 \%, \mathrm{p}<0.01)$ and prolonged episodes with
$\mathrm{SpO}_{2}<88 \%$, duration $>60 \mathrm{sec}(115[67-240]$ vs. 54 [7-184], p<0.01) and $>180$ sec $(13$ [6-39] vs. 2 [0-5], p<0.01) significantly decreased during the automated period. Time with $\mathrm{SpO}_{2}>96 \%$ decreased during automated period $(6.6 \pm 4.4 \%$ vs. $10.4 \pm 3.3 \%, p=0.02)$. There was no significant difference in $\mathrm{FiO}_{2}$ exposure. The median area under the curve below and above the defined $\mathrm{SctO}_{2}$-threshold did not differ between the two periods (15.8 [12.1-69.4] vs. 21.0 [2.9-99.7], $\mathrm{p}=0.89 ; 18.7$ [3.0-140.3] vs. 14.1 $[1.5-163.8], \mathrm{p}=0.17)$.

Conclusion. Automated $\mathrm{FiO}_{2}$ control in preterm infants with frequent $\mathrm{SpO}_{2}$ fluctuations significantly increased time within intended $\mathrm{SpO}_{2}$ target range, reduced the incidence of prolonged hypoxemic events compared to manual $\mathrm{FiO}_{2}$-adjustment, but did not affect cerebral tissue oxygenation, suggesting that mechanisms of cerebral autoregulation may be effective in infants with frequent hypoxemic episodes.

\section{P072}

Non-neurological, steroid-related adverse events in very low birth weight infants: a prospective audit

S. Röhr', L. Gortner', S. Gräber ${ }^{3}$, H. Nunold', H. Abdul-Khaliq ${ }^{5}$, H. Sauer' S. Meyer ${ }^{2}$

'Unversitätsklinik für Kinder und Jugendmedizin Gebäude ${ }^{9}$, Homburg, Deutschland, ${ }^{2}$ Universität des Saarlandes, Klinik für Allgemeine Pädiatrie und Neonatologie, Homburg, Deutschland, ${ }^{3}$ Universtätsklinikum des Saarlandes, Institut für Biometrie, medizinische Informatik und Epidemiologie, Homburg, Deutschland, ${ }^{4}$ Unversitätsklinik für Kinder und Jugendmedizin Gebäude ${ }^{9}$, Klinik für Allgemeine Pädiatrie und Neonatologie, Homburg, Deutschland, ${ }^{5} U n i-$ versitätsklinikum des Saarlandes, Klinik für Pädiatrische Kardiologie, Homburg, Deutschland

Background and purpose. To assess the incidence of cardiac and metabolic adverse events in VLBW infants receiving systematic steroids. Patients and methods. Prospective single center audit in VLBW infants ( $<1500$ grams) at the NICU at the University Childen's Hospital of Saarland, Germany.

Results. 72 VLBW infants ( 38 female) were included in this report (mean birth weight: $967 \pm 338 \mathrm{~g}$; range: $320-1490 \mathrm{~g}$ ). Birth weight, gestational age and APGAR scores were significantly lower in the steroid group $(\mathrm{p}<0.01)$. Mortality rate was $8 / 72$ ( $7 / 34$ in the steroid treated vs. nontreated 1/38; OR: 9.6; 95\% CI 1.1-82.6, $\mathrm{p}=0.02$ ). In 34/72 infants, steroids were given ( 22 hydrocortisone alone; 12 combination of hydrocortisone and dexamethasone). The most common indication for use of steroids was RDS and respiratory insufficiency (30/34). Adverse events that occurred more often in the steroid group included hypertrophic cardiomyopathy (14/34 vs. o/38; p<0.001); thrombus formation ( $8 / 34$ vs. $1 / 38$; Odd's ratio: 11.4; 95\% CI 1.3-96.6; p<0.05), hyperglycemia (27/34 vs. 3/38; Odd's ratio: 45.0; 95\% CI 10.6-190.4; $\mathrm{p}<0.01$ ), hypernatremia (15/34 vs. 7/38; Odd's ratio: 3.5; 95\% CI 1.2-10.1; $\mathrm{p}<0.05)$, and sepsis/infections (8/34 vs. $1 / 38$; Odd's ratio: 11.4 ; $95 \%$ CI 1.3-96.6; $\mathrm{p}<0.05$ ). No significant differences were seen between hydrocortisone alone and the combination of hydrocortisone with dexamethasone. Birth weight and severity of RDS were predictors of steroid use $(\mathrm{p}<0.01)$.

Conclusions. The use of steroids was significantly associated with severe short-term adverse events - most importantly hypertrophic cardiomyopathy and thrombus formation. These complications must be taken into consideration when administering steroids to VLBW infants. 


\section{P073}

\section{Mit Hydroxyethylstärke oder Dextran kann Surfactant gespart werden}

\section{G. Stichtenoth' ${ }^{1}$, T. Curstedt ${ }^{2}$, E. Herting ${ }^{3}$}

'Universitätsklinikum Schleswig Holstein, Kinderklinik, Lübeck, Deutschland, ${ }^{2}$ Karolinska Institutet, Department of Molecular Medicine and Surgery, Solna, Schweden, ${ }^{3}$ Universitätsklinikum Schleswig-Holstein Campus Lübeck, Direktor der Klinik für Kinder- und Jugendmedizin, Lübeck, Deutschland

Fragestellung. In einem Tiermodell des neonatalen Atemnotsyndroms sollte untersucht werden, ob eine Mischung aus modifiziert natürlichem Surfactant und ionischen bzw. nichtionischen Polymeren die Lungenfunktion verbessert.

Material und Methoden. Basierend auf Vorversuchen im In-vitro-Model, die eine deutliche Verbesserung der biophysikalischen Aktivität von modifiziert natürlichem Surfactant durch Zusatz von ionischen Polymeren [Hydroxyethylstärke (HAES); Dextransulfat] zeigte, stellten wir die Hypothese auf, dass eine Dosisreduktion von Surfactant durch Zugabe von ionischen Polymeren egalisiert werden kann. Dies sollte im Tiermodell überprüft werden. Dazu wurden neonatale unreife Kaninchen (Gestationsalter 27 Tage) nach Sektioentbindung tracheotomiert, Surfactant-behandelt, analgosediert, relaxiert und für 30 Minuten mit einer standardisierten Sequenz von Beatmungsdrücken beatmet. Die Gruppen wurden wie folgt intratracheal behandelt: (1) unbehandelte Kontrolle, (2) Curosurf $100 \mathrm{mg} / \mathrm{kg}$, (3) Curosurf $33 \mathrm{mg} / \mathrm{kg}$ plus Dextran (100.00o Da; 1\%), (4) Curosurf $33 \mathrm{mg} / \mathrm{kg}$ plus Dextransulfat (100.00o Da; 1\%), (5) Curosurf $33 \mathrm{mg} / \mathrm{kg}$ plus HAES (200.00o Da; $1 \%$ ). $\mathrm{N}=5-7$ Tiere wurden den Gruppen randomisiert zugeordnet. Das nicht-ionisches Polymer Dextran wurde als Kontrolle zu den ionischen Polymeren eingesetzt. Alle 5 Minuten wurden die erreichten Tidalvolumina plethysmographisch aufgezeichnet. Am Versuchsende wurde nach Tötung der Tiere das endexpiratorische pulmonale Gasvolumen bestimmt.

Ergebnisse. Die Tidalvolumina zeigten über 30 Minuten unter verschiedenen Beatmungsdrücken keine signifikanten Unterschiede zwischen der hochdosierten Surfactant Kontrolle (2) und den Polymer-Surfactant Gemischen (3), (4) und (5). Unbehandelte Kontrollen (1) zeigten erwartungsgemäß niedrige Tidalvolumina. Nur Lungen, die mit hochdosiertem Surfactant (2) oder mit reduzierter Surfacantdosis plus HAES (5) oder Dextran (3) behandelt wurden, zeigten eine Verbesserung der endexpiratorischen Gasvolumina. Curosurf/Dextransulfat-behandelte Tiere (4) unterschieden sich bei den endexpiratorischen Gasvolumina nicht von den unbehandelten Kontrollen (1).

Diskussion. Unsere Daten bestätigen eine Verbesserung der Lungenfunktion durch Zugabe von Polymeren. Dies kann ein physiologisches Modell für die Interaktion zwischen Surfactant und der polymerhaltigen extrazellulären alveolaren Matrix, der alveolaren Glykokalyx, sein. Obwohl in vitro ionische Polymere eine deutliche Verbesserung der Surfactantfunktion zeigten $[(4)+(5)]$, konnten diese Ergebnisse in vivo nur für Curosurf/HAES (5) bestätigt werden.

Schlussfolgerung. Durch Zusatz von Dextran oder HAES zu Surfactant können im Tiermodell äquivalente Lungenfunktionen und stabile endexpiratorische Gasvolumina bei gleichzeitiger Dosisersparnis von zwei Dritteln des Surfactant erreicht werden.

\section{P074}

Genregulation von ErbB4 durch Koffein und dessen Bedeutung für die Surfactant-Protein-B(SP-B)-Expression in Lungenepithelzellen

\section{Fehrholz', C. Speer', S. Kunzmann ${ }^{7}$ \\ 'Universitäts-Kinderklinik, Würzburg, Deutschland}

Hintergrund. Die Gabe von Steroiden und Koffein stellt eine wichtige therapeutische Option bei Lungenerkrankungen innerhalb der Neonatologie dar. Kürzlich konnten wir eine synergistische Induktion von SP-B durch eine Kombination dieser beiden Substanzen in vitro und in vitro beschreiben (Fehrholz et al. PLOS One 2012 + AJPLCMP 2014).
Methoden. In dieser Studie sollte darauf aufbauend geprüft werden, welche Bedeutung hierbei der transmembran-Rezeptor ErbB4 besitzt, welcher auch als Co-Transkriptionsfaktor für SP-B wirkt. Das ErbB-Rezeptorsystem besitzt eine tragende Rolle innerhalb der Lungenreifung. SP-B und ErbB4 mRNA wurde mittels Real-Time-PCR in H441 Zellen, welche zuvor mit $250 \mu \mathrm{M}$ Coffein und/oder $1 \mu \mathrm{M}$ Dexamethason (DEX) behandelt wurden, bestimmt. Mittels Lentiviraler-Transduktion von $\mathrm{H}_{441}$ Zellen mit zwei verschiedenen ErbB4-spezifischen shRNAs wurde die endogenen ErbB4-Expression in $\mathrm{H}_{441-Z e l l e n}$ ausgeschaltet.

Ergebnisse. Die gleichzeitige Behandlung von naiven $\mathrm{H}_{441-Z e l l e n}$ mit DEX und Koffein führte zu einer 110-fachen Induktion der SP-B mRNAExpression $(\mathrm{p}<\mathrm{o}, \mathrm{OO})$, während die alleinige Behandlung mit DEX nur einen 58 -fachen $(\mathrm{p}<0,001)$ und mit Koffein einen 1,5-fachen Anstieg bewirkte. Die Ausschaltung der endogenen ErbB4-Expression in $\mathrm{H}_{441}{ }^{-}$ Zellen führte zu einem Verlust dieses additiven Effektes von Koffein auf die DEX vermittelte SP-B-Expression ( $\mathrm{p}<0,001)$. ErbB4 Genregulationsstudien in naiven $\mathrm{H}_{441}$-Zellen zeigten, dass Koffein, jedoch nicht DEX, zu einer 5 -fachen Induktion der ErbB4 mRNA $(p<0,05)$ führte. Eine additive Wirkung von Koffein und DEX auf die ErbB4-mRNA-Expression, wie für SP-B, konnte dabei nicht festgestellt werden.

Schlussfolgerung. Zusammenfassend zeigen diese Ergebnisse eine Hochregulation der ErbB4-mRNA-Expression durch Koffein in Lungenepithelzellen, die funktionell wesentlich zu der additiven Wirkung von Koffein auf die steroidvermittelte SP-B-Generegulation beiträgt. Eine Aktivierung des endogenen pulmonalen ErbB4-Systems durch Koffein mit einer damit verbunden Induktion der Lungenreifung könnte einen neuen molekularen Wirkmechanismus von Koffein darstellen, der hilft die positive Wirkung von Koffein bei der Behandlung der BPD mit zu erklären.

\section{P075}

\section{Einfluss des Geburtsmodus auf neonatale Biomarker}

M. Blohm', J. Sandig', F. Arndt'², G. Müller', K. Hecher', D. Singer', J. Weil 'Universitätsklinik Hamburg Eppendorf, Kinderklinik, Sektion Neonatologie/ Päd. Intensivmedizin, Hamburg, Deutschland, ${ }^{2}$ Universitäres Herzzentrum Eppendorf, Klinik für Kinderkardiologie, Hamburg, Deutschland, ${ }^{3}$ Universitätsklinikum Hamburg-Eppendorf, Klinik für Geburtshilfe u. Pränatalmedizin, Hamburg, Deutschland

Hintergrund und Fragestellung. Biomarker wie mid-regional atriales natriuretisches Peptid (mr-ANP), BNP und NT-pro-BNP, Adrenomedullin (ADM), Troponin I (TNI) und Copeptin werden in der Erwachsenenmedizin $u$. a. als Herzinsuffizienzmarker eingesetzt. Diese Studie untersucht Biomarkerspiegel im Nabelschnurblut von gesunden Neugeborenen und den Einfluss von Wehentätigkeit auf die kindlichen Biomarkerspiegel.

Material und Methoden. Im Rahmen einer Beobachtungsstudie wurde bei 68 Geburten [ 51 primäre Sectiones, 13 Spontangeburten, 4 sekundäre Sectiones, entsprechend 17/68 Geburten nach stattgehabter Wehentätigkeit, normale kardiale Anatomie, Ausschluss HELLP, Geburtsgewicht im Mittel 3,28 kg (2,1-4,83 kg)] Nabelschurblut zur Bestimmung der obigen Biomarker asserviert. Es wurde der Einfluss des Gestationsalters und der stattgehabten Wehentätigkeit auf die Biomarkerspiegel untersucht.

Ergebnisse. Die Biomarker mr-ANP, BNP, NT-pro-BNP und ADM korrelierten im Nabelschnurblut untereinander. Alle untersuchten Biomarker außer TNI korrelierten invers dem Gestationsalter. Der Geburtsmodus hatte einen hoch signifikanten Effekt $(\mathrm{p}<\mathrm{0}, 001)$ mit Erhöhung der Werte nach stattgehabter Wehentätigkeit auf den Copetinspiegel. Diskussion. Die untersuchten Biomarkerspiegel im Nabelschnurblut (außer TNI) korrelierten invers mit dem Gestationsalter. Die Wehentätigkeit führte zu einer Erhöhung des Copeptinspiegels im Kind. Bei Normwerterstellungen für Neonaten diese Biomarker muss daher das Gestationsalter und der Geburtsmodus berücksichtigt werden. Möglicherweise ist die Erhöhung des Copeptinspiegels im Zusammenhang 
mit Wehentätigkeit ein sinnvoller und physiologischer Mechanismus bei der Umstellung und Adaptation und peripartalen Umstellung des neonatalen Kreislaufes.

Schlussfolgerung. Die Physiologie und Bedeutung der Biomarker differiert beim Fetus und Neonaten vom Erwachsenen.

\section{P076}

Lungenhypoplasie, BPD/CLD und kongenitale alveoläre Dysplasie ohne Misalignement der Pulmonalvenen mit sekundärer schwerer PHT unter Vollzeit-Heimbeatmung - Lungentransplantation am 3. Geburtstag?

T. Hoppen', H. Weinzheimer ${ }^{2}$, A. Reinfeld ${ }^{2}$, C. Müller ${ }^{3}$, H. Köditz', M. Sasse ${ }^{4}$, G. Warnecke ${ }^{5}$, S. Demirakca' ${ }^{6}$, T. Nüßlein', N. Schwerk ${ }^{3}$

${ }^{1} G e m e i n s c h a f t s k l i n i k u m$ Koblenz-Mayen, Kemperhof Koblenz, Klinik für Kinderund Jugendmedizin, Koblenz, Deutschland, ${ }^{2}$ Praxis für Kinderheilkunde u. Jugendmed., Allergologie, Kinderkardiologie und -pneumologie, Andernach, Deutschland, ${ }^{3}$ Medizinische Hochschule Hannover, Zentrum für Kinderheilkunde und Jugendmedizin, Klinik für Pädiatrische Pneumologie, Allergologie und Neonatologie, Hannover, Deutschland, ${ }^{4}$ Medizinische Hochschule Hannover, Zentrum für Kinderheilkunde und Jugendmedizin, Klinik für Pädiatrische Kardiologie und Pädiatrische Intensivmedizin, Hannover, Deutschland, ${ }^{5} \mathrm{Me}-$ dizinische Hochschule Hannover, Zentrum Chirurgie, Klinik für Herz-, Thorax-, Transplantations- und Gefäßchirurgie (HTTG), Hannover, Deutschland, ${ }^{6}$ Universitätsklinikum Mannheim, Klinik für Kinder- und Jugendmedizin, Mannheim, Deutschland

Einleitung. Über eine erfolgreiche Lungentransplantation (LTx) bei bronchopulmonaler Dysplasie (BPD) vor dem 5. Lebensjahr liegen kaum Erfahrungsberichte vor.

Kasuistik. Beschrieben wird ein Frühgeborenes der 35. Schwangerschaftswoche mit kongenitaler rechtsseitiger Zwerchfellhernie, konsekutiver beidseitiger Lungenhypoplasie, pulmonalarterieller Hypertonie (PHT) und chronischer Lungenerkrankung (CLD/BPD) mit langer Beatmungspflichtigkeit und durchgehender respiratorischer Partialinsuffizienz. Die Zwerchfellhernie wurde in der Neonatalperiode verschlossen. Sekundär erfolgte die Korrektur eines Vorhof- und Ventrikelseptumdefektes. Nach initialer Stabilisierung entwickelte das Kind infolge wiederholter schwerster pulmonaler Infektionen eine beatmungspflichtige respiratorische Globalinsuffizienz, was eine invasive Beatmung über eine Trachealkanüle seit dem 18. Lebensmonat notwendig machte. Der Patient wurde daher zur Doppellungentransplantation gelistet, welche kurz vor seinem dritten Geburtstag erfolgte. Zeitgleich wurde die Trikuspidalklappe rekonstruiert (Insuffizienz Grad IV). Der postoperative Verlauf war durch eine Adenovirensepsis und eine akute Transplantat-Abstoßung kompliziert. Drei Monate nach LTx konnte die stationäre Rehabilitation beginnen. Es bestand lediglich nachts noch ein geringer zusätzlicher Sauerstoffbedarf über die Trachealkanüle. Mittlerweile ist der Patient dekanülliert und weist selbst unter Belastung ohne Sauerstoffvorlage gute Sättigungswerte auf. Die histologische Beurteilung der explantierten Lunge erbrachte den zusätzlichen Befund einer kongenitalen alveolären Dysplasie (CAD) ohne Misalignement der Pulmonalvenen.

Diskussion. Die Festlegung des optimalen Zeitpunkts für die LTx-Listung war bei diesem Patienten eine große Herausforderung. Wesentliche „Pro“-Gründe waren die progrediente Lungenerkrankung, die deutlich eingeschränkte Lebensqualität und der explizite Wunsch der Angehörigen für eine LTx. Eine Einschränkung war allerdings die präLTx bestehende Beatmungspflichtigkeit sowie die schwere PHT. Die perioperative Mortalität dieser Patientengruppe beträgt $43 \%$ gegenüber $8 \%$ bei nicht beatmungspflichtigen Kindern. Generell leben weltweit nach 5 Jahren noch $52 \%$ der transplantierten Kinder und Jugendlichen, wobei in Zentren mit hohen Transplantationszahlen das 5-Jahresüberleben in den letzten Jahren erheblich gestiegen ist (bis zu $80 \%$ in der $\mathrm{MHH}$ ). Die CAD ist eine äußerst seltene Lungenerkrankung, die typi- scherweise mit einer schweren PHT einhergeht und ohne Transplantation innerhalb der ersten 4 Lebensmonate letal verläuft.

Schlussfolgerung. Kinder mit schwerer, fortschreitender Lungenerkrankung sollten frühzeitig in einem Lungentransplantationszentrum vorgestellt werden. Insbesondere bei Kleinkindern muss aufgrund mangelnder Spenderorgane mit langen Wartezeiten gerechnet werden. Dies ist der erste Bericht über einen Patienten mit einer CAD, der trotz dieser schweren Lungenerkrankung älter als 1 Jahr wurde.

\section{P077}

Iloprost (Ventavis) intratracheal in der Erstversorgung bei sekundärer Lungenhypoplasie - Fallbericht

\author{
S. Lippke' ${ }^{1}$ H. Skopnik ${ }^{1}$ \\ 'Stadtkrankenhaus Worms, Klinik für Kinder- und Jugendmedizin, Worms, \\ Deutschland
}

Einleitung. Im Rahmen eines vorzeitigen Blasensprungs mit Anhydramnion kann es zu einer sekundären Lungenhypoplasie kommen. Die Primärversorgung Frühgeborener mit Lungenhypoplasie gestaltet sich aufgrund mangelnder Oxygenierung bei persistierender pulmonaler Hypertonie schwierig. Häufig sind sehr hohe Beatmungsdrücke mit allen damit einhergehenden Risiken nötig, um eine Stabilisierung zu erreichen. Da Spezialbeatmungsverfahren wie HFO oder NO in der Erstversorgung meist nicht zur Verfügung stehen könnte intratracheales Iloprost hier eine zusätzliche Therapieoption bieten.

Kasuistiken. Wir berichten von Zwillingsfrühgeborenen $24+0$ und $24+3$ SSW.

Geminus I. Spontanpartus aus BEL nach 24+o SSW bei VBS 11 Tage antepartum. 3 Tage antepartum Kopf- und Körper bereits im Geburtskanal. Lungenreifeinduktion mit $23+5$ SSW. Postnatal kein Atemantrieb, $\mathrm{HF}<8 \mathrm{o} / \mathrm{min}$. Direkte Intubation und Beatmung über Perivent. Fraktionierte Gabe von Surfactant intratracheal, kurzzeitige pulmonale Stabilisierung. Im Verlauf O2-Bedarf bis $100 \%$. Intermittierend Durchführung einer Herzdruckmassage bei $\mathrm{HF}<6 \mathrm{o} / \mathrm{min}$. Volumengabe von $14 \mathrm{ml} / \mathrm{kg} \mathrm{NaCl}$ o,9\% i.v. Nach 15 Minuten Applikation von $1 \mu \mathrm{g} / \mathrm{kg}$ Iloprost intratracheal und gleichzeitig Suprarenin i.v., anschließend HF stabil $>100 / \mathrm{min}, \mathrm{O}_{2}$-Bedarf rasch rückläufig auf $35 \%$. Nach Übernahme auf die Frühgeborenen-ITS unter Beatmung mit HFO und NO stabile Vitalparameter.

Geminus II. Geburt per Sectio nach $24+3$ SSW bei Fieber und ansteigendem CrP der Mutter. Intubation in der 2. Lebensminute und SurfactantApplikation im Rahmen der Erstversorgung. Unter SIMV-Beatmung zunächst stabil, pulmonale Verschlechterung und ansteigender $\mathrm{O}_{2}-\mathrm{Be}-$ darf bis $100 \%$ trotz weiterer Gaben von Surfactant und Intensivierung der Beatmung durch HFO. Aufgrund der Nichtverfügbarkeit eines zweiten NO-Gerätes erfolgten stündliche Applikationen von $2 \mu \mathrm{g} / \mathrm{kg}$ bzw. $1 \mu \mathrm{g} / \mathrm{kg}$ Iloprost intratracheal. Darunter schnelle Verbesserung der Oxygenierung und $\mathrm{CO}_{2}$-Elimination. Nach 6 Stunden Umstellung auf NO-Therapie.

Schlussfolgerung. Mit Iloprost konnte bei Geminus I eine rasche Stabilisierung bereits in der Erstversorgung erreicht werden, bei Geminus II konnte die Zeit bis zum Eintreffen eines zweiten NO-Gerätes mit Iloprost erfolgreich überbrückt werden. Die bisherigen Studien berichten von inhalativer Anwendung von Iloprost zur Vermeidung einer invasiven Beatmung, oder vom Einsatz als Rescue-Therapie. In unseren Fällen konnte gezeigt werden, dass auch durch eine Gabe via Tubusbypass eine Stabilisierung extrem unreifer Frühgeborener erreicht werden kann. Der von uns beobachtete erfolgreiche Einsatz von intratrachealem Iloprost in der Primärversorgung oder zur Überbrückung bis zur Verfügbarkeit einer NO-Therapie sollte in Studien überprüft werden. 
P078

Dual closed-loop ex-vivo placental perfusion model: evidence for relevant LPS contamination and development of a procedure for depyrogenation

\section{T. Vasanthan', N. Rochow', B. DeFrance', G. Fusch', F. Mian', C. Fusch'} 'McMaster University, Division of Neonatology, Department of Pediatrics, Hamilton, Ontario, Kanada, ${ }^{2}$ McMaster University, Obstetrics and Gynocology, Hamilton, Kanada, ${ }^{3}$ McMaster University, Immunology, Hamilton, Kanada

Background. The dual ex-vivo perfusion model uses human placental tissue to mimic in-utero conditions in a laboratory setting. We have used this model in various studies, mainly to assess leptin production as well as substrate transfer. Recently, we intended to study the role of the placenta for fetal inflammation. For this purpose we were planning to expose the placenta to LPS concentrations as observed during chorioamnionitis. However, significant release of TNF- $\alpha$ was detected already during control experiments suggesting activation of the inflammatory response that may be attributed to either a basal contamination of solutions by endotoxins or to the release of stored or/and previously synthesized TNF- $\alpha$ in placental tissue.

Objective. 1) To identify the underlying source of TNF- $\alpha$ release in placental perfusions. 2) To validate a cleaning protocol using oxidative and acid-base depyrogenation procedures to assess effectiveness in LPS removal.

Methods. 1) To identify TNF- $\alpha$ release, placental buffer and its individual components, i.e. water, bovine serum albumin (BSA), cell culture media and inorganic additives were incubated $(12 \mathrm{~h})$ in mouse macrophage cells and analyzed with TNF- $\alpha$ ELISAs. 2) To assess memory effects and validate cleaning procedures a total of 46 experiments were conducted either in presence $(n=26)$ or absence $(n=20)$ of additional LPS $(30 \mathrm{EU} / \mathrm{mL})$ in new tubing that was contaminated, cleaned and analyzed for the effectiveness of LPS removal. Two cleaning methods have been tested: Oxidative cleaning $\left(1 \mathrm{hr} ; 30 \% \mathrm{H}_{2} \mathrm{OO}_{2}\right)$ and acid-base (15 min deionized $\mathrm{H}_{2} \mathrm{O} ; 30$ min $1 \% \mathrm{NaOH}$ at $45^{\circ} \mathrm{C}$; 10 min $1 \%$ citric acid; 10 min $70 \%$ ethyl alcohol).

Results. BSA containing samples and samples placed through the placenta chamber significantly contributed to TNF- $\alpha$ release, while all other samples showed minimal to no TNF- $\alpha$ release. Oxidative cleaning effectively reduced the buildup of LPS contamination in reused tubing to levels that were in accordance with FDA guidelines $(<0.5 \mathrm{EU} / \mathrm{mL})$. Oxidative depyrogenation was capable of maintaining the perfusion system „LPS-free“ for at least 24 hours post cleaning. The acid-based depyrogenation was less effective in LPS removal compared to oxidative cleaning method.

Discussion. Our findings demonstrate that to crucial to control for LPS contamination. To establish baseline values in all placental perfusions, a standardized endotoxin free BSA and a sufficient cleaning protocol is needed to prevent LPS residuals in the placenta perfusion system which could activate cytokine response leading to sepsis like condition.
P079

Empfundene Arbeitsintensität unter nCPAP und apparativer Beatmung

K. Halstenberg' ', B. Roth', L. Kuntz ${ }^{2}$, A. Allendorf ${ }^{3}$, V. Kirchberger', A. Demel ${ }^{5}$, C. Wollny ${ }^{6}$, F. Miedaner ${ }^{2}$

'Universitäts-Kinderklinik Köln, Neonatologie und pädiatrische Intensivmedizin, Köln, Deutschland, ${ }^{2}$ Universität zu Köln, Seminar für Allgemeine BWL und Management im Gesundheitswesen, Köln, Deutschland, ${ }^{3}$ Klinikum der J.W.Goethe-Univ. Zentrum der Kinderheilkunde, Neonatologie, Frankfurt, Deutschland, ${ }^{4}$ Klinikum Großhadern, Neonatologische Abteilung, München, Deutschland, ${ }^{5}$ Eberhard-Karls-Universität Tübingen, Neonatologie, Tübingen, Deutschland, ${ }^{6}$ Unversitätsklinik für Kinder und Jugendmedizin Gebäude ${ }^{9}$, Homburg, Deutschland

Hintergrund. Nasaler „continuous positive airway pressure“ (nCPAP) wird in der Versorgung von Frühgeborenen (FG) vermehrt eingesetzt und ersetzt damit vielfach apparative Beatmungstechniken. Neben der Verbesserung des medizinischen Outcomes wird auf ein günstigeres ökonomisches Outcome aufgrund geringeren Ressourcenverbrauchs sowie verminderter Arbeitsintensität hingewiesen. Beobachtungen und Erfahrungen aus dem klinischen Alltag widersprechen diesen Studien teilweise. Deshalb wurde in unserer Pilotstudie untersucht, ob die empfundene Arbeitsintensität bei der Versorgung von FG mit nCPAP geringer ist als bei apparativer Beatmung.

Methoden. Die Studie wurde von der 2009 initiierten interdisziplinären HSR-NICU-Arbeitsgruppe durchgeführt, die den Einfluss von Humanund Organisationsfaktoren auf die Leistungsfähigkeit von neonatologischen Intensivstationen (NICU) analysiert. Im Rahmen der Studie wurden die Mitarbeiter auf 5 NICUs mittels eines validierten Fragebogens im Zeitraum von 2 Monaten zu ihrer empfundenen Arbeitsintensität befragt. Des Weiteren wurden medizinische Daten von FG mit einem sehr niedrigen Geburtsgewicht (VLBW) erhoben. Statistische Analyse: ANOVA, lineare Regression (STATA 11.2). Insgesamt nahmen 178 Mitarbeiter (56 ärztliche, 122 pflegerische Mitarbeiter) an der Befragung zu ihrer empfundenen Arbeitsintensität teil.

Ergebnisse. Es konnte festgestellt werden, dass die empfundene Arbeitsintensität an den beteiligten Zentren signifikant differiert $(\mathrm{p}<0,01)$. Keine signifikanten Unterschiede gab es zwischen den beiden betrachteten Berufsgruppen. Medizinische Daten zur nCPAP-Therapie lagen aus 3 Zentren mit 174 VLBW vor. Es konnte festgestellt werden, dass die Gesamtdauer der nCPAP-Therapie ebenfalls signifikant unterschiedlich ist $(\mathrm{p}<\mathrm{O}, 001)$. Zudem konnte ein positiver Zusammenhang zwischen nCPAP-Therapie und empfundener Arbeitsintensität auf Zentrumsebene festgestellt werden ( $\mathrm{p}<\mathrm{o}, \mathrm{oo1})$. Kontrolliert wurde nach Morbiditätsstrukturen (Gestationsalter, Geburtsgewicht) auf Patientenebene und Patientenvolumen auf Zentrumsebene. Ein Zusammenhang zwischen der Gesamtdauer an apparativen Beatmungsstunden und Arbeitsintensität konnte nicht ermittelt werden.

Schlussfolgerung. Unsere Ergebnisse kontrastieren frühere Studien, die auf eine geringere tatsächlich gemessene Arbeitsintensität bei Einsatz von nCPAP hindeuten. Möglicherweise differieren tatsächliche und empfundene Arbeitsintensität. Der positive Zusammenhang zwischen empfundener Arbeitsintensität und nCPAP-Therapie sollte auf Mitarbeiterebene überprüft werden. Im Rahmen der HSR-NICU-Studie wurden an 66 Kliniken Daten erhoben, die einen Beitrag zur Beantwortung der hier diskutierten Hypothese leisten könnten. 


\section{Neonatale Chirurgie}

\section{P080 \\ Mikrolaparoskopische Versorgung eines 900-g-Frühgeborenen mit Pankreas anulare}

S. Wunderlich', J. Bauer ${ }^{2}$, A. Klee ${ }^{3}$, M. Knuff, S. Turial ${ }^{5}$

'HSK, Dr. Horst Schmidt Kliniken GmbH, Klinik für Kinder und Jugendliche, Wiesbaden, Deutschland, ${ }^{2} \mathrm{HSK}$, Dr. Horst Schmidt Kliniken GmbH, Klinik für Kinder und Jugendliche, Neonatologie, Wiesbaden, Deutschland, ${ }^{3} \mathrm{HSK}$, Dr. Horst Schmidt Kliniken GmbH, Klinik für Gynäkologie und Geburtshilfe, Wiesbaden, Deutschland, ${ }^{4} \mathrm{HSK}$, Dr. Horst Schmidt Kliniken GmbH, Klinik für Kinder und Jugendliche, Wiesbaden, Deutschland, ${ }^{5}$ Kinderchirurgie, Universitätsmedizin Mainz, Mainz, Deutschland

Fallbericht. Bei einem Frühgeborenen der $29+1 / 7$ Schwangerschaftswoche (Geburtsgewicht: $925 \mathrm{~g}$ ) bestand pränatal bereits der Verdacht auf eine Duodenalstenose bei Vorliegen eines Polyhydramnions. Eine Amnioncentese wurde zum Ausschluss chromosomaler Störungen durchgeführt. Postnatal bestand bei dem Mädchen rezidivierendes galliges Erbrechen. Radiologisch und sonographisch konnte ein „double bubble“-Phänomen als Zeichen einer Doudenalstenose gesichert werden. Die Frage eines möglichen minimal-invasiven Vorgehens bei dem zum Zeitpunkt der Operation 900 g schweren Frühgeborenen, wurde in einem interdisziplinärem Team (Kinderchirurgie, Neonatologie und Anästhesie) diskutiert und die chirurgisch-technische Machbarkeit als auch anästhesiologische Führung perioperativ erörtert. Eine mikrolaparoskopische Versorgung (die ausschließliche Verwendung von 2-mm-Instrumenten) konnte am 3. Lebenstag durch eine Duodenoduodenostomie erfolgreich durchgeführt werden. Intraoperative Komplikationen bestanden nicht und der Blutverlust konnte auf ca. $5 \mathrm{ml}$ eingeschätzt werden. In der ersten postoperativen Woche erfolgte über eine Magensonde und eine Duodenalsonde der Kostaufbau mit Muttermilch, begleitend erhielt die Patientin eine parenterale Ernährung. Der weitere Kostaufbau und Verlauf waren soweit komplikationslos.

Schlussfolgerung. Angaben über die Inzidenz dieser seltenen Fehlbildung schwanken in der Literatur beträchtlich mit einer Häufigkeit von 1:2632 bis zu 1:15.00o Geburten. Begleitende Fehlbildung wie Trisomie 21, intestinale Malformationen, Lipomyelocelen, kardiale Fehlbildungen haben einen entscheidenden Einfluss auf das operative Verfahren, den postoperativen Verlauf und Mortalität der Patienten. Die Therapie der Wahl ist der operative Eingriff. Der Trend der minimal-invasiven Chirurgie ist auch zunehmend bei der Versorgung von Neu- und Frühgeborenen in spezialisierten Zentren festzustellen. Die bekannten Vorteile der Laparoskopie wie ein geringes Zugangstrauma, geringer Blutverlust, weniger postoperative Schmerzen und ein hervorragendes kosmetisches Ergebnis werden durch die noch stärker minimalinvasive Technik der Mikrolaparoskopie vervielfacht. Zudem ist diese Technik an die physiologischen Verhältnisse der Neu- und Frühgeborenen adaptiert. Soweit uns bekannt, ist der vorgestellte Fall weltweit der erste komplex operativer Eingriff in der laparoskopischen Technik bei einem 900-g-Frühgeborenen.

\section{P081 \\ Postnatal vorgetäuschte Ösophagusatresie nach Anhydramnion - zwei Fallberichte}

\section{A. Arnoldy', C. Joiko', H. Skopnik}

'Klinikum Worms, Klinik für Kinder- und Jugendmedizin, Worms, Deutschland

Einleitung. Ein Anhydramnion nach Blasensprung oder ausgeprägter Plazentainsuffizienz kann zur fetalen Lungenhypoplasie oder DryLung-Syndrom sowie zu Gelenkkontrakturen führen. Assoziierte gastrointestinale Komplikationen treten selten auf. Wir berichten über zwei extrem unreife männliche Frühgeborene nach langbestehendem Anhydramnion mit postnataler Ösophaguspassagestörung.
Fall 1. Drittes Kind einer Fünft-Gravida. Vorzeitiger Blasensprung 19+5 SSW, Anhydramnion, Wunsch der Fortsetzung der Schwangerschaft. Sectio caesarea bei vaginalen Blutungen, $26+1$ SSW, GG 910 g. Schwierige Versorgung bei Lungenhypoplasie, Surfactant endotracheal, unter HFO und NO-Therapie Stabilisierung. Beim Legen der Magensonde Widerstand im oberen Ösophagusdrittel, DD Ösophagusatresie, radiologisch kein Blindsack darstellbar. Im Verlauf Vorschieben der Magensonde unter vorsichtigem Spülen mit $\mathrm{NaCl}$ o,9\% bis in das untere Ösophagusdrittel, KM-Gabe mit erkennbarem Abfluss in den Magen. Erst am vierten Lebenstag gelang schließlich eine korrekte Platzierung der Magensonde, im Verlauf unproblematischer enteraler Kostaufbau. Fall 2. Zweites Kind einer Zweit-Gravida. Re-Sectio bei schwerer Plazentainsuffizienz mit Anhydramnion, 25+5 SSW, GG 440 g. Primäre Intubation, Lungeneröffnung unter hohem Inspirationsdruck und nach Surfactant-Gabe bei Dry Lung Syndrom, O2-Bedarf bis 90\%, Pneumothorax rechts. Passagehindernis bei Anlage einer Magensonde. Vorsichtige Spülung des Ösophagus mit $\mathrm{NaCl}$ o, $9 \%$ über die vermeintlich im Ösophagus platzierte Sonde. Kein Vorschieben in den Magen möglich. KM-Gabe mit KM-Übertritt in das Mediastinum und Pleuraspalt rechts, Entfernung der Sonde. Bei klinisch unauffälligem Kind erfolgte bei hohem CrP-Wert mit V. a. Mediastinitis eine antibiotische Therapie. Zum Ausschluss eines Fortbestands der Ösophaguspassagestörung erfolgte am 14. Lebenstag erneut KM-Gabe in das obere Ösophagusdrittel mit regelrechtem Abfluss in den Magen. Anschließend unproblematische Platzierung der Magensonde und Beginn mit vorsichtigem enteralem Kostaufbau.

Schlussfolgerung. Ein lange vorbestehendes Anhydramnion kann postnatal zu erheblichen Problemen im Bereich des oberen Gastrointestinaltraktes führen. Passagestörungen können zur Perforation des Ösophagus, Fehllage der Magensonde im Mediastinum mit begleitender Mediastinitis und einem deutlich verzögerten enteralen Ernährungsaufbau führen. Eine eindeutige Differenzierung zwischen einer Ösophaguspassagestörung durch Ösophagusverklebung nach Anhydramnion und einer Ösophagusatresie ist bei Frühgeborenen in den ersten Tagen äußerst schwierig. Es besteht die Möglichkeit die Verklebung durch Ösophagusspülungen mit $\mathrm{NaCl}$ o,9\% zu lösen. Nach primärer kardiopulmonaler Stabilisierung sollte hier unter vollparenteraler Ernährung abwartend vorgegangen werden.

\section{P082}

Multiple konnatale Frakturen der langen Röhrenknochen als schwere Komplikation einer Arthrogryposis multiplex congenita ein Fallbericht

H. Lode', A. Wolf', K. Deeg'

'Sozialstiftung Bamberg, Klinikum am Bruderwald, Klinik für Kinder und Jugendliche, Bamberg, Deutschland

Einleitung. Die X-chromosomal vererbte Form der spinalen Muskelatrophie (XL-SMA) mit Mutation auf dem UBA1-Gen ist auch bekannt unter der Bezeichnung x-linked Arthrogryposis multiplex congenita (xl-AMC). Die Prävalenz dieser Erkrankung ist bis heute unbekannt, lediglich einzelne Fallberichte sowie eine Aufstellung von Symptomen sind bislang veröffentlicht. Die XL-SMA ist gekennzeichnet durch eine Arthrogryposis multiplex mit multiplen Kontrakturen sowie Frakturen der Extremitäten, eine muskuläre Hypotonie, Muskelschwäche, fehlende Muskeleigenreflexe, eine Facies myopathica, neurogene Muskelatrophie sowie Zeichen der Denervation im EMG und Verlust der Vorderhornzellen.

Fallbericht. Wir berichten über einen neugeborenen Jungen, der nach unauffälliger Schwangerschaft bei Geburt in einem geburtshilflichen Schwerpunkt (PNZ L3) in der SSW 37 6/7, per Sectio caesarea, mit Fraktur des linken Oberschenkels sowie Schwellung der Oberschenkel und Oberarme auffiel. Dem aus unserer Klinik hinzu gerufenen NeugeborenenNotarzt zeigte sich das Bild einer Arthrogryposis multiplex mit Fraktur beider Femurschäfte, des rechten Humerus und des linken Unterschen- 
kels, sowie in die in der Einleitung genannten neurologischen Auffälligkeiten. Die aufgrund der beschriebenen Symptome eingeleitete Gendiagnositk erbrachte den Befund einer XL-SMA mit der Mutation c.1731 C >T (p.N577N) im Exon 15 des UBA 1-Gens. Die Frakturen wurden mittels Overhead-Extension und Gipsschiene behandelt und zeigten innerhalb von zwei Wochen eine sehr gute Kallusbildung. Die begleitenden neurologischen Symptome wie Trinkschwäche und passagere Ateminsuffizienz mussten ebenso behandelt werden wie die Kontrakturen an den Extremitäten. Die Eltern wurden ausführlich über die Erkrankung und ihre Auswirkung auf das Leben und auch die Lebenserwartung des Jungen aufgeklärt und in die für die häusliche Versorgung notwendigen Pflegemaßnahmen eingewiesen. Der Junge konnte nach 5 1/2 Wochen in die häusliche Umgebung entlassen werden. Es folgte die enge Anbindung an das regionale sozialpädiatrische Zentrum.

Schlussfolgerung. Wenn ein Neugeborenes eine Arthrogryposis oder/ und konnatale Frakturen aufweist und zusätzlich weitere Symptome auf eine neuromuskuläre Erkrankung vorliegen sollte differentialdiagnostisch auch an so seltene Erkrankungen wie die XL-SMA gedacht werden. In der Folge ist neben der Behandlung der aktuellen Probleme des Kindes auch eine gute Schulung und Beratung der Eltern notwendig. Diese Aufgabe fordert das gesamte interdisziplinäre Team einer neonatologischen Einheit.

\section{P083}

\section{Abhängigkeit der Wahrnehmung von intraabdomineller Hyper- tonie (IAH) und abdominellem Kompartmentsyndrom (AKS) vom medizinischen Versorgungslevel behandelnder NICU und PICU}

T. Kaussen', M. Böhne', H. Steinherr', B. Mitzlaff', F. Schmidt', T. Jack', H. Köditz' $^{1}$ G. Steinau ${ }^{2}$, A. Schachtrupp ${ }^{2}$, M. Sasse $e^{1}$

'Kinderklinik der Med. Hochschule, Klinik für pädiatrische Kardiologie und Intensivmedizin, Hannover, Deutschland, ${ }^{2}$ Med. Einrichtungen der RWTH Aachen, Chirurgische Universitäts- und Poliklinik der RWTH Aachen, Aachen, Deutschland

Fragestellung. Obwohl die Problematik des Verschlusses von angeborenen Bauchwanddefekten als Prototypen von Erkrankungen gelten, die ein AKS hervorrufen können und die ihr zugrunde liegende IAH somit als ureigenste pädiatrische Erkrankung gelten sollte, finden Monitoring und Therapie beider Entitäten bis heute kaum Beachtung. Begründet wird dies häufig mit unklaren Grenzwerten und Therapiekonsequenzen. Ärzte kleinerer Kliniken geben immer wieder an, dass IAH und AKS ausschließlich in großen Abteilungen oder Unikliniken mit komplexem Krankengut zu erwarten seien. Ziel der hier vorgestellten Fragebogenerhebung war daher die Klärung der Frage nach einer Abhängigkeit des Bekanntheitsgrades von Definitionen, Risikofaktoren und Behandlungsmöglichkeiten von der Abteilungsgröße der versorgenden ICU.

Methodik. Im Juni 2010 wurde den Leitern aller 265 neonatologischen und pädiatrischen ICU in Deutschland, Österreich und der Schweiz ein 2-seitiger Fragebogen zugesandt

Ergebnisse. Die Rücklaufquote betrug 59\%. Mit zunehmender Größe der Abteilungen stieg die Häufigkeit und subjektiv empfundene Relevanz in der Vergangenheit diagnostizierter IAH/AKS. Die von der World Society of the Abdominal Compartment Syndrome (WSACS) publizierten Definitionen zu IAH/AKS waren bei der Mehrzahl der Teilnehmer nicht reproduzierbar. Die "gefühlte Prävalenz" nahm mit zunehmendem Patientenalter ab (Neonaten bis $27 \%$ vs. Adoleszenten ca. 1\%). Als Kardinalsymptome wurden neben abdomineller Distension eine Olig- oder Anurie sowie kardiorespiratorische Insuffizienz genannt. Regelmäßige IAD-Messungen wurden an großen Kliniken bis zu 3-mal häufiger durchgeführt als auf kleinen ICU. Direkte Druckmesstechniken wurden ausschließlich von Unikliniken genutzt; indirekter Goldstandard ist unabhängig von der Klinikgröße die Blasen- (vor der Magen-) Druckmessung. V. a. kleinere ICU gaben an, den IAD im klinischen Alltag prinzipiell häufiger bestimmen $\mathrm{zu}$ wollen, wenn es einfachere und standardisierte Diagnosemöglichkeiten und Therapievorgaben gäbe. Etwa zwei Drittel dieser Kliniken begründeten die Unmöglichkeit von IAD-Messungen mit dem Fehlen adäquaten Equipements. Bei Neonaten überwiegen abdominelle Erkrankungen als Auslöser von IAH/ACS (insbesondere angeborene Bauchwanddefekte und Zwerchfellhernien sowie nekrotisierende Enterokolitiden), bei älteren Kindern dagegen eher Sepsis/ SIRS/Kapillarlecksyndrom und Traumata sowie Operationen insbesondere unter Verwendung extrakorporaler Kreisläufe. Unikliniken führten dekompressive Laparotomien etwa 5-mal häufiger durch als Nicht-Universitätskliniken. Die Überlebensrate von unter dem Vollbild eines AKS leidenden Kindern wurde bei Anwendung dekomprimierender Therapieoptionen etwa 20\%-Punkte höher angegeben als ohne.

Diskussion. IAH-/AKS-auslösende Erkrankungen bei Kindern scheinen kaum von der Spezialisierung der behandelnden Klinik abzuhängen. Die Wahrnehmung und Behandlung von IAH und AKS nehmen aber mit der Größe der ICU zu.

Schlussfolgerung. Gemessen an den angegebenen Gründen könnten die durch die WSACS im Juli 2013 publizierten Definitionen und Therapieempfehlungen einen ersten wesentlichen Schritt zur Verbesserung aktueller Missstände darstellen. Unabdingbar sind Schulungen bezüglich einer korrekten und standardisierten Diagnostik und Therapie, eine Vereinfachung der IAD-Messtechnik sowie weiterführende Studien zur Steigerung der zugrunde liegenden Evidenz.

\section{P084}

\section{Behandlungsergebnisse für fünf große Fehlbildungen anhand der} Neonatalerhebung Hessen von 2008 bis 2012

\section{B. Wittekindt', B. Misselwitz' ${ }^{2}$ J. Großmann'2, U. Rolle ${ }^{3}$, R. Schlößer ${ }^{4}$}

${ }^{1}$ Klinikum der J.W.Goethe-Univ. Zentrum für angeborene Fehlbildungen, Frankfurt am Main, Deutschland, ${ }^{2} \mathrm{GQH}$ - Geschäftsstelle Qualitätssicherung Hessen, Eschborn, Deutschland, ${ }^{3}$ Universitätsklinikum der Johann-Wolfgang-GoetheUniversität Frankfurt am Main, Klinik für Kinderchirurgie, Frankfurt, Deutschland, ${ }^{4}$ Klinikum der J.W.Goethe-Universität, Zentrum der Kinderheilkunde, Abteilung für Neonatologie, Frankfurt am Main, Deutschland

Fragestellung. Es gibt nur wenige Daten aus Fehlbildungsregistern in Deutschland, die außerdem auf Inzidenzen und teratogene Risiken fokussieren. Ziel der Studie ist es, für fünf ausgewählte Fehlbildungen Behandlungsergebnisse aus der Neonatalerhebung Hessen zu identifizieren und zu analysieren.

Material und Methoden. Das Register der Neonatalerhebung Hessen wurde für die Jahre 2008 bis 2012 auf die Q-Diagnosen untersucht, die für fünf große Fehlbildungen stehen: Ösophagusatresie, Gastroschisis, Omphalozele, Zwerchfellhernie (CDH) und Anorektale Fehlbildungen. Es wurde der Ort der Behandlung identifiziert, wobei die behandelnden Krankenhäuser pseudonymisiert blieben. Außerdem wurden die spezifische Mortalität sowie die mittlere Liegedauer mit Minima und Maxima evaluiert.

Ergebnisse. Im Beobachtungszeitraum wurden in Hessen $246.352 \mathrm{Neu}-$ geborene geboren (Perinatalerhebung Hessen), von denen 32.717 als Patienten in Kinderkliniken behandelt wurden (Neonatalerhebung). 249-mal wurden die gesuchten Diagnosen gefunden und 236 Kindern zugeordnet: 44 Fälle von Gastroschisis, 39 Fälle von Omphalozele, 38 Fälle mit $\mathrm{CDH}$, 65 mit Ösophagusatresie und 63 Patienten hatten anorektale Fehlbildungen. Die Diagnosen wurden in allen 13 teilnehmenden Kliniken gemeldet, wobei sechs Kliniken mehr als 20 Fälle behandelt hatten (min. 21; max. 69). Die mittlere Verweildauer betrug für Gastroschisis und Ösophagusatresie je 35 Tage, 30 Tage für CDH, 21 Tage für Omphalozele und 16 für anorektale Fehlbildungen. Die Mortalität war am höchsten bei Patienten mit CDH (29\%) und Omphalozele (23\%). Für Ösophagusatresie (4,6\%), Gastroschisis (4,5\%)und anorektale Fehlbildungen (9,5\%). war die Mortalität deutlich geringer.

Diskussion. Auch wenn man die bekannten Schwächen der Neonatalerhebungen berücksichtigt, kann man aufgrund der Daten die unvermindert hohe Bedeutung von Patienten mit angeborenen Fehlbildungen für 
neonatale Mortalität und Morbidität erkennen. Die äußerst spezialisierten Behandlungen werden in vielen Kliniken durchgeführt. Schlussfolgerung. Daten von Patienten mit Fehlbildungen sollten in großen Registern gesammelt werden, um genauere Informationen über die Behandlungsergebnisse zu bekommen. Neben Liegedauer und Mortalität sollten ergänzende Kriterien erfasst werden, um die Behandlungsqualität von Kindern mit angeborenen Fehlbildungen zu beurteilen.

\section{P085}

\section{Hydrops fetalis bei konnatalem Verschluss der V. anonyma und Trisomie 21}

\section{A. Paulun', W. Wiebe' ${ }^{2}$, M. Ehlen'}

${ }^{1}$ Asklepios Klinik Sankt Augustin, Neonatologie und Pädiatrische Intensivmedizin, Sankt Augustin, Deutschland, ${ }^{2}$ Deutsches Kinderherzzentrum Sankt Augustin, Kinderkardiologie und angeborene Herzfehler, Sankt Augustin, Deutschland

Einführung. Wir berichten über ein Frühgeborenes von 31+6 SSW mit Trisomie 21, AVSD und Hydrops fetalis. Postnatal therapieresistenter Chylothorax bei thrombotischem Verschluss der V. anonyma mit beginnendem Kollateralkreislauf. Im Verlauf komplette Thrombosierung der V. cava superior (SVC).

Perinataler Verlauf. Mutter 28 Jahre, GII/PI, Blutgruppe O Rh pos., AKS neg. Pränatal bekannte Trisomie 21 mit AVSD, Entwicklung eines Hydrops fetalis mit beidseitigen Pleuraergüssen ab der 31. SSW. Sectio bei zunehmender Wehentätigkeit mit 31+6 SSW. APGAR 3/5/6, NapH 7,27, Geburtsgewicht 2455 g (P97), Hb 18,o g/dl. Punktion der Pleuraergüsse. Stabilisierung unter HFO-Ventilation und Katecholamintherapie.

Postnataler Verlauf. Entwicklung eines dauerhaft drainagepflichtigen Chylothorax beidseits ab Tag 9. Echokardiographisch V. a. Thrombosierung der V. anonyma. Konservative Therapie (totale parenterale Ernährung, Therapie mit Octreotid $4 \mu \mathrm{g} / \mathrm{kg} / \mathrm{h}$ über 28 Tage) erfolglos. Anhaltend hoher Chylusverlust über beide Drainagen (100 ml $/ \mathrm{kg} / \mathrm{Tag}$ ). Phlebographisch am 17. Tag kein Fluss in der V. anonyma, geringer Fluss in der SVC, venöser Abfluss über paravertebrale Kollateralen. Unauffällige Gerinnungsparameter, D-Dimere normwertig, unauffälliges Thrombophilie-Screening. Bei vermutlich bereits intrauteriner Thrombosierung keine Indikation zur Lysetherapie aber Heparinisierung (Enoxaparin) mit therapeutischer Anti-Xa-Aktivität. Am 43. Tag Thrombus in der V. cava inferior bei liegendem ZVK, erfolgreiche Lysetherapie mit r-tPA. Bei fehlender klinischer Besserung des Hydrops interdisziplinäre Entscheidung zum Versuch der interventionellen Eröffnung des thrombosierten Gefäßes an Tag 35. Hierbei keine SVC mehr darstellbar, die Sondierung des thrombosierten Gefäßgebietes gelang nicht (Abb. 1, Abb. 2). Fortführung der supportiven Therapie. Bei zunehmender oberer Einflussstauung

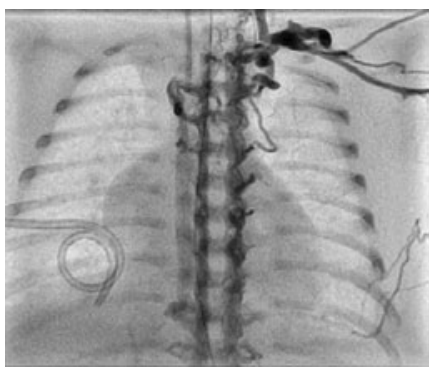

$\triangle$ Po85-Abb. 1 Herzkatheteruntersuchung: V. anonyma und SVC nicht darstellbar, venöser Abfluss über paravertebrale Kollateralen

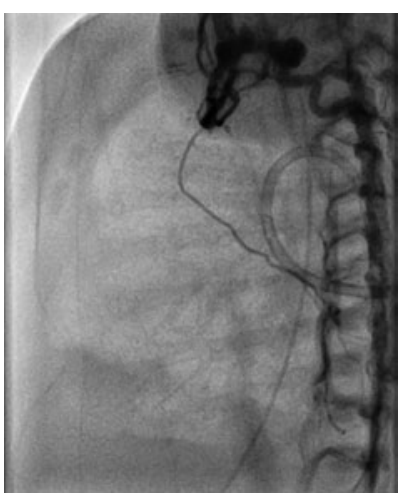

Po85 - Abb. 2 Herzkatheteruntersuchung: $\mathrm{V}$. anonyma und SVC nicht darstellbar, venöser Abfluss über paravertebrale Kollateralen und Laktatazidose interdisziplinäre Entscheidung zur Therapiebegrenzung. Das Kind verstarb an Tag 53.

Diskussion. Ein Hydrops fetalis kommt bei ca. 1:200o Geburten vor, in $75-80 \%$ der Fälle besteht ein nichtimmunologischer Hydrops unterschiedlicher Ursache. Bei unserem Patienten bestand ein Verschluss der V. anonyma und im Verlauf der SVC mit Störung des lymphatischen Abflusses trotz paravertebraler Kollateralen. Die primäre Thrombosierung ereignete sich bei Hydrops fetalis ab der 31. SSW und fehlenden anderen Ursachen wahrscheinlich pränatal. Venöse Fehlbildungen und fetale Thrombosen bei Trisomie 21 sind in der Literatur beschrieben. Therapeutische Optionen bei progredienten Gefäßverschlüssen in der Neonatalperiode wie die i.v.-Gabe von niedermolekularen Heparinen oder eine Lysetherapie zeigten sich bei unserem Patienten erfolglos. Das Risikoprofil interventioneller Verfahren erscheint ab einem Gewicht von ca. $2 \mathrm{~kg}$ akzeptabel, setzt aber eine Sondierbarkeit des betroffenen Gefäßgebietes voraus. Erfolgreiche Thrombektomien großer herznaher Venen sind bei Frühgeborenen nicht beschrieben.

\section{P086}

Volvulus bei Früh- und Reifgeborenen - eine retrospektive Fallstudie

\section{B. Albayrak', R. Tröbs'², C. Roll'}

'Vestische Kinder- und Jugendklinik Datteln, Universität Witten/Herdecke, Neonatologie und pädiatrische Intensivmedizin, Datteln, Deutschland, ${ }^{2}$ Kinderchirurgische Universitätsklinik der Ruhr Universität Bochum im Marienhospital Herne, Kinderchirurgie, Herne, Deutschland

Hintergrund. Der Volvulus ist eine seltene, gefürchtete gastrointestinale Komplikation mit foudroyantem Krankheitsverlauf. Als ursächlich gelten eine Malrotation des Darmes sowie andere Fehlanlagen des Magendarmtraktes oder der zuführenden Gefäßversorgung. Ein Teil der Volvuli bei Früh- und Reifgeborenen tritt jedoch ohne eine embryonale Fehlanlage auf. Ziel der Analyse war, typische Merkmale des Volvulus bei Früh- und Reifgeborenen am Kollektiv einer kinderchirurgischen Klinik herauszuarbeiten.

Methoden. Analyse der Patientenunterlagen von Neugeborenen und Säuglingen bis zum Alter von 6 Monaten, die zwischen dem 01.01.2006 und dem 31.12.2013 wegen eines Volvulus in die Kinderchirurgie aufgenommen wurden.

Ergebnisse. Insgesamt wurden 17 Kinder wegen eines Volvulus operativ behandelt: 10 Frühgeborene (Gestationsalter Median 31 Wochen [Range 24-36]; Alter bei Symptomatik Median 2 Tage [1-75], Gewicht bei Aufnahme 1290 g [480-2700 ]) und 7 Reifgeborene (Alter bei Symptomatik Median 3 Tage [2-8], Gewicht bei Aufnahme Median 3920 g [3030-4400]). 14 Kinder wurden innerhalb der ersten Lebenswoche symptomatisch, lediglich 3 nach der ersten Lebenswoche (1 Reifgeborenes an Tag 8, 2 Frühgeborene an Tag 35 und Tag 75). Vier Kinder hatten eine Malrotation (2-mal Nonrotation, 2-mal Malrotation I), 7 Kinder ohne Malrotation zeigten andere Fehlbildungen: 3 eine Apple-Peel-Konfiguration, 1 Ladd'sche Bänder, 1 eine Mesenteriallücke, 1 ein Mesentericum ileocolicum commune und 1 innere Hernie bedingt durch eine persistierende Art. vitelina. 6 Kinder - 3 Frühgeborene und 3 Reifgeborene - hatten keine anatomischen Auffälligkeiten. Das Leitsymptom war bei 6 der 7 Reifgeborenen galliges Erbrechen, beim siebten war galliger Magensaft $\mathrm{zu}$ aspirieren nach intrauterin abgelaufenem Volvulus. Bei den erkrankten Frühgeborenen waren die Symptome dagegen unspezifisch: distendiertes Abdomen, reduzierter Allgemeinzustand und vermehrte Apnoe-Bradykardie-Symptomatik. In 3/10 Fällen zeigten sich zusätzlich blutige Stühle. Eine Resektion von Darmanteilen war bei 7/10 Frühgeborenen und bei 1/7 Reifgeborenen notwendig. Diskussion. Die Diagnose „Volvulus“ muss bei allen Früh- und Reifgeborenen mit unklarer abdomineller Symptomatik frühzeitig differentialdiagnostisch bedacht werden, auch wenn die Symptome nicht eindeutig und die klinische und bildgebende Diagnostik nicht wegweisend sind. Bei fast $80 \%$ der Patienten unseres Kollektivs trat der Volvulus innerhalb der ersten Lebenswoche auf. Bei einem Drittel lag keine anatomische Fehlbildung des Gastrointestinaltraktes vor. 
Schlussfolgerung. Bei der Diagnose Volvulus stellt besonders die Gruppe der Frühgeborenen eine Schwierigkeit dar, da die Symptome be diesem Patientenkollektiv unspezifisch sind, die Schnelligkeit der Diagnosestellung jedoch maßgeblich über die Prognose entscheidet. Im Zweifelsfall lässt sich die Diagnose erst bei der Laparotomie stellen.

\section{P087}

Dünndarmvolvulus ohne Malrotation bei extrem unreifen Frühgeborenen - eine Fall-Kontroll-Studie

\section{Maas', S. Hammer', Y. Yarkin', H. Kirschner', C. Poets', A. Franz ${ }^{1}$}

'Eberhard-Karls-Universität Universitätsklinik für Kinderheilkunde und Jugendmedizin, Neonatologie, Tübingen, Deutschland, ${ }^{2}$ Eberhard-Karls-Universität Universitätsklinik für Kinderheilkunde und Jugendmedizin, Kinderchirurgie, Tübingen, Deutschland

Hintergrund und Fragestellung. Der Dünndarmvolvulus ohne Malrotation bei Frühgeborenen ist ein seltenes, potenziell lebensbedrohliches Krankheitsbild. Frühe Diagnosestellung und umgehendes chirurgisches Eingreifen sind erforderlich, um langstreckige Darmnekrosen und deren Folgeprobleme zu verhindern. Ziele dieser Studie waren die Erfassung potentieller Risikofaktoren bei Extrem-Frühgeborenen und möglicher früher spezifischer Warnsymptome.

Patienten und Methoden. Retrospektive, monozentrische Fall-KontrollStudie (2007-2011). Prädefinierte klinische Charakteristika der 5 Indexpatienten wurden mit denen von 25 Kontroll-Kindern mit passendem Gestationsalter bei Geburt und Geburtsgewicht aus den gleichen Jahrgängen verglichen. Zusätzlich wurden 9 Kindern mit nekrotisierender Enterokolitis (NEC) aus demselben Zeitraum analog aufgearbeitet. Daten werden als Median und Interquartilenbereich angegeben. Vergleiche zwischen Kohorten erfolgten mit dem Wilcoxon/Kruskal-Wallis-Test oder Fisher's-exact-Test. Statistische Signifikanz war definiert als p <0,05.

Ergebnisse und Diskussion. Alle Indexkinder waren Mädchen, sehr unreif, stark wachstumsretardiert bei Geburt und bei Manifestation komplett enteral ernährt. Das Gestationsalter bei Geburt lag bei 24,4 (23,6-25,5) SSW, das Geburtsgewicht betrug $480 \mathrm{~g}$ (370-530 g). Klinisch zeigte sich immer ein akutes Abdomen. 3/5 Frühgeborene verstarben an den Komplikationen des Volvulus. Das Lebensalter bei Manifestation betrug 44 (37-52) Tage. Kinder mit NEC erkrankten signifikant früher [LT 17 (297)]. Im Vergleich zu den Kontrollkindern hatten die Indexpatienten häufiger nasalen Hochfrequenz-CPAP zum Zeitpunkt der Manifestation (2/5 vs. o/25; $\mathrm{p}=0,023)$ und mehr Anwendungen eines Darmrohrs $(8-12 \mathrm{CH})$ zur Reduktion von Meteorismus [o (o-2) Anwendungen/Tag vs. o (o-o) Anwendungen/Tag; $\mathrm{p}=0,047]$. Es bestanden keine Unterschiede in der Anwendung von Bauchmassagen [5 (4-6) Anwendungen/Tag vs. 4 (o-6) Anwendungen/Tag; $\mathrm{p}=0,24]$ oder der Nahrungsverträglichkeit vor dem Ereignis. Spezifische Symptome, die eine frühe klinische Unterscheidung von der NEC ermöglichen, konnten nicht identifiziert werden.

Schlussfolgerung. Ein Volvulus ohne Malrotation bei Extrem-Frühgeborenen tritt typischerweise mehrere Wochen nach Geburt auf. Die Unterscheidung von einer NEC ist initial schwierig. Prospektive, populationsbasierte Daten sind dringend erforderlich, um mehr über Ätiologie, frühe Warnsymptome und potentielle Präventionsstrategien $\mathrm{zu}$ lernen. Eine ESPED-Erhebung hat 2014 begonnen.

\section{P088}

\section{Milzruptur bei einem extrem kleinen Frühgeborenen}

\section{P. Kutz', W. Pielemeier', L. Stüwe-Kunz', R. Tröbs', C. Roll}

'Vestische Kinder- und Jugendklinik Datteln, Universität Witten/Herdecke, Neonatologie und pädiatrische Intensivmedizin, Datteln, Deutschland, ${ }^{2}$ Kinderchirurgische Universitätsklinik der Ruhr Universität Bochum im Marienhospital Herne, Kinderchirurgie, Herne, Deutschland

Hintergrund. Bei Schocksymptomen des extrem unreifen Frühgeborenen stehen differentialdiagnostisch die schwere IVH, Lungenblutung, Sepsis, foudroyante NEC oder FIP mit Peritonitis als häufige Komplika- tionen im Vordergrund. Die Milzruptur bei Früh- wie Reifgeborenen ist dagegen eine Rarität und wird differentialdiagnostisch wenig beachtet. Fallbericht. Aufnahme der Mutter 3 Wochen vor der Geburt wegen Blasensprungs und Fruchtblasenprolaps mit Fußvorfall bei Beckenendlage des Feten. Bei ansteigenden Entzündungsparametern und V. a. Amnioninfektionssyndrom Sectio. Komplikationslose Entwicklung, spätes Abnabeln. Der Junge, Geburtsgewicht 780 g, GA 24 Wochen, war deprimiert und blass, Apgar 3/6/7. Erstversorgung mit Intubation, Surfactantgabe und Volumenbolus $10 \mathrm{ml} / \mathrm{kg} \mathrm{NaCl}$ o,9\%. Wegen Anämie ( $\mathrm{Hb} \mathrm{11,3} \mathrm{g/dl)}$ Transfusion von Erythrozytenkonzentrat. Danach gute Stabilisierung, Infektion bestätigt (IL-6 $1.38 \mathrm{o} \mathrm{pg} / \mathrm{ml}$ ). Im Alter von 38 Stunden rasche Zustandsverschlechterung, fahl-graues Hautkolorit, ansteigernder Sauerstoffbedarf, metabolische Azidose, Hb-Abfall (9 g/dl), Thrombozytopenie. Keine Hirn- oder Lungenblutung. Das Abdomen war leicht distendiert. Die Röntgenübersichtsaufnahme zeigte eine Verlagerung der Magensonde nach medial, korrekte Lage des Nabelvenenkatheters, unauffällige Darmschlingen, keine Pneumatosis, kein Anhalt für Perforation, V. a. freie Flüssigkeit. In der Abdomensonographie zeigte sich freie intraperitoneale Flüssigkeit mit Binnenechos, die Milz inhomogen mit echofreien und echoreichen Arealen. Unter der Vorstellung einer Milzruptur konservativer Therapieversuch. Darunter zunehmende Distension des Abdomens mit bläulich-grüner Verfärbung bis ins Skrotum reichend. Unter Transfusion von Erythrozytenkonzentraten (insgesamt $33 \mathrm{ml} / \mathrm{kg}$ ), Thrombozytenkonzentrat und Frischplasma innerhalb von $24 \mathrm{~h}$ anhaltende Stabilisierung. Die Blutung sistierte spontan, danach komplikationsloser Nahrungsaufbau, langsame Resorption der Blutung, unauffällige Schädelsonographie, Extubation 9. Lebenstag.

Diskussion. Initialer Schock mit Anämie direkt nach Geburt des Frühgeborenen und erneuter $\mathrm{Hb}$-Abfall am 2. Lebenstag sprechen für eine zweizeitige Milzruptur. Die Diagnose wurde durch die Sonographie der Milzloge gestellt, die bisher in unserer Abteilung nicht zum Routineprogramm bei Frühgeborenen mit abdominellen Problemen gehörte. Der (einzige) Fallbericht eines ELBW-Frühgeborenen mit Milzruptur beschreibt dieses ebenfalls mit Beckenendlage und Fußvorfall ohne schwierige Entwicklung des Kindes.

Schlussfolgerung. Die Milzruptur sollte eine weitere - wenn auch seltene - Differentialdiagnose beim akuten Schock/Hb-Abfall des Frühgeborenen sein. Sie ist sonographisch bei ausreichendem Verdacht gut zu stellen. Ein konservativer Therapieversuch scheint gerechtfertigt.

\section{Genetik}

\section{P089}

\section{XXXXY-Syndrom als seltene Ursache für DSD}

\section{S. Welcker', H. Nobel', C. Böttcher', K. Zimmer', S. Wudy', H. Ehrhardt'}

'Zentrum für Kinderheilkunde der Justus-v.-Liebig-Universität, Allgemeine Pädiatrie und Neonatologie, Gießen, Deutschland

Hintergrund. Unter Störungen der Geschlechtsentwicklung („disorders of sex development", DSD) werden angeborene Abweichungen von der normalen sexuellen Differenzierung zusammengefasst, welche ein klinisch sehr heterogenes Spektrum aufweisen. Die Inzidenz beträgt 1:450o Neugeborene. Ätiologisch können Defekte in der Biosynthese von Steroidhormonen oder deren peripheren Wirkung sowie Störungen der Gonadenentwicklung zugrunde liegen. Diese Veränderungen führen letztendlich zu einer fehlenden Übereinstimmung von chromosomalem, gonadalem und phänotypischem Geschlecht, so dass die Geschlechtseinteilung durch ein nicht eindeutiges Genitale bei Geburt erschwert sein kann.

Fallbericht. 30-jährige Mutter, GI/PI, nicht konsanguine Eltern, Geburt per sectio in der $40+5$ SSW nach unauffälliger Schwangerschaft bei pathologischem CTG und frustraner Einleitung in peripherer Klinik. APGAR 9/10/10, NapH7,34, Geburtsgewicht $2360 \mathrm{~g}\left(<\mathrm{P}_{3}\right)$, Länge $44 \mathrm{~cm}\left(<\mathrm{P}_{3}\right)$, Kopfumfang $32 \mathrm{~cm}\left(<\mathrm{P}_{3}\right)$. Die Aufnahme in der Kinderklinik erfolgte bei milder respiratorischer Anpassungsstörung und Neugeboreneninfektion. Bei der Erstuntersuchung fielen eine muskuläre Hypotonie sowie ein deutlich hyperpigmentiertes, bipartites Skrotum und ein Mikropenis 

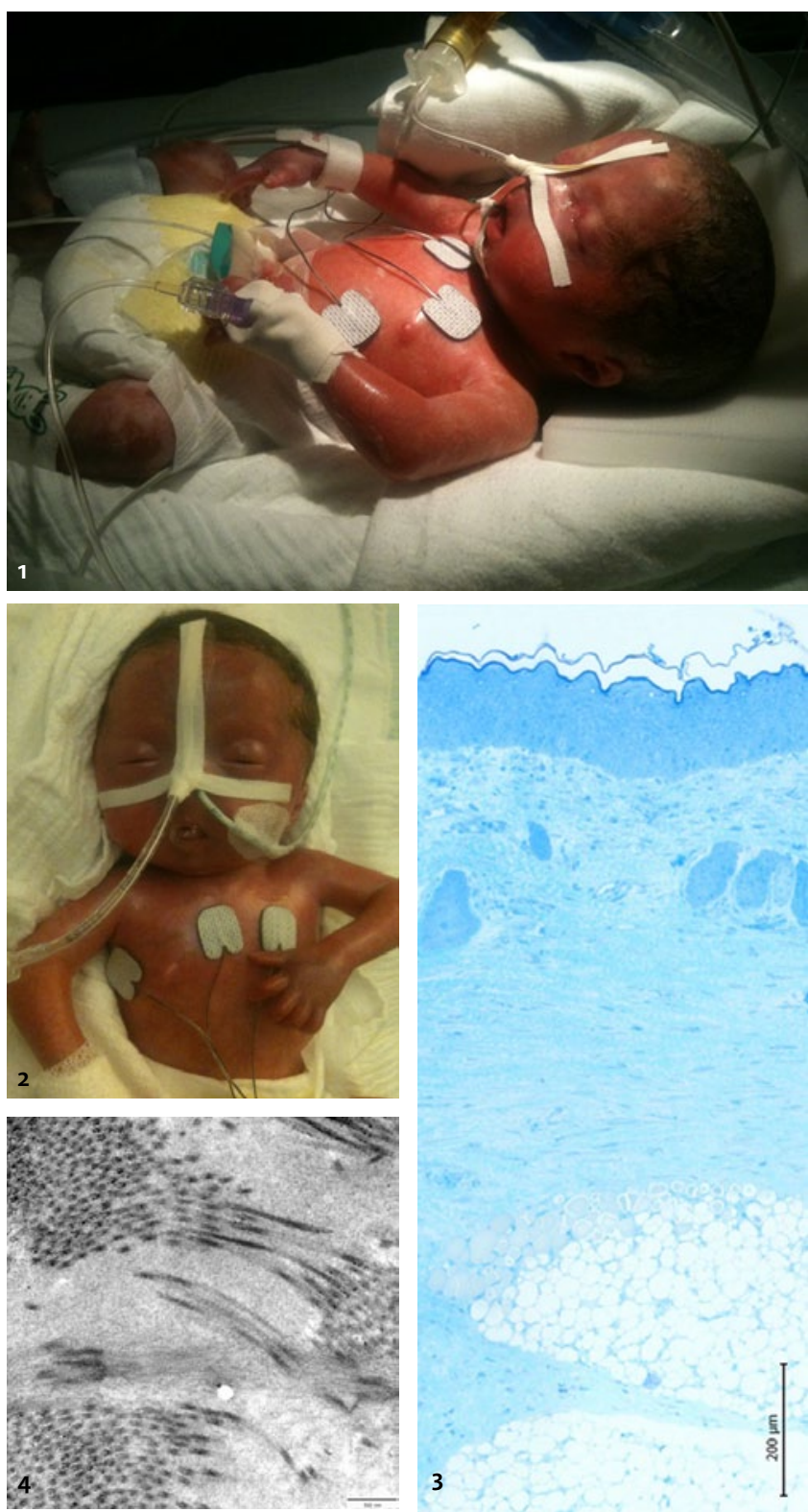

$\triangle$ Po91 - Abb. 1 Klinischer Aspekt Tag 1

$\triangle$ Po91 - Abb. 2 Klinischer Aspekt Tag 14

$\triangle$ Po91 - Abb. 3 Lichtmikroskopie

$\triangle$ Pog1-Abb. 4 Elektronenmikroskopie

auf. Aufgrund dieses nicht eindeutigen Genitales wurde eine Bestimmung des Serum-Cortisols $(1,6 \mu \mathrm{g} / \mathrm{dl})$ durchgeführt, welches trotz bestehender Infektion (CRP max. $56 \mathrm{mg} / \mathrm{l}$ ) erniedrigt war. Daher wurde zunächst der Verdacht auf eine Steroidsynthesestörung gestellt. Das Neugeborenenscreening, die Serumelektrolyte sowie wiederholte Analysen des HarnSteroidmetaboloms waren jedoch unauffällig. Sonographisch konnten beide Testes skrotal mit einem Volumen von ca. o,1 ml dargestellt werden. Müller'sche Strukturen fanden sich nicht. Die Karyotypisierung offenbarte eine extrem seltene 49,XXXXY Pentasomie, welche die unzureichende Maskulinisierung des äußeren Genitales verursacht. Die Inzidenz dieser Pentasomie beträgt 1:85.00o bis 1:100.0oo. Weitere Begleitfehlbildungen, wie in der Literatur beschrieben, fanden sich bei dem Patienten nicht.

Schlussfolgerung. Auch seltene numerische gonosomale Aberrationen wie das 49,XXXXY-Syndrom gehören zu den Differentialdiagnosen bei Vorliegen einer Störung der Geschlechtsentwicklung.

\section{P091}

Restriktive Dermopathie bei einem Frühgeborenen - ein Fallbericht

\section{Fremerey', I. Hausser², M. Kreiß-Nachtsheim ${ }^{3}$, M. Ehlen ${ }^{4}$}

'Asklepios Klinik, Neonatologie \& Pädiatrische Intensivmedizin, Sankt Augustin, Deutschland, ${ }^{2}$ Universitätsklinikum Heidelberg, Hautklinik, Heidelberg,

Deutschland, Institut für Humangenetik, Universität Bonn, Bonn, Deutschland, ${ }^{4}$ Asklepios Klinik St. Augustin, Neonatologie/Päd. Intensivmedizin, St. Augustin, Deutschland

Einführung. Wir berichten über ein Frühgeborenes mit morphologischen Auffälligkeiten und generalisierten Hautveränderungen, die zur Diagnosestellung einer Restriktiven Dermopathie führten. Die Krankheitsentität und der Verlauf unserer Patientin mit letalem Ausgang werden beschrieben. Bilder zeigen das charakteristische Aussehen und histologische Befunde.

Fallbericht. Weibliches Frühgeborenes der 30. SSW, Schwangerschaftsverlauf auffällig durch Polyhydramnion, Plazentahypertrophie und abnehmende Kindsbewegungen, Geburt durch primäre Sectio bei pathologischem CTG, Geburtsgewicht 1300 g ( $\left.\mathrm{P}_{42}\right)$. Bereits im Rahmen der Erstversorgung auffallende Gesichtsanomalien mit konstant geöffneten Augen und antimongoloider Lidachsenstellung, kleiner Nase, engen Choanen, sehr kleinem, geöffnetem Mund, Retrognathie und kleinen tiefsitzenden Ohren. Außerdem ausgeprägte Beugekontrakturen bei generalisiert straffer, glänzender Hautbeschaffenheit. Nach erschwerter nasotrachealer Intubation Übernahme beatmet auf die Neugeborenenintensivstation. Im Verlauf der ersten Lebenswochen steigender Beatmungsbedarf, keinerlei Hinweise auf Wachstum. Im Rahmen der Syndromsuche aufgrund der charakteristischen morphologischen Besonderheiten Verdacht auf das Vorliegen einer Restriktiven Dermopathie und Bestätigung dieses Verdachtes in der licht- und elektronenmikroskopischen Untersuchung eines Hautbiopsats. Auf dieser Basis gemeinsam mit den Eltern Entscheidung zur Begrenzung der Intensivtherapie, Versterben im Alter von 6 Wochen. Zwischenzeitlich molekulargenetischer Nachweis von compound-heterozygoten Mutationen im ZMPSTE24-Gen beim Kind und entsprechenden heterozygoten Genveränderungen bei beiden Eltern (Abb. 1-4).

Diskussion. Die restriktive Dermopathie (RD) ist eine seltene, autosomal-rezessiv erbliche Erkrankung. Sie beruht auf Mutationen in Proteinen, die am Lamin-A-Stoffwechsel beteiligt sind und gehört zur Gruppe der sekundären progeroiden Laminopathien. Folge der Proteinveränderung ist eine verminderte Stabilität der Zellkerne und eine gestörte Organisation des Transkriptionskomplexes während der Mitose, was wiederum zu einer Störung der Zellteilung führt. Es kommt in der 28. bis 32. Schwangerschaftswoche zu einem Entwicklungsstopp. Zentrales Merkmal der RD und Folge der gestörten Zellteilung ist eine sehr rigide, unelastische Haut, die ihrerseits zu ausgeprägten Beugekontrakturen und einer reduzierten intra- und extrauterinen Mobilität führt. Die intrauterin bereits bestehende Akinesie des Fetus führt zu Beugekontrakturen und Wachstumsrestriktion, reduzierte Mundbewegungen bedingen ein Polyhydramnion und Lungenhypoplasien. Daneben fallen die betroffenen Neugeborenen durch charakteristische Gesichtsmerkmale, sowie Skelett- und Gelenkanomalien auf. Die Prognose der RD ist infaust, meist sterben die betroffenen Kinder in den ersten Lebenstagen bis -wochen an einer Ateminsuffizienz. 
P092

Letale Sprache, letale Erkrankung? - Frühgeborenes mit sehr niedrigem Geburtsgewicht und schwerer Osteogenesis imperfecta

\section{A. Kidszun', D. Macchiella², A. Fruth ${ }^{2}$, G. Staatz ${ }^{3}$, C. Whybra-Truempler? J. Winter', N. Paul', E. Mildenberger ${ }^{1}$}

'Universitätsmedizin Mainz - Zentrum für Kinder- und Jugendmedizin, Neonatologie, Mainz, Deutschland, ${ }^{2}$ Universitätsmedizin Mainz, Klinik und Poliklinik für Geburtshilfe und Frauenkrankheiten, Mainz, Deutschland, ${ }^{3}$ Universitätsmedizin Mainz - Klinik und Poliklinik für Radiologie, Kinderradiologie, Mainz, Deutschland, ${ }^{4}$ Johannes-Gutenberg-Universität Mainz, Institut für Geschichte, Theorie und Ethik der Medizin, Mainz, Deutschland

Hintergrund. Die Osteogenesis imperfecta (OI) ist eine genetisch bedingte Knochenerkrankung mit einer Prävalenz von ca. 1:10.ooo. Sie ist durch eine ausgeprägte Neigung zu Knochenbrüchen gekennzeichnet. Eine Diagnose ist pränatal bereits vor 20 Schwangerschaftswochen (SSW) möglich. Anhand der klinischen Verlaufsform und gestützt von röntgenologischen und genetischen Befunden wird die OI klassifiziert. Der „perinatal-letale“ Typ II der Erkrankung subsummiert die schwersten Verlaufsformen. Es wird der Fall eines Frühgeborenen vorgestellt, bei dem die pränatale Klassifikation einer OI als „perinatal-letal“ nicht zutraf. Fallbericht. Bei sonst unauffälliger Schwangerschaft wird im 2. Trimester sonographisch und genetisch eine schwere OI diagnostiziert. Die vorliegende Punktmutation c.3008G >A, p.Gly1003Asp im Col1A2 Gen wurde bereits mit OI Typ II assoziiert beschrieben. Unter dem Eindruck, dass eine „perinatal-letale“ OI vorliegt, fühlt sich die werdende Mutter nicht mehr in der Lage die Schwangerschaft fortzuführen. Gemeinsam mit dem Klinischen Ethikkomitee wird die Option einer Geburtseinleitung und palliativer Versorgung des Kindes als akzeptabel erachtet. Ein Junge wird bei 31+o SSW mit 1170 g vaginal geboren und zeigt unerwartet eine unauffällige postnatale Anpassung ohne jegliche Atemunterstützung. Röntgenologisch bestätigt sich die schwere OI mit Osteopenie sowie multiplen Frakturen der Röhrenknochen und Rippen. Nach zunächst komplikationslosem Verlauf benötigt er intermittierend eine Sauerstoffvorlage und erleidet neue Knochenbrüche sowie Leistenhernien. Er ist inzwischen 3 Monate alt und gedeiht gut. Die Eltern haben eine enge Bindung zum Kind.

Diskussion. Unser Fall zeigt, wie problematisch die sprachliche Bezeichnung einer Erkrankung als „letal“ ist. Eine letale Diagnose impliziert einen unaufhaltsam tödlichen Verlauf und kann so elterliche und ärztliche Erwartungen und Entscheidungen im Sinne einer selbsterfüllenden Prophezeiung beeinflussen. Im dargestellten Fall war die pränatale Annahme einer letalen Prognose nicht zutreffend. Generell scheint die pränatale Klassifikation einer OI als „letal“ kaum möglich zu sein. Zum einen sind die phänotypischen Unterschiede zwischen „letalen“ und „nichtletalen“ Formen teilweise minimal, zum anderen bestimmt erst der klinische Verlauf die Klassifikation. Auch ein genetischer Befund determiniert aufgrund der heterogenen klinischen Ausprägung der Erkrankung nicht eine letale Prognose. Nicht zuletzt wird Letalität durch postnatal ergriffene oder unterlassene medizinische Maßnahmen aktiv mitbestimmt.

Schlussfolgerung. Die Überlebensprognose bei intrauterin diagnostizierter OI ist ungenau. In der Beratung betroffener Eltern sollten plakative sprachliche Begriffe, die eine eindeutige Prognose unterstellen, vermieden und auch bei bekannter Mutation auf die Heterogenität der klinischen Ausprägung hingewiesen werden.

\section{P093}

Kongenitale Natriumdiarrhoe mit ungewöhnlichem Phänotyp

D. Faas', J. De Laffolie', A. Zias', R. Axt-Fliedner', J. Degenhardt', K. Zimmer ${ }^{4}$ 'Zentrum für Kinderheilkunde der Justus-v.-Liebig-Universität, Allgemeinpädiatrie und Neonatologie, Giessen, Deutschland, ${ }^{2}$ Zentrum für Frauenheilkunde und Geburtshilfe, Pränataldiagnostik, Giessen, Deutschland, ${ }^{3}$ Universitätsklinikum der Justus-v.-Liebig-Universität, Abteilung Frauenheilkunde und Geburtshilfe, Giessen, Deutschland, ${ }^{4}$ Zentrum für Kinderheilkunde der Justus-v.-LiebigUniversität, Gießen, Deutschland

Einleitung. Angeborene Diarrhoen sind seltene Erkrankungen, die z. T. bereits pränatal durch dilatierte Darmschlingen und Polyhydramnion auffallen können. Es werden pathophysiologisch Defekte in Aufnahme und Transport von Nährstoffen und Elektrolyten, Enterozyten-Differenzierung und Polarisation, Differenzierung enteroendokriner Zellen und Modulation intestinaler Immunprozesse unterschieden.

Fallbericht. Wir berichten über den Verlauf eines mittlerweile 5 Monate alten Mädchens, erstes Kind konsanguiner türkischer Eltern. In der $26+3$. SSW erstmalig fetalsonographisch Nachweis von einer persistierenden Erweiterung der Darmschlingen, begleitend symptomatisches Polyhydramnion, welches 2-mal entlastet werden musste. Die Patientin kam in der $33+4 \mathrm{SSW}$ nach vorzeitigem Blasensprung und vaginalen Blutungen per Sectio zur Welt, Apgar 9/10/10, aNSpH 7,38. Es fand sich ein ausladendes Abdomen mit sonographisch weiterhin dilatierten flüssigkeitsgefüllten Darmschlingen und in den ersten Tagen 10-15 flüssigen, wässrigen Stühlen täglich. Laborchemisch fand sich eine metabolische Azidose ( $\mathrm{pH} 7,28$, BE max. -13,6) mit normwertigen Serumelektrolyten. Am 7. Lebenstag entwickelte die Patientin eine katheterassoziierte Sepsis mit Serratia marcencens und meningealer Beteiligung, von der sie sich aber unter antibiotischer Therapie rasch erholte. Der enterale Nahrungsaufbau war nach 2 Wochen erfolgreich, jedoch persistierte der häufige Stuhlgang von bis zu 10-mal tgl. Eine enterale Substitution von Natrium war phasenweise notwendig ( $\mathrm{Na}$ min. $129 \mathrm{mmol} / \mathrm{l}$ ). Darüber hinaus entwickelte sich eine persistierende Azidose ( $\mathrm{pH}$ min. 7,24, BE max. -14,7), die sich unter oraler Natriumbikarbonatsubstitution normalisierte. Der weitere Verlauf ist durch ein gutes Gedeihen gekennzeichnet (Gewicht von $\mathrm{P}_{50}-75$ bei Geburt auf $\mathrm{P}_{90}$ im 4. LM gestiegen), die Natriumbikarbonatsubstitution konnte im 4. Lebensmonat beendet werden. In der erweiterten Diagnostik zeigte sich ein unspezifisches Stuhlelektrolytmuster, keine sonstigen Begleitfehlbildungen, keine syndromale Fazies. Es erfolgte der Ausschluss von zystischen Fibrose, Microvillus inclusion disease und tuffting enteropathy mittels Laborchemie, Duodenalschleimhautbiopsie inkl. konventioneller und Elektronenmikroskopie. Kein enteraler Eiweißverlust, keine Anzeichen intestinaler Entzündung und keine Auffälligkeiten in der Gallensäurekonzentration. Es wurde molekulargenetisch eine verdächtige Mutation im Bereich eines intestinalen Natriumkanals gefunden, trotz des unspezifischen Elektrolytmusters. Als mögliche Epiphänomene fanden sich in der Duodenalbiopsie deutlich erhöhte Disaccharidaseaktivitäten sowie ein kompensatorischer sekundärer Hyperaldosteronismus.

Schlussfolgerung. Wir berichten über einen Fall kongenitaler Diarrhoe mit zunächst unspezifischen Befunden, der sich als seltene Form einer Natriumdiarrhoe herausstellt. Die beobachteten Anpassungsphänomene mit erhöhten Disaccharidaseaktivitäten und Hyperaldosteronismus könnten als kindliche Adaptationsreaktionen interpretiert werden. 


\section{P094}

Mukolipidose Typ II (i-Cell-Disease) - neonatale Manifestation mit Hyperparathyreoidismus und charakteristischen radiologischen Befunden

\section{A. Prothmann', T. Strom², J. Baumkötter', S. Burdach ${ }^{3}$}

${ }^{1}$ Kinderklinik der TU Krankenhaus München - Schwabing, Neonatologie, München, Deutschland, ${ }^{2}$ Klinikum rechts der Isar, Technische Universität München, Institut für Humangenetik, München, Deutschland, ${ }^{3}$ Kinderklinik der TU Krankenhaus München - Schwabing, München, Deutschland

Hintergrund. Die Mukolipidose Typ II (i-Cell-Disease) ist eine sehr seltene lysosomale Speicherkrankheit. Sie wurde erstmals 1967 von Leroy und DeMars beschrieben und von diesen aufgrund von Einschlusskörperchen in Fibroblasten als ,inclusion-cell disease“ bezeichnet. Die Erkrankung wird autosomal rezessiv vererbt. Durch einen Defekt der N-Acetyl-Glucosamin-1-Phosphotransferase können lysosomale Enzyme nicht mit Mannose-6-Phosphat markiert und somit nicht in die Lysosomen transferiert werden. Das Enzym N-Acetyl-Glucosamin-1Phosphotransferase besteht aus drei Untereinheiten (alpha, beta und gamma). Bei der autosomal rezessiv vererbten I-Zellkrankheit ist das GNPTA-Gen für die $\alpha$ - und $\beta$-Untereinheit auf Chromosom 12q23.3 mutiert.

Fallbericht. Wir berichten über einen Fall einer ungewöhnlichen frühen Manifestation bei einem Frühgeborenen mit dem klinischen Bild schwerer Skelettdeformitäten bereits zum Zeitpunkt der Geburt. Die klinischen Veränderungen ließen zunächst an eine schwere VDAR denken. Laborchemisch zeigten sich passend zur initialen Verdachtsdiagnose eine Hypokalzämie und ein Hyperparathyreoidismus. Die radiologischen Befunde zeigten eine schwere Entmineralisierung des gesamten Skeletts. Vor allem die langen Röhrenknochen wiesen eine charakteristische Struktur auf („cloaking“), welches für die weitere Abklärung wegweisend war. Im Rahmen einer Genomsequenzierung konnte eine 2-Basenpaar-Deletion, d. h. eine Frameshift-Mutation in GNPTAB nachgewiesen und Diagnose Mukolipidose Typ II somit molekulargenetisch bestätigt werden.

\section{P095}

\section{Kongenitales mesoblastisches Nephrom bei einem Frühgeborenen}

\section{Schreiner', A. Heydweiller', A. Müller’, P. Bartmann', S. Bagci', A. Müller'} 'Universitätsklinikum Bonn, Zentrum für Kinderheilkunde, Abteilung für Neonatologie, Bonn, Deutschland, ${ }^{2}$ Universitätsklinikum Bonn, Abteilung für Kinderchirurgie, Bonn, Deutschland, ${ }^{3}$ Universitätsklinik Bonn, Abteilung für Paidopathologie, Bonn, Deutschland

Hintergrund. Das mesoblastische Nephrom ist ein seltener niedrigmaligner Tumor. Nur $2-4 \%$ der pädiatrischen Nierentumoren sind mesoblastische Nephrome, allerdings ist das mesoblastische Nephrom der häufigste angeborene Nierentumor. 90\% der Fälle werden im ersten Lebensjahr diagnostiziert, Erkrankungen jenseits des dritten Lebensjahres sind eine Rarität. Nach histologischen Kriterien unterscheidet man klassische spindelzellige, zelluläre und gemischte Subtypen.

Fallbericht. Weibliches Frühgeborenes, Diagnose eines $6,3 \times 5,8 \times 6,2 \mathrm{~cm}$ großen soliden, stärker perfundierten Nierentumors links in der 33 . SSW, zweimalige Entlastungspunktion bei Polyhydramnion, Sectio mit $36+2$ SSW bei beginnendem high cardiac output failure. Gewicht: $3005 \mathrm{~g}$ (7o. Perz.), Länge $48 \mathrm{~cm}$ (40. Perz.), Kopfumfang 34,3 cm (70. Perz.). Bei Tachydyspnoe benötigte das Mädchen einen Tag CPAP-Atemunterstützung, ansonsten unauffällige postnatale Adaptation.

Verlauf. Bereits postnatal fiel eine arterielle Hypertonie auf (MAD bis $90 \mathrm{mmHg}$ ), die mit Amlodipin behandelt wurde. Sonographisch zeigte sich ein $12 \mathrm{~cm}$ großer Tumor mit zystischen und soliden Anteilen mit einer größeren nodulären Komponente innerhalb des Tumors. Sonographischer Befund und Tumormorphologie im nachfolgend durchgeführten MRT waren vereinbar mit der Diagnose eines Kongenitalen mesoblastischen Nephroms vom gemischtzelligen Typ, so dass am sechsten Lebenstag die linksseitige Nephrektomie erfolgte. Aufgrund einer Infiltration des Tumors ins Colon descendens wurden $15 \mathrm{~cm} \mathrm{Co-}$ lon descendens reseziert mit anschließender End-zu-End-Anastomose. Eine arterielle Hypertonie bestand postoperativ, auch nach Beendigung der antihypertensiven Therapie, nicht mehr. Der weitere postoperative Verlauf gestaltete sich komplikationslos. Die histologische Untersuchung des Resektats ergab ein die Niere zu 98\% infiltrierendes, bekapseltes mesoblastisches Nephrom vom gemischten Typ, das die Adventitia des Colons infiltrierte und bis an die Muscularis propria des Colons grenzte. Die Colonabtragungsebenen waren tumorfrei.

Diskussion. Die alleinige Nephrektomie ist die Therapie der Wahl, und das Ausmaß der Resektion ist entscheidend für die Prognose. In ca. 5\% der Fälle kommt es zu einem Lokalrezidiv, das in der Regel mit Chemotherapie, mit oder ohne Radiotherapie, behandelbar ist. Fernmetastasen in Lunge, Leber, Herz und Gehirn stellen eine Rarität dar. Das mesoblastische Nephrom kann durch eine Hyperplasie der juxtaglomerulären Zellen mit einer arteriellen Hypertonie einhergehen, die in der Regel nach Nephrektomie sistiert.

Schlussfolgerung. Das mesoblastische Nephrom ist der häufigste angeborene Nierentumor, der mit einer Hypertonie einhergehen kann und in der Regel durch alleinige Nephrektomie kurativ behandelbar ist.

\section{P096}

Sturge-Weber-Syndrom und PHACE-Syndrom bei einem Frühgeborenen der 34. SSW - Koinzidenz oder eine Erkrankung mit vielen Gesichtern?

\section{S. Disse', A. Weber' ${ }^{2}$ J. Bauer ${ }^{3}$, M. Andrassi-Darida 4 , B. Lorenz ${ }^{4}$, K. Zimmer', H. Ehrhardt ${ }^{\prime}$}

'Zentrum für Kinderheilkunde der Justus-v.-Liebig-Universität, Abteilung für Allgemeinpädiatrie und Neonatologie, Giessen, Deutschland, ${ }^{2}$ Justus-v.-Liebig Universität, Institut für Humangenetik, Giessen, Deutschland, ${ }^{3}$ Universitätsklinikum Gießen, Zentrum für Kinderheilkunde, Abteilung für Kinderkardiologie, Gießen, Deutschland, ${ }^{4}$ Universitätsklinikum Gießen und Marburg GmbH, Standort Gießen, Klinik und Poliklinik für Augenheilkunde, Gießen, Deutschland

Hintergrund. Eine klassische Differenzialdiagnose bei im Gesicht lokalisiertem Nävus flammeus und ipsilateralem Glaukom ist das Sturge-Weber-Syndrom (SWS, Inzidenz 0,5:105). Bei gemeinsamem Auftreten von im Gesicht lokalisierten Hämangiomen, okulären und kardiovaskulären Anomalien sollte ein PHACE Syndrom (PHS, ca. 300 Fälle weltweit) erwogen werden. Wir berichten über ein Frühgeborenes der 34. SSW mit klinischen Kriterien für beide Syndrome.

Kasuistik. Pränatal bekannte Fallot-Tetralogie und rechtsseitig deszendierender Aortenbogen. Geburt spontan-vaginal in der 34. SSW nach vorzeitigem Blasensprung. Postnatal Atemunterstützung mittels CPAP für 8 Stunden bei respiratorischer Anpassungsstörung. Klinisch zeigten sich ein Nävus flammeus der rechten Gesichtshälfte sowie zwei weitere Portweinflecken links thorakal und occipital. Bei massiv erhöhtem Augeninnendruck rechts zunächst konservative Therapie, aufgrund fehlenden Ansprechens im Verlauf unkomplizierte Trabekulotomie. In der kardialen MRT fiel folgendes auf: 1.) aberranter Abgang der A. subclavia links und retrograde Perfusion aus der linken Vertebralarterie, 2.) Abgang von zwei MAPCAs aus dieser linken A. subclavia, mit Mündung jeweils in die linke Pulmonalarterie bzw. in eine Thoraxvene. Dopplersonographisch retrograder Fluss aus der A. basilaris in die linke Vertebralarterie. Unkomplizierter interventioneller Verschluss der MAPCA zur linken Pulmonalarterie mittels Occluder, anschließend regelrechter, antegrader Fluss basilär. Unauffällige Schädel-MRT mit chronologisch 5 Wochen. Im kurzfristigen Verlauf über 5 Monate keine Krampfanfälle. In Blutlymphozyten kein Hinweis auf Mikrodeletion 22, andere Chromosomenaberrationen oder GNAQ Mutation (Chr. 9).

Diskussion. Im vorliegenden Fall sind sowohl die Kriterien eines „möglichen“ PHS als auch eines SWS Typ II erfüllt (bisher kein Nachweis zerebraler Angiome). Die Ausdehnung des Nävus flammeus ist jedoch im Vergleich zur Literatur ungewöhnlich: Das betroffene Gesichtsareal 
lässt sich spezifischen Segmenten der embryonalen Gesichtsentwicklung zuordnen, jedoch nicht- wie beim klassischen SWS- dem Ausdehnungsgebiet des N. trigeminus. Ein Befall spezifischer Gesichtssegmente ist typisch für die Gesichtshämangiome i. R. des PHS, dies ist jedoch nicht für das SWS beschrieben. Diverse Fallberichte deuten auf eine mögliche Überlappung zwischen PHS und SWS hin (1999: PHS \& kongenitales Glaukom; 2003: PHS \& Portweinflecken \& retinale Gefäßanomalien \& VSD; 2010: PHS \& Portweinfleck).

Schlussfolgerung. Überschneidungen zwischen SWS und PHS sind häufiger, als einer zufälligen Koinzidenz der beiden Syndrome entspricht. Dies wirft die Frage auf, ob es sich bei den beiden Phakomatosen um eine Grunderkrankung mit präferentieller Manifestation arterieller Fehlbildungen (PHS) bzw. venös-kapillärer Fehlbildungen (SWS) handeln könnte.

\section{P097}

Transienter neonataler Diabetes mellitus (TNDM) durch paternale Duplikation 6q24.2

\section{J. Wolfram ', T. Eggermann'², U. Kordaß3, C. Schröder', M. Heckmann ${ }^{5}$}

'Klinik und Poliklinik für Kinder und Jugendmedizin, Abt. Neonatologie u. Päd. Intensivmedizin, Greifswald, Deutschland, ${ }^{2}$ Uniklinik RTWH Aachen, Institut für Humangenetik, Aachen, Deutschland, ${ }^{3}$ Praxis für Humangenetik, Greifswald, Deutschland, ${ }^{4}$ Klinik und Poliklinik für Kinder und Jugendmedizin, Abt. Pädiatrie/Endokrinologie, Greifswald, Deutschland, ${ }^{5}$ Klinik und Poliklinik für Kinder und Jugendmedizin, Abt. Neonatologie u. Päd. Intensivmedizin, Greifswald, Deutschland

Einleitung. Eine gestörte Expression elterlicher Gene („genomic imprinting") durch epigenetische oder genomische Veränderungen auf molekularer Ebene führen zu sog. Imprintingerkrankungen. Zu den bisher 8 bekannten klinischen Entitäten gehört der transiente neonatale Diabetes mellitus (Inzidenz: 1/400.00o).

Kasuistik. Erstes Kind gesunder Eltern, Geburt nach 37+4 SSW durch Sectio caesarea bei intrauteriner Wachstumsretardierung und pathologischen Dopplerflüssen. Unauffälliger Schwangerschaftsverlauf sowie pränatale Diagnostik. Postnataler klinischer Befund: Gewicht: $1950 \mathrm{~g}$ (<3. Perz.), Länge: $45 \mathrm{~cm}$ (3.-10. Perz.), dystrophes Neugeborenes, außer leichter Makroglossie klinisch unauffällig. Verlauf: Entwicklung eines ANS Grad I, laborchemisch fiel eine Hypoglykämie (venös 1,9 mmol/l) auf. Unter intravenöser Glukosezufuhr rascher Anstieg der Blutglukose auf Werte zwischen 13,5 und 19,7 mmol/l. Persistieren der Hyperglykämie trotz Unterbrechung der Glukosezufuhr. Übernahme des Kindes auf die Neonatologie unserer Klinik. Beginn einer Insulintherapie zunächst intravenös, im Verlauf subkutan mit Insulinpumpe (Paradigm 554, Medtronic) und kontinuierlichem Glukosemonitoring (CGM) Rückgang des Insulinbedarfs, Normoglykämie ohne Insulin ab der 10. Lebenswoche. Aufholwachstum im Verlauf.

Diagnostik. Sonographisch Ausschluss einer Pankreasaplasie, diabetesspezifische Antikörper nicht nachweisbar. Molekulargenetische Untersuchung der ATP-sensitiven K-Kanal-Gene KCNJ11/ABCC8 ohne Mutationsnachweis, Untersuchungen der Region 6q25 (MS-SNuPE-, MS-MLPA Analytik) zeigten ein verändertes Methylierungsmuster im Sinne einer Duplikation. Eine anschließende molekulare Karyotypisierung bestätigte das Vorliegen einer $\sim 589-\mathrm{kb}$-Duplikation in der Bande 6q24.2. Die Analysen der elterlichen Proben sind aktuell noch ausstehend. Bei klinischem Verdacht eines TNDM ist vor dem Hintergrund des vorliegenden Befundes von einer molekularen Veränderung (paternale Duplikation, Methylierungsstörung, uniparentale Disomie) im Erbgut des Patienten auszugehen.

Schlussfolgerung. Im Rahmen einer neonatalen Hyperglykämie sollte immer eine Form des neonatalen Diabetes mellitus (PNDM, TNDM) in Betracht gezogen werden. Die Diagnosesicherung steht sowohl für die Therapieplanung und Prognoseabschätzung als auch für eine genetische Beratung bei weiterem Kinderwunsch der Eltern im Vordergrund.

\section{Angeborene Immunität und Infektiologie}

\section{P098}

Zelltod von Lymphozyten nach bakterieller Infektion: Die Rolle von Monozyten

\section{S. Dreschers', K. OhP', T. Orlikowsky'}

'Med. Einrichtungen der RWTH Aachen, Universitätsklinik für Kinder- und Jugendmedizin, Abt. Neonatologie, Aachen, Deutschland, ${ }^{2}$ Med. Einrichtungen der RWTH Aachen, Universitätsklinik für Kinder- und Jugendmedizin (AG PD Dr. Tenbrock), Aachen, Deutschland

Hintergrund. Lymphozyten sterben nach bakteriellen Infektionen ab, was Patienten in eine sekundäre Immundefizienz treiben kann. Monozyten könnten beteiligt sein, Signale zur Lymphozyten-Nekrose oder -Apoptose zu vermitteln. Nekrose kann im Vergleich zur Apoptose zu erhöhten inflammatorischen Immunantworten führen.

Hypothese. Monozyten sind beim lymphozytären Zelltod nach Phagozytose beteiligt. Monozytär vermittelter Zelltod von Lymphozyten ist bei Neugeborenen im Vergleich zu Erwachsenen zugunsten der Nekrose verändert.

Methodik. Mononukleäre Zellen von Erwachsenen (PBMC) und Neugeborenen (CBMC) wurden mit GFP- E. coli für 6o Minuten infiziert und bis zu 7 Tage kultiviert, nichtinfizierte Kulturen dienten als Kontrolle. Lymphozyten wurden phänotypisiert $\left(\mathrm{CD}_{3}, \mathrm{CD} 8, \mathrm{CD} 4\right.$; FACS) und Apoptose und Nekrose mittels AnnexinV/Propidiumiodid nachgewiesen. Titrieren syngener Monozyten zu CD4+ MACS-aufgereinigten Lymphozyten. Lymphozytärer Zelltod wurde als Quotient Apoptose/ Nekrose ausgedrückt $(>1=$ Apoptose überwiegt, $<1=$ Nekrose überwiegt).

Ergebnisse. Lymphozytäre Apoptose nach Kokultivierung mit Bakterien trat nicht ohne Monozyten auf (0,7 $\pm 0,3 \%$ vs. 0,2 $\pm 0,3 \%$ in Kontrolle). Die Titration von infizierten Monozyten führte - mengenabhängig - zur Verstärkung des apoptotischen Zelltodes $(5,3 \pm 2,5 \%$ bzw. 7,7 $\pm 5,8 \%$ bei 1:10 bzw. 1:5 zugegebenen Monozyten). Innerhalb von 4 Tagen wurde Zelltod (Apoptose + Nekrose) von Lymphozyten in infizierten PBMC und $\mathrm{CBMC}$ in $15,8 \pm 9,5 \%$ vs. $6,6 \pm 2,9 \%$ ( $\mathrm{p}<0,05$ vs. nichtinfizierte Kontrollen) induziert. Das Verhältnis von apoptotischen zu nekrotischen Lymphozyten war bei PBMC deutlich stärker zugunsten der Apoptose gewichtet als bei CBMC: 14-fach vs. 6-fach. Dabei starben CD8+ Lymphozyten deutlich mehr durch Apoptose als CD4+ Lymphozyten aus PBMC: $45,4 \pm 22,2 \%$ vs. $9,2 \pm 4,7 \%$. Im Vergleich zu CD8+ Lymphozyten aus $\mathrm{PBMC}$ zeigten solche aus $\mathrm{CBMC}$ eine etwas geringere Apoptoserate $(15,6 \pm 0,8 \% ; p=0,081$ vs. PBMC).

Schlussfolgerung. Nach Infektion mit E. coli findet ein geringer Zelltod von Lymphozyten direkt, d. h. unabhängig einer Phagozytose durch Monozyten statt. Monozyten verstärken diesen Prozess. Neonatale Lymphozyten versterben in unserem Versuchsmodell im Vergleich zu adulten Lymphozyten verstärkt durch Nekrose. Dies könnte als weiterer Mechanismus zur „sustained inflammation“ beitragen.

\section{P099}

Stay hungry, stay alive: Wenn Monozyten zweimal phagozytieren

\section{S. Dreschers', J. Kerth², T. Orlikowsky'}

'Med. Einrichtungen der RWTH Aachen, Universitätsklinik für Kinder- und Jugendmedizin, Abt. Neonatologie, Aachen, Deutschland, ${ }^{2}$ Med. Einrichtungen der RWTH Aachen, Universitätsklinik, Aachen, Deutschland

Hintergrund. Nach Phagozytose von Bakterien sterben etliche phagozytierende Monozyten durch Phagozytose-induzierten Zelltod (PICD) ab, lösen zuvor aber bei nicht-phagozytierenden Monozyten BystanderApoptose aus. Diese Vorgänge sind bei Neugeborenen (CBMO) im Vergleich zum Erwachsenen (PBMO) reduziert. Phagozytose ist essentiell für die Induktion von Bystander-Apoptose und CD95L bzw. TNF- $\alpha$ vermittelt. Hypothese: Monozyten, welche primär phagozytiert hatten, 
können bei Re-Infektionen Bakterien nicht in demselben Maß phagozytieren.

Methodik. Nach Anreicherung von PBMO und CBMO primäre und Re-Infektion mit verschiedenen fluorochrom-markierten E. coli. [GFPoder NilBlue A; Multiplicity Of Infection (MOI) 1:10] im 24-h-Intervall. Zwei Gruppen mit je einer singulären Infektion (do oder d1), eine Gruppe mit Re-Infektion $\left(\mathrm{do}_{\mathrm{d}} \mathrm{d}_{1}\right)$. Bestimmung von Phagozytose-Index (PI), intrazellulärem Killing (Rekultivierung aufgenommener Bakterien auf LB-Amp/Kanamycin-Platten; Protection-Assay) und Apoptose (hypodiploide DNA) mittels FACS 28 h nach erster Infektion (p.i.).

Ergebnisse. $9,4 \pm 7,7 \%$ der PBMO phagozytierten singulär an do, $17,5 \pm 5,5 \%$ an d1. Der PI bei Re-Infektion war mit $13,8 \pm 7,5 \%$ an do $+d_{1}(n=5)$ nicht erniedrigt. Korrespondierende Werte für CBMO waren $17,9 \pm 10,4 \%$ an do, $15,5 \pm 13,5 \%$ an $d_{1}, 22,2 \pm 8,9 \%$ an do und $d_{1}(n=5 ; p>0,05$ vs. PBMO). Das intrazelluläre Killing bei Primär- und Re-Infektion unterschied sich nicht signifikant zwischen PBMO und CBMO im Protection-Assay. Die Apoptoserate phagozytierender PBMO und CBMO nach ReInfektion lag bei $3,8 \pm 0,8 \%$ vs. $24,9 \pm 11,1 \%$ und war im Vergleich zur jeweiligen Einzelinfektion nicht signifikant erhöht. Bystander-Apoptose von PBMO und CBMO trat 24 h p.i. in $7,3 \pm 4,2 \%$ und $17,2 \pm 4,7 \%$ (p<0,05 vs. Kontrollen) auf.

Schlussfolgerung. Vitale PBMO und CBMO phagozytieren bei Re-Infektionen mit E. coli nach $24 \mathrm{~h}$ in demselben Maße wie bei der Primärinfektion. Die Beobachtung, dass monozytäre Apoptose im Rahmen des PICD bei Re-Infektionen im Vergleich zur primären Infektion nicht ansteigt, führt bei PBMO, nicht aber bei CBMO zu einer zytokingetriggerten, abgeschwächten Reaktion nach zweiter Phagozytose.

\section{P100}

\section{Frühgeborene Gemini mit primärer Autoimmunneutropenie}

\section{K. Wittig', W. Garbe', K. Schneider', N. Hepping'}

'St. Marien-Hospital Bonn, Neonatologie, Bonn, Deutschland

Einleitung. Die Autoimmunneutropenie (AIN) ist in der Neonatalperiode eine Rarität, bisher gibt es nur einzelne Fallbeschreibungen im Säuglingsalter. Ursächlich sind Auto-Antikörper gegen Oberflächenmarker der ganulozytären Zellmembran.

Fallvorstellung. Wir berichten über dichoriale-diamniote Zwillinge geboren mit einem Gestationsalter von 30+1 SSW. Vorzeitiger Blasensprung des ersten Zwillings mit 26+5 SSW. Geburt per Sectio caesarea bei beginnendem Amnioninfektionssyndrom und pathologischem CTG des 2. Zwillings. Erster Geminus (APGAR 7/8/9, Nabel-Arterien$\mathrm{pH}$ 7,36, BE -1,0 mmol/l, Geburtsgewicht $1195 \mathrm{~g}$ ) zeigte unter empirischer antibiotischer Therapie mit Ampicillin/Sulbactam und Tobramycin am 5. Lebenstag eine klinische Verschlechterung und erstmalig eine deutliche Leukopenie und Neutropenie. Unter antibiotischer Therapie mit Vancomycin und Meropenem jedoch rasche Besserung und unkomplizierter Verlauf. Zweiter Geminus (APGAR 7/8/9, Nabel-Arterien-pH 7,34, BE o mmol/l, Geburtsgewicht $1300 \mathrm{~g}$ ) zeigte unter empirischer antibiotischer Therapie mit Ampicillin/Sulbactam und Tobramycin einen Anstieg des CRP auf maximal 1,3 mg/dl am 2. Lebenstag. Am 3. Lebenstag erstmals Leukopenie mit Neutropenie. In der zweiten Lebenswoche klinische Verschlechterung im Rahmen einer NEC (Stadium 1 nach Bell), die unter Vancomycin und Meropenem konservativ therapiert werden konnte. Beide Patienten zeigten ab der ersten Lebenswoche rezidivierende schwere Neutropenien, mit Neutrophilenzahlen unter 50o/ $\mu \mathrm{l}$. Im weiteren Verlauf mehrfache Gaben von GCSF bei beiden Patienten mit adäquaten Anstiegen der Neutrophilenzahlen für wenige Tage. In der dritten Lebenswoche erfolgte die Diagnosestellung einer Autoimmunneutropenie mit typischer Antikörperkonstellation. Bei beiden Patienten konnten Antikörper gegen das granulozytäre Glykoprotein CD16b nachgewiesen werden. Typischerweise konnten die Antikörper bei der Mutter nicht nachgewiesen werden. In den infektionsfreien Intervallen wurde auf eine antibiotische Prophylaxe verzichtet, die Fluconazol-Prophylaxe wurde in der 5 . Lebenswoche be- endet. Entlassung der Kinder in eine ambulante pädiatrisch-hämatologische Betreuung in der 7. Lebenswoche.

Schlussfolgerung. Im Gegensatz zur sekundären Form tritt die primäre Autoimmunneutropenie (AIN) nicht in Folge einer bereits bestehenden Grunderkrankung auf. Am häufigsten wird sie bei Kindern im Alter von 5-15 Monaten diagnostiziert. Bei Frühgeborenen ist sie eine absolute Rarität, sollte aber neben der Alloimmunneutropenie, der neonatalen Neutropenie und reaktiven Veränderungen als Differentialdiagnose nicht unbeachtet bleiben. Die Erkrankung ist oft nach einigen Monaten selbstlimitierend. Eine frühzeitige antibiotische Therapie bei Infektionen ist empfohlen. Eine antibiotische und antimykotische Prophylaxe steht aktuell in der Diskussion.

\section{P101}

\section{Vici-Syndrom bei monochorialen Zwillingsfrühgeborenen (OMIM} 242840)

\section{A. Stein', R. Mein'2, U. Felderhoff-Müser ${ }^{3}$, H. Jungbluth', U. Schara}

'Klinik für Kinder und Jugendmedizin der Universität, Klinik für Kinderheilkunde I, Essen, Deutschland, ${ }^{2}$ Guy's Hospital, GSTS Pathology LLP, DNA Laboratory, London, United Kingdom, ${ }^{3}$ Klinik für Kinder und Jugendmedizin der Universität, Essen, Deutschland, 'St Thomas' Hospital, King's College London, Children's Neuroscience Centre, London, United Kingdom

Fallbericht. Die eutrophen monochorialen-diamnialen Gemini wurden mit einem Gestationsalter von 30+1 Schwangerschaftswochen wegen unstillbarer Wehen und vorzeitigem Blasensprung geboren (Geburtsgewicht $1370 \mathrm{~g}$ und $1495 \mathrm{~g}$ ). Die unmittelbar postnatale Versorgung verlief ohne Komplikationen (Apgar $7 / 7 / 8$ und $7 / 8 / 8$ ). Beide Kinder erhielten bei RDS III $^{\circ}$ einmalig porcines Surfactant mit anschließender Atemunterstützung mittels Infantflow bei Raumluft. Ein persistierender Ductus arteriosus wurde durch 3 Gaben Indomethacin verschlossen. Sonographisch fiel bei beiden Kindern eine Balkenagenesie auf. Bis auf einen hohen Gaumen und eine einseitige Vierfingerfurche beim 2. Zwilling gab es keine Dysmorphiezeichen. Eine Chromosomenanalyse war unauffällig. Die Eltern sind türkischer Nationalität und konsanguin (Cousin/Cousine). Zwei Geschwister des Vaters sind vor ca. 20 Jahren im Alter von 9 und 15 Lebensmonaten verstorben. Die Grunderkrankung blieb unklar. Die Todesursache konnte nicht genauer eruiert werden. Differentialdiagnostisch standen ein Stoffwechseldefekt oder eine Mitochondropathie im Raum. Bei beiden Kindern bestand ebenfalls eine Balkenagenesie. Im Verlauf entwickelten beide Zwillinge rezidivierend Septitiden jeweils nach nur wenigen Tagen ohne Antibiotika, eine CMV-Infektion sowie eine progrediente hypertrophe Kardiomyopathie. Es bestand bei beiden Kindern eine Papillenhypoplasie mit nicht ableitbaren VEP. Respiratorisch waren die Kinder bei gehäuften Apnoen schwer vom CPAP zu entwöhnen und benötigten im Verlauf dauerhaft Sauerstoff. Bei Trinkschwäche und Gedeihstörung war dauerhafte Sondierung notwendig. Der entscheidende diagnostische Hinweis ergab sich aus der genauen Betrachtung der Kinder. Beide waren sehr hellhäutig und hatten rötliches Haar. Die Kombination der Symptome: rote Haare, Balkenagenesie, Immundefekt führte zur Verdachtsdiagnose Vici-Syndrom, was im weiteren Verlauf molekulargenetisch gesichert wurde. Auch die beiden verstorbenen Geschwisterkinder des Vaters hatten struppige rote Haare. Somit lag auch hier sehr wahrscheinlich ein Vici-Syndrom vor.

Schlussfolgerung. Aktuell sind nur 2o Patienten mit dieser Erkrankung beschrieben. Die Vererbung ist autosomal-rezessiv. Es handelt sich um eine Multisystemerkrankung mit Balkenagensie, Pigmentierungsstörung, Kardiomyopathie, Krampfleiden, schwerer psychomotorischer Retardierung, Muskelhypotonie und Lungenhypoplasie. Die meisten Kinder versterben im Kleinkindalter bei rekurrienden Infekten im Rahmen des kombinierten Immundefekts. Auch die Zwillinge verstarben unter palliativer Therapie mit einem korrigierten Alter von 1 und 6 Monaten. 


\section{P102}

\section{Changes in hemodynamic and peripheral perfusion in suspected neonatal sepsis}

\section{Ahmad Hassan', M. Schmid', C. Hanke', M. Mendler ${ }^{2}$, M. Waitz ${ }^{2,}$ L. Huang ${ }^{3}$, H. Hummler ${ }^{2}$}

'Division of Neonatology and Pediatric Critical Care, Department of Pediatrics and Adolescent Medicine, University Ulm, Germany; Department of Pediatric, Sohag University, Sohag, Egyp, Ulm/Sohag, Deutschland, ${ }^{2}$ Division of Neonatology and Pediatric Critical Care, Department of Pediatrics and Adolescent Medicine, University, Ulm, Ulm, Deutschland, ${ }^{3}$ Division of Neonatology and Pediatric Critical Care, Department of Pediatrics and Adolescent Medicine, Ulm University, Ulm, ${ }^{89075}$, Germany and Department of Pediatric, Zhongda Hospital, South east University, Nanjing, China, Ulm/Zhongda, Deutschland

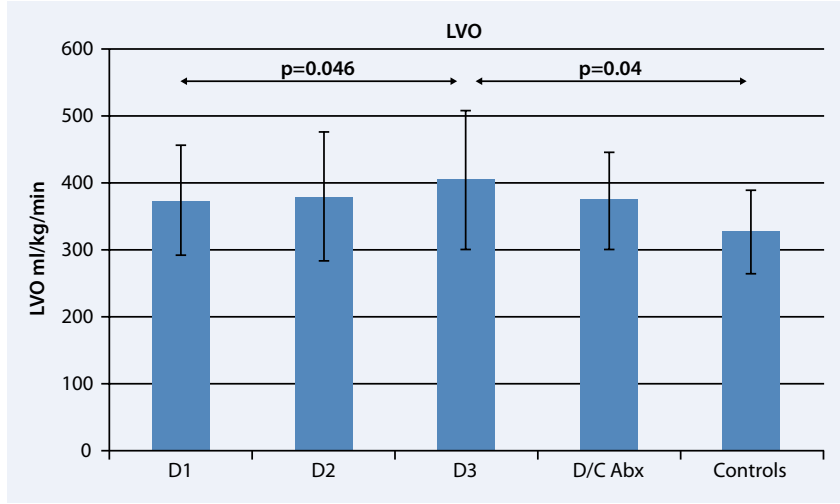

$\Delta$ P102-Abb. 1

Background. Hemodynamic support is important in the clinical management of neonatal sepsis as mortality is increased in patients with impaired hemodynamics.

Objective. to study the changes in hemodynamics and peripheral perfusion over time in premature infants treated for clinically suspected sepsis and to compare these changes with stable matched controls.

Design. Prospective case control study.

Methods. Left (LVO) and right ventricular output (RVO), and superior vena cava (SVC) flow were measured using echocardiography in newborn infants with suspected sepsis after DOL 7 and measurements were repeated 3 times $\mathrm{q} 24 \mathrm{~h}$ and when antibiotics were discontinued. Anterior cerebral artery resistance index and tissue oxygenation of the brain and the kidney were measured by near infrared spectroscopy (NIRS), and blood pressure and other vital parameters were monitored. Control subjects were matched with cases for GA, BW, postnatal age and body weight and were studied only once.

Results. Twelve preterm infants (GA $26.8 \pm 2.5$ wks; BW $843 \pm 225 \mathrm{~g}$ ) were studied at postnatal age 13.5 (7-60) days and compared to 12 stable matched controls. Four cases had a positive blood culture. Six of the other 8 infants had an elevated CrP, and 2 had an elevated IL8 only. LVO showed an increase over time reaching a maximum of $404 \pm 104 \mathrm{ml} / \mathrm{kg} /$ min on the 3 rd measurement point ( $\mathrm{p}=0.046$ compared to the 1 st measurement) and was higher compared to controls ( $\mathrm{p}=0.04$; Abb. 1). SVC flow showed a trend towards increase over time. The control group had a higher SVC flow than the initial measurement $(p=0.05)$. There was no significant change in RVO, in NIRS and Doppler parameters of peripheral perfusion, and in blood pressure over time or compared to controls, although five cases required circulatory support either by volume therapy, catecholamines or both.

Conclusions. Suspected sepsis was associated with hemodynamic changes which may have some implications for clinical management.

\section{P103}

Erfolgreiche Therapie einer metamizolinduzierten Agranulozytose bei septischem Schock mit Sargramostim

\section{A. Winkler', S. Kietz' ${ }^{2}$, H. Bahlmann', H. Lode' ${ }^{2}$, M. Heckmann ${ }^{7}$}

'Universitätsklinik Greifswald, Klinik für Kinder- und Jugendmedizin, Neonatologie und pädiatrische Intensivmedizin, Greifswald, Deutschland, ${ }^{2}$ Universitätsklinik Greifswald, Klinik für Kinder- und Jugendmedizin, Pädiatrische Hämatologie und Onkologie, Greifswald, Deutschland

Einleitung. Eine metamizolinduzierte Agranulozytose (neutrophile Granulozyten $<0,5 \mathrm{Gpt} / \mathrm{l}$ ) wird als sehr seltene unerwünschte Wirkung $(<1 / 10.000)$ angegeben. Insgesamt sind 10/1 Mio. Einwohner im Jahr (Europa) von einer medikamenteninduzierten Agranulozytose betroffen. Eine ausreichende Granulozytenzahl ist bei schwerer Sepsis und ausgedehnten Wunden unabdingbar. Bisher liegen keine Berichte in der Literatur zur Therapie der metamizolinduzierten Agranulozytose mit humanen rekombinanten Wachstumsfaktoren vor.

Kasuistik. Eine 14-jährige Patientin wurde mit schwerem septischem Schock 4 Wochen nach elektiver Exzision eines Pilonidalsinus aufgenommen. In den Wochen vor Aufnahme erfolgte eine Schmerztherapie mit Metamizol (Novalgin Tropfen $3 \times 40=3 \times 1000 \mathrm{mg}$ p.o./Tag) bei progredienter Wundheilungsstörung. Es fanden sich ausgeprägte Nekrosen und eitrige Wunden im Sakral- und Genitalbereich. Der Wundabstrich enthielt eine anaerob-aerobe Mischkultur u. a. mit Pseudomonas aeruginosa, der auch in der Blutkultur nachweisbar war. Es erfolgte eine Sepsistherapie mit Volumen-, Katecholamin- und antibiotischer Therapie, Beatmung und kinderchirurgischer Wundversorgung. Das Blutbild zeigte: Leukozyten o,6 Gpt/l mit absoluter Neutropenie (o,o1 Gpt/l) und Lymphopenie (o,56 Gpt/l), Thrombozyten $150 \mathrm{Gpt} / \mathrm{l}$, Erythrozyten 2,5 Tpt/l und Anämie mit $\mathrm{Hb}$ 4,2 mmol/l, Hkt 0,21. Die Knochenmarkpunktion ergab: o\% Granulopoese, 59\% Erythropoese, 1\% Thrombopoese, 33,5\% Lymphozyten und Plasmazellen, 4,5\% Monozyten, Befund am ehesten entsprechend einer medikamenteninduzierten Agranulozytose. Aufgrund der schweren Sepsis mit ausgedehnten Nekrosen wurde eine Therapie mit Neupogen ${ }^{\circledR}$ (Filgrastim, Granulozyten-Kolonie stimulierender Faktor, G-CSF, bis $18 \mu \mathrm{g} /$ $\mathrm{kg} / \mathrm{Tag}$ ) über 8 Tage begonnen. Weder darunter noch durch Leukozytentransfusionen stiegen die neutrophilen Granulozyten an (o,o1 bis max. o,07 Gpt/l). Eine Spendertypisierung zur Vorbereitung einer Knochenmarktransplantation wurde eingeleitet. Erst nach Wechsel auf Leukine ${ }^{\circ}$ über 7 Tage (Sargramostim, Granulozyten/Makrophagen-Kolonie stimulierender Faktor, GM-CSF, Absolutdosis 250 \%g/Tag), stiegen die Neutrophilen (nach 6 Tagen o,11 Gpt/l, nach 7 Tagen 1,19 Gpt/l) und die Monozyten an. Unter dieser Therapie konnte die Patientin nach 17 Tagen extubiert, die Katecholamintherapie nach 14 Tagen beendet werden. Die Wundheilung setzte unter regelmäßiger chirurgischer Vakuumtherapie ein.

Diskussion. Bei der Patientin fand sich im peripheren Blut und im Knochenmark eine isolierte Agranulozytose nach 4 Wochen langer Metamizoltherapie in sehr hohen Dosen. Ein sepsisbedingtes Knochenmarkversagen schied aus, da weitere Zellreihen nicht betroffen waren. Erst Sargramostim (GM-CSF) war effektiv in der Stimulation der Granulopoese, vermutlich da es in der Hämatopoese bereits eine Zellreihe früher (am Myeloblasten) als Filgrastim (am Promyelozyten) stimuliert. Schlussfolgerung. Sargramostim (GM-CSF) ist eine wirksame Therapie der metamizolinduzierten Agranulozytose. 


\section{P104}

Antiphospholipid-Antikörperassoziierter zerebraler Infarkt bei neonataler Enterobacter cloacae Meningitis

\section{S. Welcker', T. Bakchoul', K. Linnemann ${ }^{3}$, M. Heckmann ${ }^{3}$}

'Zentrum für Kinderheilkunde der Justus-v.-Liebig-Universität, Allgemeine Pädiatrie und Neonatologie, Gießen, Deutschland, Institut für Immunologie und Transfusionsmedizin, Abteilung Transfusionsmedizin, Greifswald, Deutschland, ${ }^{3}$ Klinik und Poliklinik für Kinder und Jugendmedizin, Abt. Neonatologie u. Päd. Intensivmedizin, Greifswald, Deutschland

Hintergrund. Zerebrale Infarkte ereignen sich selten in der Kindheit und weisen unterschiedliche Ätiologien auf. Im Rahmen bakterieller Meningitiden können gelegentlich Infarkte auftreten. Der Pathomechanismus ist unklar. Bei einigen Infektionskrankheiten wird von einer transienten Induktion von Antiphospholipid-Antikörpern (APL-AK) berichtet. In der Mehrzahl der Fälle kommt es jedoch nicht zu einer klinischen Manifestation eines Antiphospholipid Syndrom (APS).

Fall 1. Sectio bei pathologischem CTG und Doppler in der 29+5. SSW, 980 g, APGAR 5/8/9, NapH 7,14. Am 23. LT akute klinische Verschlechterung mit Apnoen, Bradykardien, Mikrozirkulations- und Temperaturregulationsstörung und Berührungsempfindlichkeit. Bei negativen Entzündungswerten (CRP 3,1 mg/l, IL-6 <50 pg/ml, IT-Quotient 0,04) erfolgte eine Therapie mit Ceftazidim, Vancomycin. Am Folgetag CRP 118 mg/l, Liquor: Zellen 22.00o/ml, Glukose o,1 mmol/l, Eiweiß $3223 \mathrm{mg} / \mathrm{l}$, Nachweis von Enterobacter cloacae. Im MRT an 38. LT zeigte sich eine Kolliquationsnekrose nahezu des gesamten Posteriorstromgebiet links mit zystischer Degeneration und Rand-Enhancement sowie Kommunikation zum Hinterhorn des linken Seitenventrikels.

Fall 2. Geminigravidität, IUGR, Oligohydramnion, reduzierter enddiastolischer Fluss im Doppler des männlichen Feten. Sectio in der 26+2 SSW bei diskordanten Wachstum und Bradykardie im CTG. APGAR 9/9/9, NapH 7,34, GG 465 g. Am 94. LT akute klinische Verschlechterung mit Apnoen, Bradykardien und erhöhter Körpertemperatur. Bei erhöhten Entzündungswerten (CRP 208 mg/l, IL-6 31.941pg/ml) antibiotische Therapie mit Ceftazidim, Vancomycin, Gentamycin und Meropenem. Im Liquor: Zellen 1472/ml, Glukose o,1 mmol/1, Eiweiß 4646 mg/l, Nachweis von Enterobacter cloacae. Am 3. Tag der Infektion zeigte sich im MRT ein ausgedehnter subakuter Infarkt im hinteren Media- und Posteriorstromgebiet rechts mit hämorrhagischer Transformation, deutlichem Ödem und Kompression des rechten Seitenventrikels.

Ergebnisse. Im Verlauf Entstehung einer umschriebenen zystischen Enzephalomalazie rechts parieto-okzipital mit Beteiligung hinterer Balkenanteile. Im Alter von 4 Jahren (Fall 1) und 4 Monaten (Fall 2) erfolgte die Bestimmung prothrombotischer APL-AK. In Fall 1 waren beta-2-Glykoprotein 1 IgG und in Fall 2 sowohl Cardiolipin-IgG als auch beta-2-Glykoproteinı IgG nachweisbar. Bei beiden Müttern fielen die Titer Bestimmungen der APL-AK negativ aus, so dass eine diaplazentare Übertragung mütterlicher Antikörper ausgeschlossen werden kann. Schlussfolgerung. Wir berichten erstmalig von zwei Fällen eines Enterobacter cloacae assoziierten APS, welches zu einem ischämischen Infarkt im Rahmen einer neonatalen Meningitis führte.

\section{P105}

Der seltene Fall: Diskussion der STIKO-Empfehlungen zur Prävention des neonatalen Varizellenyndroms (NVS)

\section{Laugwitz', S. Akanbi', L. Garten', C. Bührer', M. Berns ${ }^{1}$}

'Charité - Universitätsmedizin Berlin, Klinik für Neonatologie, Berlin, Deutschland

Übersicht. Die Primärinfektion mit dem Varicella-zoster-Virus (VZV) während der Schwangerschaft ist in Deutschland ein seltenes Ereignis (Inzidenz: 0,7-3 pro 1000 Geburten), nachdem $97 \%$ der Frauen im gebärfähigen Alter bereits VZV-spezifische Antikörper besitzen. Infektionswege für das neonatale Varizellensyndrom (NVS) sind pränatal eine diaplazentare, perinatal eine aufsteigende oder postnatal eine Tröpfcheninfektion. Die klinische Manifestation des NVS sind bis zu 12 Tage nach der Geburt auf einen diaplazentaren Infektionsweg zurückzuführen. Der schwerste Krankheitsverlauf des NVS (20\% Letalität) kann sich entwickeln, wenn die Mutter fünf Tage vor bis zwei Tage nach Geburt die für die Primärinfektion spezifischen Hauteffloreszenzen zeigt.

Fallbericht. Eine 22-jährige vietnamesische Erstgebärende wurde drei Tage vor Geburt mit einer floriden VZV-Primärinfektion vorstellig. Nach sekundärer Sectio wegen pathologischem CTG wurde ein eutrophes Reifgeborenes (38+o/7 SSW) ohne Effloreszenen entwickelt (Apgar 4/6/8 nach 1/5/10 min). Innerhalb der ersten Lebensstunde fand gemäß STIKO-Empfehlung eine postexpositionelle Prophylaxe durch passive Immunisierung mit Varizella-zoster-Immunglobulin (VZIG) statt. Am zweiten Lebenstag fiel das Neugeborene durch schwere zentrale Apnoen und Bradykardien auf. Unter dem klinischen Verdacht einer neonatalen VZV-Infektion mit begleitender Enzephalitis wurde eine intravenöse Aciclovir-Therapie eingeleitet. Die Infektion wurde durch eine positive PCR auf VZV-DNA im Serum bestätigt. Der Verdacht einer VZV-Enzephalitis bestätigte sich bei negativer VZV-PCR im Liquor nicht. Unter Aciclovir-Therapie für 9 Tage sistierten die Apnoen rasch. Im Verlauf traten keine weiteren VZV-typische Symptome auf.

Diskussion. Im berichteten Fall wurde das therapeutische Stufenschema der STIKO-Empfehlung eingehalten und die Aciclovir-Therapie bei klinischer Symptomatik begonnen. Auf die unerwartet schweren Apnoen konnte im stationären Kontext freilich adäquat reagiert werden. So ist zu diskutieren, ob trotz PEP nicht eine stationäre Überwachung zu empfehlen ist und warum nicht eine sofortige Aciclovir-Therapie bei florider Infektion mit Virämie empfohlen wird.

\section{P106}

Neonatale Coxsackie B4-Infektion mit dem klinischen Bild einer neonatalen Hämochromatose

\section{T. Dresbach', A. Eis-Hübinger', K. Schneider ${ }^{3}$, J. Schmitt', A. Müller ${ }^{7}$}

'Universitätsklinikum Bonn, Zentrum für Kinderheilkunde, Abteilung für Neonatologie, Bonn, Deutschland, ${ }^{2}$ Universitätsklinikum Bonn, Institut für Virologie, Bonn, Deutschland, ${ }^{3}$ St. Marien-Hospital Bonn, Neonatologie, Bonn, Deutschland, ${ }^{4}$ St. Marien-Hospital, Kinderklinik, Bonn, Deutschland

Fallbericht. Wir berichten über einen Patienten, der am 5. Lebenstag ein progredientes Leberversagen mit Hämolyse und disseminierter Koagulopathie entwickelte. Sonographisch zeigte sich eine Hepatomegalie und mäßig viel Aszites. Bei steigenden Leberenzymen, rasch progredienten Hämolysezeichen und beginnender disseminierter intravasaler Gerinnung erfolgte die Verlegung am 7. LT. in unsere Klinik. Bei Aufnahme zeigten sich deutlich erhöhte Werte der Transaminasen, Ferritin und Troponin-I, bei nur moderat erhöhten Entzündungsparametern. Bei Verdacht auf eine neonatale Hämochromatose erfolgte am 7. LT eine Austauschtransfusion sowie eine Therapie mit Immunglobulinen, NAcetylcystein, Selen und Desferroxamin. Die differentialdiagnostisch eingeleiteten virologischen Untersuchungen konnten Coxsackie $\mathrm{B}_{4}$ in Blut, Trachealsekret, Urin und Stuhl nachweisen. Auch bei den Eltern und Geschwistern konnte Coxsackie B4 im Stuhl nachgewiesen werden. Unter symptomatischer Therapie normalisierten sich die Transaminasen bis zum 10. LT. Während des stationären Aufenthaltes kam es zu einer Cholestase, die mit Ursodeoxycholsäure behandelt wurde, sowie zu rezidivierenden Hypoglykämien, die nach Supplementierung der Nahrung sistierten. Im Verlauf kam es zur Restitutio ad integrum. Diskussion. Die Ursachen für das neonatale Leberversagen umfassen neben Stoffwechseldefekten, die neonatale Hämachromatose und verschiedene Virusinfektionen. Bei unserem Patienten bestand zunächst der Verdacht auf eine neonatale Hämochromatose und wurde entsprechend der aktuellen Empfehlungen behandelt. Die weiteren Untersuchungen ergaben jedoch ein Leberversagen auf dem Boden einer Enterovirusinfektion. Enteroviren sind als Erreger von nosokomialen Infektionen im Neugeborenenalter bekannt. Die Übertragung erfolgt fäkal-oral, mit einer Inkubationszeit zwischen 2 und 14 Tagen. Vor allem der aus der Gattung der Enteroviren stammende Coxsackie-B-Virus kann in den ersten Tagen nach Geburt beim Neonaten zu generalisierten Infektionen mit Multiorganbeteiligung führen, die häufig letal 
enden. Die häufigsten klinischen Manifestationen sind Myokarditis, Hepatitis mit intravasaler Koagulopathie und Meningoenzephalitis. Die höchste Mortalität wird bei Patienten ohne protektive mütterliche Antikörper mit Myokarditis und fulminanter Hepatitis beschrieben. Der beschriebene Fall zeigt die Notwendigkeit einer frühzeitigen kalkulierten virologischen Diagnostik bei unklaren Sepsis-ähnlichen Krankheitsbildern in der Neonatalperiode. Auch aus krankenhaushygienischen Gesichtspunkten ist ein frühzeitiger Erregernachweis von großer Bedeutung, um mögliche Ausbrüche von nosokomialen Infektionen verhindern zu können. Durch die Kontamination beim Windelwechseln können Enteroviren bei Nichteinhalten von Hygienestandards leicht auf andere Patienten übertragen werden.

\section{P107}

Toxoplasma-Screening als Präventivmaßnahme aus gesundheitsökonomischer Sicht

\section{A. Prusa', E. Walter', M. Hayde', A. Pollak', A. Weinhandl', D. Kasper' \\ ${ }^{1}$ Medizinische Universität Wien, Universitätsklinik Klinik für Kinder- und Ju- gendheilkunde, Wien, Österreich, ${ }^{2}$ Institut für Pharmaökonomische Forschung, Wien, Österreich}

Fragestellung. In Österreich wurde 1974 für alle schwangeren Frauen der Mutter-Kind-Pass als Vorsorgeprogramm eingeführt. Dieses Programm beinhaltet die systematische Untersuchung auf Toxoplasma-Infektionen bei Schwangeren. Als Präventivmaßnahme werden Frauen routinemäßig serologisch gescreent. Im Falle einer frischen Infektion wird die Schwangere behandelt, um die Transmission zu verhindern. Kommt es zu einer Infektion des Ungeborenen, so wird das Kind im ersten Lebensjahr behandelt, um die klinische Manifestation zu beeinflussen. In dieser Studie wurden die Kosten des nationalen Toxoplasma-Screenings erhoben. Im Vergleich dazu wurden die Kosten der erkrankten Kinder ohne Präventivmaßnahmen kalkuliert.

Material und Methode. Österreich ist ein Land mit einer moderaten Inzidenz an Toxoplasma-Infektionen und ca. 77.00o Geburten pro Jahr. Die Daten für Inzidenz, Therapie, medizinische Interventionen, Folgeuntersuchungen und klinische Manifestation bei Kindern mit einer angeborenen Toxoplasmose wurden einer nationalen Erhebung aus zwei Jahrzehnten entnommen. Das Übertragungsrisiko der parasitären Infektion von Mutter auf Kind, wird ohne Therapie der Schwangeren mit 50\% angenommen. Die Tarife für erbrachte Leistungen und Kosten wurden für das Jahr 2012 kalkuliert. Das Modell inkludiert direkte Kosten wie Untersuchungen, stationäre Aufnahme und medizinische Behandlungen; und indirekte Kosten wie beruflicher Ausfall der Kindeseltern, Lebensqualität der Kinder, Beeinträchtigung des Kindes. In diesem Modell werden die Kosten zwei unterschiedlicher Strategien gegenübergestellt: 1) Prävention mittels Toxoplasma-Screening, medikamentöser Therapie und Management nach österreichischem Standard; 2) Folgekosten von Kindern mit angeborener Toxoplasmose ohne Screening-Maßnahmen.

Ergebnisse. Österreich verbrauchte für das Toxoplasma-Screening nach Standard $0,5 \%$ des Jahresbudgets, welches für das Mutter-Kind-Pass Programm ausgegeben wurde. Betrachtet man die Lebenskosten für erkrankte Kinder mit einer angeborenen Toxoplasmose, so ist eine Kosteneinsparung in der Screening-Gruppe um den Faktor 30 zu erwarten. Schlussfolgerung. Das Toxoplasma-Screening ist aus gesundheitsökonomischer Sicht eine sinnvolle Präventivmaßnahme, da die Streichung des Screenings eine spürbare Mehrbelastung des Gesundheitsbudgets erwarten lässt.

\section{P108}

Herpes-simplex-Sepsis trotz mütterlicher Antikörper - das Versagen des Nestschutzes bei Frühgeborenen!

M. Niewisch', H. Fuchs', R. Hentschel

'Universitätsklinikum Freiburg, Zentrum für Kinder- und Jugendmedizin, Freiburg, Deutschland, ${ }^{2}$ Universitätsklinikum Freiburg, Zentrum für Kinder- und Jugendmedizin, Funktionsbereich Neonatologie/Intensivmedizin, Freiburg, Deutschland

Hintergrund. Herpes-simplex-Infektionen beim Neugeborenen weisen eine hohe Mortalität und Morbidität auf. Die Transmission erfolgt meist perinatal nach einer maternalen Primärinfektion mit Herpes genitalis (meist Herpes-simplex-Virus Typ 2), selten antenatal (5\%) oder postnatal (10\%, bei Kontakt mit infektiösem Sekret). Klinisch manifestiert sich eine neonatale Herpes-Infektion als lokalisierte mukokutane (Haut, Auge, Mund), ZNS- oder disseminierte Infektion. Die Diagnose einer disseminierten Herpes-simplex-Infektion wird durch das unspezifische klinische Bild, ähnlich einer bakteriellen Sepsis, und der oftmals fehlenden Herpestypischen Bläschenbildung erschwert. Die unbehandelte, disseminierte Herpes-Infektion geht mit einer Mortalität bis zu 85\% einher.

Kasuistik. Wir berichten über ein Frühgeborenes der 25+3/7 SSW (Geburtsgewicht 800 g, APGAR 1/6/6), bei dessen Mutter ein durchgemachter Herpes genitalis bekannt war, zum Zeitpunkt der Geburt bestanden jedoch keine Symptome. Die pränatal durchgeführte Virus-Serologie ergab einen positiven Befund für Herpes-IgG. Im Rahmen der Erstversorgung erfolgte die Intubation und Surfactant-Gabe, es war bereits am 1. Lebenstag eine kreislaufunterstützende Therapie notwendig. Initial erfolgte eine antibiotische Therapie über 5 Tage mit Tazobactam/Tobramycin. Am 6. Lebenstag kam es zur Allgemeinzustandsverschlechterung mit ansteigenden Leukozyten, und es wurde eine erneute Antibiotikatherapie begonnen (Vancomycin/Meropenem). Hierunter normalisierten sich die Leukozyten, in der Blutkultur konnte jedoch kein Keim nachgewiesen werden. Am 10. Lebenstag traten im Gesicht und am Thorax Hauterosionen ohne Bläschenbildung auf, und es entwickelte sich ein mäßiger Aszites. Am 12. Lebenstag kam es zu einer nicht beherrschbaren metabolischen Azidose und zum Exitus letalis. Postmortal wurde eine Herpes-simplex-Infektion vermutet und eine Diagnostik veranlasst. Die Herpes-simplex-Typ-1-PCR war im Plasma positiv (334.500 copies/ml) und bestätigte den Verdacht einer neonatalen, disseminierten Herpessimplex-Infektion. Eine nachträglich durchgeführte Herpes-PCR von der am 1. Lebenstag abgenommenen Trockenblutkarte zur Stoffwechseldiagnostik war negativ. Es ließ sich retrospektiv kein Kontakt des Patienten mit einer symptomatisch an Herpes erkrankten Person feststellen.

Schlussfolgerung. Bei einer neonatalen Sepsis mit negativen Blutkulturen und weiterer Allgemeinzustandsverschlechterung unter antibiotischer Therapie sollte eine Herpes-simplex-Infektion auch ohne das Vorhandensein von typischen Bläschen in Betracht gezogen werden. Eine Normalisierung der Leukozytenzahlen unter Antibiotikatherapie kann trügerisch sein und schließt eine virale Ursache nicht aus, zudem ist bei Frühgeborenen das Risiko einer viralen Infektion aufgrund des unzureichenden Nestschutzes erhöht. Bei Herpes labialis von Kontaktpersonen sind Präventionsmaßnahmen (abdeckende Zinkcreme, Mundschutz und ggf. lokale Behandlung mit Acyclovircreme) einzuhalten 


\section{P109}

Retrospektive Diagnose von konnatalen CMV-Infektionen bei zwei Säuglingen, deren Neugeborenen-Hörscreenings bereits auffällig waren

\section{H. Buxmann', B. Wittekindt' ${ }^{1}$, P. Hofstetter ${ }^{2}$, R. Schlößer', K. Hamprecht ${ }^{3}$, H. Rabenau ${ }^{4}$}

${ }^{1}$ Klinikum der J.W. Goethe-Universität, Klinik für Kinder- und Jugendmedizin, Neonatologie, Frankfurt am Main, Deutschland, ${ }^{2}$ Klinikum der J.W. Goethe-Universität, Klinik für Kinder- und Jugendmedizin, Pädiatrische Neurologie, Frankfurt am Main, Deutschland, ${ }^{~}$ Eberhard-Karls-Universität, Institut für Medizinische Virologie und Epidemiologie der Viruskrankheiten, Tübingen, Deutschland, ${ }^{4} \mathrm{Klini}-$ kum der J.W. Goethe-Universität, Institut für Medizinische Virologie, Frankfurt, Deutschland

Einleitung. Sensorineurale Hörstörungen zählen zu den häufigsten Symptomen bei Neugeborenen mit konnataler CMV-Infektion. Aktuell sieht das Vorgehen beim Neugeborenen-Hörscreening eine zeitnahe Diagnostik auf konnatale CMV-Infektionen bei Neugeborenen mit bestätigt auffälligen Hörscreenings nicht regelhaft vor.

Fallberichte. Patient 1 wird im Alter von 9 Monaten mit folgenden Symptomen vorgestellt: seit Geburt bestehende Hörminderung links, statomotorische Entwicklungsverzögerung, Mikrocephalie, Fieberkrämpfe und Hepatosplenomegalie. Die weiterführende Diagnostik erbringt den Nachweis von CMV im Urin (Zellkultur). Im Blut: CMV-PCR negativ, CMV-IgG positiv und CMV-IgM negativ. Im Liquor negative CMVPCR. Die retrospektive Untersuchung der Neugeborenen-Screeningkarte ergibt eine positive CMV-PCR. Der Patient leidet an einer symptomatischen konnatalen CMV-Infektion. Patientin 2 wird im Alter von 10 Monaten mit folgenden Symptomen vorgestellt: Seit Geburt bestehende Hörstörung links mit Surditas im Verlauf, rechts postnatale Normakusis mit Entwicklung einer Hörstörung im Verlauf, statomotorische Entwicklungsverzögerung. Die weiterführende Diagnostik erbringt den Nachweis von CMV im Urin (Zellkultur). Im Blut: CMV-PCR positiv, CMVIgG positiv und CMV-IgM negativ. Im Liquor negative CMV-PCR. Auf der NG-Screeningkarte sind nur Blutreste verfügbar. Der Nachweis von CMV mittels PCR gelingt im Speziallabor. Die Patientin leidet an einer symptomatischen konnatalen CMV-Infektion. Es wird mit Zustimmung der Eltern eine Off-label-Therapie mit Valganciclovir durchgeführt.

Diskussion. Bei Neugeborenen mit bestätigt auffälligem Hörscreening findet sich in rund $20 \%$ der Fälle eine konnatale CMV-Infektion als Ursache. Diese Diagnose wurde in den beiden beschriebenen Fällen mit einer zeitlichen Latenz von mehreren Monaten gestellt. Wird eine konnatalen CMV-Infektion in den ersten drei Lebenswochen diagnostiziert, dann kann mittels antiviralem Therapiestart in den ersten 28 Lebenstagen das Risiko einer weiter voranschreitenden Symptomatik durch die konnatale CMV-Infektion vermindert und in einigen Fällen eine Verbesserung des Hörvermögens erreicht werden.

Schlussfolgerung. Im Rahmen prospektiver multizentrischer Studien ist zu untersuchen, ob bei frühzeitiger Kontrolle von auffälligen Hörscreening-Befunden mit sich direkt anschließender CMV-Diagnostik bei bestätigter audiometrischer Auffälligkeit Neugeborene mit behandelbaren symptomatischen konnatalen CMV-Infektionen identifiziert werden können.

\section{Entwicklung}

\section{P110}

Entwicklungsuntersuchung nach 3,5 Jahren bei Frühgeborenen der randomisierten Studie zur plazentaren Transfusion durch verzögerte Abnabelung (VA) oder Ausstreichen der Nabelschnur (AN)

\section{H. Rabe', A. Sawyers' ${ }^{2}$ P. Amess 3 , E. Emery ${ }^{4}$, S. Ayers ${ }^{5}$}

'Brighton \& Sussex Medical School, Neonatology, Brighton, United Kingdom, ${ }^{2}$ University of Sussex, Falmer, United Kingdom, ${ }^{3}$ Brighton \& Sussex University Hospitals, Neonatology, Brighton, United Kingdom, ${ }^{4}$ Brighton \& Sussex University Hospitals, Brighton, United Kingdom, ${ }^{5}$ University of London, Psychology, London, United Kingdom

Hintergrund und Fragestellung. Plazentofetale Transfusion bei der Geburt von sehr kleinen Frühgeborenen entweder durch VA oder AN bewirkt eine Reduktion von intraventrikulären Blutungen und den Bedarf an Bluttransfusionen. Der protektive Effekt wird aller Wahrscheinlichkeit nach durch eine verbesserte Kreislaufstabilisierung nach der Geburt verursacht. In der vorliegenden prospektiven Studie berichten wir über die neurologische Entwicklung von überlebenden Frühgeborenen $<33$ Schwangerschaftswochen, die an unserer randomisierten Studie zum Vergleich 30 Sekunden VA und viermaliges AN teilgenommen hatten (Rabe Obstet \& Gynecol 2011).

Material und Methode. Prospektive Kohortenstudie, Bayley-III-Untersuchungen im korrigierten Alter von 3,5 Jahren; Statistische Auswertung mit ANCOVA (Signifikanz Level für alpha 0,05). Die Statistikerin war bezüglich der Gruppenallokation geblindet.

Ergebnisse. Es konnten 29/51 (56\%) überlebender Kindern nachuntersucht werden. Verglichen mit der VA Gruppe (5 männl., 6 weibl.) hatte die AN Gruppe (9 männl., 9 weibl.) bessere Scores für Sprachentwicklung (115 SD18 vs. 105 SD23), kognitive Entwicklung (127 SD20 vs. 120 SD27) und motorischer Entwicklung (113 SD23 vs. 108 SD21). Diese Unterschide waren nicht signifikant. Die ANCOVA Analysen ergaben keine signifikante Korrelationen bezüglich Hintergrunddaten mütterlichem Body-Mass-Index, Rauchen in der Schwangerschaft, Gestationsalter bei der Geburt, Geburtsgewicht, Kopfumfang, Geschlecht, korrigiertes Alter und Intervention auf die Bayley-Scores.

Diskussion. Bisher sind nur von einer Studie (Mercer 2010) neurologische Entwicklungsdaten mit Bayley-III-Untersuchungen publiziert worden. Die VA-Gruppe hatte in dieser Studie im Vergleich zu ehemaligen Frühgeborenen mit kurzer Abnabelungszeit bessere motorische Entwicklung Scores im korrigierten Alter von 7 Monaten. Unsere Studie ist die erste mit Daten im Alter von 3,5 Jahren. Ausstreichen der Nabelschnur wird als unphysiologische Intervention diskutiert. Unsere Pilotstudie zeigt, dass kein erhöhtes Risiko für typische Komplikationen der Frühgeburtlichkeit aufgetreten ist und dass die neurologische Entwicklung im Alter von 3,5 Jahren vergleichbar ist mit der Gruppe, die plazentare Transfusion durch verzögerte Abnabelung erhalten hat. Schlussfolgerung. Ausstreichen der Nabelschnur kann eine sichere Alternative zu verzögerter Abnabelung darstellen. Studien mit größerer Fallzahl zur Verifizierung der Ergebnisse sind erforderlich.

\section{P111}

Das intrauterine Wachstum und damit die fetale Programmierung unterliegt geschlechtsspezifisch unterschiedlichen Steuerungsmechanismen

\section{B. Brune', D. Nähter', S. Kropf', G. Jorch', T. Brune}

'Universitätskinderklinik, Magdeburg, Deutschland, ${ }^{2}$ Institut für Biometrie, Magdeburg, Deutschland, ${ }^{3}$ Klinikum Lippe, Kinderklinik, Detmold, Deutschland

Einleitung. In früheren Studien konnten wir nachweisen, dass sich das Risiko, im 6. LJ Übergewicht zu entwickeln, für männliche Neugeborenen mit einem Geburts-BMI <10 P im Vergleich zu weiblichen Neugeborenen mit einem Geburts-BMI <10 P verdoppelt. Als Haupteinflussfaktor für 


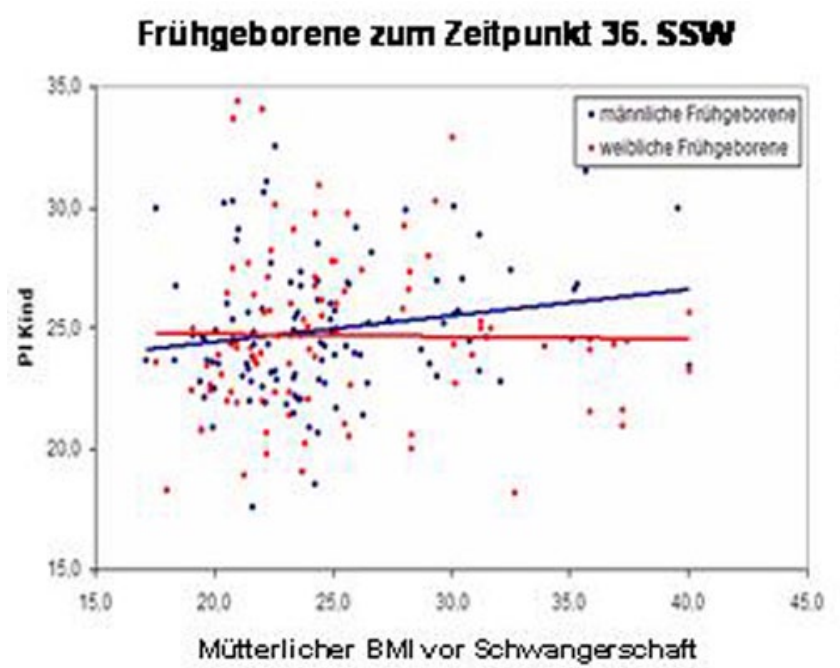

Reifgeborene zum Zeitpunkt 36. SSw

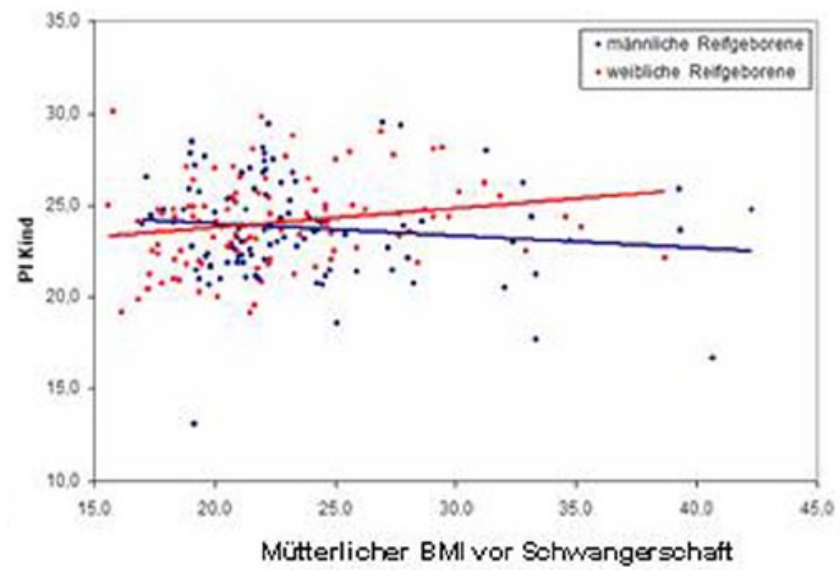

$\triangle P_{111}-A b b .1$

Übergewicht im 6. LJ konnten wir geschlechtsunabhängig den BMI der Mutter identifizieren [1]. Dies führt zu der Hypothese, dass der BMI der Mutter das Geburtsgewicht geschlechtsspezifisch beeinflusst.

Methoden. In die Studie eingeschlossen wurden 202 Reifgeborene (101 m, 101 w) 206 Frühgeborene [109 m (GA: 30/2 ), 97 w FG (GA: 30/2, Mean Ga 30/3)]. Mittels der Praderperzentilen wurden Gewicht und Länge bei den
Reifgeborenen auf die 36 SSW, die der Frühgeborenen auf die 30. SSW korrigiert. Die Frühgeborenen wurden nach der Geburt nach Standartregime der Klinik ernährt und Gewicht und Länge in der 36. SSW erhoben. Die jeweilige Ponderalindex (PI) wurde berechnet und mittels univariater Kovarianzanalyse mit dem Ponderalindex als Zielgröße und dem Geschlecht beziehungsweise dem Reifegrad als feste Faktoren und dem BMI der Mutter vor der Schwangerschaft als Kovariable geprüft, ob ein Unterschied zwischen den Wechselwirkungen des PI in Abhängigkeit vom mütterlichen Body-Mass-Index und dem Geschlecht des zum Zeitpunkt der korrigierten 30. und 36. Schwangerschaftswoche bei den Frühgeborenen bzw. zur korrigierten 36. Schwangerschaftswoche bei den Reifgeborenen besteht.

Ergebnisse. Die Abbildung zeigt, dass der mütterliche BMI bei den weiblichen Feten signifikant positiv mit ihrem Gewicht und Ponderalindex am Ende der Schwangerschaft korreliert. Bei zu früh geborenen Töchtern, die postnatal eine standardisierte Nährstoffversorgung erhalten, verläuft die Gewichtsentwicklung dagegen entgegengesetzt: 6 Wochen postnatal korreliert bei ihnen das Gewicht negativ mit dem BMI der Mutter. Bei männlichen Feten korreliert der mütterliche BMI signifikant positiv mit der Körperlänge, mütterliches Übergewicht führt so am Ende der Schwangerschaft zu einem niedrigeren PI der betroffenen Kinder. Zu früh geborene Söhne übergewichtiger Frauen, die diesem intrauterinen Einfluss nicht mehr unterliegen, holen postnatal signifikant an Gewicht auf: je höher der mütterliche BMI, desto höher ist nach 6 Wochen das Gewicht, aber nicht die Körperlänge der betroffenen Jungen Dieses Geschlechtsspezifisch unterschiedlicher Wachstumsmuster ist signifikant ( $\mathrm{p}=0,012 ; \mathrm{Abb} .1$ ).

Diskussion. Unsere Ergebnisse zeigen, dass die intra (plazentar)- bzw. extrauterine (genetisch) Gewichts- und Längenentwicklung des Kindes den mütterlichen BMI geschlechtsspezifisch unterschiedlich beeinflusst wird. Diese Beobachtung bestätigt die These der geschlechtsspezifischen fetale Programmierung.

1. Brune B et al (2010) Obesity $18: 798-802$

\section{P112}

Bei einem Geburts-BMI <10 P haben männliche Neugeborene im Vergleich zu weiblichen ein 2-fach höheres Risiko für Übergewicht im Einschulungsalter: Ist die fetale Programmierung geschlechtsspezifisch?

\section{B. Brune', G. Jorch', T. Brune}

'Universitätsklinikum, Kinderklinik, Magdeburg, Deutschland, ${ }^{2}$ Klinikum Lippe, Kinderklinik, Detmold, Deutschland

Einleitung. Der Zusammenhang zwischen niedrigem Geburtsgewicht und einem erhöhten Risiko für die Entwicklung von Übergewicht ist

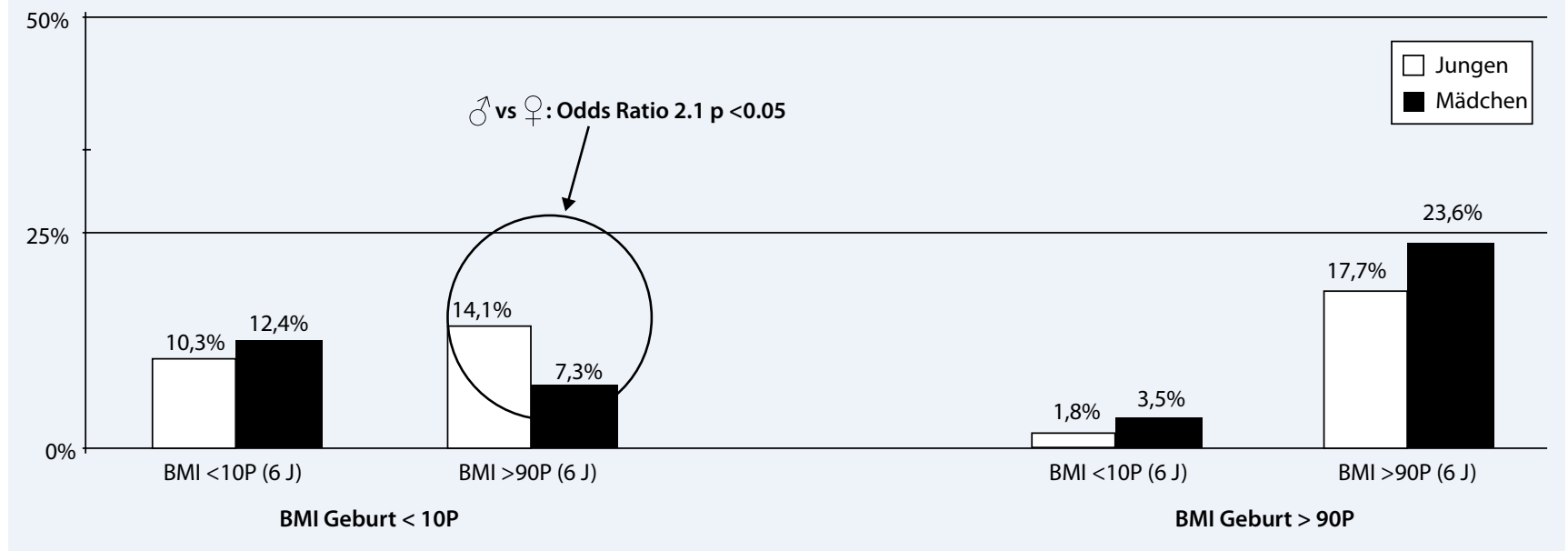

$\Delta P_{112}-A b b .1$ 
in vielen Studien eindeutig gezeigt worden. Hypothesen zur Erklärung dieses Zusammenhangs sind jedoch noch kontrovers. In der vorliegenden Studie haben wir untersucht, ob ein extremer Geburts- BMI nach normalem Schwangerschaftsverlauf einen Einfluss auf die Entwicklung von Übergewicht schon im Einschulungsalter hat und ob es geschlechtsspezifische Unterschiede gibt.

Patienten und Methoden. Insgesamt wurden 5433 Probanden (2793 2640) in die Studie eingeschlossen. Ausschlusskriterien waren Mehrlings- und Frühgeburten, gravierende kindliche und mütterliche Erkrankungen, angeborene Fehlbildungen sowie nichtdeutsche Nationalität (mindestens ein Elternteil). Für alle Probanden wurde der BMI bei Geburt sowie im Alter von 72 Monaten berechnet und dem jeweiligen Perzentil nach Kromeyer-Hauschild zugeordnet.

Ergebnisse. Bei normalem Geburts-BMI $(>10$ P $<90$ P) korreliert bei beiden Geschlechtern der Geburts-BMI linear mit dem BMI zum Zeitpunkt des 6. LJ. Bei den Mädchen ist dieser lineare Zusammenhang auch auf die Bereiche Geburts-BMI $<10 \mathrm{P}$ und $>90 \mathrm{P}$ ausgedehnt, während bei den Jungen insbesondere bei einem Geburts-BMI $<10$ P der BMI im 6. LJ nahezu invers zum Geburts-BMI korreliert. Die Graphik zeigt, dass männliche Neugeborene mit einem Geburts-BMI <10 P im 6 LJ doppelt so häufig übergewichtig sind im Vergleich zu den Mädchen. $(14,1 \%$ vs. $7,3 \%$; $<<0,05)$. Insgesamt zeigte sich, dass bei den Mädchen in allen Fällen der Geburts-BMI eine signifikant höhere prädiktive Aussage für den BMI im Einschulungsalter hat als bei den Jungen (Abb. 1). Diskussion. Die vorliegende Studie zeigt, dass bei einem hohen GeburtsBMI ein sehr ausgeprägter Trend besteht, auf diesem hohen BMI zu verharren. Durch die hohe Fallzahl der untersuchten Kinder konnten erstmalig auch genaue Aussagen über die Gruppe von Kindern gemacht werden, deren BMI innerhalb der ersten 6 Lebensjahre von einem extrem niedrigen bzw. hohen Perzentil bei Geburt zu dem entgegengesetzten extremen Perzentil zum Zeitpunkt der Einschulung wechselte. Hier konnten wir einen signifikanten Geschlechtsunterschied darstellen: Jungen mit einem niedrigen Geburts-BMI $(<10 \mathrm{P})$ haben gegenüber Mädchen ein doppelt so hohes Risiko, zum Zeitpunkt der Einschulung übergewichtig zu sein ( $\mathrm{p}<0,05$; OR: 2,1). Diese Ergebnisse lassen auf eine geschlechtsabhängige fetale Programmierung schließen.

\section{P113}

Entwicklungsverlauf von extrem kleinen Frühgeborenen nach LISA: Ergebnisse der Wiener Kohorte 2009-2012

\section{S. Brandstetter', R. Fuiko', K. Klebermass-Schrehof', A. Berger' \\ 'Medizinische Universität Wien, Neonatologie, pädiatrische Intensivmedizin und Neuropädiatrie, Wien, Österreich}

Hintergrund. Ein proaktiver Ansatz zur Reanimation und Intensivpflege von extrem kleinen Frühgeborenen ( $<27$ Schwangerschaftswochen) mit LISA hat die Überlebensrate dieser Kinder in den letzten Jahren deutlich erhöht. Diese neue Methode der weniger invasiven Surfactant-Gabe ohne Intubation („less invasive surfactant administration“ - LISA) wurde bereits beschrieben. Allerdings stellt sich die Frage, ob eine höhere Überlebensrate zu vermehrten Entwicklungsbeeinträchtigungen führt.

Ziel. Die Entwicklung der extrem kleinen Frühgeborenen wurde mit einem und zwei Jahren (korrigiert) untersucht.

Patienten und Methoden. 336 Kinder der Schwangerschaftswochen 23 bis 27 von 2009 bis 2012 wurden mit LISA erstversorgt. Für 178 Kinder konnten Entwicklungsdaten erhoben werden. Die mentale und motorische Entwicklung wurde mit den Bayley Scales of Infant Development II untersucht. Eine klinische und neurologische Untersuchung wurde durchgeführt.

Ergebnisse. Mit einem medianen Alter von 12,4 Monate (korrigiert) wurden 178 extrem kleine frühgeborene Kinder evaluiert. Der Mittelwert der mentalen Entwicklung lag bei 84, der mittlere psychomotorische Entwicklungsindex betrug 81. Die Zahl der Kinder, die keine oder nur eine milde Entwicklungsbeeinträchtigung aufwiesen nahm mit steigender Schwangerschaftswoche $\mathrm{zu}$ (55\% in 23 Schwangerschaftswochen, $74 \%$ in $24,75 \%$ in $25,88 \%$ in 26 und $79 \%$ in 27 für die mentale
Entwicklung und $50 \%$ in $23,79 \%$ in $24,82 \% 25,86 \%$ bin $26,80 \%$ in 27 ). Ähnliche Ergebnisse wurden für das Alter von zwei Jahren (korrigiert) festgestellt.

Schlussfolgerung. Rund 70\% der extrem kleinen Frühgeborenen, die mit LISA erstversorgt wurden, wiesen im Alter von einem Jahr korrigiert keine oder leichte Entwicklungsbeeinträchtigungen auf, im Alter von zwei Jahren korrigiert waren es rund 83\%. Dieser Prozentsatz nahm mit jeder Schwangerschaftswoche weiter zu. Diese Ergebnisse sind für Ärzte und Familien relevant.

\section{P114 \\ Mütterliches Belastungserleben nach Entlassung des frühgebore- nen Kindes aus der Kinderklinik}

J. Hennig', J. Reichert', M. Rüdiger' ${ }^{1}$, P. Hinner ${ }^{1}$

'Universitätsklinikum Carl Gustav Carus Kinderklinik, Neonatologie/Pädiatrische Intensivmedizin, Dresden, Deutschland

Fragestellung. Nicht selten erleben Eltern die Frühgeburt ihres Kindes bzw. ihrer Kinder als kritisches Lebensereignis, das sich im weiteren Verlauf zu einer Belastung entwickeln und sich im elterlichen Verhalten und der Beziehung zum Kind niederschlagen kann. Eine adäquate soziale Unterstützung sowie die wahrgenommene partnerschaftliche Qualität werden in der Literatur als Ressourcen für die Belastungsbewältigung beschrieben; das Bestehen einer frühkindlichen Regulationsstörung hingegen kann als Risikofaktor für das Belastungserleben betrachtet werden. Die Studie stellt die Frage nach dem Belastungserleben von Müttern Frühgeborener nach Entlassung der Kinder von der neonatologischen Station unter Berücksichtigung ausgewählter Merkmale sozialer Unterstützung, partnerschaftlicher Qualität und frühkindlicher Regulationsstörungen.

Methode. Studienteilnehmende sind 49 Mütter, deren Kinder am Perinatalzentrum Dresden stationär behandelt wurden; es handelt sich um eine anfallende Stichprobe. Ausgeschlossen wurden Mütter, die psychopathologisch auffällig waren und /oder deren Muttersprache nicht deutsch war. Die Befragung erfolgte in der vierten Woche nach Entlassung des Kindes vorzugsweise durch einen Online-Fragebogen bzw. nach Übermittlung eines Papier-Bleistift-Fragebogens. Erfragt wurden das mütterliche Belastungserleben (Eltern-Belastungs-Inventar; Tröster, 2011), die soziale Unterstützung (Fragebogen zur sozialen Unterstützung; Frydrich, Sommer \& Brähler, 1999), die partnerschaftliche Qualität (Dyadic Coping Inventory; Bodenmann, 2008) und das kindliche Schlaf-, Fütter- und Schreiverhalten.

Ergebnisse. 24\% der befragten Mütter fühlen sich stark bzw. sehr stark belastet. Ein Großteil kann auf ein zuverlässiges soziales Netzwerk und eine unterstützende Partnerschaft zurückgreifen. Signifikante Zusammenhänge zwischen sozialer Unterstützung und partnerschaftlicher Qualität auf der einen und mütterlichem Belastungserleben auf der anderen Seite finden sich nicht. Insgesamt 20\% der Kinder weisen Anzeichen für eine oder mehrere frühkindliche Regulationsstörungen auf. Mütter, deren Kinder Anzeichen für Regulationsstörungen aufweisen, erweisen sich als signifikant belasteter als Mütter mit Kindern ohne diese Anzeichen.

Diskussion. Ein Teil der Mütter zeigt sich nach Entlassung des Kindes belastet. Da der Großteil beschreibt, auf ein verlässliches soziales Netzwerk und einen stützenden Partner zurückgreifen zu können, generiert sich die Belastung vermutlich aus kindlichen Faktoren wie z. B. deren Anpassungs- und Regulationsfähigkeit in der Phase der häuslichen Adaptation.

Schlussfolgerung. Bei Müttern frühgeborener Kinder sollte insbesondere in der Phase der Entlassung des Kindes von der Station in die häusliche Umgebung nicht allein auf die Sicherung der sozialen bzw. auch partnerschaftlichen Unterstützung fokussiert werden. Da sich Belastungen vornehmlich aus der kindlichen Anpassungs- und Regulationsfähigkeit ergeben, muss eine allgemeine Unterstützung der Mutter durch ihre gezielte Vorbereitung ergänzt werden, ihr Kind bei der häuslichen Adaptation adäquat begleiten zu können. 


\section{P115}

Effekte eines videogestützten Feinfühligkeitstrainings auf elterliche Kontroll- und Kompetenzüberzeugung

\author{
J. Bonitz', J. Reichert', M. Rüdiger', P. Hinner' \\ 'Universitätsklinikum Carl Gustav Carus Kinderklinik, Neonatologie/ Pädiatri- \\ sche Intensivmedizin, Dresden, Deutschland
}

Fragestellung. Geprüft wird die Wirksamkeit eines videobasierten Trainingsprogramms für Eltern frühgeborener Kinder im stationären Setting auf die elterlichen Kompetenz- und Kontrollüberzeugungen. Dabei wird auch kindspezifisches Wissen der Eltern zu relevanten Entwicklungssignalen von Frühgeborenen untersucht. Beide Konstrukte werden als Einflussfaktoren für die Qualität der Eltern-Kind-Interaktion betrachtet.

Material und Methode. Teilnehmer der Studie sind 50 Eltern, deren frühgeborene Kinder $(\mathrm{SSW} \leq 32)$ am Perinatalzentrum Dresden stationär versorgt werden. Sie durchlaufen, randomisiert aufgeteilt in Interventions- und Kontrollgruppe, ein fünf Einzelsitzungen umfassendes Trainingsprogramm sowie drei begleitende Datenerhebungen. Das Training beruht auf Modellvideoaufnahmen frühgeborener Kinder. Mittels mehrfaktorieller Varianzanalyse mit Messwiederholung wird ein trainingsbedingter Effekt auf elterliche Kontroll- und Kompetenzüberzeugungen sowie auf kindspezifisches Wissen geprüft. Weitere einflussnehmende Faktoren wie Geburtsmerkmale des Kindes, soziale Merkmale der Eltern und Teilnahme an psychosozialen Angeboten werden berücksichtigt.

Ergebnisse. Erste Auswertungen verweisen auf eine signifikante Zunahme der elterlichen Kompetenzerwartung über die Zeit und eine trainingsbedingte Zunahme des kindspezifischen Wissens. So zeigen in der Interventionsgruppe 65\% der Teilnehmer einen bedeutsamen Wissenszuwachs ( $\geq 5$ von 47 Punkten) wohingegen Eltern der Kontrollgruppe nur in $37 \%$ der Fälle einen bedeutsamen Wissenszuwachs erreichen Weiter kann gezeigt werden, dass Zufriedenheit und Akzeptanz der Eltern mit dem Programm gegeben sind. Regressionsanalysen zur Veränderung der elterlichen Kontroll- und Kompetenzerwartung über die Zeit unter Beachtung der möglichen, bereits beschriebenen Einflussfaktoren werden aktuell durchgeführt.

Diskussion. Erste Ergebnisse zeigen, dass die Teilnehmer sich in ihrer Elternrolle über die Zeit zunehmend als kompetent betrachten. Bei Trainingsteilnahme kommt ein höheres Wissen über spezifische Verhaltenssignale der Frühgeborenen in der Eltern-Kind-Interaktion hinzu. Die Bedeutung dieses Wissenszuwachses für die Entwicklung der elterlichen Kompetenz- und Kontrollüberzeugung ist Ziel weiterer statistischer Auswertungen.

Schlussfolgerung. In den Studienergebnissen ist erkennbar, dass Eltern frühgeborener Kinder durch ein videobasiertes Trainingsprogramm einen Lernfortschritt erzielen und es als Angebot akzeptieren. Es ist somit ein Programm, das im stationären Alltag implementiert werden kann. Eine Ausweitung des Angebotes auf weitere Perinatalzentren ist anzustreben. Fortführende Studien sind erforderlich, um mögliche weitere Wirkfaktoren zu erschließen bzw. die bereits Beschriebenen auf Langzeiteffekte hin zu überprüfen.

\section{P116}

Is early detection of attention problems in preterm children possible? The value of neuropsychological assessment at preschool age

\section{Giordano', L. Ulrike², S. Brandstetter', M. Hayde', K. Klebermass-Schre- hof', R. Fuiko', W. Liesa'}

'Universitätsklinik für Kinder- und Jugendheilkunde, Ableitung für Neonatologie, Pädiatrische Intensivmedizin und Neuropädiatrie, Wien, Österreich, 'Universitätsklinik für Kinder- und Jugendheilkunde, Pädiatrische Neuron-Oncologie, Wien, Österreich

Background. Advances in neonatal intensive care have improved the survival rate of preterm babies. However, despite the increased survival rate of these babies, the risk for cognitive and behavioral problems at school age has increased. These problems are often attributable to specific attentional deficits. The early detection of attentional problems is therefore a challenging but important endeavor for clinicians.

Objective. The investigation of attentional functioning in all its subcomponents in preterms at preschool age.

Methods. Preterm $(\mathrm{N}=56)$ and full-term children $(\mathrm{N}=56)$ between $5 ; 5$ and 6;11 years (average 5;7), matched for age, were assessed for the following attentional components: alertness, sustained attention, processing speed, orienting, perception, focused attention, go/nogo, distractibility, divided attention and flexibility. Each construct was assessed through subtests of the following neuropsychological batteries: KITAP/TAP and HAWIK-IV. Further factors such as parental ratings and descriptive item-based evaluation of the child's behavior during the neuropsychological assessment were also considered.

Results. Preterms show poor attentional performance in sustained attention, focused attention and distractibility, as well as reduction of processing speed in a visual search task, divided attention and flexibility. Decrease of volitional attention compared to automatic attention was also identified. No problems were detected in alertness and inhibition (go/nogo). Additionally, a higher rate of test-aborts, decreased motivation, and poor parental ratings were detected among the preterm population.

Conclusion. The neuropsychological results highlight the difference of attentional functioning between preterm and full-term and permit an early detection of attention deficits.

\section{P117}

\section{Wirkungsmöglichkeiten eines Tagebuches für Eltern frühgeborener} und kranker neugeborener Kinder

\section{Hócza' ${ }^{1}$ J. Reichert ${ }^{1}$, M. Rüdiger ${ }^{1}$}

'Universitätsklinikum Carl Gustav Carus Kinderklinik, Neonatologie \& Pädiatrische Intensivmedizin, Dresden, Deutschland

Hintergrund. Eine zu frühe Geburt oder die Geburt eines kranken Kindes führt bei vielen Eltern zu einer starken Belastung. Die Trennung vom Kind zieht eine Beeinträchtigung in der Ausübung der Elternrolle nach sich. Dabei spielt gerade der elterliche Umgang mit ihrem Kind eine wichtige Rolle in der Bewältigung der zu frühen Geburt oder der Auseinandersetzung mit gesundheitlichen Einschränkungen des Kindes. Oftmals konnten Eltern eines frühgeborenen Kindes vor der Geburt noch keine deutliche Vorstellung vom Aussehen und Wesen ihres Kindes entwickeln oder sich verstärkt mit ihrer zukünftigen Elternschaft auseinandersetzen. Dies kann den Beziehungsaufbau zum Kind zusätzlich erschweren. Zur Förderung des Verarbeitungsprozesses wurde im November 2012 auf der Kinderintensivstation des Universitätsklinikums Dresden ein Tagebuch für Eltern eingeführt. Das Schreiben eines Tagebuches soll den Eltern ermöglichen, ihre Gedanken und Gefühle auszudrücken, besondere Momente und Aktivitäten mit ihrem Kind festzuhalten sowie die Ereignisse rund um Geburt und Klinikaufenthalt ihres Kindes zu reflektieren. Nach der „Facilitated Sensemaking Theory" (Davidson, 2010) kann diese Form der Intervention Eltern unterstützen, ihren Erfahrungen einen Sinn zu geben und sich an ihre neue Rolle anzupassen.

Fragestellung. Findet das Tagebuch die Akzeptanz der Eltern? Wie beschreiben Eltern den persönlichen Nutzen des Tagebuches?

Methodik. Ein Beurteilungsbogen für Eltern wurde nach einer inhaltsanalytischen Voruntersuchung erstellt: Akzeptanz des Tagebuches und persönlicher Nutzen des Tagebuches. Einschlusskriterium: Eltern eines frühgeborenen oder kranken neugeborenen Kindes, anfallende Stichprobe, $n=20$; Ausschlusskriterium: Aufenthaltsdauer des Kindes von $\leq 1$ Woche.

Ergebnisse. Die Inhaltsanalyse des Beurteilungsbogens bestätigt eine Zustimmung in Bezug auf Inhalt, Gestaltung und Struktur des Tagebuches. Vordergründig sehen Eltern den persönlichen Nutzen in der 
Möglichkeit der Auseinandersetzung mit ihren Gedanken und Gefühlen, des Festhaltens von Erinnerungen sowie der Förderung des Verständnisses und der Nachvollziehbarkeit der Ereignisse. Hinweise auf die Wirkungsmöglichkeiten des Tagebuchschreibens ergeben sich aus einer weiterführenden Analyse der Daten.

Diskussion. Die Ergebnisse dienen der weiteren Anpassung des Tagebuches an die elterlichen Bedürfnisse im Rahmen des psychologischen und sozialmedizinischen Angebots an Eltern frühgeborener und kranker neugeborener Kinder. Im Vordergrund steht die Frage nach dem Anteil des Tagebuches am Verarbeitungsprozess der Ereignisse sowie der Schaffung optimierter Bedingungen für den Beziehungsaufbau zwischen Eltern und Kind.

\section{P118 \\ Vorstellung neuer gesamtdeutscher Perzentilwerte für die Körper- maße von Einlingen}

\section{Voigt', N. Rochow ${ }^{2}$, K. Schneider ${ }^{3}$, H. Hagenah ${ }^{4}$, R. Scholz ${ }^{5}$, D. Olbertz ${ }^{6}$ 'Inst. für Perinatale Wachstumsforschung, Sievershagen, Deutschland, ${ }^{2}$ McMaster Children's Hospital, Hamilton, Kanada, ${ }^{3}$ Frauenklinik, Perinatalme- dizin, München, Deutschland, ${ }^{4}$ Klinikum Rotenburg, Geburtshilfe, Rotenburg, Deutschland, ${ }^{5}$ Max-Planck-Institut, Rostock, Deutschland, ${ }^{6}$ Klinikum Südstadt Rostock, Abteilung für Neonatologie und Neonatologische Intensivmedizin/ Kinderschlafmedizin, Rostock, Deutschland}

Zielstellung. Es werden neue geschlechtsspezifische Perzentilwerte für die Körpermaße (Geburtsgewicht, Länge, Kopfumfang, längenbezogenes Geburtsgewicht) von Einlingen vorgestellt und mit den Werten von 1995-2000 verglichen.

Material und Methode. Für die Jahre 2007-2011 standen Daten der deutschen Perinatalerhebung aus allen 16 Bundesländern mit 3.187.920 Einlingen zur Verfügung. Zugang zu den Daten von 2007-2011 wurde uns durch das AQUA-Institut in Göttingen ermöglicht. Als Vergleichspopulation dienten 1.815.318 Einlinge aus der deutschen Perinatalerhebung der Jahre 1995-200o.Die Berechnung der Perzentilwerte (3.,10.,25.,50.,75.,90.,97.)erfolgte mittels kumulativer Häufigkeiten für 21-43 vollendete Wochen. Selektionen wurden nicht vorgenommen.

Ergebnisse. Mit den großen Fallzahlen von 2007-2011 konnten die Perzentilwerte aktualisiert und präzisiert werden. Die neuen Werte der 10. Perzentile von 2007-2011 liegen beim Geburtsgewicht, bei der Länge und beim längenbezogenen Geburtsgewicht etwas höher als die Werte von 1995-2000. Beim Geburtsgewicht ergeben sich folgende Differenzen: bei den Mädchen, die mit 31 SSW geboren wurden, waren es $+55 \mathrm{~g}$ und bei 35 SSW +45 g; bei den Knaben analog +67 g bzw. 62 g. Bei 39 und 40 Schwangerschaftswochen sind die Werte in beiden Zeiträumen nahezu angeglichen. In der 50. und 9o. Perzentile zeigen die Werte ab etwa 30 Schwangerschaftswochen im Vergleich beider Zeiträume ebenfalls eine gute Übereinstimmung.

Schlussfolgerung. Die neuen gesamtdeutschen Perzentilwerte der Jahre 2007-2011 für die Körpermaße von Einlingen wurden unter Berücksichtigung aller 16 Bundesländer berechnet. Sie sollten in Zukunft zur Klassifikation des somatischen Entwicklungsstandes der Einlinge in Deutschland verwendet werden.

\section{P119 \\ Individueller Verlauf von Schädeldeformitäten extrem unreifer Frühgeborener bis zum korrigierten Alter von 6 Monaten}

\section{S. Ifflaender', M. Unrath', G. Thein', M. Rüdiger', W. Burkhardt ${ }^{1}$ 'Universitätsklinikum Carl Gustav Carus an der TU Dresden, Abteilung für Neo- natologie und Pädiatrische Intensivmedizin, Dresden, Deutschland}

Hintergrund. Schädeldeformitäten sind eng mit einer beeinträchtigten neurologischen Entwicklung von Säuglingen und Kleinkindern assoziiert. Neben anderen prä- und postnatalen Einflüssen stellt die Frühgeburtlichkeit einen erheblichen Risikofaktor für die Entwick- lung von lagebedingten Schädeldeformitäten dar. Eine hohe Zahl von Frühgeborenen weist bereits zum Zeitpunkt der Entlassung eine Schädeldeformität auf. So zeigen $38 \%$ der extrem unreifen Frühgeborenen einen moderaten bis schweren Plagiozephalus, $73 \%$ einen moderaten bis schweren Dolichozephalus. Quantitative Daten zum Verlauf von Schädeldeformitäten bei Frühgeborenen nach der stationären Betreuung sind bisher nicht verfügbar.

Fragestellung. 1.) Longitudinale Quantifizierung von Schädelproportion und -symmetrie extrem unreifer Frühgeborener bis zum korrigierten Alter von 6 Monaten; 2.) Untersuchung des prädiktiven Wertes entsprechender Indices zur Entlassung für das spätere Auftreten von Schädeldeformitäten.

Methoden. Im Rahmen einer longitudinalen Beobachtungsstudie wurden Frühgeborene $<32$ Schwangerschaftswochen (SSW), die zwischen März 2011 und Dezember 2012 in einem Level 1 Perinatalzentrum behandelt wurden, eingeschlossen. Daten aus Routineuntersuchungen mittels 3 D Laserscanner bei Entlassung, sowie im korrigierten Alter (CA) von 3 und 6 Monaten wurden ausgewertet. Etablierte Indices (Cranial Vault Asymmetry Index - CVAI, Cranial Index - CI) dienten der Quantifizierung von kranieller Proportion und Symmetrie. Anhand von Referenzwerten aus einer Normalpopulation wurden Prävalenz und Prädiktion von Schädeldeformitäten berechnet.

Ergebnisse. 56 Patienten (m 30, w 26, Geburtsgewicht 1313 \pm 398 g, Gestationsalter 29,5 $\pm 2,1 \mathrm{SSW}$ ) wurden an drei Terminen untersucht. Der CVAI war 3,9\% zur Entlassung, 4,5\% mit 3 Monaten CA und 3,7\% mit 6 Monaten CA. $34 \%$ der Kinder hatten einen moderaten bis schweren Plagiozephalus zur Entlassung, 46\% mit 3 Monaten, $27 \%$ mit 6 Monaten CA. Der CI war $71,4 \%$ zur Entlassung und fiel stetig bis 6 Monate CA. $77 \%$ der Kinder hatten bei Entlassung einen Dolichozephalus, die Prävalenz fiel auf $21 \%$ mit 6 Monaten CA. Während ein schwerer Plagiozephalus zur Entlassung prädiktiv für eine anhaltende Schädeldeformation mit 6 Monaten war (PPV o,78), entwickelten auch 46\% der Kinder ohne Plagiozephalus eine spätere Deformation.

Diskussion. Trotz einer hohen Prävalenz von lagebedingten Schädeldeformitäten zur Entlassung reduziert sich das Auftreten von Plagiozephalus und Dolichozephalus bei extrem unreifen Frühgeborenen bis zum korrigierten Alter von 6 Monaten. Dennoch sind Veränderungen von kranieller Proportion und Symmetrie für den einzelnen Patienten schlecht vorhersehbar. Eine akkurate Messung etablierter Indices um Schädeldeformitäten zu erkennen, zu behandeln und im Verlauf zu beurteilen, sollte Teil der Nachsorge von Hochrisikopatienten wie extrem unreifen Frühgeborenen sein.

Die vorliegende Arbeit wurde durch die Else-Kröner-Fresenius-Stiftung unterstützt.

\section{P120}

Vorstellung neuer gesamtdeutscher Perzentilwerte für die Körpermaße von Zwillingen

\section{Voigt' ${ }^{1}$ N. Rochow ${ }^{2}$, K. Schneider ${ }^{3}$, H. Hagenah ${ }^{4}$, R. Scholz 5 , D. Olbertz ${ }^{6}$} ${ }^{1}$ Inst. für Perinatale Wachstumsforschung, Sievershagen, Deutschland, ${ }^{2}$ McMaster University, Division of Neonatology, Department of Pediatrics, Hamilton, Ontario, Kanada, ${ }^{3}$ Frauenklinik, Perinatalmedizin, München, Deutschland, ${ }^{4} \mathrm{Kli}-$ nikum Rotenburg, Geburtshilfe, Rotenburg, Deutschland, ${ }^{5}$ Max-Planck-Institut, Rostock, Deutschland, ${ }^{6}$ Klinikum Südstadt Rostock, Abteilung für Neonatologie und Neonatologische Intensivmedizin/Kinderschlafmedizin, Rostock, Deutschland

Zielstellung. Es werden neue geschlechtsspezifische Perzentilwerte für die Körpermaße (Geburtsgewicht, Länge, Kopfumfang, längenbezogenes Geburtsgewicht) von Zwillingen vorgestellt und mit den Werten von 1990-1994 verglichen.

Material und Methode. Für die Jahre 2007-2011 standen Daten der deutschen Perinatalerhebung aus allen 16 Bundesländern mit 110.313 Zwillingen zur Verfügung. Die Daten erhielten wir vom AQUA-Institut in 
Göttingen. Als Vergleichspopulation dienten 81.986 Zwillinge der Jahre 1990-1994. Diese Daten der Perinatalerhebung wurden uns damals durch die Landesärztekammern zur Verfügung gestellt. Die Berechnung der Perzentilwerte (3., 10., 25., 50., 75., 90., 97.) erfolgte mittels kumulativer Häufigkeiten für 21-40 vollendete Schwangerschaftswochen. Selektionen wurden nicht vorgenommen.

Ergebnisse. Die neuen Werte der 10.Perzentile von 2007-2011 liegen beim Geburtsgewicht, bei der Länge und beim längenbezogenen Geburtsgewicht etwas höher als die Werte von 1995-200o. Beim Geburtsgewicht zeigen sich für die Mädchen folgende Differenzen: bei ${ }_{31}$ SSW +50 g, bei 35 SSW +30 g und bei 40 SSW +80 g. Bei den Knaben betragen die korrespondierenden Werte $+30 \mathrm{~g},+40 \mathrm{~g}$ und $+90 \mathrm{~g}$. Abweichungen existieren auch bei den anderen Körpermaßen im Vergleich beider Zeiträume, nach oben bzw. nach unten. Die Längenperzentilwerte von 2007-2011 wurden mit einer Kommastelle berechnet und verlaufen damit harmonischer als die Kurven von 1990-1994, bei denen die Länge in vollen Zentimeterangaben ausgewiesen wurde.

Schlussfolgerung. Die neuen gesamtdeutschen Perzentilwerte der Jahre 2007-2011 für die Körpermaße von Zwillingen wurden unter Berücksichtigung aller 16 Bundesländer berechnet. Sie sollten in Zukunft zur Klassifikation des somatischen Entwicklungsstandes der Zwillinge in Deutschland verwendet werden.

\section{Neurologie 2}

\section{P121}

\section{Erythropoietin schützt die weiße Substanz vor sauerstoffbedingter} Toxizität im neonatalen Rattenmodell

\section{Brait', I. Bendix', M. Serdar', M. Dzietko', K. Straßer', J. Fandrey', U. Fel- derhoff-Müser \\ 'Universitätsklinikum Essen, Klinik für Kinderheilkunde I, Neonatologie, Essen, Deutschland, ${ }^{2}$ Universitätsklinikum Essen, Institut für Physiologie, Essen, Deutschland}

Hintergrund und Fragestellung. Aufgrund der Anfälligkeit des unreifen Gehirns gegenüber schädlichen Einflüssen haben frühgeborene Kinder ein erhöhtes Risiko für die Entwicklung neurologischer und kognitiver Langzeitschäden. Innerhalb der letzten Jahre konnte durch tierexperimentelle Studien gezeigt werden, dass erhöhte Sauerstoffkonzentrationen (Hyperoxie) zur neuronalen Degeneration und somit zur Pathogenese der neonatalen Hirnschädigung beitragen. Erythropoietin (EPO), ein essentieller Wachstums- und Überlebensfaktor für ErythrozytenVorläuferzellen, findet in seiner rekombinanten Form (rEPO) schon seit geraumer Zeit Anwendung in der Behandlung der Früh- und Neugeborenen-Anämie. Es ist darüber hinaus bekannt, dass EPO neuroprotektive Eigenschaften in unterschiedlichen Modellen der experimentellen Hirnschädigung besitzt. Das Ziel der Studie ist die Untersuchung der Effekte von EPO im exprimentellen Modell der Hyperoxie-induzierten Schädigung der neugeborenen Ratte im Hinblick auf die weiße Subs$\operatorname{tanz}$ sowie die kognitive und motorische Langzeitentwicklung.

Material und Methoden. Wistar-Ratten erhielten an Lebenstag 5 eine intraperitoneale Injektion mit 20.00o I.E. rEPO oder isotoner Kochsalzlösung und wurden anschließend einer 24-stündigen Hyperoxie bei $80 \%$ Sauerstoff ausgesetzt. Kontrolltiere wurden in dieser Zeit bei normaler Raumluft gehalten. Im Anschluss erfolgte eine Aufarbeitung der Gehirne mittels Proteinanalysen und Immunhistochemie bezüglich verschiedener Oligodendrozyten-Marker (Olig-2, MBP). Des Weiteren erfolgte ab einem Alter von 30 Tagen die Evaluation der kognitiven und motorischen Langzeitentwicklung mittels adäquater Verhaltenstests (Open Field, Novel Object Recognition).

Ergebnisse. Sowohl in molekularbiologischen und histologischen Untersuchungen der weißen Substanz als auch in den durchgeführten Verhaltenstests ergeben sich Hinweise auf die protektive Wirkung von
rEPO im Rahmen der Hyperoxie-vermittelten Schädigung des unreifen Gehirns.

Diskussion und Schlussfolgerung. Unsere Untersuchungen zeigen erste Ergebnisse, die eine protektive Wirkung von rEPO bezüglich der weißen Substanz vermuten lassen und seine Bedeutung in klinischen Untersuchungen zur Neuroprotektion bei Frühgeborenen unterstützt.

\section{P122}

Visuelle Aufmerksamkeit VLBW-Frühgeborener in den ersten Lebenswochen - eine Grundlage früher Eltern-Kind-Interaktion

\section{P. Hinner', J. Reichert ${ }^{1}$, M. Rüdiger ${ }^{7}$}

'Universitätsklinikum Carl Gustav Carus Kinderklinik, Neonatologie/Pädiatrische Intensivmedizin, Dresden, Deutschland

Fragestellung. Ein wesentlicher Schritt in der Entwicklung der ElternKind-Interaktion ist das Herstellen gegenseitiger Aufmerksamkeit (shared attention; Pauen \& Rauh, 2007). Dies bedarf seitens der Bezugsperson intuitiver Kompetenzen und feinfühligen Verhaltens und seitens des Säuglings unter anderem die Fähigkeit zur sozialen Aufmerksamkeit. Diese Fähigkeit lässt sich bei reifgeborenen Kindern an verschiedenen Verhaltensmarkern wie z. B. Kopf- und Blickbewegung in Richtung der Bezugsperson ablesen. $\mathrm{Zu}$ früh geborene Kinder werden dieser Entwicklungsaufgabe unter Bedingungen physiologischer Unreife und eingeschränkter Regulationsfähigkeit ausgesetzt. So ist die Frage zu stellen, ob auch Frühgeborene bereits Verhaltenssignale einer sozialen Aufmerksamkeit vor dem errechneten Geburtstermin zeigen und inwieweit diese Signale an elterliche Interaktionskompetenzen gekoppelt sind.

Material und Methode. Von 49 Mütter wurden Videoaufnahmen mit ihren frühgeborenen Kindern $<1500 \mathrm{~g}$ in einer definierten Versorgungssituation erstellt und mittels eines Experten-Fremdratings analysiert. Das Lebensalter zum Untersuchungszeitpunkt lag für alle Kinder unter der 40. SSW. Die deskriptiven Auswertungen der 2-minütigen Videoaufnahmen beziehen sich auf Verhaltensmerkmale des Kindes (Augenund Kopfbewegungen), der Eltern (Sehabstand und Ansprache) sowie den dyadischen Blickkontakt (visuelle Aufmerksamkeit) als Merkmale gegenseitiger sozialer Aufmerksamkeit. Ausschlusskriterien waren infauste Prognose des Frühgeborenen, Psychopathologie und sprachliche Einschränkungen der Eltern.

Ergebnisse. Zwei Drittel der Kinder zeigten wiederholt visuelle Aufmerksamkeit durch geöffnete Augen; bei mehr als einem Drittel war gleichzeitig eine Blickhinwendung oder eine Kopfbewegung zur Mutter zu beobachten. Unter angemessenen Sehabstand der Mutter hatten knapp ein Drittel der ausgewerteten Eltern-Kind-Dyaden Blickkontakt; bei 1/10 trat dieser wiederholt und über mehrere Sekunden auf. War der Sehabstand zu groß und/oder keine Ansprache durch die Mutter gegeben, fand kein Blickkontakt statt.

Diskussion. Ein Großteil der Frühgeborenen zeigt bereits im Lebensalter unter der 40. SSW Signale für Wachheit und Aufmerksamkeit. Für ein Drittel der Dyaden lassen sich erste Anzeichen einer gegenseitigen Aufmerksamkeit beobachten, die mit elterlichen Kompetenzen wie Ansprache und Sehabstand verbunden sind.

Schlussfolgerung. Die visuelle Aufmerksamkeit stellt auch für ein frühgeborenes Kind die Grundlage für die Face-to-face-Interaktion mit der Bezugsperson dar. Sind zeitgleich entsprechende elterliche Kompetenzen vorhanden, können Momente gegenseitiger (sozialer) Aufmerksamkeit entstehen. Folgende Untersuchungen innerhalb der Studie konzentrieren sich deshalb auf den Einfluss früher Interventionen auf die Befähigung der Eltern zur Förderung der Aufmerksamkeit des Kindes in sozialer Interaktion.

1. Pauen S, Rauh H (2007) Frühe Kindheit. In Hasselhorn M, Silbereisen R (Hrsg) Enzyklopädie der Psychologie (Band 4). Entwicklungspsychologie des Säuglings- und Kleinkindalters. Hogrefe, Göttingen 


\section{P123}

\section{Deutsches Hypothermienetzwerk Hypothermiebehandlung bei neonatalen und pädiatrischen Patienten}

\section{K. Brommer', S. Brenner', A. Koch', J. Dinger', M. Rüdiger ${ }^{1}$ \\ 'Universitätskinderklinik C.G. Carus Dresden, Fachbereich Neonatologie und Pädiatrische Intensivmedizin, Dresden, Deutschland}

Einleitung. Die therapeutische Hypothermie ist bei Neugeborenen mit perinataler Asphyxie und Enzephalopathie heute eine Routinebehandlung (klassische Indikation). Bei fehlendem Nachweis einer Wirksamkeit wird dieses Vorgehen für Säuglinge oder Kinder mit akuter hypoxischer ZNS-Schädigung noch nicht empfohlen, allerdings im Rahmen individueller therapeutischer Heilversuche gelegentlich angewendet. Fragestellung. Wie groß ist die Zahl pädiatrischer Patienten mit Hypothermiebehandlung? Unterscheiden sich die Kurzzeitergebnisse von neonatalen und pädiatrischen Patienten?

Methodik. Das deutsche Hypothermienetzwerk erfasst in den beteiligten Einrichtungen Daten von Patienten mit therapeutischer Hypothermie. Zur Auswertung kommen die kompletten Daten für die Jahre 2011-2013. Es wird unterschieden zwischen „neonatalen“ (klassische Indikation bei perinataler Asphyxie und Enzephalopathie) und „pädiatrischen" Patienten (Kinder jeden Alters mit Kühlung außerhalb der klassischen Indikation).

Ergebnisse. Am Hypothermienetzwerk beteiligen sich mittlerweile 102 Einrichtungen. Im Untersuchungszeitraum wurden insgesamt 612 neonatologische sowie 123 pädiatrische Patienten gekühlt (im Mittel 6,8 Patienten pro Zentrum/Jahr). Die neonatologischen Patienten wurden in 50\% der Fälle außerhalb des Kühlungszentrums geboren, das hypoxische Ereignis bei den pädiatrischen Patienten trat in $74 \%$ außerhalb der Klinik ein. Während $88 \%$ der neonatalen Patienten überlebten, waren es nur $57 \%$ der pädiatrischen Patienten. Von den Überlebenden wurde die Mehrheit der Neonaten nach Hause entlassen ( $76 \%$ ), wohingegen es bei den pädiatrischen Patienten nur 33\% waren. Zum Zeitpunkt der Entlassung waren $87 \%$ der neonatalen Patienten komplett oral ernährt, bei den pädiatrischen Patienten nur $36 \%$ - ein indirekter Hinweis für das schlechtere neurologische Outcome bei letzteren.

Diskussion. Trotz fehlendem Nachweis einer Wirksamkeit erfolgt auch außerhalb der klassischen Indikation eine Kühlungstherapie bei pädiatrischen Patienten. Insgesamt scheint die Effektivität dieser Therapie aber nicht so gut zu sein wie bei neonatalen Patienten. Vor einer allgemeinen Etablierung der Hypothermie bei pädiatrischen Patienten ist auf die Ergebnisse der derzeit laufenden großen klinischen Studien zu warten.

\section{(Weitere Details unter www.hypothermienetzwerk.de)}

\section{P124}

Erfolgreiche therapeutische Hypothermie bei Krampfanfall assoziierter neonataler ischämischer Enzephalopathie

\section{Schmidt ', H. Bahlmann', N. Utzig' ${ }^{2}$, S. Langner ${ }^{3}$, M. Heckmann ${ }^{7}$ \\ 'Klinik und Poliklinik für Kinder und Jugendmedizin, Abt. Neonatologie u. Päd. Intensivmedizin, Greifswald, Deutschland, ${ }^{2}$ Klinik und Poliklinik für Kinder und Jugendmedizin, Abt. für Neuropädiatrie und Stoffwechselerkrankungen, Greifswald, Deutschland, ${ }^{3}$ Institut für diagnostische Radiologie und Neurora- diologie, Greifswald, Deutschland}

Einleitung. Die Hypothermiebehandlung (HT) nach perinataler Asphyxie ist ein anerkanntes Verfahren mögliche neurologische Schäden zu mindern. Nach Herz-Kreislauf-Wiederbelebung wird die HT von den Fachgesellschaften ebenfalls empfohlen. Studienergebnisse zum Einsatz der HT bei Schädelhirntrauma und zerebralen Infarkten sind bisher widersprüchlich. Zum Einsatz bei Krampfanfall-assoziierter Enzephalopathie liegen bisher beim Neonaten keine Fallberichte vor. Kasuistik. Ein 7 Tage altes, männliches Neugeborenes wurde nach mindestens 12 Stunden andauerndem prolongierten Krampfanfall aufge- nommen. Am Vortag zeigte es Faszikulationen in den Unterarmen, die als Einschlafmyoklonien gewertet wurden. Die Intensität und Frequenz der Zuckungen nahm in der Nacht zu und wurde mindestens 10-mal von den Eltern beobachtet. Nach Schwangerschaft mit diätetisch eingestelltem GDM war die postnatale Adaptation des eutrophen Reifgeborenen unauffällig (Apgar 9/10/10, art NS-pH 7,34). Die Familienanamnese auf zerebrale Krampfanfälle oder Stoffwechseldefekte ist leer. Das EEG war hochpathologisch mit einer Theta-Grundaktivität um 5/s, Suppressionsstrecken bis 8 Sekunden Dauer, multifokalen „sharp waves“ und dem Auftreten eines über eine Minute andauernden Krampfanfalls über der linken Hemisphäre mit generalisierten Sharp-wave-Serien um 2-3/s. Nach unzureichender klinischer und enzephalographischer Besserung unter Phenobarbital (kumulative Dosis $30 \mathrm{mg} / \mathrm{kgKG} / \mathrm{Tag}$ ) wurden Midazolam $(2,4 \mu \mathrm{g} / \mathrm{kgKG} / \mathrm{min}$ DTI) und Phenytoin (maximal $10 \mathrm{mg} / \mathrm{kgKG} /$ Tag) eingesetzt. Im cMRT zeigte die Diffusionsbildgebung multiple ausgedehnte frische ischämische Areale in den beiden Temporalpolen sowie temporomesial, frontal und im parietalen Marklager. Periventrikulär fanden sich petechiale Einblutungen. Das Akutlabor, die Liquoranalyse, die Stoffwechsel- (Aminosäuren im Liquor und Plasma, organische Säuren, TBK, $\mathrm{NH}_{3}$, Laktat) und infektiologische (TORCH, Parvovirus B19, Entero-, Adenoviren. Blutkultur) Diagnostik waren unauffällig. Aufgrund der ausgeprägten im MRT nachgewiesen frischen Ischämien nach prolongiertem Krampfanfall wurde eine HT für 72 Stunden mit $32-33^{\circ} \mathrm{C}$ durchgeführt. Unter dieser Therapie sistierten die klinischen Krampfanfälle nach 12 Stunden und das EEG normalisierte sich innerhalb von 20 Tagen vollständig. Eine cMRT mit 6 Monaten war unauffällig. Die Phenobarbitaltherapie wurde bis zum 7. Lebensmonat fortgeführt, Krampfanfälle traten nicht mehr auf und der neuropädiatrische Status war im Folgenden bis zum Alter von 17 Monaten stets altersgerecht.

Schlussfolgerung. Die therapeutische Hypothermie kann bei Krampfanfall-assoziierter neonataler ischämischer Enzephalopathie möglicherweise das neurologische Outcome verbessern.

\section{P125}

Korrelation zur peripheren arteriellen Sättigung und Vergleich der Werte der zerebralen Oxygenierung von 4 Nahinfrarotspektroskopie Geräten beim Frühgeborenen

A. Schneider', B. Minnich'², E. Hofstätter', C. Weisser', J. Brandner', M. Wald'

'Universitätsklinikum Salzburg, Neonatologie - Univ. Klinik für Kinder- und Jugendheilkunde, Salzburg, Österreich, ${ }^{2}$ Naturwissenschaftliche Fakultät Salzburg, Abteilung für Zellbiologie, Salzburg, Österreich

Hintergrund. Die Nahinfrarotspektroskopie (NIRS) ermöglicht es, die lokal vorherrschende Gewebesättigung ( $\mathrm{rSO}_{2}$ ) non- invasiv zu messen. Die vorliegende Studie vergleicht die Messwerte von 4 gängigen NIRS Geräten (NIRO 200, INVOS 5100c, Fore-Sight und SenSmart X-100) und untersucht die Korrelation zur peripher arteriellen Sauerstoffsättigung. Material und Methoden. Um jede mögliche Gerätepaarung zu testen, wurden insgesamt 6o Messungen an 10 Frühgeborenen durchgeführt. Hierfür wurde jeweils ein Sensor von je einem Gerät frontoparietal am Kopf des Kindes angebracht und insgesamt pro Gerät 3600 Messminuten gesammelt. Die Korrelation jedes einzelnen Gerätes zur peripheren arteriellen Sättigung, die mittels Pulsoxymeter routinemäßig gemessen wird, wurde berechnet. Um zu prüfen ob die zerebrale NIRS Messung früher auf Entsättigungen reagiert als die periphere $\mathrm{SpO}_{2}-\mathrm{Messung}$, wurden die NIRS-Werte einerseits mit den simultan gemessenen $\mathrm{SpO}_{2}$ Werten korreliert, andererseits mit $\mathrm{SpO}_{2}$-Werten die 1 Minute, 2,3,4 und 5 Minuten später gemessen wurden. Alle Rohdaten der Messungen eines Gerätes wurden mit denen der anderen 3 Geräte verglichen.

Ergebnisse. Der Korrelationskoeffizient zwischen $\mathrm{rSO}_{2}$ und $\mathrm{SpO}_{2}$, bei Verwendung der simultan gemessenen Werte variiert zwischen 0,0277 (NIRO20o) und o,351 (Fore-Sight). Bei Verwendung der $\mathrm{rSO}_{2}$ und den Werten der $\mathrm{SpO}_{2}$, die 2 Minuten später gemessen wurden entspricht der Korrelationskoeffizient 0,0614 (NIRO20o) und 0,483 (Fore-Sight). Die Messdifferenzen zwischen den Werten der einzelnen Geräte variieren zwischen $3,23 \%$ und $14,41 \%$. 
Diskussion. Bei Auswertung der Ergebnisse wurde ersichtlich, dass die Korrelation zwischen zerebralen und peripheren Werten am höchsten ist, wenn man einen $\mathrm{rSO} 2$-Wert nimmt und diesen mit dem 2 Minuten später gemessenen $\mathrm{SpO}_{2}$-Wert korreliert, was bedeutet, dass der periphere Wert sich erst zwei Minuten später an den zerebral gemessenen Wert annähert und man somit daraus schließen kann, dass die zerebrale Gewebeoxygenierung früher auf Entsättigungen reagiert bzw. die Werte der peripheren Sättigung der zerebralen nachhinken. Warum das so ist kann man sich folgendermaßen erklären: Wenn der frei gelöste Sauerstoff weniger wird, greift der Organismus auf den an Hämoglobin gebundenen Sauerstoff bzw. auf den venösen Pool zurück. Da die NIRS-Messung 70:20 (bzw. 75:25 bei INVOS) venös-arteriell gewichtet ist, fällt diese somit schneller $\mathrm{ab}$ als die rein arterielle periphere Sättigung.

Schlussfolgerung. In der Studie konnte die frühere Reaktion auf Entsättigungen der zerebralen NIRS Messungen, als ein weiterer Vorteil der Messmethode dargestellt werden. Ein Problem bleibt aber weiterhin, dass eine routinemäßige und therapieentscheidende Verwendung der Werte der zerebralen NIRS Messung, aufgrund der großen Unterschiede in den Messwerten der verschiedenen Geräte und der hohen Artefaktanfälligkeit nicht möglich ist.

\section{P126}

\section{Hydrocephalus bei Achondroplasia - Pathogenese und neurochir-} urgische Interventionsmöglichkeiten an Hand eines Fallberichtes

\section{Haftel', S. Asgari', F. Müller ${ }^{3}$}

'Kindersarzthaus St.Gallen, St.Gallen, Schweiz, ${ }^{2}$ Klinikum Ingolstadt, Neurochirurgie, Ingolstadt, Deutschland, ${ }^{3}$ Kinderklinik St. Elisabeth, Neuburg/Donau, Deutschland

Hintergrund. Die Achondroplaia ist die häufigste Form eine Knochendysplasie. Die Ausbildung eines Hydrocephalus wird bei den Patienten zwar sehr häufig beobachtet, die Notwendigkeit eine neurochirurgische Intervention ist aber selten indiziert. Nur bei entsprechender Symptomatik wie zum Beispiel Apnöen, Ataxie oder Kompression des Stammhirns ist so eine Intervention notwendig. Das Risiko der Ausbildung eines Hydrocephalus besteht prinzipiell lebenslang wird jedoch vor allem in den ersten zwei Lebensjahren beobachtet. Bezüglich der Genese existieren verschiedene Theorien: exzessives Wachstum der Schädelkalotte in Sinne eines Kompensationsversuches bei kleiner Schädelbasis, Obstruktionen der Cisterna magna und der Foramina Luschkae und Magendie aufgrund Distortionen des Hirnstammes bei häufig zu engem Foramen magnum, Stase des venösen Rückstroms und erhöhen des intrakraniellen venösen Druck.

Fallbericht. Nach unauffälligem SS-Verlauf, wurde auf Grund Makrozephalie, Ventrikulomegalie und pathologischer Hüfte-Sonographie der Verdacht auf Achondroplasie gestellt. Es wurden zwei De-novoMutationen im FGFR3-Gen nachgewiesen werden. Diese Mutationen sind mit der Symptomatik einer Hypochondroplasie, Achondroplasie Verlaufsformen verknüpft. Im Verlauf wurden vermehrte Apnöe-Ereignisse beobachtet und die MRT Untersuchung zeigte eine ausgeprägte

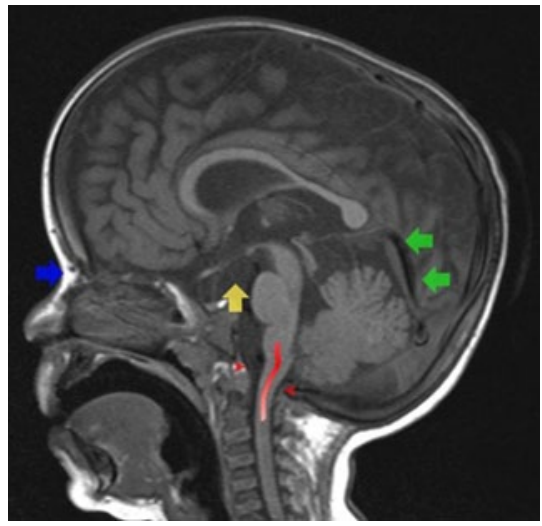

$\varangle P_{126}-$ Abb.
Enge des Formanen magnum ohne venöse Stase. Nach subokzipitaler Dekompression zeigte sich zunächst eine leichte Besserung der klinischen Symptome, bei jedoch ausgeprägtem triventrikulärem Hydrocephalus erfolgte eine endoskopische Ventrikulostomie am Boden des 3. Ventrikels. Die erfolgten bildgebenden Verfahren zeigte eine deutliche radiologische Verbesserung, und der kleine Patient konnte ohne klinische Apnoen nach Hause entlassen.

Schlussfolgerung. Hydrocephalus bei Achondroplasia ist ein komplexes Krankheitsbild, die eine sorgfältige Evaluation benötigt, um die genau Ursache zu diagnostizieren und behandeln. Die übliche ventrikuloperitoneale Shunt-Behandlung des Hydrocephalus ist bei vielen dieser Fälle nicht nötig Abb. 1).

1. Menenez AH (2009) Achondroplasia. J Neurosurg Pediatrics 4:295-296

2. Etus V, Ceylan S (2005) The role of endoscopic third ventriculostomy in the treatment of triventricular hydrocephalus seen in children aiwth achondroplasia. J Neurosurg Pediatrics 3:260-265

\section{P127}

Schwere intrauterine Asphyxie durch spontanes Nabelschnurhämatom mit Mazeration des Feten und schwerer multizystischer Enzephalomalazie des Neugeborenen

\section{N. Lorenz', A. Nordwig' ${ }^{2}$ S. Schmidt ${ }^{3}$, W. Möhr', D. Golde ${ }^{4}$, O. Holotiuk ${ }^{5}$, K.} Friedrich $^{6}$, G. Heubner ${ }^{3}$

'Städtisches Krankenhaus, Zentrum für Kinder- und Jugendmedizin, Dresden, Deutschland, ${ }^{2}$ Städtisches Krankenhaus Dresden-Neustadt, Klinik für Kinderund Jugendmedizin, Dresden, Deutschland, ${ }^{3}$ Städtisches Krankenhaus, Kinderklinik, Dresden, Deutschland, ${ }^{4}$ Diakonissen-Krankenhaus, Frauenklinik, Dresden, Deutschland, ${ }^{5}$ Praxis für Pathologie am, Städtischen KH Dresden Neustadt, Dresden, Deutschland, ${ }^{6}$ Institut für Pathologie, TU Dresden, Medizinische Fakultät Carl Gustav Carus, Dresden, Deutschland

Einleitung. Ein spontanes intrauterines Hämatom der Nabelschnur ist nach einer Fallserie aus John Hopkins von DIPPEL (1) ein eher seltenes (1:5500), für den Feten dramatisches Ereignis, das durch massive Beeinträchtigung der Nabelschnurzirkulation zu schwerem fetalem Distress und in $50 \%$ zum Absterben des Feten führt (1). Typischerweise rupturiert die Nabelvene. Risikofaktoren sind Chorioamnitis, Aneurysmata der Nabelschnurgefäße sowie eine kurze Nabelschnur.

Fallbericht. 1. Kind einer 1.-Gravida, Eltern konsanguin, Gestationsalter $40+5$ SSW, primäre Sectio in auswärtiger Einrichtung bei pathologischem CTG und verminderten Kindsbewegungen. APGAR: 3/8/8, NabelarterienpH: 7,25. Geburtsgewicht: 4220 g, Oligohydramnion Fruchtwasser: klar. Keine Feindiagnostik. Postnatum mazeriertes, deprimiertes NG, suffiziente Eigenatmung ab 5 min, Nabelschnurrest mazeriert mit Hämatom. Im Verlauf respiratorische Insuffizienz mit CPAP-Bedarf. Kein Hinweis für Gerinnungsfaktorenmangel, Schädelsonographie am 1. LT: Hirnödem Stadium 2, subkortikal Verdacht auf Infarzierungen und einlaufende multizystische Enzephalomalazie, aEEG: Burst Supression. NSE und S1oo massiv erhöht: 105,3 $\mu \mathrm{g} / \mathrm{l}$; bzw. 14,9 $\mu \mathrm{g} / \mathrm{l}$. Histologie: Regressive Veränderungen der Plazenta, Chorioamnitis. 3 Nabelschnurgefäße, Nabelschnurbindegewebe mit massiver Hämorrhagie mit Ödem und Zeichen der Mazeration. Keine nennenswerten entzündlichen Veränderungen. Antiepileptische Therapie, Frührehabilitation, schweres neurologisches Defizit.

Diskussion. Bei jährlich etwa 680.00o Neugeborenen in Deutschland sollten ca. 70 Fälle/Jahr von überlebenden Neugeborenen mit einem spontanen Nabelschnurhämatom auftreten. Obwohl DIPPEL eine Prävalenzrate von 1:5500 angibt(1), verwundert, dass es sich bei diesem Fall, unseres Wissens nach, um den ersten publizierten deutschen Fall handelt. Eine Querschnittsuntersuchung könnte geeignet sein, die tatsächliche aktuelle Prävalenz zu ermitteln und epidemiologische Daten zu gewinnen, die möglicherweise geeignet sind, die ernste Prognose dieser Feten und Neugeborenen zu verbessern.

1. Dippel AL (1940) Hematomas of the umbilical cord. Surg Gynecol Obstet 70:51-57 


\section{P128}

Auswirkungen von standardisierter akustischer Stimulation bei Frühgeborenen: eine randomisierte kontrollierte Studie

L. Wirth', F. Dorn', M. Wege', B. Lemmer' ${ }^{2}$, S. Gorbey' ${ }^{2}$, N. Timmesfeld ${ }^{3}$, M. Zemlin', R. Maier ${ }^{2}$

'Klinik für Kinder- und Jugendmedizin der Philipps-Universität Marburg, Marburg, Deutschland, ${ }^{4}$ Institut für Exp. \& Klin. Pharmakologie \& Toxikologie Mannheim der Universität Heidelberg, Mannheim, Deutschland, ${ }^{3}$ Institut für Medizinische Biometrie und Epidemiologie der Philipps-Universität Marburg, Marburg, Deutschland

Hintergrund. Anders als intrauterin sind Frühgeborene im stationären Umfeld zahlreichen lauten, unregelmäßigen und ungezielten akustischen Stimuli ausgesetzt. Eine angemessene sensorische Stimulierung wie im Mutterleib scheint aber für eine normale neurophysiologische Entwicklung notwendig zu sein.

Fragestellung. Wie wirkt sich eine standardisierte akustische Stimulation auf die Herzfrequenz, die Atemfrequenz und die Aktivität bei Frühgeborenen aus?

Methodik. 62 Frühgeborene mit einem Gestationsalter von $30<37$ Wochen und einem postnatalen Alter $<10$ Tagen wurden mittels Randomisierung auf drei Gruppen verteilt. In zwei Gruppen erfolgte 14 Tage lang täglich zwischen 20 und $21 \mathrm{Uhr}$ eine standardisierte akustische Stimulation von jeweils 30 Minuten Dauer. Die Stimulation erfolgte entweder mit Wiegenliedern (Gruppe I) oder mit gesprochener Mutterstimme (Gruppe II) jeweils von einem CD-Player über Passivlautsprecher mit einer Lautstärke von 55 bis $65 \mathrm{~dB}(\mathrm{~A})$. Die dritte Gruppe erhielt keine standardisierte Stimulation und diente als Kontrollgruppe. Herz- und Atemfrequenz wurden jeweils 15 Minuten vor, 30 Minuten während und 15 Minuten nach Intervention minütlich erfasst. Zusätzlich wurde an den Studientagen 1, 7 und 14 jeweils vor, während und nach der Stimulation die Aktivität mittels Aktometer gemessen.

Ergebnisse. Im Vergleich zur Kontrollgruppe reduzierten Wiegenlieder die Herzfrequenz während bzw. 15 Minuten nach Stimulation um (95\% Konfidenzintervall) 6,23-3,32 bzw. 10,72-6,95 Schläge/Minute und die Mutterstimme um 6,5-3,24 bzw. 10,41-6,72 Schläge/Minute (jeweils $\mathrm{p}<0,01$ im Vergleich zur Kontrollgruppe). Auch die Atemfrequenz sank während bzw. 15 Minuten nach Stimulation bei Wiegenliedern um $(95 \%$ Konfidenzintervall) 5,51-1,61 bzw. 5,90-2,09 Atemzüge/Minute und bei Mutterstimme um 4,44-1,29 bzw. 4,82-1,15 Atemzüge/Minute (jeweils $\mathrm{p}<0,01$ im Vergleich zur Kontrollgruppe). Zwischen den beiden Formen der akustischen Stimulation zeigte sich kein signifikanter Unterschied. Ein „Gewöhnungseffekt“ im Laufe des 14-tägigen Studienzeitraumes wurde bei keiner der beiden Interventionen beobachtet. Frühgeborene mit einem höheren Gestationsalter reagierten mit einer deutlicheren Senkung der Herzfrequenz als unreifere Frühgeborene $(p<0,05)$. Die Aktivität der Frühgeborenen war während Stimulation reduziert (Wiegenlieder vs. Kontrollgruppe: $\mathrm{p}=0,04$; Mutterstimme vs. Kontrollgruppe: $p<0,01)$, wobei die Mutterstimme einen stärkeren Effekt zeigte (Mutterstimme vs. Wiegenlieder: $\mathrm{p}=0,04$ ).

Schlussfolgerung. Eine regelmäßige standardisierte akustische Stimulation wirkte sich in unserer Studie beruhigend auf die Frühgeborenen aus.

\section{P129}

Methylreiche Kost in der Schwangerschaft führt im Mausmodell beim Nachwuchs zu dauerhaft erhöhter DNA-Gesamtmethylierung und verändertem Genexpressionsmuster im Hypothalamus

\section{B. Brune', R. Linke², S. Jakubiczka³, M. Brunner-Weinzierl'4, T. Brune \\ 'Universitätskinderklinik, Magdeburg, Deutschland, ${ }^{2}$ Universitätsklinikum, Ins- titut für Anatomie, Magdeburg, Deutschland, ${ }^{3}$ Universitätsklinikum, Institut für Humangenetik, Magdeburg, Deutschland, ${ }^{4}$ Universitätsklinikum, Kinderklinik, Magdeburg, Deutschland, ${ }^{5}$ Kinderklinik Klinikum Lippe, Detmold, Deutschland}

Fragestellung. Verändert eine methylreiche Ernährung während der Schwangerschaft dauerhaft das Epigenom der Nachkommen?

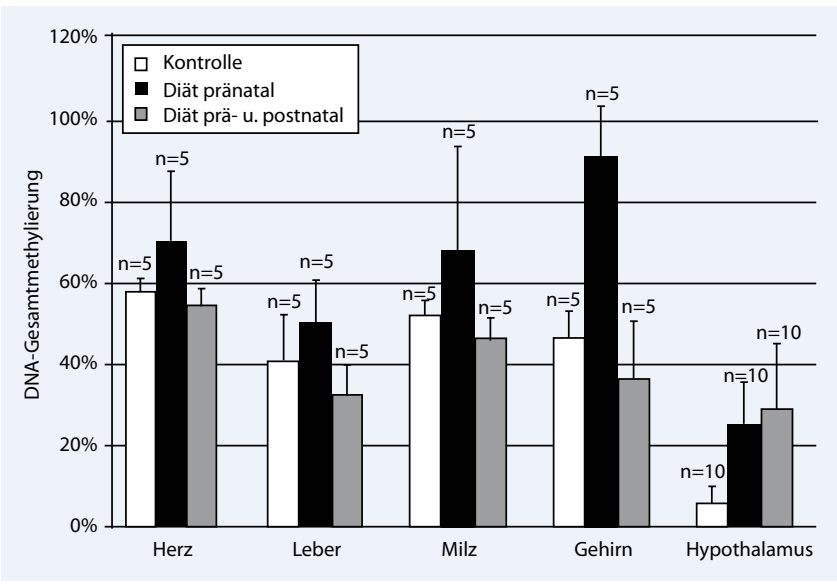

$\Delta \mathrm{P} 129-\mathrm{Abb} .1$

\section{P129 - Tab. 1}

Gen

Expressionsveränderung gegenüber Kontrolle

Lysin(K)-spezifische Demethylase

$-10 x$

Serine bzw. Zystein Peptidase Inhibitor

$+7,3 x$

N-Acetyltransferase 3

$+4,3 x$

Folsäurerezeptor alpha

$+3,8 x$

Material und Methoden. Weibliche Mäuse (C57/BL6) wurden während der Schwangerschaft mit einer methylreichen Diät gefüttert. Die Nachkommen wurden in 2 Gruppen eingeteilt: postnatal erhielt die eine Gruppe ebenfalls eine methylreiche Diät, die andere Normalfutter. Als Kontrolle dienten schwangere Mäuse, die ausschließlich normales Futter erhielten. Bei den Nachkommen wurde im Alter von 3 Monaten die DNA von Herz, Leber, Milz, Gehirn und Hypothalamus isoliert und ihr jeweiliger Gesamtmethylierungsgrad bestimmt. Im Hypothalamusgewebe wurden zusätzlich die Genexpressionsmuster zwischen Verum und Kontrolle verglichen.

Ergebnisse. Der DNA-Gesamtmethylierungsgrad der einzelnen Organe ist in der folgenden Grafik dargestellt. Eine methylreiche Diät während der Schwangerschaft führt zu einer signifikant höheren Gesamtmethylierung in den untersuchten Organen. Ein Weiterführen der Diät postnatal senkt in allen untersuchten Organen mit Ausnahme des Hypothalamusgewebes die Werte wieder auf das Ausgangsniveau (Abb. 1). Im Hypothalamus der Diätgruppe war die Expression von insgesamt 19 Gene mindestens 3-fach und signifikant gegenüber der Kontrollgruppe verändert. Die Gene, die in epigentische Prozesse eingreifen, sind in Tab. 1 aufgeführt.

Schlussfolgerung. Eine methylreiche Diät während der Schwangerschaft führt im Vergleich zu normaler Ernährung bei den Nachkommen zu einer dauerhaft höheren DNA-Gesamtmethylierung. Dieser Effekt ist besonders stark ausgeprägt im Hypothalamus und führt hier zu einer dauerhaften Über- bzw. Unterexpression von Genen, die das Epigenom beeinflussen. Diese Ergebnisse lassen darauf schließen, dass diätetische Einflüsse während der Schwangerschaft das Epigenom der Nachkommen insbesondere im Hypothalamus dauerhaft verändern können. 


\section{Hämodynamik}

\section{P130}

Ligatur des persistierenden Ductus arteriosus bei Frühgeborenen vor der 30. Schwangerschaftswoche - eine retrospektive 4-JahresAnalyse der Abteilung für Neonatologie der Medizinischen Universität Wien

\section{S. Baumgartner', U. Salzer-Muhar ${ }^{2}$, M. Steiner ${ }^{1}$, M. Wald ${ }^{3}$, G. Fischer', M. Olischar', A. Berger}

'Universitätsklinik für Kinder- und Jugendheilkunde, Medizinische Universität Wien, Klinische Abteilung für Neonatologie, Pädiatrische Intensivmedizin und Neuropädiatrie, Wien, Österreich, ${ }^{2}$ Universitätsklinik für Kinder- und Jugendheilkunde, Medizinische Universität Wien, Abt. für Kardiologie, Wien, Österreich, ${ }^{3}$ Salzburger Universitätsklinikum, Neonatologie, Salzburg, Österreich, ${ }^{4}$ Institut für Medizinisches Informationsmanagement und Bildverarbeitung, Medizinische Universität Wien, Wien, Österreich, ${ }^{5}$ Universitätsklinik für Kinderund Jugendheilkunde Wien, Abt. f. Neonatologie, angeborene Störungen und Intensivmedizin, Wien, Österreich

Hintergrund. Das therapeutische Vorgehen bei persistierendem Ductus arteriosus (PDA) bei Frühgeborenen (FG) ist nach wie vor umstritten. Fragestellung. Gibt es zum Zeitpunkt der Fragestellung „Ductusligatur ja oder nein" einen Unterschied im klinischen Zustandsbild zwischen jenen FG, bei denen der hämodynamisch wirksame Ductus (hPDA) chirurgisch ligiert wurde („Ligatur-Gruppe“) und jenen FG, bei denen nach 3 Zyklen Ibuprofen trotz hPDA keine weitere Intervention mehr erfolgte („keine Ligatur-Gruppe“)? Gibt es einen Unterschied im Outcome dieser beiden Gruppen?

Methode. Über einen 4-Jahres-Zeitraum (Oktober 2009 bis Dezember 2013) wurden insgesamt 461 FG aus der Schwangerschaftswoche (SSW) 23+0-29+6 der Abteilung für Neonatologie der Medizinischen Universität Wien retrospektiv analysiert. Erhoben wurden sowohl klinische als auch echokardiographische Parameter. Eingeschlossen wurden FG mit Ductusligatur und FG mit hPDA nach $>3$ Zyklen Ibuprofen ohne weitere Intervention. Der PDA wurde als hämodynamisch relevant eingestuft, wenn der enddiastolische Fluss in der LPA (LPAdia) $=0,2 \mathrm{~m} / \mathrm{s}$ betrug oder die Ratio linker Vorhof/Aorta (LA/Ao-Ratio) $=1,4$ gemessen wurde. Für den Vergleich wurde in der "Ligatur-Gruppe“ die HerzultraschallUntersuchung vor Ligatur erhoben und in der „keine Ligatur-Gruppe die Herzultraschall-Untersuchung nach dem letzten Ibuprofen-Zyklus. Ergebnisse. In die „Ligatur-Gruppe“ konnten 39 FG eingeschlossen werden, in die „keine Ligatur-Gruppe“ 14 FG. Signifikante Unterschiede zwischen den beiden Gruppen konnten bei folgenden Parametern erhoben werden: diastolischer und systolischer Blutdruck; Zeitraum bis zum Erreichen einer rein enteralen Ernährung; LPAdia; LA/Ao-Ratio; „backward flow" in der Aorta abdominalis und dem Schlagvolumen. Weitere Unterschiede konnten beschrieben werden für Beatmung, Flow Pattern über dem hPDA, SSW, Rate der intraventrikulären Hämorrhagie und Rate der nekrotisierenden Enterokolitis (NEC). Die Morbidität sowohl vor als auch nach Ductusverschluss war in der „Ligatur-Gruppe“ schlechter. Verstorben sind nach Ductusligatur 5 FG, in der „NichtLigatur-Gruppe“ 3 FG.

Diskussion. Die Entscheidung, einen hPDA bei FG zu ligieren, mit dem Risiko eines Post-Ligationssyndroms oder ihn nicht zu verschließen, mit dem Risiko eine NEC oder eine bronchopulmonale Dysplasie zu begünstigen, stellt Neonatologen und Kardiologen auch heute noch vor eine schwierige Entscheidung. In unserer Analyse konnten wir beobachten, dass der PDA der "Ligatur-Gruppe“ nach echokardiographischen Kriterien hämodynamisch relevanter und die FG klinisch morbider waren. Die Mortalität war jedoch in der „Nicht-Ligatur-Gruppe“ höher.

Schlussfolgerung. Mit einer Ligaturrate von $6,5 \%$ bei FG $<$ SSW 30 liegen wir unterhalb der $10 \%$ Grenze im internationalen Vermont Oxford Vergleich. Somit zeigt sich an unserer Abteilung ein Trend den hPDA nicht zu ligieren. Inwieweit aber die Entscheidung zur Ligatur das Outcome der FG beeinträchtigt ist weiterhin unklar.
P131

Verschluss des Ductus arteriosus (PDA) mit einem neuen Amplatzer-Occluder bei kleinen ( $<5 \mathrm{~kg}$ ) Säuglingen

\section{F. Riede', J. Volkmann', R. Wagner', F. Löffelbein', I. Dähnert ${ }^{1}$}

${ }^{1}$ Klinik f. Kinderkardiologie, Herzzentrum, Universität Leipzig, Leipzig, Deutschland

Einleitung. Der katheterinterventionelle Verschluss ist die Therapie der Wahl des hämodynamisch bedeutsamen PDA bei sonst gesunden Kindern. Die bisherigen Verschlusssysteme sind allerdings recht steif und eher für größere Kinder geeignet. Mit dem neuen „Amplatzer Duct Occluder II additional sizes“ (ADO IIas) steht ein System zur Verfügung, das aufgrund seiner Spezifikationen (kleiner erforderlicher Gefäßzugang, flexibles Material, Schirme mit kleinem Profil) besonders für kleine Kinder geeignet erscheint.

Methodik. Retrospektive Analyse der Krankenakten aller Patienten mit einem Körpergewicht von unter $5 \mathrm{~kg}$, bei denen die Indikation zum Verschluss eines hämodynamisch bedeutsamen PDA mit einem ADO IIas gestellt wurde.

Ergebnisse. In der Zeit vom 01.01.2012 bis zum 31.12.2013 wurde bei 5 Kindern mit einem $\mathrm{KG}<5 \mathrm{~kg}$ die Indikation zum Verschluss eines PDA mit einem ADO IIas gestellt. Alle Kinder waren ehemalige Frühgeborene (mittleres Gestationsalter: 25 SSW (24-27); mittleres Geburtsgewicht: $645 \mathrm{~g}$ (400-900). Alle Patienten hatten eine pulmonale Hypertension und eine noch offene Vorhofkommunikation (PFO $n=4$, ASD n=1). Weitere Diagnosen waren bronchopulmonale Dysplasie $(n=4)$, Retinopathie $(\mathrm{n}=1)$, andere $(\mathrm{n}=2)$. Die Eingriffe wurden im mittleren (unkorrigierten) Lebensalter von 5,4 Monaten $(3,7-7,3)$ mit einem mittleren Gewicht von $3,6 \mathrm{~kg}(2,6-4,9)$ in Analgosedierung durchgeführt. Die Sondierung des PDA und Platzierung des Schirmes erfolgten über einen femoralvenösen Zugang (4 French) unter echokardiographischer Kontrolle. Bei zwei Patienten wurden zusätzlich Angiographien über einen femoralarteriellen Zugang (4 French) durchgeführt. Alle Eingriffe verliefen erfolgreich und ohne Komplikationen. Durchleuchtungszeit und Flächendosisprodukt betrugen im Mittel 8,4 min (7-10,7) bzw. 51,8 cGy ${ }^{*} \mathrm{~cm} 2$ (21-118). Die mittlere applizierte Kontrastmittelmenge lag bei $3,4 \mathrm{ml} / \mathrm{kg}(2-5,1)$. Postinterventionell war bei keinem Patienten ein Rest-Shunt nachweisbar. Während des mittleren Follow-up von 7,6 Monaten $(4,6-10,1)$ wurden keine interventionsbezogenen Komplikationen beobachtet. Eine Patientin verstarb im Verlauf an den Folgen einer Gallengangsatresie.

Schlussfolgerung. In Anbetracht der vielversprechenden ersten Ergebnisse stellt der interventionelle PDA-Verschluss mit dem ADO IIas eine Alternative zum chirurgischen Vorgehen auch für kleine Patienten dar - insbesondere für ehemalige Frühgeborene nach erfolglosem medikamentösen PDA-Verschluss. Mit wachsender Erfahrung mit der Methode kann vermutlich bei der Mehrzahl der Kinder zugunsten einer echokardiographischen Darstellung auf die Durchführung von Angiographien über einen arteriellen Zugang verzichtet werden, was die Invasivität des Eingriffes weiter reduziert.

\section{P132}

Wirksamkeit der oralen Ibuprofentherapie zum Ductusverschluss bei extrem unreifen Frühgeborenen

\section{H. Sallmon', N. Karg', P. Barikbin', T. Klippstein', C. Bührer', P. Koehne}

${ }^{1}$ Charité - Universitätsmedizin Berlin, Klinik für Neonatologie, Berlin, Deutschland

Einleitung. Ein PDA kommt bei bis zu 70\% aller ELBW-Frühgeborenen mit einem Gestationsalter $<28$ Wochen vor. Neuere Studien zeigen für Ibuprofen eine mindestens äquivalente Wirksamkeit der oralen Applikationsform im Vergleich zur intravenösen Gabe. Hier stellen wir präliminäre Daten zur Wirksamkeit einer oralen Ibuprofentherapie an unserer Klinik vor.

Methodik. Bisher retrospektive Datenanalyse von 76 ELBW-Frühgeborenen, die zwischen Oktober 2011 bis Dezember 2012 geboren wurden. 
Ausgewertet wurde der Behandlungserfolg bei allen pharmakologisch therapierten ELBW-Frühgeborenen.

Ergebnisse. In der bisherigen Datenanalyse zeigte sich ein Trend zu höheren PDA-Verschlussraten bei oraler Gabe im Vergleich zur i.v.-Applikation. Ursächlich dafür, dass unser Therapieerfolg aber unter dem bereits publizierter Studien liegt, ist mit hoher Wahrscheinlichkeit das niedrigere Geburtsgewicht im Vergleich zu Vorgängerstudien (VLBW) Schlussfolgerung. Trotz der geringeren Kosten des oralen Ibuprofens und der guten Ergebnisse müssen randomisierte Doppelblind-Studien mit einer größeren Studienpopulation durchgeführt werden um Wirksamkeit und Nebenwirkungen einer oralen Therapie sicher zu eruieren.

\section{P133}

Sicherung der Lungenperfusion mittels Stentimplantation in einen linken und einen rechten Ductus arteriosus bei einem Neugeborenen mit Pulmonalatresie und Ventrikelseptumdefekt

\section{J. Volkmann', F. Gräfe', F. Riede', I. Dähnert ${ }^{1}$ \\ ${ }^{1}$ Herzzentrum Leipzig, Kinderkardiologie, Leipzig, Deutschland}

Einleitung. Die Pulmonalatresie mit Ventrikelseptumdefekt (PA/VSD) gehört zur Gruppe der zyanotischen Herzfehler, welche in Abhängigkeit der Morphologie und Perfusion der Lungengefäße ein breites klinisches Spektrum darstellen. Bei ductusabhängiger Lungenperfusion ist entweder eine operative Shuntanlage oder eine interventionelle Stentimplantation in den Ductus (PDA) möglich. In dieser Fallvorstellung soll von einem Patienten mit PA/VSD berichtet werden, dessen Lungenperfusion über einen rechten und linken Ductus arteriosus erfolgreich mittels beidseitiger Stentimplantation gesichert wurde.

Fallvorstellung. Ein Neugeborenes mit Atresie der Pulmonalklappe, des -stammes und der zentralen Pulmonalarterien mit VSD zeigte als Besonderheit eine alleinige Lungenperfusion der rechten Pulmonalarterie über einen rechtsseitigen PDA aus dem deszendierenden Bogen und der linken Pulmonalarterie über einen linksseitigen PDA aus dem Truncus brachiozephalicus bei Rechtsaortenbogen. Zur Sicherung der Lungenperfusion erfolgte am 5. Lebenstag eine bds. Stentimplantation in die Ductus. Der Patient konnte mit Sauerstoffsättigungen $\left(\mathrm{SaO}_{2}\right)$ von 85-94\% nach Hause entlassen werden. Im 3. LM war bei Verschlechterung der $\mathrm{SaO}_{2}$ auf $70-75 \%$ eine erneute Herzkatheteruntersuchung mit Nachdilatation beider Stents und Implantation eines weiteren Stents erforderlich. Bei stabiler $\mathrm{SaO}_{2}$ und gutem Gedeihen ist im zweiten Lebenshalbjahr eine operative biventrikuläre Korrektur geplant.

Diskussion. Bei jedem Patienten ist in Abhängigkeit der anatomischen Strukturen und des klinischen Bildes eine individuelle Abwägung des therapeutischen Vorgehens notwendig. Unter engmaschigen Kontrollen ist zu jedem Zeitpunkt eine Neubewertung des aktuellen Verlaufs notwendig, so dass der nächste interventionelle/operative Schritt geplant werden kann.

Schlussfolgerung. Bei günstiger Anatomie ist eine interventionelle Therapie zur Sicherung der Lungenperfusion bei PA/VSD schon im Neugeborenenalter erfolgreich möglich und vermeidet eine frühe Operation.

\section{P134}

\section{Dopplersonographische Normalwerte für Blutflussparameter der} Niere bei gesunden Neugeborenen am ersten und dritten Lebenstag

\section{Korten', E. Robel-Tillig ${ }^{2}$ \\ 'Universitätsklinik und Poliklinik für Pädiatrische Kardiologie, Halle, Deutsch- land, ${ }^{2}$ Klinikum St. Georg gGmbH, Neonatologie, Leipzig, Deutschland}

Fragestellung. Eine Vielzahl von Faktoren kann am Lebensanfang eine ausreichende Perfusion der Niere gefährden, bzw. ihre Funktion beeinträchtigen. Umgekehrt spiegelt eine suffiziente Nierendurchblutung meist auch eine stabile Hämodynamik des Neugeborenen wieder. Somit ist es gerade beim Neugeborenen von großer Wichtigkeit die Nieren- funktion im klinischen Alltag überprüfen zu können. Ziel dieser Studie war die Erstellung von dopplersonographischen Normalwerten der Blutflussparameter beim gesunden Neugeborenen am ersten und dritten Lebenstag.

Material und Methode. Untersucht wurden 50 reife, gesunde Neugeborene. Am ersten und dritten Lebenstag wurden die systolische, diastolische und mittlere Flussgeschwindigkeit erfasst, der Widerstandsindex und der Pulsatilitätsindex ermittelt und die sonographischen Längenund Volumenmaße der Niere bestimmt.

Ergebnisse. Mittelwerte (SD) 1. Lebenstag: Vsys 43 (10,5), Vdias 9,5 (2), Vmean 20 (4), RI o,8 (0,06), PI 1,7 (0,4). Mittelwerte (SD) 3. Lebenstag: Vsys 49,5 (10), Vdias 10,5 (2,3), Vmean 23,4 (4,5), RI o,8 (0,05), PI 1,7 (0,3) Nierenmessung 1. Tag (in mm bzw. ml): Länge 41,5 (3,5), Volumen 11,5 $(2,5)$ Nierenmessung 3. Tag: Länge $43(4,1)$, Volumen $12,5(6,5)$ rechte Niere, $13(5,8)$ linke Niere. Zwischen männlichen und weiblichen Neugeborenen wurde kein Unterschied gemessen. Auch die dopplersonographischen Untersuchungen der linken und rechten Niere zeigten keinen Unterschied. Alle drei Flussgeschwindigkeiten zeigten einen signifikanten Anstieg zwischen dem 1. und 3. Lebenstag. PI und RI änderten sich nicht, Nierenlänge und Nierenvolumen nahmen zu. Eine Korrelation von GA, Körpergewicht oder Körperlänge mit den gemessenen Werten konnte nicht gefunden werden. Eine positive Korrelation zeigte sich zwischen Nierenvolumen und Nierenlänge und besonders der systolischen Flussgeschwindigkeit. Es wurde eine relativ große Spannbreite der Parameter zwischen den einzelnen Individuen gemessen.

Diskussion. Die ermittelten Messgrößen können als orientierende dopplersonographische Referenzparameter der Durchblutung der Niere am Lebensanfang dienen. Aufgrund einer relativ hohen Spannbreite sind zwei weitere Faktoren wichtig, die in dieser Arbeit nachgewiesen werden konnten: Die Messgrößen der linken und rechten Niere stimmen überein und bei jedem Neugeborenen kann ein Anstieg der Flussgeschwindigkeiten verzeichnet werden. Eine seitendifferente Perfusion der Nieren kann auf einen pathologischen Prozess hinweisen. Fallende Flussgeschwindigkeiten sowie steigende Widerstandsindizes im Verlauf können Anzeichen für eine Störung der Nierenperfusion und somit der systemischen Hämodynamik sein.

Schlussfolgerung. Es konnten dopplersonographische Referenzparameter der Durchblutung der Niere ermittelt werden, die als unkompliziert messbare Parameter in der neonatologischen Praxis zur Einschätzung systemisch hämodynamischer Veränderungen sowie funktioneller Störungen der Niere bis zum akuten Nierenversagen benutzt werden können.

\section{P135}

Rezidivierende obstruktive Bronchitis im frühen Säuglingsalter ein Warnsignal für bronchotracheale Engstellen durch Gefäßanomalien

\section{Frank', J. Hoenecke', O. Rompel', R. Cesnejvar ', W. Rascher', H. Topf',} T. Völkl'

'Universitäts-Kinderklinik, Neonatologie und pädiatrische Intensivmedizin, Erlangen, Deutschland, ${ }^{2}$ Universitäts-Kinderklinik, Pädiatrische Radiologie, Erlangen, Deutschland, ${ }^{3} U$ niversitäts-Kinderklinik, Kinderherzchirurgische Abteilung, Erlangen, Deutschland

Hintergrund. Eine pulmonale Symptomatik, die in der allgemeinpädiatrischen Praxis als „Tracheomalazie“, „obstruktive Bronchitis“ oder „rezidivierender oberer Atemwegsinfekt“ diagnostiziert wird, kann sich bei genauer Untersuchung als Stridor darstellen, welcher der weiteren Abklärung bedarf. Wir präsentieren zwei aktuelle Fälle (2013/2014); erstens einen dramatischen Verlauf mit ECMO bei verzögerter Diagnosestellung und kontrastierend einen zweiten Fall mit komplikationslosem Verlauf durch rechtzeitige Diagnose und Therapie.

Fall 1. Der männliche Säugling ( 5 Monate) wurde in einer auswärtigen Kinderklinik mit klinischem Bild eines Infektes der oberen Luftwege antiobstruktiv behandelt. Anamnestisch waren eine Pneumonie im Alter von 3 Monaten und „rezidivierende obstruktive Bronchitis“ beschrieben. 
Im Verlauf kam es zu einer respiratorischen Verschlechterung mit massiver Hyperkapnie ( $\mathrm{pCO}_{2}>100 \mathrm{mmHg}$ ). Nach erfolgter Intubation und Beatmung zeigte sich eine schwer führbare Beatmung bei hohen Beatmungsdrücken; Verlegung per RTH in unsere Klinik. Hier nach wenigen Stunden erneuter massiver pCO2-Anstieg $(>150 \mathrm{mmHg})$ mit Indikationsstellung einer arteriovenösen ECMO-Therapie; problemloser Einbau und Betrieb über 21 Tage. Im Schnelltest konnte eine RSV-Infektion nachgewiesen werden, in der PCR Rhinoviren. Nach ECMO-Ausbau kam es trotz repetitiver Inhalation mit Adrenalin zu einem persistierenden Stridor. Im Thorax-CT konnte ein „pulmonary sling“ mit hypoplastischem Trachealsegment diagnostiziert werden. Es erfolgte die kardiochirurgische Korrektur mit Resektion des Trachealsegmentes. Klinisch präsentierte sich der Patient vor Verlegung zur Neurorehabilitation mit zentraler Koordinationsstörung ohne Krampfanfälle bei pathologischem EEG und subduralen Hämatomen (Residuen) im Schädel-MRT. Eine antikonvulsive Therapie wurde durchgeführt.

Fall 2. Bei dieser weiblichen Patientin ( 3 Monate) bestand bei stationärer Aufnahme ein seit 5 Wochen persistierender Husten sowie das klinische Bild einer „obstruktiven Bronchitis“. Eine antiobstruktive Therapie wurde begonnen und eine im stationären Verlauf entwickelte Bronchopneumonie i.v.-antibakteriell behandelt. Unter dieser Therapie kam es im Verlauf zu einer Besserung des Allgemeinzustandes. Ein nach Therapieende weiter bestehender inspiratorischer Stridor bestand laut Mutter „schon immer". Im daraufhin durchgeführten Thorax-CT zeigte sich ein symmetrischer doppelter Aortenbogen. Die Patientin erhielt eine operative Korrektur und es kam zu einer vollständigen Genesung ohne Residuen. Schlussfolgerung. Diese Fälle zeigen eindrucksvoll, dass sich hinter dem vermeidlich harmlosen klinischen Diagnosespektrum „obstruktive Bronchitis" insbesondere im frühen Säuglingsalter angeborene Engen des bronchotrachealen Systems, z. B. durch Gefäßanomalien, verbergen können. Neben einer exakten klinischen Beurteilung (Stridor?) ist eine zeitnahe Durchführung erweiterter Diagnostik (Echo, CT) bei persistierender Symptomatik zwingend.

P136

Der Einfluss des persistierenden Foramen ovale (PFO) auf die zerebrale Oxygenierung bei reifen Neogeborenen 15 Minuten nach Geburt

\section{N. Baik', B. Schwaberger', C. Binder', T. Freidl', B. Urlesberger', G. Pichler' \\ 'Universitäts Klinik für Kinder- und Jugendheilkunde Graz, Abteilung für Neo- natologie, Graz, Österreich}

Fragestellung. Ziel der Studie war es, 15 Minuten nach Geburt den Einfluss eines persistierenden Formaen ovale (PFO) auf die zerebrale Oxygenierung, welche mittels Nah-Infrarotspektroskopie (NIRS) gemessenen wurde, zu untersuchen.

Material und Methoden. In einer prospektiven Beobachtungsstudie wurden reife Neogeborene ( $>37$ Schwangerschaftswoche) 15 Minuten nach Geburt nach einer elektiven Kaiserschnittentbindung und nach unauffälliger Adaptation ohne medizinische Unterstützung eingeschlossen. Die zerebrale Oxygenierung (TOI) wurde mit NahinfrarotSpektroskopie (NIRS) rechts frontal mit dem NIRO 200NX gemessen. Der Durchmesser des PFO wurde im Rahmen einer Echokardiographie erhoben. Ein möglicher Zusammenhangzwischen TOI und Durchmesser des PFO wurde mittels Korrelationsanalyse untersucht.

Ergebnisse. Es wurden 25 reife Neogeborene mit unauffälliger Adaptation (APGAR in Median: 9/10/10) inkludiert. Das Gestationsalter

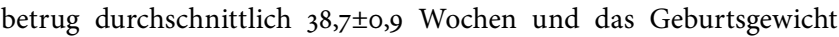
3114,0 $\pm 423,9$ g Dier zerebrale Oxygenierung betrug durchschnittlich $76,6 \pm 8,9 \%$ und der Durchmesser und des PFO 2,3 $\pm 0,7 \mathrm{~mm}$. Es bestand ein Trend zu einer negativen Korrelation zwischen zerebraler Oxygenierung und Durchmesser des PFO, diese Korrelation zeigt sich jedoch statistisch nicht signifikant

Diskussion. In der vorliegenden Studie konnte gezeigt werden, dass es keine statistisch signifikante Korrelation zwischen zerebraler Oxyge- nierung und Durchmesser des PFO in reifen Neogeborenen nach 15-minütiger unauffälliger Adaptation besteht.

Schlussfolgerung. Die Größe des PFO hat keinen Einfluss auf die zerebrale Oxygenierung 15 Minuten nach der Geburt

\section{P137}

Wie schnell kann die zerebrale Oxygenierung bei Anwendung in der Erstversorgung unmittelbar nach der Geburt erhoben werden? E. Ziehenberger', B. Urlesberger', C. Binder', B. Nariae', B. Schwaberger',
G. Pichler'

'Univ. Klinikum für Kinder- und Jugendheilkunde, Medizinische Universität Graz, Klinische Abteilung für Neonatologie, Graz, Österreich

Hintergrund. Die Nahinfrarot Spektroskopie (NIRS) ermöglicht ein nicht-invasives Monitoring zur Feststellung der regionalen zerebralen Oxygenierung (zerebrale Tissue Oxygenation Index: zTOI bzw. zerebrale regionale Sauerstoffsättigung: $\mathrm{zrSO}_{2}$ ) in der Neugeborenen Reanimation.

Fragestellung. In der vorliegenden Studie wurde untersucht, wie lange es nach der Geburt des Neugeborenen dauert, bis der erste Wert der zerebralen Oxygenierung gemessen werden kann.

Patienten. Neugeborene nach elektiven Kaiserschnitt.

Methode. Retrospektive Beobachtungsstudie bei reifen Neugeborenen und Frühgeborenen, bei denen im Rahmen von prospektiven Studien mit NIRS während der Erstversorgung aufgezeichnete Videos analysiert wurden. NIRS Messungen wurden mittels NIRO 220 NX (zTOI) und INVOS 5100 (zrSO2) durchgeführt. Folgende Zeitintervalle wurden erhoben: Gesamtzeit Geburt bis ersten Wert der zerebralen Oxygenierung (zTOI, zrSO2), Geburt bis Eintreffen am Erstversorgungstisch, Eintreffen am Erstversorgungstisch bis Anlegen der Sensoren, Anlegen der Sensoren bis ersten Wert der zerebralen Oxygenierung (zTOI, $\mathrm{rSO} 2$ ).

Ergebnisse. Es wurden 38 Neugeborene in die Studie eingeschlossen (Gestationsalter 38,3 $\pm 1,6$ Wochen, männlich/weiblich: 16/22, Geburtsgewicht $3135 \pm 559$ g, Frühgeborene/ reife Neugeborene: 7/31). Mit dem NIRO $200 N X$ wurden 20 Neugeborene gemessen, mit dem INVOS 5100 13 Neugeborene und mit NIRO 200NX und INVOS 5100 gleichzeitig wurden fünf Neugeborene gemessen. Das Zeitintervall von der Geburt bis zum ersten Wert der zerebralen Oxygenierung betrug $113 \pm 17 \mathrm{~s}$ (zTOI $101 \pm 46 \mathrm{~s}$ vs. $\mathrm{zrSO} 2125 \pm 64 \mathrm{~s} ; \mathrm{p}=0,14)$. Der initiale zTOI betrug $48 \pm 15 \%$ und $\mathrm{zrSO}_{2} 47 \pm 27 \%$. Die Zeitintervall zwischen der Geburt und dem Eintreffen am Erstversorgungstisch betrug $54 \pm 35 \mathrm{~s}$ und vom Eintreffen am Reanimationstisch und Anlegen der Sensoren 13 \pm 5 s. Das Zeitintervall vom Anlegen der Sensoren bis zum ersten Wert der zerebralen Oxygenierung betrug insgesamt $42 \mathrm{~s}$ (zTOI $33 \pm 28 \mathrm{~s}$ vs. $\mathrm{rSO}_{2} 50 \pm 33 \mathrm{~s}$; $\mathrm{p}=0,35)$.

Schlussfolgerung. Im Rahmen dieser Studie konnte erstmals die benötigte Zeit bis zum Erreichen eines NIRS Signals/Wertes bei der Anwendung in der Erstversorgung unmittelbar nach der Geburt erhoben werden, wobei sich keine signifikanten Unterschiede zwischen den verschiedenen Geräten zeigten.

\section{P138}

Infusothorax als lebensbedrohliche Komplikation einer sekundären Nabelvenenkatheterdislokation bei einem extrem untergewichtigen Frühgeborenen

\section{O. Polley', H. Sallmon', P. Barikbin', A. Pohl-Schickinger', C. Bührer',} C. Czernik

'Charité - Universitätsmedizin Berlin, Klinik für Neonatologie, Berlin, Deutschland

Einleitung. Nabelvenenkatheter (NVK) bieten in den ersten Lebenstagen die Möglichkeit, einen zentralvenösen Zugang technisch relativ einfach zu etablieren. Fehllagen des Katheters können zu verschiedenen 
Komplikationen führen. Wir berichten über einen lebensbedrohlichen Infusothorax durch NVK-Dislokation bei radiologisch und sonographisch als korrekt angesehener Lage.

Kasuistik. Hypotrophes weibliches Frühgeborenes (Geburtsgewicht 530 g, Gestationsalter 25 Wochen), Geburt per Sectio bei Eklampsie der Mutter. Initial Intubation, Surfactantgabe und NVK-Anlage. Die Spitze des Nabelvenenkatheters lag sonographisch in der unteren Hohlvene unmittelbar am Eingang des rechten Vorhofs, in der a.p.-Aufnahme projizierte sie sich knapp unterhalb der Zwerchfellkuppel. Am 3. Lebenstag akute kardiopulmonale Dekompensation unter Beatmung, Gabe von Adrenalin und Thoraxkompressionen, diaphanoskopischer Verdacht auf Pneumothorax, bei der Punktion entleerte sich seröse Flüssigkeit. Anlage von Pigtail-Thoraxdrainagen beidseits, das geförderte Sekret wirkte chylös. Nach Beginn der Gabe von Erythrozytenkonzentrat über den NVK wurde das Sekret auf der rechten Seite sofort blutig, nach Spülen des NVK wurde das Sekret wieder klar. Der NKV wurde zunächst in situ belassen, bei der nächsten sonographischen Kontrolle lag die Spitze intraluminal. Der NVK wurde danach komplikationslos gezogen.

Diskussion. Nabelgefäßkatheter können zu vielfältigen Komplikationen führen. Neben häufiger berichteten Fehllagen (z. B. in V. portae oder den Vorhöfen) kann auch eine Dislokation in die Pleura bei primär korrekter Lage auftreten, welche in unserem Fall zu einem reanimationspflichtigen Ereignis führte. Dies illustriert die Notwendigkeit sorgfältiger Kontrolle und kritischer Indikationsstellung zentraler Gefäßkatheter.

\section{Pflege}

\section{Pfl.P001}

\section{Der pflegerische Beitrag zur ethischen Entscheidungsfindung}

\section{B. Dinten-Schmid', L. Stoffel', C. Zimmermann ${ }^{3}$}

${ }^{1}$ Frauenklinik, Neonatologie, Zürich, Schweiz, ${ }^{2}$ Medizinische Universitätskinderklinik, Inselspital Bern, Neonatologie, Bern, Schweiz, ${ }^{3}$ Inselspital Bern, Stationsleitung Neonatologie B2, Bern, Schweiz

Hintergrund. In den Abteilungen für Neonatologie der Universitätsspitäler Bern und Zürich finden in kritischen Patientensituationen ethische, interprofessionelle Gespräche zur Entscheidungsfindung statt. Pflegefachpersonen übernehmen dabei eine wichtige Aufgabe. Sie bringen die pflegerische Anamnese in den ethischen Entscheidungsfindungsprozess ein. Eine Analyse ergab, dass Pflegefachpersonen ihre Einschätzung im ethischen Entscheidungsfindungsprozess unstrukturiert und unvollständig darstellen.

Ziel. Entwicklung und Implementierung einer Checkliste, die Pflegefachpersonen eine systematische und objektive Herangehensweise ermöglicht.

Fragestellung. Welche objektiven Parameter unterstützen Pflegende um ihre Einschätzung bezüglich der ethischen Entscheidungsfindung systematisch und objektiv darzustellen?

Methode. Das Projekt wurde nach den 7 Schritten des IOWA-Modells entlang der methodischen Schritte von "evidence-based nursing“ durchgeführt (Titler et al. 2001).

1) Problemidentifikation

2) Literatursuche

3) Überprüfung der relevanten Literatur

4) Einbezug von Expertenmeinung

5) Umsetzung der Veränderung

6) Implementierungsprozess

7) Ergebnisevaluation

Ergebnisse.

1) Eine Dokumentenanalyse und die Befragung bei 41 Pflegefachpersonen bestätigte eine unstrukturierte Herangehensweise

2) Es steht keine relevante Literatur zur Verfügung, hinsichtlich Checkliste, welche eine strukturierte Herangehensweise ermöglicht

3) Aufgrund fehlender Literatur wurde eine Checkliste mit aussagekräftigen Kriterien erstellt
4) Die Checkliste wurde von den jeweiligen Ethikgruppen kritisch überprüft und genehmigt

5) Die Pflegedienstleitung beider Abteilungen unterstützte den Veränderungsprozess

6) Die Implementierung erfolgte mittels Schulung der Pflegenden. Zudem durch Coaching in der Umsetzung und Fallbesprechungen

7) Eine erneute Dokumentenanalyse und Befragung von 10 Pflegefachpersonen nach Einführung der Checkliste zeigte, dass sich die Fakten der pflegerischen Anamnese mit Hilfe der Checkliste im Gespräch zur ethischen Entscheidungsfindung strukturiert benennen lassen. Die Pflegefachpersonen fühlen sich sicher und können die Anliegen des Kindes besser vertreten.

Schlussfolgerung. Mithilfe der Checkliste lassen sich die Fakten der pflegerischen Anamnese im Gespräch zur ethischen Entscheidungsfindung strukturiert und objektiv benennen. Dadurch gewinnen die Pflegefachpersonen Selbstsicherheit und sind in ihrer Rolle im interprofessionellen Entscheidungsfindungsprozess gestärkt.

\section{Pfl.PO02}

Komplementäre Pflege auf Station 5. Ein prospektives Projekt

\section{S. Fritsche', S. Kowol', H. Wolff-Teßmann'}

'UKK Bonn, Station 5, Bornheim, Deutschland

Hintergrund. Die komplementäre Pflege ist nicht gleichzusetzen mit alternativer Pflege. Komplementär heißt ergänzend, in diesem Fall zur Schul-/Intensivmedizin. Eine Harmonisierung von Körper, Geist und Seele soll hergestellt werden.

Methodik. Drei Schwestern der Station 5 haben im Rahmen einer naturheilkundlichen Ausbildung, ein Standardheft speziell für unsere stationären Bedürfnisse entworfen, welches sich mit Schmerzlinderung, Wundheilung und Fiebersenkung befasst. Methoden können sein: Wickel/Auflagen, Massagen/Einreibungen, Öle/Heilpflanzentees.

Schlussfolgerung. Was spricht dafür?

- Wird von Eltern gerne angenommen

- Kann von Eltern nach Anleitung selber durchgeführt werden, fördert die Eltern Kind Beziehung

- Massagen und Einreibungen geben das Gefühl von besonders intensiver Zuwendung

- Qualitative Berührung ist immer mit Nähe verbunden

- Düfte sprechen die Sinne an und steigern das Wohlbefinden

- Harmonisierung

- Erspart unter Umständen Medikamentengaben

\section{Pfl.PO03}

\section{Basale Stimulation auf Station 5}

\section{Spoden ${ }^{1}$, S. Durmaz ${ }^{2}$}

'Universitätsklinik Zentrum f. Kinderheilkunde, Station5, Königswinter, Deutschland, ${ }^{2}$ UKB, Station 5, Bonn, Deutschland

Definition. Basal: die Basis bildend, an der Basis ansetzen Stimulation: Reize setzen, stimulieren; Basale Stimulation kann man auch als Kommunikationskonzept bezeichnen

Wahrnehmungsbereiche. Somatische Wahrnehmung, orale Wahrnehmung, auditive Wahrnehmung, vestibuläre Wahrnehmung.

Methodik. Durch Maßnahmen wie Waschungen, gezielte Lagerungen, (unterstützte) Bewegungen, besondere Mundpflege individuelle Maßnahmen, wie z. B. gezielte Musikstücke laufen lassen, Mobilisation.

Ziele. Aktivierung der Wahrnehmungsbereiche und die Anregung primärer Körper-und Bewegungserfahrungen. Angebote zur Herausbildung einer individuellen non-verbalen Mitteilungsform bei Menschen, deren Eigenaktivität aufgrund ihrer mangelnden Bewegungsfähigkeit eingeschränkt ist. 


\section{Pfl.PO04}

Implementierung eines Bezugspflegesystems in Bezugnahme auf das Pflegekonzept "Primary Nursing“ von Marie Manthey auf der neonatologischen Intensivstation der Universitätskinderklinik Bonn

\section{A. Müller-Helm \\ 'Universitätsklinik Zentrum f. Kinderheilkunde, Neonatologie, Bonn, Deutsch- land}

Fragestellung. Die Mitte des letzten Jahrhunderts stattgefundene Entprofessionalisierung der Pflege wurde durch das von Marie Manthey Ende der 1970er Jahre in den USA entwickelte „Primary-Nursing-Konzept“ wieder in eine Professionalisierung umgewandelt. Auf der Neonatologischen Intensivstation der Universitätskinderklink Bonn werden Neonaten mit allen neonatologischen und chirurgischen Krankheitsbildern der Perinatalperiode behandelt. Etwa $72 \%$ des Pflegepersonals haben mehr als fünf Jahre Berufserfahrung in diesem Bereich und somit kann man von dem großen Potential an Regelwissen und vor allem der Erfahrung profitieren. Etwa 50\% der Pflegenden arbeiten in Teilzeit mit unterschiedlichen Stundenkontingenten. Im ärztlichen Bereich gibt es aufgrund eines Rotationsverfahrens innerhalb der Abteilung oft wechselnde Betreuung. Beide Punkte erschweren eine durchgängige Kommunikation. Zielsetzung der Implementierung war zum einen mehr Kontinuität in der medizinisch- pflegerischen Versorgung $\mathrm{zu}$ schaffen, sowie geregelte Kommunikationswege zu etablieren. Zudem sollte ein höheres Verantwortungsbewusstsein in der Betreuung der Patienten durch die „Primary Nurse“ geschaffen werden.

Methoden. Nach ausführlicher Literaturrecherche wurde ein Konzept erstellt, sowie Assessmentdokumente entworfen. Im Zeitraum von Juli 2013 bis Februar 2014 wurde das Konzept bei 16 Patienten umgesetzt. Die Anwendung beschränkte sich dabei auf Risikopatienten mit einer Verweildauer von mehr als vier Wochen. Nach acht Monaten erfolgte eine Evaluation.

Ergebnisse. Das Bezugspflegesystem in Anlehnung an das „Primary Nursing Konzept“ von Marie Manthey konnte erfolgreich umgesetzt werden. Mit der Implementierung konnte der Pflege innerhalb ihrer Professur mehr Verantwortung übertragen werden und der Pflegeprozess wurde kontinuierlicher, was zu einer höheren Zufriedenheit des Personals führte. Die Kommunikationsstruktur ist klareren Regeln unterworfen. Den Angehörigen konnten feste Ansprechpartner zugewiesen werden, was deren Zufriedenheit steigerte. Ergebnisse der Evaluation liegen zum Zeitpunkt der Abstract-Einreichung noch nicht vor. Schlussfolgerung. Die interdisziplinäre Zusammenarbeit mit dem Konzept erfordert ein intensiveres Miteinander der Professuren, welches, bei korrekter Umsetzung, auch zu einer Verbesserung der medizinischpflegerischen Versorgung führt. Das Bezugspflegekonzept erfordert ein hohes Maß an Disziplin in der Dokumentation und Kommunikation. Das evidenzbasierte Wissen kann damit erhalten und weitergegeben werden.

Quellen- und Literaturangaben über die Autorin

\section{Pfl.PO05 \\ Zwei Welten der Elternschaft - Wie kann das Pflegepersonal der neonatologischen Intensivstation Eltern von Früh- bzw. kranken Neugeborenen beim Finden der Elternrolle unterstützen?}

\section{U. von der Wense', K. Schmitz ${ }^{1}$ \\ 'Universitätsklinik Zentrum f. Kinderheilkunde, Neonatologie Station 6, Bonn, Deutschland}

Hintergrund. Schon die Geburt eines gesunden, reifen Neugeborenen ist ein einschneidendes Ereignis. Der Übergang von Schwangerschaft zur Elternschaft ist eine große Herausforderung für jedes Elternpaar. Wenn es, häufig schon nach komplizierter Schwangerschaft, zur Frühgeburt kommt, ist das Maß an Belastung für die Eltern enorm. Eltern eines
Frühgeborenen oder kranken Neugeborenen befinden sich mit dem „Frühstart“ bzw. so ganz anderen Start ihres Kindes in einem „Ausnahmezustand im Ausnahmezustand“, was die Übernahme der Elternrolle zusätzlich erschwert.

Fragestellung. Was können wir als Pflegepersonal auf der neonatologischen Intensivstation konkret tun, um den Eltern zu helfen nicht nur de facto Eltern zu sein, sondern sich auch als Eltern zu fühlen? Legen wir „Trittsteine“ in der Betreuung oder „Stolpersteine“ auf dem Weg zur gesunden Elternschaft?

Methode. Literaturrecherche, Erkenntnisse aus spezifischen Fort- und Weiterbildungen, aber auch langjährige Erfahrung im Umgang mit den Eltern sind Grundlage unserer Arbeit. Die beiden Welten der Elternschaft werden einander gegenüber gestellt. Wir zeigen Wege zur Unterstützung der Eltern.

Ergebnis. Das Pflegepersonal kann durch die Etablierung der entwicklungsfördernden, familienzentrierten Pflege in der Neonatologie neue Wege zur Findung der Elternrolle aufzeigen. Daraus resultiert ein neues Rollenverständnis der Pflege. Fazit: Die wirkliche Übernahme der Elternrolle ist der erste Schritt zu einer gesunden Eltern-Kind-Bindung. Um die Eltern darin zu unterstützen muss das Pflegepersonal für die besonderen Aspekte sensibilisiert werden und die Bereitschaft haben, sich auf das Umdenken und die neue Rolle einzulassen. Darüber hinaus gibt es weitere Faktoren wie ausreichend und gut ausgebildetes Personal, spezielle bauliche Aspekte, Raumausstattung, Klinikphilosophie sowie das Etablieren einer psychosozialen Betreuung, die den Findungsprozess für die Eltern beeinflussen. Der Start ins Leben hat eine so wichtige Bedeutung für die Entwicklung eines jeden Kindes, dass es sich lohnt, dafür Zeit und Energie zu investieren.

\section{Pfl.PO06}

\section{"Liebe auf den ersten Blick" - Bonding im Kreißsaal bei extremen} Frühgeborenen

\section{T. Bender', E. Säger', S. Rathgeber', C. Bender', M. Henschen}

'Klinik für Kinderheilkunde, Jugendmedizin und Kinderchirurgie, VillingenSchwenningen, Deutschland

Hintergrund und Einleitung. „Bonding“ umschreibt die intensive, innige Beziehung und emotionale Verbindung, die sich zwischen Mutter/Eltern und neugeborenem Kind entwickelt. Kurz nach der Geburt intensiviert sich diese Bindung durch die körperliche Nähe, Wärme, Bewegungen, Geruch und Augenkontakt. Dieses gegenseitige kennen lernen zwischen Mutter/Eltern und Neugeborenem sorgt für eine erste einzigartige soziale Bindung. Durch den direkten Kontakt wird bei der Mutter wie auch beim Kind das Hormon Oxytocin ausgeschüttet, das einerseits für Wohlbefinden und Entspannung sorgt und andererseits den Milchfluss fördert. Das Bonding sollte schnellstmöglich nach der Geburt stattfinden. Ein späteres Känguruhing auf der Station ist kein Ersatz für das Bonding direkt nach der Geburt.

Fragestellung. Ist das direkte Bonding nach der Erstversorgung auch von sehr kleinen Frühgeborenen möglich und wie soll es bestmöglich umgesetzt werden? Welchen Einfluss hat dieser erste Kontakt im Kreißsaal auf das Frühgeborene, seine Eltern und die Zusammenarbeit mit den Eltern auf der neonatologischen Intensivstation?

Methode. Die Mutter/die Eltern werden in unserer Klinik in aller Regel vor der Entbindung über die Bedeutung und den Ablauf des Bondings informiert. Nach der Erstversorgung wird das Frühgeborene im Kreißsaal unter Fortführung der Therapie und Überwachung nackt der Mutter auf die Brust gelegt oder, wenn es der Zustand des Kindes/der Mutter in Ausnahmefällen nicht erlaubt, wenigstens versucht einen ,ersten Blickkontakt“ und das „Beschnuppern“ zu ermöglichen. Wichtig sind hierbei: Ruhige Atmosphäre, Wärme, bequeme Lagerung, Vermeidung von grellem Licht, ausreichend Zeit, bei stabilen Frühgeborenen wenn möglich erster Stillversuch.

Ergebnisse und Schlussfolgerung. Ja, das Bonding ist auch bei sehr kleinen Frühgeborenen möglich. Dieser erste Kontakt hilft den Eltern, bei 
Ankunft auf der Intensivstation jetzt vorrangig ihr Kind zu sehen und nicht nur „ein kleines, verkabeltes Wesen inmitten der Intensivmedizin“. Die Berührungsängste sind geringer, die Eltern können schneller in die Pflege ihres Kindes integriert werden und auch die Zusammenarbeit mit den Eltern und die Kommunikation mit ihnen sind deutlich einfacher. Man kennt sich eben schon. Seit wir das „Bonding“ nach der Erstversorgung auch bei den extremen Frühgeborenen begonnen haben, sind nicht nur die Rückmeldungen der betroffenen Eltern sehr positiv, sondern auch das Kreißsaalteam sowie wir als erstversorgendes neonatologisches Team sind davon überzeugt, dass das „Bonding“ nach der Erstversorgung eine extrem wichtige Maßnahme ist. Wir glauben ebenso, dass dieser erste frühe Kontakt auch eine Verbesserung der Prognose der extremen Frühgeborenen bedeutet, auch wenn wir das erst durch Studien belegen müssen. Tatsache ist zumindest, dass die Frühgeborenen in den meisten Fällen jetzt etwa eine Woche früher aus der stationären Therapie entlassen werden können.

\section{Pfl.PO07}

Pflege und sozialmedizinische Nachsorge bei einem Frühgeborenen in der 28. SSW mit Klippel-Trenaunay-Weber-Syndrom und Hydrops fetalis

\section{H. Akduman', A. Mueller' I. Orth}

'Bunter Kreis Bonn, Koblenz, Deutschland, ${ }^{2}$ Zentrum für Kinderheilkunde, Universitätsklinikum Bonn, Abt. Neonatologie, Bonn, Deutschland, ${ }^{3}$ Vorstandsvorsitzende Bunter Kreis Bonn-Ahr-Rhein-Sieg e.V., Bonn, Deutschland

Hintergrund. A., ein Frühgeborenes der 28 o/7 SSW mit pränatal bekanntem Klippel-Trenaunay-Weber-Syndrom (hämangiomatöse Lymphangiektasie linkes Bein) und intrauteriner Pigtailkatheter-Anlage zur Aszitesdrainage bei Hydrops fetalis. Das Klippel-Trenaunay-Weber-Syndrom ist ein angeborenes Fehlbildungssyndrom der Gefäße, es wird auch als Angiodysplasie bezeichnet. Merkmale wie z. B. Hämangiomatöse Lymphangiektasien, Naevus flammeus (kapillare Fehlbildung), partieller Riesenwuchs mit Beteiligung aller Gewebe vervollständigen das Krankheitsbild. Dieses Syndrom tritt sporadisch auf. In der Literatur sind weit über 1500 Fälle beschrieben. Unter einem Hydrops fetalis versteht man eine generalisierte Flüssigkeitsansammlung in serösen Körperhöhlen und Weichteilen. Häufigste Ursachen sind fetale Anämie, Hypoproteinämie, Herzinsuffizienz, Chromosomenstörungen oder organische Fehlbildungen.

Pflege. A. wurde fünf Monate auf einer Neonatologischen Intensivstation betreut. Im Vordergrund stand die intensivmedizinische Therapie. Zur Stabilisierung des Kreislaufes wurden verschiedene Katecholamine eingesetzt. Die anfänglich nötige Beatmung erfolgte unter HFO Modus mit Einsatz von Stickoxid (NO). Im Verlauf wurde eine Atemunterstützung durch Masken-CPAP und High-Flow-Brille durchgeführt. Die anhaltende pulmonale Hypertension wurde durch eine Stickoxidtherapie behandelt, im weiteren Verlauf durch die orale Gabe von Sildenafil (Revatio) weitergeführt. Zur Entlastung wurden mehrere Aszitispunktionen notwendig. Unterstützend kam die Physiotherapie, Lymphdrainage sowie die Anpassung eines Kompressionsstrumpfes für das linke Bein hinzu. Diese Zeit bedeutet für die Eltern eine hohe emotionale Belastung. Frühzeitig erfolgte die Anbindung an den Bunten Kreis, dieser bietet eine sozial medizinische Familiennachsorge nach dem Augsburger Modell. Durch den frühzeitigen Kontakt der Nachsorgeschwester mit der Familie konnte die Entlassung von A. gut vorbereitet werden. Die Hauptaufgabe war die Vernetzung zu einem Kinderkardiologen, Neonatologen, sowie eines Physiotherapeuten, Kinderorthopäde. Ebenso die Hilfe bei der sozialrechtlichen Beratung, sowie die Unterstützung in der Pflege. Durch regelmäßige Hausbesuche und Telefonate wird die Elternkompetenz stetig gefördert. Schlussfolgerung. Bei komplexen Fehlbildungspatienten kann die Einbindung einer sozial-medizinischen Nachsorge schon frühzeitig während des stationären Aufenthaltes den Übergang von stationärer zur ambulanten Versorgung für Eltern und Kind einfacher gestalten. Dies wird erreicht durch professionelle ärztliche sowie pflegerische Bera- tung, zeitiger Einbindung der Eltern in die Pflege sowie Koordination der Zusammenarbeit zwischen Klinik und niedergelassenen Ärzten. Schlüsselwörter. Klippel-Trenaunay-Weber-Syndrom, Nachsorge

\section{Pfl.PO08}

Am Anfang war es nur ein Traum: Erfahrungen mit dem pflegerischen Behandlungs- und Betreuungskonzept der neonatologischmaternalen Einheit (NME) am Klinikum Aschaffenburg

\section{Stumpf', D. Steiner', T. Karen², C. Wieg²}

'Klinikum Aschaffenburg, Neonatologie, Aschaffenburg, Deutschland, ${ }^{2}$ Klinikum Aschaffenburg, Neonatologie un pädiatrische Intensivmedizin, Aschaffenburg, Deutschland

Fragestellung. Wie ermöglichen wir eine gemeinsame stationäre Versorgung von Mutter und Kind, wenn ein krankes Neugeborenes oder spätes Frühgeborenes nach der Geburt einer nicht-intensivmedizinischen Behandlung bedarf?

Material und Methode. Errichtung einer neonatologisch-maternalen Einheit für Früh- und Neugeborene in einem Level-1-Perinatalzentrum, die direkt nach der Geburt neonatologisch, aber nicht intensivmedizinisch behandelt werden müssen. Besonderes Augenmerk dabei liegt auf der pflegerischen Betreuung von Mutter und Kind aus einer Hand.

Ergebnisse. Wir berichten über unsere Erfahrungen seit 2006. Durch die Errichtung unserer Mutter-Kind-Einheit bekommen die Kinder die bestmögliche medizinisch-neonatologische Behandlung, während die Mutter gleichzeitig die speziellen Pflegeangebote einer Wochenstation erhält. Durch das „Rooming-in“ können Trennungsängsten, Schuldgefühlen und nachgeburtlicher Erschöpfung vorgebeugt und somit die Elternkompetenz und -zufriedenheit gestärkt werden. Dadurch, dass auch mehr Frühgeborene ( $>33$. SSW) hier behandelt werden können, kam es seitdem zu einer Entlastung der neonatologisch-pädiatrischen Intensivstation. Die integrative Pflege von Müttern und ihren kranken Neugeborenen oder Frühgeborenen mit ihren speziellen Bedürfnissen ist eine große Herausforderung, die vom gesamten Pflegeteam gut umgesetzt wurde und zu einer erhöhten Mitarbeiterzufriedenheit innerhalb des Pflegeteams führte.

Schlussfolgerung. Durch die Einzigartigkeit dieser neonatologischmaternalen Einheit in Deutschland ist eine Trennung von Mutter und Kind nach der Geburt bei vielen Erkrankungen nicht mehr erforderlich.

\section{Pfl.P009}

Elternberatung in der neonatologischen Intensivversorgung - Evaluation eines Beratungsmodells

\section{Thierfelder ${ }^{1}$}

${ }^{1}$ Charite - Universitätsmedizin Berlin Institut für Gesundheits- und Pflegewissenschaft, Berlin, Deutschland

- siehe Freie Vorträge, Komplementäre Therapie, FV26 -

\section{Pfl.P010}

Ich bin in meinem ganzen Leben noch nie so hilflos gewesen. Die Gefühle und das Erleben von Vätern frühgeborener Kinder während deren stationärer Versorgung auf einer neonatologischen Intensivstation - eine qualitative Untersuchung

\section{J. Binter', T. Garzuly-Rieser}

'SALK - Salzburger Universitätsklinikum, Division für Neonatologie, Hallein, Österreich, ${ }^{2}$ SALK - Salzburger Universitätsklinikum, Division für Neonatologie, Salzburg, Österreich

- siehe Freie Vorträge, Komplementäre Therapie, FV23 - 


\section{Abstracts}

\section{Pfl.P011}

Pflege von Kindern mit komplexen angeborenen Fehlbildungen am Beispiel von Kindern mit intrauterin bekanntem Neuralrohrdefekt.

C. Ditscheid', B. Wiebe', M. Messing-Jünger', M. Ehlen'

${ }^{1}$ Asklepios Klinik St. Augustin, Neonatologie und Pädiatrische Intensivmedizin, St. Augustin, Deutschland, ${ }^{2}$ Asklepios Kinderklinik Sankt Augustin, Abteilung für Kinderneurochirurgie, Sankt Augustin, Deutschland

- siehe Freie Vorträge, Komplementäre Therapie, FV25 -

\section{Pfl.P012}

Szenen der Eltern-Kind-Interaktion in der Versorgungssituation

\section{Baden}

M. Sommerfeld', J. Bonitz', A. Freymann', F. Fischer', J. Reichert', M. Rüdiger

'Universitätsklinikum Carl Gustav Carus Kinderklinik, Neonatologie/ Pädiatrische Intensivmedizin, Dresden, Deutschland

- siehe Freie Vorträge, Komplementäre Therapie, FV27 -

\section{Pfl.P013}

Einarbeitungskonzept Neonatologie B2 Universitätskinderkliniken Bern, Inselspital Bern, $\mathrm{CH}$

\section{Zimmermann', A. Frohloff ${ }^{2}$}

'Inselspital Bern, Stationsleitung Neonatologie B2, Bern, Schweiz, ${ }^{2}$ Inselspital Bern, Neonatolgie B2, Bern, Schweiz

- siehe Freie Vorträge, Intensivpflege, FVo1 - 


\section{Autorenindex}

A

$\begin{array}{ll}\text { Abdul-Khaliq, Hashim } & \text { P072 } \\ \text { Ahmad, Hassan Mohammad } & \text { P102 } \\ \text { Akanbi, Sandra } & \text { P105 } \\ \text { Akduman, Hava } & \text { Pfl.PO07 } \\ \text { Albayrak, Bilge } & \text { P086 } \\ \text { Alejandre Alcazar, Miguel } & \text { FV07 } \\ \text { Ali, Anaam } & \text { FV54 } \\ \text { Ali, Muhammed } & \text { P004 } \\ \text { Allendorf, Antje } & \text { FV51, P079 } \\ \text { Altuntas, Özlem } & \text { FV56, P051 } \\ \text { Alvira, Cristina M. } & \text { FV70 } \\ \text { Amess, Philip N } & \text { P110 } \\ \text { Andrassi-Darida, Monika } & \text { P096 } \\ \text { Ankerne, Janina } & \text { FV06 } \\ \text { Appel, Sarah } & \text { FV06 } \\ \text { Arenz, Stephan } & \text { FV77 } \\ \text { Arenz, Tina } & \text { FV77 } \\ \text { Armbrust, Sven } & \text { P026 } \\ \text { Arndt, Florian } & \text { P075 } \\ \text { Arnoldy, Andrea } & \text { P081 } \\ \text { Asgari. Siamak } & \text { P126 } \\ \text { Avian, Alexander } & \text { FV61 } \\ \text { Axt-Fliedner, Roland } & \text { P093 } \\ \text { Ayers, Susan } & \text { P110 } \\ \text { Aziz, Khalid } & \text { FV63 } \\ \text { Azpeitia, Agueda } & \text { FV66 }\end{array}$

B

$\begin{array}{ll}\text { Baden, Winfried } & \text { FV39 } \\ \text { Bae-Gartz, Inga } & \text { FV07 } \\ \text { Bagci, Soyhan } & \text { FV50, FV56, P051, } \\ & \text { P064, P095 } \\ \text { Bahlmann, Hagen } & \text { P103, P124 } \\ \text { Baik, Nariae } & \text { FV62, FV64, P136 } \\ \text { Bailey, Douggl } & \text { P052 } \\ \text { Bakchoul, Tamam } & \text { P104 } \\ \text { Barikbin, Payman } & \text { P132, P138 } \\ \text { Bartmann, Peter } & \text { FV44, FV50, FV56, } \\ & \text { FV67, P015, P051, } \\ \text { Bauer, Jacqueline } & \text { P095 } \\ \text { Bauer, Jürgen } & \text { P080 } \\ \text { Baumann, Philipp } & \text { P096 } \\ \text { Baumgartner, Sigrid } & \text { FV69 } \\ \text { Baumkötter, Jochen } & \text { FV36, P130 } \\ \text { Beblo, Skadi } & \text { P094 } \\ \text { Beckenbach, Elisabeth } & \text { P055 } \\ \text { Beckené, Stéphanie } & \text { FV61 } \\ \text { Bender, Christian } & \text { FV50 } \\ \text { Bender, Tina } & \text { Pfl.PO06 } \\ \text { Bendix, Ivo } & \text { Pfl.PO06 } \\ \text { Bensch, Jürgen } & \text { FV80, FV83, P121 } \\ \text { Berberich, Lisa } & \text { P001 } \\ & \text { P031 }\end{array}$

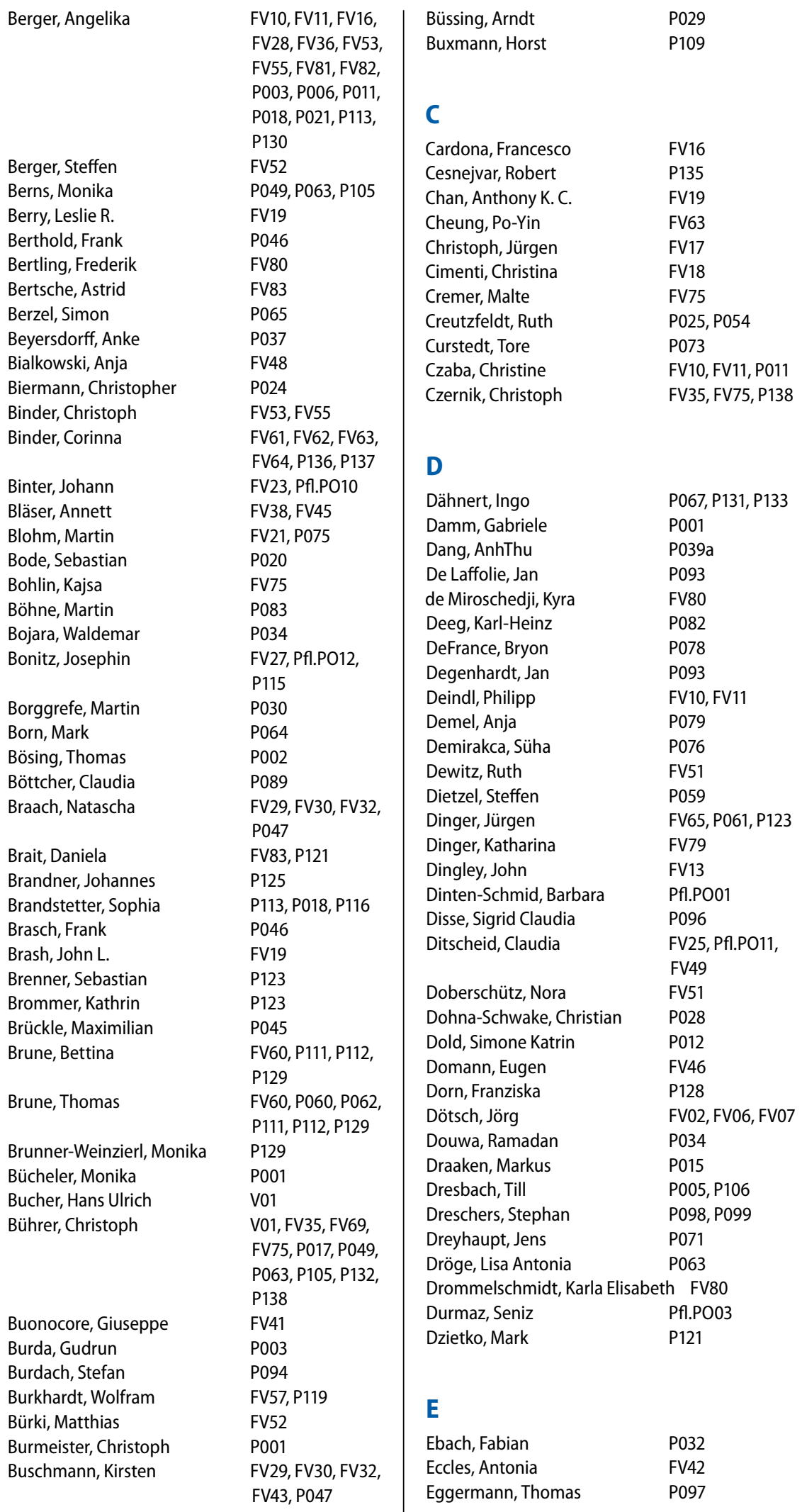




\section{Autorenindex}

\begin{tabular}{|c|c|c|c|c|c|}
\hline \multirow[t]{3}{*}{ Ehlen, Michael } & FV25, Pfl.P011, & Gembruch, Ullrich & P015 & Henschen, Matthias & Pfl.PO06 \\
\hline & FV49, P032, P085, & Genschaft, Marina & P008 & Hentschel, Roland & FV74, P022, P025, \\
\hline & P091 & Gentner, Sarah & FV73 & & P052, P054, P069, \\
\hline \multirow[t]{2}{*}{ Ehrhardt, Harald } & FV70, FV71, FV72, & Gerull, Roland & FV77 & & P108 \\
\hline & P089, P096 & Giebel, Bernd & FV80 & Hepping, Nico & P040, P065, P066, \\
\hline Eichhorn, Joachim G. & $\mathrm{FV} 40$ & Gill, Hannah & FV13 & & P068, P100 \\
\hline Eisert, Albrecht & P039, P042, P043 & Gille, Christian & FV14, FV33, FV34 & Herebian, Diran & FV04 \\
\hline Eis-Hübinger, Anna Maria & FV44, P106 & Gimm, Tina & FV70, FV71 & Hermanspann, Theresa & P039, P042, P043 \\
\hline Elstad, Maja & FV84 & Giordano, Vito & FV10, FV11, FV82, & Hermes, Katharina & P039a \\
\hline Emery, Elisabeth & P110 & & P011, P116 & Herrmann, Nina & FV46 \\
\hline Endress, David & FV55 & Gloßmann, Antje & FV09 & Hertfelder, Hans-Jörg & P064, P065 \\
\hline Engel, Veronika & FV52 & Goeral, Katharina & FV82 & Herting, Egbert & V02, P014, P069, \\
\hline Enke, Christian & P009 & Golde, Dorothea & P127 & & P073 \\
\hline \multirow[t]{3}{*}{ Essig, Andreas } & FV22 & Göpel, Wolfgang & V02, P014, P069 & Herz, Josephine & FV83 \\
\hline & & Göral, Katharina & P003 & Heubner, Georg & $\mathrm{P} 127$ \\
\hline & & Gorbey, Stefan & P128 & Hewson, Michael & FV42 \\
\hline $\mathbf{F}$ & & Gortner, Ludwig & FV59, FV71, FV81, & Heydweiller, Andreas & FV50, P095 \\
\hline Faas, Dirk & P093 & & P069, P072 & Hilgendorff, Anne & FV70, FV71, FV72 \\
\hline Fandrey, Joachim & $\mathrm{P} 121$ & Goswami, Ipsita & FV03 & Hilger, Alina & P015 \\
\hline Fatehpour, Davud & P030, P034 & Grabenhenrich, Jana & P049 & Hinner, Patricia & P114, P115, P122 \\
\hline Fehrholz, Markus & P074 & Grabenhenrich, Linus & P049 & Hócza, Cesryn & P117 \\
\hline \multirow[t]{2}{*}{ Felderhoff-Müser, Ursula } & FV80, FV83, P069, & Gräber, Stefan & P072 & Hoenecke, Johannes & P135 \\
\hline & $\mathrm{P} 101, \mathrm{P} 121$ & Gräfe, Florentine & P133 & Hoffmann, Georg F. & P057 \\
\hline Fischer, Doris & P013 & Grass, Beate & FV69 & Hofstädter, Kathrin & FV33 \\
\hline Fischer, Franziska & FV27, Pfl.P012 & Griese, Matthias & P066 & Hofstätter, Edda & P125 \\
\hline Fischer, Georg & P130 & Groneck, Peter & P019 & Hofstetter, Peter & P109 \\
\hline Fischer, Hans-Peter & P056 & Großmann, Jasmin & P084 & Höhn, Thomas & FV04, FV37 \\
\hline Fischer, Hendrik & FV75 & Grylli, Chryssa & P021 & Höland, Anita & FV71 \\
\hline Flatebø, Torun & FV84 & Guthmann, Florian & FV17 & Holl, Ulla & P026 \\
\hline Fouzas, Sotirios & FV69 & & & Holotiuk, Olaf & $\mathrm{P} 127$ \\
\hline Frank, Christina & P135 & & & Hopfner, Reinhard & FV09 \\
\hline \multirow[t]{2}{*}{ Franz, Axel } & FV39, FV48, FV50, & $\mathbf{H}$ & & Hoppen, Thomas & P001, P030, P033, \\
\hline & P087 & Haberl, Hannes & P017 & & P034, P076 \\
\hline Freidl, Thomas & P136 & Haftel, Lior & P126 & Horn, Peter & FV80 \\
\hline Fremerey, Christian & P091 & Hagenah, H.-P. & P118, P120 & Hossain, Hamid & FV71 \\
\hline Freymann, Annett & FV27, Pfl.PO12 & Hagmann, Cornelia & V01 & Huang, Li & P102 \\
\hline Friedrich, Katrin & P127 & Haiden, Nadja & FV16, FV28, FV53, & Hudalla, Hannes & FV29, FV30, FV43 \\
\hline Fritsche, Sabine & Pfl.PO02 & & FV55, P018 & Hummler, Helmut & FV09, FV22, FV66, \\
\hline Fröhlich, Susanne & FV04 & Hallauer, Johannes & P026 & & FV73, P014, P071, \\
\hline Fröhlke, Lena & P044 & Haller, Ulrike & P048 & & P102 \\
\hline Frohloff, Anke & FV01, Pfl.PO13 & Halstenberg, Katrin & P079 & Hünseler, Christoph & FV08, FV12, P045 \\
\hline \multirow[t]{2}{*}{ Frommhold, David } & FV29, FV30, FV32, & Hamelmann, Eckard & $\mathrm{P} 002, \mathrm{P} 048$ & Hürtgen, $M$. & P033 \\
\hline & FV43, P047 & Hammer, Stefanie & P087 & Huth, Ralf G. & P034 \\
\hline Fruth, Anja & P092 & Hamprecht, Klaus & P109 & Hütten, Matthias & FV41 \\
\hline \multirow[t]{3}{*}{ Fuchs, Hans } & FV09, FV74, P020, & Handwerk, Marion & FV06 & Huttner, Kenneth & P039a \\
\hline & P022, P052, P071, & Hanke, Christof A & P102 & & \\
\hline & P108 & Hanke, Kathrin & V02 & & \\
\hline \multirow[t]{2}{*}{ Fuiko, Renate } & FV11, P018, P113, & Hansmann, Andreas & P056 & I & \\
\hline & P116 & Härtel, Christoph & V02, P014, P069 & Ifflaender, Sascha & FV57, FV65, P119 \\
\hline \multirow[t]{2}{*}{ Fusch, Christoph } & FV03, FV19, FV20, & Hartung, Julia Christine & FV78 & Isermann, Berend & FV30, FV32 \\
\hline & FV54, FV58, P078 & Hartz, Annika & V02 & Ittermann, Till & P044 \\
\hline \multirow[t]{4}{*}{ Fusch, Gerhard } & FV03, FV20, FV54, & Hassalll, Oliver & P062 & & \\
\hline & FV58, P078 & Hattesohl, Akira & FV46 & & \\
\hline & & Hausser, Ingrid & P091 & J & \\
\hline & & Hayde, Michael & P107, P116 & Jack, Thomas & P083 \\
\hline G & & Hecher, Kurt & P075 & Jahn, Peter & FV40, P019 \\
\hline \multirow[t]{2}{*}{ Garbe, Werner } & P040, P065, P066, & Heckmann, Matthias & P037, P044, P097, & Jain, Noopur & FV72 \\
\hline & P068, P100 & & P103, P104, P124 & Jakubiczka, Sibylle & P129 \\
\hline Garnys, Lukas & P003 & Heep, Axel & FV05 & Janoschek, Ruth & FV06, FV07 \\
\hline Garritsen, HSP & P060 & Heimann, Konrad & P016, P035 & Jobe, Alan $\mathrm{H}$. & FV41 \\
\hline Garten, Lars & P105 & Heitmann, Sebastian & P050 & Joiko, Cornelia & P081 \\
\hline Garzuly-Rieser, Teresa & FV23, Pfl.PO10 & Helfer, Sven & FV35 & Jorch, Gerhard & P060, P062, P111, \\
\hline Gebauer, Corinna & FV38, FV45, P055 & Helling, Astrid & FV40 & & P112 \\
\hline Gehrisch, Siegmund & P061 & Henjes, Sonja & P055 & Jungbluth, Heinz & P101 \\
\hline Gehrlein, Britta & P013 & Henneke, Philipp & P022, P025 & & \\
\hline \multirow{2}{*}{$\begin{array}{l}\text { Genrieln, Britta } \\
\text { Geipel, A }\end{array}$} & P056 & Hennig, Judith & P114 & & \\
\hline & & Henrich, Wolfgang & P063 & & \\
\hline
\end{tabular}




\begin{tabular}{|c|c|c|c|c|c|}
\hline \multicolumn{2}{|l|}{$\mathbf{K}$} & \multicolumn{2}{|l|}{$\mathbf{L}$} & Messing-Jünger, Martina & \multirow{2}{*}{$\begin{array}{l}\text { FV49, FV25, Pfl. } \\
\text { P011 }\end{array}$} \\
\hline \multicolumn{2}{|l|}{ aiser, Jan } & \multicolumn{2}{|l|}{ L } & & \\
\hline Kallapur, Suhas & FV41 & Lange, Anja & P044 & $\begin{array}{l}\text { Metzger, Marie-Sophie } \\
\text { Meyer, Ansqar }\end{array}$ & $\begin{array}{l}F V 29 \\
\text { P019 }\end{array}$ \\
\hline Kappler, Gregor & FV11 & Lange, Mandy & FV76 & & $\begin{array}{l}\text { P019 } \\
\text { P020, P072 }\end{array}$ \\
\hline Karen, Tanja & Pfl.PO08, P031 & Langer, Laura & FV37 & $\begin{array}{l}\text { Meyer, Sascha } \\
\text { Mian, Firoz }\end{array}$ & $\begin{array}{l}\text { P020, PO/2 } \\
\text { P078 }\end{array}$ \\
\hline Karg, Nadine & P132 & Langgartner, Michaela & FV16, FV36, P021 & Michael, Bianca & P067 \\
\hline Kasper, David & P107 & Langhammer, Kristina & FV12 & $\begin{array}{l}\text { MIIChael, Blanca } \\
\text { Miedaner, Felix }\end{array}$ & $\begin{array}{l}\text { P06/ } \\
\text { P079 }\end{array}$ \\
\hline Kattner, Evelyn & FV17 & Langner, Sönke & P124 & $\begin{array}{l}\text { Miedaner, Felix } \\
\text { Mildenberger. Eva }\end{array}$ & $\begin{array}{l}\text { P0/9 } \\
\text { P092 }\end{array}$ \\
\hline Katzer, David & FV56, P051 & Lassay, Lisa & P035 & $\begin{array}{l}\text { Milldenberger, Eva } \\
\text { Minnich, Bernd }\end{array}$ & $\begin{array}{l}\text { PU92 } \\
\text { P125 }\end{array}$ \\
\hline Kauppinen, Risto A & FV05 & Laugwitz, Lucia & P105 & $\begin{array}{l}\text { Mınnıch, Bernd } \\
\text { Misselwitz, Björn }\end{array}$ & $\begin{array}{l}\mathrm{P} 125 \\
\mathrm{P} 084\end{array}$ \\
\hline Kaussen, Torsten & FV47, P083 & Lausch, Ekkehart & P020 & $\begin{array}{l}\text { MlsselWItZ, BJorn } \\
\text { Mitra, Souvik }\end{array}$ & $\begin{array}{l}\text { FV58 } \\
\text { FV }\end{array}$ \\
\hline Keller, Matthias & FV80 & Laux, Reinhard & FV08 & Mitzlaff, Bernd & P083 \\
\hline Keller, Titus & FV68. FV79 & Lehnhardt, Anja & FV21 & Moerth, Susanne & P018 \\
\hline Kemp, Matthew W & FV41 & Leiber, Anja & FV34 & Mögel, Michael & P007 \\
\hline Kerth, Janna-Lina & P099 & Lemmer, Björn & P128 & Möhr, Winfried & $\begin{array}{l}\text { P00/ } \\
\text { P127 }\end{array}$ \\
\hline Kidszun, André & P092 & Leung, Jennifer M. & FV19 & $\begin{array}{l}\text { Mionr, Vintried } \\
\text { Mollenhauer, Jan }\end{array}$ & $\begin{array}{l}\text { PIII } \\
\text { FV72a }\end{array}$ \\
\hline Kiefer, Friedemann & P059 & Liesa, Weiler & P116 & Möller Jens & $\begin{array}{l}\text { FV72a } \\
P 001\end{array}$ \\
\hline Kietz, Silke & P103 & Liese, Jan & FV14 & Jens & $\begin{array}{l}\text { P001 } \\
\text { P003 }\end{array}$ \\
\hline Kindorff, Ulrich & P001 & Lindinger, Franziska & P011 & Mö & P003 \\
\hline Kipfmueller, Florian & FV44, FV67 & Lindner, Martin & P057 & Monkman, Shelley & $\begin{array}{l}\text { FV20 } \\
\end{array}$ \\
\hline Kipp, Frank & P023, P027 & Lindner, Wolfgang & FV22 & Müller, Andreas & FV44, FV50, FV56, \\
\hline Kirchberger, Valerie & P079 & Linduska, Nina & P006 & & FV67, P005, P051, \\
\hline Kirschner, Hans-Joachim & P087 & Linke, Rüdiger & P129 & & P056, P064, P095, \\
\hline Kirschner, Janbernd & P020 & Linnemann, Knud & P104 & & P106, Pfl.PO07 \\
\hline \multirow{3}{*}{ Klebermaß-Schrehof, Katrin } & FV36, FV82, P003, & Lippach, Gregor & FV02 & Müller, Annette M. & P095 \\
\hline & P011, P018, P113, & Lippke, Samuel & P077 & Müller, Carsten & P076 \\
\hline & P116 & Lippmann, Norman & FV45 & Müller, Fabian & P126 \\
\hline Klee, Andreas & P080 & Liu, Xun & FV13 & Müller, Götz & P075 \\
\hline Klippstein, Tamara & P132 & Lode, Hans-Martin & P082 & Müller, Hanna & FV72a \\
\hline Klotz, Daniel & FV74 & Lode, Holger & P103 & Müller-Helm, Alexandra & Pfl.PO04 \\
\hline Knuf, Markus & P080 & Löffelbein, Florian & P067, P131 & Muth, Lajos & P031 \\
\hline Knüpfer, Matthias & FV38, FV45, P055 & Lohse, Peter & P066 & Mütze, Jens & P019 \\
\hline Koch, Arite & P007, P123 & Long, Frederic & FV40 & & \\
\hline Koch, Lutz & FV29, FV30 & Lorenz, Birgit & P096 & $\mathbf{N}$ & \\
\hline Koczulla, Andreas Rembert & FV46 & Lorenz, Norbert & P127 & $\mathbf{N}$ & \\
\hline Köditz, Harald & P076, P083 & Loukanov, Tsvetomir & FV40 & Nagel, Christian & FV72a \\
\hline Koehne, Petra & P132 & Lücke, Thomas & P048 & Nähter, Diana & P111 \\
\hline Kohl, Eva Maria & P039, P042 & Lückemann, Laura & FV83 & Nariae, Baik & P137 \\
\hline Koller, Brigitte & V01 & Ludwig, Ann-Kathrin & FV80 & Nawroth, Peter & FV30 \\
\hline Konstantelos, Dimitrios & FV65, P007 & Ludwig, Michael & P015 & Nelle, Mathias & FV77 \\
\hline Kordaß, Ulrike & P097 & Luyt, Karen & FV05 & Newnham, John P. & FV41 \\
\hline Korsch, Eckhard & FV08 & & & Niemann, Nadja & P001 \\
\hline Korten, Insa & P134 & & & Niemarkt, Hendrik & FV68 \\
\hline Koschlig, Markus & FV70, FV72 & M & & Niesse, Oliver & FV69 \\
\hline Kossert, Melina & FV70 & Maas Chrictonh & & Niewisch, Marena Rebekka & P054, P108 \\
\hline Köstlin, Natascha & FV33,FV34 & IVdas, Cnilstopn & $808 /$ & Nijhof, Sarah & P041 \\
\hline Kowol, Sarah & Pfl.PO02 & Macchiella, Doris & P092 & Nilles, K. Louise & P030 \\
\hline Kramer, Boris W. & FV41, FV68 & Madi, Marwan & P037 & Nitsch- Felsecker, Patrizia & P036 \\
\hline Krebs, Lena & FV04 & Maes, Elke & FV84 & Nobel, Hannes & P089 \\
\hline Kreissl, Alexandra & FV28, FV53, FV55 & Maier, Barbara & P022 & Nordwig, Antje & P127 \\
\hline Kreiß-Nachtsheim, Martina & P091 & Maier, Rolf Felix & FV46, P12 & Nöthen, Markus M. & P015 \\
\hline Kremer, Ludmilla & P059 & Malchau, Gebhart & FV68 & Nunold, Holger & P072 \\
\hline Kreth, Ulrich & P002 & Manser, Helen & FV77 & Nüsken, Eva & FV02 \\
\hline \multirow[t]{3}{*}{ Kribs, Angela } & FV12, FV68, FV79, & $\begin{array}{l}\text { Margraf, Andreas } \\
\text { Marschal Matthias }\end{array}$ & $\begin{array}{l}\text { P059 } \\
\text { FV14 }\end{array}$ & Nüsken, Kai-Dietrich & FV02 \\
\hline & P009, P024, P045, & Marschitz Ingrid & FV18 P038 P058 & Nußbaum, Claudia & P059 \\
\hline & P069 & Matysiak-Scholze, Uta & $\begin{array}{l}\text { P020 } \\
\text { P020, }\end{array}$ & Nüßlein, Thomas & P030, P033, P034, \\
\hline Krieg, Peter & P039a & Mayatepek, Ertan & FV37 & & P076 \\
\hline Kropf, Siegfried & P111 & Mehler, Katrin & FV68, FV79 & & \\
\hline Krüger, Carsten & P004 & Mein, Rachael & P101 & & \\
\hline Krutzke, Sophia Karoline & P015 & Mende, Christina & P014 & 0 & \\
\hline Kuntz, Ludwig & P079 & Mendler, Marc & P102 & O'Reilly, M'egan & FV63 \\
\hline Künzl, Kira & FV44 & Mendler, Marc Robin & P071 & Oak, Prajakta & FV72 \\
\hline Kunzmann, Steffen & P074 & Menke, Thomas & P041 & Oberthür, Andre & FV68, FV79, P046 \\
\hline Kuß, Navina & FV29 & Mense, Lars & P061 & Obwegeser, Eva & P011 \\
\hline Küster, Helmut & FV15, FV77, P010 & Merz. Waltraut & P015 & Oechsle, Oliver & P060, P062 \\
\hline Kutz, Patrizia & P041, P050, P088 & & & Oh, Jun & FV21 \\
\hline
\end{tabular}




\section{Ohl, Kim}

Olbertz, Dirk Manfred

Olischar, Monika

Orlikowsky, Thorsten W.

Orth, Inka

Osredkar, Damjan

Ott, Torsten

P

Pagel, Friederike

Paul, N.W.

Paulun, Annika

Pecks, Ulrich

Pflaum, Johanna

Pichler, Gerhard

Pick, Robert

Pielemeier, Wolfgang

Pillekamp, Frank

Pimpel, Birgit

Pocivalnik, Mirjam

Poets, Christian

Pogodda, Sven

Pohl-Schickinger, Anja

Pollak, Arnold

Polley, Oliver

Poralla, Silvia

Pöschl, Johannes

Prager, Sebastian

Pramana, Isabelle

Predescu, Dragos

Preusche, Antonio

Preuß, Michael

Prothmann, Anke

Prusa, Andrea-Romana

Pulzer, Ferdinand

Q

Quackenbush, Elizabeth

\section{R}

Rabe, Heike

Rabenau, Holger

Rabsilber, Angelika

Radauer, Wolfgang

Rascher, Wolfgang

Rathgeber, Sandra

Reichert, Jörg

Reicherzer, Tobias

Reinboth, Barbara Simone

Reinfeld, Andreas H.

Reinprecht, Andrea

Reinsberg, Jochen

Repa, Andreas

Reus, Erik
P098

P118, P120

FV10, FV11, FV82,

P011, P130

FV41, P016, P035,

$\mathrm{P} 039, \mathrm{P} 042, \mathrm{P} 043$,

P098, P099

Pfl.PO07

FV84

P070

P069

P092

P085

FV41

FV30

FV61, FV62, FV63,

FV64, P136, P137

FV32

P050, P088

FV37

FV28

FV61

FV14, FV33, FV34,

FV39, FV48, P087

P026

P017, P138

FV10, FV81, P107

P138

P005

FV29, FV30, FV32,

FV43, FV72a,

$\mathrm{P} 047$

FV80

FV69

FV20

FV39

P069

P094

P107

FV38, FV45, P055

P059

P110
P109
P060
P058
P135
Pfl.PO06
FV27, Pfl.PO12,
P114, P115, P117,
P122
FV71, FV72
FV83
P076
P011
FV56, P051
FV53, FV55
FV59

Reuter, Christina $\quad$ FV15

Reutter, Heiko

Rhode, Stefanie

Riede, Frank-Thomas

Rieger-Fackeldey, Esther

Ring, Stefan

Robel-Tillig, Eva

Rochow, Niels

Rödl, Siegfried

Roehr, Charles Christoph

Rogosch, Tobias

Röhr, Sebastian

Rohwedder, Ina

Roll, Claudia

Rolle, Udo

Römer, Susanne

Rompel, Oliver

Rose, Elmar

Roth, Bernhard

Rother, Eva

Rüdiger, Mario

Rüegger, Christoph

Ryschich, Eduard

\section{$S$}

Sabir, Hemmen FV13, FV84

Säger, Eva

Sallmon, Hannes

Salzer-Muhar, Ulrike

Sandig, Jan

Sasse, Michael

Sauer, Harald

Sawyers, Alexandra

Schachtrupp, Alexander

Schara, Ulrike

Schenk, Jens-Peter

Schimpf, Rainer

Schlößer, Rolf

Schmalisch, Gerd

Schmelter, Viola

Schmid, Manuel

Schmidt, Florian

Schmidt, Martin

Schmidt, Sarah

Schmidt, Stefan

Schmidtke, Susanne

Schmitt, Joachim

Schmitt-Kästner, Sophie

Schmitz, Kirstin

Schmitz, Lothar

Schmölzer, Georg

Schneider, Anna

Schneider, Holm

Schneider, K.T.M.
P015

FV35

$\mathrm{P} 131, \mathrm{P} 133$

$\mathrm{P} 023, \mathrm{P} 027, \mathrm{P} 070$

FV18, P038

P008, P134

FV03, FV19, FV20,

FV54, FV58, P078,

$\mathrm{P} 118, \mathrm{P} 120$

FV18, P038, P058

FV78

FV46

P072

P059

P041, P050, P086,

P088

FV51, P084

$\mathrm{P} 063$

P135

P016

FV08, FV12, FV68,

FV79, P024, P036,

P045, P046, P079

FV06, FV07, FV68

FV27, Pfl.PO12,

FV57, FV65, P007,

P061, P069, P114,

P115, P117, P119,

$\mathrm{P} 122, \mathrm{P} 123$

V01

FV32

Pfl.PO06

P132, P138

FV36, P130

P075

FV47, P076, P083

P072

P110

P083

P101

FV40

P030

FV51, P013, P084,

P109

FV35, FV75, FV78

FV04

FV09, P014, P071,

$\mathrm{P} 102$

P083

P124

P059

P127

FV76

P040, P068, P106

$\mathrm{P} 034$

Pfl.PO05

FV35

FV63

P125

P039a

$\mathrm{P} 118, \mathrm{P} 120$

Schneider, Katja P040, P065, P066,

P068, P100, P106

$\mathrm{P} 039 \mathrm{a}$

$\mathrm{P} 016, \mathrm{P} 035, \mathrm{P} 039$,

$\mathrm{P} 042, \mathrm{P} 043$

FV71

P118, P120

P095

P097

FV08

$\begin{array}{ll}\text { Schüller, Simone } & \text { FV81 } \\ \text { Schulz, Matthias } & \text { P017 }\end{array}$

$\begin{array}{ll}\text { Schüller, Simone } & \text { FV81 } \\ \text { Schulz, Matthias } & \text { P017 }\end{array}$

Schumann, Madita Magdalena P015

Schwaberger, Bernhard FV61, FV62, FV63,

FV64, P136, P137

Schwarz, Christoph E. $\quad$ FV39

Schwarz, Julian FV34

Schwerk, Nicolaus $\quad$ P076

Schwindt, Jens $\quad$ FV16, P003

Seewald, Markus $\quad$ FV60

Seidel, Vera $\quad$ FV76

Selvaganapathy, Ravi $\quad$ FV19

Serdar, Meray $\quad$ FV80, P121

Sieg, Franz

Singer, Dominique

FV38

FV08, FV21, FV76,

$\mathrm{P} 053, \mathrm{P} 075$

Skopnik, Heino

Smith-Collins, Adam PR

P001, P077, P081

FV05

FV27, Pfl.PO12

Sommerfeld, Mario

FV69

Speer, Christian P.

Sperandio, Markus

P074

P059

Sperling, Gisela

FV17

P067

FV81

Spittler, Andreas

Spoden, Claudia

Spring, Bärbel

Staatz, Gundula

Pfl.PO03

FV33, FV34

P092

FV30

FV74, P022

Stavropoulou, Dimitra

P101

P083

Pfl.PO08

FV16, FV36, P130

$\mathrm{P} 083$

P073

Pfl.PO01

FV83, P121

P094

Pfl.PO08

P088

P050

FV36

T

Tantcheva-Poor, lliana $\quad$ P024

Taylan, Christina P036

te Pas, Arjan FV78

Teig, Norbert

Tekaat, Maria

Thanhäuser, Margarita

$\mathrm{P} 002, \mathrm{P} 048$

P070

FV53, FV55 


\begin{tabular}{|c|c|c|c|}
\hline Thome, Ulrich & $\begin{array}{l}\text { FV38, FV45, FV73, } \\
\text { P055 }\end{array}$ & $\begin{array}{l}\text { Weitkämper, Almut } \\
\text { Welcker, Silvia }\end{array}$ & $\begin{array}{l}\text { P048 } \\
\text { P089, P104 }\end{array}$ \\
\hline Thoresen, Marianne & FV13, FV84 & Wellmann, Sven & V01, FV69 \\
\hline Thyrian, Rene & P044 & Weninger, Manfred & FV10, FV82, P011 \\
\hline Timmesfeld, Nina & P128 & Wenzlaff, Paul & P001 \\
\hline Topf, Hans-Georg & P135 & Werther, Tobias & FV10, FV11 \\
\hline Treichel, Markus & FV40 & Whybra-Truempler, Catherine & P092 \\
\hline Trepels-Kottek, Sonja & P016, P035 & Wiebe, Beatrix & FV25, Pfl.PO11, \\
\hline Treszl, Andras & FV08 & & FV49 \\
\hline Tröbs, Ralf-Bodo & P086, P088 & Wiebe, Walter & P085 \\
\hline Tschada, Raphaela & FV29 & Wieg, Christian & P031, Pfl.P008 \\
\hline Turial, Salmai & P080 & Wiegand, Susanne & P048 \\
\hline Tutdibi, Erol & FV59 & Wien, Sebastian & P033 \\
\hline & & Wilden, Elisabeth & P002 \\
\hline & & Wilken, Meike & FV21 \\
\hline $\mathbf{U}$ & & Willers, Sabine & P053 \\
\hline Uhlig, Ulrike & FV73 & Willruth, Arne & FV56, P051 \\
\hline Ulreich, Raphael & P038, P058 & Winking, Torben Christian & P023, P027 \\
\hline Ulrike, Leiss & P116 & Winkler, Annegret & P103 \\
\hline Unrath, Manja & FV57, P119 & Winter, Julia & P092 \\
\hline Unterasinger, Lukas & FV11 & Wirth, Lara & P128 \\
\hline Urlesberger, Berndt & FV61, FV62, FV63, & Wisgrill, Lukas & FV81 \\
\hline & FV64, P136, P137 & Wittekindt, Boris & P084, P109 \\
\hline Utzig, Norbert & P124 & Wittig, Kathrin & P100 \\
\hline & & Wohlfarth, Maria & FV02 \\
\hline & & Wolf, Alfons & P082 \\
\hline $\mathbf{V}$ & & Wolf, Martin & FV39 \\
\hline Valls-i-Soler, Adolf & & Wolff-Teßmann, Heidrun & Pfl.PO02 \\
\hline Vasanthan, Tarushika & $\begin{array}{l}\text { rVbo } \\
8070\end{array}$ & Wolfram, Juliane & P097 \\
\hline Verde, Pablo & FU/8 & Wolfs, Tim G.A.M. & FV41 \\
\hline Verjans, Eva & 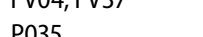 & Wollny, Caroline & P079 \\
\hline Verlohren, Stefan & $\begin{aligned} 5063 \\
6030\end{aligned}$ & Woopen, Christiane & P009 \\
\hline Vezyroglou, Katharina & $\begin{array}{ll}\text { P063 } \\
\text { FY12 }\end{array}$ & Wördehoff, Ursula & P064 \\
\hline Vierzig, Anne & FVIL & Wrobel, Marc & FV59 \\
\hline Vohlen, Christina & $\begin{array}{l}\text { Fy } \\
\text { Fy }\end{array}$ & Wudy, Stefan A. & P089 \\
\hline Voigt, Manfred & P118, P120 & Wüllenweber, Jörg & P023, P027 \\
\hline Volk, Thomas & FV59 & Wunderlich, Saskia & P080 \\
\hline Völkl, Thomas M.K. & P135 & & \\
\hline Volkmann, Julia & P131, P133 & $\mathbf{Y}$ & \\
\hline von Andrian, Ulrich & P059 & & \\
\hline von Baum, Heike & FV22 & Yarkin, Yasemin & P087 \\
\hline von Buch, Christoph & P001 & & \\
\hline von der Wense, Axel & FV08 & & \\
\hline von der Wense, Ursula & Pfl.PO05 & $\mathbf{L}$ & \\
\hline & & Zemlin, Michael & FV46, P128 \\
\hline & & Zias, Anastasios & P093 \\
\hline $\mathbf{W}$ & & Ziegler, Andreas & P069 \\
\hline Wagner, Michael & P003 & Ziehenberger, Evelyn & FV61, P137 \\
\hline Wagner, Robert & P131 & Zimmer, Klaus-Peter & P089, P093, P096 \\
\hline Waitz, Markus & P071, P102 & Zimmermann, Claudia & FV01, Pfl.P013, \\
\hline Wald, Martin & $\mathrm{P} 125, \mathrm{P} 130$ & & Pfl.PO01 \\
\hline Waldecker, Karin & P020 & Zobel, Gerfried & FV18, P058 \\
\hline Waldhör, Thomas & FV11, FV36 & Zwiauer, Valentina & FV53 \\
\hline Walter, Evelyn & P107 & & \\
\hline Walzog, Barbara & FV32 & & \\
\hline Wang, Hongjie & FV30, FV32 & & \\
\hline Warnecke, Gregor & P076 & & \\
\hline Weber, Axel & P096 & & \\
\hline Weber, Michael & FV55 & & \\
\hline Wege, Mirjam & P128 & & \\
\hline Weil, Jochen & P075 & & \\
\hline Weinhandl, Anja & P107 & & \\
\hline Weinzheimer, Hermann R. & P076 & & \\
\hline Weise, Christian & P060 & & \\
\hline Weiß, Christel & FV72a & & \\
\hline Weisser, Christof & P125 & & \\
\hline
\end{tabular}

